\title{
Global Millimeter VLBI Array Survey of Ultracompact Extragalactic Radio Sources at $86 \mathrm{GHz}$
}

\author{
INAUGURAL-DISSERTATION \\ zur \\ Erlangung des Doktorgrades \\ der Mathematisch-Naturwissenschaftlichen Fakultät \\ der Universität zu Köln
}

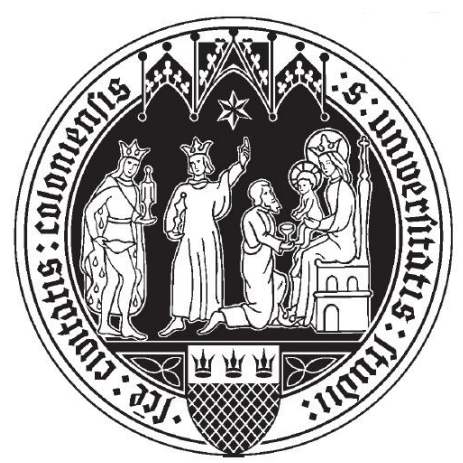

vorgelegt von

Dhanya G. Nair

aus Alleppey, India

Köln, 2019 
Berichterstatter:

Prof. Dr. Andreas Eckart

Prof. Dr. J. Anton Zensus

Tag der letzten mündlichen Prüfung: 08.03.2018 
To my dearest Father and Mother 


\section{ABSTRACT}

Ultracompact extragalactic radio sources, representing the inner parsec and sub-parsec scales of Active Galactic Nuclei (AGN), form the best laboratory to test extreme physics and understand our universe. Studies of ultracompact extragalactic radio sources have revealed the morphology and kinematics of AGN from sub-parsec to Mega-parsec scales, the powerful energy emission ranging from radio to $\gamma$-rays, the galaxy evolution, the important role of accretion disk and supermassive black hole in the formation, acceleration and collimation of relativistic jets and many other interesting facts about AGNs. A substantial fraction of these studies has been enabled by the advent and continued developments of the technique of very long baseline interferometry (VLBI) which combines radio telescopes across the entire Earth into a single instrument. VLBI observations at $86 \mathrm{GHz}$ (wavelength, $\lambda=$ $3 \mathrm{~mm}$ ) reach a resolution of about $50 \mu$ as, and can probe the collimation and acceleration regions of relativistic outflows in the vicinity of supermassive black holes in AGN. In this context, a high resolution $86 \mathrm{GHz}$ VLBI survey of a representative sample of ultracompact extragalactic radio sources provides the best statistical tool, to investigate the underlying physics of AGN and their relativistic jets.

This dissertation presents results from a large global VLBI survey of compact radio sources carried out in 2010-2011 with the Global Millimeter VLBI Array. The main goals of the survey are to extend VLBI imaging surveys at $86 \mathrm{GHz}$ to fainter sources, to develop a comprehensive sample of ultracompact radio sources imaged at $86 \mathrm{GHz}$, and to study the morphology and the distribution of brightness temperatures. The survey data reaches a typical baseline sensitivity of $0.1 \mathrm{Jy}$ and a typical image sensitivity of $5 \mathrm{mJy} /$ beam. A total of 162 unique compact radio sources have been observed in this survey. All the sources are detected and imaged, signifying a nearly twofold increase in the total number of sources ever imaged with VLBI at $86 \mathrm{GHz} .138$ sources are imaged for the first time with VLBI at $86 \mathrm{GHz}$ through this survey.

Gaussian model fitting of the visibility data is applied to represent the structure of the observed sources and to estimate the flux densities and sizes of distinct emitting regions (components) in their jets. These estimates are used to calculate the brightness temperature $\left(T_{\mathrm{b}}\right)$ at the jet base (core) and in the next closest regions (jet components) downstream of the core. These model fit based estimates of $T_{\mathrm{b}}$ are compared to the estimates of brightness temperature limits made directly from the visibility data, demonstrating a good agreement between the two methods. The apparent brightness temperature estimates for the jet cores in our sample ranges from $1.1 \times 10^{9} \mathrm{~K}$ to $5.5 \times 10^{12} \mathrm{~K}$, with the mean brightness temperature of $1.8 \times 10^{11} \mathrm{~K}$. The apparent brightness temperature estimates for the nearest jet components in our sample ranges from $5.8 \times 10^{7} \mathrm{~K}$ to $4.1 \times 10^{11} \mathrm{~K}$. A population model with a single intrinsic value of brightness temperature, $T_{0}$, is applied to reproduce the 
observed distribution. It yields $T_{0}=\left(3.77_{-0.14}^{+0.10}\right) \times 10^{11} \mathrm{~K}$ for the jet cores, implying that the inverse Compton losses dominate the emission. In the nearest jet components, $T_{0}=$ $\left(1.42_{-0.19}^{+0.16}\right) \times 10^{11} \mathrm{~K}$ is found, which is slightly higher than the equipartition limit of $\sim$ $5 \times 10^{10} \mathrm{~K}$ expected for these jet regions. A correlation between $T_{0}$ and Lorentz factor, $\gamma_{\mathrm{j}}$ inherent to the population model description precludes fitting for $T_{0}$ and $\gamma_{\mathrm{j}}$ simultaneously. We find that a relation $T_{0}[\mathrm{~K}] \approx\left(7.7 \times 10^{8}\right) \gamma_{\mathrm{j}}^{2.7}$ is implied for this modelling framework by the survey data. The approach used to derive the intrinsic brightness temperature at $86 \mathrm{GHz}$ through population modelling is applied to the database of VLBI survey at lower frequencies $(2,8,15 \mathrm{GHz})$. This enables us to investigate the multifrequency aspects of $T_{\mathrm{b}}$. The intrinsic brightness temperature obtained for cores at $86 \mathrm{GHz}$ is found to be lower than that at $2 \mathrm{GHz}, 8 \mathrm{GHz}$ and $15 \mathrm{GHz}$. Such a decrease of $T_{0}$ will provide a hint in favor of the decelerating jet model or particle-cascade model. A decreasing $T_{0}$ would also have consequences for higher frequency VLBI. This has to be further investigated by compiling the jet speeds, $\beta_{\text {app }}$ with observed $T_{\mathrm{b}}$.

The intrisic $T_{0}$ and the observed $T_{\mathrm{b}}$ in core and jets show that the brightness temperature drops by approximately a factor of $\sim(2-10)$ already on sub-parsec scales in the jets. This evolution might occur with the inverse Compton, synchrotron, and adiabatic losses subsequently dominating the energy losses. A basic evolutionary scheme in which the changes observed in the jet emission are caused by the adiabatic energy losses in relativistic shocks is investigated. For objects with sufficient structural detail detected, the adiabatic energy losses are shown to dominate the observed variations of brightness temperature along the jet implying that the jet components can be viewed as adiabatically expanding relativistic shocks. Under the equipartition condition between the magnetic field energy and particle energy density, the absolute distance of the VLBI core from the central engine can be estimated. The brightness temperature measurements obtained from this survey at $86 \mathrm{GHz}$ are combined with the database of VLBI survey at lower frequencies $(2,8,15 \mathrm{GHz})$ to study the brightness temperatures in source frame in the sub-parsec scale of the ultracompact radio sources. From the vicinity of the central engine, the brightness temperature increases slowly and then rises with a steeper slope and then slow down. This gives a strong implication that the jets are accelerated and collimated by the magnetically driven force, as predicted by the relativistic inner jet models for the magnetic acceleration. 


\section{ZUSAMMENFASSUNG}

Ultrakompakte, extragalaktische Radioquellen, die die inneren Parsec und sub-parsec Regionen von Aktiven Galaktischen Kernen (AGN) darstellen, sind die beste Forschungsmöglichkeit um Physik unter extremen Bedingungen zu testen und um unser Universum $\mathrm{zu}$ verstehen. Untersuchungen von ultrakompakten, extragalaktischen Radioquellen haben die Morphologie und Kinematik von AGN von sub-parsec bis Mega-parsec Längenskalen, die gewaltige Energieabstrahlung die vom Radiobereich bis zur Gammastrahlung reicht, die Galaxieentwicklung, die wichtige Rolle von Akkretionsscheibe und supermassiven schwarzen Löchern bei der Bildung, Beschleunigung und Kollimierung von relativistischen Jets, sowie viele weitere interessante Fakten über AGN gezeigt. Ein wesentlicher Anteil dieser Studien wurde durch das Aufkommen und die kontinuierlichen Weiterentwicklungen der Technik der Very-Long-Baseline-Interferometrie (VLBI) ermöglicht, bei dieser werden Radioteleskope auf der ganzen Erde zu einem einzigen Instrument kombiniert. VLBI-Beobachtung bei $86 \mathrm{GHz}$ (Wellenlänge $\lambda=3 \mathrm{~mm}$ ) erreichen eine Aufösung von etwa $50 \mu$ as und ermöglichen eine Untersuchung der Kollimierungs- und Beschleunigungsregionen von relativistischen Ausströmungen in der nahen Umgebung von supermassiven schwarzen Löchern in AGN. In diesem Zusammenhang stellt eine hochauflösende $86 \mathrm{GHz}$-VLBI-Studie eines repräsentativen Samples von ultrakompakten extragalaktischen Radioquellen die beste statistische Methode zur Untersuchung der zugrundeliegenden Physik von AGN und ihren relativistischen Jets dar.

Diese Dissertation zeigt Ergebnisse einer großen, globalen VLBI-Survey von kompakten Radioquellen, die im Zeitraum 2010-2011 mit dem Global-Millimeter-VLBI-Array durchgeführt wurde. Die wichtigsten Ziele der Studie sind eine Ausweitung der 86 GHz-Abbildungen auf schwächere Quellen, Entwicklung eines umfangreichen Samples von ultrakompakten Radioquellen die bei $86 \mathrm{GHz}$ abgebildet sind, sowie eine Untersuchung der Morphologie der Quellen und der Verteilung der Helligkeitstemperaturen. Die Daten der Studie erreichen eine typische Baseline-Empfindlichkeit von 0.1 Jy und eine typische Empfindlichkeit in den Abbildungen von $5 \mathrm{mJy} /$ beam. Insgesamt wurden in dieser Studie 162 verschiedene kompakte Radioquellen beobachtet. Alle Quellen wurden detektiert und abgebildet, das bedeutet nahezu eine Verdopplung der Anzahl der Quellen die jemals mit VLBI bei $86 \mathrm{GHz}$ abgebildet wurden. Durch diese Studie wurden 138 Quellen erstmals mit VLBI bei $86 \mathrm{GHz}$ abgebildet.

Eine Modellierung der Visibility-Daten mit Gauß-Profilen wurde angewendet, um die Struktur der beobachteten Quellen abzubilden und die Flussdichten und Größen der einzelnen Emissionsregionen (Komponenten) in deren Jets abzuschätzen. Diese Abschätzungen werden verwendet um die Helligkeitstemperatur $\left(T_{\mathrm{b}}\right)$ der Jetbasis (Kern) und der stromabwärts 
angrenzenden Emissionsregionen (Jetkomponenten) zu berechnen. Die auf der Modellierung basierenden Abschätzungen von $T_{\mathrm{b}}$ werden mit Grenzwerten der Helligkeitstemperatur, welche direkt anhand der Visibility-Daten ermittelt wurden, verglichen, dabei zeigt sich eine gute Übereinstimmung zwischen beiden Methoden.

Die Abschätzung der scheinbaren Helligkeitstemperatur der Kerne der Jets in unserem Sample reichen von $1.1 \times 10^{9} \mathrm{~K}$ bis $5.5 \times 10^{12} \mathrm{~K}$. Die mittlere Helligkeitstemperatur beträgt $1.8 \times 10^{11} \mathrm{~K}$. Die Abschätzung der scheinbaren Helligkeitstemperatur der Jet-Komponenten die sich am nähesten am Kern befinden reicht in unserem Sample von $5.8 \times 10^{7} \mathrm{~K}$ bis $4.1 \times 10^{11} \mathrm{~K}$. Ein Modell mit einem einzigen intrinsischen Wert der Helligkeitstemperatur, $T_{0}$, wird verwendet um die beobachtete Verteilung zu reproduzieren. Es liefert $T_{0}=\left(3.77_{-0.14}^{+0.10}\right) \times 10^{11} \mathrm{~K}$ für die Kerne der Jets, das impliziert, dass die Emission durch den inversen Compton-Effekt dominiert wird. In den nähesten Jetkomponenten findet sich $T_{0}=\left(1.42_{-0.19}^{+0.16}\right) \times 10^{11} \mathrm{~K}$. Dieser Wert liegt etwas oberhalb des in diesen Jetbereichen erwarteten Äquipartionslimits von $\sim 5 \times 10^{10} \mathrm{~K}$. Eine Korrelation zwischen $T_{0}$ und dem Lorentzfaktor, $\gamma_{\mathrm{j}}$, der im Modell beinhaltet ist, verhindert simultanes Fitten von $T_{0}$ und $\gamma_{\mathrm{j}}$. Wir zeigen, dass die Survey-Daten bei dieser Modellierung eine Beziehung $T_{0}[\mathrm{~K}] \approx\left(7.7 \times 10^{8}\right) \gamma_{\mathrm{j}}^{2.7}$ impliziert. Der Ansatz, die intrinsische Helligkeitstemperatur bei $86 \mathrm{GHz}$ durch Modellierung zu ermitteln, wird auf den Datenbestand der VLBI-Survey bei niedrigeren Frequenzen $(2,8,15 \mathrm{GHz})$ angewendet. Das ermöglicht es uns, die Multiwellenlängenaspekte von $T_{\mathrm{b}}$ zu untersuchen. Die intrinsische Helligkeitstemperatur, die für Kerne bei $86 \mathrm{GHz}$ erhalten wird, ist niedriger als die bei $2 \mathrm{GHz}, 8 \mathrm{GHz}$ und $15 \mathrm{GHz}$. Eine derartige Verkleinerung von $T_{0}$ deutet auf das Decelerating-Jet-Modell oder TeilchenKaskaden-Modell hin. Ein abnehmendes $T_{0}$ hätte auch Konsequenzen für VLBI bei höheren Frequenzen. Das muss weiter untersucht werden, indem die Jetgeschwindigkeiten, $\beta_{\text {app }}$, und die beobachteten Werte $T_{\mathrm{b}}$ betrachtet werden.

Das intrinsische $T_{0}$ und das beobachtete $T_{\mathrm{b}}$ in Kern und Jets zeigt, dass die Helligkeitstemperatur in Jets bereits auf sub-parsec Längenskalen um einen Faktor $\sim(2-10)$ abfällt. Dieses Entwicklung könnte auftreten, indem die Energieverluste aufeinanderfolgend von inversen Compton-, Synchrotron-, und adiabatischen Verlusten dominiert werden. Ein einfaches Schema, in dem die beobachteten Veränderungen der Jet-Emission durch adiabatische Energieverluste in relativistischen Schocks verursacht werden, wird untersucht. Für Quellen deren Struktur mit einem ausreichenden Detailgrad detektiert wurde, wird gezeigt, dass die adiabatischen Energieverluste die beobachteten Veränderungen der Helligkeitstemperatur entlang des Jets dominieren und eine Jetkomponente somit als adiabatisch expandierender, relativistischer Schock betrachtet werden kann. Unter der Äquipartionsbedingung der Energiedichte zwischen Magnetfeld und Teilchen kann die absolute Entfernung vom Kern in der VLBI-Beobachtung abgeschätzt werden. Die Helligkeitstemperaturen die in dieser Studie bei $86 \mathrm{GHz}$ gemessen wurden werden mit dem Datenbestand von VLBI-Surveys 
bei niedrigeren Frequenzen $(2,8,15 \mathrm{GHz})$ verglichen, um die Helligkeitstemperaturen im Bezugssystem der Quelle innerhalb der sub-parsec Längenskalen der ultrakompakten Radioquellen zu untersuchen. Ab der nahen Umgebung des Zentrums der Quelle steigt die Helligkeitstemperatur zunächst leicht an und erhöht sich dann mit einer größeren Steigung. Das stellt eine klare Implikation dar, dass die Jets durch das Magnetfeld beschleunigt und kollimiert werden, wie es durch Modelle für die inneren relativistischen Jets für magnetische Beschleunigung vorhergesagt wird. 


\section{Contents}

Abstract

Zusammenfassung $\quad$ v

List of Figures $\quad$ xi

List of Tables $\quad$ xvi

1 Active Galactic Nuclei 1

1.1 Historical Background \& Physical Classification . . . . . . . . . . . . . . 2

1.1.1 Seyfert Galaxies . . . . . . . . . . . . . . . . . . 3

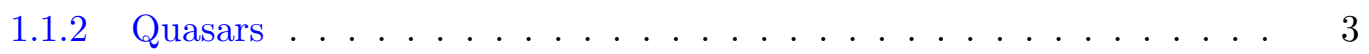

1.1.2.1 Discovery \& Understanding of Quasars . . . . . . . . . . 3

1.1.3 Radio Galaxies . . . . . . . . . . . . . . . . . . . . 4

1.1.3.1 Luminosity Classification of Radio Galaxies . . . . . . . . . 5

1.1.3.2 Extended/Compact Components . . . . . . . . . . . . 5

1.1 .4 Blazars . . . . . . . . . . . . . . . . 6

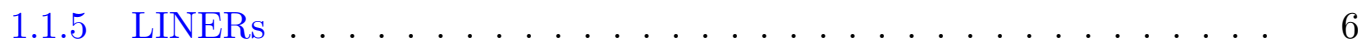

1.2 A Unified Model for AGNs . . . . . . . . . . . . . . . 6

1.3 The Different AGN Constituents . . . . . . . . . . . . . . . 8

1.3.1 The Supermassive Black hole . . . . . . . . . . . . . . . 9 9

1.3.1.1 Virial Theorem Argument . . . . . . . . . . . . . . . 9

1.3.1.2 Eddington Luminosity Argument . . . . . . . . . . . . . . 10

1.3.1.3 Rapid Variability Argument . . . . . . . . . . . . . . . 11

1.3.1.4 Observational Evidence for SMBH . . . . . . . . . . . . 12

1.3.2 The Accretion Disk . . . . . . . . . . . . . . . . . . . . . 13

1.3.2.1 Fueling the Accretion Disk . . . . . . . . . . . . . . . 13

1.3.3 The Broad and Narrow Line Regions . . . . . . . . . . . . . . . . . . . . 15

1.3.4 Molecular Torus . . . . . . . . . . . . . . . . . . . 15

1.3.5 Relativistic Jets from AGN . . . . . . . . . . . . . . . . . 17

1.3.6 Radio Lobes . . . . . . . . . . . . . . . . . . . . . . . 18

1.4 Brightness Temperature . . . . . . . . . . . . . . . . . . . . . . 19

1.5 Visibility based $T_{\mathrm{b}}$ Estimates $\ldots \ldots \ldots \ldots \ldots \ldots$

1.5.1 Minimum Brightness Temperature . . . . . . . . . . . . . . 20

1.5.2 Maximum Measurable Brightness Temperature . . . . . . . . . . . 21 
1.6 Relativistic and Geometrical Effects . . . . . . . . . . . . . . . . . . 22

1.6.1 Superluminal Velocities . . . . . . . . . . . . . . . . 22

1.6.2 Relativistic Beaming . . . . . . . . . . . . . . . . . 24

2 Ultracompact Extragalactic Radio Sources 26

2.1 Radiative Processes . . . . . . . . . . . . . . . . . . . . . . . 27

2.1.1 Synchrotron Radiation . . . . . . . . . . . . . . . . . . . . 27

2.1.1.1 Synchrotron Radiation Spectrum . . . . . . . . . . . . . 28

2.1.1.2 Synchrotron Self Absorption _... . . . . . . . . 29

2.1 .2 Inverse Compton . . . . . . . . . . . . . . . . . . . . 31

2.2 Brightness Temperature Limits . . . . . . . . . . . . . . . . . . . 32

2.2.1 Compton Catastrophe and Inverse Compton Limits . . . . . . . . 32

2.2.2 Equipartition Brightness Temperature . . . . . . . . . . . . . . 34

2.2.3 The Brightness Temperature Problem . . . . . . . . . . . . . . 34

2.3 Relativistic Jet Formation, Acceleration \& Collimation . . . . . . . . . . . . 35

2.3 .1 Jet Formation . . . . . . . . . . . . . . . . . . . . . . . . . 36

2.3.2 Acceleration and Collimation of Jets . . . . . . . . . . . . . . 37

2.3.3 Jets and Magnetic Fields . . . . . . . . . . . . . . . . . . . . . . . . . . . . . 38

2.3.4 The Radio-Loud / Radio-Quiet Dichotomy . . . . . . . . . . . . . . 39

2.3.5 Plasma Instabilities . . . . . . . . . . . . . . . . . . . . 41

2.3.6 Jet Composition . . . . . . . . . . . . . . . . . . . . 41

2.3.6.1 Light Jets . . . . . . . . . . . . . . . . . . . . . 41

2.3.6.2 Heavy Jets . . . . . . . . . . . . . . . . . . . . 42

2.3.6.3 Poynting Flux Dominated Jets . . . . . . . . . . . . . . 42

2.4 Ultracompact Radio Sources - Innermost Region of Relativistic Jets . . . . 42

2.4 Shock-Shock Interactions _ . . . . . . . . . . . . . . . . . 43

2.4 .2 Spine-Sheath Configuration . . . . . . . . . . . . . . . . 44

2.4.3 Accelerating and Decelerating Inner Jet Models . . . . . . . . . . . . 44

2.5 VLBI Observations To Investigate Physics of Extragalactic Jets . . . . . . . 45

2.5.1 VLBI Surveys . . . . . . . . . . . . . . . . . . . . . 44 45

2.6 Motivation for this Dissertation . . . . . . . . . . . . . . . . 48

3 Radio Interferometry $\quad \mathbf{5 0}$

3.1 Atmospheric Window . . . . . . . . . . . . . . . . . . . 51

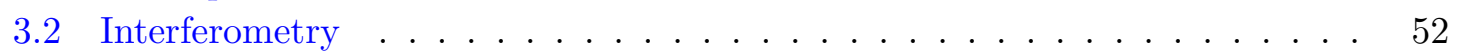

3.2 .1 van Cittert-Zernike Theorem . . . . . . . . . . . . . 53

3.2 .2 Aperture Synthesis . . . . . . . . . . . . . . . . . . 55

3.2 .3 Two Element Interferometer . . . . . . . . . . . . . . . . . . . . . . . . . . . . . 55

3.2 .4 Correlation . . . . . . . . . . . . . . . . 57

3.3 Phase and Amplitude Calibration . . . . . . . . . . . . . . 57

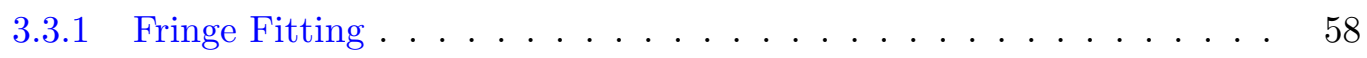

3.3.2 Amplitude Calibration . . . . . . . . . . . . . . . . . . 59

3.4 Imaging . . . . . . . . . . . . . . . . . . . . . . . 60

3.4.1 Sampling Function \& Fourier Inversion . . . . . . . . . . . . . . . 60

3.4 .2 Deconvolution . . . . . . . . . . . . . . . . . . . 61

3.4 .3 Closure Quantities . . . . . . . . . . . . . . . . . . . . 61

3.4.4 Self-Calibration \& Hybrid Mapping . . . . . . . . . . . . . . . 62 
3.5 Model Fitting . . . . . . . . . . . . . . . . . . . . . . . 63

3.5.1 Error Estimation . . . . . . . . . . . . . . . . . . . 64

4 Global 3 mm-VLBI Array Survey $\quad 65$

4.1 Introduction . . . . . . . . . . . . . . . . . 65

4.2 GMVA Survey of Compact AGN . . . . . . . . . . . . . . . 66

4.2 .1 Source Selection . . . . . . . . . . . . . . . . . 68

4.2 .2 Observations . . . . . . . . . . . . . . . . . . . 69

4.3 Data Processing . . . . . . . . . . . . . . . . . . . . . 73

4.3.1 Parallactic Angle Correction . . . . . . . . . . . . . 73

4.3.2 Fringe Fitting the Data with "FRING" . . . . . . . . . . . . . 73

4.3.2.1 Manual Phase Calibration ... . . . . . . . . . . 74

4.3.2.2 Reference Antenna and Solution Interval . . . . . . . . . . . . 74

4.3.2.3 Global Fringe Fitting . . . . . . . . . . . . . . . . 75

4.3.3 Amplitude Calibration . . . . . . . . . . . . . . . . . . 75

4.4 Imaging . . . . . . . . . . . . . . . . . . . 76

4.4.1 Hybrid Mapping and Model Fitting . . . . . . . . . . . . . . . . 77

4.5 Results of the Survey . . . . . . . . . . . . . . . . . . . . . . . . . . . . . . . . . .

4.5.1 Images . . . . . . . . . . . . . . . . . . . . 80

4.6 Discussion . . . . . . . . . . . . . . . . . . . . . . . . . . 134

4.6.1 Model fit based Brightness Temperatures . . . . . . . . . . . . . 134

4.6.2 Visibility based Brightness Temperatures . . . . . . . . . . . . . . . . . 135

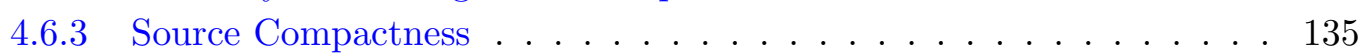

4.7 Summary . . . . . . . . . . . . . . . . . . . . . 140

5 Modelling the Observed Brightness Temperatures $\quad \mathbf{1 4 5}$

5.1 Introduction . . . . . . . . . . . . . . . . . . . 145

5.2 Theory of Population Modelling of $T_{\mathrm{b}} \ldots \ldots \ldots \ldots \ldots$

5.3 Population Modelling of Observed $T_{\mathrm{b}} \ldots \ldots \ldots \ldots \ldots$. . . . . . . . . . . . . . . . . . . 147

5.4 Results and Discussion . . . . . . . . . . . . . . . . . . . . . 148

5.4.1 Comparison of Modelling Results with Low Frequency Surveys . . . 152

5.4 .2 Constraints of the Population Model . . . . . . . . . . . . . . . 152

5.5 Testing the Adiabatic Expansion of Jets . . . . . . . . . . . . . . 153

5.6 Conclusion . . . . . . . . . . . . . . . . . . . 154

6 Intrinsic Properties of Ultracompact Radio Sources $\mathbf{1 5 6}$

6.1 Intrinsic Brightness Temperature from Jet Speeds and $T_{\mathrm{b}} \ldots \ldots \ldots$

6.2 Ultracompact Jets . . . . . . . . . . . . . . . . . . . . . . . . . . . . . . . . . . . . . . . . . . . . .

6.2 .1 Theory - Ultracompact Jets . . . . . . . . . . . . . . . . 159

6.3 Results and Discussion . . . . . . . . . . . . . . . . . . . . 160

6.3.1 Modelling the Radial Dependence of $T_{\mathrm{b}} \ldots \ldots \ldots$. . . . . . . 161

6.4 Conclusion . . . . . . . . . . . . . . . . . . . . . . . 162

7 Conclusions \& Outlook $\quad 169$ 
Thank you | Vielen Dank | Dhanyawad | Nandhi 182

Erklärung 


\section{List of Figures}

1.1 The radio map of Cygnus A at $11.06 \mathrm{GHz}$ with a resolution of 2.25 arcseconds (Perley \& Butler 2017). . . . . . . . . . . . . . . . . .

1.2 A schematic representation of the current AGN unification scheme. The AGN type we observe depends up on the viewing angle. The radio-loud and radio-quiet AGNs are shown in the upper and lower part, respectively (Beckmann \& Shrader 2012). . . . . . . . . . . . . . . . . . . . .

1.3 Optical/UV spectrum for optically-selected quasars in the SDSS adopted from Vanden Berk et al. (2004). Permitted broad lines and semiforbidden broad line CIII and the forbidden OIII narrow line can be seen. . . . . . . .

1.4 Radio images of the galaxy M87 at different scales. Credit: National Radio Astronomy Observatory/AUI - F. Owen, J. Biretta, J. Eilek, \& N. Kassim .

1.5 The Inner Jet of the Radio Galaxy M87 at $15 \mathrm{GHz}$. The x-axis is right ascension and $y$ axis is declination, both in in milliarcsecond unit. Credit: Y.Y. Kovalev, MPIfR Bonn. . . . . . . . . . . . . . . . . . . 18

1.6 Superluminal motion observed in 3C 273 (Pearson et al. 1981). . . . . . . . 22

1.7 Left top: The apparent velocity $\beta_{a p p}$ versus the viewing angle $\theta$ for different values of Lorentz factor, $\gamma$, as shown. Left bottom: the amplification caused by fourth power of Doppler factor, $\delta^{4}$ versus $\theta$, for the same $\gamma$ as shown. Right: Geometry of superluminal motion. Both figures taken from Ghisellini (2013) . . . . . . . . . . . . . . . . . . .

2.1 The synchrotron spectrum from a partially self absorbed source. The region on the left side and right side of dashed line represents optically thick region (self absorbed part) and optically th region, respectively. $\nu_{t}$ represents the turnover frequency. Observations of the self absorbed part could determine magnetic field, B. Observations of the thin part can then determine constant $C$ and the electron slope $s$ (Ghisellini 2013) . . . . . . . . . . .

2.2 An illustration of the structure of magnetosphere with field lines threaded around the black hole. The accretion flow extends to the plunching region (shown as wedge ends pointing toward the black hole) makes the magnetic flux threading the black hole higher. The angular velocity of the black hole and the magnetic flux determines the jet power. Credit: Serguei Komissarov

2.3 A schematic representation of the frequency dependence position shift of the VLBI core (Lobanov 1996). . . . . . . . . . . . . . . . . . .

2.4 Left: Schematic representation of accelerating and decelerating jet model. Right Top: Sketch of the synchrotron spectrum of a self-similar relativistic jet. Right Bottom: The flat spectrum formation at the jet base. Figures taken from Marscher (1995). . . . . . . . . . . . . . . 
2.5 The maps of Cygnus A produced at different radio wavelengths from $6 \mathrm{~cm}$ $(\sim) 5 \mathrm{GHz}$ to $3 \mathrm{~mm}(\sim 86 \mathrm{GHz})$ and different baselines. Note that as the frequency of observation and baseline increases, we can zoom into the inner parsec scale regions close to the supermassive black hole (ly implies light year). Picture taken from Boccardi et al. (2017). . . . . . . . . . . . . .

3.1 Percentage transmission through the Earths atmosphere, in the vertical direction. Credit: Ulaby F. Long, University of Michigan. . . . . . . . . . .

3.2 Left: An incoherent radio source A and a simple geometry of an interferometer. Right: The direction cosines $(l, m, n) \cdot l^{2}+m^{2}+n^{2}=1$. Credit: Lectures in NRAO Synthesis Imaging Summer School 2013 by Rick Perley.

3.3 A schematic representation of two types of two element interferometer - Product Interferometer on the left and Sum Interferometer on the right. Credit: http://www.radioastrolab.com/radio-astronomy/radio-interferometry. . . .

4.1 The Global Millimeter VLBI Array (GMVA) with its participating telescopes, operating at the frequency of $86 \mathrm{GHz}$. Credit: Helge Rottmann,

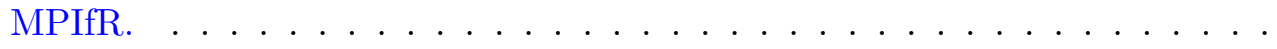

4.2 The sky distribution of the $86 \mathrm{GHz}$ VLBI sources in this survey. Symbols: circles are quasars, triangles are blazars, squares are galaxies and diamonds are unidentified sources. . . . . . . . . . . . . . .

4.3 Distribution of the total single dish flux density of the sources, measured at $86 \mathrm{GHz}$ at Pico Veleta or Plateau de Bure during the observations,, $S_{86}$ broken down along different host galaxy types and compared to the respective distribution for the sources from the sample of Lee et al. (2008). The present survey provides a nearly twofold increase in the number of objects imaged with VLBI at $3 \mathrm{~mm} . \ldots \ldots \ldots \ldots \ldots$

4.4 Examples of $u v$-coverages of the survey observations for a low declination source (left; J1811 $+1704, \delta=+17^{\circ}$ ) and a high declination source (right; $\left.\mathrm{J} 0642+8811, \delta=+88^{\circ}\right) . \ldots \ldots \ldots \ldots \ldots \ldots$

4.5 Amplitudes and phases of baseline visibilities between Pie Town (Pt) and other VLBA antennas before (top panel) and after (bottom panel) fringe fitting and amplitude calibration for the source 3C279 in Epoch C. The phases are plotted against channels of each IF in degrees (from $-200^{\circ}$ to $+200^{\circ}$ ) in both top and bottom panels. The amplitude plotted against channels of each IF on top panel is in units of micro Jansky (before calibration), and on bottom panel is in units of Jansky (after calibration). . . . . . . . . . . .

4.6 Distribution of the ratios of the visibility amplitudes obtained without and with the antenna gain correction, on short $B_{\mathrm{S}}\left(R_{S}=S_{S} / S_{S}^{\prime}\right)$ and long $B_{\mathrm{L}}$ $\left(R_{L}=S_{L} / S_{L}^{\prime}\right)$ baselines, shown respectively, on the left and right panel. The median and mean of $R_{S}$ is 1.15 and 1.24, respectively and the median and mean of $R_{L}$ is 0.99 and 1.01 , respectively. . . . . . . . . . .

4.7 Distribution of the noise quality factor $\xi_{\mathrm{r}}$ for the residual images of all the sources in the survey. . . . . . . . . . . . . . . .

4.8 Distribution of the imaging signal-to-noise ratios (SNR) of all the sources in the survey calculated from the ratio of the peak flux density of the map, $S_{\mathrm{p}}$ to the r.m.s noise in the map, $\sigma_{\text {rms }}$. The mean and median value of the SNR distribution are 42 and 36.5 , respectively. . . . . . . . . . 
4.9174 contour maps of 162 unique sources imaged at $3 \mathrm{~mm}$ in this survey (left panel), shown together with the respective radial amplitude distributions (right panel) and $u v$-coverages (inset in the right panel) of the respective visibility datasets. The contouring of images is made at $3 \sigma_{\mathrm{rms}} \times(-1,1, \sqrt{2}, 2, \ldots)$ levels, with $\sigma_{\text {rms }}$ representing the off-source r.m.s noise in the residual image. 91

$4.103 \mathrm{~mm}$ maps continued . . . . . . . . . . . . . . . . . . . . . . . 92

$4.113 \mathrm{~mm}$ maps continued . . . . . . . . . . . . . . . . . . . 93

$4.123 \mathrm{~mm}$ maps continued . . . . . . . . . . . . . . . . . . . . . . 94

$4.133 \mathrm{~mm}$ maps continued . . . . . . . . . . . . . . . . . . . . . . . . 95

$4.143 \mathrm{~mm}$ maps continued . . . . . . . . . . . . . . . . . . . 96

$4.153 \mathrm{~mm}$ maps continued . . . . . . . . . . . . . . . . . . . . . . . . . . . 97

$4.163 \mathrm{~mm}$ maps continued . . . . . . . . . . . . . . . . . . . . . . . . . . . . . . . .

$4.173 \mathrm{~mm}$ maps continued . . . . . . . . . . . . . . . . . . . . . . . . . . . . . . . . .

$4.183 \mathrm{~mm}$ maps continued . . . . . . . . . . . . . . . . . . 100

$4.193 \mathrm{~mm}$ maps continued . . . . . . . . . . . . . . . . . . . . . . . . . . . . . . . . . 101

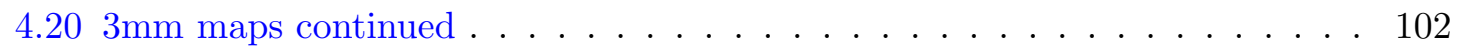

$4.213 \mathrm{~mm}$ maps continued . . . . . . . . . . . . . . . . . . . . . . . . . . . . . . . . . . . . . . . .

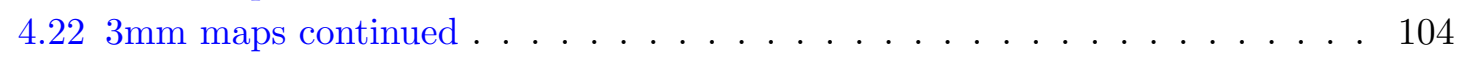

$4.233 \mathrm{~mm}$ maps continued . . . . . . . . . . . . . . . . . . 105

$4.243 \mathrm{~mm}$ maps continued . . . . . . . . . . . . . . . . . 106

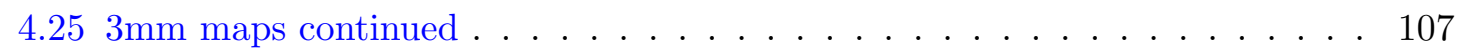

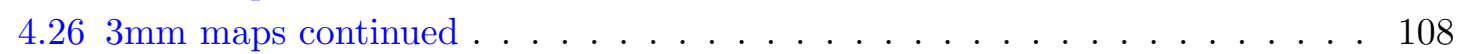

$4.273 \mathrm{~mm}$ maps continued . . . . . . . . . . . . . . . . . . . . . . . . . . . . . . . . . . . . . . . . . .

$4.283 \mathrm{~mm}$ maps continued . . . . . . . . . . . . . . . . . . . . . . . . . . . . . . . . . . 110

$4.293 \mathrm{~mm}$ maps continued . . . . . . . . . . . . . . . . . . . . . . . . . . . . . . . . . . 111

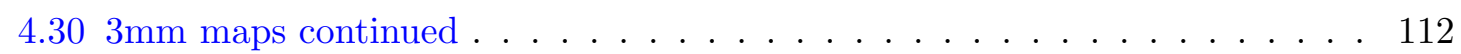

$4.313 \mathrm{~mm}$ maps continued . . . . . . . . . . . . . . . . . . . . . . . . . . . . . . . . . . . . . . . . . . .

$4.323 \mathrm{~mm}$ maps continued . . . . . . . . . . . . . . . . . . . . . . . . . . . . . . . . . . . . . . . . . .

$4.333 \mathrm{~mm}$ maps continued . . . . . . . . . . . . . . . . . . 115

$4.343 \mathrm{~mm}$ maps continued . . . . . . . . . . . . . . . . . . . . . . . . . . . . . . 116

$4.353 \mathrm{~mm}$ maps continued . . . . . . . . . . . . . . . . . . . . . . . . . . . . . . . . . 117

$4.363 \mathrm{~mm}$ maps continued . . . . . . . . . . . . . . . . . . . . . . . . . . . . . . . 118

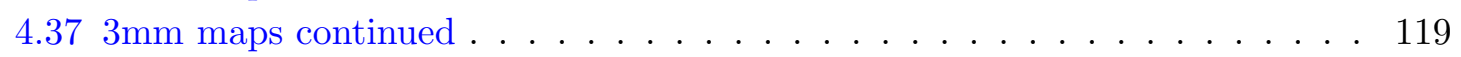

$4.383 \mathrm{~mm}$ maps continued . . . . . . . . . . . . . . . . . . . . . . . . . . . . . . . . . . . 120

$4.393 \mathrm{~mm}$ maps continued . . . . . . . . . . . . . . . . . . . . . . . . . . . . . . . . . 121

$4.403 \mathrm{~mm}$ maps continued . . . . . . . . . . . . . . . . . . . . . . . . . . . . . . . . . . 122

$4.413 \mathrm{~mm}$ maps continued . . . . . . . . . . . . . . . . . . . . . . . . . . . . . . . . . . 123

$4.423 \mathrm{~mm}$ maps continued . . . . . . . . . . . . . . . . . . . . . . . . . . . . . . . . . 124

$4.433 \mathrm{~mm}$ maps continued . . . . . . . . . . . . . . . . . . . . . . . . . . . . . . . . . . . . . . . . . . . .

$4.443 \mathrm{~mm}$ maps continued . . . . . . . . . . . . . . . . . . . 126

$4.453 \mathrm{~mm}$ maps continued . . . . . . . . . . . . . . . . . . . . . . . . . . . . . . . . 127

$4.463 \mathrm{~mm}$ maps continued . . . . . . . . . . . . . . . . . . . . . . . . . . . . . . . . . 128

$4.473 \mathrm{~mm}$ maps continued . . . . . . . . . . . . . . . . . . . . . . . . . . . . . . . . . 129

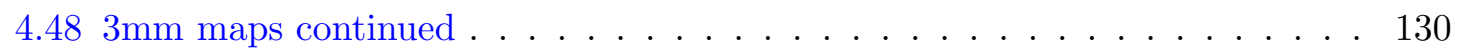

$4.493 \mathrm{~mm}$ maps continued . . . . . . . . . . . . . . . . . . 131

$4.503 \mathrm{~mm}$ maps continued . . . . . . . . . . . . . . . . . . . . . . . . . . . . . . . . . . . . . . . . .

$4.513 \mathrm{~mm}$ maps continued . . . . . . . . . . . . . . 133 
$4.523 \mathrm{~mm}$ maps continued . . . . . . . . . . . . . . . .

4.53 Comparison of $T_{\mathrm{b} \text {,mod }}$ measured from circular Gaussian representation of source structure and $T_{\mathrm{b}, \lim }$ estimated from the interferometric visibilities at $u v$-radii within $10 \%$ of the maximum baseline $B_{\max }$ in the data for a given source (top panel). Correlation between the two distribution is illustrated by the residual logarithmic distribution of $T_{\mathrm{b} \text {,mod }} / T_{\mathrm{b} \text {,limit }}$ ratio (bottom panel) which is well approximated by the Gaussian distribution with mean value, $\mu=0.001$ and standard deviation, $\sigma=0.46 \ldots \ldots \ldots \ldots$

4.54 Comparison of $T_{\mathrm{b} \text {, mod }}$ measured from circular Gaussian representation of source structure and $T_{\mathrm{b} \text {,min }}$ estimated from the interferometric visibilities in the data for a given source (top panel). The residual logarithmic distribution of the $T_{\mathrm{b}, \text { mod }} / T_{\mathrm{b}, \min }$ ratio is approximated by the Gaussian PDF with $\mu=$ 0.354 and $\sigma=0.42$ (bottom panel). . . . . . . . . . . . .

4.55 The compactness parameters $S_{86} / S_{\text {CLEAN }}$ and $S_{\text {core }} / S_{\text {CLEAN }}$ on the left and right panel respectively. The Pearson correlation coefficients, $\rho_{\left(S_{86}, S_{C L E A N}\right)}$ $=0.908$ and $\rho_{\left(S_{C O R E}, S_{C L E A N}\right)}=0.874$ are obtained for the combined data from this survey and Lee et al. (2008). . . . . . . . . . . . . .

4.56 Distribution of the total CLEAN image flux density, $S_{\text {clean }}$, of the survey targets broken down along different host galaxy types and compared with respective distribution for the sources from the sample of Lee et al. (2008).

4.57 The distribution of the compactness indices on milli arcsecond scales $S_{\text {CLEAN }} / S_{86}$ (top left), the core dominance indices $S_{\text {CORE }} / S_{\text {CLEAN }}$ (top right), the correlated flux density on long baselines $S_{\mathrm{L}}$ (bottom left) and the compactness indices on sub-milli arcsecond scales $S_{\mathrm{L}} / S_{\mathrm{S}}$ (bottom right). . . . . . . .

4.58 The distribution of the total flux density $S_{\mathrm{CORE}}$ (top panel) and the angular size of the core components (middle and bottom panel). In the bottom panel, the green color represents lower limits on size $d_{\min }$ estimated for unresolved core components and the red color represents measured sizes $d$ for resolved core components. . . . . . . . . . . . . . . . . .

4.59 The distribution of the brightness temperature in the core components of different classes of the sources in this survey - quasars (top left), BL Lac objects (top right), galaxies (middle left), unidentified sources (middle right) and that of all the classes of sources together (bottom panel). The green color represents lower limits on $T_{\mathrm{b}}$ estimated for the minimum resolvable size of a core component and the red color represents measured $T_{\mathrm{b}}$ in resolved core components. . . . . . . . . . . . . . . . . .

4.60 The distribution of the brightness temperature in the core components estimated from visibility. The limiting resolved $T_{\mathrm{b}, \mathrm{lim}}$ is shown in top panel and the minimum $T_{\mathrm{b}, \text { min }}$ is shown on the bottom panel. The green color represents lower limits on size $d_{\min }$ for unresolved core components and the red color represents measured sizes $d$ for resolved core components, estimated from Gaussian model fitting. . . . . . . . . . . . . . . . . .

5.1 Distribution of the brightness temperatures, $T_{\mathrm{b}}$ measured in the core components and represented by the population models calculated for $\gamma_{j}=10$ and different values of $T_{0}$. The best approximation of the observed $T_{\mathrm{b}}$ distribution is obtained with $T_{\mathrm{o} \text {,core }}=\left(3.77_{-0.14}^{+0.10}\right) \times 10^{11} \mathrm{~K}$. Note that for better viewing of the observed distribution, one core component with a very high $T_{\mathrm{b}}=5.5 \times 10^{12} \mathrm{~K}$ is not shown but is included in the modelling. . . . . . 
5.2 Distribution of the brightness temperatures, $T_{\mathrm{b}}$ measured in the inner jet components and represented by the population models calculated for $\gamma_{\mathrm{j}}=$ 10 and different values of $T_{0}$. The best approximation of the observed $T_{\mathrm{b}}$ distribution is obtained with $T_{\mathrm{o} \text {,jet }}=\left(1.42_{-0.19}^{+0.16}\right) \times 10^{11} \mathrm{~K} . \ldots \ldots$

5.3 The two-dimensional $\chi^{2}$ distribution plot in the $\gamma-T_{0}$ space, calculated for the brightness temperatures measured in the VLBI cores. The blank area (in white) shows the ranges of the parameter space disallowed by the observed distribution. The distribution of the $\chi^{2}$ values indicates a $T_{0}-\gamma$ correlation, with $T_{0}[\mathrm{~K}] \approx\left(7.7 \times 10^{8}\right) \gamma_{\mathrm{j}}^{2.7}$, thus precluding a simultaneous fit for $\gamma_{\mathrm{j}}$ and $T_{0} .151$

5.4 Changes of the brightness temperature as a funcion of jet width for four sources $-\mathrm{J} 2322+5037$, J1033+6051, 3C345 and 0716+714 from this survey. Blue squares denote the measured $T_{\mathrm{b}}$ from this survey. The red circles, connected with a dotted line represent theoretically expected $T_{\mathrm{b}}$ under the assumption of adiabatic jet expansion. The initial brightness temperature in each jet is assumed to be same as that measured in the VLBI core. . . . . . 154

6.1 Plot of the apparent jet speed versus the observed brightness temperature for the sources in our $86 \mathrm{GHz}$ sample. The dashed line represents sources observed at the critical angle that have the intrinsic brightness temperature of $T_{0}=6.2 \times 10^{9} \mathrm{~K} . \ldots \ldots \ldots \ldots \ldots \ldots$

6.2 Brightness temperatures in source frame as a function of frequencies in source frame (top panel) and of absolute position of the VLBI core components (middle and bottom panel) at 2, 8, 15 and $86 \mathrm{GHz}$. The absolute position of the VLBI core is derived based on $\gamma_{\mathrm{j}}=10, \phi_{\mathrm{o}}=1 / \gamma_{\mathrm{j}}^{2}, B=1 \mathrm{G}, N_{1}=$ $5 \times 10^{3} \mathrm{~cm}^{-3}$, and $r_{\max } / r_{\min }=100$. Nearby sources are marked as - squares for NGC 1052, pentagons for M87, stars for NGC 315, diamonds for Mrk421, triangles for $3 \mathrm{C} 84$ and left triangles for $3 \mathrm{C} 120 \ldots \ldots \ldots$

6.3 Brightness temperature $T_{\mathrm{b}}$ as a function of core position $r$ fitted with single power law (top panel), three power law functions (middle panel) and empirical model given by equation 6.14 (bottom panel). . . . . . . . . . 165 


\section{List of Tables}

3.1 Radio Bands . . . . . . . . . . . . . . . . . . . . . . 52

4.1 VLBI surveys at $86 \mathrm{GHz} \ldots \ldots \ldots \ldots \ldots \ldots \ldots$

4.2 Participating Telescopes . . . . . . . . . . . . . . . . . . . 67

$4.3 \log$ of survey observations . . . . . . . . . . . . . . . . . . 69

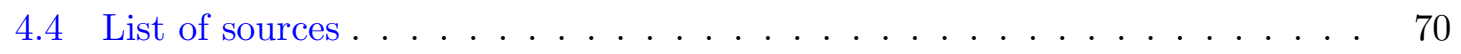

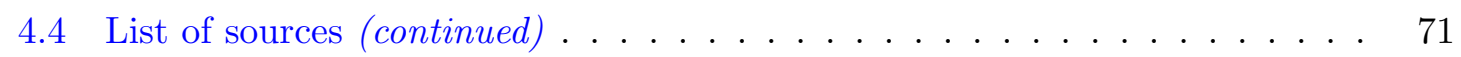

4.4 List of sources (continued) . . . . . . . . . . . . . . . . . . . . . . . . . 72

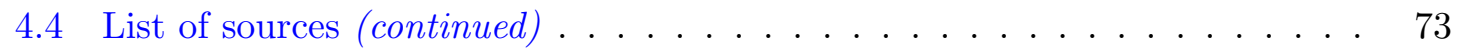

4.5 Average Antenna Gain Corrections . . . . . . . . . . . . . . . . . . . . . 76

4.6 Image Parameters . . . . . . . . . . . . . . . . . . . . . . . . . . . . . . . . . . . . . .

4.6 Image Parameters continued . . . . . . . . . . . . . . . . . . . . . 82

4.6 Image Parameters continued . . . . . . . . . . . . . . . . . . . . . 83

4.6 Image Parameters continued . . . . . . . . . . . . . . . . . . . . . . . . . . . . . . 84

4.7 Model Fit Parameters . . . . . . . . . . . . . . . . . . . . . 84

4.7 Model fit parameters continued . . . . . . . . . . . . . . . . . . . . . . . . . . . . . . 85

4.7 Model fit parameters continued . . . . . . . . . . . . . . . . . . 86

4.7 Model fit parameters continued . . . . . . . . . . . . . . . . . 87

4.7 Model fit parameters continued . . . . . . . . . . . . . . . . . . . . . . . . . . . . . 88

4.7 Model fit parameters continued . . . . . . . . . . . . . . . . . . . . 89

4.7 Model fit parameters continued . . . . . . . . . . . . . . . . . 90

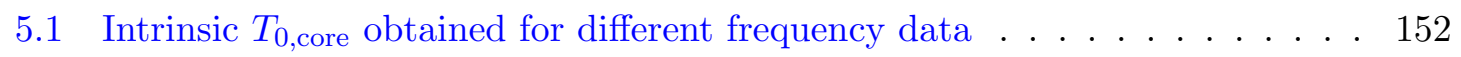

6.1 Synchrotron Luminosity . . . . . . . . . . . . . . . . . . . . 166

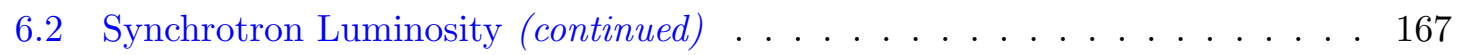

6.3 Synchrotron Luminosity (continued) . . . . . . . . . . . . . . . 168 


\section{Chapter 1}

\section{Active Galactic Nuclei}

Galaxies are astronomical systems consisting of billions of stars, gas, dust, interstellar medium (ISM) and dark matter held together by the force of gravity. About two-thirds of the galaxies have spiral shape and many galaxies have supermassive black hole at the center. The Milky Way galaxy in which our solar system, including the Sun and its eight planets exist also has a supermassive black hole of mass $\sim 10^{6} M_{\odot}$ at the center where $M_{\odot}$ is one solar mass ( 1 solar mass $\sim 1.99 \times 10^{30} \mathrm{~kg}$ ). The observable universe was believed to contain 200 million galaxies from the studies of Hubble's Ultra Deep Field conducted in mid 1990's. Recently it is found that there are 2 trillion galaxies in the universe, ten times more than previously thought (Conselice et al. 2016). Thanks to Hubble Space Telescope (HST), the space telescope which led astronomers to this breakthrough discovery!

In a normal galaxy, most of the light comes from the stars, ISM and HII regions in visible wavelengths and is evenly distributed throughout the galaxy. But when some galaxies are observed, interestingly it was seen that intense amount of light is coming from their nuclei. These galaxies emit tremendous amount of energy in the form of radio, infrared, optical, ultra-violet, X-ray and gamma ray, apparently from the nucleus. These are called Active Galaxies, which represent only less than ten percentage of all the galaxies in our universe. Their overall luminosities range from $10^{37}$ watts to more than $10^{40}$ watts which is several thousand times that of the Milky Way Galaxy that outshine their central host galaxy (Nicolson 1999). Active Galactic Nucleus (AGN) is the compact extremely luminous region at the centre of active galaxies which is powered by conversion of gravitational potential energy into radiation via the accretion of matter on a hot accretion disk surrounding a supermassive black hole (SMBH; Zel'dovich \& Novikov 1964) of mass in the range of $10^{6} M_{\odot}-10^{10} M_{\odot}$. Most of the energy radiated from AGN is non thermal radiated by charged particles moving at high speeds in magnetic fields rather than processes like nuclear fusion that happens in stars. An exotic feature called jets are observed in many cases which are highly energetic plasma ejected as two-sided or one-sided.

Quasars, which is a dominant class of AGNs, are mostly found in high redshifts thereby representing the early universe. The most distant known quasar to date is J1342+0928 at redshift $z=7.54$ with a bolometric luminosity of $4 \times 10^{13} L_{\odot}$, where $L_{\odot}$ is the luminosity of Sun. It is interesting to think that the light emitted from this quasar at a redshift of 7.54 represent light as old as 690 million years i.e. within the first Giga Year (GYr)of formation of the Universe which is just $5 \%$ of the current age of the Universe and the supermassive black hole in this quasar is the most distant black hole identified to date with a mass $\sim 8 \times 10^{8} M_{\odot}$ (Bañados et al. 2017). Therefore AGNs can tell us a lot about the physics 
and conditions that triggered its formation and existence at the earliest cosmic epochs of the universe. The study of AGNs from the beginning of universe (high redshifts) till today (low redshifts) can help to understand a lot about galaxy evolution and how the evolution is likely to vary at different redshift. AGNs represent the extragalactic objects on large scales. The powerful energy generation ranging from low to high frequencies, the accretion process and the conditions of supermassive black hole formation and its evolution, the relativistic jet formation and its composition, the origin of the magnetic field, the role of merger events of galaxies in AGN feedback are among the key questions astronomers address. Therefore $A G N s$ and their jets form the best laboratory to test extreme physics and understand our universe.

\subsection{Historical Background \& Physical Classification}

AGNs exist in different types and flavours due to the difference in their intrinsic luminosity, orientation of the source with respect to the line of sight of observer, spectral and polarization characteristics. The two major division of AGNs evolved on the basis of how strong is the radio flux of a source with respect to its optical flux. They are called Radio-loud AGN ( $R L A G N)$ and Radio-quiet $A G N$ (RQ AGN). Radio loudness parameter $(R)$ is defined as the ratio of radio $(5 \mathrm{GHz})$ to optical (B band) spectral flux densities centered at the wavelength $\lambda=4400 \AA$ i.e.

$$
R=\frac{f_{5 \mathrm{GHz}}}{f_{B}}
$$

Kellermann et al. (1989) established $R=10$ as the borderline between RQ and RL AGNs based on a study done using optically selected Palomar Green (PG) catalog of quasars i.e. the sources with $R<10$ is classified into RQ AGN and the rest with $R \geq 10$ is classified into RL AGN. Around $10-15 \%$ of AGNs are radio-loud. The existing bimodality in radio flux distribution in RL and RQ AGNs can be linked to the existence of radio jets because of the general argument that the power from the RL AGNs is dominated by jet whereas the power from RQ AGNs is dominated by the accretion disk (Chiaberge \& Marconi 2011). The radio-loud AGNs are also 3 times brighter in X-rays than radio-quiet AGNs. A detailed discussion of radio-loud/radio-quiet dichotomy is done in section 2.3.4 in Chapter 2.

Both the radio-loud AGNs and radio-quiet AGNs are further classified into type1, type2 and Intermediate objects depending on the emission line strength in their optical spectra. In type 1 objects, both broad and narrow emission lines are present in their spectra with a typical line width of broad emission line extending up to $10,000 \mathrm{~km} \mathrm{~s}^{-1}$ because of the fact that the compact central nucleus of such objects are seen face on. In type2 objects, only narrow lines are present in their spectra with a line width of the order of $1000 \mathrm{~km} \mathrm{~s}^{-1}$ because the compact central nucleus is observed edge on thereby hindering the broad emission lines from the fast rotating central broad line region close to the accretion disk. In Intermediate objects, the spectral characteristics are intermediate between type1 and type2 objects.

A broad classification is based on the fundamental difference in the amount of radiation emitted by the compact central source i.e. their "luminosity". Based on luminosity, radioquiet AGNs are classified into Seyfert Galaxies and Quasi Stellar Objects (Quasars or QSOs). The total energy emitted by the AGN in Seyfert galaxies at visible wavelength is comparable to energy emitted by all of the stars in the galaxy i.e. $\sim 10^{11} L_{\odot}$, but in a typical quasar the nuclear source is brighter than the stars by a factor $\geq 100$ i.e. of the order $\geq 10^{13} L_{\odot}$. The Seyfert galaxies are mostly spiral galaxies whereas the host galaxies 
of quasars are mostly elliptical galaxies. Quasars are found at higher redshifts and the strong emission from them outshines the host galaxy. Seyferts are found at low redshift. A detailed discussion on the discovery and characteristics of Seyfert galaxies and quasars follows in the next sections 1.1 .1 and 1.1.2, respectively.

\subsubsection{Seyfert Galaxies}

In 1908, Edward A. Fath was observing the spectra of a spiral nebulae called "NGC 1068" which showed six bright emission lines. This was the discovery of a first AGN. In 1926, Edwin Hubble also recorded the emission lines of NGC 1068 and two other galaxies. Later, in 1943, Carl K. Seyfert reported that a small percentage of galaxies have very bright nuclei which is the source of their broad emission lines (Seyfert 1943). These nuclei were stellar in appearance and the full width half maximum (FWHM) of the broad emission lines extended up to $8500 \mathrm{~km} \mathrm{~s}^{-1}$. Today these objects are known as Seyfert galaxies. Seyfert galaxies are lower luminosity AGNs (Schmidt \& Green 1983) with nuclear magnitude,

$$
M_{\mathrm{B}}<-21.51+5 \log \mathrm{h}_{\mathrm{o}}
$$

where $h_{o}$ is the Hubble constant. They are categorized into two based on their spectra Seyfert 1 and Seyfert 2. Seyfert 1 galaxies belong to type1 objects having two types of emission lines. One type of lines originates from a low density ionized gas (electron density, $n_{\mathrm{e}} \approx 10^{3}-10^{6} \mathrm{~cm}^{-3}$ ) with FWHM corresponding to velocities of $\sim 100 \mathrm{~km} / \mathrm{sec}$ containing both permitted lines (HI, HeI, HeII) and forbidden lines (like OIII) and they are called as "narrow" lines. The other type of "broad" lines are also observed with widths of upto $10^{4}$ $\mathrm{km} / \mathrm{sec}$, but only in the permitted lines. The absence of broad forbidden lines is a direct implication that the broad line gas originate from a region of high density $\left(n_{\mathrm{e}} \approx 10^{9} \mathrm{~cm}^{-3}\right)$ (Peterson 1997). But in Seyfert 2 which belong to type2 objects, only narrow lines are observed. These lines are broadened due to Doppler broadening. The spectrum of Seyferts also shows a featureless continuum, that does not contain any emission lines which is a characteristic of the central source. Weak absorption lines are also seen in the spectra of both type1 and type 2 Seyfert galaxies.

\subsubsection{Quasars}

Quasars comprise the most luminous subclass of AGNs (Schmidt \& Green 1983) with nuclear magnitude,

$$
M_{\mathrm{B}}>-21.51+5 \log \mathrm{h}_{\mathrm{o}} .
$$

where $h_{o}$ is the Hubble constant. Their characteristics are large ultra-violet (UV) flux, broad emission lines, time variable continuum flux and large redshift.

\subsubsection{Discovery \& Understanding of Quasars}

In the early 1960s, some radio sources were shown to coincide in position with objects that looked like stars. These became known as quasars, the name deriving from "Quasi stellar radio source. Later, it was discovered that only one in ten of these objects is a strong radio emitter and the term quasi stellar object (QSO) was used to describe the radio-quiet versions, the term quasar is still widely used to describe both kinds of objects. The first 
quasar to be identified was 3C 48. In 1960, Thomas Matthews and Allan Sandage used the $5.1 \mathrm{~m}$ telescope on Mount Palomar in California to show that this source coincide with what looked like a star, the spectrum of which consisted of a set of emission lines which were not properly identified and understood then (Matthews \& Sandage 1963). In 1963, Maarten Schmidt of the California Institute of Technology realized that the lines were the emission lines of hydrogen which was Doppler shifted by a factor of 0.16, i.e. each lines wavelength had been stretched to 16 percent longer than normal (Schmidt 1963). If it was assumed that the redshift was a Doppler effect, then the results implied that 3C 273 was receding at about 16 percent of the speed of light from earth. According to this, 3C 273 was at a distance of 2 billion light-years. In order to appear as bright as they do, existing at such enormous distances, quasars must be extremely luminous than a conventional galaxy like the Milky Way. During that time, many astronomers were doubtful about how such a remote small object can emit so much energy.

Studies in 1980s and 1990s found that quasars are surrounded by fuzzy patches of light whose spectra contain stellar type absorption lines with the same redshifts as the quasars within them. This indicated that quasars are residing within remote galaxies. Also, quasars showed characteristics similar to AGN in general, for example multi wavelength emissions, characteristic synchrotron spectrum, brightness varying over periods equal to weeks or even days, compact central engine and presence of jets coming from their center (Nicolson 1999). All the observed features of quasars finally convinced astronomers that a quasar is simply a remote and highly luminous AGN. No known quasar has a red shift less than 0.06, and quasar numbers seem to be highest at redshifts of around (2 - 3) (Nicolson 1999). There are many measurements for quasar clustering at redshift, $z<2.2$ (Ross et al. 2009), and a few at $z>2.9$, (Shen et al. 2007) studied using Sloan Digital Sky Survey (SDSS). Also, Eftekharzadeh et al. (2014) found a clustering of quasars at $z=2.5$. These studies indicate that quasar activity must have been dominant among galaxies billions of years ago, when the universe was younger than the present.

The radio-loud AGNs can be mainly classified into two - Radio Galaxies and Blazars. A detailed discussion on the discovery and characteristics of radio galaxies and blazars is followed in the next sections 1.1.3 and 1.1.4, respectively.

\subsubsection{Radio Galaxies}

Cygnus A in the constellation of Cygnus was the first radio galaxy to be identified in 1951. Much before that in 1940s, it was detected by Grote Reber as a radio source. In 1951, it was found that Cygnus A coincides in position with an elliptical galaxy at a distance of 750 million light years (Nicolson 1999). Following this, many radio galaxies have been found and studied. A particular characteristic of a radio galaxy is that most of its emission comes from its huge radio lobes, consisting of energetic charged particles on either side of the central visible galaxy situated at kiloparsecs (kpc) up to megaparsecs (Mpc) distances. Jets are usually seen pointing in opposite directions towards the radio lobes. Spots of enhanced intensity known as Hotspots are often seen where jet particles fall into the radio lobes. These features of radio galaxy, Cygnus A are clearly seen in figure 1.1. The spectrum of a normal galaxy rises steeply at the short wavelengths, reaches a peak in the visible regime, and then falls in the infrared indicating its continuum consisting of stars. But the spectra of a radio galaxy is much flatter decreasing in flux from low to high frequencies. This type of spectrum is characteristic of non-thermal synchrotron radiation emitted by relativistic charged particles influenced by a magnetic field. A detailed discussion on synchrotron 


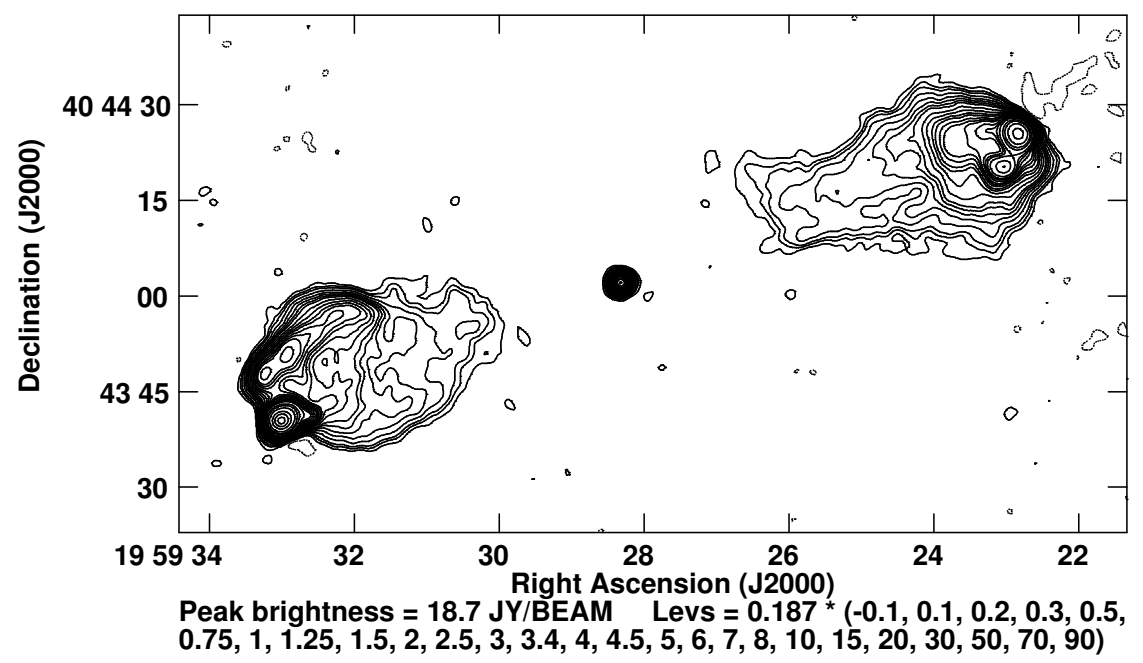

Figure 1.1: The radio map of Cygnus A at $11.06 \mathrm{GHz}$ with a resolution of 2.25 arcseconds (Perley \& Butler 2017).

radiation is given in section 2.1.1 in Chapter 2. The spectra of radio lobes and jets follow a power law, with a typical spectral index of $\alpha \approx-0.65$. The presence of power law spectra and a high degree of linear polarization strongly suggests that the energy emitted by the lobes and jets comes from synchrotron radiation. Strong radio emissions are observed from elliptical galaxies such as Messier 87 (M 87 or Virgo A) or Centaurus A (NGC 5128), which appears to be formed from a merger between an elliptical galaxy and dusty spiral galaxy (Israel 1998). Some radio galaxies have several lobes, aligned along an axis; for example, Centaurus A has inner lobes close to the visible galaxy, and a pair of outer lobes, which span $250 \mathrm{kpc}$ (Israel 1998).

\subsubsection{Luminosity Classification of Radio Galaxies}

The radio galaxies are classified as Fanaroff-Riley I (FR I) and Fanaroff-Riley II (FR II) galaxies based on their luminosity at $1.4 \mathrm{GHz}$ (Fanaroff \& Riley 1974). FR I are weaker radio galaxies which are bright in the centre and decreasing brightness towards edges with luminosities less than $10^{32} \mathrm{erg} / \mathrm{sec} / \mathrm{Hz}$ (Bridle \& Perley 1984). FR II are limb brightened radio galaxies with enhanced emission at the edge of the radio structure or within the radio structure with luminosities greater than $10^{32} \mathrm{erg} / \mathrm{sec} / \mathrm{Hz}$ (Bridle \& Perley 1984; Peterson 1997). In FRI, the ratio of the distance between the brightest spots of radio emission on either side of the center to the full extent of the radio source is typically less than 0.5 while in FR II, the same ratio is greater than 0.5 (Carroll \& Ostlie 1996). FR I have two recognizable radio jets whereas FR II have mostly a single identifiable jet. FR I have curved jets and FR II have straight jets. The best example for FR I type is NGC 1265 and that of FR II type is Cygnus A.

\subsubsection{Extended/Compact Components}

The radio morphology of quasars and radio galaxies is mainly described in terms of two components as extended (or spatially resolved) and compact (or spatially unresolved) with different spectral characteristics (Peterson 1997). The extended component is double i.e. 
with two radio lobes of emission on either side of optical quasars or center of radio galaxies (refer to section 1.1.3 and 1.1.2). Quasars usually coincide in position with compact radio sources. The extended sources can be as large as megaparsecs. The extended component is optically thin to its own radio energy synchrotron emission whereas the compact sources are not. A very detailed discussion on the physics of compact radio source is given in Chapter 2 .

\subsubsection{Blazars}

Blazars are a subclass of AGN which are radio-loud and its jet is oriented close to the line of sight of the observer. They were discovered in 1929 (Loreta 1929) and were thought to be a variable star and was named BL Lacertae for the first time. Later in 1986, it was found to be a variable radio source. Blazars display rapid variability (Heidt \& Wagner 1996) across the entire electro magnetic spectrum going from radio to $\gamma$ rays and emit polarized synchrotron radiation (Fan et al. 1997). High resolution radio images suggest that blazars are core-dominant with most of the emission coming from the parsec scale structures rather than extended components. Blazars have a double-peaked structure in their spectral energy distribution (SED). They are classified as BL Lacertae objects (BL Lacs) and Flat-Spectrum Radio Quasars (FSRQs) based on their optical spectra and luminosity. The spectra of BL Lacs is devoid of absorption and emission lines. Sometimes they show very weak emission lines. This is due to the fact that when we look straight down, the continuum will get boosted in that direction; so even if emission and absorption lines are there, we will not see them in the spectra. But FSRQs have broad emission lines in their spectra. Optically Violent Variable Quasars (OVVs) are also included in FSRQs. FSRQs have higher luminosity than BL Lacs.

\subsubsection{LINERs}

A very low luminosity class of Low Ionization Nuclear Emission Line Region (LINERs) galaxies was identified by Heckmann in 1980 (Heckman et al. 1980). Spectroscopically they resemble Seyfert 2 galaxies except that the low ionization lines for example [OI] $\lambda 6300$ and $[\mathrm{NII}] \lambda \lambda 6548,6583$ are relatively strong for LINERs. This help us to infer that the source is weak in LINERS. Seyfert 2 galaxies, LINERs and HII region can be distinguished from each other by the inspection of their spectra. The ratios $[\mathrm{OIII} / \mathrm{H} \beta]$ and $[\mathrm{NII} / \mathrm{H} \alpha]$ is greater for Seyfert 2 galaxies than LINERs and HII region. The ratio of $[\mathrm{NII} / \mathrm{H} \alpha]$ is greater for LINERs than HII region.

\section{$1.2 \quad$ A Unified Model for AGNs}

A unification model for all the different type of AGNs we observe, which would bring together the wide collection of radio sources - quasars, radio galaxies, Seyfert galaxies, blazars and the compact sources and the varieties within them came into existence with the knowledge that viewing angle of the source with respect to the line of sight of the observer can strongly affect the observational properties of the source. In the past, it appeared like an impossible task. Scheuer \& Readhead (1979) was the first to attempt a unification for AGNs. They proposed that FSRQs can be the relativistically beamed version of radio-quiet 
quasars. Today, a well accepted unification scheme is established by the works of Barthel (1989), Antonucci (1993) and Urry \& Padovani (1995). Most of the discussions in this section are based on the papers of Blandford \& Königl (1979), Meier (2001) and on the monographs by Nicolson (1999) and Burke \& Graham-Smith (1997). A synopsis of what the unified model tells us about an AGN and its energy machine is discussed below with a detailed discussion followed in section 1.3 on different AGN constituents.

In the simplified unification model, an AGN has a central massive object, usually a black hole with a mass $\sim 10^{6}-10^{9} M_{\odot}$ within $0.01 \mathrm{pc}$ or even less i.e. about the size of the Solar System. Because galaxies rotate, matter falling toward the central black hole will conserve angular momentum and will form a rapidly spinning disk of gas called as an accretion disk rather than falling directly into the black hole. The energy of the AGN is derived from the gravitational potential energy of the surrounding material released as it falls into the black hole. A considerable fraction, around $10 \%$ of the rest mass of this material is released in the process (Soltan 1982). The material may be gas from the interstellar medium but it may also include stars which are disrupted as they pass within a Roche limit of the black hole. Angular momentum of the in falling material concentrates the in falling material to an accretion disk with steep gradients of angular velocity. Further collapse occurs through frictional dissipation of the differentially rotating disk, and by turbulent dissipation through a magneto-rotational instability (Balbus \& Hawley 1991). Kinetic energy released by in falling matter, and frictional effects within the accretion disk, raise the temperature of the inner parts of the disk to enormous values and provide plenty of energy to power AGNs on all scales from Seyferts to quasars. Surrounding the thin accretion disk, there is a thicker torus of accreting material, which is cooler and sufficiently opaque to make the thin disk invisible from the side. CO (1 to 0$)$ emission was detected from the infrared quasar IRAS 07598+6508 and the luminous Seyfert galaxies IRAS 08572+3915 and Markarian 463 (Sanders et al. 1989) which indicated the strong presence of gas and dust in torus and disk. The central regions can only be observed from polar directions, not from edge-on. The inner rim of the accretion disk, together with surrounding gas and magnetic fields (Blandford \& Königl 1979; Meier 2001), forms a nozzle that confines the outward flow of energetic particles into narrow collimated flow that shoot out perpendicularly to the plane of the disk, thereby creating the jets that are observed in AGNs and that supply energy and mass to their distant radio lobes.

Figure 1.2 gives an overall picture of our understanding on the unification scheme. Many of the observational differences may arise from intrinsic differences in the strength of emission from the core, the disc, the jets and the lobes. But the most important distinctions arise from the differences between the polar and the edge on aspects from which such a complex object may be viewed (Urry \& Padovani 1995). The dividing angle between pole-on and edge-on is around $45^{\circ}$ from the pole. Seen edge-on, the torus may obscure the core and disk. Seen pole-on, the brightness of the approaching jet, in which material is moving with high velocity, may be greatly enhanced by relativistic beaming (see section 1.6.2). So if we are looking straight down, or very close to the axis of the jet, our view is dominated by jet and its synchrotron emission and the emission from the outer lobes, which lie along the same line of sight, which is much feebler and may not be detected. In these circumstances, we will see a violently variable source with no obvious spectral lines i.e. a blazar. Looking at a moderate angle to the axis of the jets, we see a relatively unobscured compact source i.e a quasar inside the torus of the AGN. From a viewpoint closer to the plane of the torus, the central engine is hidden from direct view and we see instead the jets and lobes of a radio galaxy (Beckmann \& Shrader 2012). A similar picture applies to the less energetic Seyferts, with a Seyfert 2 being seen when the less massive central engine and the fast 


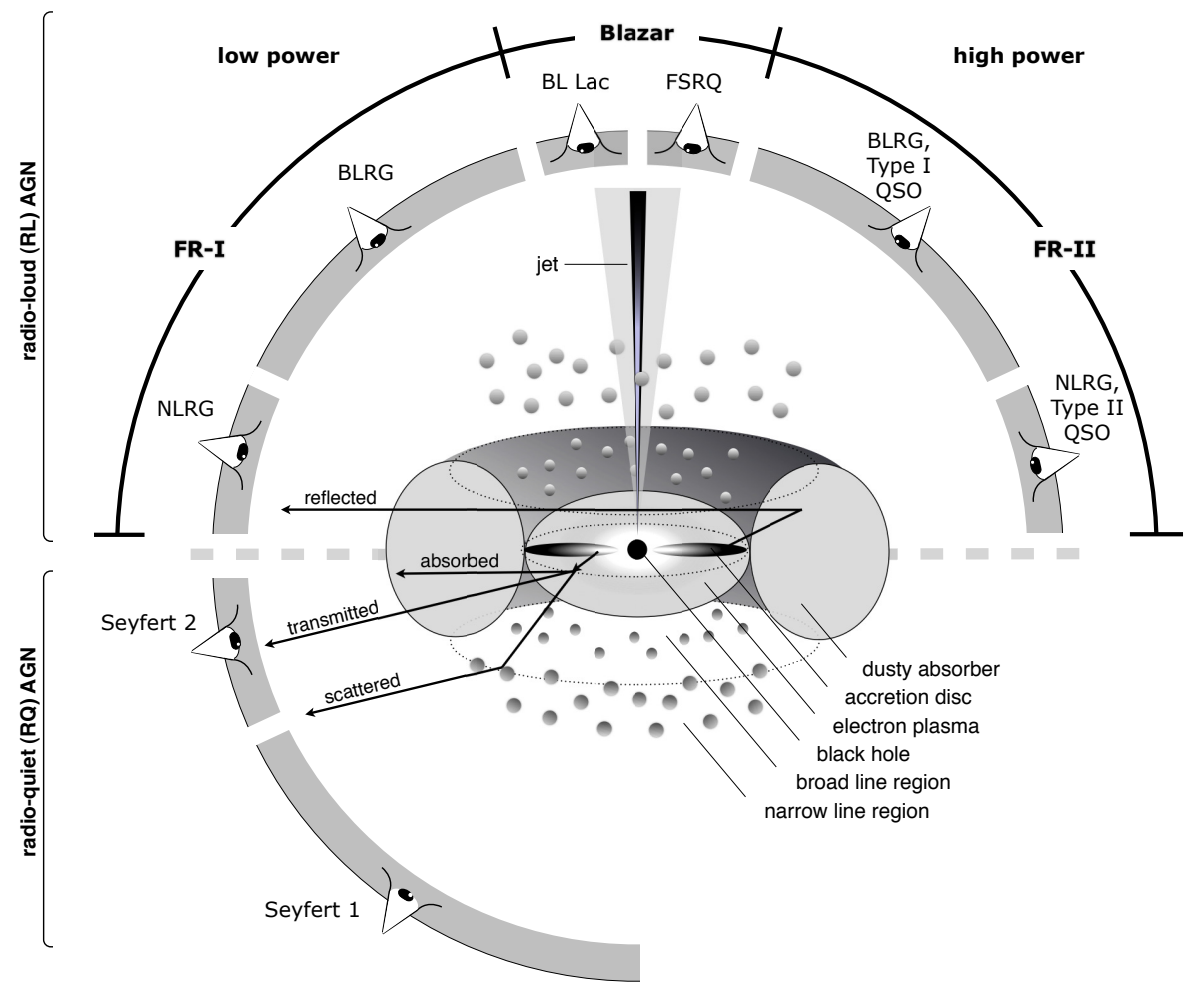

FIGURE 1.2: A schematic representation of the current AGN unification scheme. The AGN type we observe depends up on the viewing angle. The radio-loud and radio-quiet AGNs are shown in the upper and lower part, respectively (Beckmann \& Shrader 2012).

moving inner clouds (the broad line region) are hidden by the torus, and a Seyfert 1 when our line of sight looks over the rim of the torus toward the central regions. Further support of this model come from observations of certain Seyfert 2 galaxies in which light from their innermost regions has been scattered towards us by dust clouds located above and below the plane of the torus. Spectra of this light, which originates in fast moving clouds close to the central engine display the broad lines that are normally associated with Type 1 Seyferts (Smith et al. 2004).

\subsection{The Different AGN Constituents}

As discussed in section 1.2, the summary of the established model for an AGN phenomenon is a central engine that consists of a hot accretion disk surrounding a supermassive blac khole, where energy is generated by gravitational infall of matter which is heated to high temperature in a dissipative accretion disk on to a supermassive blackhole. Now, I will review the different AGN constituents - the supermassive black hole, the accretion disk, the broad-line region, the narrow-line region, the torus, the relativistic jets and radio lobes in detail. Most of the discussions below are based on the monograph by Nicolson (1999), Peterson (1997) and Burke \& Graham-Smith (1997). 


\subsubsection{The Supermassive Black hole}

Every active galactic nucleus contains a supermassive black hole with a mass of $\sim 10^{6}-$ $10^{10} M_{\odot}$ at the center. A peculiar surface called Event Horizon is a region around a black hole whose radius depends on the mass of the black hole. It is a boundary in space-time beyond which any event cannot affect an observer from outside. The radius of the event horizon is known as Schwartzchild Radius, $R_{\mathrm{S}}=2 G M_{B H} / c^{2}$ where G is the gravitational constant, $\mathrm{c}$ is the speed of light and $M_{B H}$ is the mass of the black hole. The escape velocity in event horizon is greater than the speed of light which makes it "invisible". It means that all lightlike paths within an event horizon in the forward light cone are twisted such that matter fall into the event horizon. When a particle is trapped inside event horizon, it actually moves forward in time and thereby moves into the black hole depending on the spacetime coordinate system (Hawking \& Ellis 1975). A rotating black hole is known as Kerr black hole and the spin, $\mathrm{s}$ is given as $s=(J c) /\left(G M_{B H^{2}}\right)^{2}$ where $\mathrm{J}$ is the angular momentum of rotation of the black hole (Visser 2007). A rotating black hole has an elliptical region around the event horizon known as Ergosphere. A particle entering the ergosphere can still escape from the black hole by acquiring energy from the rotational energy of the black hole. A very detailed discussion of this phenomena is presented in section 2.3.1 of Chapter 2 while discussing the jet formation. In the following section, four fundamental arguments which can prove that a supermassive black hole exists at the centre of an AGN is presented.

\subsubsection{Virial Theorem Argument}

Since the material in the nucleus of an AGN is gravitationally bound, the mass of the nucleus can be estimated. According to Virial theorem given by:

$$
\begin{aligned}
2<T>+<V> & =0 \\
2 \times \frac{m v^{2}}{2} & =\frac{G M m}{r} \\
M & =\frac{v^{2} r}{G}
\end{aligned}
$$

where $v$ is the velocity dispersion of the gas and it can be taken as $10^{3} \mathrm{~km} / \mathrm{s}$ from the widths of broad emission lines (section 1.1.1). The upper limit for size of an AGN is roughly $100 \mathrm{pc}$. Thus the mass of the nucleus comes out as $10^{9} M_{\odot}$. So in a tiny volume of cubic parsec, an extreme mass of $10^{9} M_{\odot}$ packed implies the existence of a supermassive black hole candidate. This method of estimating the black hole mass from gas dynamics is known as Reverberation Mapping (Peterson \& Bentz 2006; Peterson et al. 2014; Blandford $\&$ McKee 1982). Naturally, a question may arise in the mind of readers about why one has to estimate mass from Virial theorem in a region of gas radiation pressure where a Maxwell Boltzmann distribution given by:

$$
\frac{1}{2} m v^{2}=K_{B} T,
$$

has to be considered by classical gas dynamics? If we use Maxwell Boltzmann relation in this region with gas velocity $v=1000 \mathrm{~km} / \mathrm{s}$ and mass of hydrogen, $m \approx 1.627 \times 10^{-27}$ $\mathrm{kg}$, then an unrealistic temperature, $\mathrm{T}=58982246.38$ kelvin $\approx 60$ million kelvin comes out. This is an unrealistic temperature, impossible from thermal emission. This gives an 
indication that the velocity of gas in the central engines in AGNs is not of thermal origin but of some other non thermal origin.

There are other methods as well to estimate the black hole mass. The orbit of companion star of black hole which orbit around it can be determined. Thus once we know the orbital velocity of companion star (this can be obtained from the spectra of star), then using Keplerian law of motion or by using Virial theorem, the black hole mass can be estimated. More precisely to get the mass of the central black hole, the broad emission line width and the distance of the broad line region from the center together with the assumption of Keplerian motion can be used. But this may be affected by the errors in the assumption of BLR kinematics, dynamics and radius. The $H_{\beta}$ line width and continuum luminosity was used by Laor (1998) to derive black hole mass. The motion of single stars in the galactic center are studied and the black hole mass has been estimated by Eckart (2002).

There exists a correlation between the black hole mass and the bulge mass of normal galaxies (Laor 1998, 2000) which can be also used to estimate the black hole mass in the center of galaxies. This correlation is similar to the black hole mass versus bulge luminosity correlation found by Magorrian et al. (1996) in a sample of 32 nearby normal galaxies using the data from Hubble Space Telescope. John Magorrian showed a surprising result between the mass of the central object and the properties of surrounding galaxies. In every case, the central black hole comprises about $0.5 \%$ of the mass of the stars in the spheroid of the galaxy. This was further investigated by Magorrian \& Tremaine (1999). Later Merritt \& Quinlan (1998) showed that the black hole mass is several times greater than $0.5 \%$ of spheroid mass. Later, Merrit along with Ferrarese proposed the correlation (Ferrarese \& Merritt 2000) between the black hole mass, $M_{B H}$ and stellar velocity in the bulge, $\sigma_{b}$ given by:

$$
M_{B H}=3.1 \times 10^{8}\left(\frac{\sigma_{b}}{200 \mathrm{~km} \mathrm{sec}}\right)^{4} M_{\odot} .
$$

All these studies clearly show the unavoidable role of black hole in a galaxy formation and its evolution. There are two possibilities - one is that the black holes were there at first in the center of host galaxies and accreted a closely proportional amount of matter to make stars or the other possibility is that the galaxy was there at first and grew a black hole whose size always stayed a constant fraction of the stellar mass.

\subsubsection{Eddington Luminosity Argument}

Assume isotropic and completely ionized gas (for example hydrogen gas) system. Under equilibrium, the outward force of radiation pressure must be counter balanced by the inward force of gravity given by:

$$
\begin{aligned}
\text { Gravitational force, } F_{G} & =\frac{G M\left(m_{p}+m_{e}\right)}{r^{2}} \\
\text { The outward momentum flux, } P_{\text {rad }} & =\frac{F}{c}=\frac{L}{4 \pi c r^{2}} \\
\text { Radiation Force, } F_{\text {rad }} & =\sigma \frac{L}{4 \pi c r^{2}}
\end{aligned}
$$

The outward radiation force on a single electron is obtained by multiplying its cross section of interaction with a photon. It is significant only for electron not proton (because $\sigma$ is inversely proportional to square of mass). For the source to remain stable, the inward 
gravitational force should balance or exceed the outward radiation force which is given by:

$$
\begin{aligned}
F_{r a d} & \leq F_{G} \\
\sigma \frac{L}{4 \pi c r^{2}} & \leq \frac{G M m_{p}}{r^{2}} \\
L & \leq \frac{4 \pi G c m_{p} M}{\sigma} \\
L_{E d d} & \cong 1.26 \times 10^{31} \frac{M}{M_{\odot}} \text { Watts }
\end{aligned}
$$

This $L_{E d d}$ is called the Eddington limit or Eddington luminosty, which is the upper limit to the luminosity that can be achieved in a system where the radiation pressure and gravitational pressure are balanced (Rybicki \& Lightman 1979). In the case of AGNs, the energy production mechanism is the accretion of matter on to a supermassive black hole. If the radiation pressure force exceeds the gravitational force, accretion stops in the case of AGNs. $\mathrm{L}_{E d d}$ can be used to estimate the minimum mass to support an AGN. So for a typical quasar of luminosity of $10^{39}$ watts, the upper limit on mass, $\mathrm{M}$ can be estimated as:

$$
M \geq \frac{L}{1.26 \times 10^{31}} M_{\odot} \geq 3.96 \times 10^{8} M_{\odot}
$$

This is the lower limit on the mass of a system reached Eddington luminosity. At the same time, the mass of a black hole, $M_{B H}$ with a typical Schwartzchild radius $R_{s}=3.0 \times 10^{11}$ meters can be estimated as:

$$
M_{B H}=\frac{R_{s} c^{2}}{2 G} \cong 3.7 \times 10^{8} M_{\odot}
$$

The two mass estimates above are of the same order and this is good enough to support that a supermassive black hole is powering the AGN at the centre.

\subsubsection{Rapid Variability Argument}

The rapid variability of AGN implies that their central energy sources must be very small (Peterson 2001) on the cosmic scale. Gaskell \& Klimek (2003) describes that AGN shows variability from optical to X-ray in every bands. Because no signal can travel faster than light, no source of light can vary substantially in brightness in a period of time shorter than the time taken for light to travel from one side of it to the other. For example, imagine a spherical light source of diameter ' $\mathrm{D}$ ' that emits a brief flash of light simultaneously from every point on its surface. The time interval, $\delta \mathrm{t}$, between the arrival of light from the centre of the front hemisphere of the source at a distant observer and the arrival of light from the centre of the far side will be equal to the time taken by light to cross the diameter of the object given by $\delta \mathrm{t}=\mathrm{D} / \mathrm{c}$. The observer will not see a brief flash of light, but will instead see the change in brightness spread out over the time interval $\delta$ t. The diameter of the light source must be less than or equal to the speed of light multiplied by the duration of the fluctuation i.e D must be less than or equal to $c \delta$ t. For example, if a quasar undergoes a substantial variation in brightness in 1 year, its energy source can be no longer than 1 light year across. If it fluctuates in a day, the diameter of the energy source is less than 1 light day and so on (Nicolson 1999).

Because the under lying black hole has to be smaller than the accretion disk from which the energy is escaping, the timescale of variability provides an upper limit to its size and 
mass. As we have seen, if the brightness fluctuates in 1 day (approximately $10^{5}$ seconds), the energy source and hence the size of the black hole has to be less than 1 light day. In $10^{5}$ seconds, light travels a distance, $\mathrm{D}=\mathrm{c} \delta \mathrm{t}=3 \times 10^{5} \times 10^{5}=3 \times 10^{10} \mathrm{~m}$. Because the radius $\mathrm{R}$, of a black hole of mass $\mathrm{M}$ (expressed in solar masses) is " $3 \mathrm{M}$ " $\mathrm{km}$, then the mass of a black hole of radius $\mathrm{R}$ is $\mathrm{R} / 3$. If the radius of the black hole were $3 \times 10^{10} \mathrm{~km}$, it's mass would be $10^{10}$ solar masses. As a result, the mass of the black hole that may lie at the centre of a source that fluctuates in brightness in 1 day must be less than $10^{10}$ solar masses. We can also estimate the minimum mass for the black hole from Eddington limit using equation 1.9. So for an AGN with luminosity of $10^{39}$ watts (W), the mass of the underlying black hole would have to be at least $10^{39} / 10^{31}=10^{8}$ solar masses and for a luminosity of $10^{40} \mathrm{~W}$, at least $10^{9}$ solar masses, and so on. Taken together these two approaches point to there being supermassive black holes ranging in mass $10^{6}-10^{9} M_{\odot}$ at the center of AGNs.

\subsubsection{Observational Evidence for SMBH}

The fourth argument is nothing but the observational evidences for the presence of massive compact objects in AGNs comes from high resolution imaging and spectroscopy. For example, the optical images of the central regions of many active galaxies reveal a tiny bright spot where the intensity of light reaches a sharp peak. This implies that stars are concentrated together much more closely than would be the case if they were not being herded together in the gravitational well of a supermassive compact body. If a supermassive compact object is present in the centre of a spiral or disk shaped galaxy, the orbital speeds of stars and gas clouds will rise sharply to a peak as the centre is approached. Because stars and gas clouds on one side of the centre will be approaching the observer, whereas those on the other side will be receding, spectroscopic observations will reveal a sharply rising blue shift as the centre is approached from one side and a sharply rising red shift as it is approached from the other. In active elliptical galaxies where individual stars and gas clouds move in random directions and orientations, high speeds in their central regions will result in spectral lines being broadened rather than being shifted to longer or shorter wavelengths (Nicolson 1999).

For example, M 87 has a central intensity spike and rationally broadened lines consistent with stars being concentrated together and revolving within the gravitational field of a compact mass believed to be a supermassive black hole of $\sim(2-3) \times 10^{9} M_{\odot}$. Observations made in 1994 with the Hubble Space Telescope resolved a gaseous disk at the centre of M 87 and determined its rotational velocity as $750 \mathrm{~km} / \mathrm{sec}$ (Nicolson 1999). The results implied that a mass equivalent to $2.4 \times 10^{9} M_{\odot}$ was contained within a region of space less than 20 light years across and were entirely consistent with the presence of a central supermassive black hole. Since then, various active and radio galaxies have been shown to have flattened disks of gas and dust around their central engines. For example, Doppler measurements of the rotational speeds of gas clouds in the core of the spiral galaxy M 84 (Laing \& Bridle 1987) imply the presence of a compact central body of $\sim 300$ million solar masses, whereas the core of NGC 6251 has been shown to contain a twisted dusty disk or ring that appears to be illuminated by intense ultraviolet light (Crane \& Vernet 1997) originating from the vicinity of a central black hole. An X-ray spectrograph from the Japanese ASCA satellite has detected X-ray emission line from ionized iron $\left(K_{\alpha}\right.$ line) from the galaxy MCG-6-30-15 that appears to originate from extremely hot gas in the inner part of an accretion disk. There is a marked asymmetry towards energies lower than the rest-energy of the emission line $(6.4 \mathrm{keV})$. This asymmetry is most likely caused by 
gravitational and relativistic Doppler shifts near the black hole at the center of the galaxy (Tanaka et al. 1995; Iwasawa et al. 1996). The overall profile of the line is consistent with its being distorted by the gravitational redshift, as would be expected if the radiation originated from deep within the gravitational space-time that surrounds a massive black hole, extending between $\left(3-10 R_{s}\right)$. These and other examples provide strong circumstantial evidence in favor of the hypothesis that AGNs are indeed powered by supermassive black holes.

\subsubsection{The Accretion Disk}

A very crucial component of the AGN unified model is the accretion disk. Provided that the orientation allows direct observation, optical and X-ray spectra, and polarization give a detailed account of conditions in this compact and very energetic region of discs. The whole disk is only $\sim 10^{-3}-10^{-2}$ pc. Radiation from the disk is thermal and the continuum spectra should therefore provide a measure of the temperature. A detailed discussion on the energy generation in accretion disk via the accretion of matter was given in section 1.2. The observed spectra of the disk composites of thermal radiation over a range of temperatures typically $10^{4}-10^{6} \mathrm{~K}$ calculated from the relation:

$$
T(R)=\left[\frac{3 G M_{B H} \dot{M}}{8 \pi R^{3} \sigma}\right]^{1 / 4},
$$

where the temperature depends on the radius of the accretion disk, $\mathrm{R}$ and mass accretion rate, $\dot{M}$. This relation is an outcome of the assumption that a classical optically thick and geometrically thin accretion disk follows the black body spectrum. $T_{\text {disk }} \propto \dot{M}^{1 / 4}$ indicates that for less massive black holes, the accretion disk temperature is quite large. Typically, AGN accretion disks emit a large amount of energy as optical-UV forming the "blue bump" and soft X-ray emission photons forming the "big blue bump" in the spectra. The upper limit to the luminosity that can be reached in the accretion process is given in section 1.3.1.2. Optical spectral lines are observed from AGNs, predominantly from a small number of atomic species, for example, $\mathrm{H}, \mathrm{He}, \mathrm{C}, \mathrm{N}, \mathrm{O}, \mathrm{Mg}$, Ne as seen in $\mathrm{H}$ II regions. Their characteristic is an extreme broadening which can be accounted to the rotation and turbulent motion in the disk.

\subsubsection{Fueling the Accretion Disk}

The fundamental process at work in an AGN is conversion of mass into energy as discussed in section 1.2 which takes place in accretion disks. This is done with efficiency $\eta$ so that energy available from a mass, $\mathrm{M}$ is $E=\eta M c^{2}$. The rate at which energy is emitted by the nucleus is given by:

$$
\begin{aligned}
& L=\frac{d E}{d t} \\
& L=\eta \dot{M} c^{2}
\end{aligned}
$$

where

$$
\dot{M}=\frac{d M}{d t}, \text { is the mass accretion rate. }
$$

We know gravitational potential, $U=-G M m / r$. The rate at which potential energy of infalling material on to the supermassive black hole that can be converted to radiation is 
given by:

$$
\begin{aligned}
& L=\frac{d U}{d t} \\
& L=\frac{G \dot{M} m}{r} .
\end{aligned}
$$

Therefore,

$$
\eta \propto(m / r),
$$

i.e. efficiency is proportional to compactness of system. The compactness is maximized in the case of black hole. The energy available for a particle falling within Schwartzchild radius say $5 R_{s}$ is:

$$
U=\frac{G M m}{5 R_{s}}=\frac{G M m}{\left(\frac{10 G M}{c^{2}}\right)}=0.1 m c^{2},
$$

i.e. efficiency of the accretion process $\eta=0.1$ is much greater than the efficiency of nuclear fusion whose efficiency is 0.007 . For example, the efficiency for accretion on to a $0.85 M_{\odot}$ white dwarf is $1.9 \times 10^{-4}$. Since Eddington luminosity for quasars is $\approx 10^{46} \mathrm{erg} / \mathrm{sec}$, the mass accretion rate $\dot{M} \approx 2.2 \times 10^{8} M_{\odot} /$ year. The factor $\eta$ depends on the compactness of the system and characteristics of the accretion disk. The efficiency is maximum if the disk is optically thick and geometrically thin (Shakura \& Sunyaev 1973) whereas the optically thin and geometrically thick accretion disks are very inefficient in accretion process. Soltan (1982) has also calculated the efficiency of conversion of mass into radiation in AGNs as $10 \%$. Assuming $10 \%$ efficiency, Soltan (1982) calculated the mass contained in the dead quasars in $1 \mathrm{Gpc}^{3}$ is $8 \times 10^{13} M_{\odot}$. By equating equations 1.14 and 1.8 , a limit on the accretion rate can also be obtained known as the Eddington accretion rate given by:

$$
\dot{M}_{E d d}=\frac{4 \pi G m_{p} M_{B H}}{\eta c \sigma} .
$$

There are observational evidences for super-Eddington accretion rates for example Pounds \& Page (2004) observed super-Eddington accretion rates in the quasar PG1211+143 and Jin et al. (2017) in quasar RX J0439.65311I. Collin et al. (2002) coined out that accretion proceeds at Eddington or super-Eddington rates through slim or thick disks. Another possibility suggested by Collin et al. (2002) is that the optical luminosity is not produced by the gravitational release of energy, but by a mechanism for example, by the reprocessing of X-ray photons from the central source in a geometrically thin disk and therefore, superEddington rates are not required.

In advection-dominated accretion flows (ADAF), most of the energy viscously dissipated in the differentially rotating flow is stored as entropy rather than being radiated (Narayan et al. 1994). Narayan \& Yi (1994) stated that advection-dominated accretion may provide an explanation for the slow spin rates of accreting stars and the widespread occurrence of outflows and jets in accreting systems. Thus the less efficient ADAFs can be used to explain also the low-luminosity AGNs observed. Meier (2001) presented a model in which the strong jets are produced when the accretion is geometrically thick (for example in an advectiondominated or a convection dominated accretion flow) and associated with a rotating black hole. 


\subsubsection{The Broad and Narrow Line Regions}

Often the lines from the centre of AGNs show a spectrum in which they have two distinct components (Khachikian \& Weedman 1974), one with width corresponding to velocities ranging from $(1000-25,000) \mathrm{km} / \mathrm{s}$. This originates in Broad Line Region or BLR. The other with width corresponding to less than $500 \mathrm{~km} / \mathrm{sec}$ originating from Narrow Line Region or $N L R$. It has been proposed that the BLR is the accretion disk itself at a typical distances of $\sim 0.01-1 \mathrm{pc}$ with electron number density $\geq 10^{9} \mathrm{~cm}^{-3}$, while the NLR is a more extended region, extending high above the disk at a distance of $\sim 100-1000 \mathrm{pc}$ with electron number density $10^{3}-10^{5} \mathrm{~cm}^{-3}$, which can be observed even if the dusty torus (see section 1.3.4) obscures the accretion disk. The mass inside the BLR is of the order $10^{3}-10^{4} M_{\odot}$ and that of NLR is of the order $10^{6} M_{\odot}$. The typical temperature in BLR region ranges from $10^{4}-10^{5} \mathrm{~K}$ (Peterson 1997). The narrow line region spectrum contains both permitted lines (HI, HeI, HeII) and forbidden lines (like OIII) But the BLR spectrum contains mostly permitted lines like hydrogen Lyman alpha (Ly $\alpha$ ), CIV, MgII only which is an indication of its high electron number density and sometimes semi forbidden lines like CIII is also seen as seen in its spectrum in figure 1.3. The size of BLR is related to the AGN continuum luminosity as

$$
R_{B L R} \propto L^{1 / 2},
$$

which is used to determine the black hole mass by reverberation mapping as discussed in section 1.3.1.1.

The line profile for both the BLR and the NLR may be asymmetrical, extending towards the blue, indicating an outflow towards the observer. Continuum radiation from the BLR extends up to the $\mathrm{keV} \mathrm{X}$-ray region; above $3 \mathrm{keV}$ there is featureless continuum while at lower X-ray energies absorption can be seen, giving steps in the continuum. BLR radiation is usually taken as the distinguishing characteristics of quasars and Seyferts. When the model AGN is seen from the side, the torus obscures the direct radiation from the BLR, while the NLR is still observable. Part of the continuum radiation from the BLR can, however be observed as scattered radiation from above the obscuring torus. This accounts for a lower level of optical continuum which is highly polarized (about 15-20\%; Antonucci \& Miller 1985); the polarization is usually wavelength independent as expected from scattering by electrons. Part of the BLR X-rays are also scattered; this again implies that the scattering region is ionized, and suggests that it may be identified with the NLR itself. The NLR is the silent feature in Seyfert galaxies, where it may be resolved observationally into a region several hundred parsecs across.

\subsubsection{Molecular Torus}

As discussed in the previous section, the central engine of an AGN can be obscured by a region of very dense clouds of dust which resembles the geometry of a torus. This region known as Molecular Torus, is more of a deduction from the observations. There is an excess of infrared radiation from AGNs, which indicates the presence of a dust cloud, and several AGNs contain huge $\mathrm{H}_{2} \mathrm{O}$ masers, analogous to those observed in molecular clouds around some stars in the Milky Way. The torus is therefore depicted as a molecular cloud with both hot and warm dust with temperature ranging from $10^{2}-10^{3} \mathrm{~K}$.

Direct observation of the torus in a typical AGN requires an angular resolution of the order of one milli arc second. Thanks to VLBI with which this resolution can be achieved. Also, 


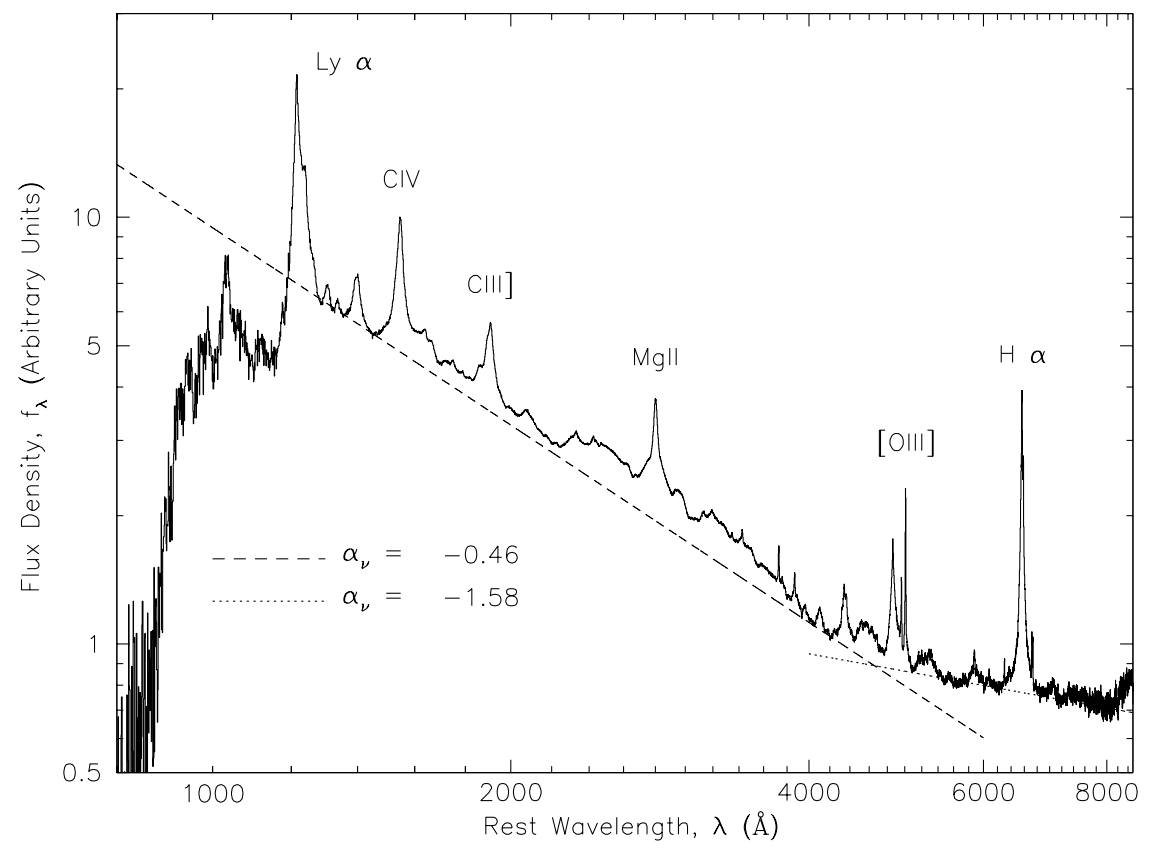

FIGURE 1.3: Optical/UV spectrum for optically-selected quasars in the SDSS adopted from Vanden Berk et al. (2004). Permitted broad lines and semiforbidden broad line CIII and the forbidden OIII narrow line can be seen.

the narrowness of the water maser lines allow the dynamics of the torus to be revealed from the distribution of spectral components across the central regions of the AGN. This has been achieved by Miyoshi et al. (1995) in the nucleus of the spiral galaxy NGC 4258 (M 106), where the water maser sources are seen to be strung along a line, and their velocities vary progressively along the line, towards us at one end and away at the other. They are convincingly represented as located in a flattened inner part of the torus, which is rotating at velocities up to $900 \mathrm{~km} / \mathrm{s}$ round a massive central object, with a mass of 40 million solar masses. The water masers of NGC 4258 illustrate well the origin of maser emission, which may either be the selectivity amplified emission from a continuum source lying behind the maser, or it may be self generated within the maser itself. The torus as a ring round a compact central source, cannot itself be seen directly. The spectrum of NGC 4258 has components at velocities around $\pm 900 \mathrm{~km} / \mathrm{s}$, which derive from the edges of the rotating torus, where the line of sight is a maximum length.

Further VLBI observations (Herrnstein et al. 1999) have provided measurements of the lateral movement of the central components, giving their orbital velocities. Combining the angular size and the velocities gives a distance $7.2 \pm 0.3 \mathrm{Mpc}$ to NGC 4258 , which is both precise and independent of all other distance determinations, the spin axis of the AGN is aligned with the radio jets which are seen at much larger distances, about $500 \mathrm{pc}$, from the nucleus. The radiation axis of the whole galaxy is, however at an angle of $119^{\circ}$ to that of the nucleus and its jets. Such misalignment seems to be common; the behavior of the AGN is independent of the alignment of the main bulk of the galaxy (Burke \& Graham-Smith 1997). The typical column density in this region is of the order $\sim 10^{21-22} \mathrm{~cm}^{-2}$. These column densities are high enough to obscure the AGN. Molecular hydrogen, $\mathrm{H}_{2}$, is detected in nine Seyfert 1 galaxies studied by Hicks \& Malkan (2008). The size scales of torus ranges from 10-60 pc. The torus is geometrically thick with the disk scale heights of $10 \mathrm{~s}$ of pc. 


\subsubsection{Relativistic Jets from AGN}

Jets are collimated flows of plasma, observed in young stars, Gamma Ray Burst (GRB), AGNs, X-ray binaries etcJets were discovered in 1918 for the first time associated with an elliptical galaxy M 87 in the Virgo cluster. Understanding the physics of jet formation in AGN puzzled astronomers for years and it still remains as an open question. How the relativistic jets are accelerated and collimated to large distances?. How the mechanism of jet launching is connected to the properties of host galaxy and how does the jet affects the surroundings during its propagation?. How does the relativistic plasma in the jet overcome the kink instability? Why jets are not seen in all of the AGNs?. However, over the past few decades, our understanding of the dynamics of jet formation has changed. The seat of all activity of an AGN is believed to be a supermassive black hole and the wide aspect of thinking of many astronomers that how the various properties of supermassive black holes can be linked to the production and propagation of jets has become the core studies. In all of the astrophysical sources where jets are observed, one thing is observationally clear that accretion takes place onto the central compact object, a star or a black hole. Therefore, it is obvious that accretion also play an important role in the production of jets.

In AGNs, the jets are mainly made of relativistic plasma (Walker et al. 2001) consisting of at least a magnetic field $(\geq 1 \mu \mathrm{G})$ and they show "superluminal" motion (see section 1.6.1). The apparent velocities, $\beta_{a p p}$ as high as $\sim 50 \mathrm{c}$ (Lister et al. 2009) have been observed in jets while studying a sample of 135 radio-loud AGN from a 13 year monitoring programme called "Monitoring Of Jets in Active Galactic Nuclei with VLBA Experiments (MOJAVE)". The observation of synchrotron radiation from jets confirms that the jets consist of magnetized plasma. The detection of low-linear polarization (than synchrotron radiation) implies that the plasma in relativistic jets is highly hydro magnetically turbulent. There is observational evidence that the relativistic jets originate in sub-parsec scales and can be accelerated and collimated to large distances up to kiloparsecs and even Megaparsecs (Mpc) as seen in the case of sources like M87 (Biretta et al. 1983), Cygnus A (Perley et al. 1984), J2345-0449 (Bagchi et al. 2014) etc. A substantial fraction of accretion energy is carried away by jets and it is generally believed that the jet emission we see come mostly from relativistic electrons. The various plausible jet compositions are discussed in detail in section 2.3.6 in Chapter 2.

According to Rees (1971) and Scheuer (1974), the jets are powered by central black holes and associated accretion disks and the jets themselves transport energy, momentum, and angular momentum over vast distances from black holes to radio hot spots, hot spot complexes and lobes upto megaparsec. The optimal combination of a rapidly spinning supermassive black hole and magnetic field is important in building up a jet triggering mechanism. The rotational energy of a rapidly spinning black hole acts as the immense energy source behind jets and the energy of a spinning black hole can be extracted via Blandford and Znajeck mechanism (Blandford \& Znajek 1977) or Penrose mechanism (Penrose 1969; Penrose \& Floyd 1971), and theoretically up to $29 \%$ of the energy of a Kerr black hole (i.e. spinning black hole) can be extracted (Christodoulou \& Ruffini 1971; Li \& Paczyski 2000). The mechanism of jet formation, acceleration and collimation is discussed in detail in section 2.3 in Chapter 2. 


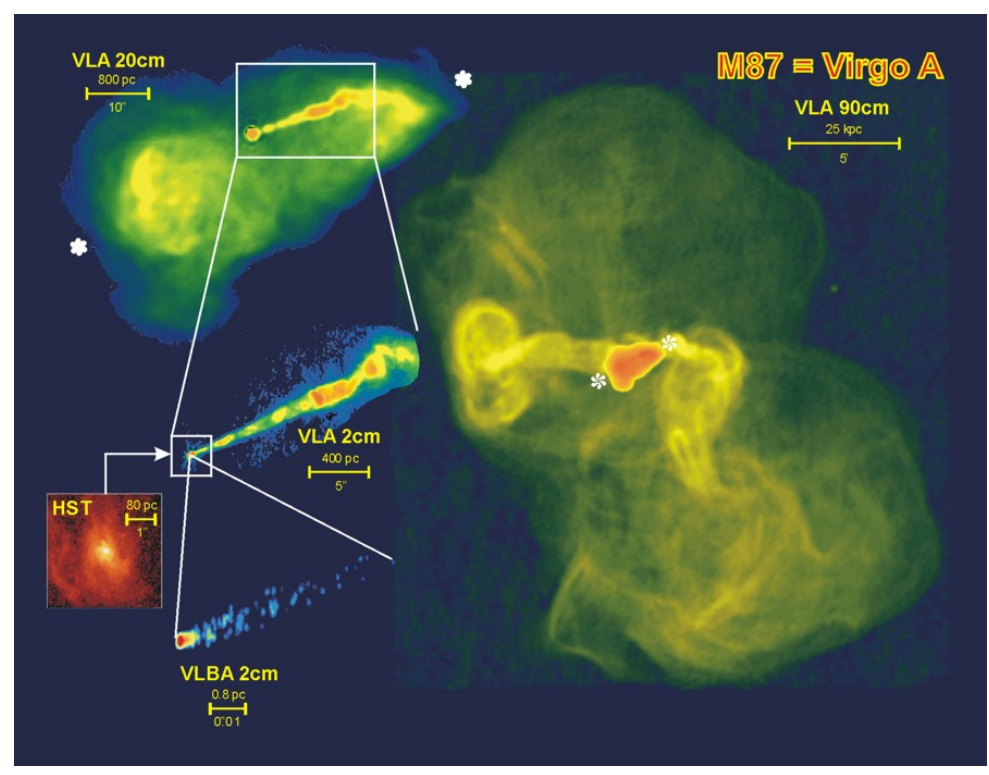

Figure 1.4: Radio images of the galaxy M87 at different scales. Credit: National Radio Astronomy Observatory/AUI - F. Owen, J. Biretta, J. Eilek, \& N. Kassim

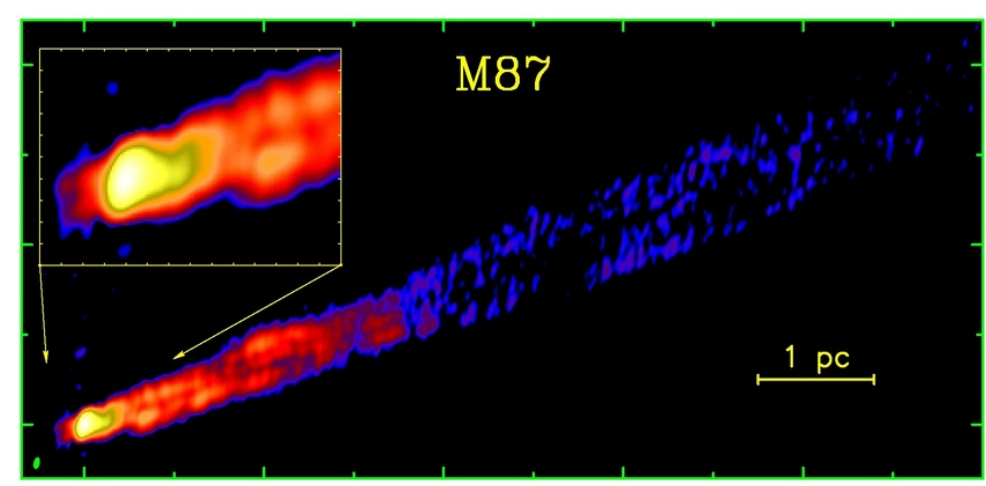

Figure 1.5: The Inner Jet of the Radio Galaxy M87 at $15 \mathrm{GHz}$. The x-axis is right ascension and y axis is declination, both in in milliarcsecond unit. Credit: Y.Y. Kovalev, MPIfR Bonn.

\subsubsection{Radio Lobes}

A very detailed discussion on the relativistic jets formation, acceleration and collimation is given in section 2.3 in Chapter 2. As a jet of material travels outwards, its energy primarily resides in the kinetic energy of the particles. However the jet encounters the resistance as it penetrates the interstellar medium within the host galaxy and the interstellar medium beyond. As a result, the material at the head of the jet is slowed, and a shock front forms there (Carroll \& Ostlie 1996). The accumulation and deceleration of the particles at the shock front causes the directed energy may be shared equally by the kinetic and magnetic energy. Shock waves may accelerate charged particles by magnetically squeezing them, reflecting them back and forth inside the shock. The problem of calculating the motion of a jet through the intergalactic medium is so complicated that extensive numerical simulations are required to model the process. The motion of the charged particles and the magnetic fields within the lobes of radio-loud objects contain an enormous amount of energy. For example in Cygnus A, the energy of each lobe is estimated to be $10^{53}$ to $10^{54}$ Joules, 
equivalent to energy emitted by $10^{7}$ supernovae. The radio lobes of Cygnus A can be clearly seen in figure 1.1 and in Very Large Array (VLA) $6 \mathrm{~cm}$ image shown on top in figure 2.5. In radio lobes, the energy is equally shared between kinetic and magnetic energy which means that half of the energy in $V_{\text {lobe }}$ is magnetic i.e. in $V_{\text {lobe }} / 2$, where $V_{\text {lobe }}$ is the volume of the lobe. Therefore, the total magnetic energy contained in a radio lobe can be expressed as:

$$
\frac{V_{\text {lobe }}}{2}=\mu V_{\text {lobe }}=\left(\frac{B^{2}}{2 \mu_{o}}\right) V_{\text {lobe }}
$$

where the magnetic energy stored per unit volume, $\mu=\frac{B^{2}}{2 \mu_{o}}$.

\subsection{Brightness Temperature}

The spectral distribution of the radiation of a black body in thermal equilibrium with its surroundings, $I_{\nu}$ is given by the Planck law :

$$
I_{\nu}(T)=\frac{2 h \nu^{3}}{c^{2}} \frac{1}{e^{\frac{h \nu}{k_{B} T}}-1},
$$

where $I_{\nu}$ is the specific intensity, $T$ the temperature, $\nu$ the frequency, $c$ the velocity of light and $h$ the Planck constant. In the radio regime since $h \nu<<K_{B} T$, Planck's law can be expanded using a taylor expansion as:

$$
e^{\frac{h \nu}{k_{B} T}} \cong 1+\frac{h \nu}{k_{B} T}+{\frac{h \nu}{k_{B} T}}^{2}+\ldots .
$$

which modifies equation 1.20 into:

$$
I_{\nu}(T)=\frac{2 \nu^{2}}{c^{2}} k_{B} T
$$

which is referered to as Rayleigh-Jeans approximation. So brightness temperature $T_{b}$ can be defined as:

$$
T_{b}=\frac{c^{2}}{2 k_{B}} \frac{1}{\nu^{2}} I_{\nu}=\frac{\lambda^{2}}{2 k_{B}} I_{\nu}
$$

As a result, for a source with a flux density $S_{\nu}$ and solid angle $\Omega$, the brightness temperature is given by:

$$
T_{b}=\frac{1}{2 k_{B}} \frac{S_{\nu \lambda^{2}}}{\Omega}
$$

where $I_{\nu}=\frac{S_{\nu}}{\Omega}$. Equation 1.24 holds for black body emitters. Historically, brightness temperature has been used also for non thermal sources which follows a power law spectrum. So brightness temperature can be treated as a virtual temperature which corresponds to the temperature that the source should have as if it was a black body to radiate the specific intensity $I_{\nu}$. The extremely high observed brightness temperatures measured in relativistic jets lead us to the understanding that the processes involved in jets are of non-thermal nature.

If the source can be represented as a circular Gaussian with a full width at half maximum $\theta_{\text {FWHM }}$, then the solid angle of the source is:

$$
\Omega=\frac{\pi}{4 \ln 2} \theta_{\mathrm{FWHM}}^{2} .
$$


So brightness temperature of the emission region represented by the Gaussian component with the total flux density $S_{\text {tot }}$ and the angular size $d\left(\equiv \theta_{\mathrm{FWHM}}\right)$ is given by:

$$
T_{b}=\frac{2 \ln 2}{\pi k_{B}} \frac{S_{t o t} \lambda^{2}}{d^{2}}(1+z) .
$$

The factor $(1+\mathrm{z})$ in equation 1.26 reflects the cosmological effect on the observed brightness temperature. For the sources with unknown redshift, the brightness temperature can be simply calculated in the observer's frame of reference. The above formula can be simplified as:

$$
T_{\mathrm{b}}[\mathrm{K}]=1.22 \times 10^{9}(1+z)\left(\frac{S_{t o t}}{\mathrm{mJy}}\right)\left(\frac{d}{\mathrm{mas}}\right)^{-2}\left(\frac{\nu}{\mathrm{GHz}}\right)^{-2} .
$$

where the total flux density $S_{t o t}$ is measured in Jy, the size of the circular Gaussian component $d$ in mas, and the observing frequency $\nu$ in GHz. If the size of the Gaussian component, $d$ is less than the minimum resolvable size of the Gaussian component, $d_{\min }$, given by equation (3.42), the latter is used for estimating the lower limit on $T_{\mathrm{b}}$.

\subsection{Visibility based $T_{\mathrm{b}}$ Estimates}

As discussed in section 1.4, the brightness temperature can be usually estimated by fitting Gaussian components on the visibility data. But in fact, one has to deal with difficult cases of interferometric observations such as millimeter and space VLBI measurements which do not provide enough data to fit models on the visibility data due to lack of short baseline measurements and extremely complex fine structures in the target sources. Lobanov (2015) introduced a different approach to estimate the brightness temperature which is based on individual visibility measurements and their errors. In this section, we discuss how a minimum and maximum brightness temperature can be calculated from a visibility data following Lobanov (2015).

Consider an emitting region with a brightness distribution, $I_{r}$, observed at a wavelength, $\lambda$, by an interferometer which consists of two receiving elements (telescopes) separated by a baseline distance, $B$. The interferometric visibility is described as $V=V_{q} e^{-i \phi_{q}}$ where $V_{q}$ is the amplitude and $\phi_{q}$ is the phase. The subscript $q$ corresponds to a single spatial (Fourier) frequency $q=B / \lambda$ where $B$ is the baseline. Let $\sigma_{q}$ and $\sigma_{\phi}$ be the errors in $V_{q}$ and $\phi_{q} . V_{q}$ depends on the shape and angular extent of the brightness distribution, and $\phi_{q}=\phi_{\mathrm{p}}+\phi_{\mathrm{o}}$ depends on position and geometry. $\phi_{\mathrm{p}}$, which is the position-dependent term of the phase is relative and it can be zeroed by an appropriate shift applied to the visibility. $\phi_{\mathrm{o}}$, which is the geometry-dependent term depends on the structure of the brightness distribution and its orientation with respect to the projection of the interferometric baseline on the image plane (Lobanov 2015). If we consider a circularly symmetric or axially symmetric brightness temperature distribution, then it is independent of baseline orientation and can be taken as $\phi_{\mathrm{o}} \equiv 0$.

\subsubsection{Minimum Brightness Temperature}

If we don't know the structure of brightness distribution, $I_{r}$, then a symmetric brightness distribution can be assumed. In that case, the angular extent of the emission $\theta_{r}$ can be 
estimated from $V_{q}$ alone. Such estimates require knowledge of the zero-spacing visibility, $V_{0}$. In the case of a circular Gaussian distribution, $V_{q}$ is given by

$$
V_{q}=V_{0} \exp \left(-\frac{\pi^{2} \theta_{r}^{2} q^{2}}{4 \ln 2}\right)
$$

and from equation $1.28, \theta_{r}$, of the emitting region can be estimated as:

$$
\theta_{r}=\frac{2 \sqrt{\ln 2}}{\pi} \frac{\lambda}{B} \sqrt{\ln \left(V_{0} / V_{q}\right)} .
$$

Using equations 1.28 and 1.29 with equations 1.26 from section 1.4 , the brightness temperature can be estimated as,

$$
T_{\mathrm{b}}=\frac{\pi}{2 k} \frac{B^{2} V_{0}}{\ln \left(V_{0} / V_{q}\right)} .
$$

Brightness temperature is a function of the ratio $V_{0} / V_{q}$ between the zero spacing flux density and visibility flux density measured at a given spatial frequency $q$. The minimum value of the brightness temperature $T_{\mathrm{b}, \min }$, corresponds to equation 1.30 obtained, when $V_{0}=e V_{q}$, which is given as,

$$
T_{\mathrm{b}, \min }=\frac{\pi}{2 k} B^{2} e V_{q} \approx 3.09\left(\frac{B}{\mathrm{~km}}\right)^{2}\left(\frac{V_{q}}{\mathrm{mJy}}\right)[\mathrm{K}] .
$$

Equation 1.31 gives the absolute minimum of the brightness temperature that can be obtained from the measured visibility amplitude $V_{q}$ under the assumption that the brightness distribution is well approximated by a circular Gaussian.

\subsubsection{Maximum Measurable Brightness Temperature}

The expression for $T_{\mathrm{b}, \text { min }}$ given by equation 1.31 is independent of $V_{0}$, but in order to derive the maximum brightness temperature, we should have an idea on $V_{0}$. The maximum $T_{\mathrm{b}}$ can only be derived from upper limits on $V_{0}$ for $V_{0}>e V_{q}$ and from lower limits on $V_{0}$ for $V_{0}<e V_{q}$. The zero-spacing visibility $V_{0}$ is usually approximated by the total flux density, $S_{\text {tot }}$, measured at a single receiving telescope. But for extremely poor coverages of the Fourier domain, a satisfactory estimate of $V_{0}$ is difficult to obtain. So, a lower limit of $V_{0}=V_{q}+\sigma_{q}$ can be used. This limit is based on the requirement that $V_{q}$ probes a structure in the source that is marginally resolved, which is justified for visibility measurements made at sufficiently long baselines, where the visibility amplitude is dominated by the most compact structure in the target source (Lobanov 2015). $V_{q} \equiv$ constant for a point source. The requirement of marginal resolution of the observed structure put a lower limit on the size given by,

$$
\theta_{\lim }=\frac{2 \sqrt{\ln 2}}{\pi} \frac{\lambda}{B} \sqrt{\ln \left(\frac{V_{q}+\sigma_{q}}{V_{q}}\right)},
$$

which in turn put an upper limit on the brightness temperature of this feature as,

$$
\begin{aligned}
T_{\mathrm{b}, \lim } & =\frac{\pi B^{2}\left(V_{q}+\sigma_{q}\right)}{2 k}\left[\ln \frac{V_{q}+\sigma_{q}}{V_{q}}\right]^{-1} \\
& =1.14\left(\frac{V_{q}+\sigma_{q}}{\mathrm{mJy}}\right)\left(\frac{B}{\mathrm{~km}}\right)^{2}\left(\ln \frac{V_{q}+\sigma_{q}}{V_{q}}\right)^{-1} \cdot[K] .
\end{aligned}
$$


Equation 1.33 is also based on the assumption that the respective brightness distribution is circularly Gaussian;

Equations 1.31 and 1.33 provide a robust bracketing for the brightness temperature obtained from interferometric measurements that have a limited sampling of the visibility distribution of the target source. The respective expressions for minimum and maximum brightness temperature for other patterns of brightness distributions can be seen in Lobanov (2015). For further details on this section 1.5, we refer the reader to Lobanov (2015).

\subsection{Relativistic and Geometrical Effects}

\subsubsection{Superluminal Velocities}

In 1969, motions with velocities faster than light was observed in the very-long-baseline interferometry (VLBI) maps of blazar 3C 273 (Gubbay et al. 1969). Later it was observed by Cohen et al. (1971) and Whitney et al. (1971) also. Much before all these observations, Rees predicted it (Rees 1966) in 1966 itself. This phenomenon is now known as Superluminal motion. Although the standard model of jets and radio lobes requires a steady supply of charged particles moving at relativistic speeds, evidence for such high velocities is difficult to obtain. This effect is observed within about $100 \mathrm{pc}$ of the AGN's center and probably continues farther out. By now, the apparent velocities, $\beta_{\text {app }}$ as high as $\sim 50 \mathrm{c}$ (Lister et al. 2009) have been observed in jets. Let us look into the geometry of superluminal motion taking the example of quasar 3C 273. Figure 1.6 shows the radio view of $3 \mathrm{C} 273$ showing a blob of radio emission moving away from the nucleus with an angular velocity $\mu$ $=0.0008 "$ /yr. Assume that the radio knot is traveling in the plane of the sky, perpendicular

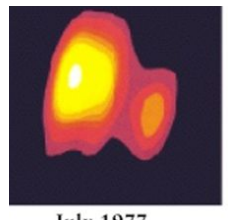

July 1977

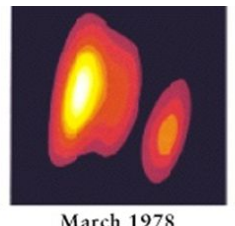

March 1978

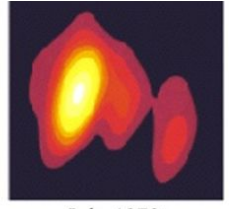

July 1979

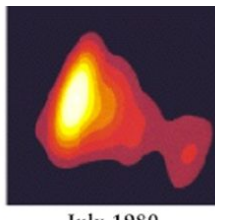

July 1980

Figure 1.6: Superluminal motion observed in 3C 273 (Pearson et al. 1981).

to the line of sight, and distance, $\mathrm{d}=440 \mathrm{~h}^{-1} \mathrm{Mpc}$ for $3 \mathrm{C} 273$, then apparent transverse velocity of the blob away from the nucleus is:

$$
v_{\text {app }}=d \mu=1.67 \times 10^{9} \mathrm{~h}^{-1} \mathrm{~m} / \mathrm{s}=5.57 \mathrm{~h}^{-1} c .
$$

If $\mathrm{h}=[\mathrm{h}]_{W M A P}$ where $[\mathrm{h}]_{W M A P}$ is the $\mathrm{h}$ value calculated by Wilkinson Microwave Anisotropy Probe (WMAP), then $v_{a p p}=7.85 \mathrm{c}$. This is clearly unphysical, and so the assumption of motion perpendicular to the line of sight must be wrong. This unusual phenomena can be explained as follows. Consider two photons emitted at time $\mathrm{t}=0$ and $\mathrm{t}=t_{e}$ by a source moving with a speed $v$ as shown in the right panel of figure 1.7 from the core situated at $\mathrm{A}_{1}$. The first photon travels through path $\mathrm{A}_{1} \mathrm{H}$ (by geometry, $\mathrm{A}_{1} \mathrm{H}=v t_{e} \cos \theta$ ) and reach earth at time $t_{1}$ and the second photon travels through path $\mathrm{A}_{1} \mathrm{~B}_{1}$ arrives at earth at time 
$t_{2}$ which can be calculated as:

$$
\begin{aligned}
& t_{1}=\frac{d}{c} \\
& t_{2}=t_{e}+\left[\frac{d-v t_{e} \cos \theta}{c}\right]
\end{aligned}
$$

By the time second photon is emitted, the source travels a distance with velocity $v$. The time on earth between the reception of the two photons is thus:

$$
\begin{aligned}
\Delta t & =t_{2}-t_{1} \\
& =t_{e}\left(1-\frac{v \cos \theta}{c}\right)
\end{aligned}
$$

The perpendicular distance between $\mathrm{A}_{1} \mathrm{H}$ and $\mathrm{A}_{1} \mathrm{~B}_{1}$ is $v t_{e} \sin \theta$. Therefore, the apparent transverse velocity measured on earth is:

$$
v_{\text {app }}=\frac{v t_{e} \sin \theta}{\Delta t}
$$

Substituting equation 1.36 in equation 1.37 and replacing $v / c$ represented as $\beta$ and $v_{\text {app }} / \mathrm{c}$ represented as $\beta_{\text {app }}$, the apparent speed of the source (or the blob) can be obtained as:

$$
\beta_{\text {app }}=\frac{\beta \sin \theta}{1-\beta \cos \theta}
$$

Equation 1.38 implies that $\beta_{\text {app }}$ can be much larger than 1 for high intrinsic values of speeds $\beta$ and small viewing angles as seen on the top of right panel of figure 1.7.

Solving equation 1.37 for $v / c$ or $(\beta)$ results in,

$$
\beta=\frac{v}{c}=\left[\frac{\frac{v_{a p p}}{c}}{\sin \theta+\frac{v_{a p p}}{c} \cos \theta}\right]
$$

and the smallest possible value of $v / c$ for the source is:

$$
\frac{v}{c}=\sqrt{\frac{\frac{v_{a p p}^{2}}{c^{2}}}{1+\frac{v_{a p p}^{2}}{c^{2}}}}
$$

which occurs at an angle $\theta_{\min }$ which is given by:

$$
\cot \left(\theta_{\min }\right)=\frac{v_{a p p}}{c}
$$

This minimum value of $v / c$ corresponds to a minimum Lorentz factor, $\Gamma_{\min }$ of the source given by:

$$
\Gamma_{\text {min }}=\sqrt{\frac{1}{1-\frac{v_{\min }^{2}}{c^{2}}}} \approx \sqrt{1+\frac{v^{2}}{c^{2}}}=\frac{1}{\sin \left(\theta_{\min }\right)}
$$

Now if we take the earlier example of $3 \mathrm{C} 273$ and use equation 1.41,then,

$$
\theta_{\text {min }}=\cot ^{-1}\left(\frac{v_{a p p}}{c}\right)=7.26^{\circ}
$$



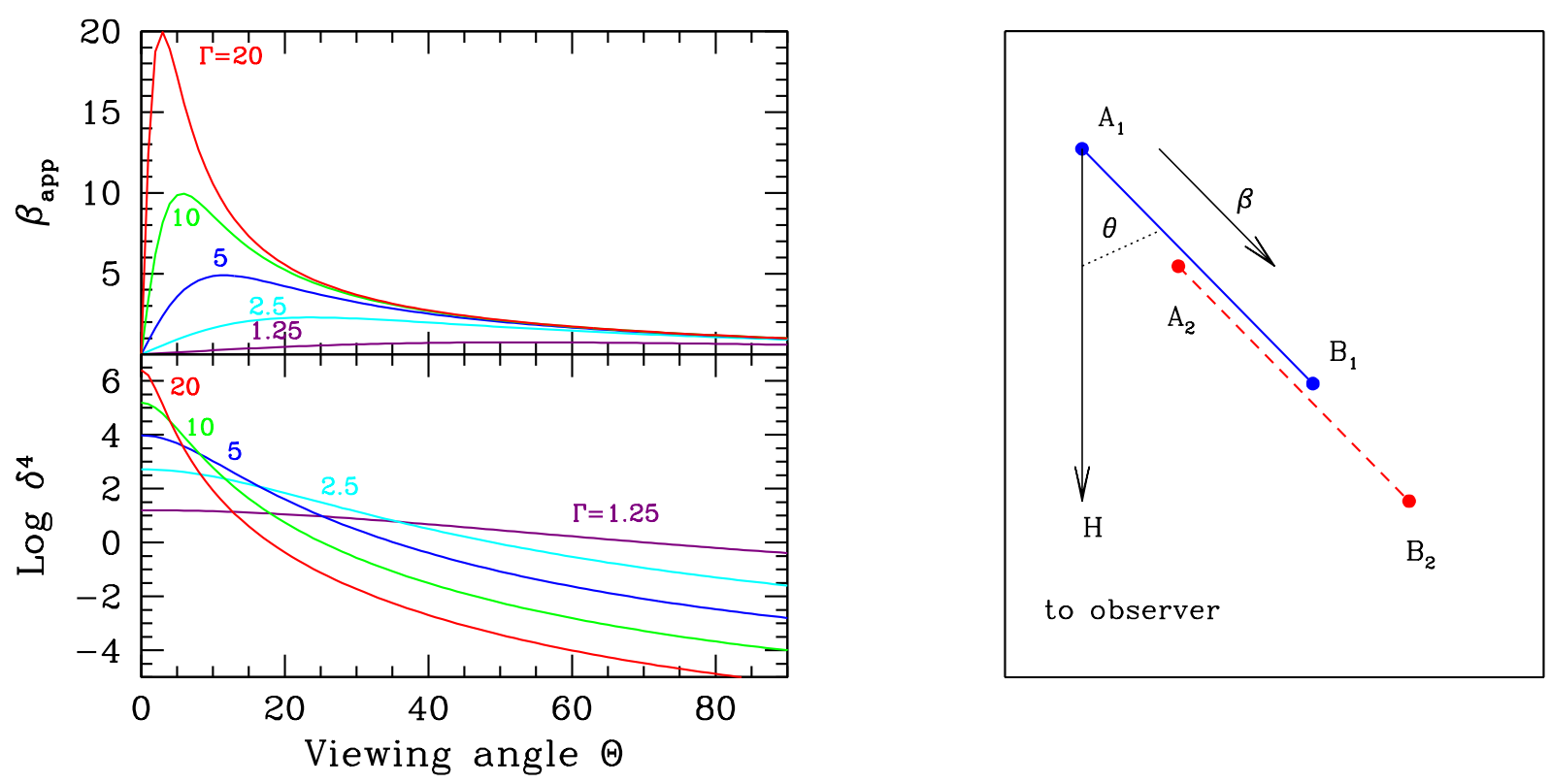

Figure 1.7: Left top: The apparent velocity $\beta_{a p p}$ versus the viewing angle $\theta$ for different values of Lorentz factor, $\gamma$, as shown. Left bottom: the amplification caused by fourth power of Doppler factor, $\delta^{4}$ versus $\theta$, for the same $\gamma$ as shown. Right: Geometry of superluminal motion. Both figures taken from Ghisellini (2013).

Thus the radio knot of $3 \mathrm{C} 273$ must be approaching earth within $7.26^{\circ}$ of the line of sight, the lower limit of the knot's speed should be $v_{\min }=0.992 \mathrm{c}$. Thus the problem of extreme velocities can be solved by understanding them on the basis of superluminal motion. In short, the source just appears to move faster than light due to the reduction in the time intervals between the emission of two successive photons.

\subsubsection{Relativistic Beaming}

If the jet is approaching towards us at relativistic speed and very close to our line of sight, then the resulting flux density and luminosity is changed by relativistic time dilation. Extreme brightness is observed and on the other hand, the jet going away from our line of sight in the opposite direction is deboosted. This effect is known as relativistic beaming.

The radiation emitted will be collimated into a cone with opening angle in the direction of motion as

$$
\theta \sim \frac{1}{\gamma}
$$

where, $\gamma$ is the bulk Lorentz factor. The Lorentz factor, which is a parameter that quantifies the relativistic beaming is defined as

$$
\gamma=\left(1-\beta^{2}\right)^{-1 / 2}
$$

While observing, we could measure only the apparent jet speeds, flux, luminosities and brightness temperatures, not their intrinsic values in the source's frame of reference. The observed and intrinsic parameters can be related to each other by a factor called Doppler 
factor, $\delta$ defined as:

$$
\delta=\frac{1}{\gamma(1-\beta \cos \theta)} .
$$

The above relativistic effects results in the fact that the intrinsic values are affected by Doppler factor and that is what we measure on Earth. The observed frequency and observed time is given as:

$$
\begin{gathered}
\nu=\delta \nu^{\prime} \\
\Delta t=\frac{\Delta t^{\prime}}{\delta} .
\end{gathered}
$$

The primed values refers to the source's frame of reference. This results in the modification of the observed flux and observed luminosity in our frame as:

$$
\begin{aligned}
& S_{\nu}=\delta^{n-\alpha} S_{\nu^{\prime}} \\
& L_{\nu}=\delta^{n-\alpha} L_{\nu^{\prime}}
\end{aligned}
$$

where $\mathrm{n}$ depends on the jet emission and $n=2$ for continuous jet (or steady state; Scheuer $\&$ Readhead 1979) and $n=3$ for a jet with optically thick spherical blobs (or optically thin "plasmoids") i.e. moving structure. Since blazars are the class of AGNs observed most close to the line of sight (see section 1.1.4), they are severely affected by relativistic effects.

The brightness temperature is related to observed flux density and observed frequency. So, $T_{b}$ is also modified by the relativistic effects as:

$$
\begin{aligned}
T_{b} & =K \frac{S_{\nu}}{\nu^{2} d^{2}} \\
& =K \frac{\delta^{n-\alpha} S_{\nu^{\prime}}}{\left(\delta \nu^{\prime}\right)^{2} d^{\prime 2}} \\
& =\delta^{n-2-\alpha} T_{b}^{\prime}
\end{aligned}
$$

where $d=d^{\prime}$, since the size of a sphere would not be affected by relativistic abberation.

Even though jet is intrinsically symmetric and is seen on large scales so, they appear onesided because of relativistic beaming effect on parsec scales. The difference in the observed flux for the approaching and receding jet can be derived as:

$$
R=\left[\frac{1+\beta \cos \theta}{1-\beta \cos \theta}\right]^{n-\alpha}
$$

where $n=2$ for continuous jet and $n=3$ for blobby jet (jet with spherical blobs) as discussed above. 


\section{Chapter 2}

\section{Ultracompact Extragalactic Radio Sources}

Compact radio sources are described as radio sources for which the flux is dominated by a single bright component which is smaller than $\sim 1 \mathrm{Kpc}$ in size at an intermediate radio frequency like $1 \mathrm{GHz}$ (Blandford \& Königl 1979). These compact radio sources are characterized by flat radio spectrum which can be explained as the superposition of different synchrotron self-absorbed components from relativistic electrons in a conical geometry (Marscher 1995; Boccardi et al. 2017). In compact radio sources, the jets move discontinuously as blobs or knots originated due to pressure differences between the jet plasma and the surrounding medium. Enhanced polarization and strong variability (Brandie et al. 1974) are seen in the shocked regions of such compact radio sources. The ratio of optical to radio power tends to be higher in compact radio sources than in steep spectrum sources (Usher 1975). Superluminal motion with very high speeds typically order of $\sim 10 \mathrm{c}$ (Lister et al. 2016) is a characteristic feature of the blobs seen in such compact radio sources. The fractional linear polarization measured in VLBI cores of compact radio sources are below $5 \%$, which might be due to the random orientation of the magnetic field (Hughes 2005). At large distances from the jet base, up to tens of percent of polarization is measured (Lister $\&$ Homan 2005). The base of such ultra-compact jets exhibit a spectral index of $\sim 0.62$.

Most jets are believed to be two sided intrinsically because the outer radio structures are generally symmetric. But on parsec scales, jets appear one-sided because of relativistic boosting i.e. when $\theta \leq \gamma^{-1}$, where $\theta$ is the angle between jet and line of sight of the observer and $\gamma$ is the Lorentz factor. Thus we detect only the approaching jet and their apparent luminosities which are boosted by a factor of $2 \gamma$. Because of this, the surveys down to a fixed flux density level are strongly biased and contain one-sided sources out of their actual numbers in the sky (Boettcher et al. 2012). Counterjets which are expected to be present in radio galaxies and blazars are seen rarely because they are relativistically deboosted. Such rare two sided parsec scale jets are seen in sources like M87 (Kovalev et al. 2007), NGC 1275 (3C 84; Walker et al. 2000), Centaurus A (Horiuchi et al. 2006) and are studied by Lister et al. (2009) through survey resolving parsec scales. NGC 1052 shows counter jet even at $7 \mathrm{~mm}$ and $3 \mathrm{~mm}$ VLBI maps with jet orientation of $64^{\circ}<\theta<87^{\circ}$ and a size smaller than 1.9 light days i.e. $100 R_{s}$ (Baczko et al. 2016). The magnetic field strength in such compact radio sources is $\sim 10^{3-4}$ Gauss as calculated from the GMVA studies of OJ 287 (Hodgson et al. 2017) and NGC 1052 (Baczko et al. 2016) as predicted by theoretical models (Blandford \& Znajek 1977). 
In this chapter, I discuss the physics aspects of ultracompact radio sources covering radiative processes - synchrotron \& inverse Compton, brightness temperature limits, jet composition, radio-loud/radio-quiet dichotomy, relativistic jet formation, acceleration and collimation. I also present a discussion on relativistic jets as ultracompact radio sources and how VLBI observations could yield information on parsec scale jets focusing on surveys from low frequencies to high frequencies. Finally, I conclude this chapter with a motivation for this dissertation focused on high frequency VLBI survey discussion.

\subsection{Radiative Processes}

AGN emits radiation in the entire electromagnetic spectrum from the radio to the high energy $\gamma$-ray region. Although the continuum radiation spectra has a complex shape in totality, we can approximate a simple power law for the spectra in wide wavelength intervals. The emission we receive from AGN mainly constitutes thermal radiation, synchrotron radiation, inverse Compton emission and Bremsstrahlung. This is also added with the emission due to scattering, absorption and reemission. This will be discussed in detail in section 2.1.1 and in section 2.1.2. The low frequency radio emission from AGNs is from the synchrotron radiation from kiloparsec $(\mathrm{kpc})$ scale jet and the high frequency radio and optical emission is from the inner parsec scales of the jet. The thermal radiation including optical and UV is emitted from the accretion disk and the high energy $\gamma$ and X-rays are produced by the inverse Compton scattering of synchrotron photons or by the scattering of photons (optical/ultra-violet) from accretion disk, BLR etc. Most of the discussions in this chapter are based on the monographs by Boettcher et al. (2012), Kembhavi \& Narlikar (1999) and Robson (1996).

\subsubsection{Synchrotron Radiation}

When a relativistic particle (like electron or positron) is accelerated by a magnetic field, photons are emitted with a wide range of energies. This radiation is called as Synchrotron Radiation. Synchrotron radiation is highly collimated and polarized. It was Alfvén \& Herlofson (1950) who correctly proposed the source of synchrotron radiation as radio stars in their article on cosmic radiation and radio stars. In the following section, let's take a look at the synchrotron emission from a single charged particle and the power radiated. We follow here mainly the discussions from Rybicki \& Lightman (1979) and Jackson (1975). By classical electrodynamics, the motion of a charged particle in a magnetic field, $\mathbf{B}$ is given by the Lorentz force equation

$$
\frac{d}{d t}(\gamma m v)=\frac{e}{c}(v \times \mathbf{B})
$$

where $e$ and $m$ are the charge and rest mass of the particle, $v$ is the velocity and $\gamma=$ $\left(1-v^{2} / c^{2}\right)^{-1 / 2}$. Since the acceleration $d v / d t$ is perpendicular to the velocity, $v$ and $\gamma$ are constant. There is no force acting on the particle in the direction of $\mathbf{B}$ and this makes the velocity component parallel to it, $v_{\|}$constant. Since $v=$ constant, the component of velocity which is normal to the magnetic field, $v_{\perp}$ is also constant. The result of $v_{\perp}$ and $d v / d t$ is that it induces a helical path motion for the particle. The gyration frequency, i.e. the frequency of the projected orbit on a plane normal to $\mathbf{B}$ which is equivalent to the 
frequency at which the synchrotron radiation is emitted is given by

$$
v_{g}=\frac{e B \gamma^{2}}{2 \pi m c} .
$$

The total power emitted by an accelerated charged particle is obtained from Larmor's formula as

$$
P=\frac{2 e^{2}}{3 c^{3}} \gamma^{4}\left(a_{\perp}^{2}+\gamma^{2} a_{\|}^{2}\right)=\frac{2 e^{2}}{3 c^{3}} \gamma^{4}\left(\frac{e B}{\gamma m c}\right)^{2} v_{\perp}^{2}=\frac{2}{3} r_{0}^{2} c \beta^{2} \gamma^{2} B^{2} \sin ^{2} \alpha
$$

where $r_{0}=e^{2} / m c^{2}, \beta=v / c$ and $\alpha=\cos ^{-1}\left(v_{\|} / v\right)$ is the pitch angle between the velocity and magnetic field. When particle velocities are isotropically distributed, the distribution of $\alpha$ is

$$
p(\alpha) d \alpha=\frac{1}{2} \sin (\alpha) d \alpha,
$$

with $\alpha$ going from $[0, \pi]$. When averaged over the pich angle $\alpha, \sin ^{2} \alpha=2 / 3$. Therefore equation 2.3 transforms as

$$
P_{\text {Synch }}=\frac{4}{3} \sigma_{T} c \beta^{2} \gamma^{2} U_{B}
$$

where $\sigma_{T}=8 / 3 \pi r_{0}^{2}$ is the Thompson cross section and $U_{B}=B^{2} / 8 \pi$ is the energy density in the magnetic field.

The power given by equation 2.5 is emitted mainly in a narrow cone of opening angle $\approx$ $1 / \gamma$ around an axis which coincide with the instantaneous velocity vector. We know that a radiating electron loses energy at the rate $d E / d t=-P$. From equation 2.5, it is clear that $d E / d t=-P=E^{2} B_{\perp}^{2}$ since $\gamma \propto E$ (keep in mind $E \propto \gamma m c^{2}$ ) and $U_{B} \propto B^{2}$.

\subsubsection{Synchrotron Radiation Spectrum}

Now, when we consider synchrotron emission from an ensemble of electrons which is the reality in relativistic jets from AGN, we have to make the generic assumption that the radiation follows a simple power law which is seen in observations as

$$
F_{\nu}=C \nu^{-\alpha}
$$

where $\mathrm{F}_{\nu}$ is the specific flux in units of $\mathrm{erg} / \mathrm{cm}^{2} / \mathrm{Hz}, \nu$ is the frequency and $\alpha$ is the spectral index. Over a frequency range $\nu_{1}-\nu_{2}$, the total power received is: 


$$
\begin{array}{rlrl}
P\left(\nu_{1}-\nu_{2}\right) & =\int_{\nu_{1}}^{\nu_{2}} F_{\nu} d \nu & \\
& =C \int_{\nu_{1}}^{\nu_{2}} \nu^{-\alpha} d \nu & \\
& =\frac{C}{1-\alpha} \times\left[\nu_{2}^{1-\alpha}-\nu_{1}^{1-\alpha}\right] & & \text { for } \quad \alpha \neq 1 \\
& =C \times \ln \left(\frac{\nu_{2}}{\nu_{1}}\right) & & \text { for } \quad \alpha=1
\end{array}
$$

Mathematically, if we try to understand $\left(\log \nu F_{\nu}\right.$ vs $\left.\log \nu\right)$, then the result is a horizontal line for $\alpha=1$ which represents a flat spectrum and for $\alpha \neq 1$, the spectrum rises with frequency, typically lies close to 1 . The typical spectral index of synchrotron radiation observed in astrophysical sources, $\alpha$ is less than 0.7 for flat spectra, between 0.7 to 1 for moderate spectra and between 1 and 2 for very steep spectra. The observations show that the extended structures have steep spectra while the compact sources have flat spectra. The reason for this will be discussed in the next section 2.1.1.2.

For a homogeneous source with constant magnetic field $\mathbf{B}$, a power law continuum spectra can be generated by the synchrotron mechanism by an initial power law distribution of electron energies $\mathbf{E}$ of the form

$$
N(E) d E=N_{0} E^{-s} d E \quad \text { where, } \quad \alpha=\frac{s-1}{2} .
$$

Therefore equation 2.7 reduces to

$$
P\left(\nu_{1}-\nu_{2}\right)=C \int_{\nu_{1}}^{\nu_{2}} \nu^{-\left(\frac{1-s}{2}\right)} d \nu
$$

where $\mathrm{s}$ is the particle distribution index. Such power law distribution of electrons is produced by acceleration mechanisms like Fermi-acceleration through shocks (Blandford et al. 1990). Synchrotron radiation is also characterized by high degree of polarization.

\subsubsection{Synchrotron Self Absorption}

An observer can see such a power law spectrum from an emitting region only if there is no absorption by the emitting region or by an absorbing matter in the host galaxy of AGN, or intermediate galaxy or our own galaxy in between emitter and observer. We often observe a typical observed value of the power law index $\alpha=0.7$, so $<\mathrm{s}>\approx 2.4$ (in other words a steep spectrum) for the extended component i.e. large scale jets of radio sources and radio lobes from AGN. But for the compact regions, we often see a flat or inverted spectrum. If the basic radiation process in both the regions is synchrotron, then it is clear that the different shape seen for the former is because of absorption or scattering.

In the presence of a magnetic field, an electron can absorb a photon and make a transition to higher energy state or stimulate further emission of photons into the same state. At lower frequencies, the photons emitted will be self absorbed by the electrons ensemble. This process is called as Synchrotron Self-Absorption. At lower frequencies, the emitting 


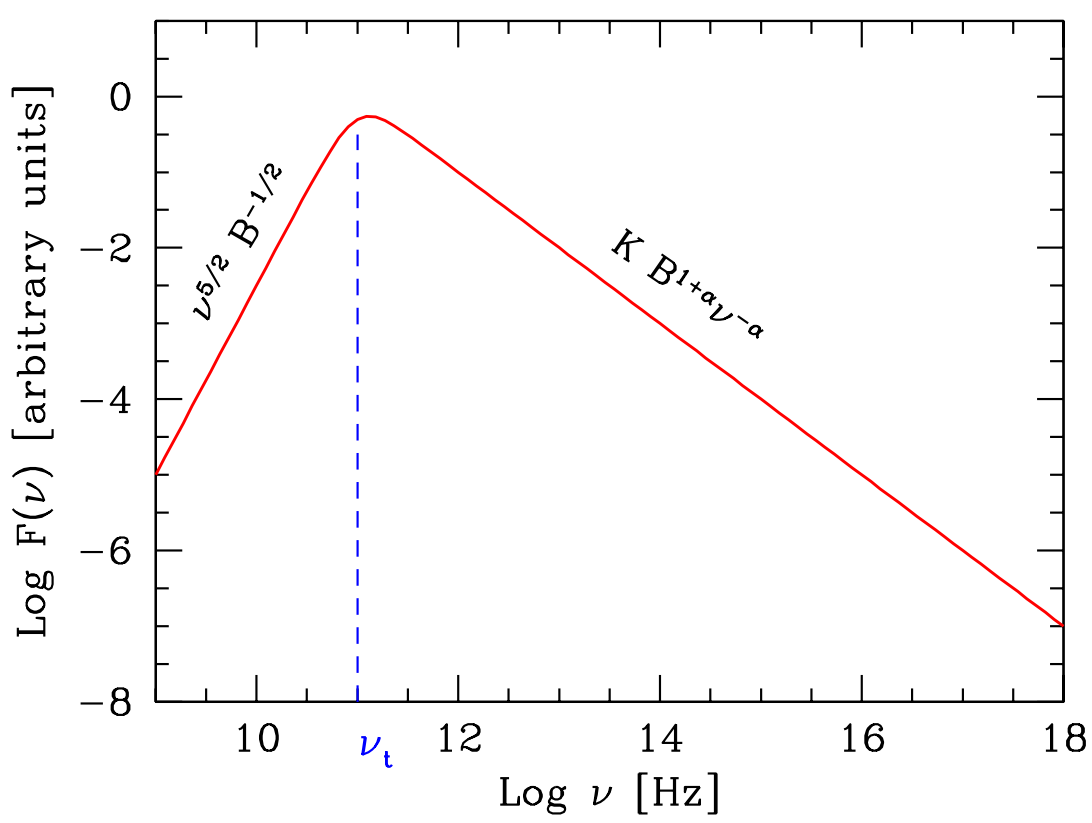

FiguRE 2.1: The synchrotron spectrum from a partially self absorbed source. The region on the left side and right side of dashed line represents optically thick region (self absorbed part) and optically th region, respectively. $\nu_{t}$ represents the turnover frequency. Observations of the self absorbed part could determine magnetic field, B. Observations of the thin part can then determine constant $C$ and the electron slope $s$ (Ghisellini 2013).

gas is optically thick and the trend towards increasing flux with decreasing frequency turns over to yield

$$
F_{\nu}=\nu^{-5 / 2} \text {. }
$$

This will result in the characteristic self-absorption turnover in the spectrum as shown in figure 2.1. The frequency at which this self-absorption turnover occurs in the synchrotron spectrum is called as turn over frequency, $\nu_{m}$. A given source will be optically thick below this frequency at which $\tau_{\nu} \approx 1$. The turn over frequency increases with the density of relativistic electrons in the source. Even though the electrons do not have a thermal spectrum (Maxwell-Boltzmann energy distribution), we can associate a kinetic temperature, $T_{e}=E / k_{B}$. According to thermodynamics, the brightness temperature of the radiation, $T_{b}$ cannot exceed the kinetic temperature of electrons, $k T_{b} \leq k T_{e}$ and the radiation becomes self-absorbed when $k T_{b} \approx k T_{e}$ i.e.

$$
E \approx\left(\frac{3 e}{4 \pi m^{3} c^{5}}\right)^{-1 / 2} B^{-1 / 2} \nu_{m}^{1 / 2}
$$

Therefore, the condition for avoiding synchrotron self-absorption is

$$
T_{\mathrm{b}}[\mathrm{K}] \leq 9 \times 10^{13}\left(\frac{B}{\mu \mathrm{G}}\right)^{-1 / 2}\left(\frac{\nu}{\mathrm{GHz}}\right)^{1 / 2}
$$

wher $\nu_{m}$ is the turnover frequency. This gives an upper limit for the brightness temperature, $T_{b}$. Practically equation 2.12 can be used to calculate the magnetic field. The angular size of a source that becomes self-absorbed at $\nu_{m}$ is

$$
\theta \approx c\left(\frac{3 e}{4 \pi m^{3} c^{5}}\right)^{1 / 2} B^{1 / 4} S_{m}^{1 / 2} \nu_{m}^{-5 / 4}
$$


The power law slope of self-absorbed synchrotron emission can never exceed $5 / 2$. Though this limit is widely used, it is true only for single power law, but for a two power-law distribution that is concave i.e. steeper at low energies, it is possible to have a slope exceeding 5/2 (de Kool \& Begelman 1989; Ghisellini et al. 1988). This is rarely seen as the very steep slopes for radio-quiet quasars and AGNs in submillimeter region. Schlickeiser et al. (1991) explained the large slopes of 2.4 and 4.6 (a concave synchrotron spectrum) observed in the case of Seyfert galaxies Mrk 104, I Zw 1 and NGC 4151 by considering a combination of influences like stochastic acceleration and Coulomb interaction losses. It should be also noted that the radio spectrum sometimes curve downward at higher frequencies. The basic reason for this is that electrons radiate at frequencies proportional to their energies $\mathrm{E}$ and the rate at which energy loss takes place is proportional to $E^{2}$. Thus the highest energy electrons radiate away their energy the most rapidly, thus depleting the emitted spectrum at the high frequency end first and if no replenishment of the high energy electrons occurs.

\subsubsection{Inverse Compton}

The scattering of an electromagnetic wave incident on an electron, with photon energy $h \nu<<m_{e} c^{2}$ described as Thomson scattering can be treated classically. The total scattering cross section summed over all the angles is

$$
\sigma_{T}=\frac{8 \pi r_{0}^{2}}{3}=6.65 \times 10^{-25} \mathrm{~cm}^{2} .
$$

But in the case of compact radio sources, there are highly energetic photons and relativistic electrons that result in the scenario no longer to be treated as classical. An electron at rest that scatters a photon will gain energy from the collision and acquire a recoil velocity to conserve the angular momentum. This is called Compton Scattering. This results in the loss of energy of photon and the final wavelength of the photon will be greater than the initial wavelength by

$$
\Delta \lambda=\lambda_{f}-\lambda_{i}=\frac{h}{m_{e} c}(1-\cos \theta),
$$

where $\theta$ is the angle between the incident photon and scattered photon. When we deal with relativistic electrons in ultra-compact radio sources, it is obvious that the elctrons have energy comparable to photons which results in the inverse of Compton Scattering called as inverse Compton Scattering. The energy loss by a single elctron or the rate of the net energy output is

$$
P_{\text {Comp }}=\frac{4}{3} \sigma_{T} c \beta^{2} \gamma^{2} U_{p h}
$$

where $U_{p h}$ is the photon energy density. The total number of incident photons per unit time is $c \sigma_{T} n_{t o t}$, where $n_{t o t}=U_{p h} /\langle\epsilon\rangle$, and $\langle\epsilon\rangle$ is the average incident photon energy. Therefore, the average incident photon energy for $\beta=1$ is

$$
<\epsilon_{1}>=\frac{4}{3} \gamma^{2}<\epsilon>\text {. }
$$

The number density of photons is conserved in the interaction and the frequency of upscattered photons, $\nu_{f}$ is increased by a factor of $\gamma^{2}$ given as,

$$
\nu_{f}=\gamma^{2} \nu_{i},
$$


where $\nu_{i}$ is the frequency of the incident photon. The equation 2.16 is analogous to equation 2.5 except the magnetic field density, $U_{B}$ is replaced by photon energy density, $U_{p h}$. This can be written as,

$$
\frac{P_{C o m p}}{P_{\text {sync }}}=\frac{U_{p h}}{U_{B}} .
$$

The inverse Compton spectrum is also a power law index spectrum with spectral shape depends only on the electron energy distribution. The number of electrons with $\gamma$ ranging from $\gamma$ to $\gamma+d \gamma$ per unit volume is

$$
N(\gamma) d \gamma=C \gamma^{-p}
$$

where $p$ is photon index. Then for $\beta=1$, the total Compton spectrum can be obtained by integrating $P_{C o m p}$ for a single electron scattering over the total number of electrons given by equation 2.1 .2 as,

$$
\begin{aligned}
P_{t o t} & =\int P_{C o m p} N(\gamma) d \gamma \\
& =\frac{4}{3} \sigma_{T} c U_{p h} C(3-p)^{-1}\left(\gamma_{\max }^{3-p}-\gamma_{\min }^{3-p}\right)
\end{aligned}
$$

\subsection{Brightness Temperature Limits}

\subsubsection{Compton Catastrophe and Inverse Compton Limits}

As discussed in section 2.16 and 2.18, the synchrotron photons are upscattered by the ensemble of electrons which produced them through inverse Compton scattering by a factor of $\gamma^{2}$. This process is known as Synchrotron Self-Compton (SSC). If the seed photons are external photons from the accretion disk or broad line regions which are upscattered by the ensemble of electrons through inverse Compton scattering, then the process is known as External Comptonization (EC). If the seed photons are from the infra red emission from circumnuclear dust, then it is called as Infra-Red Comptonization (IRC).

Let's take the most dominant process of SSC. The ratio of the luminosity produced by an ensemble of electrons in the inverse Compton process, $L_{I C}$ and synchrotron process, $L_{S}$ is proportional to the ratio of photon energy density to magnetic field energy density as shown in equation 2.19. In compact radio sources, the high energy photons produced by SSC can be upscattered again due to the high radiation energy density in them and so on until the condition $\gamma \epsilon<<m c^{2}$ is violated. If the energy density of once-scattered radiation exceeds that of the magnetic field, the energy density of the twice scattered photons exceeds that of the once scatterd photons (Kembhavi \& Narlikar 1999). This multiple upscattering of same photons results in extinguishing the source and this is known as Compton catastrophe (Hoyle \& Burbidge 1966). Such a catastrophe can be avoided if the energy density in the once-scattered photons is less than that in the magnetic field. Kellermann and Pauliny-Toth have derived a condition for this (Kellermann \& Pauliny-Toth 1969):

$$
L_{s}=4 \pi D^{2} F_{s}=4 \pi\left(\frac{r^{2}}{\theta^{2}}\right) F_{s},
$$

where $F_{s}$ is the synchrotron flux, $r$ is the radius of the source, $D$ is the distance of the source and $\theta$ is the angular size of the source. Using equation 2.19, we have 


$$
\frac{L_{I C}}{L_{S}}=\frac{U_{p h}}{U_{B}}=\frac{\frac{L s}{4 \pi r^{2} c}}{\frac{B^{2}}{8 \pi}}=\frac{8 \pi F_{s}}{B^{2} \theta^{2} c} .
$$

For the turn over frequency, $\nu_{m}$, equation 2.23 reduces to

$$
\frac{L_{I C}}{L_{S}}=\frac{8 \pi F\left(\nu_{m}\right) \nu_{m}}{B^{2} \theta^{2} c} .
$$

Now using the fact that the intensity of radiation $I(\nu)=F_{\nu} \theta^{2}$ can be written in terms of $T_{b}$ and the turn over frequency can be expressed in terms of energy $E=k T$ as described by equations 2.11 and 2.12 , we have

$$
\frac{L_{I C}}{L_{S}} \approx 16 \pi\left(\frac{3 e}{4 \pi m^{3} c^{5}}\right)^{2} \frac{\left(k T_{b}\right)^{5}}{c^{3}} \nu_{m}=\left(\frac{T_{b}}{10^{12.1} \mathrm{~K}}\right)^{5} \frac{\nu_{m}}{100 \mathrm{GHz}} .
$$

It is clear from equation 2.25 that for $\nu_{m} \approx 100 \mathrm{GHz}$, we have $L_{I C}<<L_{S}$ for $T<10^{12} \mathrm{~K}$ and the synchrotron emission dominates. When $T \geq 10^{12} \mathrm{~K}$ the $L_{I C}$ dominates $L_{S}$ and the second order scattering becomes prevalent. Considering this prevalence of inverse Compton radiation equation 2.25 reduces to:

$$
\frac{L_{I C}}{L_{S}} \approx\left(\frac{T_{b}}{10^{12.1} \mathrm{~K}}\right)^{5} \frac{\nu_{m}}{100 \mathrm{GHz}}\left[1+\frac{T_{b}}{10^{12.1} \mathrm{~K}}\right]^{5}
$$

Since $T_{b} \geq 10^{12} \mathrm{~K}$ the second order term dominates and

$$
\frac{L_{I C}}{L_{S}} \approx\left(\frac{T_{b}}{10^{12.1} \mathrm{~K}}\right)^{10} .
$$

The situation induced by equation 2.27 results in Compton catastrophe and most of the energy will be radiated away as X-rays in a very short time scale of a day. Kellermann \& Pauliny-Toth (1969) showed that for a sample of resolved opaque radio sources, lie in the maximum range of $T_{b}$ from $10^{11} \mathrm{~K}$ to $10^{12} \mathrm{~K}$. The lower limits on $T_{b}$ corresponds to $10^{11} \mathrm{~K}$ for all the unresolved sources. They explained the high $T_{b}$ observed at $10^{12} \mathrm{~K}$ called as the Inverse Compton limit as a rapid inverse Compton cooling of the sources in which the brightness temperature exceeded $\approx 10^{12} \mathrm{~K}$. Readhead (Readhead 1994) studied samples from three surveys at low and high frequencies including $81.5 \mathrm{MHz}, 430 \mathrm{MHz}$ and $5 \mathrm{GHz}$ and proved that the upper cut off in the brightness temperature in emission rest frames occur at $10^{11.5} \mathrm{~K}\left(3.16 \times 10^{11} \mathrm{~K}\right)$ for compact powerful extragalactic radio sources (Readhead 1994). On this evidence, they re-derived equation 2.26 for powerful extragalactic radio sources into:

$$
\frac{L_{I C}}{L_{S}} \approx\left(\frac{T_{b}}{10^{11.45} \mathrm{~K}}\right)^{5}\left[1+\frac{T_{b}}{10^{11.45} \mathrm{~K}}\right]^{5},
$$

for an object at $\mathrm{z}=1$, with $\alpha=-0.75$, the frequency of the peak in the spectrum, $\nu_{o p}=3.25$ $\mathrm{GHz}$ and the upper cut-off frequency in the spectrum, $\nu_{\text {high }}=100 \mathrm{GHz}$. Their conclusion was that brightness temperature measured at high frequencies by interferometry techniques was Doppler boosted to few times $10^{12} \mathrm{~K}$ and this coincided with maximum brightness temperature measured in high redshift sources as reported by Kellermann \& Pauliny-Toth (1969). The brightness temperature limit was showed as $3.16 \times 10^{11}$ by modifying the timescales for radiative cooling by combined synchrotron and inverse Compton radiation 
as equation (2) in Readhead (1994). This occurs for the maximum life time. For $T_{b} \leq$ $3.16 \times 10^{11} \mathrm{~K}$, the life time is decided by the synchrotron loss time scales and lifetime, $t_{1 / 2} \propto T_{b}^{3}$. For an object showing synchrotron self-absorption, $B \propto T_{b}^{-2}$ i.e. in order to keep the frequency of the peak flux density constant, magnetic field has to decrease with increasing brightness temperature. At the same time, the magnetic field energy density, $U_{B} \propto\left(B^{2} / 8 \pi\right) \propto T_{b}^{-4}$.

\subsubsection{Equipartition Brightness Temperature}

Readhead (1994) argued that if there is an equipartition between the particle energy density and the magnetic energy density, then the peak brightness temperature is an order lower than $10^{12} \mathrm{~K}$ corresponding to inverse Compton limit as found by Kellermann \& PaulinyToth (1969). This assumption of equipartition and the maximum $T_{b}$ was based on Burbidge \& Burbidge (1957) and Scott \& Readhead (1977) who introduced it to minimize the excessive energy content in extended radio galaxies NGC 5128 and NGC 1316 with ages of $10^{8-9}$ years. Readhead (1994) applied the same to parsec scale jets as well in synchrotron self-absorption dominated radio sources at frequencies less than $1 \mathrm{GHz}$ and the equipartition brightness temperature obtained from Rayleigh-Jeans formula is defined as:

$$
T_{e q}=\left[\frac{2 c^{2}}{\pi k_{B}}\right]\left[\frac{S}{\theta_{e q}^{2}} \nu^{-2}\right]
$$

where $\theta_{e q}$ is the equipartition angular size of the source.

The equipartition brightness temperature formulated by Readhead (1994) is:

$$
T_{e q}=4.7 \times 10^{10} h^{-2 / 17} \delta^{13-2 \alpha} \mathrm{K},
$$

for $\alpha=1, \mathrm{z}=1$, peak frequency of the source, $\nu_{\text {peak }}=3.5 \mathrm{GHz}$ and flux density of the source, $S=1 \mathrm{Jy}$ and $H_{0}=100 \mathrm{hm} \mathrm{s}^{-1} \mathrm{Mpc}^{-1}$ assuming Einstein-de Sitter universe. In most of the cases, radio sources radiate at an equipartition brightness temperature of $5 \times 10^{10} \mathrm{~K}$. The ratio of particle energy density, $u_{p}$ to magnetic energy density, $u_{B}$ is formulated as:

$$
\frac{u_{p}}{u_{B}}=\left(\frac{T_{e q}}{T_{B}}\right)^{17 / 2} .
$$

But we need to think that it is unlikely that equipartition exists in parsec scale jets where a highly collimated ejection of relativistic plasma takes place. It is natural to expect an excess of particle energy density than magnetic field energy in parsec scales of AGNs where the ejection of jet particles occur. The age of parsec scale jets might be just $\sim 1-100$ years also much lesser than totally evolved kiloparsec jets of age $\sim 10^{8-9}$ years.

\subsubsection{The Brightness Temperature Problem}

Most of the ground based brightness temperature, $T_{b}$ measurements give a value ranging from $10^{11}-10^{12} \mathrm{~K}$. But in realistic cases and observations, many sources deviate from the theoretical predictions of inverse Compton and equipartition brightness temperature limits. In the following, I discuss such cases and possible explanations for them. 
For Intra-Day Variable (IDV) sources, showing variability time scales of hours in the radio band, the observed $T_{b}$ can exceed $10^{16} \mathrm{~K}$, a value much larger than the theoretical limit. If the variability is not due to interstellar scintillation and it is intrinsic, only very high Doppler boosting can explain it and a Doppler factor larger than hundreds is needed to explain $T_{b}$ exceeding limits (Ghisellini 2013). But now it is also clear that interstellar scintillations by ionized clouds can play a big role in the observed high $T_{b}$ and is well discussed for the case of IDV source PKS 0405-385 for which the actual $T_{b}$ can be corrected for scintillation effect by $10^{13} \mathrm{~K}$ from the very high observed value of $5 \times 10^{14} \mathrm{~K}$ (Protheroe 2003).

The inverse Compton limit applies to incoherent synchrotron radiation only. So coherent processes like coherent plasma waves and stimulated synchrotron emission from masers can explain extreme $T_{b}$ (Setti \& Swings 2001). Also it is possible to maintain a large $T_{b}$ for short periods of time if the initial energy in the jet is sufficiently high (Setti \& Swings 2001), for example, $T_{b}$ can be higher than $10^{15} \mathrm{~K}$ for approximately a month and can exceed $10^{12} \mathrm{~K}$ for more than 100 years at $10 \mathrm{GHz}$.

The maximum $T_{b}$ that can be measured depends mainly on two factors - the baseline length and the flux density of the source. With a baseline of $\sim 10,000 \mathrm{~km}$ and few Jansky flux source, we already measure a $T_{b}=10^{12} \mathrm{~K}$. So it is possible to test inverse Compton limit from extremely long baselines. Thanks to "RadioAstron" space telescope (Kardashev et al. 2013) with long baselines extended to space (see section 2.5.1) with which $T_{b}>10^{13} \mathrm{~K}$ is observed. Pilipenko et al. (2018) has measured $T_{b}>10^{13} \mathrm{~K}$ for the source B0529+483 even after taking into account the refractive scattering and they suggested this as a clear dominance of the particle energy density over the magnetic field energy density in the core. Extreme $T_{b}$ is reported by Johnson et al. (2016) in 3C 273 also.

Synchrotron emission from relativistic protons can give a higher $T_{b}$ than that from relativistic electrons. Jukes (1967) firstly discussed the synchrotron emission from protons. Kardashev (2000) suggested that the brightness temperature limits can scale to mass of the particle as $T_{b} \propto m^{9 / 7}$. So the composition of relativistic protons in the jets rather than electrons, can raise the theoretical brightness temperature limit by a factor of

$$
\left(\frac{m_{p}}{m_{e}}\right)^{9 / 7}=1.5 \times 10^{4},
$$

resulting in a $T_{b}$ limit of $1.5 \times 10^{16} \mathrm{~K}$. This explanation is used as a strong argument for the extreme $T_{b}$ measured in 3C 273 by RadioAstron in Kovalev et al. (2016). They also suggested perhaps there is sufficient injection or acceleration of relativistic electrons to balance losses due to inverse Compton cooling (Kovalev et al. 2016).

\subsection{Relativistic Jet Formation, Acceleration \& Collimation}

The relativistic jets as compact radio sources have been widely studied by observations and simulations in order to develop a theoretical model which explains all the observed properties in AGN. From the extensive studies from past years, it is very clear that a poloidal magnetic field associated with accretion disk which acts like a conductive charged object and a rotating supermassive black hole at the centre are the basic requirements for jet launching mechanism. The discussion presented below is based on Blandford \& Znajek (1977); Penrose (1969); Penrose \& Floyd (1971); Blandford \& Payne (1982), the review by Dadhich (2012) and the monograph by Boettcher et al. (2012). 


\subsubsection{Jet Formation}

In 1969, Penrose showed that a spinning black hole has free energy i.e. in principle, available to be tapped (Penrose 1969). This free energy is tapped from the ergosphere which is a region lying outside the event horizon with radius $2 r_{g}$ in the equatorial plane and radius $r_{g}$ in the polar direction (see section 1.3.1). This has led to the popular idea that the energy source behind relativistic jets is the rotational energy of the accreting black hole. This was proposed by Blandford and Znajeck. Magnetic field lines are kept confined around the black hole by an accretion disc. The rotation of space time near the black hole twists these lines into helical magnetic springs which expand under their own pressure and accelerate any attached plasma. In the process, energy is extracted from the spinning black hole and is transported out along the magnetic field, making a relativistic jet. The Blandford-Znajeck (BZ) mechanism is an acceptable idea since magnetic fields are common in astrophysical accretion discs and so the requirements for this mechanism are easily met. If $\Omega$ is the angular velocity of the system perpendicular to the plane of the rotating disc, then the charged particles on the disk surface experience a Lorentz force, $\mathbf{L}_{\mathbf{f}}=q(\mathbf{v} \times \mathbf{B})$. This force results in a quadrupole electric field. On the surface of the disk current will flow from pole to equator and the Lorentz force will give a net torque i.e. a rotational force which taps the rotational energy of the spinning black hole.

According to the Blandford-Payne model (Blandford \& Payne 1982), a rotating magnetised accretion disk also plays an inevitable role in the jet launching and acceleration along with the central spinning black hole. Considering a cylindrical coordinate system $(z, r, \phi)$, the magnetic field vector can be decomposed into a poloidal component, $B_{p}=B_{r}(r, z) \vec{r}+$ $B_{z}(r, z) \vec{z}$ and a toroidal component $B_{\phi}=B_{\phi}(r, z) \vec{\phi}$ where $z$ is the axis of rotation, and $(r, \phi)$ is the plane of the disk. Blandford \& Payne (1982) suggested that the magnetic field can be advected inwards by the accretion disk and the field lines get coordinated with the central spinning black hole and this magnetosphere rotate along with it. The inertia i.e. the tendency of magnetosphere to follow the rotational motion of the central rotating object deviates the magnetosphere from the force free equilibrium condition given by:

$$
\rho_{q} \mathbf{E}+\frac{1}{c}(\mathbf{j} \times \mathbf{B})=0,
$$

where first term is the electrostatic force and second term is Laplace force which is given by:

$$
(\mathbf{j} \times \mathbf{B})=\mathbf{i}_{\mathbf{n}} \frac{1}{R_{c}} \frac{B^{2}}{4 \pi}+\mathbf{i}_{\mathbf{t}} \frac{d}{d s}\left(\frac{B^{2}}{8 \pi}\right)-\nabla\left(\frac{B^{2}}{8 \pi}\right),
$$

where $\mathbf{i}_{\mathbf{t}}=d r / d s$ is the unit tangent vector along the magnetic field and $\mathbf{i}_{\mathbf{n}}$ is the unit vector towards the center of curvature of magnetic field lines. The first two terms are called as magnetic tension and $\nabla\left(\frac{B^{2}}{8 \pi}\right)$ is the gradient of magnetic pressure, $p_{m}=B^{2} / 8 \pi$, the effect of which will be discussed in 2.3.2.

When equilibrium defined by equation 2.33 is lost, the poloidal current and poloidal magnetic field are no longer parallel; instead it crosses the poloidal magnetic field lines and creates a closed circuit inside the magnetosphere. Then the Laplace force has a component which is parallel to this poloidal magnetic field which accelerates the magnetized plasma, converting the Poynting flux into the kinetic energy of bulk motion. This results in the formation of winds and jets in magnetically driven outflows. In BP mechanism, the jet is composed of electrons and positrons as they are launched from a black hole's ergosphere 


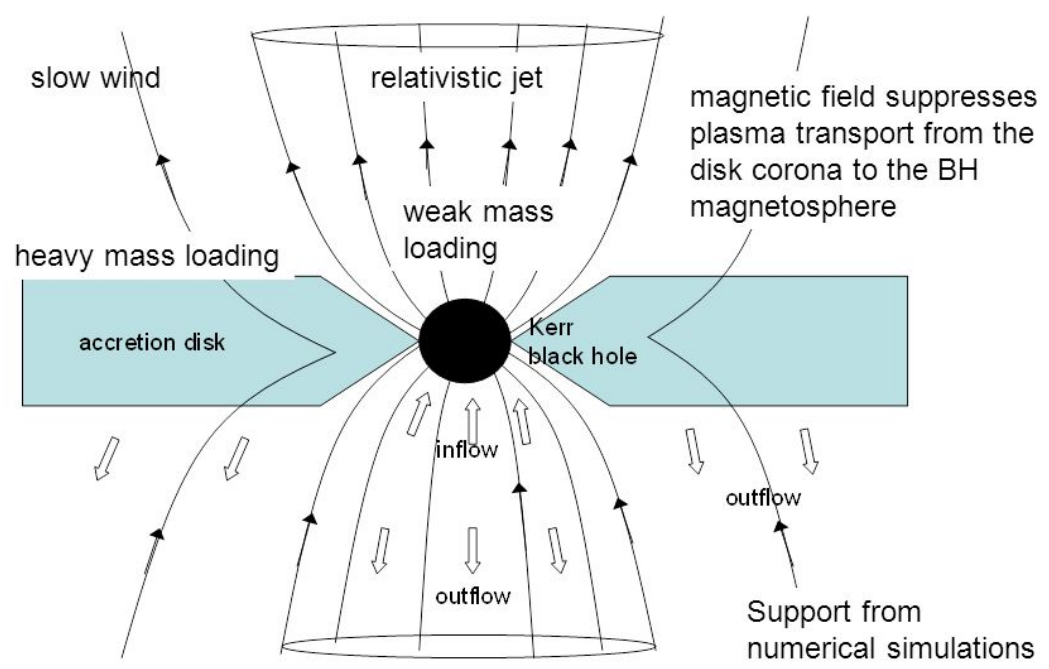

Figure 2.2: An illustration of the structure of magnetosphere with field lines threaded around the black hole. The accretion flow extends to the plunching region (shown as wedge ends pointing toward the black hole) makes the magnetic flux threading the black hole higher. The angular velocity of the black hole and the magnetic flux determines the jet power. Credit: Serguei Komissarov

while in the BZ mechanism, the jet consists of protons as they are launched from the accretion disc.

\subsubsection{Acceleration and Collimation of Jets}

The launching and acceleration is initiated by a magneto centrifugal mechanism (Blandford \& Payne 1982). The steady wind solutions result in the flow become faster, accelerated to high bulk Lorentz factor with their distance. The flow cross three critical surfaces slow magnetosonic, Alfvénic and fast magnetosonic (Boettcher et al. 2012; Meier 2012) which are the major acceleration zones for the flow, which are discussed below. At the launching point, close to the accretion disk, the gas pressure is important and provides an initial acceleration. The Lorentz factor of the flow, $\gamma$ at the launch point is of the order of 1.0 and there Poynting flux is dominated. Right after this is the region of low density and the magnetic pressure (recall equation 2.34) dominates gas pressure and therefore the disk and the flow co-rotates. This results in a centrifugal force which accelerates the plasma parallel to the field. When seen from an observer's frame of reference, there is an azimuthal magnetic field component and therefore the gradient of magnetic pressure, $\nabla\left(\frac{B^{2}}{8 \pi}\right)$ (see equation 2.34) will be the reason for acceleration in this region. This is the slow magnetosonic regime. Next is the Alfvénic region where the flow speed is lesser than the Alfvén speed. In this region, the co-rotation of the field lines stops and the field start lagging behind the flow thus giving rise to a toroidal magnetic field component resulting in magnetic helical springs. In this axisymmetric configuration, the gradient of magnetic pressure, $\nabla\left(\frac{B^{2}}{8 \pi}\right)$ give rise to hoop stress forces that is associated with azimuthal component of magnetic field and accelerates the magnetized flow to fast magnetosonic speeds in the fast magnetosonic regime. At the fast magnetosonic regime, the Lorentz factor becomes, $\gamma \sim \mu^{1 / 3}$ where $\mu$ is the ratio of the Poynting (magnetic) energy flux to kinetic energy flux called as magnetization parameter. The maximum Lorentz factor that can be reached is 
$\gamma_{\max } \sim \mu^{1 / 3}$ because of the force free equilibrium achieved by magnetic pressure term and magnetic tension given in equation 2.34 (Michel 1969). This is because in split-monopole geometry of magnetic field lines, Poynting energy flux cannot be fully converted to kinetic energy flux. But in parabolic geometry, the equipartition between Poynting and kinetic energy can be achieved and Lorentz factor can exceed $\mu$. It is found observationally (Homan et al. 2015) and through simulations (Komissarov et al. 2007) that the acceleration scale can reach large distances of $\sim 100 \mathrm{pc}\left(10^{3}-10^{4} \mathrm{R}_{\mathrm{s}}\right)$.

The AGN jets are highly collimated. In the case of M 87, its jet shows an extraordinary collimation from an angle of $60^{\circ}$ on the scales $0.01 \mathrm{pc}\left(50 \mathrm{r}_{\mathrm{g}}\right)$ to $5^{\circ}$ on kpc scale (Biretta et al. 2002). The collimation could be due to a component of the central engine that limits the volume occupied by relativistic flow and this component could be the disk wind in the parabolic solution shown in Blandford \& Znajek (1977) or the inter stellar medium (ISM) of the host galaxy or the ambient medium in combination with the jet cocoon (Spruit 2010; Boettcher et al. 2012). The high Lorentz factors of the order of 10 seen in the case of blazars can be explained by the gradual increase of volume confinement and collimation that are essential for magnetic acceleration and also, on the geometry of field lines. After this stage of acceleration and collimation that takes place from a region of $10^{3}-10^{5} R_{s}$, a large amount of magnetic energy is converted to kinetic energy and kinetic-flux dominated jets are seen between $10^{5}-10^{9} R_{s}$ (Boccardi et al. 2017). Later the collimation is lost due to various plasma instabilities (discussed in section 2.3.5) and energy is lost in the form of radiation. Finally, the jet terminates when its kinetic energy equals that of the ambient intergalactic medium forming a termination shock seen as radio lobes (see section 1.3.6).

\subsubsection{Jets and Magnetic Fields}

In the $\mathrm{BZ}$ mechanism, the rate at which rotational energy of the black hole $(\mathrm{BH})$ is extracted, $\mathrm{P}_{\mathrm{BZ}}$ or the jet power, $L_{J}$ is given in Gaussian cgs units (Tchekhovskoy et al. 2010) as:

$$
\mathrm{P}_{\mathrm{BZ}}=L_{J}=\frac{\kappa}{4 \pi \mathrm{c}} \Omega_{\mathrm{H}}^{2} \phi_{\mathrm{BH}}^{2} \mathrm{f}\left(\Omega_{\mathrm{BH}}\right),
$$

where $\kappa$ is a numerical constant whose value depends on the magnetic field geometry $(\kappa$ is 0.053 for a split monopole geometry and 0.044 for a parabolic geometry), $\Omega_{\mathrm{H}}=\mathrm{ac} / 2 \mathrm{r}_{\mathrm{H}}$ is the angular frequency of the $\mathrm{BH}$ horizon, a is the spin parameter, $\mathrm{r}_{\mathrm{H}}$ is the radius of the event horizon, $\phi_{\mathrm{BH}}$ is the magnetic flux accumulated. Equation 2.35 for the jet power is valid for Blandford-Payne (BP) mechanism also in the sense that the jet power depends on $\Omega_{\mathrm{H}}$ and $\phi_{\mathrm{BH}}$.

For both BZ or BP meachanism of jet launching, it is clear from equation 2.35 that

$$
\mathrm{P}_{\mathrm{BZ}} \propto \Omega_{\mathrm{H}}^{2}, \text { and } \Omega_{\mathrm{H}}^{2} \propto a^{2} .
$$

Therefore, equation 2.36 implies that the power extracted which is the power for triggering jets is proportional to square of spin parameter given by:

$$
\mathrm{P}_{\mathrm{BZ}} \propto a^{2} .
$$

The efficiency with which the $\mathrm{BH}$ generates jet power, $\eta_{B Z}$, is defined as the ratio of the time average electromagnetic power that flows out of the $\mathrm{BH},\left\langle\mathrm{P}_{\mathrm{BZ}}\right\rangle$, to the time average 
rate at which rest-mass energy flows into the $\mathrm{BH},\left\langle\dot{M}>c^{2}\right.$ is:

$$
\begin{aligned}
\eta_{B Z} & =\frac{\left\langle\mathrm{P}_{\mathrm{BZ}}>\right.}{<\dot{M}>c^{2}}, \\
& =\left[\frac{\kappa}{4 \pi \mathrm{c}}\right]\left[\frac{\Omega_{H} r_{g}}{c}\right]^{2} \phi_{\mathrm{BH}}^{\dot{2}} \mathrm{f}\left(\Omega_{\mathrm{BH}}\right) \times 100 \text { percent },
\end{aligned}
$$

where $\phi_{\mathrm{BH}}=\phi_{\mathrm{BH}} /\left(<\dot{M}>r_{g} c\right)^{1 / 2}$ is the dimensionless magnetic flux threading the $\mathrm{BH}$ and $\langle.$.$\rangle is a time average. Thus the efficiency with which a spinning BH can generate$ jet power depends on $\mathrm{BH}$ spin parameter 'a' via the angular frequency, $\Omega_{\mathrm{H}}$ and on the dimensionless magneticflux, $\phi_{\mathrm{BH}}$. The strength of $\phi_{\mathrm{BH}}$ is very uncertain (Tchekhovskoy et al. 2010).

Even though it is agreed that $\phi_{\mathrm{BH}}$ is non-zero, since magnetic flux is transported to the accreting BH by turbulent accretion, the key elements of this is not well understood. This leads to a large uncertainty in the value of $\eta_{B Z}$. Recent time dependent general relativistic magneto hydrodynamic (GRMHD) numerical simulations have found a rather low efficiency, $\eta_{B Z} \leq 20$ per cent, even when the central $\mathrm{BH}$ is nearly maximally spinning (De Villiers et al. 2005). Observationally, some AGN in the universe are found to have extremely efficient jets that require $\eta>100$ per cent (Rawlings \& Saunders 1991). A non-spinning $\mathrm{BH}$ usually has $\eta_{B Z}<10$ per cent, and might under special circumstances have $\eta \approx 10$ per cent (Narayan, Igumenshchev and Abramowicz 2003). However, a non-spinning BH can never give $\eta>100$ per cent, since this requires the system to produce more energy than the entire rest mass energy supplied by accretion. Values of $\eta>100$ per cent are possible only by extracting energy from the spin of the BH. Thus, taken at face value, any robust observation of $\eta>100$ per cent in an AGN implies that the Penrose/BZ process must be operating.

In 2011, a team of Alexander Tchekhovskoy, Ramesh Narayan and Jonathan C. McKinney (Tchekhovskoy et al. 2011) worked on the 3D, time-dependent, non-radiative, generalrelativistic, magneto hydrodynamic simulations of accreting black holes (BHs). They designed the simulations in such a way to transport a large amount of magnetic flux to the centre, more than the accreting gas can force into the BH. The excess magnetic flux remains outside the $\mathrm{BH}$, impedes accretion, and leads to a magnetically arrested disk (MAD). Their result showed powerful outflows like for a $\mathrm{BH}$ with spin parameter $a=0.5$, the efficiency is $\eta \approx 30$ per cent and for $a=0.99$, they found $\eta \approx 140$ per cent, which means that more energy flows out of the BH than flows in. The only way this can happen is by extracting spin energy from the $\mathrm{BH}$ via some mechanism like the Penrose or BZ mechanism. A possibility they suggest is that the magnetically arrested accretion might explain this high power and efficiency of black holes to power jets.

\subsubsection{The Radio-Loud / Radio-Quiet Dichotomy}

The existing bimodality in radio flux distribution into radio-loud (RL) and radio-quiet (RQ) AGNs can be linked to the jet because of the general argument that the power from the RL AGNs is dominated by jet produced via the synchrotron radiation whereas the power from RQ AGNs is dominated by the accretion disk (Chiaberge \& Marconi 2011). This has motivated many people to study the link between the radio loudness and the black hole properties. This has motivated many people to study the link between the radio loudness and the black hole properties. Radio loudness parameter defined by equation 1.1 as the ratio 
of radio ( $5 \mathrm{GHz}$ ) to optical (B band) spectral flux densities (Kellermann et al. 1989) and they established $R=10$ as the borderline between RQ and RL AGNS. The radio dichotomy can be directly linked to the efficiency of jet production (Sikora et al. 2008). Laor (2000) established the correlation between the radio loudness bimodality and the black hole mass in Boroson \& Green Palomar catalog. 87 quasars whose red shift lesser than red shift 0.5 was studied by him and the border line established for RQ AGNs was to be less massive than $3 \times 10^{8} M_{\odot}$. It is well seen that there is no population of RL AGNs with supermassive black holes $<10^{8} M_{\odot}$ (Dunlop et al. 2003; Best et al. 2005; Chiaberge et al. 2005). When $M>10^{8} M_{\odot}$, the tidal radius lies outside the Schwartzchild radius and so stars are easily captured and become radio-loud and since most of the QSOs have black hole mass less than critical mass $\left(\sim 10^{8} M_{\odot}\right)$, most of them are RQ (Gopal-Krishna et al. 2008). However black hole mass alone cannot explain the radio loudness and the jet triggering efficiency. It has explicit connection to the black hole spin (Blandford et al. 1990; Chiaberge et al. 2005; Garofalo 2009; Tchekhovskoy et al. 2011). As a supportive observational evidence, even though RL and RQ AGNs have supermassive black holes, only $10 \%$ of the AGNs are radio-loud and produce radio jets. So the optimal combination of a rapidly spinning black hole and supermassive black hole is important in building up jet triggering mechanism as discussed in section 2.3 .

The launching of jets is also linked to the properties of host galaxy. The radio-loud AGNs are mostly associated with elliptical galaxies and radio quiet galaxies are associated with spiral galaxies although there are few exceptions as in the case of J2345 - 0449 studied by Bagchi et al. (2014) where powerful radio jets are seen in a spiral galaxy. This may be linked to the formation of the galaxy and the merger events it has gone through. The accretion process is efficient in gaining up angular momentum for the spinning up of the black hole (Bardeen 1970) and the higher spin is attained in the case of elliptical galaxies than spiral galaxies due to the different merger events they had in their formation and if two galaxies with comparable mass merges, then only effectively fast rotating black holes can be created (Sikora et al. 2007). These kind of galaxy mergers are rare events and those lesser chances of merging events can be linked to the less number of radio-loud AGNs seen observationally in the universe. The thin accretion disks which are most common in nature is inefficient in depositing magnetic flux near the black hole whereas the thick disk is capable of effective magnetic flux deposition during a hot accretion phase that is associated with a major merger event (Sikora \& Begelman 2013). Thin disk being associated with almost all the AGNs in the universe, the no. of RQ AGNs are larger than their RL cousins.

It is clearly seen in the equation 2.38 that the efficiency with which the black hole generates jet is directly linked to the average value of square of magnetic flux, $\phi_{\mathrm{BH}}^{2}$. Sikora \& Begelman (2013) explained that magnetic flux threading in the black hole is the dominant factor for producing jets and thus the radio loudness of BHs. Since the thin accretion disks that feed most of the AGNs are inefficient in deposing magnetic flux close to the black hole, most of the AGNs are radio-quiet. Flux accumulation is more likely to occur in a hot accretion phase, and we know that radio-loud quasars and radio galaxies occur when a massive, cold accretion event follows an episode of hot accretion. Such an event might be triggered by the merger of a giant elliptical galaxy with a disk galaxy. While the strong jets are explained from the accumulation of magnetic flux, moderate jet activity can also be triggered by magnetic flux fluctuations deposited by turbulent, hot inner regions of otherwise thin accretion disks, or by the dissipation of turbulent fields in accretion disk coronae. These processes might be responsible for jet triggering in Seyferts, low luminosity AGNs and in X-ray binaries. It is already established that about $10 \%$ of the QSOs known in $z \leq 6$ are detected and bright in radio (Haiman et al. 2004). 


\subsubsection{Plasma Instabilities}

There are instabilities occurring in the plasma flow especially when they reach distance of kpc or Mpc. The two most common instabilities associated with plasma flow are CurrentDriven (CD) "kink" instability (Hardee et al. 2010) and Kelvin-Helmoltz (K-H) instability. The kink mode of the CD instability disturbs the fluid's center of mass and thereby perturbs the cylindrical flow of the plasma. This operates in the Poynting flux dominated regime of the jet (Mizuno et al. 2012; Moser \& Bellan 2012). The KH instability occurs as a result of the pressure difference acting between the jet and the external medium due to the viscosity difference in the fast jet and slowly moving external medium (Lobanov \& Zensus 2001). This is clearly seen in the bend of the jet in 3C 273 when it interacts with the surrounding medium in $15 \mathrm{GHz}$ maps from MOJAVE. These plasma instabilities have significant role in disturbing the collimation of the jets especially in kiloparsec scales i.e. at a distance of $>10^{9} R_{s}$ and finally dissipating all of its energy into radiations.

\subsubsection{Jet Composition}

One of the key questions addressed in jet physics is "What is the composition of relativistic jets in compact radio sources which are responsible for the transport of energy to megaparsec scales?". The different possibilities for the jet composition are either pairs or normal plasma - more precisely cold (thermal) or hot (relativistic) protons, normal electron plasma, cold electrons/positrons pair, and Poytnting flux (Boettcher et al. 2012). Neutrons and lowfrequency electro magnetic (EM) radiation were also suggested but they were ruled out since it is difficult to explain the bending of jets and the jet genesis. The different works tried to explain the jet composition by the general approach of estimating the total power requirements of the jet, and then find an estimate on how many electrons are there in the jet.

\subsubsection{Light Jets}

The study by Wardle et al. (1998) detected circularly polarized radio emission from the jets of 3C 279. The circular polarization produced by Faraday rotation of linearly polarized radiation set constrains for an $\left(e^{+} e^{-}\right)$pair by establishing the minimum of the electron energy distribution as $\gamma_{\min }<20$. Similar detections in three other radio sources gave evidence that extragalactic radio jets are composed mainly of an $\left(e^{+} e^{-}\right)$plasma. Dunn et al. (2006) showed results that the radio emitting region of the jet is electron-positron dominated for M87 and Perseus (3C 84) assuming that the minimum of the electron energy distribution, $\gamma_{\min }=1$. This result was valid only for low energy radio sources. Reynolds et al. (1996) also suggests that jet is composed of electrons and positrons.

Sikora \& Madejski (2000) investigated X-ray observations from optically violent variable quasars (see section 1.1.4) and showed that the jets contain electron-positron $\left(e^{+} e^{-}\right)$pairs more than protons but they are dynamically dominated by protons. We should keep in mind that there is uncertainty in the number of electrons (or particles) derivation process mentioned in the above papers. All these studies are based on the assumption of the steep power law spectrum which is electron ensemble's energy distribution with no spectral breaks at low energies. But, Blundell et al. (2006) discovered the low energy cut off in a giant radio galaxy $6 \mathrm{C} 0905+3955(z=1.88)$. The X-ray emission from inverse Compton 
scattering of cosmic microwave background $(\mathrm{CMB})$ radiation in the radio lobes were found to be coming from electrons with Lorentz factor, $\gamma=10^{3}$. This is an evidence for large number of $\gamma=10^{3}$ particles in the old plasma in the lobes than the freshly accelerated plasma from hotspots, which was $80 \mathrm{kpc}$ away from radio lobe in this radio galaxy.

\subsubsection{Heavy Jets}

One supportive study for heavy jets (protojets or electron-proton plasma) is done by Celotti \& Fabian (1993) where they estimated the bulk kinetic energy and the number flux of particles in parsec scale jets using the synchrotron self-Compton emission model. The results showed that there have to be more electrons and positrons to be delivered from the central compact object if the energy transport is due to pairs than if most of the energy is carried by protons.

\subsubsection{Poynting Flux Dominated Jets}

Sikora et al. (2005) addressed the question of matter content in jets in quasars. They found that in quasars the conversion from Poynting flux-dominated to matter-dominated jets is very likely to take place closer to the black hole than in the region where most of the Doppler-boosted radiation observed in blazars is produced (Sikora et al. 2005). While jets are dominated by Poynting flux (Poynting flux means the cross product of electric fields, $\mathbf{E}$ and magnetic fields, B. It is the electromagnetic energy radiated out through a surface at the cost of the energy stored in the fields) initially, latter they become dominated by protons.

Harris \& Krawczynski (2007) ruled out the possibility for hot electrons/positrons to be the energy carriers in jets. Such hot electrons/positrons plasma with $\gamma>2000$ would loose energy before they reach the end of the jet flow and so they cannot transport energy to large distances.

\subsection{Ultracompact Radio Sources - Innermost Region of Rel- ativistic Jets}

As discussed in section 2.3.2, the acceleration scale can reach large distances of $\sim 100 \mathrm{pc}$, observationally showed by Homan et al. (2015) and proved through simulations by Komissarov et al. (2007) whereas the jets are formed and acceleration begins at a scale of the order $\sim 0.1-10 \mathrm{pc}$ (Lobanov et al. 2006). This region or scale is called as Ultracompact jets. According to Meier (2001), the jet launching takes place at a distance of $\leq 10^{2} R_{\mathrm{s}}$ and the acceleration and collimation span an interval between few and $\left(10^{3}-10^{4} \mathrm{R}_{\mathrm{s}}\right)$ (Vlahakis \& Königl 2004; Komissarov et al. 2007). From the discussion in section 2.3.2, it is clear that the acceleration and collimation of the plasma and the immense electromagnetic radiation observed from radio to $\gamma$ energies take place in a region close to the supermassive black hole on parsec scales. VLBI studies by Krichbaum et al. (1992), Lister et al. (2009), Mertens et al. (2016), Boccardi et al. (2017) etc. have shown that the physics of ultracompact radio jets can be studied through very-long-baseline interferometry (VLBI) studies. 
What we usually see in a VLBI observation is the most upstream feature in the radio jet usually called as VLBI core and an extended jet on parsec scales. The VLBI core is the most bright feature we see, which is usually unresolved. The position of VLBI core does not coincide with the central engine of the AGN, instead it represents a region where the optical depth to synchrotron emission, $\tau$, becomes unity for a given observing wavelength (Königl 1981). For different frequencies, the distance of these VLBI core positions will be different. The position of the VLBI core, $r$ depends on frequency as $r \propto \nu^{-1 / k r}$ where $\nu$ is the observing frequency and $k_{r}$ is 1 for a synchrotron self absorbed core in equipartition (Königl 1981; Lobanov 1998). This effect is known as core-shift which is schematically illustrated in figure 2.3. This makes it particularly important to conduct multi-frequency observations of same source. The $T_{b}$ measured in the core at different frequencies represents actually physically different regions. The combined studies of the positions of the VLBI cores at different frequencies or core-shift studies can help to investigate the magnetic field strength, the actual distance to the central engine, testing the inner jet models, studying the acceleration in the inner jets etc.

\section{Frequency dependent position shift of VLBI core.}

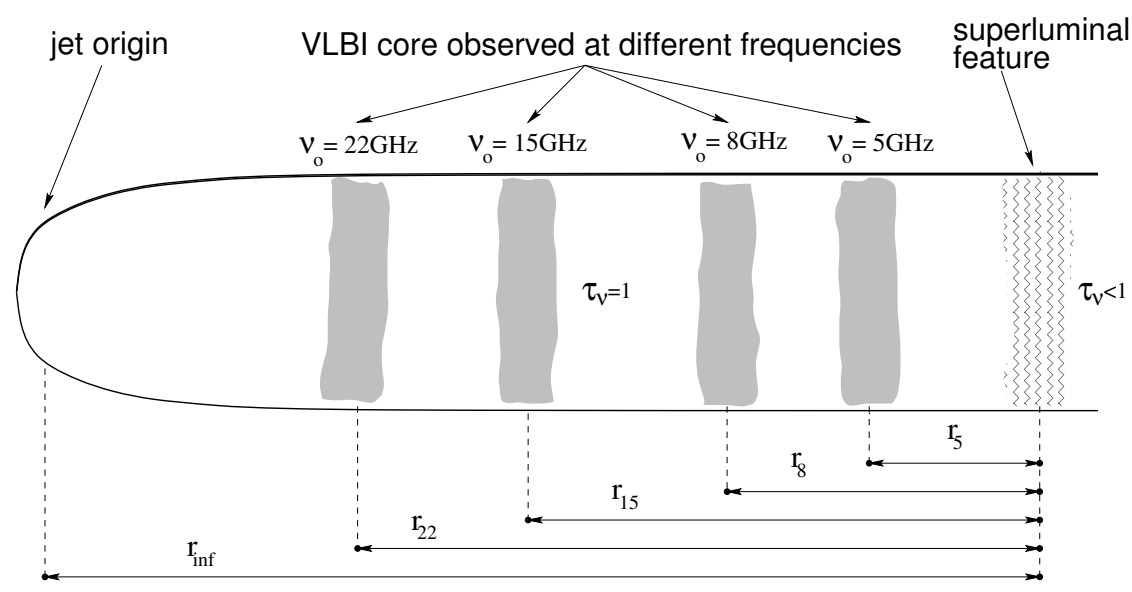

Figure 2.3: A schematic representation of the frequency dependence position shift of the VLBI core (Lobanov 1996).

\subsubsection{Shock-Shock Interactions}

It is discussed in Marscher \& Gear (1985) that energy (or plasma flow) is transferred in the form of disturbances or shocks from the central engine to the VLBI radio core and accelerated and collimated by magneto hydrodynamic accretion disks along with the rotational energy extracted from the spinning black hole in the center which was discussed in detail in section 2.3. The spectrum of the ultracompact sources steeps at a frequency, $\nu_{s}$ which lies somewhere between $\sim 10-1000 \mathrm{GHz}$. Shock-shock interactions or an interaction between a stationary shock with moving shocks can accelerate particles and such stationary features (which are standing recollimation shocks) are often seen in ultracompact jets (Fromm et al. 2013). The VLBI core itself we see can be considered as a conical recollimation shock that can accelerate particles according to Daly \& Marscher (1988) through Fermi mechanism (Bell 1978; Blandford et al. 1990) and the local magnetic field. The shock wavefronts interactions were used to explain the high $\mathrm{TeV}$ energy flares seen in the $\gamma$ energy regime of compact jets. Extensive studies have been done to investigate the origin of such gamma 
ray flares and to understand whether it coincides with such VLBI cores (Fuhrmann et al. 2014) in the compact jets. Hodgson et al. (2017) showed $\gamma$ ray flares appeared to correlate with the mm core region and a downstream stationary feature in OJ 287.

\subsubsection{Spine-Sheath Configuration}

Ultracompact jets can be well understood by their Spine-Sheath configuration (Laing \& Bridle 2002; Meier 2003; McKinney 2005). The best example is the edge-brightened transverse velocity gradient structure seen in the jets of $\mathrm{M} 87$ in its $15 \mathrm{GHz}, 43 \mathrm{GHz}$ and $86 \mathrm{GHz}$ VLBI maps (Kovalev et al. 2007; Mertens et al. 2016) which can be explained by the transverse velocity gradient in the jet i.e. the speed of jet region on the axis, called as Spine, is faster than that of the funnel shaped edge region, called as Sheath. Mertens et al. (2016) clearly showed the displacement of features along three main filaments of the flow, two outer limbs and a central body while studying their 2-D velocity field in the parsec scales of M 87. This transverse velocity gradient supporting spine-sheath configuration in an inner jet is also seen in the $43 \mathrm{GHz}$ map of $3 \mathrm{C} 84$ (Nagai et al. 2014) and in the $43 \mathrm{GHz}$ map of Cygnus A (Boccardi et al. 2016). If the viewing angle is greater than the critical angle for the central spine, $\theta>\theta_{\text {crit,spine }}$, then the spine is deboosted and if $\theta<\theta_{\text {crit,spine, }}$, then the limb is enhanced. The spine consists relativistic electron-positron pairs whereas the sheath contains normal matter like electrons and protons. Cohen et al. (2007) suggested that the strong radio lobes of Cygnus $\mathrm{A}$ is an indication of its relativistic spine but the large viewing angle makes it practically impossible to see the spine. But its mildly relativistic sheath is observed and that produces its weak jet and counter jet. The flux density of a source that has spine-sheath configuration depends on the viewing angle, $\theta$ and the flux density varies more slowly with $\theta$ than the canonical Doppler factor, $\delta^{p}$, with $p=2$ or 3 (Boettcher et al. 2012).

\subsubsection{Accelerating and Decelerating Inner Jet Models}

According to the accelerating jet model (Blandford \& Rees 1974; Marscher 1995), a relativistic neutron beam is launched from the central machine which decay into protons and electrons constituting the plasma flow. The acceleration starts from the jet base with a magneto hydrodynamic pressure and the Lorentz factor increases along the jet due to the conversion of the rest mass energy of the plasma to kinetic energy of the plasma flow. In this model, the synchrotron emission at UV, optical and IR is confined near the central black hole, opaque to radio emission. The radio emission is generated outside this region of confinement with the maximum intensity occurring where the Lorentz factor, $\gamma$ is highest.

According to the decelerating jet model, the central engine launches a decelerating flow of collimated electron positron pair (Melia \& Königl 1989), the jets are accelerated to superrelativistic Lorentz factors by electromagnetic or hydromagnetic forces taking place near black hole and are compton dragged to $\gamma_{\infty} \leq 10$ (Lee et al. 2008). The electron-positron pair up-scatter the UV photons in the accretion disk to X-rays and $\gamma$-rays, which in turn decelerates the beam and so decreases the Lorentz factor along the jet (Marscher \& Gear 1985; Marscher 1995; Lee et al. 2008). Melia \& Königl (1989) proposed that the thermal radiation field produced by accretion in an AGN can be very effective in decelerating ultra relativistic jets that are accreted. The terminal Lorentz factors as inferred by them was consistent with the values seen in superluminal radio sources. The intensity profiles and 
the resulting brightness temperatures along the jet are significantly different and have a characteristic shape, in each of these models (Medici \& Lobanov 2002). As shown in figure 2.4, the relative position of the emission regions at each wavelength is also model dependent. This will help to distinguish between the emission coming from accelerating or decelerating model and from electron-positron or electron-proton plasma.
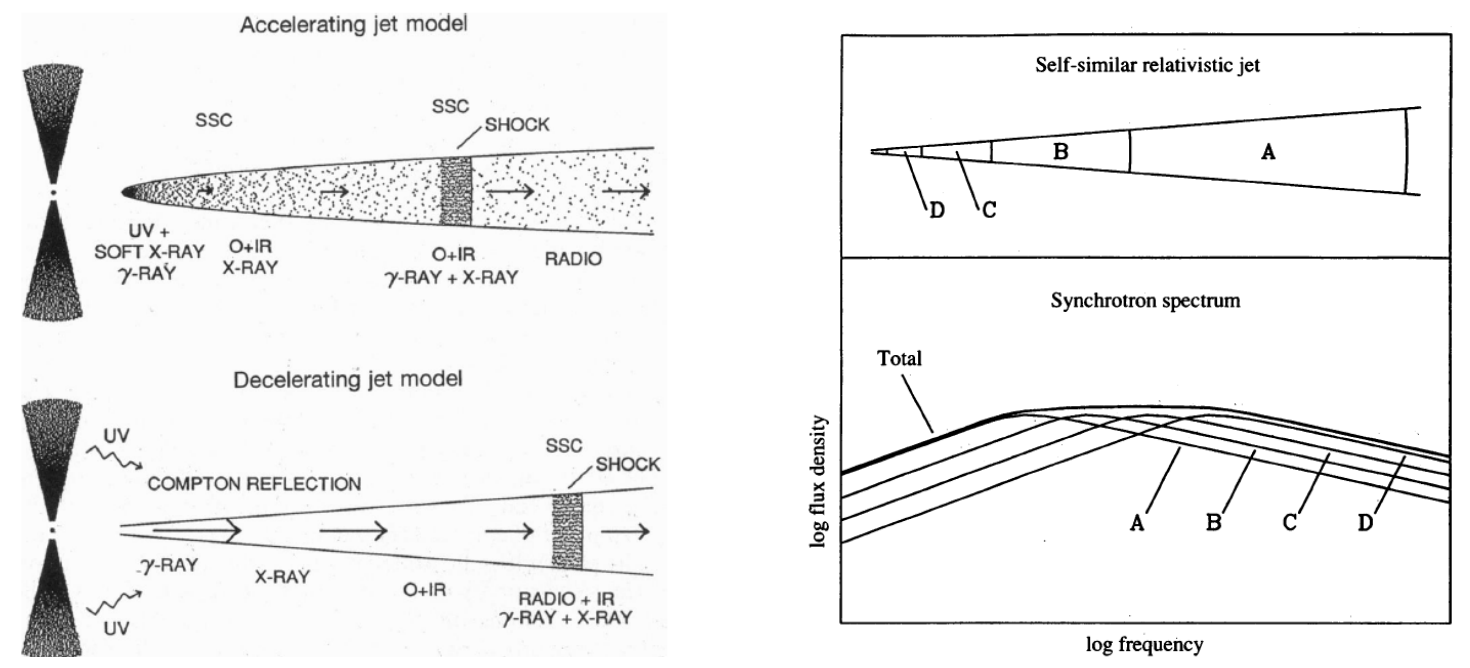

Figure 2.4: Left: Schematic representation of accelerating and decelerating jet model. Right Top: Sketch of the synchrotron spectrum of a self-similar relativistic jet. Right Bottom: The flat spectrum formation at the jet base. Figures taken from Marscher (1995).

\subsection{VLBI Observations To Investigate Physics of Extragalac- tic Jets}

Of course, a big achievement by VLBI is that one can directly probe a region close to the supermassive black hole in AGNs upto a region of 100s of Schwartzchild radii $\left(R_{s}\right)$ (Hada et al. 2016; Nagai et al. 2014). A good example is the angular resolution obtained for $86 \mathrm{GHz}$ VLBI map of Cygnus A $(z=0.056)$ which is at a distance of $249 \mathrm{Mpc}$, is $\sim 90 \mu a s$ Boccardi et al. (2015). This corresponds to a linear scale of 400 Schwartzchild radii and a linear region of $\sim 0.15$ pc. Even though centimeter VLBI (Lister et al. 2009) have given a wealth of information on parsec scales, one has to go to still higher frequencies (or shorter wavelength) like $43 \mathrm{GHz}(\sim 7 \mathrm{~mm}), 86 \mathrm{GHz}(\sim 3 \mathrm{~mm})$ etc. to overcome the synchrotron self absorbed regions which are opaque to centimeter radiation but optically thin to millimeter and sub-millimeter radiation. Now there are ongoing promising projects like Event Horizon Telescope (EHT) to understand ultracompact jet flow in the immediate vicinity of supermassive black holes at $230 \mathrm{GHz}(\sim 1 \mathrm{~mm})$. EHT observations are already successful for the nearby source M87 (Doeleman et al. 2012).

\subsubsection{VLBI Surveys}

In order to understand the AGN physics completely, it is important to study them throughout the entire electro magnetic spectrum from infra-red to $\gamma$-ray bands. There are many surveys done in different electro magnetic regime or frequencies to study the AGN emission. Surveys allow a statistical study of the population they are representing by modelling the 
results and get estimates on general kinematics and intrinsic properties of the extragalactic jets. The popular surveys conducted in different energy bands are Sloan Digital Sky Survey (SDSS; Gunn et al. 1998; Ahn et al. 2014; Blanton et al. 2017) NRAO VLA Sky Survey (NVSS; Condon et al. 1998), VLA FIRST survey (Becker et al. 1994), Spitzer surveys (Fadda et al 2004), Fermi, Chandra, Hubble Space Telescope (HST) etc. SDSS is a legacy optical survey started in 1994 when a large-format mosaic CCD camera for the SDSS was constructed (Gunn et al. 1998) . Their first Data Release 1 (DR1) provided images, catalogs, spectra, and redshifts. SDSS is a multi-spectral imaging and spectroscopic redshift survey using a dedicated 2.5-m wide-angle optical telescope at Apache Point Observatory in New Mexico, United States. Data Release 14 (DR14) is the most latest data release from SDSS camera, BOSS and APOGEE combined. NVSS is a $1.4 \mathrm{GHz}$ continuum survey covering the entire sky north of $-40^{\circ}$ declination with an angular resolution of $\sim 45^{\prime \prime}$. VLA FIRST is a systematic survey of the northern sky at $20 \mathrm{~cm}$ wavelength with an angular resolution of $\sim 5^{\prime \prime}$.

The famous VLBI surveys of compact radio sources done so far are discussed below where the sources are mainly selected on the basis of compactness, flux density and high-energy emission. PR Survey was the first imaging survey conducted in centimeter VLBI with 65 powerful extragalactic radio sources at $5 \mathrm{GHz}$ (Pearson \& Readhead 1988). They obtained a resolution of $0.001^{\prime \prime}$ for 37 sources in the sample and they showed that majority of sources showed a "core-jet" structure. The sources were classified as compact, asymmetric, compact double, irregular flat-spectrum, compact steep-spectrum, and lobe-dominated. They studied the radio polarization, variability and alignment of large-scale and small-scale structure in each of the classes. Their results showed that the nuclear jets are fairly highly polarized, in the range of 1-5 percent. Superluminal motion was also found in almost all the classes and explained how Doppler boosting results in the asymmetric core-jet structure observed.

The $5 \mathrm{GHz}$ survey called Caltech-Jodrell Bank Flat-Spectrum Survey (CJF) was presented in a series of two surveys CJ1 and CJ2 (Taylor et al. 1994; Xu et al. 1995; Henstock et al. 1995; Polatidis et al. 1996; Thakkar et al. 1996). The CJF consists of 293 sources and present at least 3 epochs of data at $5 \mathrm{GHz}$. The images and kinematic results are given in Britzen et al. (2007). The flux density limit was $S \geq 0.35 \mathrm{Jy}$. The sample contains flat-spectrum or gigahertz-peaked-spectrum sources selected at $4850 \mathrm{MHz}$. They obtained a resolution of $\sim 1$ mas with a global array. The PR survey combined with CJ1 and CJ2 surveys gave the final complete sample presented as CJF survey at $5 \mathrm{GHz}$ in Taylor et al. (1996). Soon after CJF release, Shen et al. (1997) released a $5 \mathrm{GHz}$ southern hemisphere VLBI survey of compact extragalactic radio sources which was conducted with the SHEVE array plus Shanghai station in 1992. 20 images were made from observations of 22 sources which revealed corejet structure and some sources showed bent structures. The dominance of highly polarized quasars were also found by them. The VLBA Imaging and Polarimetry Survey (VIPS; Helmboldt et al. 2007) presented 1127 sources with flat radio spectra obtained from $5 \mathrm{GHz}$ VLBI survey. This survey subsumes the CJF survey. An optical counterpart was searched from the SDSS survey. The mean ratio of the polarized to total $5 \mathrm{GHz}$ flux density for VIPS sources with detected polarized flux density was found to range from $1 \%$ to $20 \%$.

MOJAVE was a major breakthrough survey at $15 \mathrm{GHz}(\sim 2 \mathrm{~cm}$ wavelength) using VLBA which revealed the physics and kinematics of blazar jets on parsec scales (Lister \& Homan 2005; Homan et al. 2006; Kovalev et al. 2005; Lister et al. 2016). First time, 133 sources were presented with declination, $\delta>20^{\circ}$ and correlated $15 \mathrm{GHz}$ Very Long Baseline Array (VLBA) flux density exceeding 1.5 Jy. A strong circular polarization $(>=0.3 \%$ ) was found in $15 \%$ of the sample. Evidences for jet collimation at projected distances of $\leq 10 \mathrm{pc}$ and 
bending accelerations were found by Homan et al. (2015). The analysis combining the jet speeds and observed brightness temperatures by Homan et al. (2006) showed that intrinsic brightness temperatures was centered on a characteristic temperature, $T_{\text {int }} \approx 3 \times 10^{10} \mathrm{~K}$, which is close to the value of equipartition brightness temperature, when the energy in the radiating particles and the energy stored in the magnetic fields are equal. They also found that the intrinsic brightness temperature is greater than $2 \times 10^{11} \mathrm{~K}$, excess of the equipartition temperature. Hovatta et al. (2013) showed that the mean intrinsic brightness temperature is near the equipartition brightness temperature of $\sim 10^{11} \mathrm{~K}$ for 14 sources by combined analysis of MOJAVE survey and 15 light curves obtained from OVRO 40 m telescope using a new Bayesian Markov Chain Monte Carlo method to deconvolve the blazar light curves.

The other significant surveys done with VLBI for various motives are TANAMI (8.4, $22 \mathrm{GHz})$, VCS $(2,8 \mathrm{GHz})$, RRFID $(2,8 \mathrm{GHz})$. The VLBA-BU Blazar Monitoring Program in the Boston University have successfully obtained the total and polarized images of many sources giving a wealth of information regarding the jet collimation and acceleration at $43 \mathrm{GHz}$ (Q band) Jorstad \& Marscher (2016); Jorstad et al. (2017). A tight connection between $\gamma$ ray outbursts and parsec scale jet activity was found in quasar 3C 454.3 (Jorstad et al. 2013). Another successful $43 \mathrm{GHz}$ VLBI survey was done by Petrov et al. (2012) using Korean-VLBI network (KVN) whose maximum baseline is $480 \mathrm{~km}$. KVN Calibrator Survey (KVNCS), a single dish radio survey conducted at 22 (K band) and $43 \mathrm{GHz}(\mathrm{Q}$ band) simultaneously using KVN from 2009 to 2011 is also a major breakthrough with a total of 1533 sources in the K band with a flux density limit of 70 mJy and 553 sources in the Q band with a flux density limit of 120 mJy (Lee et al. 2017). The KVN team have developed a new millimeter wave receiver system with input optics that support simultaneous observation in four bands of $22,43,86$, and $129 \mathrm{GHz}$ and a 4 band fringe detection survey is ongoing.

The details of previous $86 \mathrm{GHz}$ (W band) surveys will be discussed in detail in section 2.6. As we have discussed in section 2.3.1, one thing is clear that the fundamental mechanism of wide range of energy production and jet formation takes place in the immediate vicinity of black holes in parsec scales or comparable to Schwartzchild radius. So it is important to carry out VLBI observations which result in high angular resolution to get a full picture of AGN physics. The two important well established VLBI arrays are Very Long Baseline Array (VLBA) and the European VLBI Network (EVN). The angular resolution depends on two factors - the baseline distance and the frequency of observation. Therefore a smart way to achieve extreme resolution is by increasing the baseline by putting one radio telescope in the VLBI array in a satellite in space. They are known as Space telescopes. This was successfully done for the first time with the VLBI Space Observatory Program (VSOP) (Hirabayashi \& Hirosawa 2000) using $1.6 \mathrm{GHz}$ and $5 \mathrm{GHz}$ receivers. A maximum baseline $\sim 360,000 \mathrm{~km}$, equivalent to distance between earth and moon is achieved recently by the RadioAstron space telescope (Kardashev et al. 2013). RadioAstron space telescope is equipped with a $10 \mathrm{~m}$ radio telescope and receivers at $\mathrm{L}(1.6 \mathrm{GHz}), \mathrm{C}(5 \mathrm{GHz})$ and $\mathrm{K}$ $(22 \mathrm{GHz})$ bands. The polarization maps at extreme resolution is a great achievement of RadioAstron (Lobanov et al. 2015). One of the most important result of RadioAstron is that they are able to measure extremely high brightness temperatures exceeding inverse Compton limit from space. Nowadays it is also possible to attain high resolutions by using high frequency receivers in VLBI arrays on earth itself. For example, Global Millimeter VLBI Array (GMVA) can attain a maximum baseline of $\sim 10,000 \mathrm{~km}$ across the trans-Atlantic baseline and observe with extremely high frequencies like $86 \mathrm{GHz}$ (millimeter bands) and $235 \mathrm{GHz}$ (sub-millimeter bands). This results in a resolution of $\sim 50 \mu$ as which is double 


\section{Cygnus A}

\section{VLA $6 \mathrm{~cm}$}

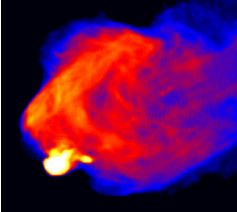

35000 ly

VLBI $6 \mathrm{~cm}$

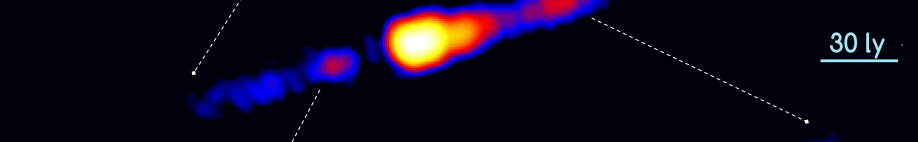

VLBI $2 \mathrm{~cm}$

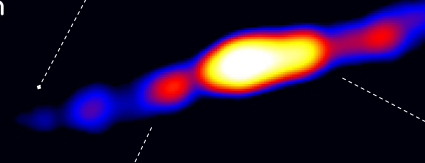

12 ly

VLBI $7 \mathrm{~mm}$

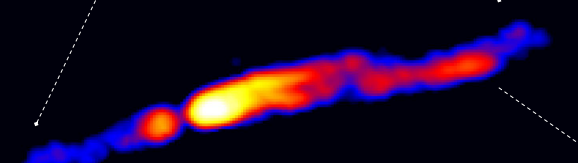

3 ly

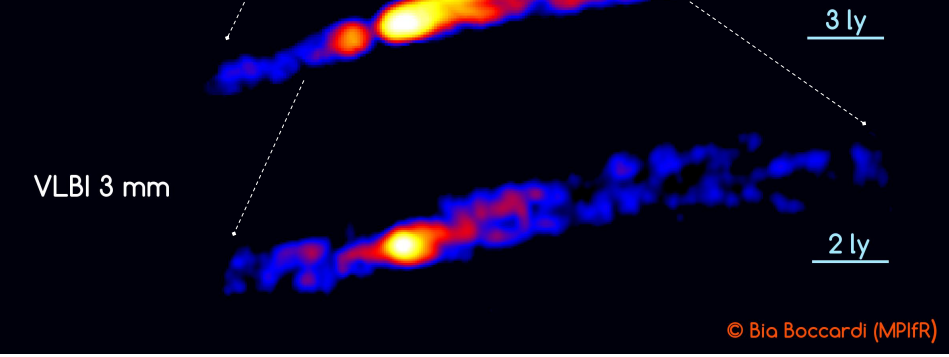

Figure 2.5: The maps of Cygnus A produced at different radio wavelengths from $6 \mathrm{~cm}$ $(\sim) 5 \mathrm{GHz}$ to $3 \mathrm{~mm}(\sim 86 \mathrm{GHz})$ and different baselines. Note that as the frequency of observation and baseline increases, we can zoom into the inner parsec scale regions close to the supermassive black hole (ly implies light year). Picture taken from Boccardi et al.

(2017).

that of the angular resolution achievable by space VLBI RadioAstron (Kardashev et al. 2013 ) at $1.6 \mathrm{GHz}$ and comparable to that achieved at $5 \mathrm{GHz}$ calculated for its maximum baseline of $\sim 360,000 \mathrm{~km}$.

\subsection{Motivation for this Dissertation}

Very Long Baseline Interferometry (VLBI) observations at high frequencies for e.g. $86 \mathrm{GHz}$ (wavelength, $\lambda=3 \mathrm{~mm}$ ) enable detailed studies to be made of compact radio sources at a resolution of $\sim(40-100) \mu$ as. This resolution corresponds to linear scales as small as $10^{3}-$ $10^{4}$ Schwarzschild radii and uncovers the structure of the jet regions where acceleration and collimation of the flow takes place (Vlahakis \& Königl 2004; Lee et al. 2008, 2016; Asada et al. 2014; Mertens et al. 2016). As seen in the maps of Cygnus A in figure 2.5, inner 
parsec scale regions close to the supermassive black hole are zoomed as one goes from lower frequency to higher frequency of observations by increasing the baseline.

To date, five $86 \mathrm{GHz}$ VLBI surveys have been conducted (Beasley et al. 1997; Lonsdale et al. 1998; Rantakyro et al. 1998; Lobanov et al. 2000; Lee et al. 2008, see Table 1), with the total number of objects imaged reaching just over a hundred. No complete sample of objects imaged at $86 \mathrm{GHz}$ has been established so far. Recent works (e.g., Homan et al. 2006; Cohen et al. 2007; Lister et al. 2016) have demonstrated that high-resolution studies of complete (or nearly complete) samples of compact jets yield a wealth of information about the intrinsic properties of compact extragalactic flows.

Measuring brightness temperature in a statistically viable sample enables performing detailed investigations of the physical conditions in this region. As discussed in section 2.2, the upper limit on intrinsic brightness temperature in inner jets of AGN always puzzled astronomers and this question can be addressed by a statistical approach to model the observed brightness temperature distribution and thereby test the theoretical values given by inverse Compton conditions and equipartition conditions.

The understanding of the distribution of intrinsic brightness temperature in the compact radio sources can also be used to test the jet models proposed for the inner jets in active galactic nuclei especially the two main inner jet models - accelerating and decelerating jet models (see section 2.4.3). The intensity profiles and the resulting brightness temperatures along the jet are significantly different and have a characteristic shape, in each of these models (Medici \& Lobanov 2002). The relative position of the emission regions at each wavelength is quite model dependent for the accelerating and decelerating. These will help to distinguish between the emission coming from accelerating or decelerating model.

Another interesting fact is that $T_{b}$ measured in the core is frequency dependent and $T_{b}$ measured at different frequencies represents actually physically different regions as discussed in section 2.4. The distribution of observed brightness temperatures, $T_{\mathrm{b}}$, derived at $86 \mathrm{GHz}$ can be combined with the $T_{\mathrm{b}}$ distributions measured at lower frequencies (e.g., Kovalev et al. 2005). This can help constraining the bulk Lorentz factor, $\gamma_{\mathrm{j}}$, and the intrinsic brightness temperature, $T_{0}$, of the jet plasma, using different types of population models of relativistic jets (Vermeulen \& Cohen 1994; Lobanov et al. 2000; Lister 2003; Homan et al. 2006; Lee 2013). The frequency-dependence of $T_{0}$ in the compact jets can be used to distinguish between the emission coming from accelerating or decelerating jet model and from electron-positron or electron-proton plasma. Theoretical models predict $T_{0} \propto \nu^{\epsilon}$, with $\epsilon \approx 2.8$, below a critical frequency $\nu_{\text {break }}$ at which energy losses begin to dominate the emission (Marscher 1995). Above $\nu_{\text {break }}, \epsilon$ can vary from -1 to +1 , depending on the jet composition and dynamics. Measuring this break and the power-law slopes above and below enables to distinguish between different physical conditions in the compact jets.

Previous studies (Lobanov et al. 2000; Lee et al. 2008) indicate that the value of $\nu_{\text {break }}$ is likely to be below $86 \mathrm{GHz}$. Indeed, a compilation of brightness temperatures measured at $2,8,15$, and $86 \mathrm{GHz}$ (Lee et al. 2016) indicates that brightness temperatures measured at $86 \mathrm{GHz}$ are systematically lower and $\nu_{\text {break }}$ can be as low as $20 \mathrm{GHz}$. This needs to be confirmed on a more complete sample observed at $86 \mathrm{GHz}$. If $T_{0}$ starts to decrease at $86 \mathrm{GHz}$, there will be only a few sources suitable for VLBI $>230 \mathrm{GHz}$ and higher frequencies. Such a decrease of $T_{0}$ will also provide a strong argument in favor of the decelerating jet model or particle-cascade models as discussed by Marscher (1995). In view of these arguments, it is important to undertake a dedicated $86 \mathrm{GHz}$ VLBI study of a larger sample of extragalactic radio sources and this is implemented in this dissertation. 


\section{Chapter 3}

\section{Radio Interferometry}

For thousands of years all the information that astronomers gathered about the universe was based on ordinary visible light. In the twentieth century, however, astronomers first began to explore the non visible electromagnetic radiation coming from astronomical objects by the discovery of extraterrestrial radio emission by Karl Jansky in 1931 (Jansky 1933), which was later confirmed to be coming from the center of Milky Way. The result has been an opening of astronomers eyes to more of the cosmos than could ever be seen using optical telescopes alone. It is no exaggeration to say that todays astronomers learn as much about the universe using telescopes for non visible wavelengths as they do using visible light. Observational astronomy may be divided according to the observed region of the electromagnetic spectrum. The prominent branches of astronomy based on classification are Radio Astronomy, Infrared Astronomy, Optical Astronomy, Ultraviolet Astronomy, $\mathrm{X}$-ray Astronomy and Gamma ray Astronomy. Radio astronomy is the study of distant objects in the universe by collecting and analyzing the radio waves emitted by those objects. Multi-wavelength astronomy is the way to understand an object fully. The luminosity, size, morphology etc. differ from band to band; each tells us something about the origin of object. Putting everything together in one perspective makes the full understanding of the object.

Radio waves were the first non-visible part of the electro magnetic spectrum to be used for astronomy. Radio waves penetrate much of the gas and dust in space as well as the clouds of planetary atmospheres and pass through the terrestrial atmosphere with little distortion. Radio astronomy can therefore obtain a much more intricate picture of stars and galaxies than is possible by means of optical observation. Radio astronomy studies radiation with wavelengths greater than one millimeter. Electromagnetic radiation, with wavelengths in the range from about $0.3 \mathrm{~mm}$ to about $30 \mathrm{~cm}$, is conventionally referred to as microwave, and radiation longer than $30 \mathrm{~cm}$ as radio, but the term radioastronomy is often used to embrace the entire range. The observed radio waves can be treated as waves rather than as discrete photons and this makes radio astronomy different from other forms of observational astronomy. Hence, it is relatively easier to measure both the amplitude and phase of radio waves, whereas this is not as easy at shorter wavelengths. A major fraction of the observed radio emission is produced by non-thermal processes, most notably by the synchrotron radiation. Also, spectral lines produced by ISM and many molecules for example, the hydrogen spectral line at $21 \mathrm{~cm}$, are observable at radio wavelengths. Supernovae, interstellar gas, pulsars and AGNs are observable in radio. Radio observations resulted in the discovery of exciting objects such as pulsars (Hewish et al. 1968) and quasars 
(Seyfert 1943) and revealed the remnants of the Big Bang and afterglows of Gamma Ray Bursts.

This dissertation make use of data from radio interferometric techniques at a frequency of $86 \mathrm{GHz}$. In the following, I will discuss the basics of radio telescopes, interferometry, and the principle of aperture synthesis employed for imaging the data from radio interferometric observations. A specific attention will be paid to describe the technique of very-long baseline interferometry (VLBI) used for achieving unprecedentedly high angular resolution of radio observations. In the second half of the chapter, I will discuss the calibration and imaging of VLBI data. The discussion are mainly based on Thompson et al. (2001), Taylor et al. (1999) and the online materials from the 15th Synthesis Imaging Workshop by NRAO (https://science.nrao.edu/science/meetings/2016/ 15th-synthesis-imaging-workshop/lectures) and from the school on low frequency radio astronomy at NCRA (http://gmrt.ncra.tifr.res.in/gmrt_hpage/Users/doc/WEBLF/LFRA/ index.html).

\subsection{Atmospheric Window}

The Earth's atmosphere absorbs most of the infrared, ultraviolet, X-ray, and gamma-ray wavelengths, so ground-based astronomy can be done mainly in two atmospheric windows - the radio and visible wavebands and a small region in the near infrared. The visible window is roughly (3000 $\AA-7000 \AA)$ which corresponds to spectral peaks of thermal emission with black body temperature ranging from $3000 \mathrm{~K}$ to $10000 \mathrm{~K}$ which comes mainly from stars. But the radio window which ranges from $10 \mathrm{MHz}-300 \mathrm{GHz}$, is much wider than the optical window allowing observations of many astronomical sources and emission mechanisms. Vibrational transitions of molecules like $\mathrm{CO}_{2}, \mathrm{O}_{2}$, and $\mathrm{H}_{2} \mathrm{O}$ have energies $E=h \nu$ comparable with those of mid infrared photons and therefore absorb most of the infra-red (IR) waves. Due to the lower energy rotational transitions of atmospheric molecules equal to far IR band and short wavelengths of radio, those frequencies are also absorbed or partially absorbed by atmosphere. This happens above $30 \mathrm{GHz}(\sim 1 \mathrm{~cm})$. In order to observe at frequencies above this, one has to install radio telescopes in very high and dry places for example, Atacama Large Millimeter Array (ALMA) at $5000 \mathrm{~m}$ in Chile.

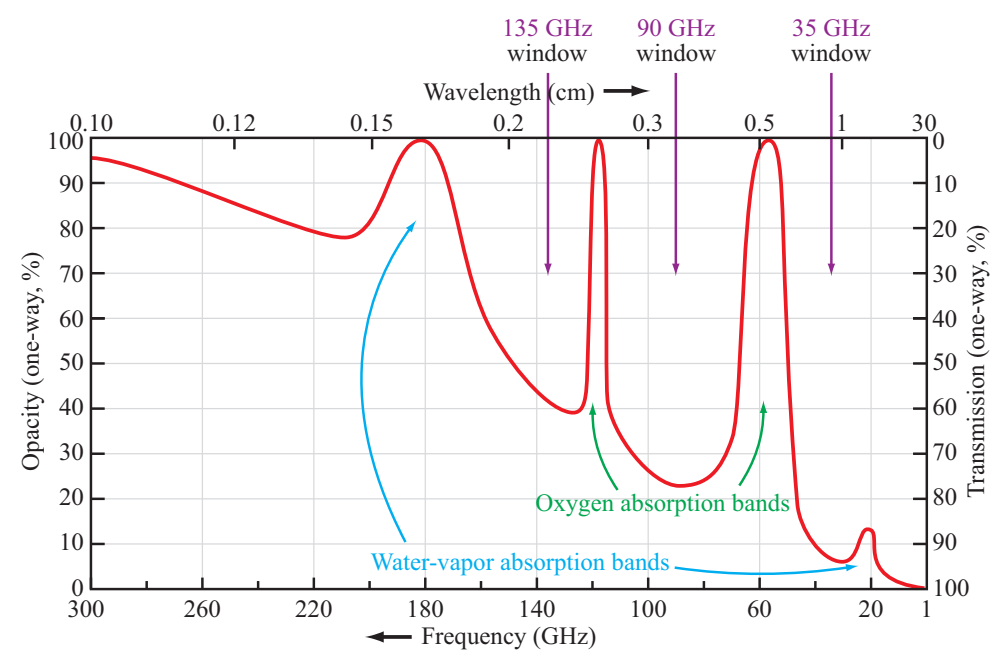

FiguRE 3.1: Percentage transmission through the Earths atmosphere, in the vertical direction. Credit: Ulaby F. Long, University of Michigan. 
At $300 \mathrm{MHz}(\sim 1 \mathrm{~m})$, there occurs severe ionospheric refraction and radio waves at frequency below $10 \mathrm{MHz}(\sim 30 \mathrm{~m})$ are reflected back into space by ionosphere and so observations below $10 \mathrm{MHz}$ is not possible from earth. Now in the case of ultraviolet photons, they have energies close to the binding energies of the valence electrons in atoms which results in the electronic transitions in atoms. This results in high ultraviolet opacity of the atmosphere. Higher energy nuclear transitions result in X-ray and gamma ray absorption. So spacecrafts are needed to do observations in gamma rays, X-rays, ultra-violet (UV), and IR. Sun is a barrier for the daytime optical observations because of the Rayleigh scattering of sunlight by atmospheric dust at optical and UV wavelengths. But this is not a barrier to radio wavelengths being much longer than the size of these dust particles and the Sun is not a bright radio source, making radio observations possible day and night. There are also disturbances produced by military and civil radio signals like mobile phones, Frequency Modulation (FM) radios, televisions etc. which is often referred to as Radio Frequency Interference (RFI). In order to minimize RFI as much as possible, there are certain reserved radio bands for radio astronomy outside the frequencies used by others. The most common radio band names in which astronomers use their radio telescopes and their features are given in table 3.1 .

TABle 3.1: Radio Bands

\begin{tabular}{lcc}
\hline \hline Band & Wavelength $(\lambda)$ & Frequency $(\nu)$ \\
\hline P-band & $90 \mathrm{~cm}$ & $327 \mathrm{MHz}$ \\
L-band & $20 \mathrm{~cm}$ & $1.4 \mathrm{GHz}$ \\
C-band & $6 \mathrm{~cm}$ & $5.0 \mathrm{GHz}$ \\
X-band & $3.6 \mathrm{~cm}$ & $8.5 \mathrm{GHz}$ \\
U-band & $2.0 \mathrm{~cm}$ & $15 \mathrm{GHz}$ \\
K-band & $1.3 \mathrm{~cm}$ & $23 \mathrm{GHz}$ \\
Q-band & $7 \mathrm{~mm}$ & $45 \mathrm{GHz}$ \\
W-band & $3 \mathrm{~mm}$ & $86 \mathrm{GHz}$ \\
\hline
\end{tabular}

\section{$3.2 \quad$ Interferometry}

A radio telescope is a type of directional radio antenna which can take variety of forms and is used singly or in an array. The most common form is the steerable dish, a concave reflector that brings waves to a focus and which can be pointed in any direction. The largest steerable dish is the Green Bank telescope with a diameter of $\sim 110 \mathrm{~m}$. The largest non-steerable telescope is Five-hundred-meter Aperture Spherical radio Telescope (FAST).

The biggest problem faced by the early radio astronomers was resolution. The resolving power of any telescope depends on its aperture size and on the observing wavelength of radiation - the longer the wavelength, the poorer the resolution. In order to achieve a resolving power equivalent to that of naked eye, the radio telescope should have an aperture of $\sim 1$ $\mathrm{km}$. A solution to this was found later by the introduction of interferometry principle. If two radio telescopes, separated by a known distance called the baseline observe the same source, then depending on whether the wavefronts reaching each telescope are in phase or not, they either add on to form an interference pattern or cancel out, respectively. The interference fringes can be carefully analyzed to study the physics of the source. Such a 
device, known as interferometer, can achieve a resolution much greater that that of a single dish. In such arrays, the spatial resolution is determined not by the size of the individual radio telescopes, but rather by the maximum separation between the radio telescopes (baseline). With an interferometer, the diffraction limit $\theta$ is:

$$
\theta=\frac{\lambda}{B}
$$

where $\mathrm{B}$ is the baseline which can extend to many thousands of kilometers. Very Long Baseline Interferometers, employing radio telescopes located across the entire globe, can achieve resolutions of the order of $\sim 40 \mu$ as at short wavelength $\sim 3 \mathrm{~mm}$.

\subsection{1 van Cittert-Zernike Theorem}

In this section, I discuss the van Cittert-Zernike Theorem (van Cittert 1934; Zernike 1938), which forms the basics of intensity measurements in radio interferometry. Consider an incoherent source A and the observer's plane B. The monochromatic component of an electromagnetic field represented by $\mathbf{E}(\mathbf{r})$ and frequency $\nu$ where $\mathbf{R}$ is the radio source's location and $\mathbf{r}$ is the observer's location can be defined as:

$$
\mathbf{E}(\mathbf{r})=\int \frac{\mathbf{E}(\mathbf{R}) e^{2 \pi i \nu|\mathbf{R}-\mathbf{r}| / c} d S}{|\mathbf{R}-\mathbf{r}|}
$$

where electric field travels from $\mathbf{R}$ to $\mathbf{r}$ and $d S$ is the element of area integration. Consider two points $B_{1}$ and $B_{2}$ whose positions are represented by $\mathbf{r}_{1}$ and $\mathbf{r}_{2}$ in the observer's plane (left panel of figure 3.2), then the fields at $\mathbf{r}_{1}$ and $\mathbf{r}_{2}$ is correlated and this correlation function is defined as:

$$
V\left(\mathbf{r}_{1}, \mathbf{r}_{2}\right)=<\mathbf{E}\left(\mathbf{r}_{1}\right) \mathbf{E}^{*}\left(\mathbf{r}_{2}\right)>.
$$

$V\left(\mathbf{r}_{1}, \mathbf{r}_{2}\right)$ is known as the Spatial coherence function of an electromagnetic field or Visibility function. An interferometer is simply a device for measuring this spatial coherence function. Substituting equation 3.2 in $<\mathbf{E}\left(\mathbf{r}_{\mathbf{1}}\right) \mathbf{E}^{*}\left(\mathbf{r}_{\mathbf{2}}\right)>$ gives:

$$
<\mathbf{E}\left(\mathbf{r}_{\mathbf{1}}\right) \mathbf{E}^{*}\left(\mathbf{r}_{\mathbf{2}}\right)>=\iint \frac{\mathbf{E}\left(\mathbf{R}_{\mathbf{1}}\right) e^{2 \pi i \nu\left|\mathbf{R}_{\mathbf{1}}-\mathbf{r}_{\mathbf{1}}\right| / c}}{\left|\mathbf{R}_{\mathbf{1}}-\mathbf{r}_{\mathbf{1}}\right|} \frac{\mathbf{E}\left(\mathbf{R}_{\mathbf{2}}\right) e^{2 \pi i \nu\left|\mathbf{R}_{\mathbf{2}}-\mathbf{r}_{\mathbf{2}}\right| / c}}{\left|\mathbf{R}_{\mathbf{2}}-\mathbf{r}_{\mathbf{2}}\right|} d S_{1} d S_{2} .
$$

If the emission from the source is spatially incoherent i.e. $\left\langle\mathbf{E}\left(\mathbf{R}_{\mathbf{1}}\right) \mathbf{E}^{*}\left(\mathbf{R}_{\mathbf{2}}\right)>=0\right.$, then $|\mathbf{E}|\left|\mathbf{E}^{*}\right|=\mathbf{E}^{2}=\mathrm{I}$, the intensity of the radiation. Then equation 3.4 reduces to:

$$
<\mathbf{E}\left(\mathbf{r}_{1}\right) \mathbf{E}^{*}\left(\mathbf{r}_{\mathbf{2}}\right)>=\iint I(\mathbf{s}) \frac{e^{2 \pi i \nu\left|\mathbf{R}_{1}-\mathbf{r}_{1}\right| / c}}{\left|\mathbf{R}_{\mathbf{1}}-\mathbf{r}_{1}\right|} \frac{e^{2 \pi i \nu\left|\mathbf{R}_{2}-\mathbf{r}_{2}\right| / c}}{\left|\mathbf{R}_{\mathbf{2}}-\mathbf{r}_{\mathbf{2}}\right|} d S_{1} d S_{2} .
$$

where $\mathbf{s}$ is a unit vector defined as $\mathbf{s}=\mathbf{R} /|\mathbf{R}|$ in the direction of $\mathbf{R}$. Now substituting the element of solid angle, $d \Omega$ instead of $d S$, the element of area integration, results in:

$$
V\left(\mathbf{r}_{1}, \mathbf{r}_{2}\right)=\iint I(\mathbf{s}) e^{-2 \pi i \nu \mathbf{s} .\left(\mathbf{r}_{1}-\mathbf{r}_{2}\right) / c} d \Omega
$$

$\mathbf{r}_{1}-\mathbf{r}_{\mathbf{2}}$ is the baseline if we consider two antennas separated by a baseline distance $\mathbf{b}$. So equation 3.6 can be rewritten as:

$$
V(\mathbf{b})=\iint I(\mathbf{s}) e^{-2 \pi i \nu \mathbf{s} \cdot \mathbf{b} / c} d \Omega .
$$




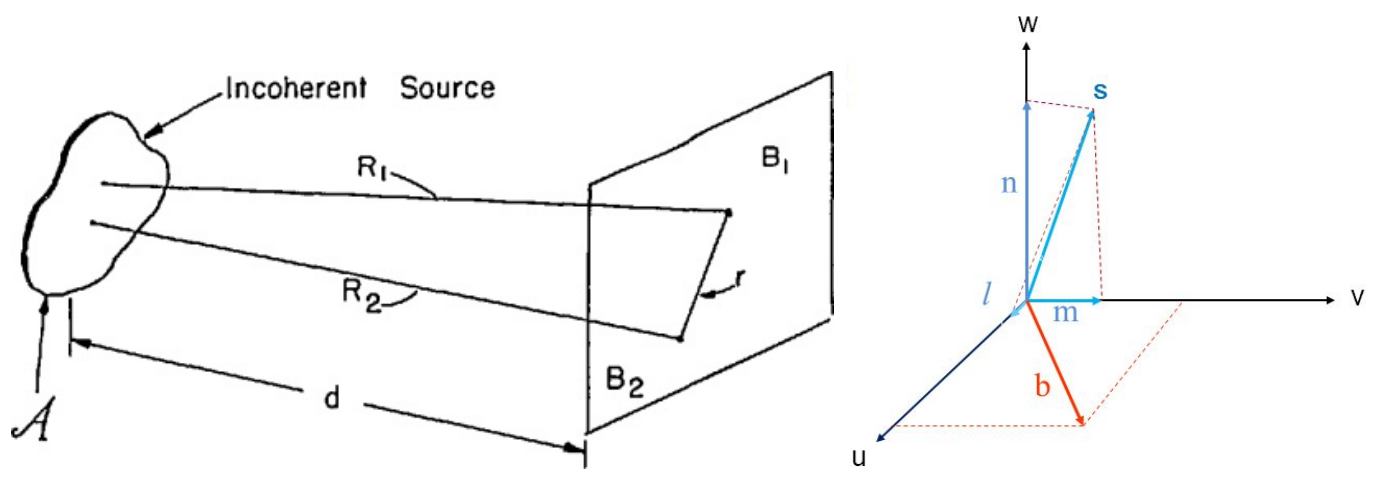

FiguRE 3.2: Left: An incoherent radio source A and a simple geometry of an interferometer. Right: The direction cosines $(l, m, n) . l^{2}+m^{2}+n^{2}=1$. Credit: Lectures in NRAO Synthesis Imaging Summer School 2013 by Rick Perley.

van Cittert-Zernike theorem simply represented by Equation 3.6 correlates the spatial coherence function (visibility) to the distribution of intensity of the incoming radiation $I(\mathbf{s})$. So the source brightness can be obtained from an inverse Fourier of complex visibility function.

If a new coordinate system $(u, v, w)$ defined by the direction cosines $(l, m, n)$ is introduced as shown in the right panel of figure 3.2, then $\mathbf{r}_{1}-\mathbf{r}_{2}=\mathbf{b}=(u, v, w) c / \nu$ and $d \Omega=d l d m / \sqrt{1-l^{2}-m^{2}}$. $u$ axis points towards east, $v$ axis points towards the north celestial pole and $w$ axis points towards the source tracking it over time. The $2 \mathrm{D}$ plane formed by $u$ and $v$ is called $u v$-plane (in the units of wavelength) and is always perpendicular to the source direction. Equation 3.7 reduces to:

$$
V(u, v)=\iint \frac{I(l, m)}{\sqrt{1-l^{2}-m^{2}}} e^{-i 2 \pi(l u+m v+n w)} d l d m .
$$

So visibility is the Fourier transform of the modified brightness distribution $\frac{I(l, m)}{\sqrt{1-l^{2}-m^{2}}}$. Equation 3.8 refers to the basic relation between spatial frequency domain $(u, v, w)$ and image domain $(l, m, n)$. Equation 3.8 reduces to

$$
V(u, v)=\iint \frac{I(l, m)}{\sqrt{1-l^{2}-m^{2}}} e^{-i 2 \pi(l u+m v)} d l d m
$$

by setting $w=0$. For a parabolic antenna whose primary beam $<1^{\circ}, \sqrt{1-l^{2}-m^{2}}=n \approx$ 1 , equation 3.7 reduces to:

$$
V(u, v)=\iint I(l, m) e^{-i 2 \pi(l u+m v)} d l d m .
$$

As more and more points are sampled by the interferometer, the source function can be calculated with better precision. Each $u v$-data point contains a visibility representing amplitude and phase for each integration of equation 3.7. The filling of the $u v$-plane is called as uv-coverage which is constrained by the antenna's position and number. In fact, antennas is not a point element. It has some size which has some sensitivity to the direction of arrival of the radio waves. So the primary beam or normalised reception pattern $G_{\nu}(l, m)$ or $G_{\nu}(\theta)$ has to be added to the integral in equation 3.10 to account for the complex visibility relative to the chosen phase tracking center. From the inverse 
relationship of Fourier conjugate variables, it implies that the short baselines are sensitive to large angular structures in the source and that long baselines are sensitive to short or compact scale structure.

\subsubsection{Aperture Synthesis}

The $u v$-plane is filled smartly by a technique called Aperture Synthesis, the basis of which can be understood as follows: If two radio telescopes, $\mathrm{A}$ and $\mathrm{B}$ at different locations on Earth observe same source in the sky for 12 hours, then the Earth rotation causes B to trace out a semicircle around A, effectively tracing out half the surface of a radio dish which is equivalent in radius to the separation between the two telescopes. The other half of the dish can be synthesized mathematically in a computer. This means, as seen from a distant radio source, the baseline vector between two antennas on the Earth is continuously changing because of the Earth's rotation. For an array of $n$ antennas in different parts of Earth, $n C_{2}$, i.e. $n(n-1) / 2$ combinations of visibilities $V_{i j}$ can be measured and therefore that many Fourier inversions can be done to recover the source brightness. As the Earth rotates, $u v$-plane is filled more and more. This technique of using the Earth's rotation to improve $u v$-coverage is known as "aperture synthesis". The process of aperture synthesis using many interferometer pairs to construct a large aperture were found by Ryle \& Hewish (1960) and later contributed by Scott et al. (1961) and Ryle (1962). This discovery of aperture synthesis led to Nobel prize in 1974. In fact, it was the first Nobel prize for any research in astronomy.

\subsubsection{Two Element Interferometer}

An important difference between radio and optical telescopes is the use of coherent amplifiers in radio telescopes. Radio waves from the distant radio source impinges a fluctuating voltage at the antenna terminals. Let these voltages at the two telescopes in an interferometer be $V_{1}$ and $V_{2}$. This voltage varies at the same frequency of the incoming radio wave, referred to as the Radio Frequency (RF). The radiation is first focused in a device called feed. The voltage is first amplified by the front end (or Radio Frequency) amplifier. The signal is weakest at this point and so it is important that the amplifier introduce as little noise as possible. So front end amplifiers are equipped with low noise solid state devices, High Electron Mobility Transistors (HEMTs), often cooled to liquid helium temperatures.

After amplification, the signal goes into a mixer which is a device that alters the frequency of the input signal. Mixers have two inputs, one for the signal whose frequency is to be changed (the RF signal), and the other input for a pure sine wave generated by a tunable signal generator called the Local Oscillator (LO). The output of the mixer is produced at the beat frequency of the radio frequency and the local oscillator frequency. After mixing, the signal is at a lower frequency than the RF, this frequency is called the Intermediate Frequency (IF). The main reason for mixing or heterodyning is that even though most of the radio telescopes operate at different radio frequencies, the processing required at each of these frequencies is identical. So it is economically good to convert each of these incoming radio frequencies to a standard IF and then to use the exact same back end equipment for all RF frequencies that the telescope operates. In telescopes that use co-axial cables to transport the signal across long distances, the IF frequency is selected such that it minimizes transmission loss in the cable. Sometimes there is more than one mixer along the signal 


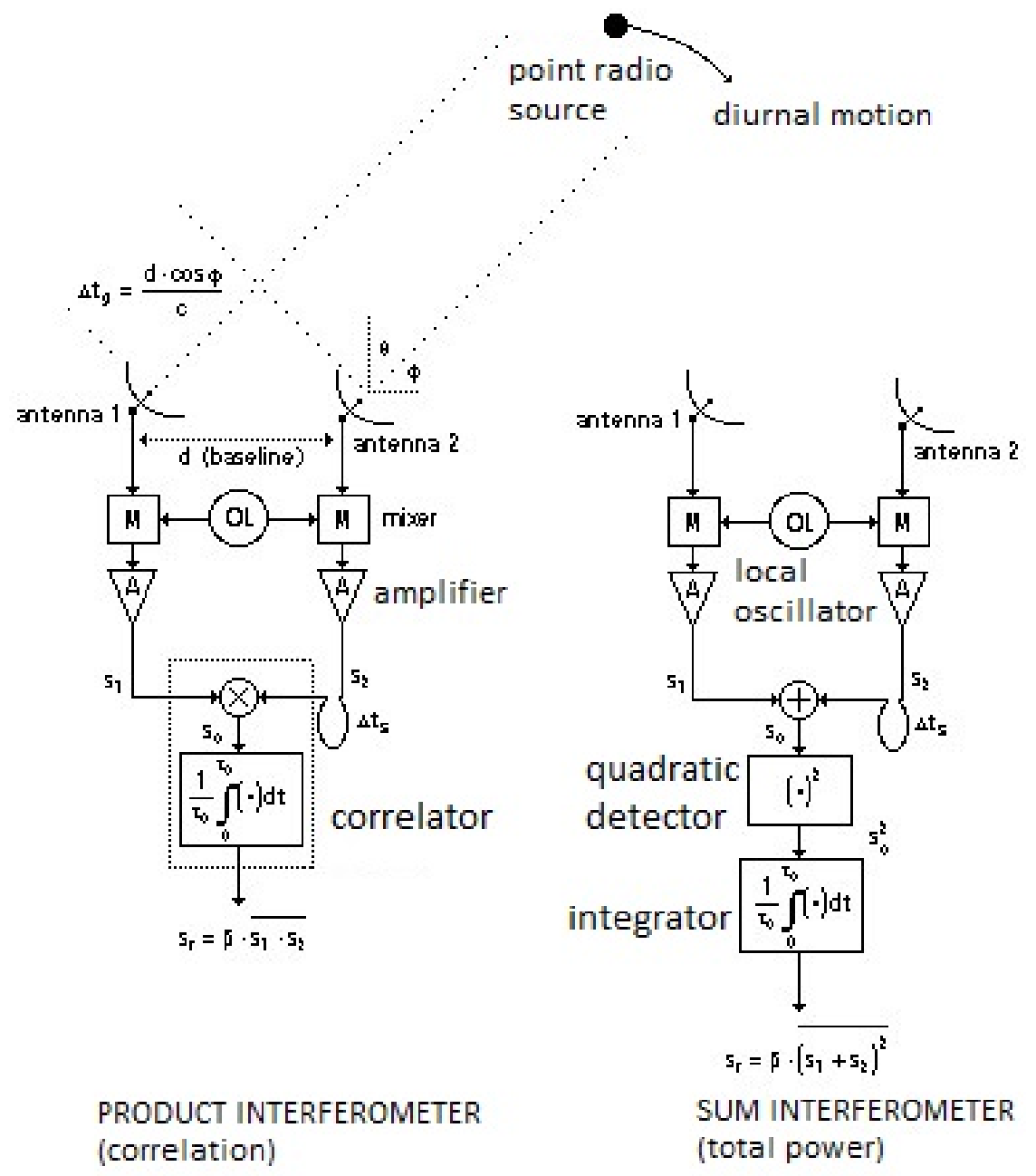

Figure 3.3: A schematic representation of two types of two element interferometer Product Interferometer on the left and Sum Interferometer on the right. Credit: http: //www.radioastrolab.com/radio-astronomy/radio-interferometry.

path, resulting in a series of IF frequencies, one of which is optimum for signal transport, another which is optimum for amplification etc. This is known as "super-heterodyne" system.

Once the IF conversion is done, the signal is again amplified, and then mixed to a frequency called base band. Then the signal is fed into the backend. The back end equipments is based on the observations. If the total power received by the telescope has to be measured, then the back end will be simple square law detector or quadratic detector followed by an integrator. This is known as Sum Interferometer. The signal is a voltage that is proportional to amplitude of the electric field of the incoming radiation, and since the power in the wave is proportional to the square of its amplitude, the square of the voltage is a measure of the strength of the radio source. The integrator averages the signal to improve the signal to noise ratio.

If the back end is equipped with correlator it is known as Product Interferometer. As the name indicates, correlator takes the product of voltages whereas integrator do the integration of voltages. The entire process is shown clearly in figure 3.3. 


\subsubsection{Correlation}

In VLBI consisting of antennas across continents, information is recorded separately on each antenna on disks. The disks are then transported to correlator where the signals are correlated described by equation 3.13. The signal depends on both frequency of observation and time. The correlation is discussed in detail below. The signals from the two telescopes before processing to correlator with frequency responses $H_{1}(\nu)$ and $H_{2}(\nu)$ can be represented as:

$$
\begin{aligned}
& V_{c 1}(\nu)=V_{1}(\nu) H_{1}(\nu) \\
& V_{c 2}(\nu)=V_{2}(\nu) H_{2}(\nu)
\end{aligned}
$$

where $V_{\nu}$ is the output produced from a single antenna which depends on the angular reception pattern $G_{\nu}(\theta)$ given as:

$$
V_{\nu}=\int E_{\nu}(\theta) G_{\nu}(\theta) d \theta
$$

The signals are then cross correlated in a "correlator". Cross correlation of these signals results in:

$$
r(\tau)=\lim _{T \rightarrow \infty} \frac{1}{2 T} \int V_{c 1 T}(t) V_{c 2 T}^{*}(t-\tau) d t,
$$

where $\tau$ represents the delay in the signal at the second telescope as compared to first and $T$ is the truncation applied to the time variability of the subscripted functions. At this stage the correlator corrects the total delay due to instruments, antennas clock rate, earth's rotation and atmosphere. For example, the correlator calculates a delay exactly for the central frequency of the bandwidth and with respect to the phase center, i.e. the source position. So still there remains time delays, uncertainties in models, due to antenna or source position etc. in the correlator output. In VLBI observations, the remaining delays are corrected in the step fringe fitting discussed in next section 3.3.1. If the fringes are averaged over a bandwidth it will result in an attenuation of the fringes called as bandwidth smearing. This is more for long baselines and wide bandwidths. In order to reduce smearing, bandwidth $\Delta \nu$ is divided into sub-bands and sub-bands are divided into a large number of channels. Then averaging is done over the bandwidth of a single channel by sufficiently overcoming the smearing problem.

\subsection{Phase and Amplitude Calibration}

In fact, the quantity measured by equation 3.7 is not true coherence function $V_{\nu, t}(u, v)$ but it is affected by the corruption terms introduced by the atmosphere and by instrumentation. The true and observed visibilities are related to each other as:

$$
V_{\nu, t}^{\text {obs }}=V_{\nu, t}^{\text {true }} G_{i}(\nu, t) G_{j}^{*}(\nu, t),
$$

where $\mathrm{i}$ and $\mathrm{j}$ are antennas forming a baseline. $G_{i}(\nu, t)$ and $G_{j}(\nu, t)$ have an amplitude and phase same as the visibility and are called as complex gains. The process of phase and amplitude calibration is actually done to determine and correct for the complex gains and thereby reducing the true visibility $V_{\nu, t}^{t r u e}$. For the VLBI data presented in this dissertation, this process is mainly done using Astronomical Image Processing System (AIPS, Greisen 1990) . New software packages like CASA for calibrating the data for example, from ALMA 
telescope are presently being developed. But AIPS can deal with a process called fringe fitting (see section 3.3.1) which is very important especially for high frequency VLBI data.

\subsubsection{Fringe Fitting}

As discussed in section 3.2.4, even after correlation efforts to calculate the time delays, there are still uncertainties and errors caused from instrumentation, earth model, atmosphere model, position of antenna and source, clock rates at individual antennas, time stamps etc. These errors can result in the reduction of visibility amplitude and increase the phase noise. The phase of the interferometer visibility $\phi_{\nu, t}$ is related to the interferometer delay $\tau_{t}$ by:

$$
\phi_{\nu, t}=2 \pi \nu \tau_{t} .
$$

Differentiating equation 3.15 gives the phase error $\Delta \phi_{\nu, t}$ as:

$$
\Delta \phi_{\nu, t}=2 \pi \nu \Delta \tau_{t}
$$

At the same time, in the frequency and time domain, a first order expansion of the phase error is:

$$
\Delta \phi_{\nu, t}=\phi_{0}+\frac{\partial \phi}{\partial \nu} \Delta \nu+\frac{\partial \phi}{\partial t} \Delta t
$$

where $\phi_{0}$ is the phase error at a reference time and frequency. $\left(\frac{\partial \phi}{\partial \nu}\right)$ is the delay or delay residual, and $\left(\frac{\partial \phi}{\partial t}\right)$ is the rate or delay rate. The equation 3.17 holds for each individual antenna. The motive for phase calibration is to determine the delay and rate terms so that visibilities can be integrated coherently at the end and this procedure for correcting delay and rate is called fringe fitting. A detailed review can be seen in Cotton (1995). In the fringe-fitting based on delay-rate domain, the process searches for the delay and rate corresponding to the peak SNR. This process is called fringe search and this is baselinebased (Moran 1976). The baseline dependent errors between two antennas $i$ and $j$ can be expressed as the difference in their antenna dependent errors calculated from equation 3.17 for antennas $i$ and $j$ as:

$$
\Delta \phi_{i, j}=\Delta \phi_{i}-\Delta \phi_{j}
$$

In the fringe-fitting based on time-frequency, the fringe search is done antenna-based by fitting a linear solution to the phase variations as a function of time and frequency (Schwab $\&$ Cotton 1983). The solutions are found globally for whole data and hence this method is called as global fringe fitting. An advantage of antenna-based fringe fitting is that it increases the number of detections for weak sources (Alef \& Porcas 1986). But in a baseline-based fringe fitting, if no detection is found for a particular baseline, then it is impossible to calibrate those baselines (Moran 1976). Therefore, antenna-based fringe fitting is really helpful while dealing with high frequency data like $86 \mathrm{GHz}$ data in this dissertation. A manual phase calibration and global fringe fitting i.e. an antenna based fringe fitting is applied to the $86 \mathrm{GHz}$ VLBI data in this dissertation. A detailed discussion of specific procedures applied to data reduction is presented in Chapter 4. 


\subsubsection{Amplitude Calibration}

The antenna based complex gains $G_{i}(\nu, t)$ and the complex conjugate of $G_{j}(\nu, t)$ in equation 3.14 can be expressed as:

$$
\begin{array}{r}
G_{i}(\nu, t)=a_{i}(\nu, t) e^{i \phi_{i}(\nu, t)} \\
G_{j}^{*}(\nu, t)=a_{j}(\nu, t) e^{-i \phi_{j}(\nu, t)}
\end{array}
$$

where $a_{i}(\nu, t)$ and $a_{j}(\nu, t)$ are the antennas based amplitude corrections and $\phi_{i}(\nu, t)$ and $\phi_{j}(\nu, t)$ are the antenna based phase corrections. Substituting equation 3.19 in equation 3.14 results in:

$$
V_{\nu, t}^{o b s}=a_{i}(\nu, t) a_{j}(\nu, t) e^{i\left(\phi_{i}-\phi_{j}(\nu, t)\right)} V_{\nu, t}^{\text {true }} .
$$

Let us define $A_{i j}=a_{i}(\nu, t) a_{j}(\nu, t) V_{\nu, t}^{\text {true }}$ which modifies equation 3.20 and equation 3.20 can be simply written as:

$$
V_{i, j}^{o b s}=A_{i j} e^{i \psi_{i j}}
$$

where $\psi_{i j}$ is $\left[\left(\phi_{i}-\phi_{j}(\nu, t)\right)\right]$ and $A_{i j}$ represents the observed visibility amplitude which is a dimensionless raw correlation coefficient from the correlator.

In practical data reduction, this output of the correlator, $A_{i j}$ which is dimensionless has to be converted to Jansky. A parameter quantifies the amplitude called the System Equivalent Flux Density can be defined in terms of system temperature, $T_{\text {sys }}$ and a scaling factor between temperature in Kelvin and flux density in Jansky, $A_{\text {eff }}$ as:

$$
S E F D=\frac{2 K T_{\text {sys }}}{A_{\text {eff }}}
$$

$A_{\text {eff }}$ can be obtained by observing a calibrator of known flux. So the correlated flux between the two antennas $i$ and $j$ can be expressed in Jansky, $S[J y]$ as:

$$
S[J y]=A_{i j} \frac{1}{y_{c}} \frac{\sqrt{S E F D^{i} \cdot S E F D^{j}}}{2 \Delta \nu \Delta \tau},
$$

where $y_{c}$ is a term representing the data loss due to digital sampling, $\Delta \nu$ is the bandwidth and $\Delta \tau$ is the integration time. $T_{s y s}$ and $A_{\text {eff }}$ are known from the TY and GC tables in AIPS and the conversion of flux to Jansky units can be done by the task APCAL. The task APCAL also performs the opacity correction using the weather (WX table) information. Opacity correction is crucial for high frequency data because of the fact that most of the signal is attenuated by the atmosphere before reaching the antenna receiver by a factor $e^{-\tau_{a t m}}$ where $\tau_{a t m}$ represents the atmospheric opacity. $\tau_{a t m}$ depends on the elevation of the source which can be expressed as the product of zenith opacity $\tau_{z e n}$ and the airmass which is a function of the zenith angle $\chi(z)$. Therefore, $\tau_{a t m}$ is given as:

$$
\tau_{a t m}=\tau_{z e n} \chi(z) \sec (z) .
$$

The opacity corrections are done using the information from the TY table on $T_{\text {sys }}$ and weather information from WX table. The task APCAL is started by an initial guess value for the zenith opacity (TAU0) and receiver temperature (TRECVR) which is usually set as 0.1 and $100 \mathrm{~K}$, respectively for high frequency VLBI data. Another smart way is to run a quick APCAL with these initial values for $\tau_{z e n}$ and receiver temperatures and then obtain the fitted $\tau_{z e n}$ and receiver temperatures and use them in the next round of APCAL. We have used this approach for the $86 \mathrm{GHz}$ GMVA data presented in this dissertation. If there 
are subarrays (array of antennas divided into sub groups which observe different sources at different times, very common in GMVA observations conducted in different continents) in the data, it is important to run the task APCAL separately for different subarrays since they are observed at different times. Amplitude calibration finishes with bandpass correction. The first and last channels in a band is severely attenuated by the frequency dependence of the response of the electronic system. The task BPASS is used to calculate this attenuation factor using a very strong calibrator source and records it in BP table. This will be later applied to the whole data before averaging in frequency domain.

\subsection{Imaging}

The derived image of the sky is called map in radio astronomy and the process of producing the image from the visibilities is called mapping or imaging. Once the phase and amplitude calibration is done, the visibility data set can be imaged to deduce the source brightness distribution. Imaging is done in three different steps: Inverse Fourier transform, deconvolution and self-calibration. Imaging can be done using AIPS, CASA or DIFMAP (Shepherd et al. 1994). The VLBI data presented in this dissertation is imaged by DIFMAP. In the following section, I discuss the three steps of imaging.

\subsubsection{Sampling Function \& Fourier Inversion}

The equation 3.10 can be inverted via a Fourier transform to find the actual intensity distribution of the source as:

$$
I_{\nu}(l, m)=\iint V_{\nu}(u, v) e^{i 2 \pi(l u+m v)} d u d v .
$$

$V_{\nu}(u, v)$ is not known everywhere, it is actually sampled at particular places on the $u v$ plane. Therefore, a sampling function $S(u, v)$ called as the uv-coverage has to be added to equation 3.25 as:

$$
I_{\nu}^{D}(l, m)=\iint V_{\nu}(u, v) S(u, v) e^{i 2 \pi(l u+m v)} d u d v .
$$

Equation 3.26 gives the dirty map represented as $I_{\nu}^{D}(l, m)$. It is just the Fourier transform of the $u v$-plane. $I_{\nu}^{D}(l, m)$ is known as the dirty map because it is convolved with synthesized beam or point spread function $B(l, m)$. This convolution represented by $*$ is given by:

$$
I_{\nu}^{D}(l, m)=\left[I_{\nu}(l, m)+N(l, m)\right] * B(l, m)
$$

where $N(l, m)$ is the image noise and $B(l, m)$ is:

$$
B(l, m)=\iint S(u, v) e^{i 2 \pi(l u+m v)} d u d v .
$$

The shape of the synthesized beam can be changed applying either tapering function or density weighting function. Tapering function is applied to down weight the data at the outer edge of the $u v$-plane for example a Gaussian tapering function. Using this function, we can prefer which $u v$-data point has to be weighted more while imaging. In DIFMAP, this is done by a function called uvtaper. Density weighting function $\left(D_{k}\right)$ gives a balance between the highly concentrated central $u v$ points and the less denser outer $u v$ points. This 
helps to reduce side lobes. $D_{k}=1$ is applied for natural weighting which is the best for weak sources. $D_{k}=0$ is applied for uniform weighting which is good in the case of bright sources and helps to reduce the side lobes of the dirty beam, which is nothing but the noise in the image. A natural weighing is done for the VLBI data in this dissertation since most of the sources are very weak in intensity. In DIFMAP, the visibility data is first binned into pixels by a function called mapsize which can be set manually. The total size should be an integer power of 2 (like 512, 1024, 2048 etc.) with bin size depending on the beam size, which in turn depends on the frequency of the data. For high frequency data in this dissertation, an optimal pixel size of 0.01 mas is applied while mapping most of the sources. Then a Fast Fourier Transform (FFT) algorithm is applied in DIFMAP.

\subsubsection{Deconvolution}

As described by equations 3.26 and 3.27 in section 3.4.1, the true intensity distribution is convolved with the synthesized beam. There are methods for undoing this convolution in order to recover the true intensity map. This is known as deconvolution. The two widely used deconvolution methods are the Maximum Entropy Method (MEM; Ables 1974; Ponsonby 1973) the "CLEAN" algorithm (Högbom 1974) and the non-negative, least-squares algorithm (NNLS; Lawson \& Hanson 1974). A detailed discussion on imaging and deconvolution methods can be found in Cornwell \& Fomalont (1999), Cornwell (1995) and Cornwell et al. (1999).

The CLEAN algorithm in DIFMAP is used as the deconvolution method for the whole data in this dissertation. The "CLEAN" algorithm is developed by Högbom (1974) and further developed by Clark (1980) and Schwab \& Cotton (1983). A number of deconvolution cycles are run over to find the peak in the map. Then the algorithm subtracts the dirty beam at the peak point. Equation 3.27 is solved by assuming the true intensity distribution represented by a number of model delta $(\delta)$ functions which are point sources. Each $\delta$ function has a flux. The process is iterated to find the position and flux of these delta functions. These iterations are repeated until a peak or optimum flux is left in the residual map. Finally a deconvolved map known as clean map is restored with a clean beam when an optimum model has been obtained. The clean map is a summation of all the delta functions restored with clean beam.

In maps produced by the CLEAN algorithm, the number of grid points in the $(l, m)$ and $(u, v)$ plane are equal. But not all the $(u, v)$ grid points contain visibility points. So caution has to be taken to apply CLEAN only within a limited area in the original map known as clean window. This is usually set manually.

\subsubsection{Closure Quantities}

Closure effects are relationships between visibility values for baselines that form a closed figure, for example a triangle or quadrilateral with the antennas at the vertices. Equation 3.21 gives the complex correlation coefficient corresponding to one spatial frequency. Then from equation 3.20, the closure phases from three correlator outputs of three antennas 
can be calculated as:

$$
\begin{aligned}
C_{i j k}^{o b s} & =\left(\psi_{i j}+\phi_{i}-\phi_{j}\right)+\left(\psi_{j k}+\phi_{j}-\phi_{k}\right)+\left(\psi_{k i}+\phi_{k}-\phi_{i}\right) \\
& =\psi_{i j}+\psi_{j k}+\psi_{k i} \\
& =C_{i j k}^{\text {true }}
\end{aligned}
$$

This equation 3.29 clearly shows that the antenna based errors cancel out. This means that the combination of three correlator output phases constitutes an observable quantity which depends only on the phase of the visibility and is completely error independent. This closure phase relationship was first recognized and used by Jennison (1958). Similarly the closure amplitude, $C_{m n p q}$ for four antennas can be also shown independent of the antenna based errors as:

$$
C_{m n p q}=\frac{\left|V_{m n}\right|\left|V_{p q}\right|}{\left|V_{m p}\right|\left|V_{n q}\right|}=\frac{\left|A_{m n}\right|\left|A_{p q}\right|}{\left|A_{m p}\right|\left|A_{n q}\right|} .
$$

This was first used by Twiss et al. (1960). Equation 3.29 means closure phase is a function of four variables (two positions in the $u v$-plane) whereas equation 3.30 means closure amplitude is a function of six variables (three positions in the $u v$-plane). The fraction of information that can be restored from the closure phase is $(n-2) n$ (Zensus et al. 1995) and that of closure amplitudes is $(n-3) /(n-1)$ where $n$ is the number of antennas in an array. This means that for 14 antennas used for the VLBI survey in this dissertation, $86 \%$ and $85 \%$ of information can be recovered from closure phase and closure amplitude, respectively.

\subsubsection{Self-Calibration \& Hybrid Mapping}

The quality of the map can be further increased by calibrating the phase and amplitude using the closure relationships. Such calibrations are known by a term called Adaptive calibration (Schwab 1980). Two such methods are self-calibration and hybrid mapping. In self-calibration, the complex antenna gains are taken as free parameters to be explicitly derived together with the intensity. Let us understand the process of self-calibration in the following way. We have equation 3.20 which can be simply written as:

$$
V_{i, j}^{o b s}=G_{i} G_{j}^{*} V\left(u_{i j}, v_{i j}\right) .
$$

In self-calibration (Cornwell \& Fomalont 1999), corrections are made starting from an initial model of the source itself. The model is build during the deconvolution (here 'CLEAN') and therefore, an iterative process including both CLEAN and self-calibration has to be operated to get a final best quality image (i.e. an image with absolute information on the source intensity distribution).

From the starting image, an initial value of $V\left(u_{i j}, v_{i j}\right)$ is obtained and substituted in equation 3.31 to solve for complex gains $G_{i}$ and $G_{j}$. Then the equation 3.31 is rewritten as:

$$
V\left(u_{i j}, v_{i j}\right)=\frac{V_{i, j}^{o b s}}{G_{i} G_{j}^{*}},
$$

and calculate the new $V\left(u_{i j}, v_{i j}\right)$. This is done for each set of samples. All these $V\left(u_{i j}, v_{i j}\right)$ corresponding to each set of samples are Fourier inverted to make an image and (after CLEAN algorithm), it is again used as the starting image for the next step of iteration. In fact, this process consisting of many such iterations is called self-calibration. The method 
used to solve equation 3.31 for $G_{i}$ is to minimize the square of the modulus of the difference between the observed visibility and the corresponding values for the derived model, i.e. by a least square method. The expression that is minimized is:

$$
\sum_{\text {time }} \sum_{i<j} w_{i j}\left|V_{i, j}^{o b s}-G_{i} G_{j}^{*} V\left(u_{i j}, v_{i j}\right)\right|^{2}
$$

where $w_{i j}$ is the weighting coefficient usually chosen to be inversely proportional to the variance of observed visibility. If the method used to solve equation 3.31 for $G_{i}$ is to minimize the errors in the phases of derived models given by $G_{i} G_{j}^{*} V\left(u_{i j}, v_{i j}\right)$, the process is known as Hybrid Mapping. All other steps in iterations are similar to self-calibration.

\subsection{Model Fitting}

The fitting of intensity models to visibility data known as model fitting, especially when the visibility phase was poorly calibrated or the data is not complete enough to allow imaging steps like Fourier inversion, deconvolution and adaptive calibration. There is ambiguity of $180^{\circ}$ in the position angle of the model if phase information is not available (Thompson et al. 2001). For example, the visibility data obtained from high frequency VLBI and space VLBI the $u v$-plane is not well sampled and model fitting is a best way to deal with such data. Gaussian functions are convenient model components for source intensity. This is because of the fact that they are always positive and vary smoothly with angle, as do many of the nebulae structures and radio galaxies. A circularly symmetric Gaussian function centered at $\left(l_{1}, m_{1}\right)$ is:

$$
I(l, m)=I_{0} \exp \left[\frac{-\left(l-l_{1}\right)^{2}-\left(m-m_{1}\right)^{2}}{2 \sigma^{2}}\right],
$$

where $\sqrt{8 \ln 2 \sigma}$ is the full width at half maximum (FWHM) of the Gaussian. Different model fitting methods can be seen in the review by Pearson (1999). Model fitting produces a model of the sky brightness distribution $I(l, m)$ and calculates the expected value of the measurement. Then these models are compared with the actual measurements. Then the model is adjusted to account for the differences between model and actual measurement. After the model is chosen, a function that will quantify the quality of the fit is estimated. The likelihood of the model is given by:

$$
\prod_{i=1}^{n_{d}}\left\{\exp \left[-\frac{1}{2}\left(\frac{V\left(u_{i}, v_{i}\right)-\bar{M}\left(u_{i}, v_{i} ; a_{1}, \ldots, a_{p}\right)}{\sigma_{i}}\right)^{2}\right]\right\}
$$

where $\bar{M}$ is the Fourier transform of the model having $p$ parameters, $n_{d}$ is the number of independent visibility data and $\sigma_{i}$ is the standard deviation. The basic assumption for equation 3.35 is that the noise in the $u v$-plane is of Gaussian form. The best fit is obtained when the function given by equation 3.35 is maximum. This is in principle equal to minimizing the negative $\log$ of equation 3.35 , i.e. minimizing

$$
\chi^{2}=\sum_{i=1}^{n_{d}}\left(\frac{V\left(u_{i}, v_{i}\right)-\bar{M}\left(u_{i}, v_{i} ; a_{1}, \ldots ., a_{p}\right)}{\sigma_{i}}\right)^{2} .
$$


With Gaussian errors, the method become one of the least square method. The expected minimum for $\chi^{2}$ is $n_{d}-p$ and the standard deviation of $\chi^{2}$ is $\sqrt{2\left(n_{d}-p\right)}$. So the optimized model is selected by the value of reduced chi-square $\chi_{\text {red }}^{2}$, i.e. $\chi^{2} /\left(n_{d}-p\right)$. If $\chi_{\text {red }}^{2}$ is larger, the model is far away from the actual measurement and the fitness quality is bad. If $\chi_{\text {red }}^{2}=$ 1 , it means that the error $\sigma_{i}$ is estimated correctly and therefore the fit is good. If $\chi_{\text {red }}^{2}<$ 1 , it means that the error $\sigma_{i}$ is over estimated and therefore the fit is too good to be true. Depending on the $\chi_{\text {red }}^{2}$, we have to decide whether to accept or reject a model in the map.

\subsubsection{Error Estimation}

In fact, the observed visibilities is affected by noise from sky background, atmosphere, losses in feed, spillover from ground, receiver etc. The noise in the dirty image produced by the Fourier inversion of the observed visibility function given by equation 3.27 being a linear function, is Gaussian. However, the noise in the dirty image produced by CLEAN or MEM is complex to estimate since they are non-linear in nature. Here we assume that the source intensity distribution can be represented by Gaussian components. So, the final self calibrated data fitted with circular Gaussian components were used to obtain the total, $S_{\text {tot }}$, and peak flux density, $S_{\text {peak }}$, the size, $d$, and the positional offset, (in polar coordinates $r, \theta$ ) of the component from the brightest region (core) at the base of the jet, taken to be at the coordinte origin.

The uncertainties of the model parameters can be estimated analytically, based on the SNR of detection of individual components given by (Fomalont 1999) and (Schinzel et al. 2012):

$$
\begin{gathered}
\sigma_{\text {peak }}=\sigma_{\text {rms }}\left(1+\frac{S_{\text {peak }}}{\sigma_{\text {rms }}}\right)^{\frac{1}{2}}, \\
\sigma_{\text {tot }}=\sigma_{\text {peak }}\left(1+\frac{S_{\text {tot }}^{2}}{S_{\text {peak }}^{2}}\right)^{\frac{1}{2}} \\
\sigma_{\mathrm{d}}=d\left(\frac{\sigma_{\text {peak }}}{S_{\text {peak }}}\right) \\
\sigma_{\mathrm{r}}=\frac{1}{2} \sigma_{\mathrm{d}} \\
\sigma_{\theta}=\arctan \frac{\sigma_{\mathrm{r}}}{r}
\end{gathered}
$$

where $\sigma_{\text {peak }}, \sigma_{\text {tot }}, \sigma_{\mathrm{d}}, \sigma_{\mathrm{r}}$ and $\sigma_{\theta}$ are the uncertainties in peak flux density, total flux density, size and positional offsets - radial distance and positional angle of a component, respectively and $\sigma_{\text {rms }}$ is the rms noise in the model fit map. To assess whether a given component is extended (resolved), the minimum resolvable size of the component can be calculated and compared with the size obtained with the model fitting. The minimum resolvable size, $d_{\text {min }}$ of a Gaussian component is given in Lobanov (2005) as

$$
d_{\min }=\frac{2^{(1+\beta / 2)}}{\pi}\left[\pi a b \log \left(\frac{\mathrm{SNR}+1}{\mathrm{SNR}}\right)\right]^{1 / 2},
$$

where $a$ and $b$ are the axes of the restoring beam, SNR is the signal-to-noise ratio, $\beta$ is the weighing function which is 0 for natural weighting or 2 for uniform weighting. For components which have size $d<d_{\min }$, the uncertainities should be estimated with $d=d_{\text {min }}$. 


\section{Chapter 4}

\section{Global 3 mm-VLBI Array Survey}

This Chapter is partially published in the journal Astronomy \& Astrophysics, and is reproduced with the permission of the Editor-in-Chief. Credit: Nair et al., A\&A, 622, A92 (2019), reproduced with permission (C)ESO.

\subsection{Introduction}

Very Long Baseline Interferometry (VLBI) observations at millimeter wavelengths reach a resolution of about 50 micro arcseconds, enabling us to image the regions in AGNs in the immediate vicinity of supermassive black holes on scales down to a few tens of gravitational radii. Systematic studies of radio sources on such scales is the best laboratory to understand generic properties of relativistic flows and address intricate evolution of individual objects. This information serves as a basis for investigations of the collimation and acceleration of relativistic flows.

Synchrotron radiation becomes optically thin between $1 \mathrm{~cm}(\sim 30 \mathrm{GHz})$ and $1 \mathrm{~mm}(\sim 300 \mathrm{GHz})$. So, the synchrotron self absorbed regions which are opaque to centimeter radiation but optically thin to millimeter and sub-millimeter radiation can be revealed by high frequency VLBI observations. The first detection of single baseline interference fringes of $89 \mathrm{GHz}$ (3.4 mm) observations was done by Readhead et al. (1983), demonstrating the feasibility of $3 \mathrm{~mm}$ VLBI, which was later continued with many VLBI observations at $86 \mathrm{GHz}$. Later, in 1995, the first global mm-VLBI array known as Coordinated Millimeter VLBI Array (CMVA; Rogers et al. 1995) successfully came into operation, by increasing the number of participating radio telescopes gradually to 12 . From 2002, a large millimeter VLBI array known as Global Millimeter VLBI Array (GMVA) ${ }^{1}$ is in operation with 16 radio telescopes participating in total. Detailed studies using GMVA observations by Krichbaum et al. (1992), Boccardi et al. (2016), Baczko et al. (2016), Hodgson et al. (2017), Casadio et al. (2017) etc. have shown the success of GMVA in understanding the physics of ultracompact radio jets.

In order to increase the number of objects imaged at $86 \mathrm{GHz}$, five detection and imaging surveys have been conducted to date (Beasley et al. 1997; Lonsdale et al. 1998; Rantakyro et al. 1998; Lobanov et al. 2000; Lee et al. 2008, see Table 4.1), with a total of 251 extragalactic sources observed at $86 \mathrm{GHz}$. Fringes have been detected in 165 sources and 133

\footnotetext{
${ }^{1}$ http://www3.mpifr-bonn.mpg.de/div/vlbi/globalmm/
} 
have been successfully imaged so far. A complete sample of objects imaged at $86 \mathrm{GHz}$ has not been established so far.

A large global $86 \mathrm{GHz}$ VLBI survey of compact radio sources was conducted from October 2010 to October 2011 during three sessions of the Global Millimeter VLBI Array. The main goals of the survey were to extend the existing survey at $86 \mathrm{GHz}$ to fainter sources, to develop a comprehensive sample of ultra compact radio sources imaged at $86 \mathrm{GHz}$ and to study the morphology and the distribution of brightness temperatures.

In this chapter, the results from this survey is presented. Section 4.2 describes the source selection and the survey observations. In Section 4.3, we describe the data processing, amplitude and phase calibration and in section 4.4, we describe imaging and model fitting procedures. Section 4.5 describes the images and derived parameters of the target sources. The complete set of images of all of the target sources is presented in section 4.5. Brightness temperatures of the survey sources are derived and discussed in section 4.6.1. The visibility based brightness temperature estimates is discussed in section 4.6.2 and source compactness is described in section 4.6.3.

TABLE 4.1: VLBI surveys at $86 \mathrm{GHz}$

\begin{tabular}{lcrrrrrrr}
\hline \hline $\begin{array}{l}\text { Survey } \\
(1)\end{array}$ & $\begin{array}{c}N_{\text {ant }} \\
(2)\end{array}$ & $\begin{array}{r}B_{\text {rec }} \\
(3)\end{array}$ & $\begin{array}{r}\Delta S \\
(4)\end{array}$ & $\begin{array}{r}\Delta I_{\mathrm{m}} \\
(5)\end{array}$ & $\begin{array}{r}D_{\text {img }} \\
(6)\end{array}$ & $\begin{array}{r}N_{\text {obs }} \\
(7)\end{array}$ & $\begin{array}{r}N_{\text {det }} \\
(8)\end{array}$ & $\begin{array}{r}N_{\text {img }} \\
(9)\end{array}$ \\
\hline Beasley et al. (1997) & 3 & 112 & $\sim 0.5$ & $\ldots$ & $\ldots$ & 51 & 12 & $\ldots$ \\
Lonsdale et al. (1998) & $2-5$ & $112 / 224$ & $\sim 0.7$ & $\ldots$ & $\ldots$ & 79 & 14 & $\ldots$ \\
Rantakyrö et al. (1998) & $6-9$ & 128 & $\sim 0.5$ & $\sim 30$ & 70 & 68 & 16 & 12 \\
Lobanov et al. (2000) & $3-5$ & 224 & $\sim 0.4$ & $\sim 20$ & 100 & 28 & 26 & 14 \\
Lee et al. (2008) & 12 & 256 & $\sim 0.3$ & 10 & 200 & 127 & 121 & 109 \\
This survey & $13-14$ & 512 & $\sim 0.1$ & $\sim 5$ & $>400$ & 162 & 162 & 162 \\
\hline
\end{tabular}

Columns: 1 - Survey ; 2 - number of participating antennas; 3 - recording bit rate [Mbps]; 4 - average baseline sensitivity $[\mathrm{Jy}] ; 5$ - average image sensitivity [mJy/beam]; 6 - typical dynamic range of images; 7 - number of sources observed; 8 - number of sources detected; 9 - number of sources imaged.

\subsection{GMVA Survey of Compact AGN}

Dedicated VLBI observations at $86 \mathrm{GHz}$ are made with the GMVA and with the VLBA ${ }^{2}$ working in a standalone mode (VLBA also takes part in GMVA observations). The GMVA has been operating since 2002, superseding the operations of the Coordinated Millimeter VLBI Array (CMVA; Rogers et al. 1995) and earlier ad hoc arrangements employed since the early 1980s (Readhead et al. 1983). The GMVA carries out regular, coordinated observations at $86 \mathrm{GHz}$, providing good quality images with a typical angular resolution of $\sim(50-70) \mu$ as.

The array comprises up to 16 telescopes located in Europe, USA and Korea. The following telescopes took part in the GMVA observations for this survey in 2010 and 2011: eight VLBA antennas equipped with $3 \mathrm{~mm}$ receivers, the IRAM $30 \mathrm{~m}$ telescope on Pico Veleta (Spain), the phased 6-element IRAM interferometer on Plateau de Bure (France), the MPIfR $100 \mathrm{~m}$ radio telescope in Effelsberg (Germany), the OSO $20 \mathrm{~m}$ radio telescope at Onsala (Sweden), the $14 \mathrm{~m}$ telescope in Metsähovi (Finland), and the OAN $40 \mathrm{~m}$ telescope in Yebes (Spain).

\footnotetext{
${ }^{2}$ Very Long Baseline Array of the National Radioastronomy Observatory, Socorro, NM; https://www.lbo.us/
} 


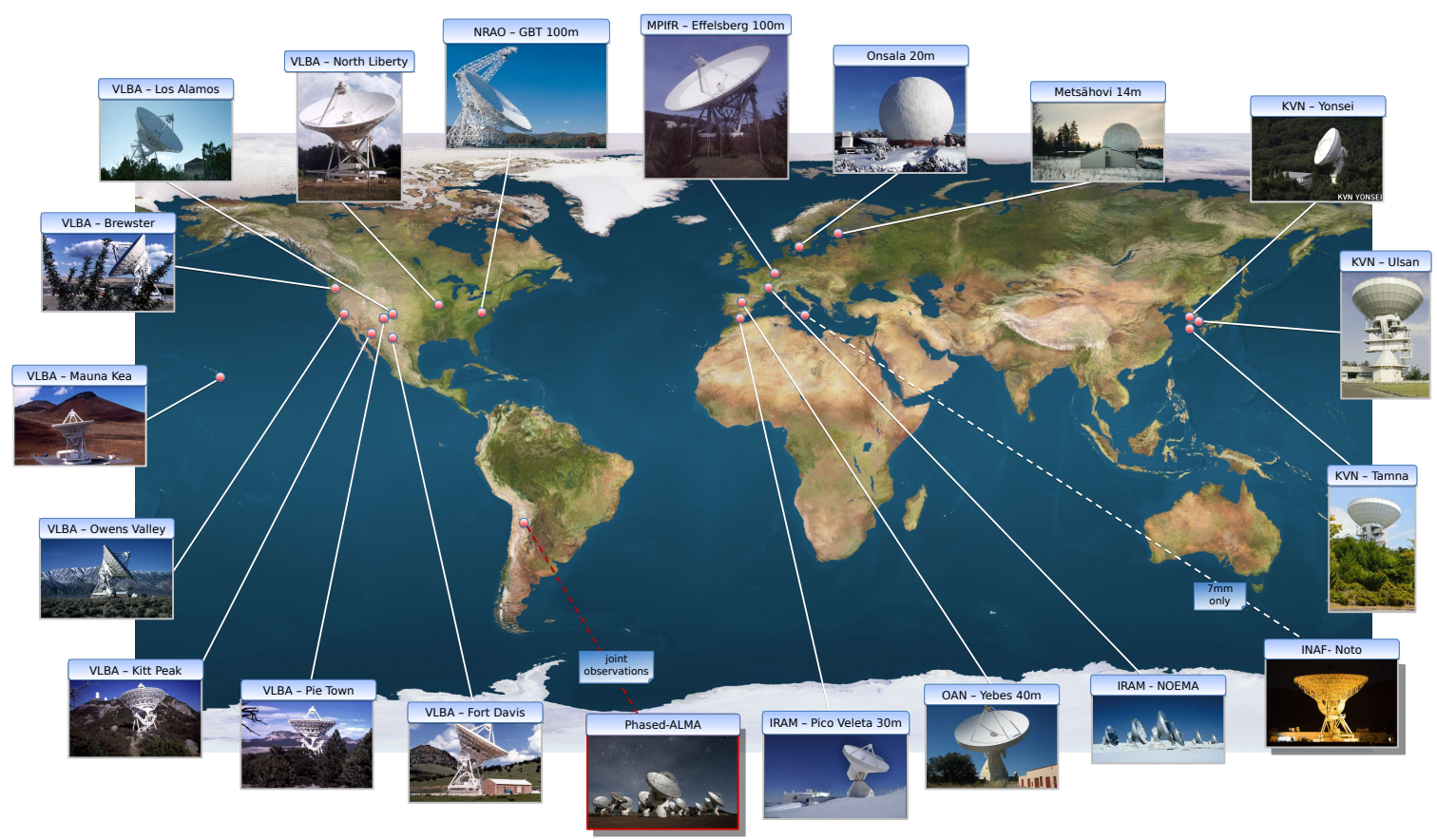

Figure 4.1: The Global Millimeter VLBI Array (GMVA) with its participating telescopes, operating at the frequency of $86 \mathrm{GHz}$. Credit: Helge Rottmann, MPIfR.

TABLE 4.2: Participating Telescopes

\begin{tabular}{lcccccccc}
\hline \hline & & $D$ & $\mathrm{G}$ & $T_{\text {sys }}$ & $\eta_{\mathrm{A}}$ & $\begin{array}{c}\text { SEFD } \\
\text { Name }\end{array}$ & $\begin{array}{c}\Delta_{512,20} \\
{[\mathrm{Jy}]}\end{array}$ & $\begin{array}{c}\sigma_{\text {rms }} \\
{[\mathrm{mJy}]}\end{array}$ \\
{$[\mathrm{mJy}]$} \\
\hline Brewster & $\mathrm{m})$ & $(3)$ & $(4)$ & $(5)$ & $(6)$ & $(7)$ & $(8)$ & $(9)$ \\
Effelsberg & $\mathrm{Br}$ & 25 & 0.033 & 110 & 0.22 & 3333.3 & 23.44 & 164.09 \\
Fort Davis & $\mathrm{Fd}$ & $80^{\dagger}$ & 0.140 & 140 & 0.08 & 1000 & 12.84 & 89.87 \\
Kitt Peak & $\mathrm{Kp}$ & 25 & 0.039 & 140 & 0.22 & 3589.7 & 24.32 & 170.28 \\
Los Alamos & $\mathrm{La}$ & 25 & 0.042 & 100 & 0.22 & 3571.4 & 24.26 & 169.85 \\
Metsähovi & $\mathrm{Mh}$ & 14 & 0.017 & 300 & 0.22 & 2857.1 & 21.70 & 151.91 \\
Mauna Kea & $\mathrm{Mk}$ & 25 & 0.019 & 100 & 0.22 & 5263.2 & 29.45 & 206.18 \\
North Liberty & $\mathrm{Nl}$ & 25 & 0.022 & 130 & 0.22 & 5909.1 & 31.21 & 218.47 \\
Onsala & $\mathrm{On}$ & 20 & 0.049 & 250 & 0.43 & 5102 & 29.00 & 203.00 \\
Owens Valley & $\mathrm{Ov}$ & 25 & 0.035 & 120 & 0.22 & 3428.6 & 23.77 & 166.41 \\
Plateau de Bure & $\mathrm{Pb}$ & $36^{\ddagger}$ & 0.22 & 180 & 0.7 & 818.2 & 11.61 & 81.29 \\
Pie Town & $\mathrm{Pt}$ & 25 & 0.044 & 100 & 0.22 & 2272.7 & 19.36 & 135.52 \\
Pico Veleta & $\mathrm{Pv}$ & 30 & 0.153 & 100 & 0.6 & 653.6 & $\ldots$ & $\ldots$ \\
Yebes & $\mathrm{Ys}$ & 40 & 0.09 & 150 & 0.2 & 1666.7 & 16.58 & 116.03 \\
\hline
\end{tabular}

Columns: 1 - telescope name; 2 - abbreviation for the telescope name; 3 -diameter; 4 - antenna gain; 5 - zenith system temperature; 6 - aperture efficiency; 7 - zenith SEFD; 8 - sensitivity on the baseline to Pico Veleta, for a $20 \mathrm{sec}$ fringe fit interval and $512 \mathrm{Mbps}$ recording rate; $9-7 \sigma$ detection threshold. Notes: ${ }^{\dagger}-$ effective diameter at $86 \mathrm{GHz} ;{ }^{\ddagger}$ - effective diameter in the phased array mode. 


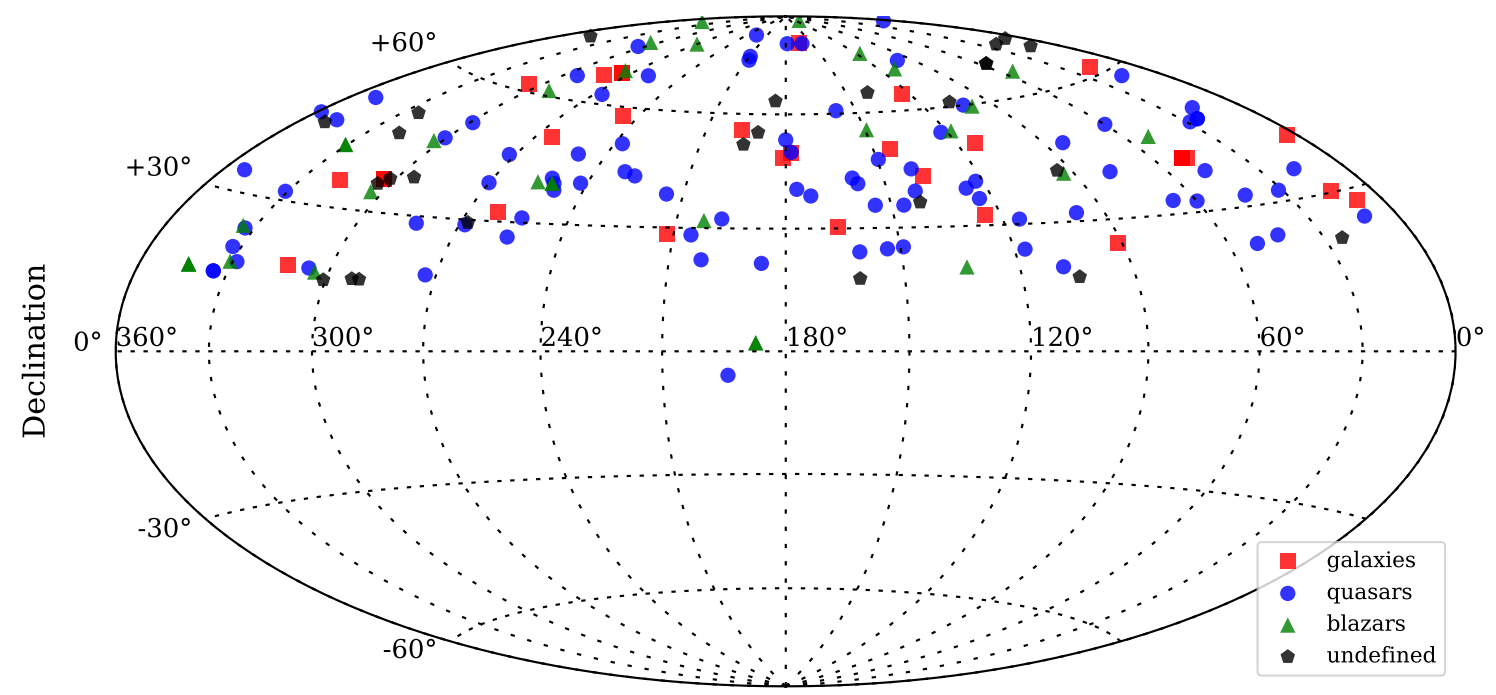

Right Ascension

Figure 4.2: The sky distribution of the $86 \mathrm{GHz}$ VLBI sources in this survey. Symbols: circles are quasars, triangles are blazars, squares are galaxies and diamonds are unidentified sources.

\subsubsection{Source Selection}

The source list has been compiled from the $15 \mathrm{GHz}$ VLBA survey (MOJAVE, $2 \mathrm{~cm}$ VLBA) database (Kovalev et al. 2005; Lister \& Homan 2005). This database includes all objects from the $15 \mathrm{GHz}$ VLBA monitoring programmes. This database was used to select a representative flux density limited sample of objects that satisfies the following selection criteria: a) the sources selected are in the statistically complete MOJAVE sample (Kovalev et al. 2005; Lister \& Homan 2005; Lister et al. 2009), allowing us to perform statistically significant analysis of the $86 \mathrm{GHz}$ VLBI data; b) additional sources selected have the $15 \mathrm{GHz}$ correlated flux density at the longest VLBA baselines $\left.(400-450 \mathrm{M} \lambda), S_{\mathrm{c}} \geq 0.5 \mathrm{Jy} ; c\right)$ compactness at longest spacings, $S_{\mathrm{c}} / S_{\mathrm{VLBA}} \geq 0.4$ where $S_{\mathrm{VLBA}}$ is the $15 \mathrm{GHz}$ total clean flux density; $d$ ) declination $\delta \geq 15^{\circ}$. A similar approach was applied to select objects for the $86 \mathrm{GHz}$ Lee et al. (2008) survey that has yielded a 95\% detection rate. The selection criteria applied optimize the chances for a source to be detected at long baselines at $86 \mathrm{GHz}$. The sample selected using this procedure should be representative down to $\sim 0.5$ Jy for declinations $\delta \geq 15^{\circ}$.

With the above mentioned selection criteria, a total of 162 unique sources have been selected, comprising 89 quasars, 26 BL Lac objects, 22 radio galaxies and 25 unidentified sources. Eight bright sources, 3C 84, OJ 287, 3C 273B, 3C 279, 3C 345, BL Lac, 0716+714 and $3 \mathrm{C} 454.3$ have been added to this list for fringe finding and calibration purposes. The basic information about the selected target sources is summarized in Table 4.4. The distribution of the total single dish flux density of the sources, measured at $86 \mathrm{GHz}$ at Pico Veleta or Plateau de Bure during the observations, is shown in Figure 4.3 and compared with the respective distribution of the source sample observed in Lee et al. (2008). This comparison shows that our survey observations probe objects down to about one order of magnitude fainter than the $3 \mathrm{~mm}$ survey by Lee et al. (2008) and provide a roughly twofold increase of the total number of objects imaged with VLBI at $3 \mathrm{~mm}$ (see Table 4.4 for details). 


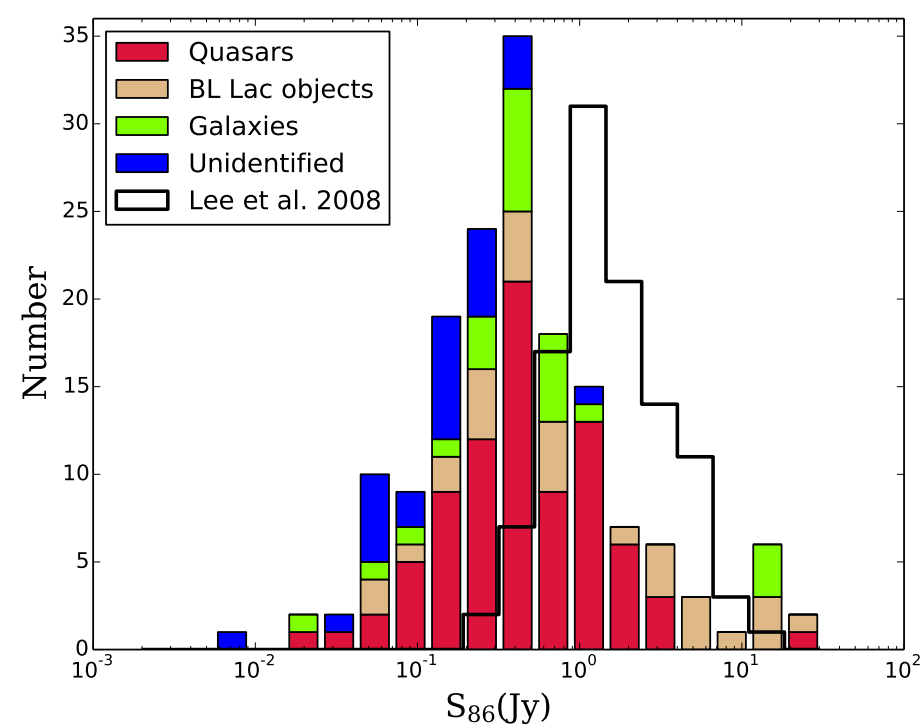

Figure 4.3: Distribution of the total single dish flux density of the sources, measured at $86 \mathrm{GHz}$ at Pico Veleta or Plateau de Bure during the observations, $S_{86}$ broken down along different host galaxy types and compared to the respective distribution for the sources from the sample of Lee et al. (2008). The present survey provides a nearly twofold increase in the number of objects imaged with VLBI at $3 \mathrm{~mm}$.

\subsubsection{Observations}

The survey observations are spread over a total of $3 \times 2$ observing days (144 hours), scheduled within three separate GMVA sessions. Up to 14 telescopes were taking part in the observations (see Table 4.3). The observations were typically scheduled with 5 scans per hour, each of 300 seconds in duration. Gaps of 5-10 minutes were introduced for antenna pointing at Effelsberg $(\mathrm{Eb})$ and Pico Veleta $(\mathrm{Pv})$ and for the phasing of the Plateau de Bure $(\mathrm{Pb})$ interferometer. This observing scheme yielded the total of 720 scans distributed between 174 observing targets (162 unique radio sources), ensuring that each object was observed with 4-5 scans distributed over a wide range of hour angles. Despite a rather modest observing time spent on each target, the large number of participating antennas ensured the achievement of good uv-coverages for all survey sources down to the lowest declinations (see Figure 4.4).

TABLE 4.3: Log of survey observations

\begin{tabular}{|c|c|c|c|c|c|c|c|c|}
\hline $\begin{array}{l}\text { Epoch } \\
\text { (1) }\end{array}$ & $\begin{array}{l}\text { Date } \\
(2)\end{array}$ & $\begin{array}{c}\mathrm{N}_{\text {sources }} \\
\text { (3) }\end{array}$ & $\begin{array}{c}\text { Bit rate } \\
{[\mathrm{Mbit} / \mathrm{s}]} \\
(4)\end{array}$ & Bandwidth & $\begin{array}{c}\text { Polarization } \\
(6)\end{array}$ & $\begin{array}{c}\text { Frequency } \\
\text { channels } \\
(7)\end{array}$ & Sampling & $\begin{array}{c}\text { Telescopes } \\
\text { (9) }\end{array}$ \\
\hline A & Oct 2010 & 68 & 512 & 128 & LCP & 32 & 2 & $8 \mathrm{VLBA}+(\mathrm{Eb}, \mathrm{On}, \mathrm{Mh}, \mathrm{Pb}, \mathrm{Pv})$ \\
\hline B & May 2011 & 46 & 512 & 128 & LCP & 64 & 2 & $8 \mathrm{VLBA}+(\mathrm{Eb}, \mathrm{On}, \mathrm{Pb}, \mathrm{Pv}, \mathrm{Mh})$ \\
\hline $\mathrm{C}$ & Oct 2011 & 60 & 512 & 128 & LCP & 32 & 2 & $8 \mathrm{VLBA}+(\mathrm{Eb}, \mathrm{On}, \mathrm{Pb}, \mathrm{Pv}, \mathrm{Mh}, \mathrm{Ys})$ \\
\hline
\end{tabular}

Columns: 1 - survey epoch; 2 - observing date; 3 - number of objects observed; 4 - recording bit rate [Mbps]; 5 polarization; 6 - bandwidth [MHz]; 7 - number of frequency channels per IF band; 8 -sampling [bits]; 9 - participated telescopes

The observations were performed at a sampling rate of $512 \mathrm{Mbit} / \mathrm{sec}$ and with a 2-bit sampling. The observations were done at left circular polarization (LCP). The total observing 

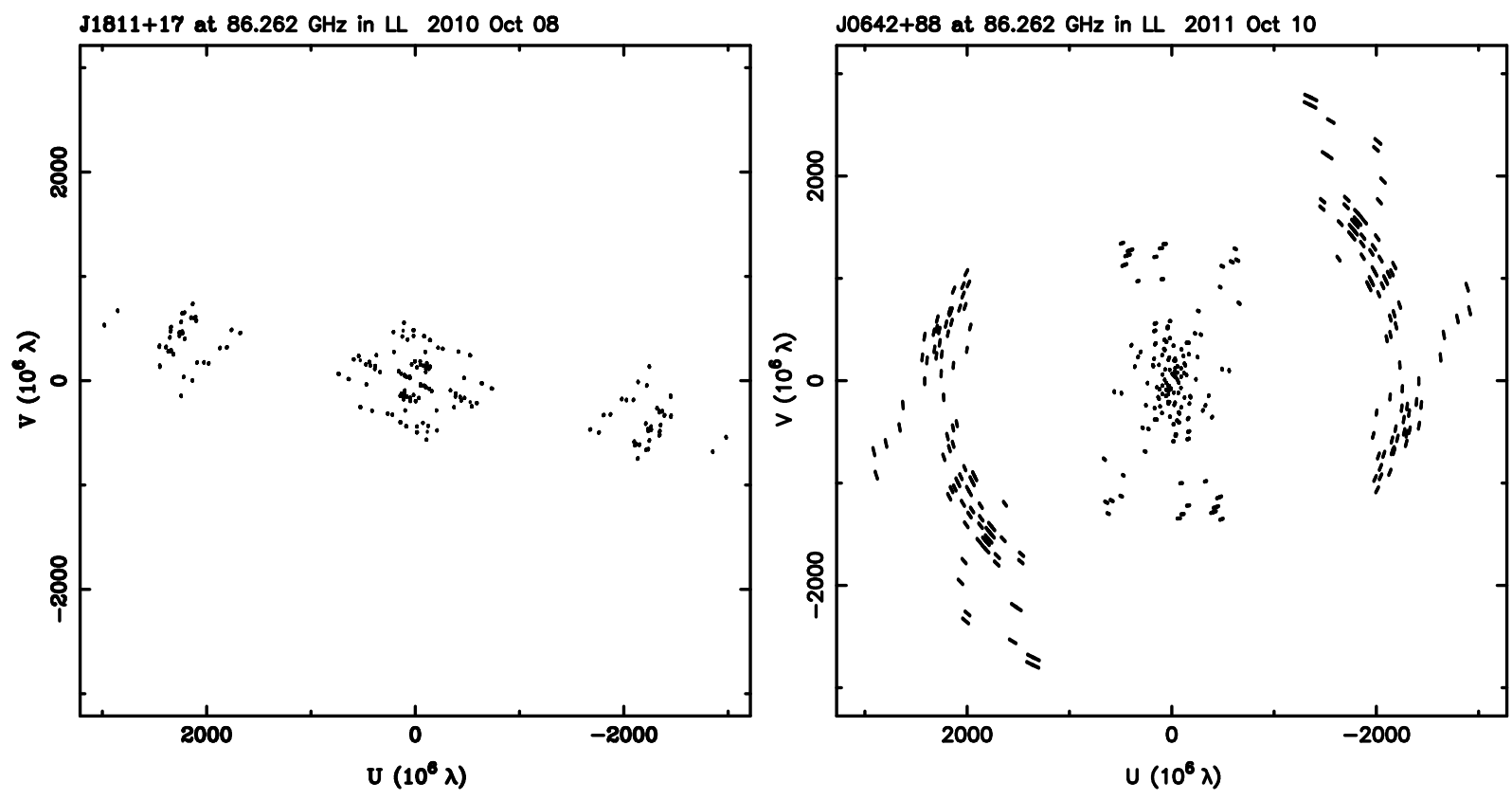

FiguRE 4.4: Examples of $u v$-coverages of the survey observations for a low declination source (left; J1811 $+1704, \delta=+17^{\circ}$ ) and a high declination source (right; J0642+8811,

$$
\left.\delta=+88^{\circ}\right) \text {. }
$$

bandwidth of $128 \mathrm{MHz}$ was split into 4 intermediate frequency (IF) bands of $32 \mathrm{MHz}$ each (64 MHz in Epoch B). The typical baseline sensitivities for a 20 -second integration time are $\approx 0.05 \mathrm{Jy}$ on the $\mathrm{Pb}-\mathrm{Pv}$ baseline, $\approx 0.1 \mathrm{Jy}$ on the $\mathrm{Eb}-\mathrm{Pv}$ baseline, $\approx 0.2 \mathrm{Jy}$ on the baselines between $\mathrm{Eb} / \mathrm{Pv}$ and other antennas, and $\approx 0.4$ Jy on the baselines between the VLBA antennas. With such baseline sensitivities and an on-source integration time of $\sim 20$ minutes (e.g. 3 scans $\times 7$ minutes), the typical point source sensitivity of a survey observation is $\approx 5 \mathrm{mJy} /$ beam, which is sufficient to obtain robust images of most of the survey sources.

TABLE 4.4: List of sources

\begin{tabular}{|c|c|c|c|c|c|c|c|c|c|}
\hline $\begin{array}{c}\text { Source (J2000) } \\
\text { (1) }\end{array}$ & $\begin{array}{c}\text { Source (B1950) } \\
(2)\end{array}$ & $\begin{array}{c}\text { Common Name } \\
(3)\end{array}$ & $\begin{array}{l}\text { Epoch } \\
\text { (4) }\end{array}$ & $\begin{array}{l}\alpha_{2000} \\
(5)\end{array}$ & $\begin{array}{c}\delta_{2000} \\
(6)\end{array}$ & $\begin{array}{c}z \\
(7)\end{array}$ & $\begin{array}{c}\text { Type } \\
(8)\end{array}$ & $\begin{array}{l}m_{\mathrm{v}} \\
(9)\end{array}$ & $\begin{array}{l}S_{86 \mathrm{GHz}} \\
(10)\end{array}$ \\
\hline $\mathrm{J} 0013+4051$ & $0010+405$ & $4 \mathrm{C} 40.01$ & $\mathrm{C}$ & 001331.130201 & +405137.14403 & 0.2560 & G & 17.9 & 0.79 \\
\hline $\mathrm{J} 0017+8135$ & $0014+813$ & & B & 001708.474904 & +813508.13656 & 3.3660 & Q & 16.5 & 0.18 \\
\hline $\mathrm{J} 0030+7037$ & $0027+703$ & & B & 003014.412959 & +703740.06069 & $\ldots$ & $\mathrm{U}$ & 17.0 & 0.34 \\
\hline J0034+2754 & $0032+276$ & & $\mathrm{C}$ & 003443.486179 & +275425.72112 & 2.9642 & G & 18.0 & 0.07 \\
\hline J0044+6803 & $0041+677$ & & B & 004450.759603 & +680302.68574 & $\cdots$ & $\mathrm{U}$ & $\ldots$ & 0.17 \\
\hline J0046+2456 & $0043+246$ & & A & 004607.825730 & +245632.52437 & 0.7467 & Q & 17.1 & 0.47 \\
\hline J0057+3021 & $0055+300$ & NGC 315 & $\mathrm{~A}$ & 005748.883342 & +302108.81194 & 0.0165 & G & 12.2 & 0.48 \\
\hline $\mathrm{J} 0102+5824$ & $0059+581$ & $7 \mathrm{C} 0059+5808$ & B & 010245.762380 & +582411.13659 & 0.6440 & Q & 17.6 & 3.11 \\
\hline $\mathrm{J} 0109+6133$ & $0106+612$ & & B & 010946.344370 & +613330.45531 & 0.7830 & $\mathrm{G}$ & 19.4 & 0.64 \\
\hline $\mathrm{J} 0112+3522$ & $0109+351$ & & $\mathrm{~A}$ & 011212.944409 & +352219.33615 & 0.4500 & Q & 17.8 & 0.53 \\
\hline $\mathrm{J} 0113+4948$ & $0110+495$ & & A & 011327.006813 & +494824.04306 & 0.3890 & Q & 18.4 & 0.61 \\
\hline $\mathrm{J} 0126+7046$ & $0122+705$ & & B & 01267.8495750 & +704652.38656 & $\ldots$ & $\mathrm{U}$ & 18.7 & 0.07 \\
\hline $\mathrm{J} 0136+4751$ & $0133+476$ & DA 55 & $\mathrm{~B}, \mathrm{C}$ & 013658.594700 & +475129.10000 & 0.8590 & Q & 18.0 & 1.86 \\
\hline $\mathrm{J} 0137+2145$ & $0134+215$ & & A & 013715.624949 & +214544.27088 & $\ldots$ & $\mathrm{U}$ & $\ldots$ & 0.07 \\
\hline $\mathrm{J} 0154+4743$ & $0151+474$ & & B & 015456.289889 & +474326.53956 & 1.0260 & Q & & $\ldots$ \\
\hline J0205+3212 & $0202+319$ & & A & 020504.925360 & +32 1230.09541 & 1.4660 & Q & 18.2 & 0.81 \\
\hline J0253+3217 & $0250+320$ & & $\mathrm{~A}$ & 025333.650138 & +321720.89168 & 0.8590 & Q & ... & 0.37 \\
\hline $\mathrm{J} 0254+2343$ & $0251+235$ & & A & 025424.718127 & +234326.47461 & 1.9870 & Q & $\cdots$ & 0.05 \\
\hline $\mathrm{J} 0310+3814$ & $0307+380$ & & B & 031049.879930 & +38 1453.83778 & 0.9450 & Q & 18.5 & 0.44 \\
\hline $\mathrm{J} 0313+4120$ & $0309+411$ & NRAO 128 & B & 031301.962125 & +412001.18349 & 0.1340 & $\mathrm{G}$ & 18.0 & 0.89 \\
\hline $\mathrm{J} 0319+4130$ & $0316+413$ & $3 \mathrm{C} 84$ & $\mathrm{~A}, \mathrm{~B}, \mathrm{C}$ & 031948.160100 & +413042.11415 & 0.0176 & G & 12.6 & 12.70 \\
\hline $\mathrm{J} 0325+2224$ & $0322+222$ & & $\mathrm{C}$ & 032536.814357 & +222400.36551 & 2.0660 & Q & 18.9 & 0.90 \\
\hline $\mathrm{J} 0325+4655$ & $0321+467$ & & $\mathrm{C}$ & 032520.303800 & +465506.63500 & $\ldots$ & $\mathrm{B}$ & 14.1 & 0.27 \\
\hline $\mathrm{J} 0333+6536$ & $0329+654$ & & $\mathrm{C}$ & 033356.737600 & +653656.18400 & $\ldots$ & B & 19.3 & 0.07 \\
\hline $\mathrm{J} 0344+6827$ & $0339+683$ & & $\mathrm{~A}, \mathrm{C}$ & 034441.441278 & +682747.81028 & $\ldots$ & $\mathrm{U}$ & $\ldots$ & 0.07 \\
\hline
\end{tabular}


TABLE 4.4: List of sources (continued)

\begin{tabular}{|c|c|c|c|c|c|c|c|c|c|}
\hline $\begin{array}{c}\text { Source (J2000) } \\
(1)\end{array}$ & $\begin{array}{c}\text { Source (B1950) } \\
(2)\end{array}$ & $\begin{array}{c}\text { Common Name } \\
\text { (3) }\end{array}$ & $\begin{array}{c}\text { Epoch } \\
(4)\end{array}$ & $\begin{array}{l}\alpha_{2000} \\
(5)\end{array}$ & $\begin{array}{c}\delta_{2000} \\
(6)\end{array}$ & $\begin{array}{c}z \\
(7)\end{array}$ & $\begin{array}{c}\text { Type } \\
(8)\end{array}$ & $\begin{array}{l}m_{\mathrm{v}} \\
(9)\end{array}$ & $\begin{array}{l}S_{86 \mathrm{GHz}} \\
(10)\end{array}$ \\
\hline J0359+3220 & $0356+322$ & & A & 035944.912917 & +322047.15548 & 1.3310 & $\mathrm{Q}$ & 19.9 & 0.36 \\
\hline J0359+5057 & $0355+508$ & NRAO 150 & A & 035929.747200 & +505750.16100 & 1.5200 & $\mathrm{Q}$ & 22.9 & 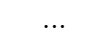 \\
\hline J0428+3259 & $0424+328$ & & A & 042805.808725 & +325952.04381 & 0.4760 & $\mathrm{Q}$ & 20.2 & 0.04 \\
\hline $\mathrm{J} 0512+4041$ & $0509+406$ & & A & 051252.542864 & +404143.62019 & $\ldots$ & $\mathrm{Q}$ & & 1.29 \\
\hline J0533+4822 & $0529+483$ & & A & 053315.865792 & +482252.80771 & 1.1620 & $\mathrm{Q}$ & 19.9 & 1.26 \\
\hline J0604+2429 & $0601+245$ & $4 \mathrm{C} 24.11$ & A & 060455.121380 & +242955.03635 & 1.1330 & G & & 0.20 \\
\hline J0612+4122 & $0609+413$ & & A & 061251.185236 & +412237.40815 & $\ldots$ & B & 15.7 & 0.27 \\
\hline J0618+4207 & $0614+421$ & & A & 061808.619909 & +420759.84609 & & $\mathrm{U}$ & & 0.05 \\
\hline J0632+3200 & $0629+320$ & & A & 063230.782861 & +3200 53.63193 & 1.8310 & $\mathrm{Q}$ & 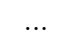 & 0.02 \\
\hline J0638+5933 & $0633+595$ & & B & 063802.871950 & +593322.21466 & 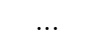 & B & & 0.39 \\
\hline J0639+7324 & $0633+734$ & & $\mathrm{C}$ & 063921.961200 & +732458.04000 & 1.8500 & Q & 17.8 & 0.56 \\
\hline J0642+8811 & $0604+882$ & & $\mathrm{C}$ & 06426.1363170 & +881155.01734 & $\ldots$ & B & 19.5 & 0.15 \\
\hline J0650+6001 & $0646+600$ & & B & 065031.254355 & +600144.55601 & 0.4550 & $\mathrm{Q}$ & 18.9 & 0.26 \\
\hline J0700+1709 & $0657+172$ & & A & 070001.525539 & +170921.70130 & $\ldots$ & $\mathrm{U}$ & 21.0 & 1.05 \\
\hline J0707+6110 & $0702+612$ & & B & 070700.615678 & +611011.60689 & 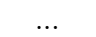 & $\mathrm{U}$ & 17.0 & 0.23 \\
\hline J0713+1935 & $0710+196$ & WB92 0711+1940 & A & 071330.782861 & +193500.40875 & 0.5400 & $\mathrm{Q}$ & 18.6 & 0.23 \\
\hline $\mathrm{J} 0721+7120$ & $0716+714$ & S5 $0716+71$ & $\mathrm{~B}, \mathrm{C}$ & 072153.448400 & +712036.36300 & 0.3000 & B & 15.5 & 4.91 \\
\hline J0733+5022 & $0730+504$ & & $\mathrm{C}$ & 073352.520500 & +50 2209.06200 & 0.7200 & Q & 19.0 & 0.26 \\
\hline J0741+3112 & $0738+313$ & OI 363 & $\mathrm{C}$ & $0741 \quad 10.703310$ & +311200.22894 & 0.6320 & $\mathrm{Q}$ & 16.7 & 0.43 \\
\hline J0747+7639 & $0740+767$ & & B & 074714.607565 & +763917.27140 & & B & 20.0 & 0.12 \\
\hline J0748+2400 & $0745+241$ & S3 $0745+24$ & $\mathrm{C}$ & 074836.109275 & +240024.11002 & 0.4092 & $\mathrm{Q}$ & 19.7 & 0.88 \\
\hline J0753+5352 & $0749+540$ & $4 C 54.15$ & $\mathrm{C}$ & 075301.384569 & +535259.63709 & 0.2000 & B & 18.5 & 0.49 \\
\hline J0808+4052 & $0805+410$ & & $\mathrm{C}$ & 080856.652043 & +405244.88880 & 1.4193 & $\mathrm{Q}$ & 19.0 & 0.51 \\
\hline J0809+5341 & $0805+538$ & & $\mathrm{C}$ & 080941.732819 & +534125.09245 & 2.1330 & $\mathrm{Q}$ & 19.8 & 0.11 \\
\hline J0814+6431 & $0810+646$ & & $\mathrm{C}$ & 081439.190224 & +643122.02696 & & B & 17.9 & $\ldots$ \\
\hline J0815+3635 & $0812+367$ & & $\mathrm{C}$ & 081525.944861 & +363515.14876 & 1.0286 & $\mathrm{Q}$ & 18.0 & $\ldots$ \\
\hline J0817+3227 & $0814+326$ & & $\mathrm{C}$ & $\begin{array}{lll}08 & 17 & 28.542305\end{array}$ & +322702.92601 & & G & 21.6 & $\ldots$ \\
\hline J0824+3916 & $0821+394$ & $4 \mathrm{C} 39.23$ & $\mathrm{C}$ & 082455.483855 & +391641.90401 & 1.2159 & $\mathrm{Q}$ & 18.3 & \\
\hline J0854+2006 & $0851+202$ & OJ 287 & B & 085448.874900 & +200630.64100 & 0.3060 & B & 14.0 & 6.70 \\
\hline J0909+4253 & $0906+430$ & & A & 090933.497140 & +425346.48213 & 0.6704 & G & 20.0 & 0.67 \\
\hline J0917+6530 & $0913+657$ & & $\mathrm{C}$ & 091755.568093 & +653015.12741 & $\ldots$ & $\mathrm{U}$ & 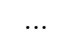 & 0.01 \\
\hline J0920+4441 & $0917+449$ & & A & 092058.458486 & +444153.98499 & 2.1890 & $\mathrm{Q}$ & 18.1 & 1.89 \\
\hline J0925+3612 & $0922+364$ & & A & 092551.851387 & +361235.67435 & 1.0150 & U & 19.3 & 0.12 \\
\hline J0927+3902 & $0923+392$ & $4 \mathrm{C} 39.25$ & $\mathrm{C}$ & 092703.013934 & +3902 20.85186 & 0.6950 & Q & 17.9 & \\
\hline J0937+5008 & $0933+503$ & & $\mathrm{C}$ & $0937 \quad 12.327300$ & +500852.09700 & 0.2757 & G & 18.9 & 0.53 \\
\hline J0945+3534 & $0942+358$ & & A & 094538.120719 & +353455.08842 & 1.1283 & $\mathrm{Q}$ & 18.8 & 0.04 \\
\hline J0956+2515 & $0953+254$ & 90 & A & 095649.875378 & +251516.04976 & 0.7120 & $\mathrm{Q}$ & 17.5 & 1.03 \\
\hline J0957+5522 & $0954+556$ & & $\mathrm{C}$ & 095738.184500 & +552257.76800 & 0.8990 & B & 17.7 & 0.54 \\
\hline J0958+4725 & $0955+476$ & OK 492 & $\mathrm{C}$ & 095819.671640 & +472507.84244 & 1.8815 & $\mathrm{Q}$ & 18.8 & 0.37 \\
\hline $\mathrm{J} 1013+2449$ & $1011+250$ & & A & 101353.428771 & +244916.44062 & 1.6360 & $\mathrm{Q}$ & 15.4 & 0.11 \\
\hline J1018+3542 & $1015+359$ & B2 1015+35B & $\mathrm{C}$ & $1018 \quad 10.988093$ & +354239.44094 & 1.2280 & $\mathrm{Q}$ & 18.6 & 0.50 \\
\hline $\mathrm{J} 1033+4116$ & $1030+415$ & & $\mathrm{C}$ & 103303.707872 & +411606.23282 & 1.1185 & $\mathrm{Q}$ & 19.6 & 0.92 \\
\hline $\mathrm{J} 1033+6051$ & $1030+611$ & & $\mathrm{C}$ & 103351.428900 & +60 5107.33500 & 1.4010 & $\mathrm{Q}$ & 18.9 & 0.66 \\
\hline $\mathrm{J} 1038+4244$ & $1035+430$ & & $\mathrm{C}$ & $1038 \quad 18.190536$ & +424442.75990 & 0.3055 & $\mathrm{Q}$ & 18.4 & 0.15 \\
\hline $\mathrm{J} 1043+2408$ & $1040+244$ & B2 $1040+24 \mathrm{~A}$ & A & 104309.035778 & +240835.40922 & 0.5590 & $\mathrm{Q}$ & 17.7 & 0.92 \\
\hline $\mathrm{J} 1044+8054$ & $1039+811$ & & B & 104423.062546 & +805439.44301 & 1.2600 & $\mathrm{Q}$ & 16.5 & 1.08 \\
\hline $\mathrm{J} 1045+1735$ & $1042+178$ & & A & 104514.359788 & +173548.08359 & 0.9210 & $\mathrm{U}$ & 21.8 & 0.28 \\
\hline $\mathrm{J} 1058+8114$ & $1053+815$ & & B & $1058 \quad 11.535395$ & +81 1432.67511 & 0.7060 & Q & 18.5 & 0.76 \\
\hline $\mathrm{J} 1103+3014$ & $1100+305$ & & B & 110313.301905 & +301442.70196 & 0.3838 & G & 18.3 & 0.48 \\
\hline $\mathrm{J} 1130+3815$ & $1128+385$ & & B & 113053.282615 & +381518.54688 & 1.7330 & $\mathrm{Q}$ & 18.6 & 1.00 \\
\hline J1146+3958 & $1144+402$ & & B & 114658.297920 & +3958 34.30434 & 1.0880 & $\mathrm{Q}$ & 18.0 & 1.60 \\
\hline $\mathrm{J} 1152+4939$ & $1149+499$ & & B & 115232.871056 & +493938.76785 & 1.0931 & $\mathrm{Q}$ & 16.6 & 0.12 \\
\hline $\mathrm{J} 1153+4931$ & $1150+497$ & $4 \mathrm{C} 49.22$ & B & 115324.466600 & +493108.83000 & 0.3334 & $\mathrm{Q}$ & 17.1 & 2.30 \\
\hline $\mathrm{J} 1153+8058$ & $1150+812$ & & B & 115312.499212 & +80 5829.15456 & 1.2500 & $\mathrm{Q}$ & 18.5 & 0.41 \\
\hline $\mathrm{J} 1200+5300$ & $1157+532$ & & $\mathrm{C}$ & 120011.384309 & +530046.87708 & 1.9970 & $\mathrm{Q}$ & 19.7 & 0.08 \\
\hline $\mathrm{J} 1203+4803$ & $1200+483$ & & $\mathrm{C}$ & 120329.853038 & +480313.62592 & 0.8133 & G & 16.4 & 0.34 \\
\hline $\mathrm{J} 1219+6344$ & $1216+640$ & & $\mathrm{C}$ & 121910.583100 & +634410.71688 & $\ldots$ & $\mathrm{U}$ & 22.5 & 0.03 \\
\hline $\mathrm{J} 1224+2122$ & $1222+216$ & 4C 21.35 & A & 122454.458398 & +212246.38854 & 0.4335 & $\mathrm{Q}$ & 17.5 & $\ldots$ \\
\hline $\mathrm{J} 1229+0203$ & $1226+023$ & $3 \mathrm{C} 273 \mathrm{~B}$ & A,B & 122906.699700 & +020308.59800 & 0.1583 & B & 12.9 & 13.81 \\
\hline $\mathrm{J} 1241+5458$ & $1239+552$ & & $\mathrm{C}$ & 124127.703846 & +5458 19.05549 & $\cdots$ & $\mathrm{U}$ & 22.1 & $\cdots$ \\
\hline J1256-0547 & $1253-055$ & 3C 279 & B & $1256 \quad 11.166500$ & -054721.52500 & 0.5362 & $\mathrm{Q}$ & 15.2 & 22.96 \\
\hline $\mathrm{J} 1259+5140$ & $1257+515$ & & $\mathrm{C}$ & 125931.173983 & +514056.26083 & & $\mathrm{U}$ & 19.9 & 0.29 \\
\hline J1306+5529 & $1303+557$ & & $\mathrm{C}$ & 130603.351107 & +552943.85746 & 1.6000 & G & 17.5 & 0.08 \\
\hline $\mathrm{J} 1310+3220$ & $1308+326$ & OP 313 & B & 131028.663800 & +32 2043.78200 & 0.9970 & Q & 19.0 & 1.37 \\
\hline $\mathrm{J} 1327+2210$ & $1324+224$ & B2 $1324+22$ & A & 132700.861314 & +221050.16277 & 1.4034 & $\mathrm{Q}$ & 17.2 & 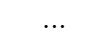 \\
\hline J1329+3154 & $1327+321$ & & A & 132952.864909 & +315411.05443 & 0.3350 & B & 20.0 & 0.38 \\
\hline $\mathrm{J} 1341+2816$ & $1338+285$ & & A & $1341 \quad 15.282755$ & +281605.07702 & 1.2750 & $\mathrm{Q}$ & 19.6 & 0.14 \\
\hline $\mathrm{J} 1353+7532$ & $1352+757$ & & B & 135323.168007 & $\begin{array}{l}+753257.73527 \\
\end{array}$ & 1.6190 & Q & 17.7 & 0.13 \\
\hline
\end{tabular}


TABLE 4.4: List of sources (continued)

\begin{tabular}{|c|c|c|c|c|c|c|c|c|c|}
\hline $\begin{array}{c}\text { Source (J2000) } \\
(1) \\
\end{array}$ & $\begin{array}{c}\text { Source (B1950) } \\
(2) \\
\end{array}$ & $\begin{array}{c}\text { Common Name } \\
(3)\end{array}$ & $\begin{array}{c}\text { Epoch } \\
(4)\end{array}$ & $\begin{array}{c}\alpha_{2000} \\
(5)\end{array}$ & $\begin{array}{c}\delta_{2000} \\
(6)\end{array}$ & $\begin{array}{c}z \\
(7) \\
\end{array}$ & $\begin{array}{c}\text { Type } \\
(8)\end{array}$ & $\begin{array}{c}m_{\mathrm{v}} \\
(9)\end{array}$ & $\begin{array}{c}S_{86 \mathrm{GHz}} \\
(10)\end{array}$ \\
\hline $\mathrm{J} 1357+7643$ & $1357+769$ & & B & 135755.371536 & +764321.05098 & 1.5850 & Q & 19.0 & 0.31 \\
\hline $\mathrm{J} 1407+2827$ & $1404+286$ & OQ 208 & $\mathrm{~A}$ & 140700.394415 & +282714.69008 & 0.0766 & $\mathrm{G}$ & 16.0 & 0.02 \\
\hline $\mathrm{J} 1419+3821$ & $1417+385$ & & $\mathrm{~A}$ & 141946.613761 & +382148.47496 & 1.8320 & $\mathrm{Q}$ & 19.3 & 0.49 \\
\hline $\mathrm{J} 1506+4239$ & $1505+428$ & & $\mathrm{~A}$ & 150653.041839 & +423923.03555 & 0.5870 & $\mathrm{Q}$ & 21.5 & 0.56 \\
\hline $\mathrm{J} 1506+8319$ & $1510+835$ & & B & 150624.715438 & +831928.03538 & 2.5770 & Q & 18.7 & 0.11 \\
\hline $\mathrm{J} 1521+4336$ & $1520+437$ & & $\mathrm{~A}$ & 152149.613879 & +433639.26807 & 2.1750 & Q & 18.4 & 0.37 \\
\hline $\mathrm{J} 1549+5038$ & $1547+507$ & & $\mathrm{C}$ & 154917.468558 & +503805.78820 & 2.1741 & $\mathrm{Q}$ & 18.9 & 0.31 \\
\hline $\mathrm{J} 1608+4012$ & $1606+403$ & 5C 13.225 & B & 160822.157698 & +401217.83288 & 0.6275 & Q & 20.8 & 0.46 \\
\hline $\mathrm{J} 1624+5741$ & $1623+578$ & & $\mathrm{C}$ & 162424.807566 & +574116.28100 & 0.7890 & G & 17.3 & 0.32 \\
\hline $\mathrm{J} 1635+3808$ & $1633+382$ & $4 \mathrm{C} 38.41$ & $\mathrm{C}$ & 163515.492900 & +380804.50000 & 1.8130 & Q & 17.7 & 2.65 \\
\hline $\mathrm{J} 1637+4717$ & $1636+473$ & $4 \mathrm{C} 47.44$ & $\mathrm{~B}$ & 163745.130554 & +471733.83090 & 0.7350 & $\mathrm{Q}$ & 17.5 & 1.01 \\
\hline $\mathrm{J} 1640+3946$ & $1638+398$ & & B & 164029.632770 & +394646.02835 & 1.6660 & Q & 18.5 & 1.00 \\
\hline $\mathrm{J} 1642+3948$ & $1641+399$ & $3 \mathrm{C} 345$ & A,B & 164258.809900 & +394836.99300 & 0.5950 & $\mathrm{~B}$ & 16.5 & 4.13 \\
\hline $\mathrm{J} 1646+4059$ & $1645+410$ & & $\mathrm{~B}$ & 164656.858690 & +405917.17204 & 0.8350 & Q & 20.7 & 0.16 \\
\hline $\mathrm{J} 1653+3107$ & $1651+312$ & & $\mathrm{~A}$ & 165329.910650 & +310756.87239 & 1.2954 & Q & 13.6 & 0.31 \\
\hline $\mathrm{J} 1659+2629$ & $1657+265$ & $4 \mathrm{C} 26.51$ & A & 165924.149448 & +262936.94297 & 0.7947 & $\mathrm{Q}$ & 18.0 & 0.35 \\
\hline $\mathrm{J} 1701+3954$ & $1659+399$ & & A & 170124.634812 & +395437.09149 & 0.5071 & $\mathrm{~B}$ & 17.4 & 0.30 \\
\hline $\mathrm{J} 1716+6836$ & $1716+686$ & & $\mathrm{C}$ & 171613.938009 & +683638.74465 & 0.7770 & Q & 18.5 & 0.36 \\
\hline $\mathrm{J} 1726+3213$ & $1724+322$ & & A & 172635.124676 & +321323.02210 & 1.0900 & G & 16.0 & 0.12 \\
\hline $\mathrm{J} 1735+5049$ & $1734+508$ & & $\mathrm{~A}$ & 173549.005166 & +504911.56578 & 0.8350 & $\mathrm{G}$ & 23.1 & 0.22 \\
\hline $\mathrm{J} 1746+6226$ & $1745+624$ & $4 \mathrm{C} 62.29$ & B & 174614.034133 & +622654.73830 & 3.8890 & Q & 19.5 & 0.08 \\
\hline $\mathrm{J} 1751+2920$ & $1749+293$ & & $\mathrm{~A}$ & 175142.683934 & +292050.20228 & $\ldots$ & $\mathrm{U}$ & $\ldots$ & 0.44 \\
\hline $\mathrm{J} 1753+2848$ & $1751+288$ & & A & 175342.473647 & +284804.93877 & 1.1180 & Q & 19.6 & 0.35 \\
\hline $\mathrm{J} 1800+3848$ & $1758+388$ & & $\mathrm{~A}$ & 180024.765361 & +384830.69742 & 2.0920 & Q & 18.0 & 0.30 \\
\hline $\mathrm{J} 1800+7828$ & $1803+784$ & & $\mathrm{C}$ & 180045.683900 & +782804.01800 & 0.6797 & $\mathrm{~B}$ & 17.0 & 2.36 \\
\hline $\mathrm{J} 1808+4542$ & $1806+456$ & & $\mathrm{C}$ & 180821.885884 & +454220.86639 & 0.8300 & Q & 19.3 & 0.44 \\
\hline $\mathrm{J} 1811+1704$ & $1809+170$ & & A & 181143.183465 & +170457.25713 & & Q & 20.5 & 0.42 \\
\hline $\mathrm{J} 1821+6818$ & $1822+682$ & & $\mathrm{~B}, \mathrm{C}$ & 182159.491723 & +681843.00867 & 1.6920 & $\mathrm{G}$ & $\ldots$ & 0.29 \\
\hline $\mathrm{J} 1823+6857$ & $1823+689$ & & B & 182332.853905 & +685752.61247 & $\ldots$ & $\mathrm{B}$ & 19.0 & 0.10 \\
\hline $\mathrm{J} 1849+6705$ & $1849+670$ & & B & 184916.072283 & +670541.68022 & 0.6570 & $\mathrm{Q}$ & 18.6 & 2.70 \\
\hline $\mathrm{J} 1850+2825$ & $1848+283$ & & $\mathrm{~A}$ & 185027.589824 & +282513.15527 & 2.5600 & $\mathrm{Q}$ & 17.1 & 0.22 \\
\hline $\mathrm{J} 1922+1530$ & $1920+154$ & & A & 192234.699313 & +153010.03193 & $\ldots$ & $\mathrm{U}$ & $\ldots$ & 0.16 \\
\hline $\mathrm{J} 1927+6117$ & $1926+611$ & & $\mathrm{C}$ & 192730.442616 & +611732.87881 & 0.4730 & $\mathrm{~B}$ & 17.5 & 0.70 \\
\hline $\mathrm{J} 1930+1532$ & $1928+154$ & $4 \mathrm{C} 15.66$ & A & 193052.766983 & +153234.42704 & & $\mathrm{U}$ & $\ldots$ & 0.40 \\
\hline $\mathrm{J} 1933+6540$ & $1933+655$ & & $\mathrm{C}$ & 193357.337206 & +654016.82845 & 1.6870 & $\mathrm{Q}$ & 18.7 & 0.13 \\
\hline $\mathrm{J} 1939+3817$ & $1937+381$ & & B & 193933.566865 & +38 1735.38833 & $\ldots$ & $\mathrm{U}$ & 17.5 & 0.06 \\
\hline $\mathrm{J} 1955+5131$ & $1954+513$ & OV 591 & B & 195542.738264 & +513148.54602 & 1.2230 & Q & 18.5 & 0.69 \\
\hline $\mathrm{J} 2002+1501$ & $2000+148$ & & $\mathrm{C}$ & 200241.999236 & +150114.57396 & $\ldots$ & $\mathrm{U}$ & $\ldots$ & 0.24 \\
\hline $\mathrm{J} 2002+4725$ & $2000+472$ & & B & 200210.418256 & +472528.77372 & 2.2660 & Q & 19.6 & 0.23 \\
\hline $\mathrm{J} 2007+3722$ & $2005+372$ & & B & 200745.397822 & +372202.31078 & $\ldots$ & $\mathrm{U}$ & $\ldots$ & 0.16 \\
\hline $\mathrm{J} 2012+4628$ & $2010+463$ & $7 \mathrm{C} 2010+4619$ & $\mathrm{C}$ & 201205.637418 & +462855.77715 & $\ldots$ & $\mathrm{B}$ & 18.2 & 0.49 \\
\hline $\mathrm{J} 2015+3410$ & $2013+340$ & & $\mathrm{C}$ & 201528.831879 & +34 1039.40992 & $\ldots$ & $\mathrm{B}$ & $\ldots$ & 0.07 \\
\hline $\mathrm{J} 2015+3710$ & $2013+370$ & & $\mathrm{~B}, \mathrm{C}$ & 201528.729778 & +371059.51495 & 0.8590 & $\mathrm{~B}$ & 21.8 & 3.07 \\
\hline $\mathrm{J} 2016+1632$ & $2013+163$ & & $\mathrm{C}$ & 201613.860029 & +163234.11306 & $\ldots$ & $\mathrm{B}$ & 1.0 & 0.27 \\
\hline $\mathrm{J} 2016+3600$ & $2014+358$ & & A & 201645.618743 & +360033.37332 & $\ldots$ & $\mathrm{U}$ & $\ldots$ & 0.15 \\
\hline $\mathrm{J} 2017+7440$ & $2017+745$ & $4 \mathrm{C} 74.25$ & A & 201713.079301 & +744047.99989 & 2.1870 & Q & 18.3 & 0.24 \\
\hline J2022+6136 & $2021+614$ & OW 637 & $\mathrm{~A}$ & 202206.681758 & +613658.80486 & 0.2270 & $\mathrm{G}$ & 19.5 & 0.41 \\
\hline $\mathrm{J} 2022+7611$ & $2023+760$ & & A & 202235.575939 & +761126.17158 & 0.5940 & B & 17.8 & 0.82 \\
\hline $\mathrm{J} 2024+1718$ & $2022+171$ & & $\mathrm{C}$ & 202456.563449 & +1718 13.19767 & 1.0500 & Q & 17.5 & 0.16 \\
\hline $\mathrm{J} 2051+1743$ & $2049+175$ & & $\mathrm{C}$ & 205135.582921 & +174336.90076 & 0.1950 & $\mathrm{G}$ & 18.0 & 0.55 \\
\hline $\mathrm{J} 2109+3532$ & $2107+353$ & B2 $2107+35 \mathrm{~A}$ & $\mathrm{~A}$ & 210931.878718 & +353257.59746 & 0.2023 & $\mathrm{G}$ & 13.9 & 0.51 \\
\hline $\mathrm{J} 2114+4634$ & $2112+463$ & & A & 211432.875692 & +463439.30126 & $\ldots$ & $\mathrm{U}$ & $\ldots$ & 0.07 \\
\hline $\mathrm{J} 2137+5101$ & $2135+508$ & & A & 213700.986190 & +510136.12894 & $\ldots$ & $\mathrm{U}$ & $\ldots$ & 0.19 \\
\hline $\mathrm{J} 2154+1727$ & $2152+172$ & & $\mathrm{C}$ & 215440.900400 & +172750.79300 & 1.0200 & Q & 18.0 & 0.13 \\
\hline $\mathrm{J} 2202+4216$ & $2200+420$ & BL Lac & $\mathrm{A}, \mathrm{C}$ & $2202 \quad 43.291371$ & +421639.97992 & 0.0686 & $\mathrm{~B}$ & 14.5 & $\ldots$ \\
\hline $\mathrm{J} 2203+1725$ & $2201+171$ & & $\mathrm{C}$ & 220326.893600 & +172548.24700 & 1.0760 & Q & 18.8 & 1.08 \\
\hline $\mathrm{J} 2203+3145$ & $2201+315$ & $4 \mathrm{C} 31.63$ & $\mathrm{C}$ & 220314.975700 & +314538.26900 & 0.2950 & Q & 15.5 & 1.56 \\
\hline $\mathrm{J} 2210+2013$ & $2208+199$ & & $\mathrm{~A}$ & 221051.652453 & +201324.05454 & 0.2820 & Q & $\ldots$ & 0.48 \\
\hline $\mathrm{J} 2212+2355$ & $2209+236$ & & $\mathrm{~A}$ & 221205.966311 & +235540.54372 & 1.1250 & Q & 19.0 & 0.34 \\
\hline $\mathrm{J} 2217+2421$ & $2214+241$ & & $\mathrm{C}$ & 221700.821100 & +242145.95700 & 0.5050 & $\mathrm{~B}$ & 17.2 & 0.55 \\
\hline $\mathrm{J} 2218+1520$ & $2215+150$ & & $\mathrm{~A}$ & 221810.913904 & +152035.71746 & 2.3350 & Q & $\ldots$ & 0.35 \\
\hline $\mathrm{J} 2253+1608$ & $2251+158$ & $3 \mathrm{C} 454.3$ & $\mathrm{~A}, \mathrm{~B}, \mathrm{C}$ & 225357.747937 & +160853.56094 & 0.8590 & $\mathrm{~B}$ & 16.1 & 17.37 \\
\hline $\mathrm{J} 2305+8242$ & $2304+824$ & & A & 230517.539999 & +824249.15557 & 0.6200 & B & $\ldots$ & 0.25 \\
\hline $\mathrm{J} 2311+4543$ & $2309+454$ & & $\mathrm{~A}$ & 231147.408964 & +454356.01658 & 1.4470 & Q & $\ldots$ & 0.36 \\
\hline $\mathrm{J} 2312+7241$ & $2310+724$ & & $\mathrm{C}$ & 231219.697802 & +724126.91735 & $\ldots$ & $\mathrm{U}$ & 19.3 & 0.13 \\
\hline $\mathrm{J} 2322+4445$ & $2319+444$ & & A & 232220.358080 & +444542.35353 & 1.3100 & $\mathrm{U}$ & 20.7 & 0.23 \\
\hline $\mathrm{J} 2322+5057$ & $2320+506$ & & $\mathrm{~A}$ & $2322 \quad 25.982171$ & +505751.96363 & 1.2790 & Q & 18.6 & 0.59 \\
\hline $\mathrm{J} 2330+3348$ & $2327+335$ & & $\mathrm{C}$ & 233013.737652 & +334836.47152 & 1.8090 & Q & 18.5 & 0.22 \\
\hline
\end{tabular}


TABLE 4.4: List of sources (continued)

\begin{tabular}{|c|c|c|c|c|c|c|c|c|c|}
\hline $\begin{array}{c}\text { Source (J2000) } \\
\text { (1) }\end{array}$ & $\begin{array}{c}\text { Source (B1950) } \\
\text { (2) }\end{array}$ & $\begin{array}{c}\text { Common Name } \\
\text { (3) }\end{array}$ & $\begin{array}{l}\text { Epoch } \\
\text { (4) }\end{array}$ & $\begin{array}{c}\alpha_{2000} \\
(5)\end{array}$ & $\begin{array}{c}\delta_{2000} \\
(6)\end{array}$ & $\begin{array}{c}z \\
(7)\end{array}$ & $\begin{array}{l}\text { Type } \\
(8)\end{array}$ & $\begin{array}{l}m_{\mathrm{v}} \\
(9)\end{array}$ & $\begin{array}{l}S_{86 \mathrm{GHz}} \\
(10)\end{array}$ \\
\hline 70254 & 2351 & $4 \mathrm{C} 45.51$ & $\mathrm{~A}$ & 235421.68021 & -455304.23640 & 1.9860 & Q & 20.6 & 0.31 \\
\hline
\end{tabular}

Columns: 1 - IAU Source name (J2000); 2 - IAU Source name (B1950); 3 - common name; 4 - observing epochs - A: October 2010; B: May 2011 and C: October 2011; 5,6 - source coordinates in J2000 epoch: right ascension and declination; 7 - redshift; 8 - optical class - Q: quasar; B: BL Lac object; G: Galaxy; U: Unidentified source; 9 - optical magnitude (information for Columns 7,8,9 obtained from the NASA/IPAC Extragalactic Database (https://ned.ipac.caltech.edu), Simbad Astronomical Database ( http://simbad.ustrasbg.fr/simbad; Wenger et al. 2000) and Sloan Digital Sky Survey (http://www.sdss.org/); 10 - total single dish flux density measured at $86 \mathrm{GHz}$ obtained from the pointing and calibration scan measurements at Pico Veleta or Plateau de Bure. (This table is also available in a machine-readable and Virtual Observatory (VO) forms in the online journal of Nair et al. (2019).)

\subsection{Data Processing}

In this section, the data reduction of the $3 \mathrm{~mm}-\mathrm{VLBI}$ survey is discussed. The data were correlated at the DiFX correlator (Deller et al. 2011) of the Max-Planck-Institut für Radioastronomie (MPIfR) at Bonn. After correlation, the data were loaded into AIPS (Astronomical Image Processing System; Greisen 1990). This is done by loading the original uv FITS file using the task FITLD in AIPS. Before proceeding for calibration of data, the data was checked for subarrays. Subarrays were present in the first and third epoch. So after creating the first calibration table, the task USUBA was run for indexing the subarrays properly. Since the survey consists of many weak sources, the sources were scheduled in the survey in such a way that each block of four sources consists of at least one bright source which can be later used for extending the phase solutions from brighter sources to nearby weak sources.

\subsubsection{Parallactic Angle Correction}

Since all the antennas participating in our survey are in Alt-Azimuth mount, the instrumental phases vary due to changing orientation of the antenna feed during the observations. With an equatorial mount, the telescope has only one rotational axis parallel to the earth's axis of rotation and it can follow celestial objects in diurnal motion easily. This is done by counter rotation of the telescope by a certain angle to cancel the effect of earth's rotation. But, in the case of Alt-Azimuth mount, the radio telescope must be simultaneously moved along two different axes, thereby changing the orientation of its feed with respect to the object tracked. Therefore, a parallactic angle correction is important for all the Alt-Azimuth mount telescopes participated in this $3 \mathrm{~mm}$ survey. This was done by the task CLCOR in AIPS. This was done before phase calibration. Pico Veleta has a Nasmyth left mount which was corrected using task TABED before parallactic angle correction.

\subsubsection{Fringe Fitting the Data with "FRING"}

Even after applying the correlator model and possible corrections during correlation, there is still residual phase errors between the Intermediate Frequencies (IFs, see section 3.2.3). 
There can be gradient of phase in frequency that is the gradient within each IF (single band delay) and the gradient between IFs (multi-band delay). This happens due to errors in the correlator model representing the overall geometry of the telescope array. In addition to this, a variety of factors (atmospheric and ionospheric delays, clock errors, variable electronic delays and others) can affect the instrumental phase at each participating antenna. As a result, the phase as well as the amplitude of the signal varies as a result of this and in $3 \mathrm{~mm}$ VLBI observations, the phases can vary on timescales of the order of $<20$ sec. Since the phase as well as amplitude remains coherent for such a short time, it is important to look scan by scan (using task POSSM in AIPS) to check whether fringe is detected. The phase varies not only as a function of frequency but also in time. This time dependent phase rates, is equivalent to the residual fringe rate. The main task of fringe fitting using AIPS task FRING is therefore, to remove the frequency dependent as well as time dependent phase errors and line up the phases in all the IFs.

\subsubsection{Manual Phase Calibration}

At the first step of the fringe fitting, manual phase calibration is done. In this process, we generated 'manual-phasecals' to line up the data from IF to IF. A scan on a strong source that give high signal-to-noise ratio (SNR) fringe solutions (the residuals, single-band delay, multi-band delay and rate) over all antennas is selected and the data is fringe fitted with a high SNR threshold (e.g. $\geq 7$ ) for a solution interval much greater than the typical coherence time $(<20 \mathrm{sec})$ at $86 \mathrm{GHz}$. Usually, if such a scan is not present, then select another strong scan where the solutions of the missing antennas are present (including reference antenna). Then we have to apply all these solutions for different antenna to the calibration table. The resulting fringe fit solutions were applied to other sources using the task CLCAL in AIPS. Before starting with manual phase calibration, a quick single-IF global fringe fitting is done using the task FRING. This step is done to see where the good SNRs for different antennas are present in time. The SNR set for this quick fringe fit should be relatively high (e.g. > 5).

\subsubsection{Reference Antenna and Solution Interval}

The reference antenna should be one of the most sensitive antenna in the VLBI stations we have and the antenna which is located at the center of the array by principle. The reference antenna selected to find fringes in the case of VLBA stations was Pie Town. Then, in order to connect VLBA stations with European stations, a single strong scan was selected between Pie Town and a European station (usually Plateau de Bure or Pico Veleta, since they are very sensitive) and fringe fit the scan with the CL table which contains the phase solutions from VLBA antennas. Then, for the remaining European stations, Plateau deBure (or Pico Veleta) was used as the reference antenna and the solution was applied to the CL table which contains the solutions to VLBA +1 European station (VLBA + PdB or Pv here).

Because of the rapid phase changes, the solution interval for the fringe fitting should be greater than the fluctuations time scale. In order to determine the appropriate solution interval, those strong scans selected for manual-phasecal were fringe fitted with different trial solution intervals starting from 0.33 minutes $(\sim 20 \mathrm{sec})$ to the full length of scan and the appropriate solution interval which gives high SNR solutions as well as coherence in 
phases were determined. It was clearly seen that, as solution interval increases, the SNR also increases. So, we kept a solution interval equal to the full length of the scan for the subsequent fringe fits.

\subsubsection{Global Fringe Fitting}

After the manual phase calibration, antennas-based global fringe fitting (Schwab \& Cotton 1983; Alef \& Porcas 1986) was performed, setting the solution interval to the full scan length in order to improve the detection. Assuming the single-band delays in each IF during a global fringe fit are the same, the difference between the model phases and measured phases is reduced by solving for the antenna-based instrumental phase, rate, and delay difference between single- and multi-band delays for each antenna. To minimize the chance of false detections, the data were fringe fitted with an SNR threshold of 5 and a search window width of $200 \mathrm{nsec}$ for the fringe delay and $400 \mathrm{mHz}$ for the fringe rate. Then the data were fringe fitted again with an SNR threshold of 4.3 by tighter windows of 100 nsec for the fringe delay and $100 \mathrm{mHz}$ for the fringe rate.

In global fringe fitting, all the baselines were jointly used to estimate the antenna phase, delay and rate relative to a reference antenna. The baseline phase error equation was solved with one of the antennas set to the reference antenna. The delay, rate and phase residuals for reference antenna were set to zero. Pico Veleta was used as the reference antenna for almost all the data of this survey. Whenever Pico Veleta was not present in the data, Plateau de Bure or Pie Town were used as the reference antennas. Since this fringe search method uses only a single baseline, it is important to set secondary reference antennas as well. If the fringe search with the primary reference antenna cannot meet the SNR threshold or if a specific antenna cannot find a path to the primary reference antenna, then the secondary reference antennas could solve it. The secondary antennas were selected after trials with all antennas and deciding which antennas give the best SNR (Alef \& Porcas 1986). The secondary antennas used were Efflesberg, Fort Davis, Kitt Peak, Onsala and Plateau de Bure.

Once the global fringe fit was done, the SNR of the fringe solutions were inspected for all the sources, and strong sources which give relatively very high SNR were determined. Whenever feasible, the solutions from those strong sources were interpolated to nearby weak sources, using a procedure similar to that adopted in earlier VLBI survey observations at $86 \mathrm{GHz}$ (cf., Lee et al. 2008). Application of the interpolated solutions lead to a further improved detection rate for all of the survey targets.

\subsubsection{Amplitude Calibration}

A priori amplitude calibration was done using the measured values of antenna gain, and system temperature stored in the TY and GC tables in AIPS, using the task APCAL in AIPS. The antab files for system temperature $T_{\text {sys }}$ and antenna gain were prepared outside AIPS and loaded to AIPS using the task ANTAB. Opacity corrections were also done for antennas where ever required. The opacity corrections were done using the information on $T_{\text {sys }}$ from the TY table and weather information from the WX table. The task APCAL was started by an initial guess value for the zenith opacity (TAU0) and receiver temperatures (TRECVR) which was set as 0.1 and $100 \mathrm{~K}$, respectively. After this, a second round of APCAL is done. In this step, the fitted values of opacity and receiver temperatures for 
each antenna were used as initial values for fitting simultaneously for zenith opacity $\tau$ and receiver temperature $T_{\text {rec }}$ again. A detailed discussion on how we did amplitude calibration is already done in section 3.3.2. By the second APCAL, the fit converges and give more appropriate values for the receiver temperature and $\tau$. The SN table produced by the second APCAL was applied on the calibration table.

The accuracy and self-consistency of the amplitude calibration was checked with a procedure developed and used in the earlier $86 \mathrm{GHz}$ VLBI survey experiments (Lobanov et al. 2000; Lee et al. 2008). The calibrated visibility data were model fitted for each of the survey targets, using circular Gaussian components and allowing for scaling the individual antenna gains by a constant factor. The resulting average gain scale corrections are listed in Table 4.5. The scale offsets are within $20 \%$ for most of the antennas, except Metsähovi which had suffered from persistently bad weather during each of the three observing sessions. The averaged offsets are within about $3 \%$ of the unity implying that there is no significant bias in the calibration and their root mean square (r.m.s) is less than $10 \%$, for each of the three observing epochs. Based on this analysis, we conclude that the a priori amplitude calibration should be accurate to about $25 \%$, providing a sufficient initial calibration accuracy for the hybrid mapping of the source structure. After phase and amplitude calibration, the data are split into single source files and saved as FITS uv files for further analysis using the task SPLIT in AIPS. The task FITTP was executed to write data to disk for imaging. The whole AIPS file data were saved into archive at this stage by the task FITAB.

TABle 4.5: Average Antenna Gain Corrections

\begin{tabular}{lccc}
\hline \hline $\begin{array}{l}\text { Telescope } \\
(1)\end{array}$ & $\begin{array}{c}\text { Epoch A } \\
(2)\end{array}$ & $\begin{array}{c}\text { Epoch B } \\
(3)\end{array}$ & $\begin{array}{c}\text { Epoch C } \\
(4)\end{array}$ \\
\hline $\mathrm{Br}$ & $1.008 \pm 0.406$ & $1.054 \pm 0.137$ & $1.010 \pm 0.192$ \\
$\mathrm{~Eb}$ & $1.103 \pm 0.264$ & $1.028 \pm 0.224$ & $0.925 \pm 0.182$ \\
$\mathrm{Fd}$ & $0.992 \pm 0.206$ & $1.105 \pm 0.289$ & $0.958 \pm 0.162$ \\
$\mathrm{Kp}$ & $0.881 \pm 0.154$ & $0.933 \pm 0.104$ & $0.931 \pm 0.102$ \\
$\mathrm{La}$ & $0.819 \pm 0.199$ & $0.940 \pm 0.172$ & $0.987 \pm 0.133$ \\
$\mathrm{Mh}$ & $\ldots$ & $\ldots$ & $1.620 \pm 0.573$ \\
$\mathrm{Mk}$ & $0.919 \pm 0.256$ & $1.188 \pm 0.438$ & $0.844 \pm 0.199$ \\
$\mathrm{Nl}$ & $1.224 \pm 0.386$ & $1.190 \pm 0.296$ & $0.798 \pm 0.264$ \\
$\mathrm{On}$ & $0.934 \pm 0.292$ & $0.935 \pm 0.114$ & $0.889 \pm 0.147$ \\
$\mathrm{Ov}$ & $0.900 \pm 0.371$ & $0.868 \pm 0.148$ & $0.939 \pm 0.382$ \\
$\mathrm{~Pb}$ & $1.081 \pm 0.146$ & $1.087 \pm 0.225$ & $1.095 \pm 0.203$ \\
$\mathrm{Pt}$ & $0.841 \pm 0.113$ & $0.878 \pm 0.109$ & $1.041 \pm 0.098$ \\
$\mathrm{Pv}$ & $1.006 \pm 0.116$ & $1.088 \pm 0.220$ & $1.010 \pm 0.086$ \\
$\mathrm{Ys}$ & $\ldots$ & $\ldots$ & $1.009 \pm 0.196$ \\
Array average & $1.037 \pm 0.084$ & $0.991 \pm 0.074$ & $1.027 \pm 0.097$ \\
\hline
\end{tabular}

Columns: 1 - abbreviation for the participating telescope; 2,3,4 - average and r.m.s of antenna gains for observing epochs A, B, and C, respectively.

\subsection{Imaging}

After the phase and amplitude calibration on the data, the visibility data were averaged over $10 \mathrm{sec}$ for most of the sources and in some cases, the data was averaged over $30 \mathrm{sec}$. The data were then processed in the Caltech DIFMAP software package (Shepherd et al. 1994) for imaging. 

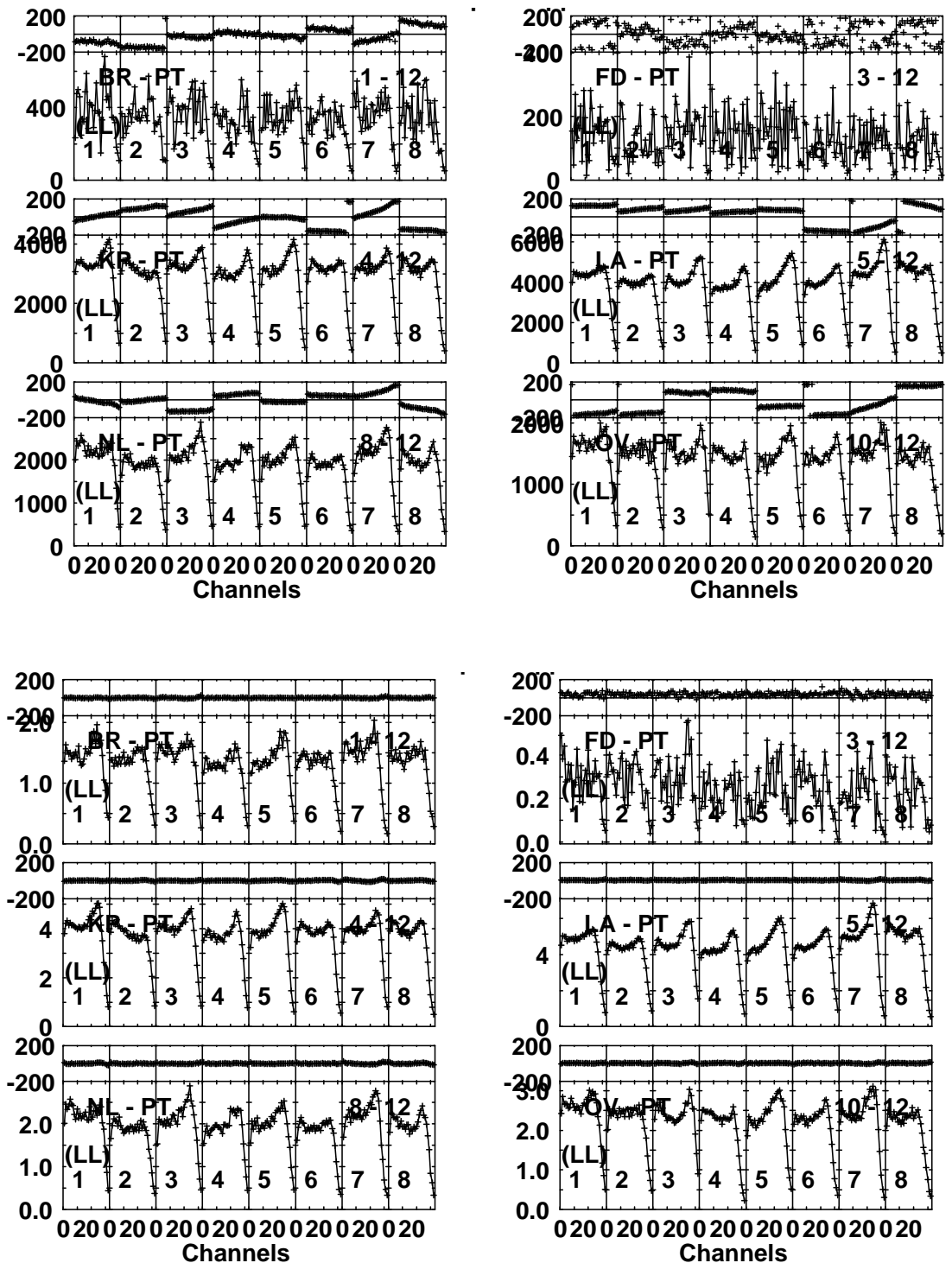

Figure 4.5: Amplitudes and phases of baseline visibilities between Pie Town $(\mathrm{Pt})$ and other VLBA antennas before (top panel) and after (bottom panel) fringe fitting and amplitude calibration for the source 3C279 in Epoch C. The phases are plotted against channels of each IF in degrees (from $-200^{\circ}$ to $+200^{\circ}$ ) in both top and bottom panels. The amplitude plotted against channels of each IF on top panel is in units of micro Jansky (before calibration), and on bottom panel is in units of Jansky (after calibration).

\subsubsection{Hybrid Mapping and Model Fitting}

The visibility data obtained from high frequency VLBI is not well sampled in the visibility domain and model fitting is a best way to deal with such data. So, in the first step, the visibility data for each source are model fitted with circular Gaussian patterns (model fitting; Fomalont 1999). The satisfactory Gaussian models were then used as starting models for hybrid mapping.

In the next step, hybrid mapping is implemented on the data. The hybrid mapping procedure comprised of the CLEAN deconvolution (Clark 1980) and self calibration (Cornwell \& Fomalont 1999; Cornwell 1995). For most of the objects, the hybrid mapping procedure was initiated with a point source model. For objects with sparse visibility data, the initial 
satisfactory Gaussian models obtained in the first step were used as the initial models. Only visibility phases were allowed to be modified during the initial iterations of the hybrid mapping. At the last step of hybrid mapping, a single, time constant antenna gain correction factor was applied to the visibility amplitudes (hence not allowing for time variable antenna gains in order to avoid the imprint of model errors into data). The data were not $u v$-tapered and the imaging was performed using the natural weighting in order to improve the sensitivity of the images. The parameters of the final images are listed in Table 4.6, together with the correlated flux densities measured on short and long baselines.

In order to check the effect of the amplitude (antenna gain) corrections applied during the final self calibration step, we compared the visibility amplitudes obtained without and with it. This was done by comparing the ratios of the visibility amplitudes obtained without $\left(S_{S}\right.$ and $\left.S_{L}\right)$ and with the antenna gain correction $\left(S_{S}^{\prime}\right.$ and $\left.S_{L}^{\prime}\right)$, on short $B_{\mathrm{S}}$ and long $B_{\mathrm{L}}$ baselines (see Table 4.6). The average of the ratios are found to be (1.24 \pm 0.18$)$ and (1.01 $\pm 0.14)$ for the short and long baselines, respectively. The distribution of these ratios are shown in figure 4.6. These ratios imply that the amplitude self-calibration did not introduce substantial gain corrections, thus further indicating the overall good quality of the a priori amplitude calibration of the survey data.
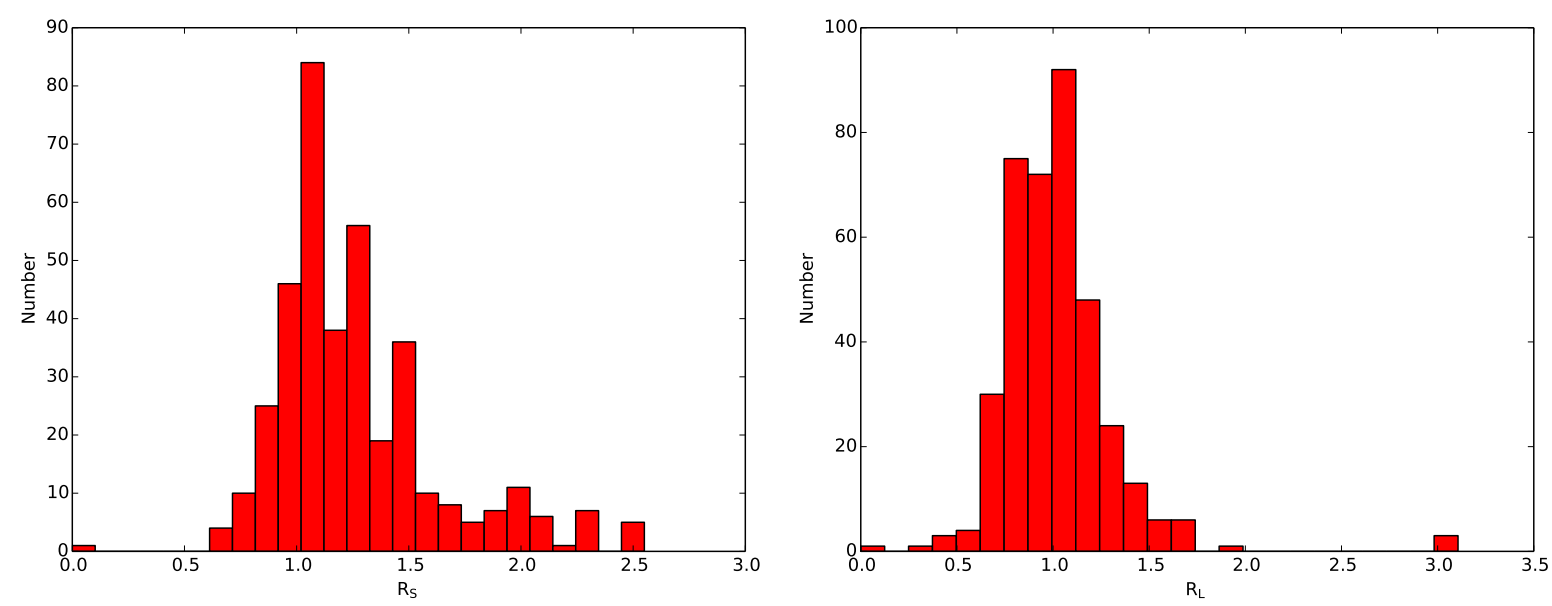

FIGURE 4.6: Distribution of the ratios of the visibility amplitudes obtained without and with the antenna gain correction, on short $B_{\mathrm{S}}\left(R_{S}=S_{S} / S_{S}^{\prime}\right)$ and long $B_{\mathrm{L}}\left(R_{L}=S_{L} / S_{L}^{\prime}\right)$ baselines, shown respectively, on the left and right panel. The median and mean of $R_{S}$ is 1.15 and 1.24 , respectively and the median and mean of $R_{L}$ is 0.99 and 1.01 , respectively.

The quality of the residual noise in the final clean image, which ideally should have a zeromean Gaussian distribution, was also checked by calculating the expectation value for the maximum absolute flux density $\left|S_{\mathrm{r}, \exp }\right|$ in a residual image,

$$
\left|S_{\mathrm{r}, \exp }\right|=\sigma_{\mathrm{r}} \sqrt{2} \ln \left(\frac{N_{\mathrm{pix}}}{\sqrt{2 \pi \sigma_{\mathrm{r}}}}\right)^{\frac{1}{2}},
$$

where $N_{\text {pix }}$ is the total number of pixels in the image (Lee et al. 2008). The quality of the residual noise is given by $\xi_{\mathrm{r}}=S_{\mathrm{r}} / S_{\mathrm{r} \text {,exp }}$ where $S_{\mathrm{r}}$ is the maximum flux density in the residual image and $\sigma_{\mathrm{r}}$ is the r.m.s noise in the residual image. When the residual noise approaches Gaussian noise, $\xi_{\mathrm{r}}$ tends to 1 . If $\xi_{\mathrm{r}}>1$, not all the structure has been adequately recovered; if $\xi_{\mathrm{r}}<1$, the image model has an excessive large number of degrees of freedom (Lobanov et al. 2006). Figure 4.7 shows the overall distribution of $\xi_{\mathrm{r}}$ for the residual maps of all the 
sources in this survey. Column 14 in Table 4.6 shows the quality factor, $\xi_{\text {r }}$ obtained for all the $3 \mathrm{~mm}$ images implying that the images adequately represent the source structure detected in the visibility data. The Gaussianity of the residual noise is also reflected in the median and the mean of the $\xi_{\text {r }}$ distributions which are within $10 \%$ of the unity factor.

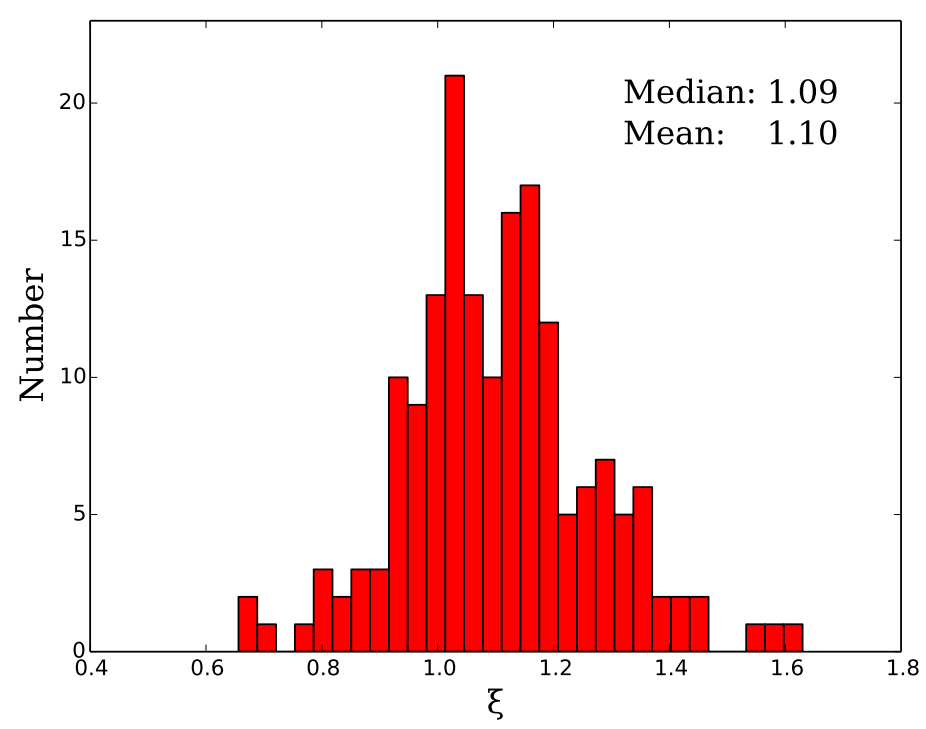

FiguRE 4.7: Distribution of the noise quality factor $\xi_{\mathrm{r}}$ for the residual images of all the sources in the survey.

In the last step, in order to extract the physical quantities out of the images, a final Gaussian model fitting was done on the self-calibrated data resulting from the hybrid mapping. Gaussian functions are convenient model components for source intensity, as they are always positive and vary smoothly with angle. The total number of Gaussian components used for model fitting a given source was determined using the $\chi^{2}$ statistics of the fits. The final models were obtained when the addition of another Gaussian component did not provide a statistically significant change of the $\chi^{2}$ agreement factors (Schinzel et al. 2012).

Once the final visibility data are represented by satisfactory single- or multi- component Gaussian components, then the image parameters i.e. the total flux density, the peak flux density, size and position of each component are obtained from the fits. Uncertainties in the models are calculated from equations 3.37-3.41. The fluxes of each components are obtained by removing each component out of the fitted image and investigating the residual image whose distribution reflects the Gaussian component. From the statistical information of pixels in the residual map, we also measured the off-source r.m.s noise. The overall noise in the images are less than $5 \mathrm{mJy} /$ beam. We use the total flux density $S_{\text {tot }}$ and size $d$ of the model fit components to estimate the brightness temperature, using equation 1.27 for the individual emitting regions in the jets. The measured image parameters and model fit parameters are given in section 4.5 .

\subsection{Results of the Survey}

In this section, we present the results on the measured image parameters, model fit parameters and $3 \mathrm{~mm}$ maps of the sources obtained from the global $86 \mathrm{GHz}$ VLBI survey 


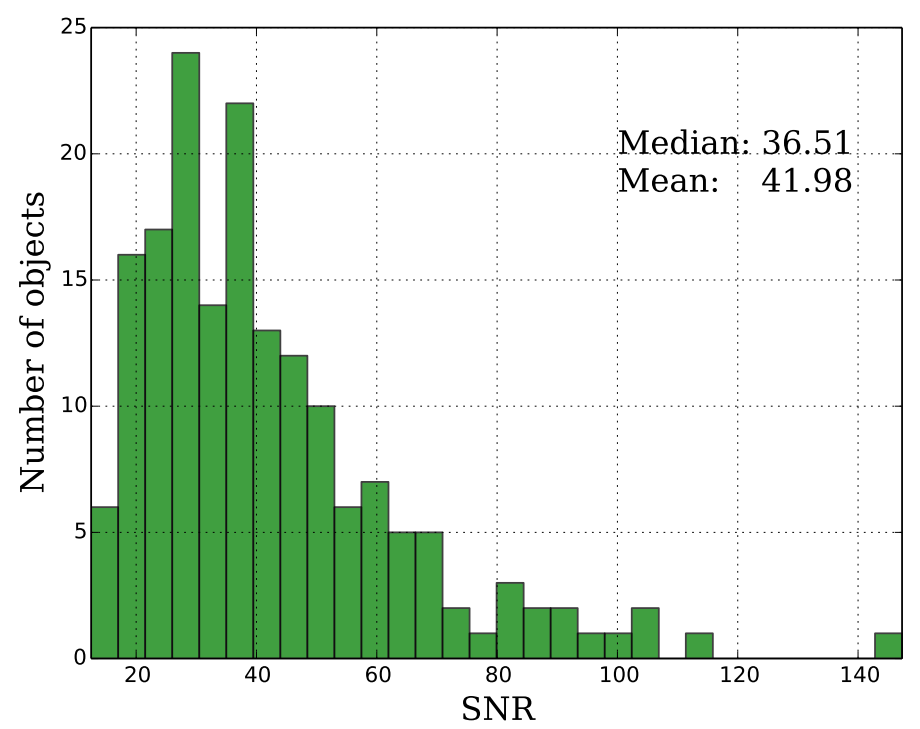

FIGURE 4.8: Distribution of the imaging signal-to-noise ratios (SNR) of all the sources in the survey calculated from the ratio of the peak flux density of the map, $S_{\mathrm{p}}$ to the r.m.s noise in the map, $\sigma_{\mathrm{rms}}$. The mean and median value of the SNR distribution are 42 and 36.5 , respectively.

of ultracompact radio sources. A total of 162 unique compact radio sources have been observed in this survey. All the sources are detected and imaged. Using the procedures described above, we have made hybrid maps of all 174 observations of 162 unique sources in this survey, with a typical image sensitivity of $5 \mathrm{mJy} /$ beam, signifying a nearly twofold increase in the total number of sources ever imaged with VLBI at $86 \mathrm{GHz}$. For 138 objects, the survey provides the first ever VLBI images made at $86 \mathrm{GHz}$. Most of the imaged sources show extended radio emission, revealing a jet morphology down to sub-parsec scales. For a small number of weaker sources, only the brightest core at the base of the jet could be imaged. The source compactness, model fit-based $T_{\mathrm{b}}$ estimates and visibilty based $T_{\mathrm{b}}$ estimates are also discussed in this section.

\subsubsection{Images}

In the set of figures from figure 4.9 to figure 4.52 , we present one contour map for each source at each epoch and two plots. The $3 \mathrm{~mm}$ maps of all of the survey observations are shown in the left panel. The respective radial amplitude distributions are shown in the right panel and $u v$-coverages of the respective visibility datasets are shown in inset on the right panel. In the left panel, the contour map of each source is shown with the $\mathrm{X}$ - and Y-axis in the units of milli arcsecond. The name and observing date of the source are put in the upper left corner of the map. The lowest contour level is also presented in the lower right corner of the map. The shaded ellipse represents the FWHM of the restoring beam in the image. The contours of each image is drawn at $3 \sigma_{\text {rms }} \times(-1,1, \sqrt{2}, 2, \ldots)$ levels, with $\sigma_{\text {rms }}$ representing the off-source r.m.s noise in the residual image of the source. The X-axis of the plot in the right panel of the visibility amplitude represents the $u v$-radius which is the length of the baseline used to obtain the corresponding visibility point. The $u v$-radius is given in the units of $10^{6} \lambda$, where $\lambda$ is the wavelength of the observing emission. The Y-axis of the plot shows the amplitude of each visibility point (i.e. correlated flux density) 
in units of Jy. The $u v$-sampling distribution in the inset in the right panel describes the overall distribution of the visibility in the $u v$-plane, whose maximum scale equals to that of $u v$-radius. Multi-epoch images are presented for sources observed more than once in the survey.

In Table 4.6, we present the basic parameters of the images, listing (1) source name, (2) observing epoch, (3) single dish $86 \mathrm{GHz}$ flux density, $S_{86}$, measured at Pico Veleta or Plateau de Bure, (4) correlated flux density on the shortest baseline, $S_{\mathrm{S}}$, (5) shortest baseline, $B_{\mathrm{S}}$, (6) correlated flux density on the longest baseline, $S_{\mathrm{L}},(7)$ longest baseline, $B_{\mathrm{L}}$, (8) major axis, $B_{\mathrm{a}}$, (9) minor axis, $B_{\mathrm{b}}$, and (10) position angle, $B_{\mathrm{PA}}$ of the major axis of the restoring beam, (11) total CLEAN flux density, $S_{\text {tot }}$, and (12) peak flux density, $S_{\text {peak }}$, in the image, (13) image r.m.s noise, $\sigma_{\mathrm{rms}}$, and (14) the quality factor of the residual noise in the image, $\xi_{\mathrm{r}}$.

Table 4.7 summarizes the model fits obtained for all of the survey sources providing (1) the source name, (2) observing epoch, (3) sequential number of the Gaussian component, (4) total flux density, $S_{\mathrm{t}}$, and (5) peak flux density, $S_{\text {peak }}$, of the component, (6) full width at half maximum (FWHM) size, $d$, of the component,(7) separation, $r$, and (8) position angle, $\theta$ of the component with respect to the brightest feature in the model (core, taken to be located at the coordinate origin), (9) brightness temperature, $T_{\mathrm{b} \text {,mod }}$, estimated from the model fit, (10) minimum, $T_{\mathrm{b} \text {, min }}$ and (11) limiting resolved, $T_{\mathrm{b}, \mathrm{lim}}$, brightness temperatures estimated from the visibility amplitudes (Lobanov 2015) at the longest baselines given in Column 7 in Table 4.6. Table 4.7 contains the model fit parameters for a total 174 VLBI cores and 205 jet components, with 42 and 37 of these unresolved, as reflected also in the lower limits of the model fit based brightness temperature estimates, $T_{\mathrm{b} \text {,mod }}$, listed for these components.

TABLE 4.6: Image Parameters

\begin{tabular}{|c|c|c|c|c|c|c|c|c|c|c|c|c|c|}
\hline $\begin{array}{l}\text { Source (J2000) } \\
(1)\end{array}$ & $\begin{array}{l}\text { Obs } \\
(2)\end{array}$ & $\begin{array}{l}S_{86 \mathrm{GHz}} \\
(3)\end{array}$ & $\begin{array}{l}S_{\mathrm{S}} \\
(4)\end{array}$ & $\begin{array}{l}B_{\mathrm{S}} \\
(5)\end{array}$ & $\begin{array}{l}S_{\mathrm{L}} \\
(6)\end{array}$ & $\begin{array}{l}B_{\mathrm{L}} \\
(7)\end{array}$ & $\begin{array}{l}B_{\mathrm{a}} \\
(8)\end{array}$ & $\begin{array}{l}B_{\mathrm{b}} \\
(9)\end{array}$ & $\begin{array}{l}B_{\mathrm{PA}} \\
(10) \\
\end{array}$ & $\begin{array}{l}S_{\mathrm{t}} \\
(11)\end{array}$ & $\begin{array}{l}S_{\mathrm{p}} \\
(12) \\
\end{array}$ & $\begin{array}{l}\sigma \\
(13) \\
\end{array}$ & $\begin{array}{l}\xi_{\mathrm{r}} \\
(14) \\
\end{array}$ \\
\hline J0013+4051 & $\mathrm{C}$ & 0.79 & $0.656 \pm 0.020$ & 56 & $0.192 \pm 0.006$ & 3136 & 159 & 35 & -16.8 & 436 & 317 & 8 & 1.43 \\
\hline $\mathrm{J} 0017+8135$ & B & 0.18 & $263 \pm 0.018$ & 60 & $075 \pm 0.004$ & 3062 & 74 & 35 & 2.1 & 143 & 81 & 2 & 1.36 \\
\hline $\mathrm{J} 0030+7037$ & B & 0.34 & $0.363 \pm 0.026$ & 65 & $0.115 \pm 0.009$ & 3070 & 76 & 36 & 66.5 & 155 & 92 & 3 & 1.34 \\
\hline J0034+2754 & $\mathrm{C}$ & 0.07 & $0.124 \pm 0.015$ & 54 & $0.156 \pm 0.021$ & 3086 & 262 & 36 & -14.2 & 159 & 60 & 2 & 1.04 \\
\hline $\mathrm{J} 0044+6803$ & B & 0.17 & $0.315 \pm 0.023$ & 65 & $0.097 \pm 0.006$ & 3034 & 75 & 36 & 70.4 & 133 & 99 & 3 & 1.15 \\
\hline J0046+2456 & $\mathrm{A}$ & 0.47 & $0.252 \pm 0.019$ & 59 & $0.137 \pm 0.009$ & 3060 & 267 & 37 & -15.0 & 235 & 160 & ; & 1.17 \\
\hline J0057+3021 & A & 0.48 & $361 \pm$ & 58 & $0.233 \pm 0.017$ & 3060 & 308 & 37 & -14.5 & 306 & 226 & 8 & 1.07 \\
\hline J0102+5824 & B & 3.11 & $3.579=$ & 44 & $0.336 \pm 0.020$ & 3050 & 59 & 43 & -19.5 & 2221 & 1075 & 9 & 1.58 \\
\hline $\mathrm{J} 0109+6133$ & B & 0.64 & $706=$ & 45 & $0.242 \pm 0.023$ & 3048 & 111 & 37 & -12.9 & 381 & 239 & 5 & 1.17 \\
\hline $\mathrm{J} 0112+3522$ & $\mathrm{~A}$ & 0.53 & $0.624 \pm 0.031$ & 58 & $0.105 \pm 0.008$ & 3054 & 205 & 37 & -18.3 & 238 & 119 & 3 & 1.08 \\
\hline $\mathrm{J} 0113+4948$ & $\mathrm{~A}$ & 0.61 & $547=$ & 56 & $0.156 \pm 0$. & 3066 & 119 & 36 & -28.2 & 269 & 234 & 0 & 1.32 \\
\hline $\mathrm{J} 0126+7046$ & B & 0.07 & $0.233 \pm 0.018$ & 48 & $0.071 \pm 0.004$ & 2474 & 115 & 36 & -13.4 & 76 & 69 & 2 & 1.20 \\
\hline $\mathrm{J} 0136+4751$ & B & 1.95 & $2.531 \pm 0$ & 33 & $1.324 \pm 0.0$ & 2388 & 157 & 43 & -14.4 & 2090 & 862 & & 1.21 \\
\hline $\mathrm{J} 0136+4751$ & $\mathrm{C}$ & 1.76 & $1.811=$ & 46 & 0.518 & 2870 & 230 & 36 & -0.5 & 1317 & 740 & 15 & 1.15 \\
\hline+2145 & $\mathrm{~A}$ & 0.07 & $289=$ & 54 & 97 & 3070 & 259 & 40 & -10.2 & 278 & 175 & 3 & 1.03 \\
\hline $\mathrm{J} 0154+4743$ & B & $\ldots$ & $0.436 \pm 0.022$ & 32 & $0.315 \pm 0.026$ & 2860 & 150 & 42 & -11.2 & 239 & 172 & 4 & 1.12 \\
\hline $205+3212$ & A & 0.81 & $530+0$ & 49 & $0.228 \pm 0$ & 3052 & 185 & 38 & -11.6 & 499 & 1 & 1 & 9 \\
\hline J0253+3217 & A & 0.37 & $0.579 \pm 0.0$ & 43 & 0.27 & 3070 & 190 & 39 & -9.0 & 259 & 205 & 5 & 1.01 \\
\hline+2343 & $\mathrm{~A}$ & 0.05 & $0.342=$ & 48 & 0.34 & 3060 & 245 & 39 & -8.7 & 186 & 170 & 5 & 0.99 \\
\hline+3814 & B & 0.44 & $0.320 \pm$ & 58 & $0.272 \pm 0.021$ & 3050 & 193 & 40 & -29.3 & 190 & 182 & 9 & 1.35 \\
\hline $\mathrm{J} 0313+4120$ & B & 0.89 & $0.823=$ & 58 & 0.294 & 3020 & 178 & 39 & -30.9 & 663 & 349 & 8 & 1.26 \\
\hline $\mathrm{J} 0319+4130$ & $\mathrm{~A}$ & 12.06 & $4.690\rfloor$ & 26 & $0.506 \pm 0.054$ & 3064 & 155 & 33 & -12.2 & 7677 & 978 & 10 & 1.16 \\
\hline $\mathrm{J} 0319+4130$ & B & 12.15 & $9.885 \pm 0.206$ & 55 & $0.357 \pm 0.074$ & 2972 & 170 & 44 & -24.1 & 8865 & 724 & 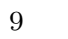 & 1.12 \\
\hline $\mathrm{J} 0319+4130$ & $\mathrm{C}$ & 13.85 & $3.290 \pm 0.126$ & 67 & $0.158 \pm 0.017$ & 1634 & 185 & 116 & 2.7 & 3504 & 625 & 6 & 0.66 \\
\hline $\mathrm{J} 0325+2224$ & $\mathrm{C}$ & 0.90 & $0.879 \pm 0.037$ & 39 & $0.266 \pm 0.010$ & 3131 & 203 & 37 & -9.0 & 621 & 370 & 12 & 1.12 \\
\hline J0325+4655 & $\mathrm{C}$ & 0.27 & $0.258 \pm 0.018$ & 33 & $0.074 \pm 0.008$ & 3135 & 127 & 40 & -9.3 & 190 & 88 & 4 & 0.81 \\
\hline J0333+6536 & $\mathrm{C}$ & 0.07 & $0.189 \pm 0.020$ & 40 & $0.158 \pm 0.013$ & 3050 & 109 & 37 & -12.0 & 77 & 64 & 3 & 1.04 \\
\hline J0344+6827 & $\mathrm{A}$ & 0.06 & $0.187 \pm 0.023$ & 62 & $0.136 \pm 0.007$ & 3138 & 122 & 39 & -35.5 & 105 & 97 & 3 & 1.10 \\
\hline J0344+6827 & $\mathrm{C}$ & 0.07 & $0.213 \pm 0.017$ & 41 & $0.205 \pm 0.018$ & 3030 & 105 & 35 & -7.7 & 159 & 66 & 2 & 1.20 \\
\hline $\mathrm{J} 0359+3220$ & $\mathrm{~A}$ & 0.36 & $0.358 \pm 0.037$ & 67 & $0.140 \pm 0.021$ & 3132 & 173 & 43 & -12.6 & 318 & 140 & 2 & 1.05 \\
\hline
\end{tabular}


TABLE 4.6: Image Parameters continued

\begin{tabular}{|c|c|c|c|c|c|c|c|c|c|c|c|c|c|}
\hline $\begin{array}{l}\text { Source }(\mathrm{J} 2000) \\
(1)\end{array}$ & $\begin{array}{l}\text { Obs } \\
(2)\end{array}$ & $\begin{array}{l}S_{86 \mathrm{GHz}} \\
(3)\end{array}$ & $\begin{array}{l}S_{\mathrm{S}} \\
(4)\end{array}$ & $\begin{array}{l}B_{\mathrm{S}} \\
(5)\end{array}$ & $\begin{array}{l}S_{\mathrm{L}} \\
(6)\end{array}$ & $\begin{array}{l}B_{\mathrm{L}} \\
(7)\end{array}$ & $\begin{array}{l}B_{\mathrm{a}} \\
(8)\end{array}$ & $\begin{array}{l}B_{\mathrm{b}} \\
(9)\end{array}$ & $\begin{array}{l}B_{\mathrm{PA}} \\
(10)\end{array}$ & $\begin{array}{l}S_{\mathrm{t}} \\
(11)\end{array}$ & $\begin{array}{l}S_{\mathrm{p}} \\
(12)\end{array}$ & $\begin{array}{l}\sigma \\
(13)\end{array}$ & $\begin{array}{l}\xi_{\mathrm{r}} \\
(14)\end{array}$ \\
\hline J0359+5057 & $\mathrm{A}$ & $\ldots$ & $1.861 \pm 0.076$ & 67 & $0.336 \pm 0.011$ & 3058 & 148 & 40 & -48.6 & 1477 & 313 & 23 & 1.12 \\
\hline & A & 0.0 & $0.325 \pm$ & 67 & $0.131 \pm$ & 3138 & 167 & 45 & -11.4 & 124 & 103 & & .94 \\
\hline $512+4041$ & A & 1.29 & $1.014 \pm 0.035$ & 53 & $0.211 \pm 0.009$ & 3070 & 159 & 38 & -22.7 & 409 & 235 & 4 & .63 \\
\hline+4822 & A & & $807 \pm 0.027$ & 51 & $0.417 \pm 0.008$ & 3065 & 138 & 38 & -25.7 & 556 & 258 & & 28 \\
\hline+2429 & A & & $3 \pm 0.024$ & 48 & $.188 \pm$ & 2474 & 262 & 35 & -10.7 & 247 & 203 & & $07>$ \\
\hline $2+4122$ & A & 7 & $51 \pm 0.025$ & 48 & $0.155 \pm$ & 3060 & 154 & 39 & -19.4 & 260 & 167 & & .17 \\
\hline-4207 & A & & $0.277 \pm 0.031$ & 49 & $0.151 \pm 0.006$ & 3055 & 148 & 38 & -18.8 & 154 & 147 & & 1.12 \\
\hline $632+3200$ & A & & $0.350 \pm 0.028$ & 44 & $0.168 \pm 0.006$ & 2473 & 208 & 37 & -11.0 & 174 & 174 & & .01 \\
\hline & B & & 8 & 42 & 0.0 & 3140 & 109 & 37 & -22.7 & 130 & 74 & & 94 \\
\hline 7324 & $\mathrm{C}$ & & $3 \pm$ & 46 & $0.186 \pm$ & 2992 & 87 & 38 & 14.5 & 374 & 131 & & 94 \\
\hline-8811 & $\mathrm{C}$ & 0 & $1 \pm 0$ & 56 & $0.096 \pm$ & 3091 & 85 & 37 & 26.5 & 121 & 74 & & .02 \\
\hline+6001 & B & 0.2 & $0.287 \pm 0.023$ & 40 & $0.091 \pm 0.005$ & 3062 & 94 & 36 & -20.6 & 149 & 92 & 4 & .99 \\
\hline & A & & $0863+$ & 47 & 8 & 2471 & 323 & 37 & -7.9 & 630 & 234 & & .05 \\
\hline 110 & B & & 0 & 38 & 0.0 & 3040 & 93 & 36 & -14.7 & 164 & - & & -1 \\
\hline & A & & 36 & 46 & 0.2 & 2471 & 329 & 37 & -5.8 & 220 & 216 & 0 & 79 \\
\hline & B & 4.91 & & 43 & 0.5 & 3135 & 55 & 40 & -65.9 & 2935 & 1284 & & .44 \\
\hline & $\mathrm{C}$ & $\ldots$ & 99 & 39 & $0.859 \pm$ & 3138 & 55 & 39 & -17.9 & 1119 & 980 & P & 0.70 \\
\hline & C & & & 23 & & 2472 & 119 & 42 & 12.6 & 156 & 109 & & .04 \\
\hline 112 & $\mathrm{C}$ & & 20 & 42 & 0.4 & 3081 & 191 & 39 & -2.5 & 45 & 268 & & 03 \\
\hline & B & & 23 & 47 & 0.0 & 3080 & 105 & 38 & -2.1 & 58 & 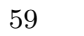 & & .03 \\
\hline & $\mathrm{C}$ & & 29 & 45 & 0.2 & 3116 & 185 & 38 & -7.6 & 1002 & 421 & 11 & .05 \\
\hline & $\mathrm{C}$ & & & 25 & & 2760 & 106 & 40 & 10.0 & 48 & 28 & & .16 \\
\hline & $\mathrm{C}$ & & & 34 & 0. & 3135 & 123 & 36 & -3.2 & 36 & 47 & & 30 \\
\hline & C & & & 35 & & 3084 & 120 & 40 & 10.0 & 10 & 3 & & .07 \\
\hline & $\mathrm{C}$ & $\ldots$ & & 45 & & & 95 & 41 & -12.6 & & 4 & & 35 \\
\hline & $\mathrm{C}$ & $\ldots$ & & 44 & & 3050 & 173 & 41 & -11.8 & 427 & 70 & & .04 \\
\hline & $C$ & 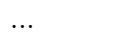 & & 46 & & 3020 & 207 & 45 & -5.1 & 52 & Y & & .00 \\
\hline & c & & & 45 & & 3075 & 128 & 36 & -10.5 & 36 & 4 & & .02 \\
\hline & B & & 1 & 64 & & 3128 & 301 & 37 & -14.5 & 4274 & 1317 & 8 & .24 \\
\hline+4253 & A & 06 & 23 & 53 & $0.146 \pm$ & 3070 & 148 & 38 & -20.9 & 232 & 155 & 2 & .14 \\
\hline & $\mathrm{C}$ & & & 50 & & & 108 & 38 & -7.1 & 24 & & & .76 \\
\hline & A & & 7 & 53 & & 3070 & 140 & 38 & -22.8 & 1018 & & & .54 \\
\hline & A & 0.1 & & 56 & & 3062 & 176 & 44 & -19.5 & 25 & & & 12 \\
\hline & $\mathrm{C}$ & & & 48 & & 3086 & 200 & 37 & -4.2 & 3286 & 447 & & .15 \\
\hline & $\mathrm{C}$ & & & 49 & & & 135 & 37 & -6.7 & 349 & & 11 & 0.97 \\
\hline & A & & 6 & 55 & & 3065 & 188 & 40 & -15.9 & & & & .06 \\
\hline & 1 & & 0 & 50 & 0. & 3050 & 239 & 36 & -13.6 & 59 & 373 & & 12 \\
\hline & $\mathrm{C}$ & & & 47 & & 3087 & 116 & 38 & -7.2 & & & & .83 \\
\hline & $\mathrm{C}$ & & 8 & 49 & 0. & 3134 & 107 & 39 & -17.0 & 368 & 251 & & 1.37 \\
\hline & A & & & 49 & & & 255 & 36 & -12.7 & & & & 18 \\
\hline & C & & & 30 & & & 163 & 37 & .1 & 4 & & & 98 \\
\hline & c & & & 29 & & 2960 & 155 & 36 & -3.8 & 6 & & & 10 \\
\hline & C & & 7 & 39 & & 3070 & 112 & 39 & 0.7 & 641 & 22 & 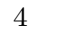 & 1.28 \\
\hline & $\mathrm{C}$ & & & 30 & & & 150 & 37 & -2.9 & & & & .01 \\
\hline & $A$ & & & 47 & & 20 & 300 & 36 & .3 & & & & 02 \\
\hline & B & & 7 & 56 & 0 . & 3052 & 104 & 33 & -41.1 & 77 & 9 & & 22 \\
\hline & A & & & 52 & & 3054 & 351 & 38 & -8.9 & 33 & 3 & & .94 \\
\hline 14 & B & & 7 & 56 & 0. & 3036 & 98 & 34 & -40.1 & 571 & 64 & & 1.20 \\
\hline & $\mathrm{B}$ & & & 48 & & & 220 & 42 & -1.8 & 482 & & & 12 \\
\hline & $\mathrm{E}$ & & & $3 s$ & & 6 & 196 & 42 & .7 & & & & \\
\hline & B & & & 3 & & 2420 & 229 & 37 & 8 & 1479 & & & 5 \\
\hline & B & & 6 & 33 & 0 . & 2400 & 200 & 36 & 12.3 & 83 & & 1 & .96 \\
\hline & B & & & 31 & & 2384 & 179 & 37 & 7.3 & 1518 & 030 & 39 & .20 \\
\hline & B & & & 55 & & & 101 & 36 & -25.9 & & & & .10 \\
\hline & c & & & 5 & & & 125 & 36 & -16 & & & & \\
\hline & $\mathrm{C}$ & & & 5 & & & 135 & 36 & & & & & 33 \\
\hline & $\mathrm{C}$ & 0.03 & & 54 & 0. & 3058 & 162 & 35 & -14.8 & 67 & 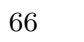 & 5 & .94 \\
\hline & A & & & 63 & & 2970 & 255 & 83 & 19.9 & & & & 90 \\
\hline & $A$ & & & 69 & & 1460 & 383 & $138>$ & -22.9 & & & & 01 \\
\hline & $\mathrm{E}$ & & & 156 & & & 1277 & 188 & & 2482 & 10 & 76 & \\
\hline & 0 & $\ldots$ & 2 & 53 & 0 . & 3010 & 149 & 37 & -12 & 71 & 66 & 4 & .28 \\
\hline & B & & .980 & 37 & 1.6 & 1676 & 311 & 91 & -21.8 & 22696 & 7250 & 205 & 1.16 \\
\hline & $C$ & 0 & & 54 & & 2890 & 114 & 38 & -14.7 & 189 & 141 & & .08 \\
\hline & & & & 61 & & 2469 & & 42 & -27.5 & 77 & & & \\
\hline & B & & & 3 & & & 17 ? & & & & & & \\
\hline 10 & A & $\ldots$ & \pm & 57 & $4 \pm$ & 3060 & 305 & 43 & -16.7 & 391 & 288 & 5 & .00 \\
\hline $\mathrm{J}$ & A & 0. & $18 \pm 0$ & 57 & $0.190 \pm 0$ & 3046 & 203 & 35 & -16.1 & 297 & 202 & 4 & 1.01 \\
\hline $\mathrm{J} 1341+2816$ & A & 0.14 & $0.310 \pm 0.023$ & 58 & $0.232 \pm 0.008$ & 3054 & 235 & 36 & -15.6 & 235 & 203 & 6 & 0.90 \\
\hline
\end{tabular}


TABLE 4.6: Image Parameters continued

\begin{tabular}{|c|c|c|c|c|c|c|c|c|c|c|c|c|c|}
\hline $\begin{array}{l}\text { Source (J2000) } \\
(1)\end{array}$ & $\begin{array}{l}\text { Obs } \\
(2) \\
\end{array}$ & $\begin{array}{l}S_{86 \mathrm{GHz}} \\
(3)\end{array}$ & $\begin{array}{l}S_{\mathrm{S}} \\
(4) \\
\end{array}$ & $\begin{array}{l}B_{\mathrm{S}} \\
(5) \\
\end{array}$ & $\begin{array}{l}S_{\mathrm{L}} \\
(6)\end{array}$ & $\begin{array}{l}B_{\mathrm{L}} \\
(7)\end{array}$ & $\begin{array}{l}B_{\mathrm{a}} \\
(8) \\
\end{array}$ & $\begin{array}{l}B_{\mathrm{b}} \\
(9)\end{array}$ & $\begin{array}{l}B_{\mathrm{PA}} \\
(10)\end{array}$ & $\begin{array}{l}S_{\mathrm{t}} \\
(11)\end{array}$ & $\begin{array}{l}S_{\mathrm{p}} \\
(12)\end{array}$ & $\begin{array}{l}\sigma \\
(13) \\
\end{array}$ & $\begin{array}{l}\xi_{\mathrm{r}} \\
(14) \\
\end{array}$ \\
\hline J1353+7532 & $\mathrm{B}$ & 0.13 & $0.286 \pm 0.023$ & 48 & $0.087 \pm 0.005$ & 2988 & 104 & 35 & -0.2 & 91 & 73 & 2 & 1.40 \\
\hline $357+7643$ & B & 0.31 & $0.371 \pm 0.024$ & 49 & $0.147 \pm$ & 2994 & 107 & 34 & -0.6 & 177 & 175 & 4 & 1.35 \\
\hline $\mathrm{J} 1407+2827$ & A & 0.02 & $0.368 \pm 0.021$ & 47 & $0.218 \pm 0.009$ & 3066 & 249 & 37 & -12.6 & 251 & 189 & & .03 \\
\hline 821 & A & 49 & $0.498 \pm$ & 45 & $0.186 \pm$ & 2840 & 234 & 36 & -12.3 & 223 & 142 & & 1.16 \\
\hline $506+4239$ & A & 56 & $0.434 \pm 0.027$ & 39 & $0.143 \pm 0.015$ & 2946 & 201 & 30 & -4.7 & 183 & 201 & & 1.27 \\
\hline $6+8319$ & B & 0.11 & $0.276 \pm 0.019$ & 52 & $0.092 \pm 0.007$ & 2974 & 105 & 35 & 17.2 & 83 & 69 & & 1.21 \\
\hline $\mathrm{J} 1521+4336$ & $\mathrm{~A}$ & 0.37 & $0.315 \pm 0.028$ & 39 & $0.208 \pm 0.010$ & 3054 & 153 & 34 & -7.7 & 212 & 211 & 9 & 1.37 \\
\hline 038 & $\mathrm{C}$ & 31 & $0.268 \pm$ & 46 & $0.191 \pm$ & 2440 & 263 & 35 & 4.3 & 320 & 253 & & .03 \\
\hline 012 & B & 46 & $0.786 \pm 0$ & 54 & $0.232 \pm$ & 3070 & 190 & 35 & -17.7 & 303 & 304 & 1 & 40 \\
\hline $1+5741$ & $\mathrm{C}$ & 0.32 & $0.257 \pm 0$ & 36 & $0.179 \pm$ & 2412 & 204 & 37 & 13.6 & 370 & 255 & & .03 \\
\hline $635+3808$ & $\mathrm{C}$ & .65 & $1.036 \pm 0.099$ & 32 & $0.814 \pm 0.014$ & 2305 & 226 & 37 & 13.0 & 1676 & 1133 & 4 & .15 \\
\hline $37+4717$ & B & 1.01 & $1.098 \pm 0.024$ & 48 & $0.201 \pm 0.018$ & 3044 & 189 & 34 & -14.8 & 751 & 407 & ; & .34 \\
\hline 46 & B & 1.00 & 22 & 46 & 0.158 & 3030 & 190 & 35 & -8.9 & 630 & 265 & & .14 \\
\hline 948 & A & 58 & $2 \pm$ & 61 & $0.194 \pm$ & 3026 & 242 & 34 & -16.6 & 1104 & 423 & 27 & 97 \\
\hline+3948 & B & 68 & $3.689 \pm 0$ & 42 & $0.419 \pm 0.021$ & 2984 & 204 & 34 & -6.9 & 2563 & 705 & 12 & 1.21 \\
\hline+4059 & B & 0.16 & $0.309 \pm 0.015$ & 44 & $0.141 \pm 0.009$ & 3000 & 184 & 36 & -7.8 & 87 & 68 & 2 & 1.06 \\
\hline & A & 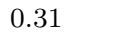 & 6 & 54 & $0.195 \pm$ & 2474 & 207 & 43 & -11.2 & 327 & 165 & & .14 \\
\hline$\$ 29$ & A & & 8 & 57 & $0.203 \pm$ & 2476 & 234 & 42 & -10.9 & 311 & 47 & & .10 \\
\hline 954 & A & 30 & \pm & 55 & $0.176 \pm$ & 2476 & 158 & 41 & -19.6 & 232 & 151 & & 21 \\
\hline & $\mathrm{C}$ & 36 & \pm & 40 & $0.817 \pm$ & 2786 & 185 & 35 & 26.4 & 252 & 227 & 0 & 1.24 \\
\hline 213 & A & 12 & 3 & 52 & $0.180 \pm$ & 2470 & 237 & 36 & -15.6 & 176 & 177 & & .86 \\
\hline 49 & A & 22 & 5 & 51 & 0.1 & 2469 & 139 & 43 & -20.5 & 229 & 00 & & .07 \\
\hline 226 & B & & 6 & 64 & $0.100 \pm$ & 3016 & 89 & 38 & -69.0 & 94 & 11 & & .20 \\
\hline 920 & A & & +8 & 52 & $0.196 \pm$ & 2464 & 256 & 36 & -14.4 & 3 & & & 10 \\
\hline+2848 & A & 0.35 & $0.362 \pm 0$ & 54 & $0.115 \pm$ & 2472 & 353 & 38 & -11.5 & 192 & 54 & & 0.96 \\
\hline & A & & 0 & 53 & 0.1 & 2444 & 221 & 36 & -18.2 & 2 & 00 & 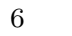 & .88 \\
\hline 328 & $C$ & & 5 & 53 & 0.2 & 3134 & 46 & 43 & -35.7 & 1736 & 18 & 1 & .13 \\
\hline 542 & C & & 8 & 18 & 0.4 & 2140 & 205 & 73 & 35.6 & 18 & & & .93 \\
\hline+1704 & A & 42 & 0 & 58 & $0.379 \pm$ & 3030 & 383 & 37 & -7.9 & 379 & & & 99 \\
\hline & B & & 2 & 62 & $0.125 \pm$ & 3061 & 82 & 37 & -64.2 & & & & .18 \\
\hline & $\mathrm{C}$ & & 1 & 51 & 0.1 & 3116 & 161 & 35 & -24.9 & 220 & 9 & & 1.17 \\
\hline 857 & $\mathrm{P}$ & 0.10 & \pm & 62 & 0.0 & 3061 & 83 & 37 & -67.3 & 98 & 7 & & 1.11 \\
\hline 705 & B & & & 59 & 0.17 & 3068 & 83 & 36 & -59.1 & 1200 & & 22 & 1.25 \\
\hline+2825 & A & 22 & 5 & 65 & $0.203 \pm$ & 2462 & 306 & 39 & -12.1 & & & 10 & 1.08 \\
\hline & A & & 3 & 66 & 0. & 2445 & 435 & 39 & -9.4 & 2 & & & .88 \\
\hline 117 & $\mathrm{C}$ & & 3 & 44 & 0.1 & 3064 & 126 & 35 & -3.9 & 4 & 19 & & .97 \\
\hline & $\Delta$ & & & 66 & & 2454 & 390 & 39 & -8.7 & 37 & & & 1.02 \\
\hline & $\mathrm{C}$ & & \pm & 46 & 0.1 & 3072 & 129 & 40 & -2.1 & 105 & 76 & & 0.97 \\
\hline & B & & 9 & 45 & & 2475 & 146 & 37 & -12.1 & 65 & & & 1.27 \\
\hline & D & & 8 & 43 & 0 . & 2472 & 126 & 36 & -8.3 & 2 & & & .12 \\
\hline 01 & $\mathrm{C}$ & 0.24 & \pm & 47 & 0.2 & 3020 & 287 & 43 & -5.6 & 252 & 3 & & .05 \\
\hline & B & & & 44 & 0.0 & 2474 & 128 & 37 & -11.7 & 98 & & & 1.03 \\
\hline & B & & 8 & 45 & 0.0 & 2475 & 149 & 37 & -10.7 & 137 & & & 1.19 \\
\hline & $C$ & & & 42 & & 2400 & 92 & 38 & -19.3 & & & & 1.27 \\
\hline & C & & & 64 & 0. & 2410 & 157 & 46 & -20.7 & 43 & & & .98 \\
\hline 10 & B & & 4 & 36 & 0 . & 3065 & 141 & 36 & -8.0 & 194 & & 5 & 1.04 \\
\hline & $\mathrm{C}$ & & \pm & 40 & $1.131 \pm$ & 3028 & 162 & 38 & -11.8 & 3115 & 2096 & 33 & 1.33 \\
\hline & $\mathrm{C}$ & & & 65 & & 3070 & 257 & 46 & -8.2 & 2 ? & & & .80 \\
\hline & $A$ & & & 60 & & & 215 & 38 & -25.0 & & & & .95 \\
\hline & A & & 1 & 57 & 0 . & 3138 & 111 & 38 & -24.7 & 1 & & & .93 \\
\hline & A & & & 59 & $2 \pm$ & 138 & 111 & 38 & -27.9 & 138 & 1 & 2 & .10 \\
\hline 611 & A & & 2 & 56 & \pm & 3138 & 104 & 38 & -31.6 & 767 & & $c$ & 1.46 \\
\hline & $\mathrm{C}$ & & & 65 & & & 255 & 49 & -9.1 & 146 & & & 0.67 \\
\hline & $\Omega$ & & & 63 & & & 252 & 45 & .4 & 5 & & & 90 \\
\hline & A & & & 5 & & 24 & 225 & 37 & -18.9 & 39 & & & 04 \\
\hline & A & & 6 & 55 & 0 . & 3010 & 178 & 36 & -35.9 & & & 2 & 1.09 \\
\hline & A & & 1 & 54 & 0 . & 3070 & 141 & 36 & -32.3 & 260 & 77 & & .30 \\
\hline & $\mathrm{C}$ & 0.1 & & 67 & & 3139 & 309 & 39 & & 6 & & & .90 \\
\hline & $A$ & $\ldots$ & & 67 & & 2854 & 165 & 35 & -3 & & 30 & 45 & .94 \\
\hline & C & & & 110 & 2. & 715 & 2399 & 210 & -74.6 & 4546 & 01 & 64 & .70 \\
\hline 1725 & $\mathrm{C}$ & 1.08 & \pm & 66 & $0.311 \pm$ & 3080 & 351 & 40 & -14.4 & 706 & 239 & 9 & 1.29 \\
\hline & $\mathrm{C}$ & & 6 & 62 & 0.1 & 3136 & 212 & 35 & -17.1 & 66 & 67 & 4 & .16 \\
\hline & A & & 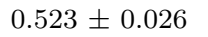 & 58 & 0 . & 3042 & 286 & 38 & & 2 & 56 & 19 & 15 \\
\hline & 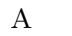 & & & 5 & & & 286 & 39 & & & & & \\
\hline 2421 & $\mathrm{C}$ & & \pm & 63 & 0 . & 3086 & 270 & 38 & -18 & 221 & 171 & 7 & 1.22 \\
\hline+1520 & A & 0 & $3 \pm 0$ & 62 & $0.293 \pm$ & 3064 & 344 & 39 & -11.2 & 283 & 222 & 7 & 0.93 \\
\hline & A & & \pm & 62 & $0.293 \pm$ & 3064 & 344 & 39 & -11.2 & 283 & 222 & 1 & 0.93 \\
\hline 1608 & A & 30.38 & $37 \pm 0.400$ & 58 & $9.270 \pm$ & 2329 & 469 & 72 & -15.7 & 2195 & 1016 & 338 & 1.10 \\
\hline
\end{tabular}


TABLE 4.6: Image Parameters continued

\begin{tabular}{|c|c|c|c|c|c|c|c|c|c|c|c|c|c|}
\hline $\begin{array}{l}\text { Source }(\mathrm{J} 2000) \\
(1)\end{array}$ & $\begin{array}{l}\text { Obs } \\
(2)\end{array}$ & $\begin{array}{l}S_{86 \mathrm{GHz}} \\
(3)\end{array}$ & $\begin{array}{l}S_{\mathrm{S}} \\
(4)\end{array}$ & $\begin{array}{l}B_{\mathrm{S}} \\
(5)\end{array}$ & $\begin{array}{l}S_{\mathrm{L}} \\
(6)\end{array}$ & $\begin{array}{l}B_{\mathrm{L}} \\
(7)\end{array}$ & $\begin{array}{l}B_{\mathrm{a}} \\
(8)\end{array}$ & $\begin{array}{l}B_{\mathrm{b}} \\
(9)\end{array}$ & $\begin{array}{l}B_{\mathrm{PA}} \\
(10)\end{array}$ & $\begin{array}{l}S_{\mathrm{t}} \\
(11)\end{array}$ & $\begin{array}{l}S_{\mathrm{p}} \\
(12)\end{array}$ & $\begin{array}{l}\sigma \\
(13)\end{array}$ & $\begin{array}{l}\xi_{\mathrm{r}} \\
(14) \\
\end{array}$ \\
\hline $\mathrm{J} 2253+1608$ & $\mathrm{~B}$ & 15.97 & $16.897 \pm 0.269$ & 63 & $1.061 \pm 0.075$ & 1770 & 249 & 88 & -17.0 & 17324 & 4550 & 74 & 1.04 \\
\hline $\mathrm{J} 2253+1608$ & $\mathrm{C}$ & 5.74 & $5.792 \pm 0.051$ & 59 & $0.476 \pm 0.030$ & 3065 & 270 & 34 & -5.0 & 4073 & 1100 & 21 & 1.03 \\
\hline $\mathrm{J} 2305+8242$ & $\mathrm{~A}$ & 0.25 & $\ldots$ & $\ldots$ & $\ldots$ & $\ldots$ & 101 & 38 & 7.1 & 145 & 145 & 3 & 1.41 \\
\hline $\mathrm{J} 2311+4543$ & $\mathrm{~A}$ & 0.36 & $0.298 \pm$ & 62 & $0.155 \pm 0.010$ & 3134 & 142 & 39 & -26.3 & 235 & 132 & 2 & 1.13 \\
\hline $\mathrm{J} 2312+7241$ & $\mathrm{C}$ & 0.13 & $0.152 \pm 0.014$ & 46 & $0.177 \pm 0.015$ & 2870 & 112 & 41 & -26.6 & 128 & 106 & 4 & 1.20 \\
\hline $\mathrm{J} 2322+4445$ & $\mathrm{~A}$ & 0.23 & $0.333 \pm 0.025$ & 63 & $0.159 \pm 0.006$ & 3130 & 147 & 38 & -25.9 & 141 & 123 & 3 & 1.13 \\
\hline $\mathrm{J} 2322+5057$ & $\mathrm{~A}$ & 0.59 & $0.391 \pm 0.030$ & 63 & $0.151 \pm 0.007$ & 3130 & 137 & 40 & -32.7 & 426 & 145 & 3 & 1.00 \\
\hline $\mathrm{J} 2330+3348$ & $\mathrm{C}$ & 0.22 & $0.159 \pm 0.013$ & 60 & $0.225 \pm 0.022$ & 3066 & 178 & 45 & -16.2 & 154 & 133 & 5 & 1.03 \\
\hline $\mathrm{J} 2354+4553$ & A & 0.31 & $0.289 \pm 0.021$ & 62 & $0.144 \pm 0.008$ & 3132 & 155 & 39 & -28.7 & 141 & 124 & 3 & 1.19 \\
\hline
\end{tabular}

Columns: (1) - IAU Source name (J2000); (2) - observing epochs - A: October 2010; B: May 2011 and C: October 2011; (3) - total single dish flux density measured at $86 \mathrm{GHz}$ obtained from the pointing and calibration scan measurements at Pico Veleta or Plateau de Bure [Jy]; (4),(6) - correlated flux densities [Jy] measured on baselines listed in columns (5) and (7) $[M \lambda]$; (8) - major axis of the restoring beam [ $\mu \mathrm{as}]$; (9) - minor axis of the restoring beam [ $\mu$ as]; (10) - position angle of the major axis [degrees]; (11) - total clean flux density [mJy]; (12) - peak flux density [mJy/beam]; (13) - off-source r.m.s noise in the residual image [mJy/beam]; (14) - quality factor of the residual noise in the image. (This table is also available in a machine-readable and Virtual Observatory (VO) forms in the online journal of Nair et al. (2019).)

TABle 4.7: Model Fit Parameters

\begin{tabular}{|c|c|c|c|c|c|c|c|c|c|c|}
\hline $\begin{array}{l}\text { Source }(\mathrm{J} 2000) \\
\text { (1) }\end{array}$ & $\begin{array}{l}\mathrm{Ob} \\
(2)\end{array}$ & $\begin{array}{l}\text { Son } \\
(3)\end{array}$ & $\begin{array}{l}S_{\text {tot }} \\
(4)\end{array}$ & $\begin{array}{l}S_{\text {peak }} \\
(5)\end{array}$ & $\begin{array}{l}d \\
(6)\end{array}$ & $\begin{array}{l}r \\
(7)\end{array}$ & $\begin{array}{l}\theta \\
(8)\end{array}$ & $\begin{array}{l}T_{\mathrm{b}} \\
(9)\end{array}$ & $\begin{array}{l}T_{\mathrm{b}, \min } \\
(10)\end{array}$ & $\begin{array}{l}T_{\mathrm{b}, \lim } \\
(11)\end{array}$ \\
\hline J0013+4051 & $\mathrm{C}$ & 1 & $288 \pm 71$ & $278 \pm 49$ & $<14$ & & $\ldots$ & $>28.642$ & 4.46 & 9.69 \\
\hline J0013+4051 & $\mathrm{C}$ & 2 & $194 \pm 61$ & $136 \pm 35$ & $34 \pm 9$ & $41 \pm 4$ & $-30.5 \pm 6.2$ & $3.428 \pm 1.647$ & ... & $\ldots$ \\
\hline J0017+8135 & B & 1 & $99 \pm 22$ & $69 \pm 12$ & $30 \pm 5$ & & $\ldots$ & $8.115 \pm 2.745$ & 1.89 & 3 \\
\hline J0017+8135 & B & 2 & $35 \pm 18$ & $11 \pm 5$ & $77 \pm 38$ & $431 \pm 19$ & $-179.3 \pm 2.5$ & $0.435 \pm 0.377$ & & \\
\hline J0030+7037 & B & 1 & $121 \pm 26$ & $90 \pm 16$ & $26 \pm 5$ & & $\ldots$ & $2.947 \pm 0.970$ & 1.78 & 2.26 \\
\hline $\mathrm{J} 0030+7037$ & B & 2 & $41 \pm 45$ & $4 \pm 4$ & $22 \pm 24$ & $53 \pm 12$ & $130.3 \pm 12.7$ & $1.395 \pm 2.651$ & & \\
\hline J0034+2754 & $\mathrm{C}$ & 1 & $72 \pm 17$ & $57 \pm 10$ & $26 \pm 5$ & & - & $6.803 \pm 2.367$ & 3.29 & 4.64 \\
\hline J0034+2754 & $\mathrm{C}$ & 2 & $88 \pm 36$ & $13 \pm 5$ & $777 \pm 316$ & $5758 \pm 158$ & $-49.2 \pm 11.8$ & $0.010 \pm 0.007$ & $\ldots$ & $\ldots$ \\
\hline J0034+2754 & $\mathrm{C}$ & 3 & $12 \pm 7$ & $14 \pm 5$ & $<41$ & $1217 \pm 8$ & $-33.5 \pm 0.4$ & $>0.494$ & $\ldots$ & $\ldots$ \\
\hline J0034+2754 & $\mathrm{C}$ & 4 & $14 \pm 8$ & $11 \pm 5$ & $59 \pm 26$ & $1713 \pm 13$ & $-30.2 \pm 0.4$ & $0.261 \pm 0.221$ & $\ldots$ & $\ldots$ \\
\hline J0034+2754 & $\mathrm{C}$ & 5 & $15 \pm 9$ & $10 \pm 5$ & $<3 \overline{7}$ & $1720 \pm 9$ & $-40.4 \pm 0.3$ & $>0.732$ & 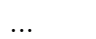 & $\ldots$ \\
\hline J0044+6803 & B & 1 & $100 \pm 24$ & $100 \pm 17$ & $<10$ & & $\ldots$ & $>16.750$ & 1.84 & 2.61 \\
\hline J0044+6803 & B & 2 & $28 \pm 14$ & $23 \pm 9$ & $22 \pm 8$ & $65 \pm 4$ & $131.2 \pm 3.6$ & $0.972 \pm 0.704$ & & \\
\hline J0046+2456 & A & 1 & $199 \pm 52$ & $157 \pm 32$ & $28 \pm 6$ & & $\ldots$ & $7.484 \pm 2.942$ & 4.11 & 6.06 \\
\hline J0046+2456 & A & 2 & $60 \pm 31$ & $39 \pm 17$ & $38 \pm 16$ & $171 \pm 8$ & $79.1 \pm 2.7$ & $1.225 \pm 0.988$ & & \\
\hline J0057+3021 & A & 1 & $298 \pm 72$ & $208 \pm 41$ & $33 \pm 7$ & $\ldots$ & $\ldots$ & $4.596 \pm 1.694$ & 9.32 & 23.1 \\
\hline J0102+5824 & B & 1 & $1030 \pm 199$ & $942 \pm 134$ & $15 \pm 2$ & $\ldots$ & $\ldots$ & $130.538 \pm 36.496$ & 8.09 & 24.2 \\
\hline J0102+5824 & B & 2 & $1633 \pm 481$ & $239 \pm 70$ & $193 \pm 56$ & $51 \pm 28$ & $92.0 \pm 28.7$ & $1.193 \pm 0.604$ & $\ldots$ & $\ldots$ \\
\hline J0102+5824 & B & 3 & $188 \pm 89$ & $166 \pm 59$ & $<18$ & $90 \pm 3$ & $-99.6 \pm 2.0$ & $>16.293$ & $\ldots$ & $\ldots$ \\
\hline J0109+6133 & B & 1 & $397 \pm 66$ & $231 \pm 33$ & $40 \pm 6$ & $\ldots$ & $\ldots$ & $7.221 \pm 1.880$ & 2.98 & 3.99 \\
\hline J0112+3522 & A & 1 & $201 \pm 38$ & $95 \pm 16$ & $52 \pm 9$ & ... & $\ldots$ & $1.790 \pm 0.544$ & 2.7 & 3.62 \\
\hline J0112+3522 & A & 2 & $24 \pm 12$ & $34 \pm 10$ & $35 \pm 10$ & $182 \pm 5$ & $-110.6 \pm 1.6$ & $0.459 \pm 0.300$ & $\ldots$ & $\ldots$ \\
\hline J0113+4948 & A & 1 & $295 \pm 75$ & $235 \pm 46$ & $24 \pm 5$ & $\ldots$ & $\ldots$ & $11.840 \pm 4.463$ & 3.34 & 6.83 \\
\hline J0126+7046 & B & 1 & $82 \pm 19$ & $68 \pm 12$ & $22 \pm 4$ & $\ldots$ & $\ldots$ & $2.719 \pm 0.923$ & 0.981 & 1.28 \\
\hline J0136+4751 & B & 1 & $960 \pm 166$ & $874 \pm 112$ & $19 \pm 2$ & $\ldots$ & $\ldots$ & $82.095 \pm 20.530$ & 3.98 & 4.71 \\
\hline J0136+4751 & B & 2 & $529 \pm 129$ & $359 \pm 72$ & $41 \pm 8$ & $195 \pm 4$ & $-80.0 \pm 1.2$ & $9.473 \pm 3.554$ & $\ldots$ & $\ldots$ \\
\hline J0136+4751 & B & 3 & $172 \pm 73$ & $154 \pm 49$ & $<26$ & $274 \pm 4$ & $-124.2 \pm 0.9$ & $>7.746$ & $\ldots$ & $\ldots$ \\
\hline J0136+4751 & B & 4 & $121 \pm 63$ & $103 \pm 41$ & $41 \pm 16$ & $508 \pm 8$ & $-58.4 \pm 0.9$ & $2.238 \pm 1.700$ & $\ldots$ & $\ldots$ \\
\hline J0136+4751 & B & 5 & $258 \pm 90$ & $191 \pm 54$ & $31 \pm 9$ & $858 \pm 4$ & $-23.5 \pm 0.3$ & $8.051 \pm 4.259$ & $\ldots$ & $\ldots$ \\
\hline J0136+4751 & $\mathrm{C}$ & 1 & $742 \pm 150$ & $737 \pm 105$ & $<14$ & & $\ldots$ & $>109.137$ & 7.68 & 14.5 \\
\hline J0136+4751 & $\mathrm{C}$ & 2 & $134 \pm 66$ & $138 \pm 48$ & $248 \pm 85$ & $221 \pm 43$ & $-101.3 \pm 11.0$ & $0.067 \pm 0.046$ & $\ldots$ & $\ldots$ \\
\hline J0136+4751 & $\mathrm{C}$ & 3 & $231 \pm 92$ & $140 \pm 48$ & $149 \pm 51$ & $892 \pm 25$ & $-8.8 \pm 1.6$ & $0.322 \pm 0.202$ & $\ldots$ & $\ldots$ \\
\hline J0136+4751 & $\mathrm{C}$ & 4 & $206 \pm 86$ & $133 \pm 47$ & $219 \pm 77$ & $1145 \pm 39$ & $0.9 \pm 1.9$ & $0.131 \pm 0.085$ & & \\
\hline J0136+4751 & $\mathrm{C}$ & 5 & $356 \pm 125$ & $157 \pm 50$ & $266 \pm 85$ & $1458 \pm 43$ & $-9.3 \pm 1.7$ & $0.154 \pm 0.088$ & $\ldots$ & 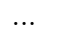 \\
\hline J0137+2145 & A & 1 & $185 \pm 34$ & $166 \pm 23$ & $22 \pm 3$ & $\ldots$ & $\ldots$ & $6.468 \pm 1.715$ & 6.47 & 16.8 \\
\hline $\mathrm{J} 0137+2145$ & A & 2 & $60 \pm 32$ & $14 \pm 7$ & $252 \pm 131$ & $1342 \pm 65$ & $-20.4 \pm 10.8$ & $0.016 \pm 0.014$ & $\ldots$ & $\ldots$ \\
\hline J0137+2145 & A & 3 & $68 \pm 31$ & $18 \pm 8$ & $262 \pm 115$ & $719 \pm 57$ & $-28.5 \pm 4.6$ & $0.016 \pm 0.013$ & $\ldots$ & . \\
\hline J0154+4743 & B & 1 & $197 \pm 41$ & $169 \pm 26$ & $23 \pm 4$ & $\ldots$ & $\ldots$ & $12.993 \pm 3.920$ & 4.64 & 6.76 \\
\hline J0154+4743 & B & 2 & $30 \pm 16$ & $31 \pm 12$ & $<32$ & $899 \pm 6$ & $-166.4 \pm 0.4$ & $>0.978$ & $\ldots$ & $\ldots$ \\
\hline
\end{tabular}


TABLE 4.7: Model fit parameters continued

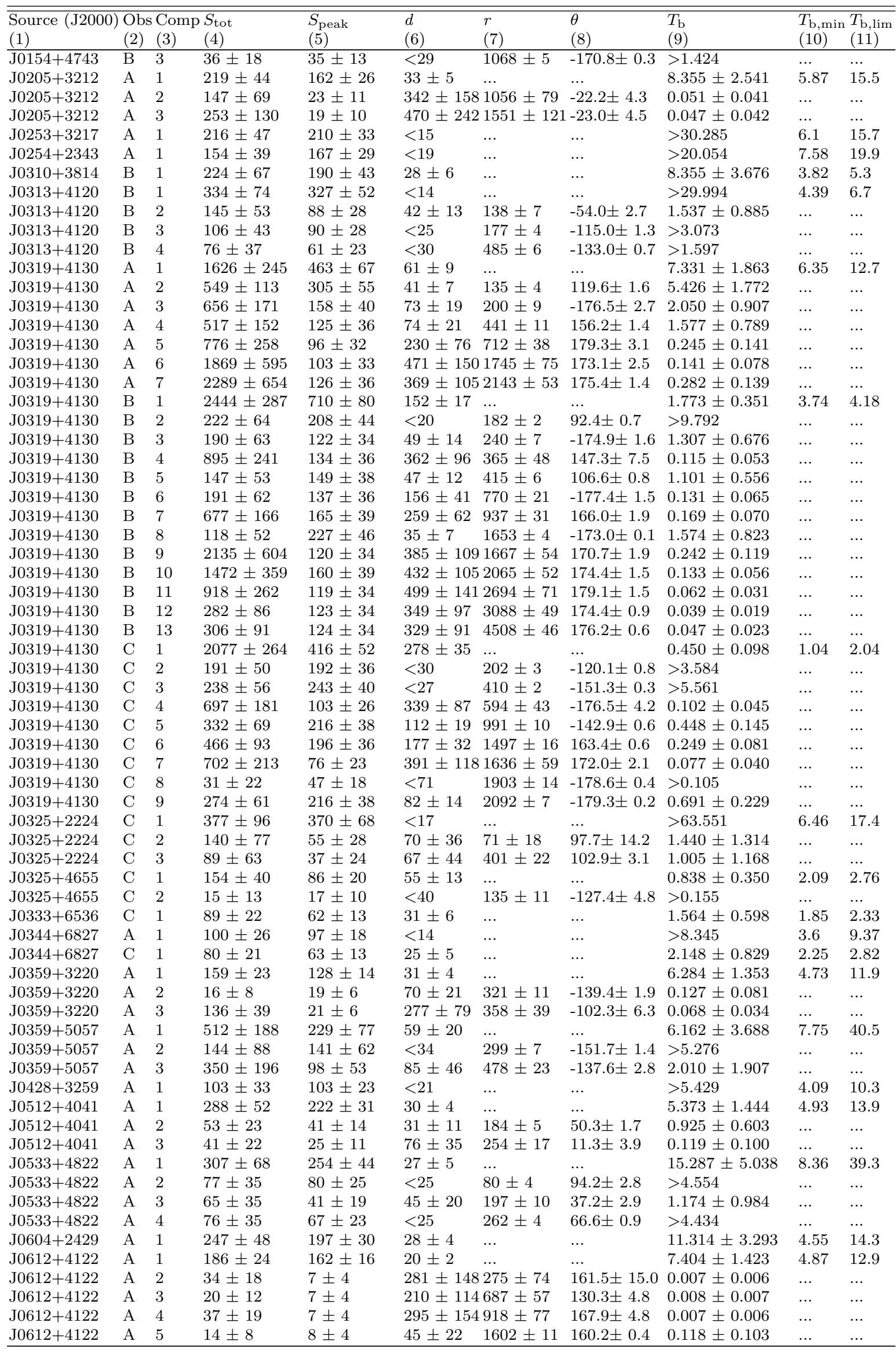


TABLE 4.7: Model fit parameters continued

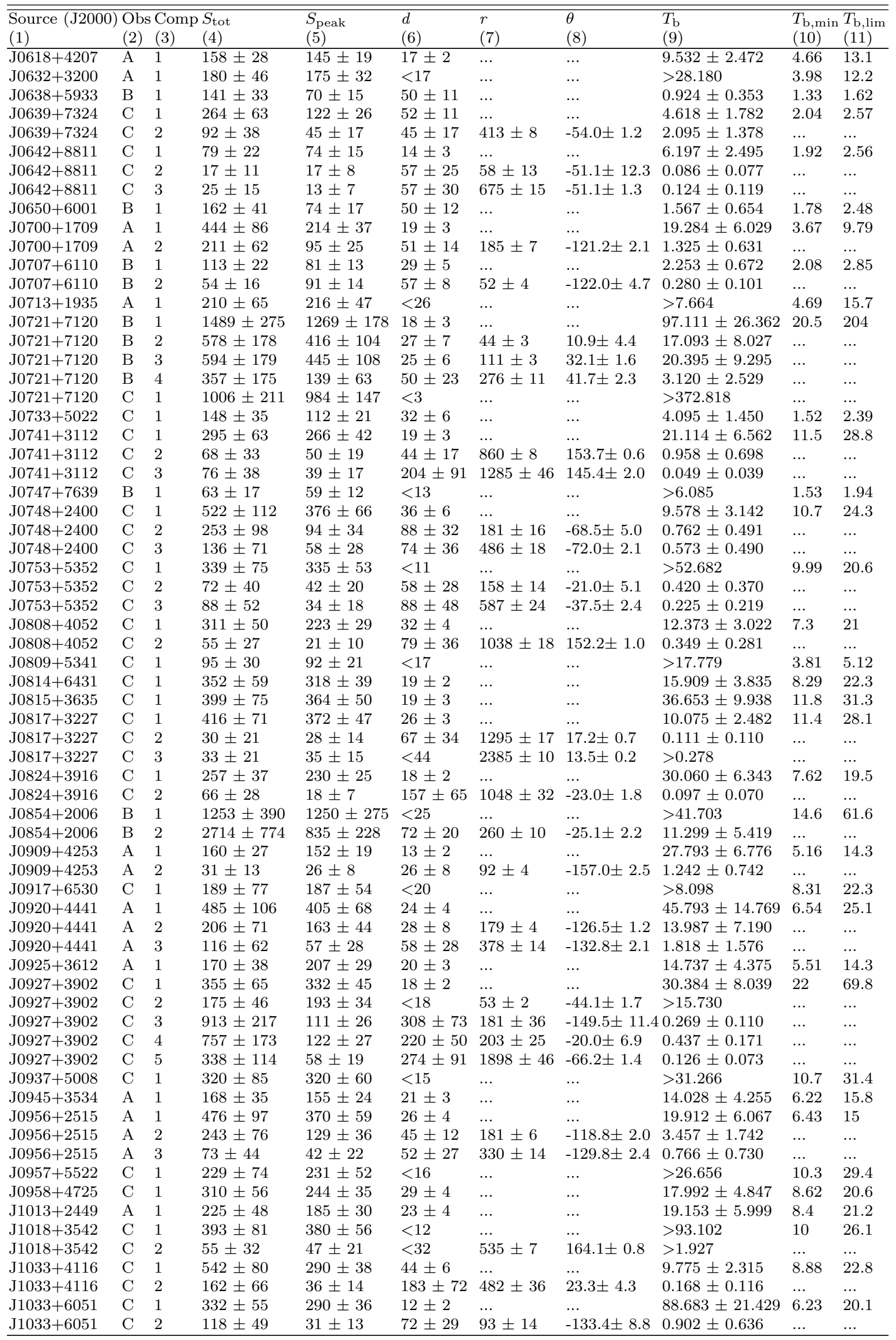


TABLE 4.7: Model fit parameters continued

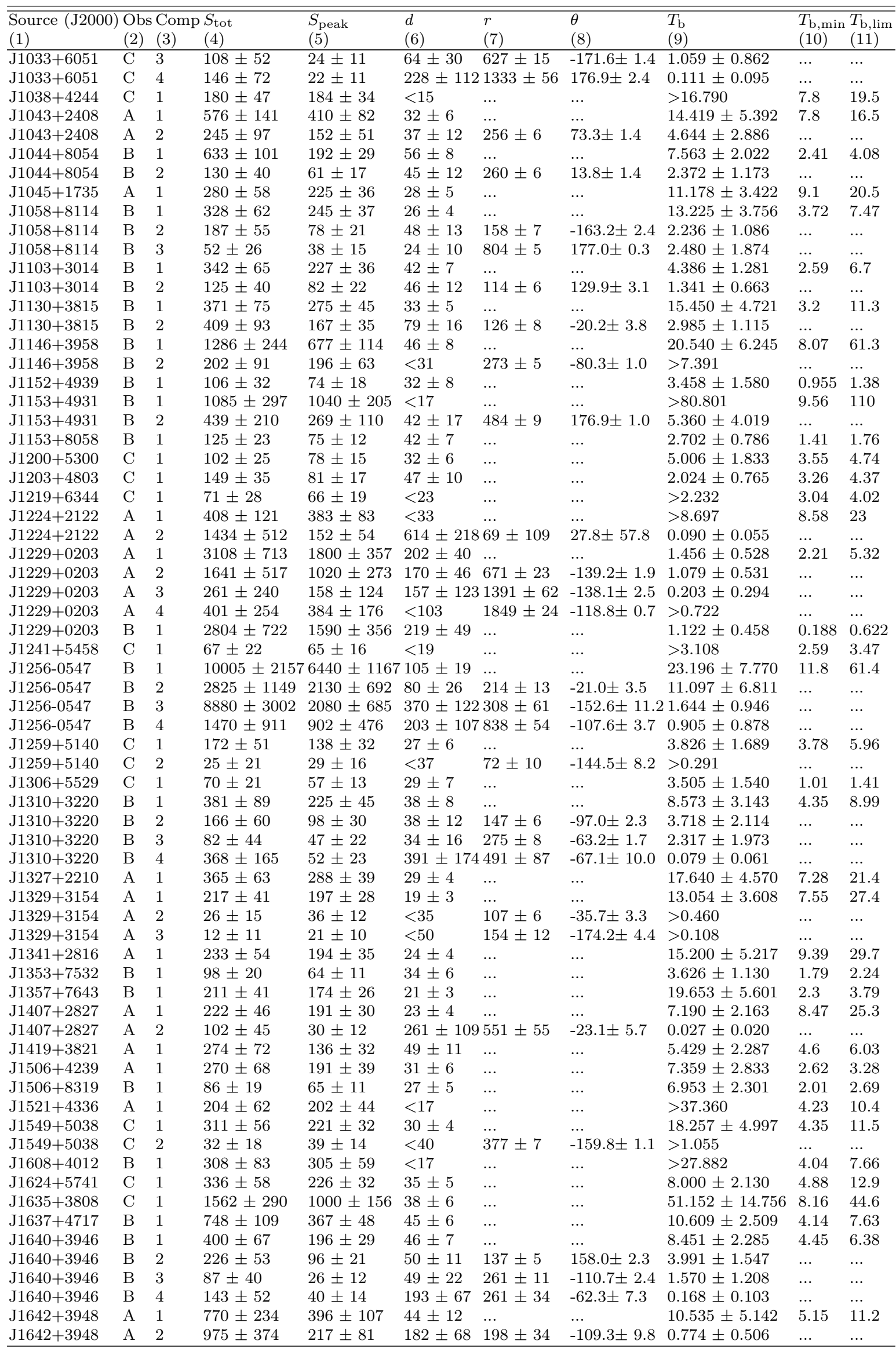


TABLE 4.7: Model fit parameters continued

\begin{tabular}{|c|c|c|c|c|c|c|c|c|c|c|}
\hline $\begin{array}{l}\text { Source }(\mathrm{J} 2000) \\
(1)\end{array}$ & $\begin{array}{l}\text { Obs } \\
(2)\end{array}$ & $\begin{array}{l}\text { Con } \\
(3)\end{array}$ & $\begin{array}{l}S_{\text {tot }} \\
(4)\end{array}$ & $\begin{array}{l}S_{\text {peak }} \\
(5)\end{array}$ & $\begin{array}{l}d \\
(6) \\
\end{array}$ & $\begin{array}{l}r \\
(7) \\
\end{array}$ & $\begin{array}{l}\theta \\
(8) \\
\end{array}$ & $\begin{array}{l}T_{\mathrm{b}} \\
(9) \\
\end{array}$ & $\begin{array}{l}T_{\mathrm{b}, \min } \\
(10)\end{array}$ & $\begin{array}{l}T_{\mathrm{b}, \lim } \\
(11) \\
\end{array}$ \\
\hline J1642+3948 & $\mathrm{A}$ & 3 & $339 \pm 176$ & $141 \pm 67$ & $37 \pm 18$ & $67 \pm 9$ & $-65.4 \pm 7.5$ & $6.571 \pm 5.598$ & $\ldots$ & $\ldots$ \\
\hline $\mathrm{J} 1642+3948$ & $\mathrm{~A}$ & 4 & $403 \pm 215$ & $127 \pm 65$ & $73 \pm 37$ & $723 \pm 19$ & $-131.1 \pm 1.5$ & $1.973 \pm 1.763$ & $\ldots$ & $\ldots$ \\
\hline J1642+3948 & $\mathrm{B}$ & 2 & $1005 \pm 258$ & $201 \pm 51$ & $127 \pm 32$ & $176 \pm 16$ & $-105.2 \pm 5.2$ & $1.628 \pm 0.715$ & $\ldots$ & $\ldots$ \\
\hline J1642+3948 & $\mathrm{B}$ & 3 & $630 \pm 234$ & $100 \pm 37$ & $257 \pm 94$ & $807 \pm 47$ & $-109.2 \pm 3.3$ & $0.251 \pm 0.160$ & $\ldots$ & 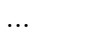 \\
\hline $\mathrm{J} 1646+4059$ & $\mathrm{~B}$ & 2 & $8 \pm 7$ & $10 \pm 5$ & $<46$ & $172 \pm 12$ & $-34.1 \pm 4.1$ & $>0.112$ & $\ldots$ & $\ldots$ \\
\hline $\mathrm{J} 1653+3107$ & $\mathrm{~A}$ & 1 & $179 \pm 23$ & $170 \pm 16$ & $16 \pm 2$ & $\ldots$ & $\ldots$ & $25.498 \pm 4.702$ & 2.5 & 8.79 \\
\hline $\mathrm{J} 1653+3107$ & $\mathrm{~A}$ & 2 & $54 \pm 14$ & $27 \pm 6$ & $81 \pm 19$ & $388 \pm 10$ & $20.4 \pm 1.4$ & $0.313 \pm 0.135$ & $\ldots$ & $\ldots$ \\
\hline $\mathrm{J} 1653+3107$ & $\mathrm{~A}$ & 3 & $63 \pm 16$ & $30 \pm 7$ & $105 \pm 24$ & $1067 \pm 12$ & $-18.5 \pm 0.6$ & $0.215 \pm 0.087$ & $\ldots$ & $\ldots$ \\
\hline $\mathrm{J} 1659+2629$ & $\mathrm{~A}$ & 1 & $197 \pm 25$ & $175 \pm 16$ & $22 \pm 2$ & $\ldots$ & $\ldots$ & $11.971 \pm 2.181$ & 2.48 & 8.81 \\
\hline $\mathrm{J} 1659+2629$ & $\mathrm{~A}$ & 2 & $66 \pm 16$ & $32 \pm 7$ & $70 \pm 15$ & $342 \pm 8$ & $23.2 \pm 1.3$ & $0.403 \pm 0.161$ & $\ldots$ & $\ldots$ \\
\hline $\mathrm{J} 1701+3954$ & $\mathrm{~A}$ & 3 & $52 \pm 22$ & $16 \pm 6$ & $179 \pm 73$ & $426 \pm 37$ & $-167.6 \pm 4.9$ & $0.040 \pm 0.029$ & $\ldots$ & $\cdots$ \\
\hline $\mathrm{J} 1716+6836$ & $\mathrm{C}$ & 1 & $273 \pm 75$ & $217 \pm 47$ & $26 \pm 6$ & $\ldots$ & $\ldots$ & $11.847 \pm 4.866$ & 14.7 & 39 \\
\hline $\mathrm{J} 1726+3213$ & $\mathrm{~A}$ & 1 & $174 \pm 42$ & $177 \pm 30$ & $<17$ & $\ldots$ & $\ldots$ & $>19.597$ & 3.41 & 11.6 \\
\hline $\mathrm{J} 1735+5049$ & $\mathrm{~A}$ & 1 & $148 \pm 22$ & $148 \pm 16$ & $<9$ & $\ldots$ & $\ldots$ & $>54.376$ & 2.51 & 8.01 \\
\hline $\mathrm{J} 1735+5049$ & $\mathrm{~A}$ & 2 & $19 \pm 9$ & $11 \pm 4$ & $98 \pm 41$ & $286 \pm 21$ & $-25.2 \pm 4.1$ & $0.060 \pm 0.045$ & $\ldots$ & $\ldots$ \\
\hline $\mathrm{J} 1735+5049$ & $\mathrm{~A}$ & 3 & $12 \pm 7$ & $11 \pm 5$ & $37 \pm 15$ & $301 \pm 7$ & $31.1 \pm 1.4$ & $0.279 \pm 0.221$ & $\ldots$ & $\cdots$ \\
\hline $\mathrm{J} 1735+5049$ & $\mathrm{~A}$ & 4 & $29 \pm 11$ & $16 \pm 5$ & $109 \pm 37$ & $915 \pm 18$ & $-3.6 \pm 1.2$ & $0.075 \pm 0.046$ & $\ldots$ & $\ldots$ \\
\hline $\mathrm{J} 1735+5049$ & $\mathrm{~A}$ & 5 & $33 \pm 12$ & $20 \pm 6$ & $108 \pm 32$ & $1047 \pm 16$ & $-19.5 \pm 0.9$ & $0.087 \pm 0.047$ & $\ldots$ & $\ldots$ \\
\hline $\mathrm{J} 1746+6226$ & $\mathrm{~B}$ & 1 & $91 \pm 21$ & $69 \pm 13$ & $30 \pm 6$ & $\ldots$ & $\ldots$ & $8.032 \pm 2.812$ & 2.08 & 2.79 \\
\hline $\mathrm{J} 1751+2920$ & $\mathrm{~A}$ & 1 & $350 \pm 72$ & $241 \pm 41$ & $35 \pm 6$ & $\ldots$ & $\ldots$ & $4.674 \pm 1.481$ & 2.64 & 7.44 \\
\hline $\mathrm{J} 1753+2848$ & $\mathrm{~A}$ & 1 & $222 \pm 57$ & $152 \pm 32$ & $34 \pm 7$ & $\ldots$ & $\ldots$ & $6.785 \pm 2.663$ & 2.69 & 5.76 \\
\hline $\mathrm{J} 1800+3848$ & $\mathrm{~A}$ & 1 & $202 \pm 52$ & $197 \pm 36$ & $<18$ & $\ldots$ & $\ldots$ & $>32.519$ & 3.43 & 11.8 \\
\hline $\mathrm{J} 1821+6818$ & B & 2 & $41 \pm 19$ & $40 \pm 13$ & $<19$ & $181 \pm 3$ & $-153.4 \pm 1.0$ & $>4.929$ & $\ldots$ & $\cdots$ \\
\hline $\mathrm{J} 1821+6818$ & $\mathrm{C}$ & 1 & $180 \pm 39$ & $168 \pm 27$ & $15 \pm 2$ & $\ldots$ & $\ldots$ & $36.726 \pm 11.548$ & 2.13 & 2.79 \\
\hline $\mathrm{J} 1821+6818$ & $\mathrm{C}$ & 2 & $34 \pm 20$ & $20 \pm 10$ & $52 \pm 26$ & $108 \pm 13$ & $-161.2 \pm 7.0$ & $0.567 \pm 0.527$ & $\ldots$ & $\ldots$ \\
\hline $\mathrm{J} 1821+6818$ & $\mathrm{C}$ & 3 & $30 \pm 18$ & $19 \pm 10$ & $42 \pm 22$ & $824 \pm 11$ & $-164.3 \pm 0.8$ & $0.767 \pm 0.734$ & $\ldots$ & $\ldots$ \\
\hline $\mathrm{J} 1823+6857$ & $\mathrm{~B}$ & 1 & $77 \pm 18$ & $70 \pm 12$ & $18 \pm 3$ & $\ldots$ & $\ldots$ & $4.065 \pm 1.381$ & 2.5 & 3.25 \\
\hline $\mathrm{J} 1823+6857$ & $\mathrm{~B}$ & 2 & $12 \pm 8$ & $14 \pm 6$ & $<25$ & $41 \pm 5$ & $-141.6 \pm 7.1$ & $>0.328$ & $\ldots$ & $\ldots$ \\
\hline $\mathrm{J} 1849+6705$ & B & 1 & $686 \pm 185$ & $472 \pm 105$ & $30 \pm 7$ & $\ldots$ & $\ldots$ & $21.367 \pm 8.852$ & 3.02 & 4.98 \\
\hline $\mathrm{J} 1849+6705$ & $\mathrm{~B}$ & 2 & $312 \pm 135$ & $179 \pm 67$ & $35 \pm 13$ & $85 \pm 7$ & $-142.0 \pm 4.4$ & $6.905 \pm 4.712$ & $\ldots$ & $\cdots$ \\
\hline $\mathrm{J} 1849+6705$ & $\mathrm{~B}$ & 3 & $213 \pm 105$ & $168 \pm 65$ & $25 \pm 10$ & $165 \pm 5$ & $-139.9 \pm 1.7$ & $9.577 \pm 7.066$ & $\ldots$ & $\ldots$ \\
\hline $\mathrm{J} 1850+2825$ & $\mathrm{~A}$ & 1 & $202 \pm 64$ & $199 \pm 45$ & $<27$ & $\ldots$ & $\ldots$ & $>16.705$ & 3.98 & 21.5 \\
\hline $\mathrm{J} 1922+1530$ & $\mathrm{~A}$ & 1 & $208 \pm 64$ & $215 \pm 46$ & $<31$ & $\ldots$ & $\ldots$ & $>3.581$ & 3.61 & 16.7 \\
\hline $\mathrm{J} 1927+6117$ & $\mathrm{C}$ & 1 & $449 \pm 116$ & $125 \pm 31$ & $74 \pm 18$ & $\ldots$ & $\ldots$ & $1.987 \pm 0.866$ & 2.7 & 3.76 \\
\hline $\mathrm{J} 1930+1532$ & $\mathrm{~A}$ & 1 & $240 \pm 50$ & $174 \pm 29$ & $33 \pm 6$ & $\ldots$ & $\ldots$ & $3.666 \pm 1.159$ & 2.69 & 6.51 \\
\hline $\mathrm{J} 1930+1532$ & $\mathrm{~A}$ & 2 & $65 \pm 26$ & $56 \pm 17$ & $<37$ & $252 \pm 6$ & $-98.6 \pm 1.3$ & $>0.785$ & $\ldots$ & $\ldots$ \\
\hline $\mathrm{J} 1933+6540$ & $\mathrm{C}$ & 1 & $110 \pm 26$ & $73 \pm 14$ & $39 \pm 8$ & $\ldots$ & $\ldots$ & $3.255 \pm 1.192$ & 2.61 & 4.63 \\
\hline $\mathrm{J} 1939+3817$ & B & 1 & $73 \pm 21$ & $64 \pm 14$ & $24 \pm 5$ & $\ldots$ & $\ldots$ & $2.180 \pm 0.915$ & 0.865 & 1.1 \\
\hline J1955+5131 & $\mathrm{B}$ & 1 & $228 \pm 51$ & $156 \pm 29$ & $33 \pm 6$ & $\ldots$ & $\ldots$ & $7.819 \pm 2.689$ & 1.83 & 4.29 \\
\hline J1955+5131 & $\mathrm{B}$ & 2 & $101 \pm 47$ & $30 \pm 13$ & $70 \pm 31$ & $514 \pm 16$ & $-23.6 \pm 1.7$ & $0.751 \pm 0.590$ & $\ldots$ & $\ldots$ \\
\hline $\mathrm{J} 2002+1501$ & $\mathrm{C}$ & 1 & $227 \pm 48$ & $180 \pm 30$ & $33 \pm 5$ & $\ldots$ & $\ldots$ & $3.539 \pm 1.119$ & 8.55 & 12.2 \\
\hline $\mathrm{J} 2002+4725$ & B & 1 & $99 \pm 24$ & $74 \pm 14$ & $32 \pm 6$ & $\ldots$ & $\ldots$ & $5.296 \pm 1.958$ & 1.01 & 1.42 \\
\hline $\mathrm{J} 2002+4725$ & B & 2 & $29 \pm 15$ & $15 \pm 7$ & $54 \pm 26$ & $331 \pm 13$ & $106.9 \pm 2.2$ & $0.525 \pm 0.449$ & $\ldots$ & $\ldots$ \\
\hline $\mathrm{J} 2007+3722$ & $\mathrm{~B}$ & 1 & $130 \pm 28$ & $59 \pm 11$ & $51 \pm 10$ & $\ldots$ & $\ldots$ & $0.829 \pm 0.288$ & 0.899 & 1.19 \\
\hline
\end{tabular}


TABLE 4.7: Model fit parameters continued

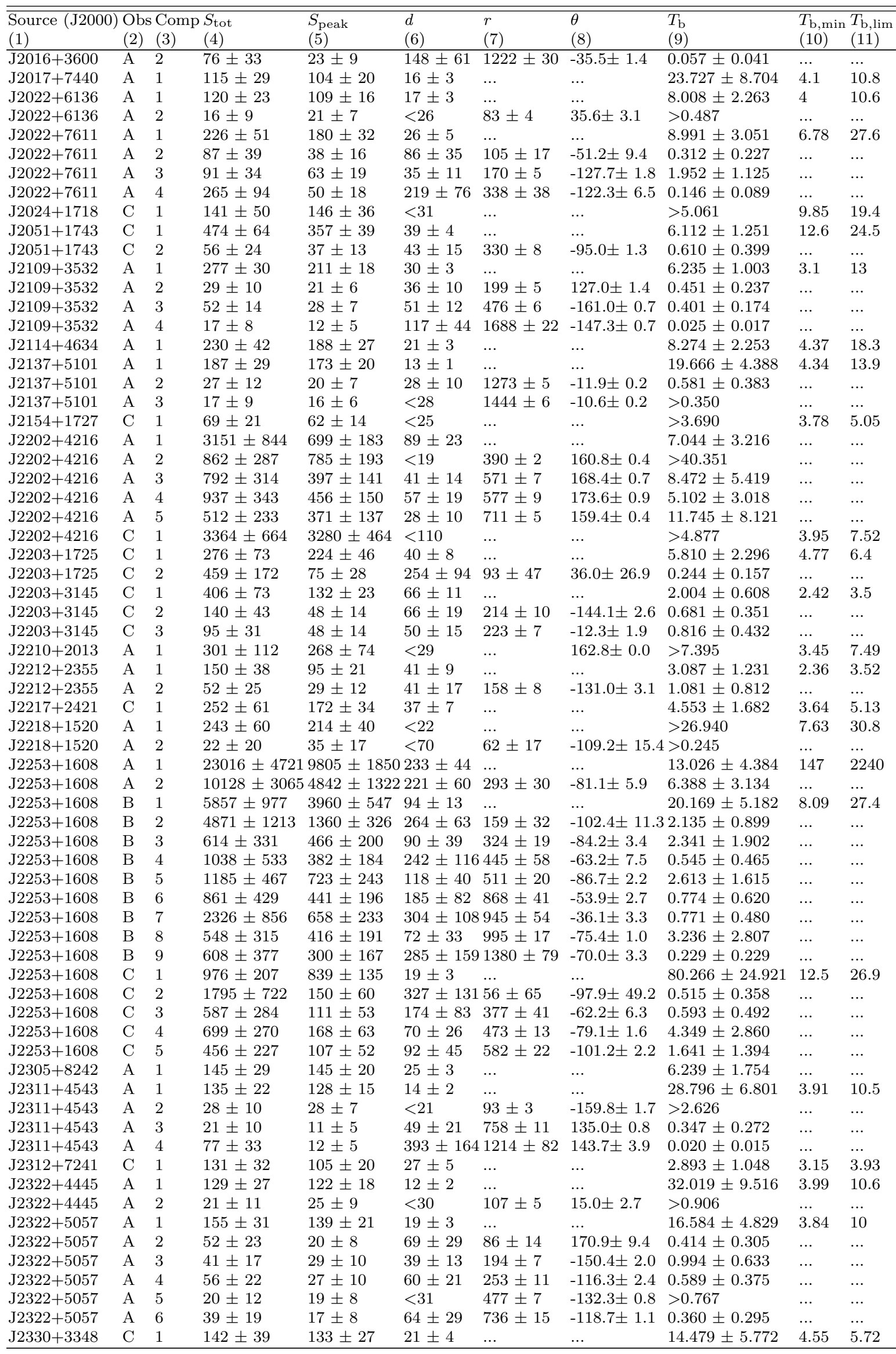


TABLE 4.7: Model fit parameters continued

\begin{tabular}{|c|c|c|c|c|c|c|c|c|c|}
\hline $\begin{array}{l}\text { Source }(\mathrm{J} 2000) \\
(1)\end{array}$ & $\begin{array}{l}\text { Obs } \\
(2)\end{array}$ & $\begin{array}{l}\text { Com } \\
(3)\end{array}$ & $\begin{array}{l}S_{\text {tot }} \\
(4)\end{array}$ & $\begin{array}{l}S_{\text {peak }} \\
(5)\end{array}$ & $\begin{array}{l}d \\
(6)\end{array}$ & $\begin{array}{l}r \\
(7)\end{array}$ & $\begin{array}{l}\theta \\
(8)\end{array}$ & $\begin{array}{l}T_{\mathrm{b}} \\
(9)\end{array}$ & $\begin{array}{ll}T_{\mathrm{b}, \min } & T_{\mathrm{b}, \lim } \\
(10) & (11)\end{array}$ \\
\hline$\overline{\mathrm{J} 2354+4553}$ & $\mathrm{~A}$ & 1 & $122 \pm 29$ & $122 \pm 20$ & $<14$ & $\ldots$ & $\ldots$ & $>29.156$ & 10.7 \\
\hline
\end{tabular}

Columns: (1) - IAU Source name(J2000); (2) - observing epochs - A: October 2010; B: May 2011 and C: October 2011; (3) - I.D. number of Gaussian model fit component; (4) - total flux density of the component [mJy]; (5) - peak flux density of the component [mJy/beam]; (6) - component size [ $\mu \mathrm{as}$ ], or upper limits; (7) - component's offset from the core $[\mu \mathrm{as}] ;(8)$ - position angle of the offset [degrees]; (9) - brightness temperature obtained from the model fit $\left[\times 10^{10} \mathrm{~K}\right]$, or lower limits; $(10)$ - visibility based estimate of the minimum brightness temperature $\left[\times 10^{10} \mathrm{~K}\right] ;(11)-$ visibility based estimate of the maximum resolved brightness temperature $\left[\times 10^{10} \mathrm{~K}\right]$. (This table is also available in a machine-readable and Virtual Observatory (VO) forms in the online journal of Nair et al. (2019).) 

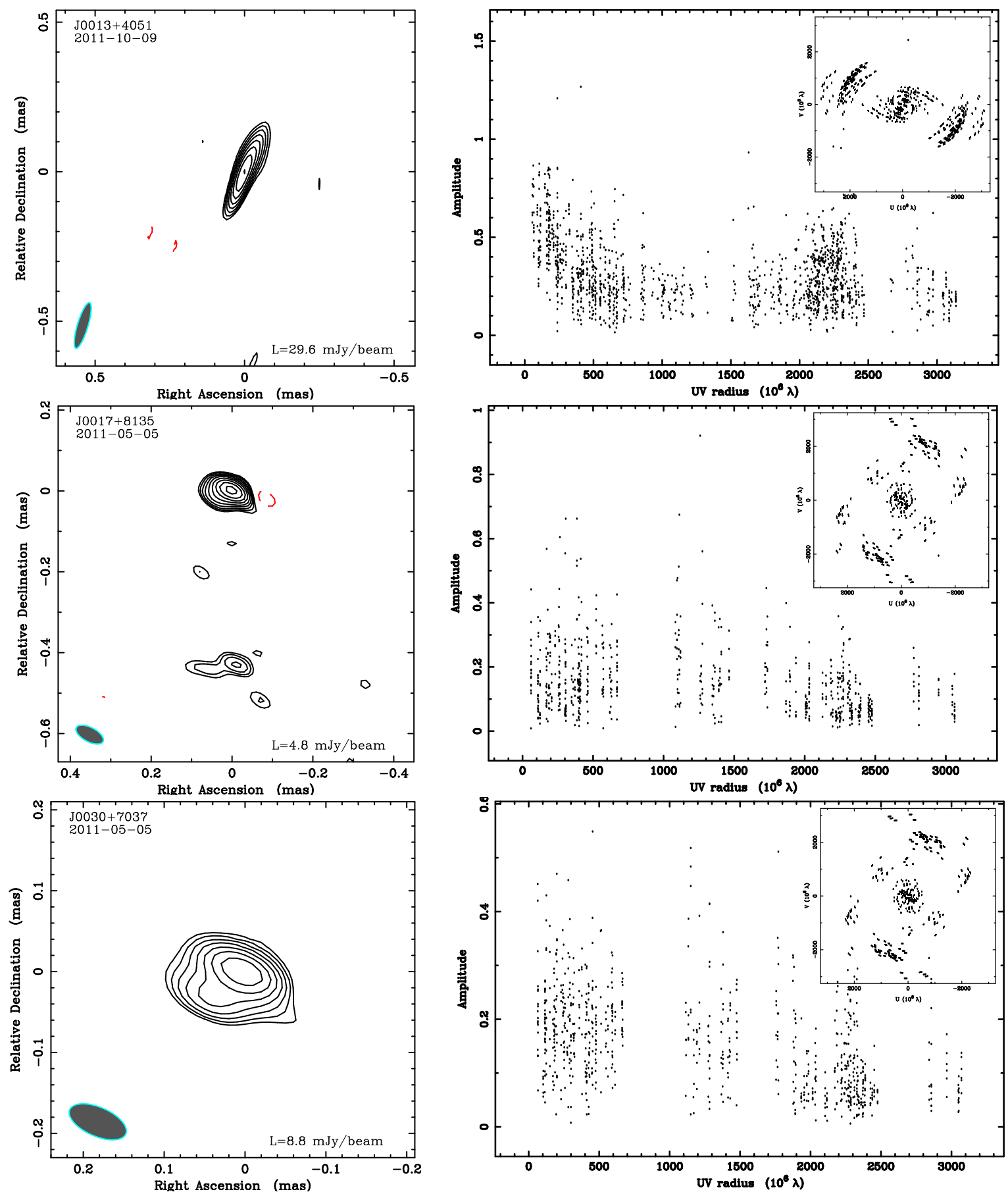

Figure 4.9: 174 contour maps of 162 unique sources imaged at $3 \mathrm{~mm}$ in this survey (left panel), shown together with the respective radial amplitude distributions (right panel) and $u v$-coverages (inset in the right panel) of the respective visibility datasets. The contouring of images is made at $3 \sigma_{\mathrm{rms}} \times(-1,1, \sqrt{2}, 2, \ldots)$ levels, with $\sigma_{\mathrm{rms}}$ representing the off-source r.m.s noise in the residual image. 

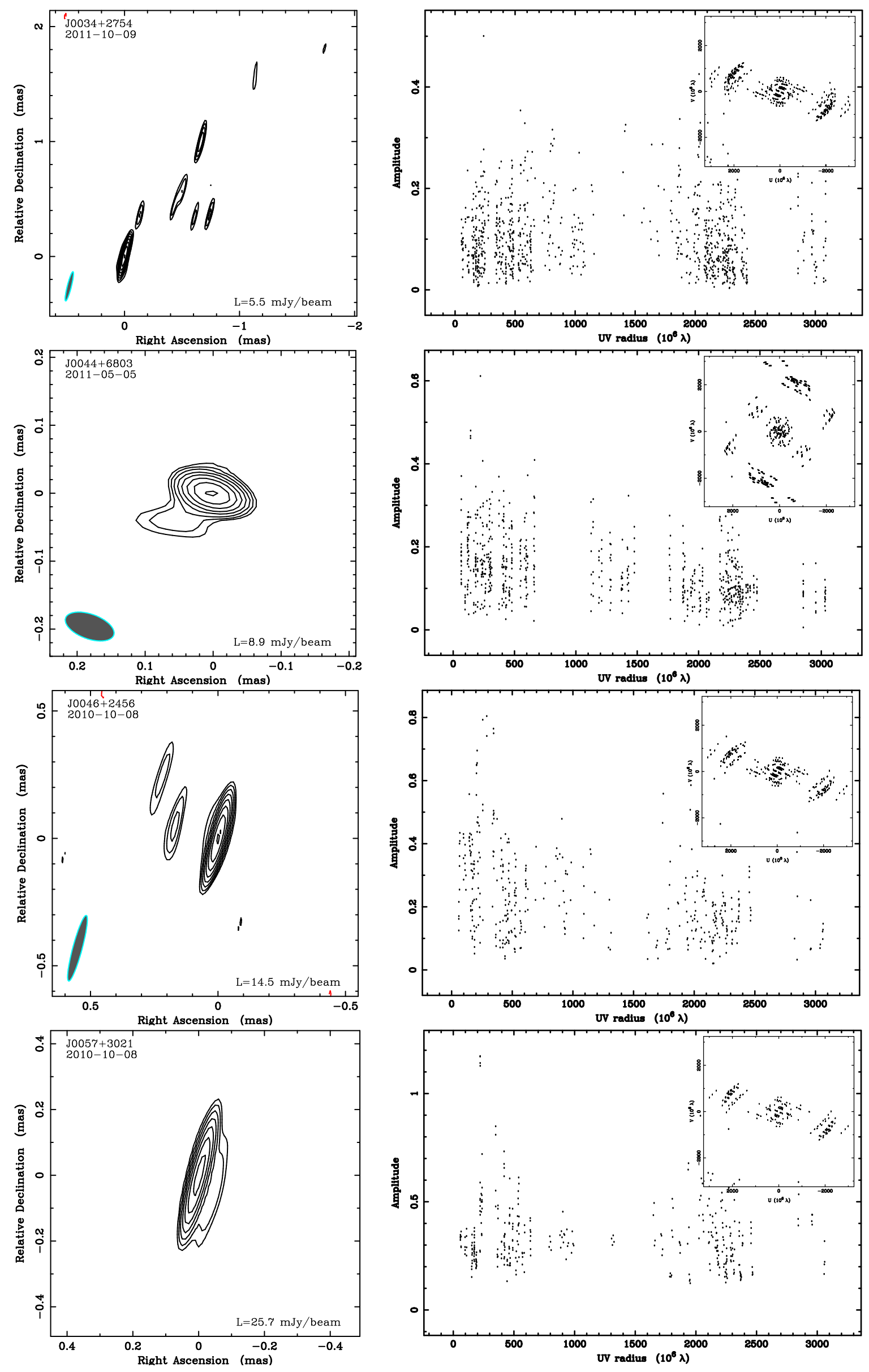

Figure 4.10: $3 \mathrm{~mm}$ maps continued 

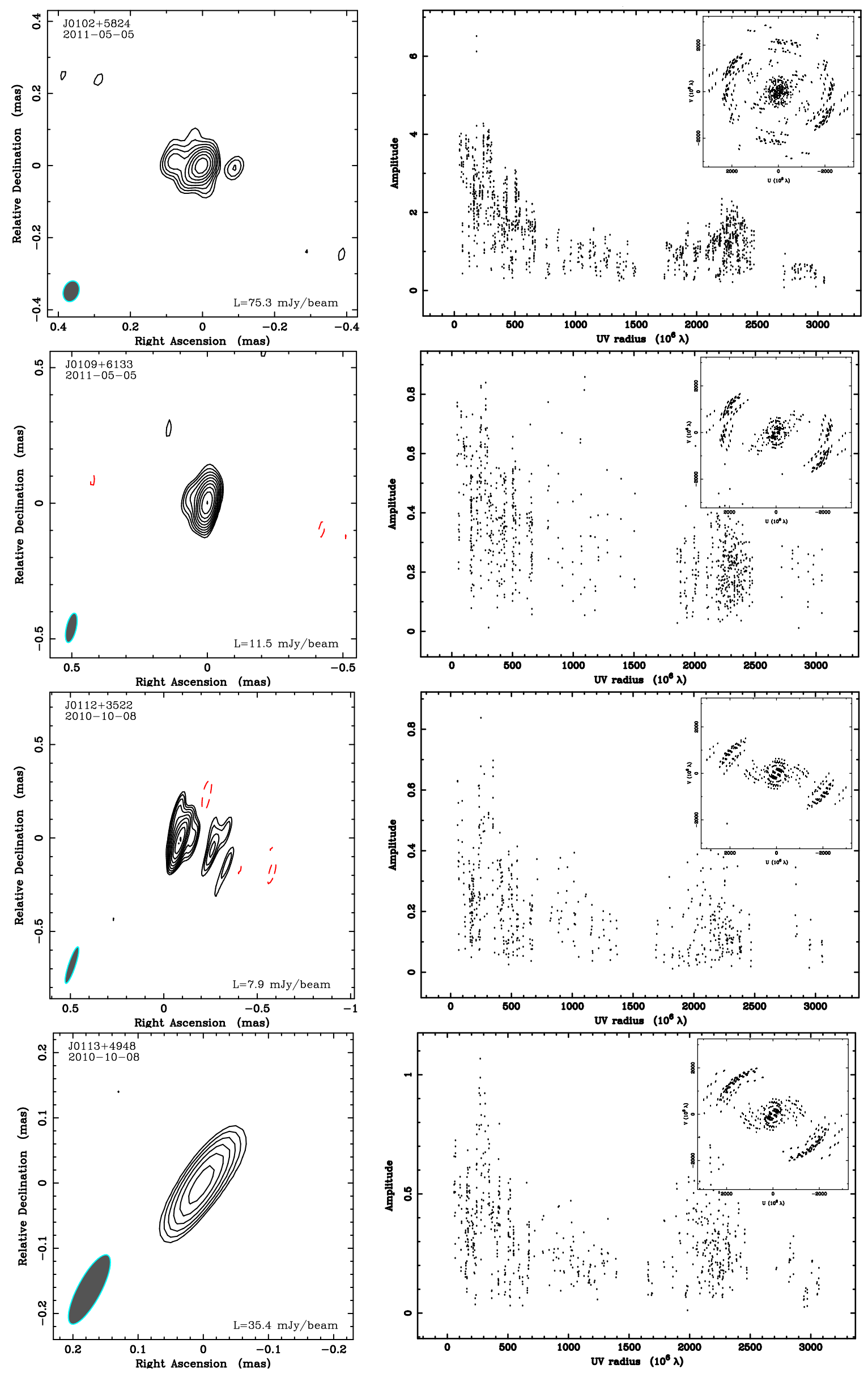

Figure 4.11: $393 \mathrm{~mm}$ maps continued 

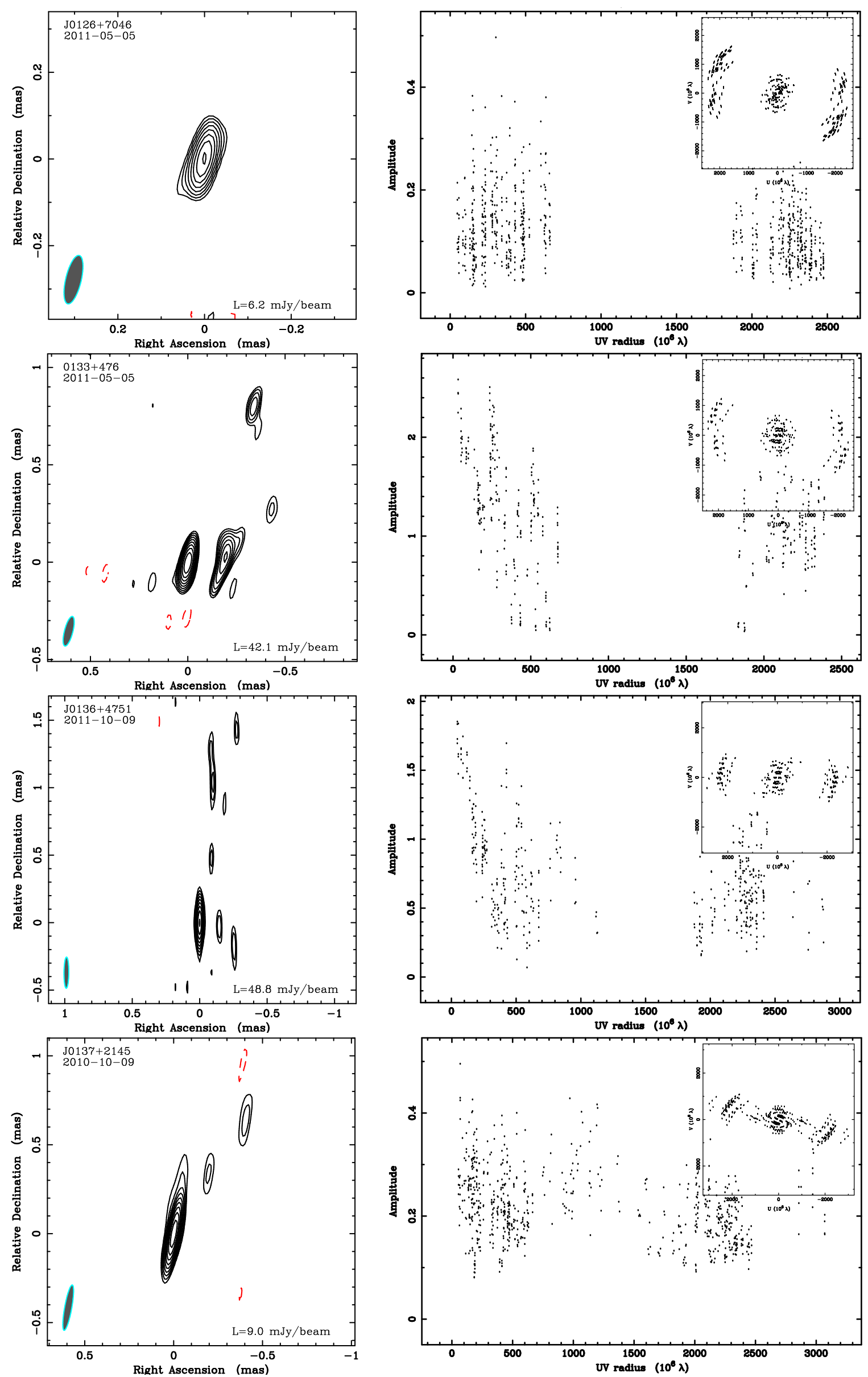

FIGURE 4.12: $394 \mathrm{~mm}$ maps continued 

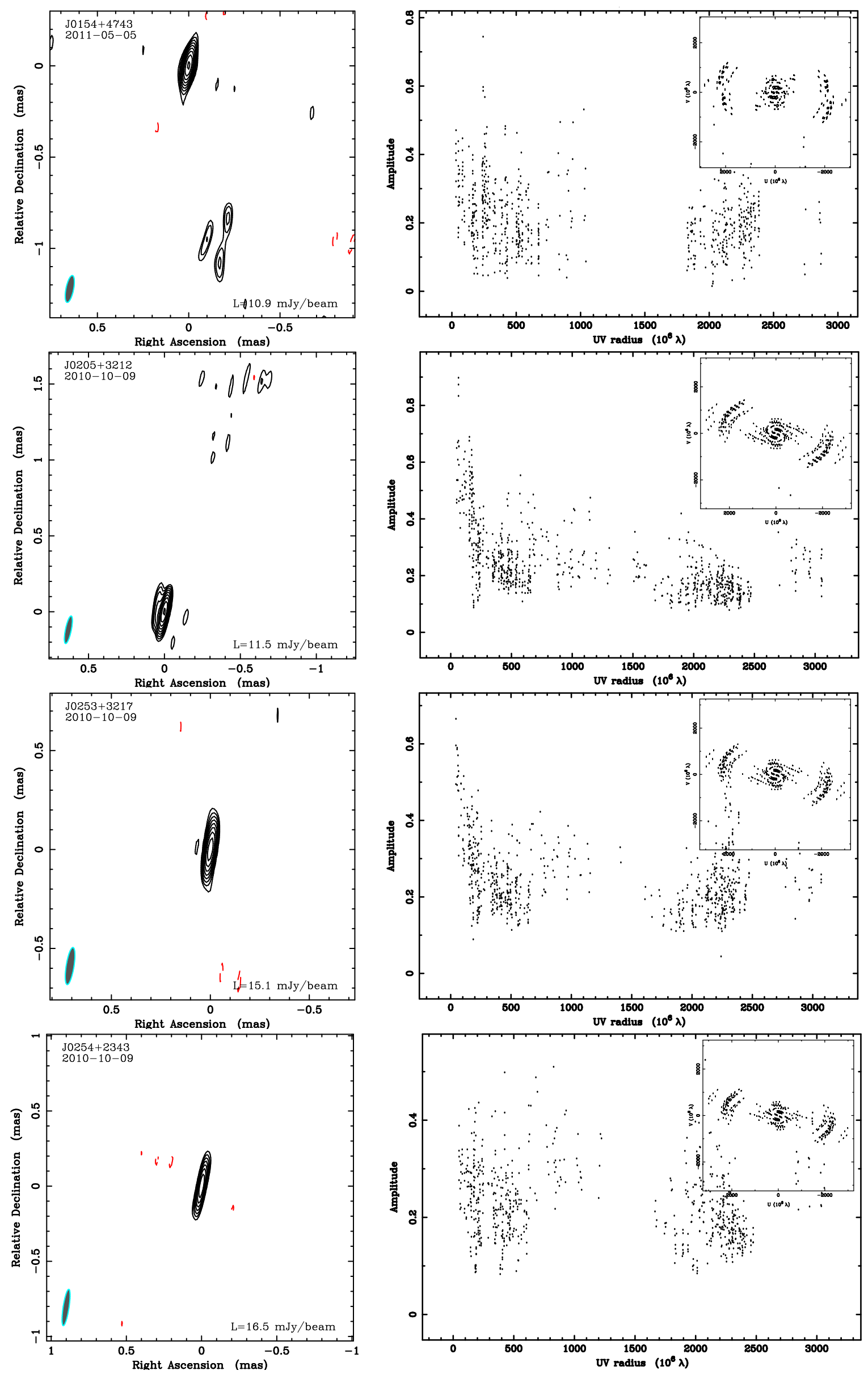

FIGURE 4.13: 395 maps continued 

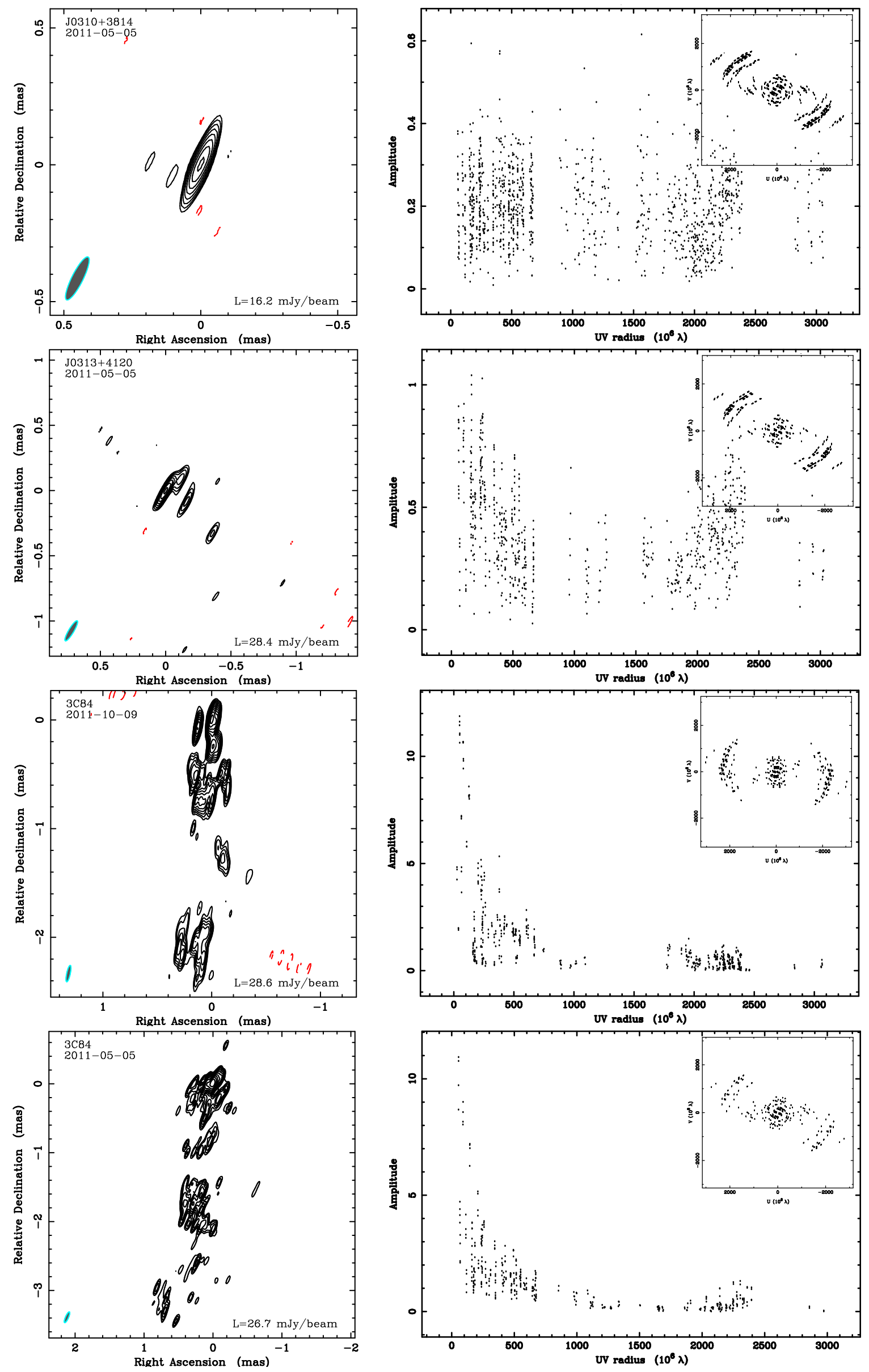

Figure 4.14: $3 \mathrm{~mm}$ maps continued 

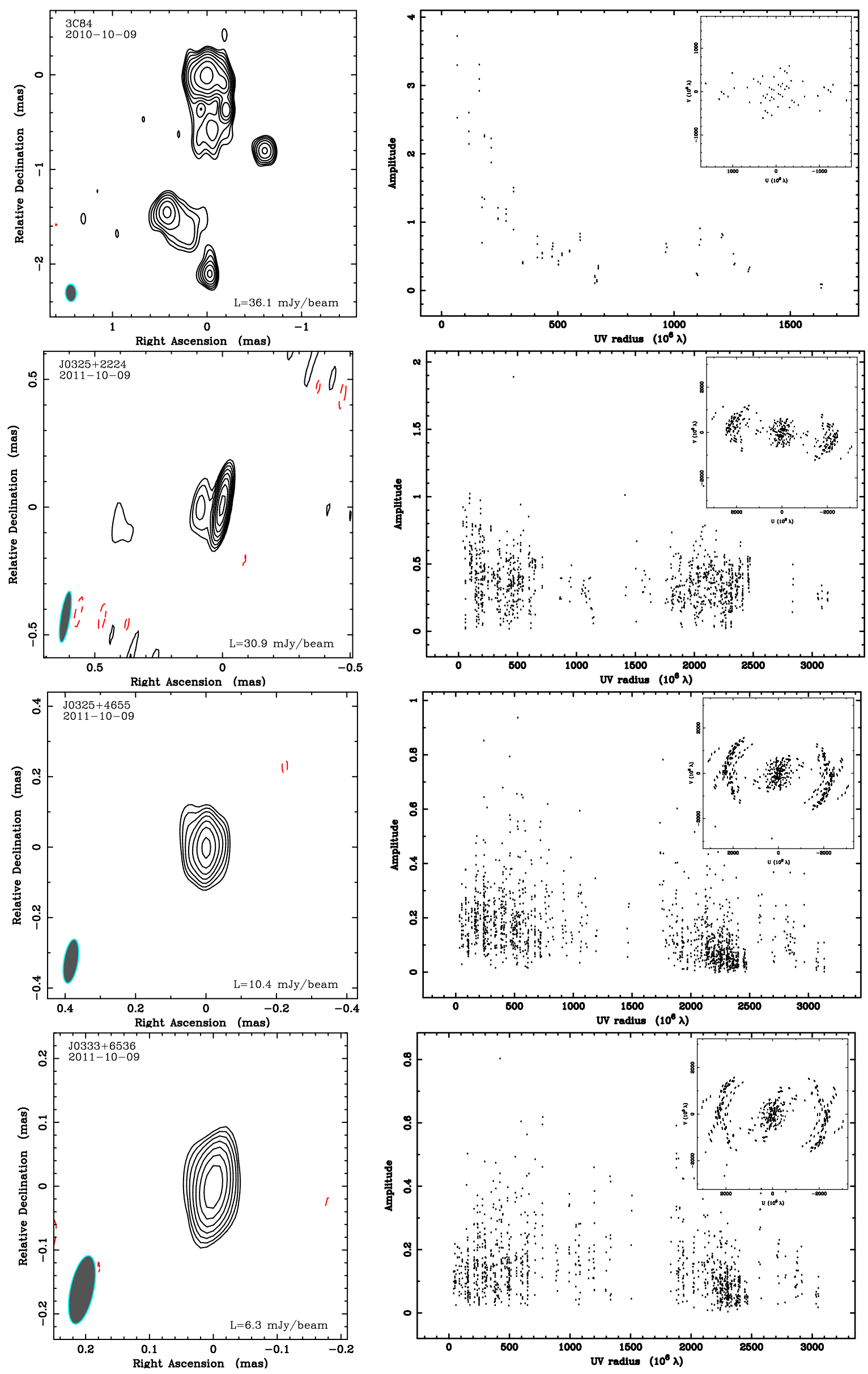

Figure 4.15: 397 maps continued 

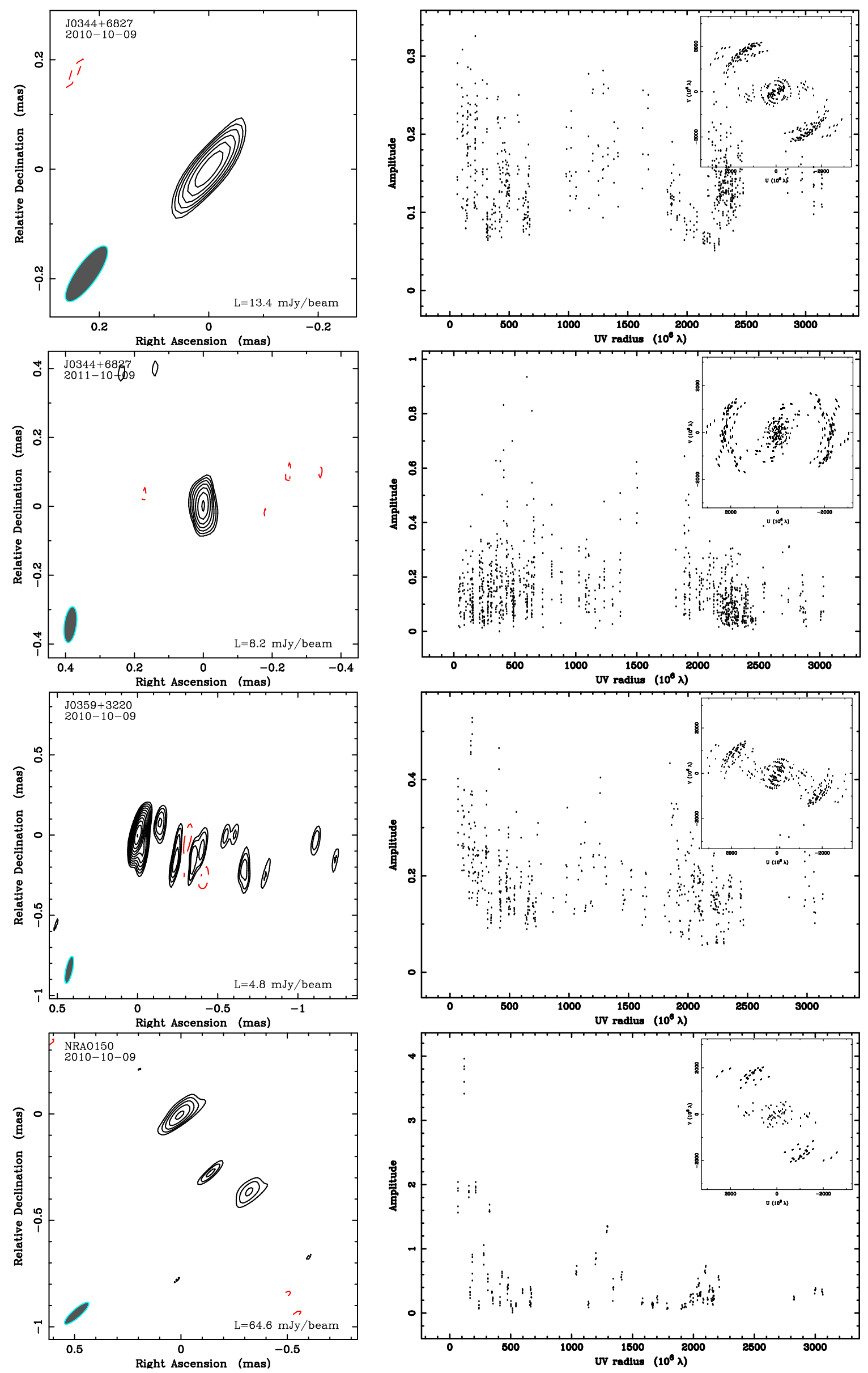

Figure 4.16: 398 maps continued 

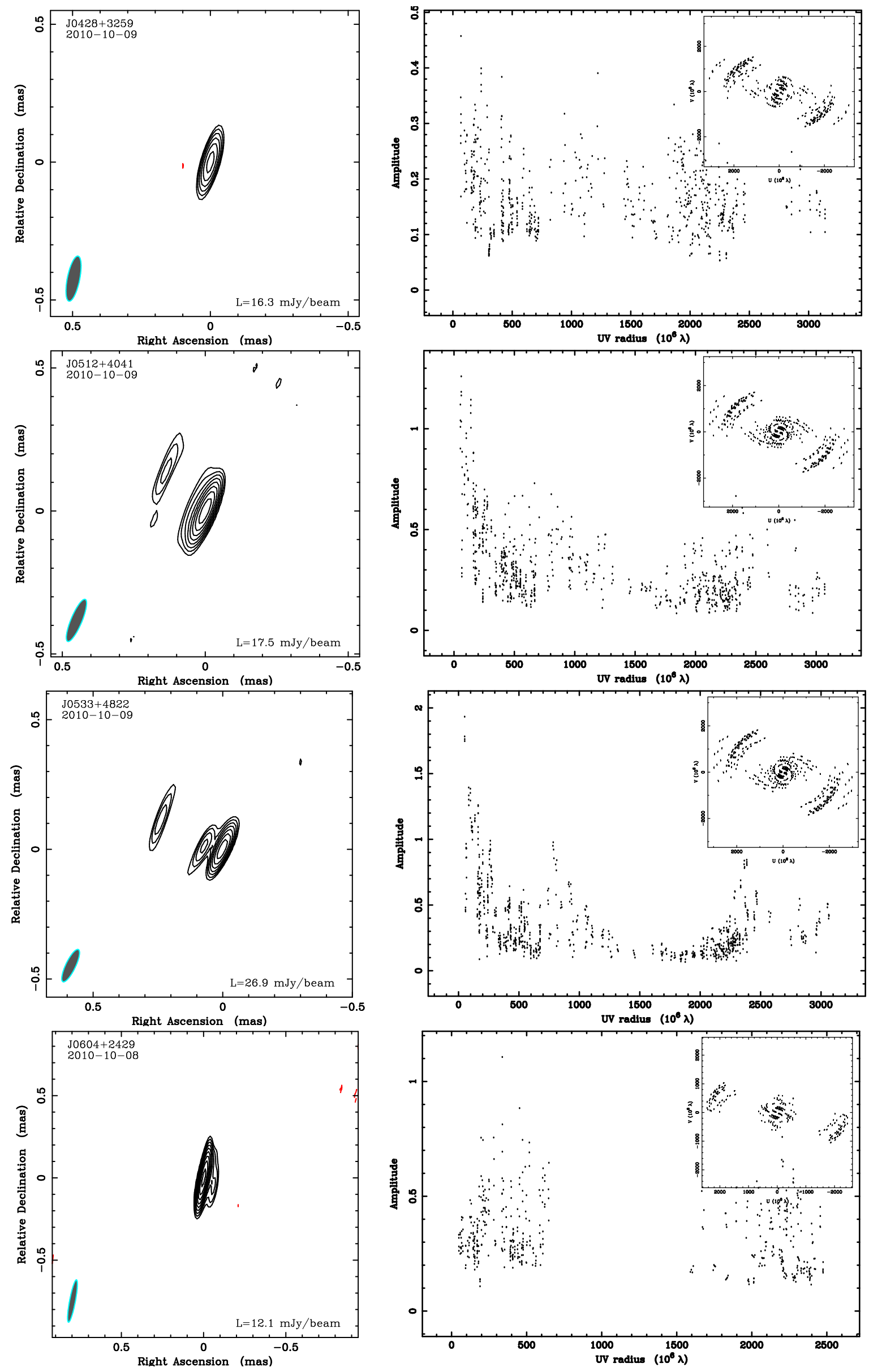

Figure 4.17: $3 \mathrm{~mm}$ maps continued 

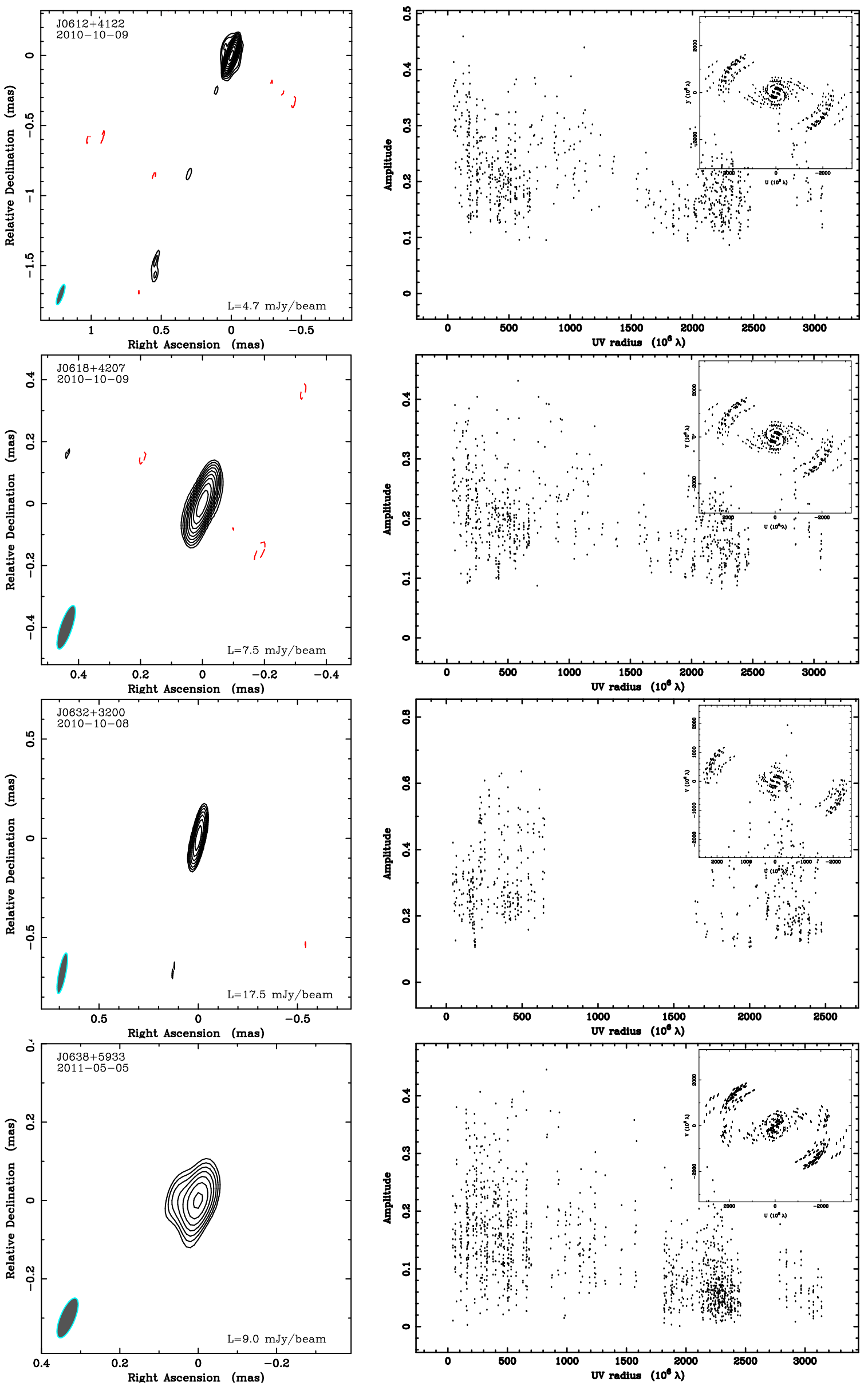

Figure 4.18: 3100 maps continued 

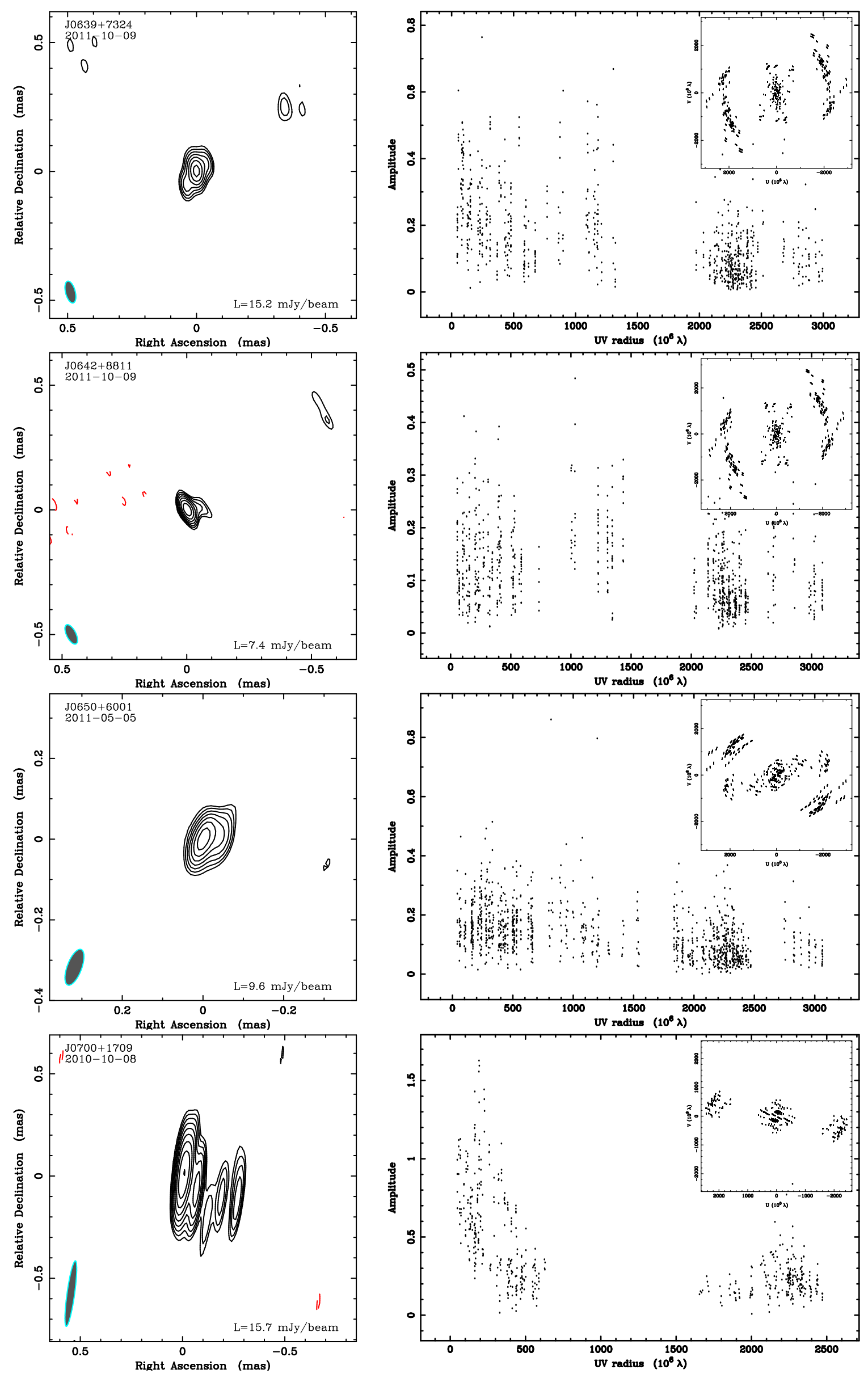

Figure 4.19: $3 \mathrm{~mm}$ maps continued 

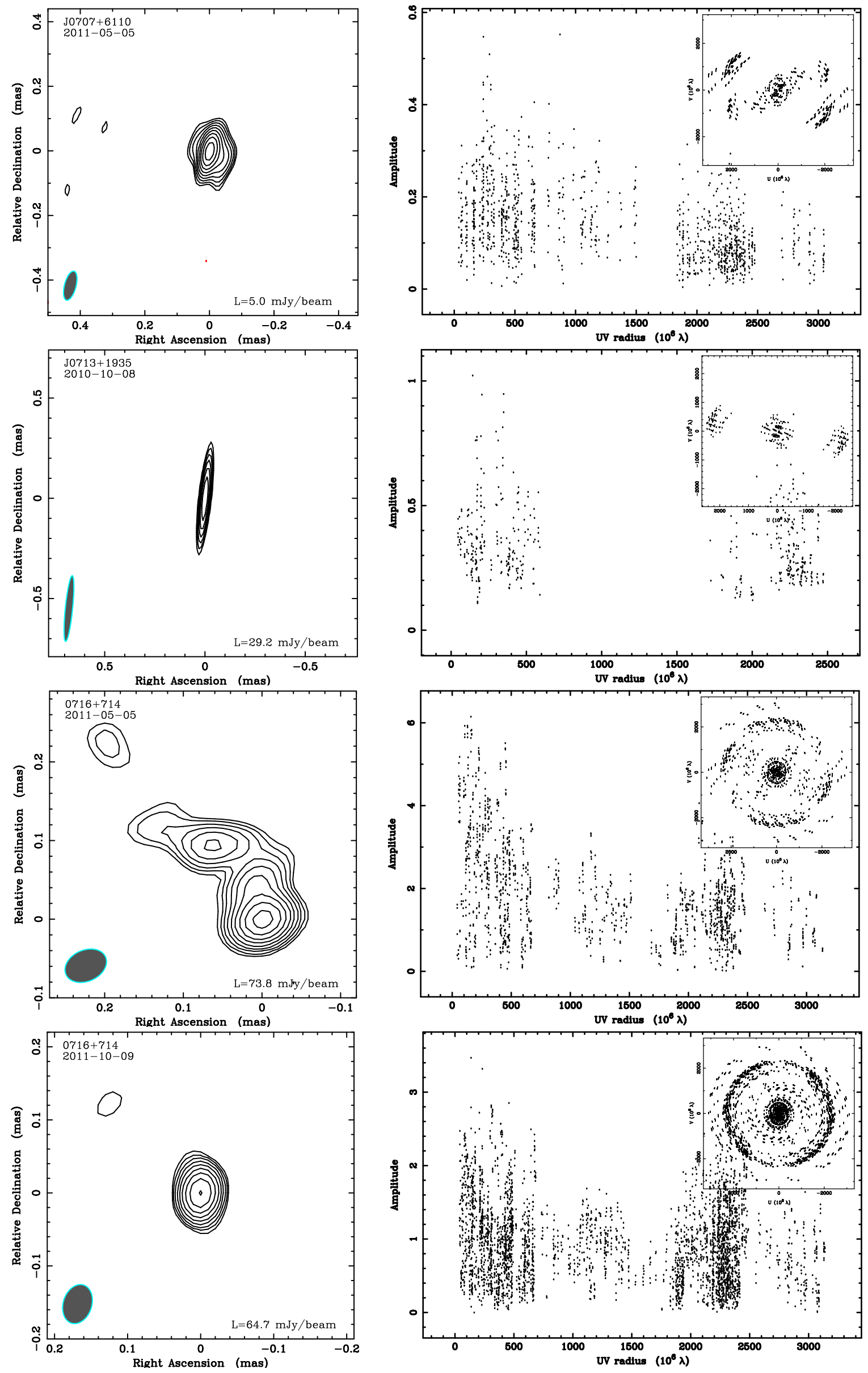

Figure 4.20: $3 \mathrm{~mm}$ maps continued 

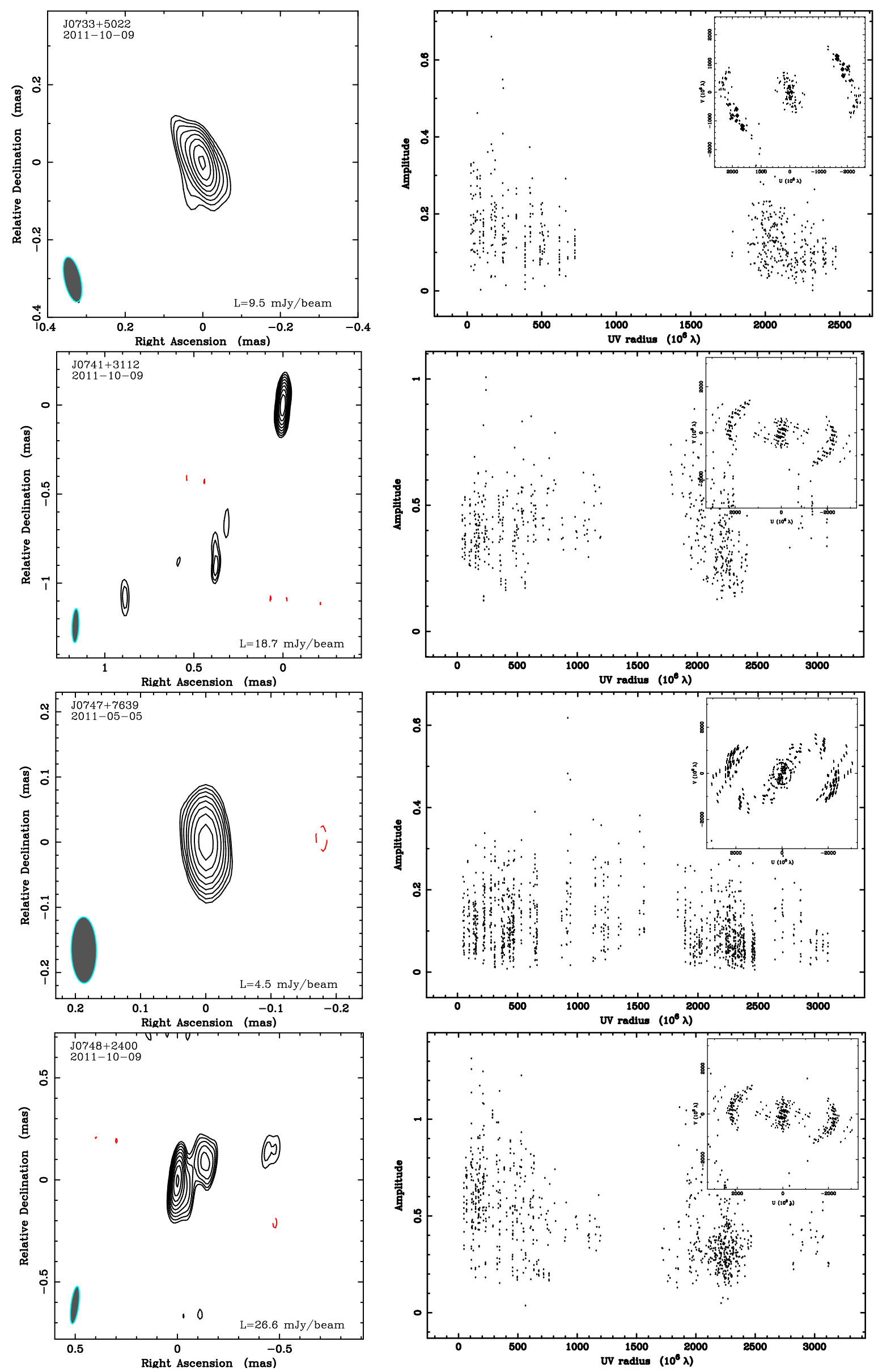

Figure 4.21: 3103 maps continued 

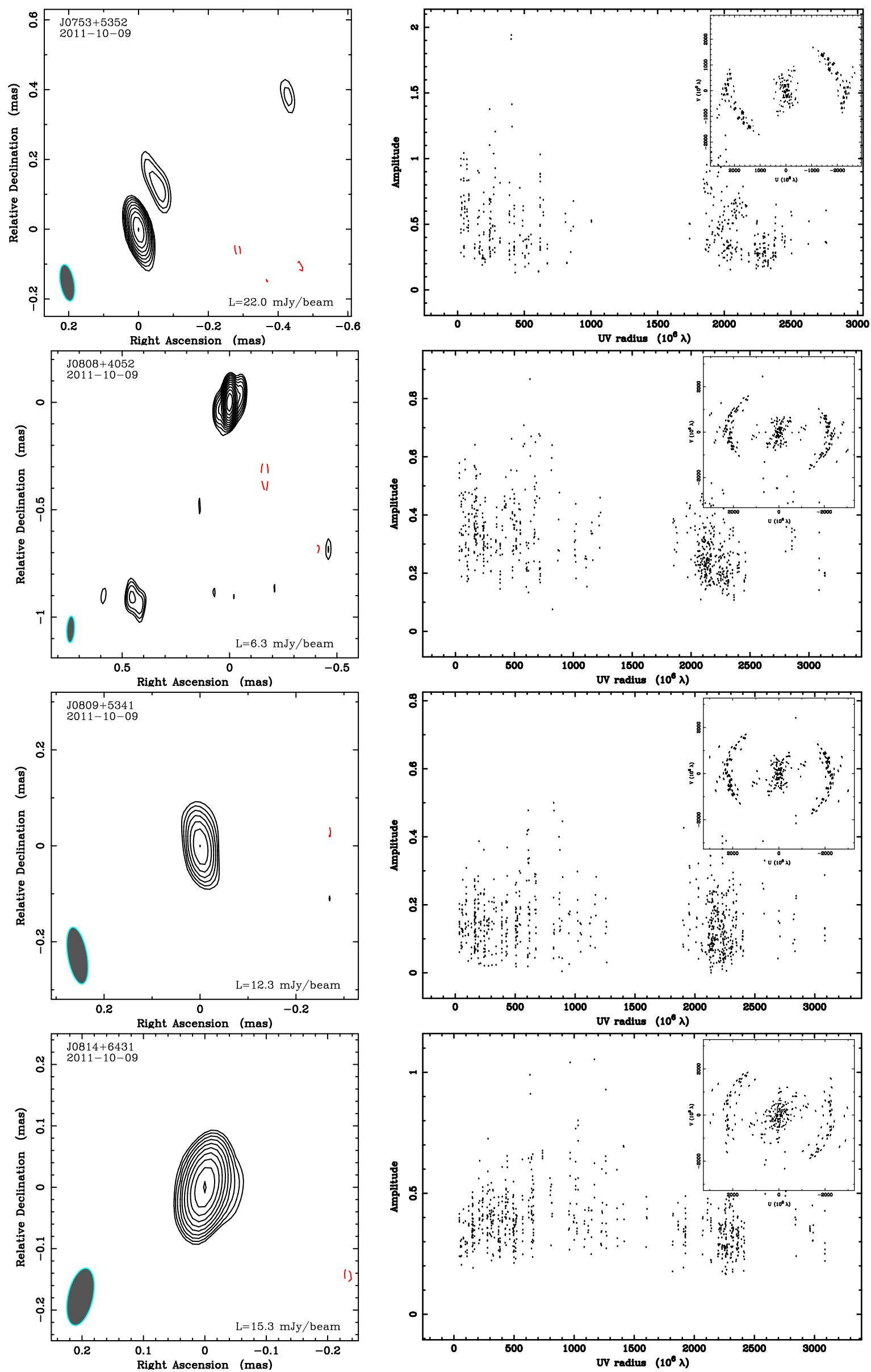

Figure 4.22: 3 mm maps continued 

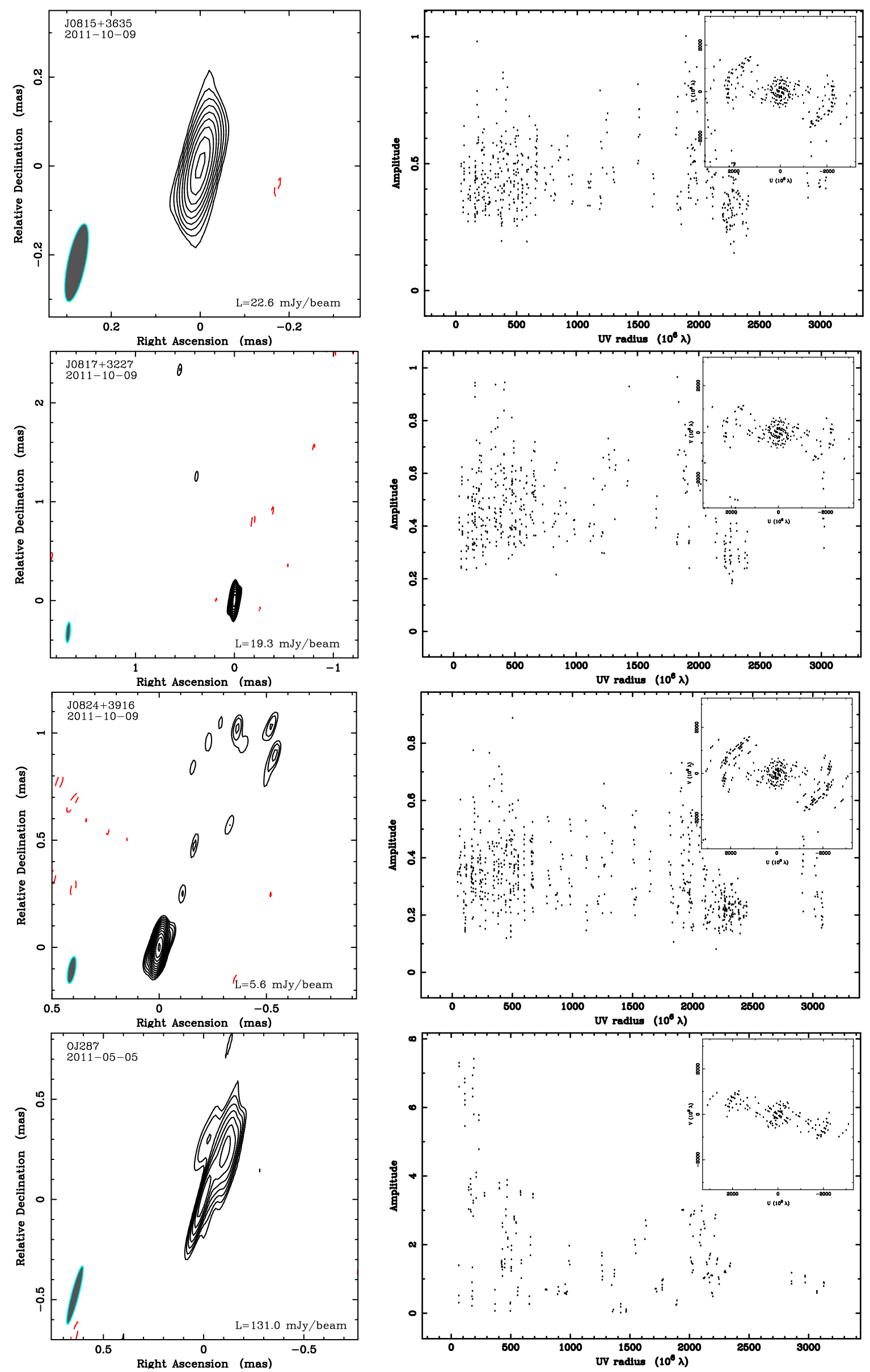

Figure 4.23: $3 \mathrm{~mm}$ maps continued 

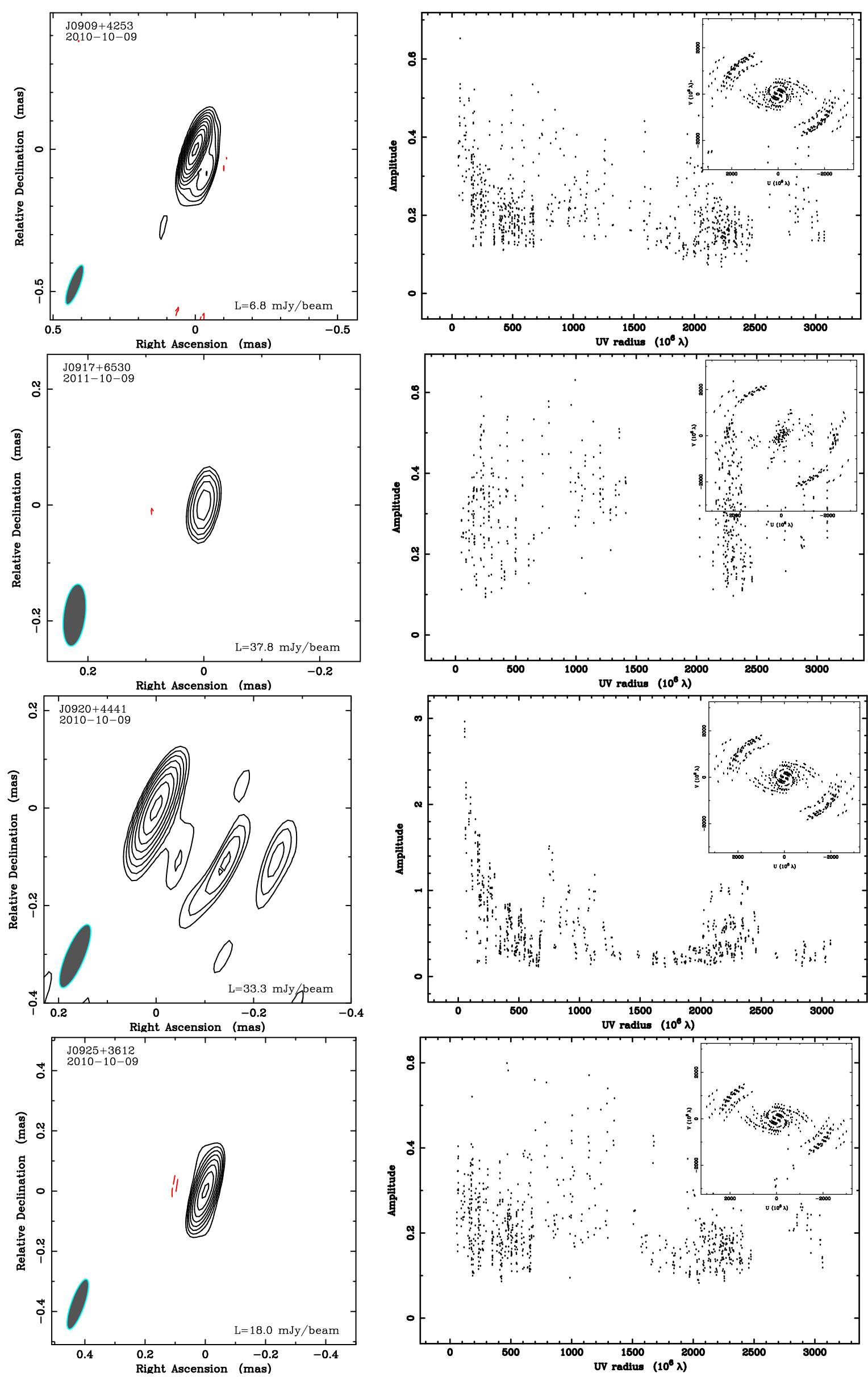

Figure 4.24: 3106 maps continued 

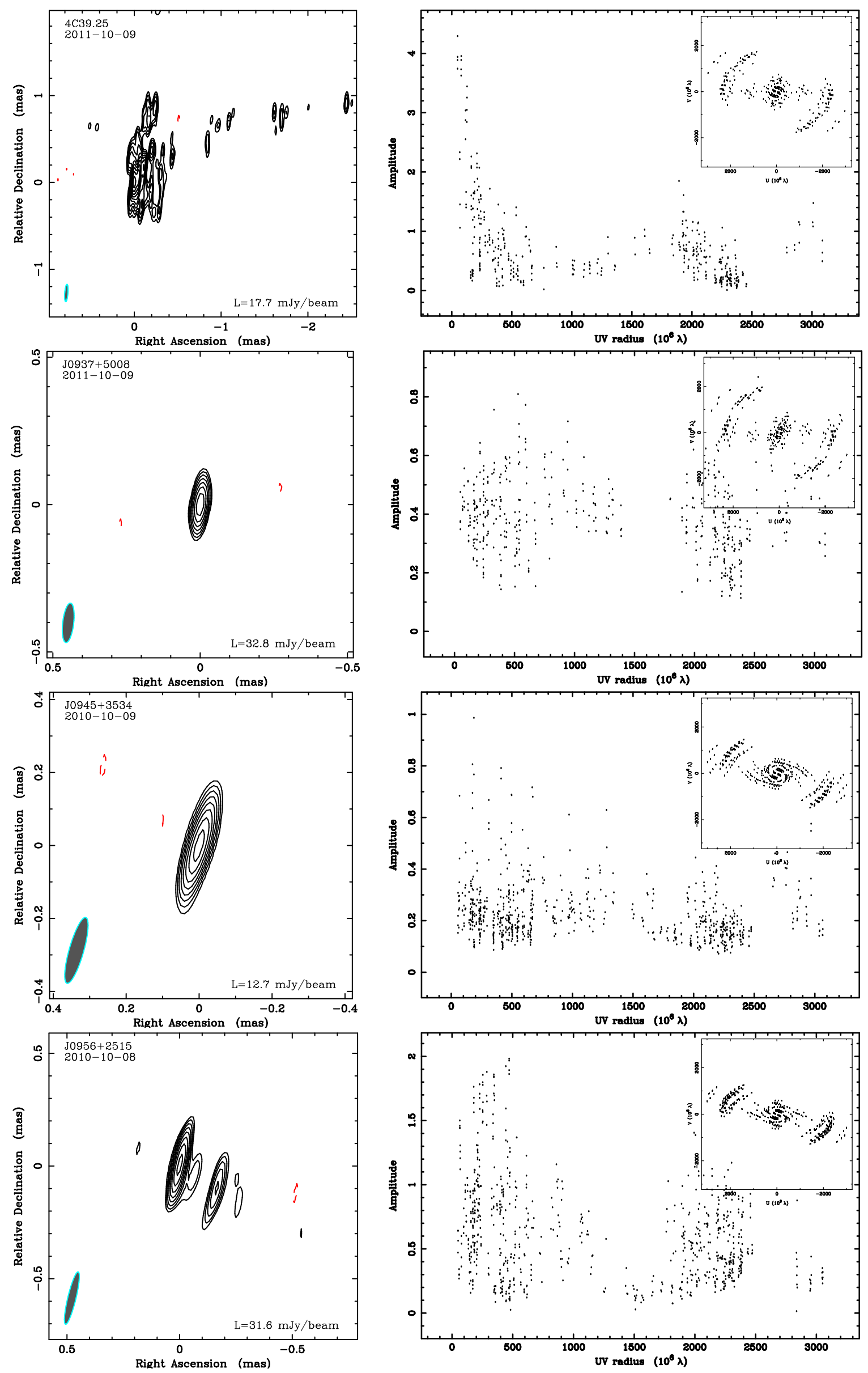

Figure 4.25: 3107 maps continued 

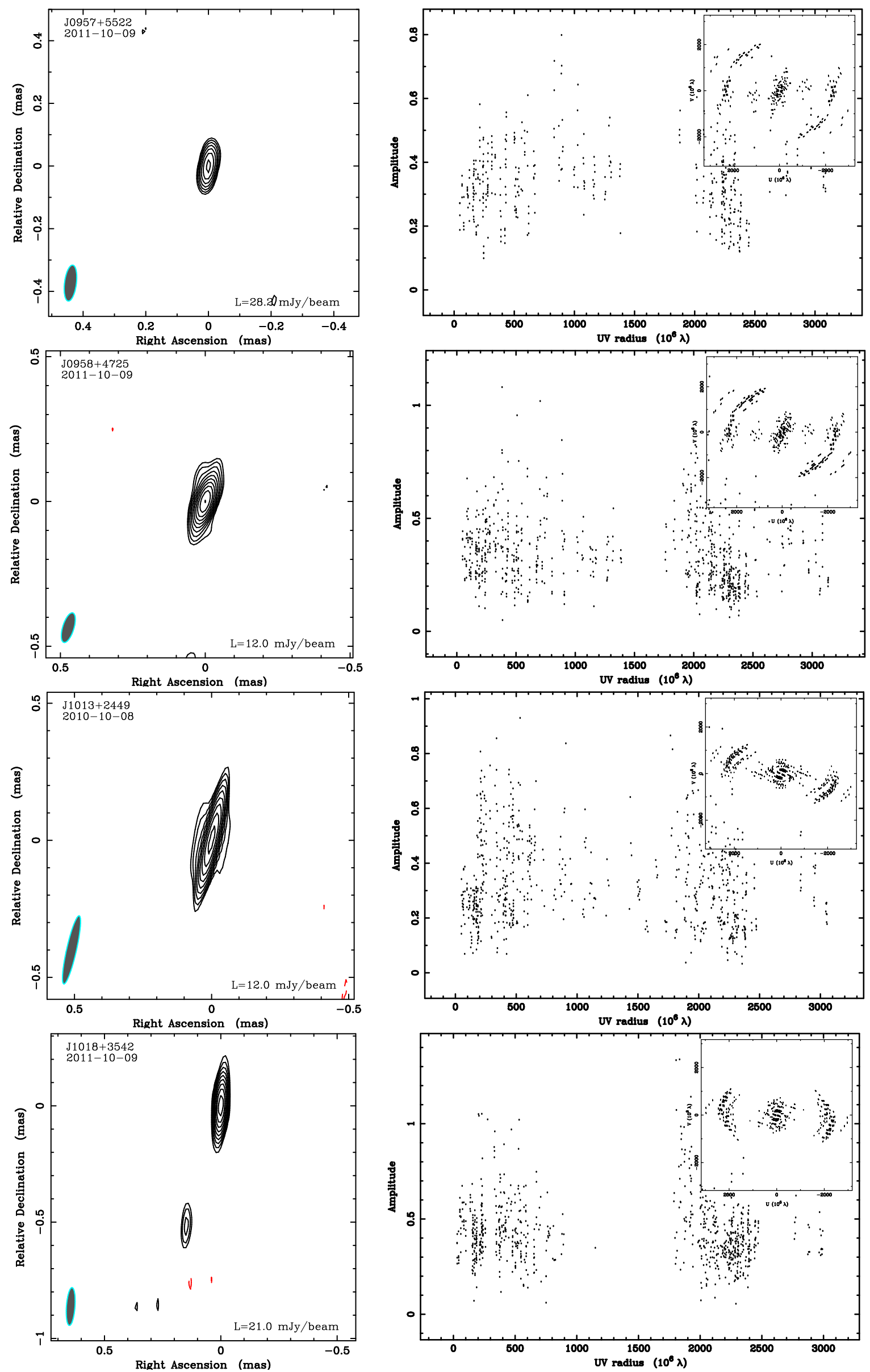

Figure 4.26: $3 \mathrm{~mm}$ maps continued 

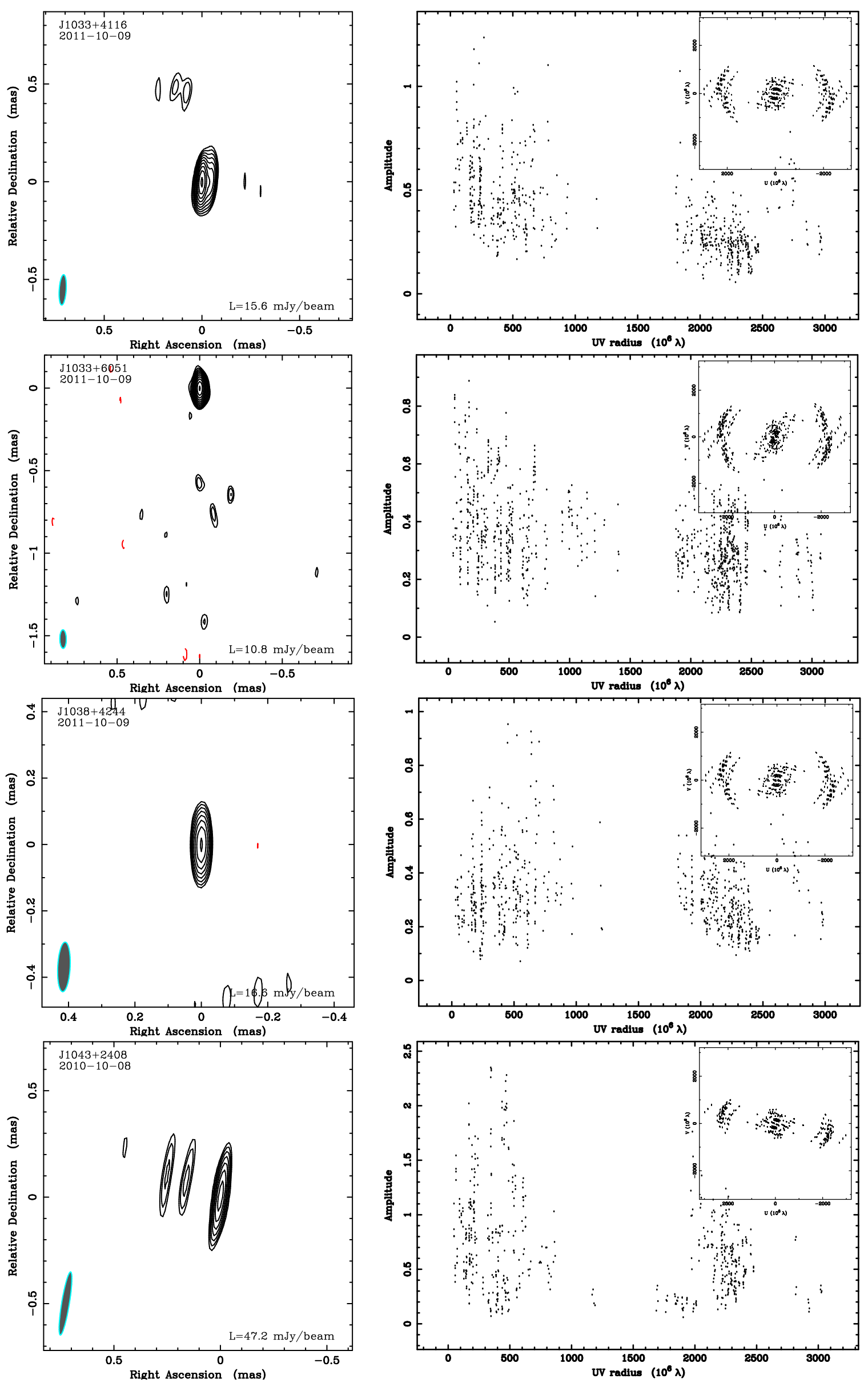

Figure 4.27: 3109 maps continued 

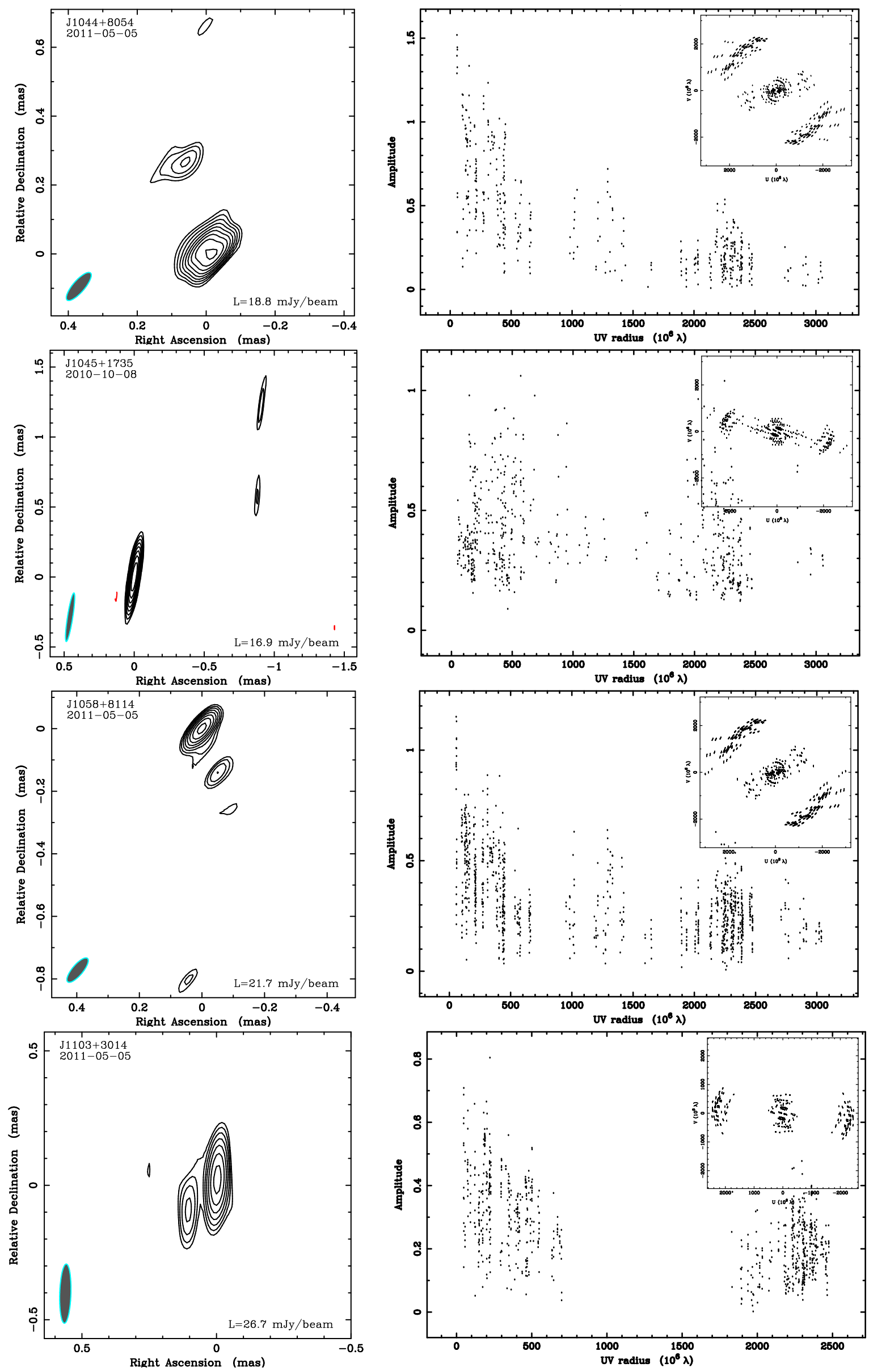

Figure 4.28: $3 \mathrm{~mm}$ maps continued 

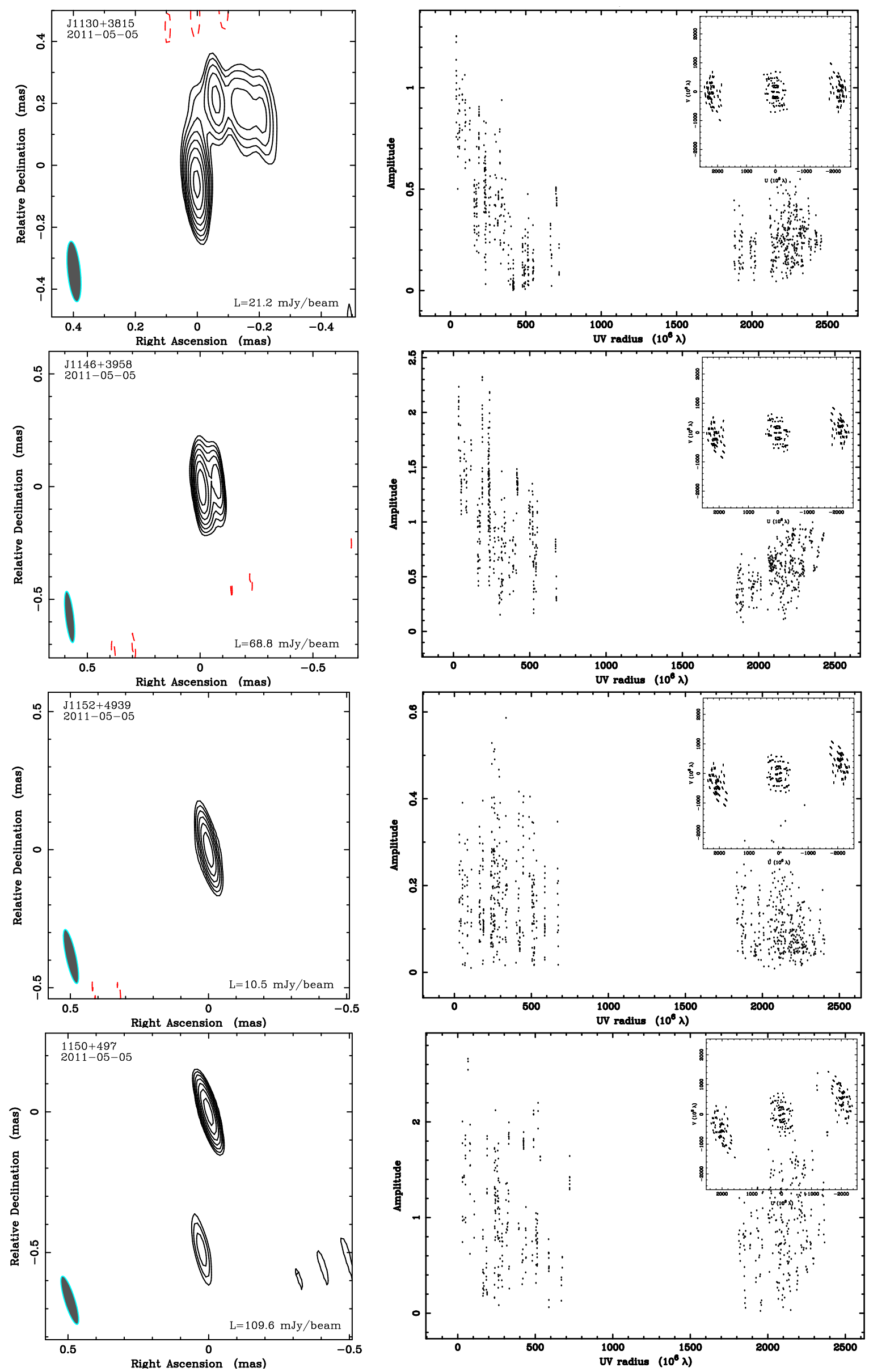

Figure 4.29: 3111 maps continued 

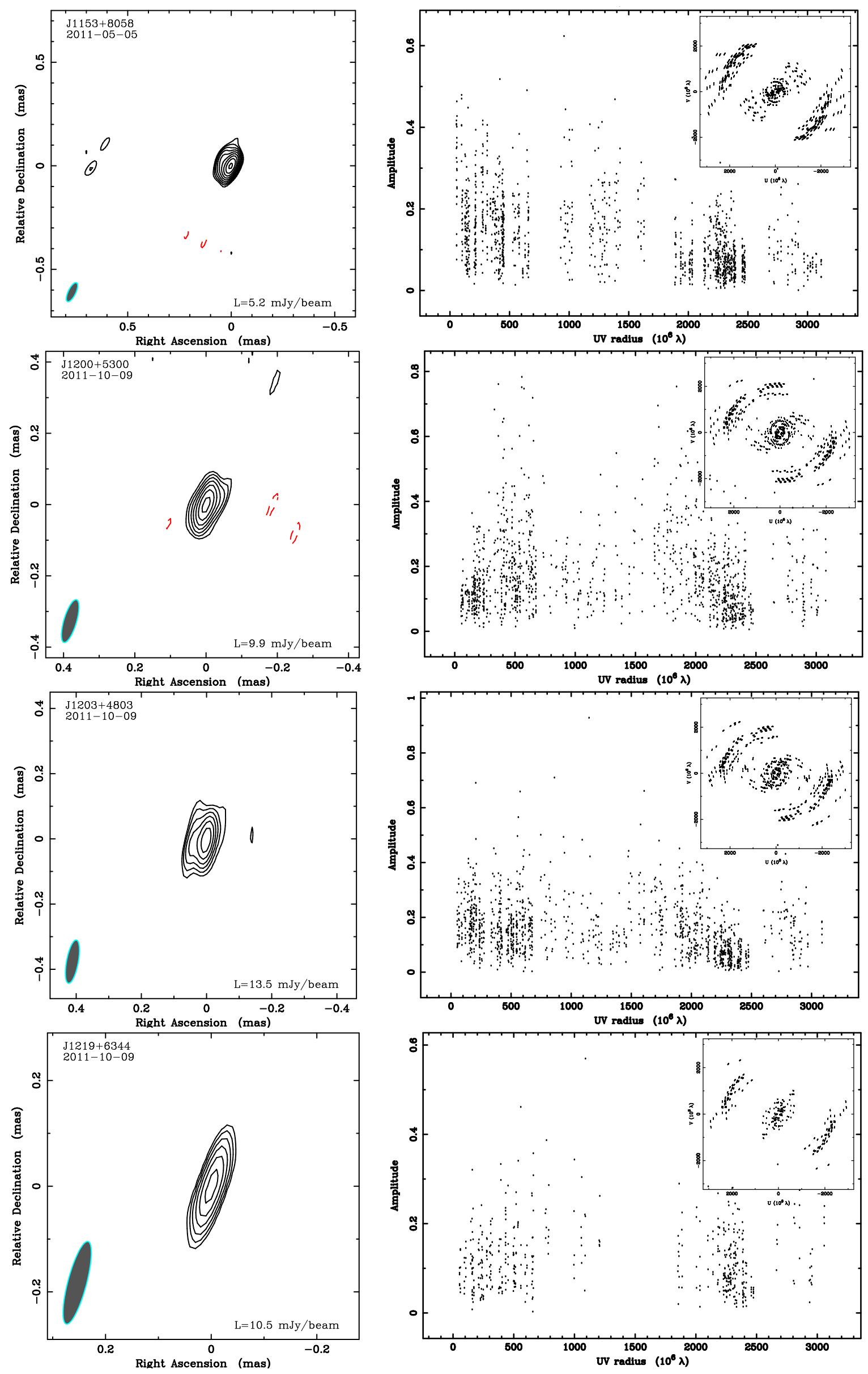

Figure 4.30: $3 \mathrm{~mm}$ maps continued 

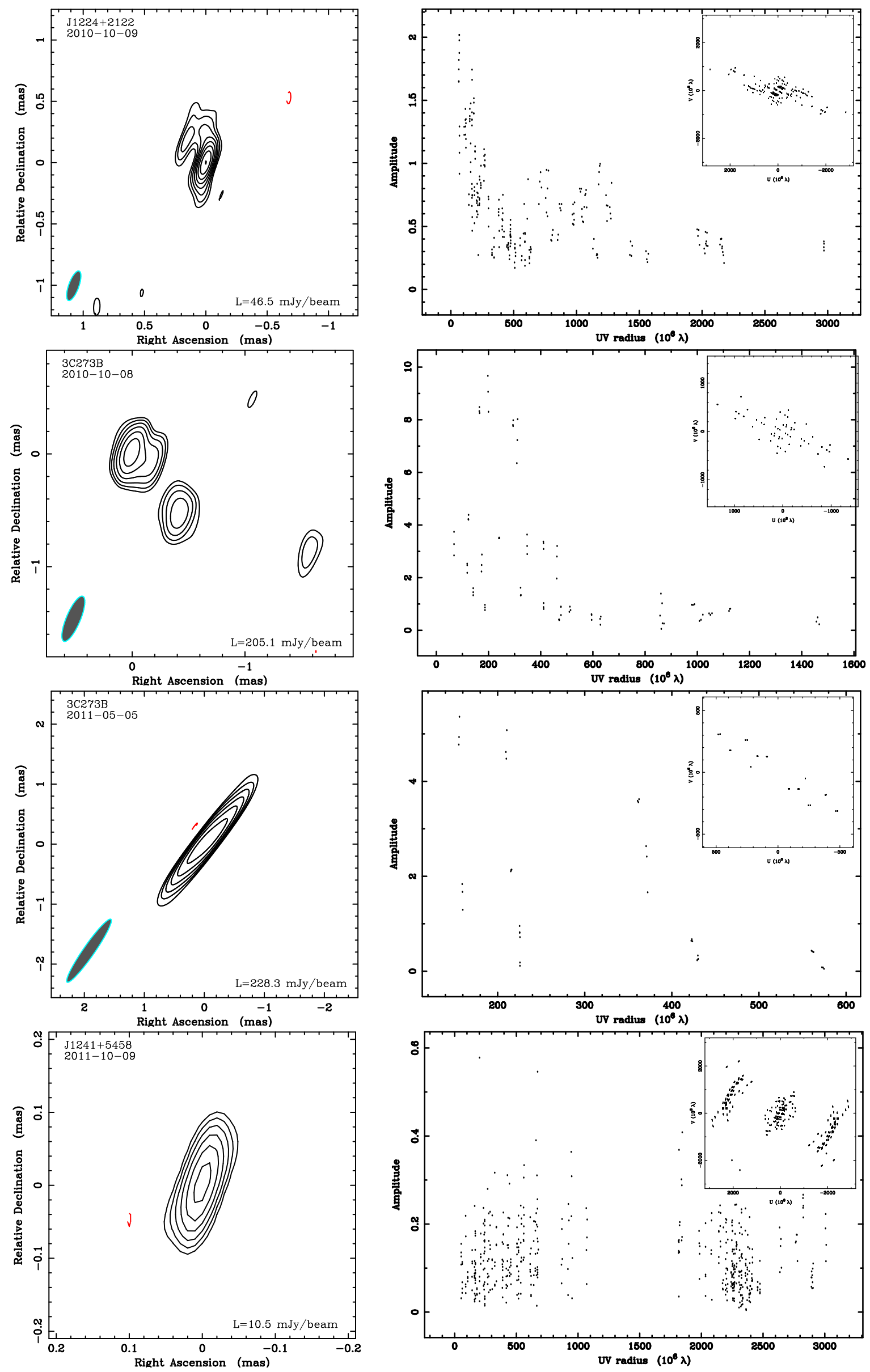

Figure 4.31: $3 \mathrm{~mm}$ maps continued 

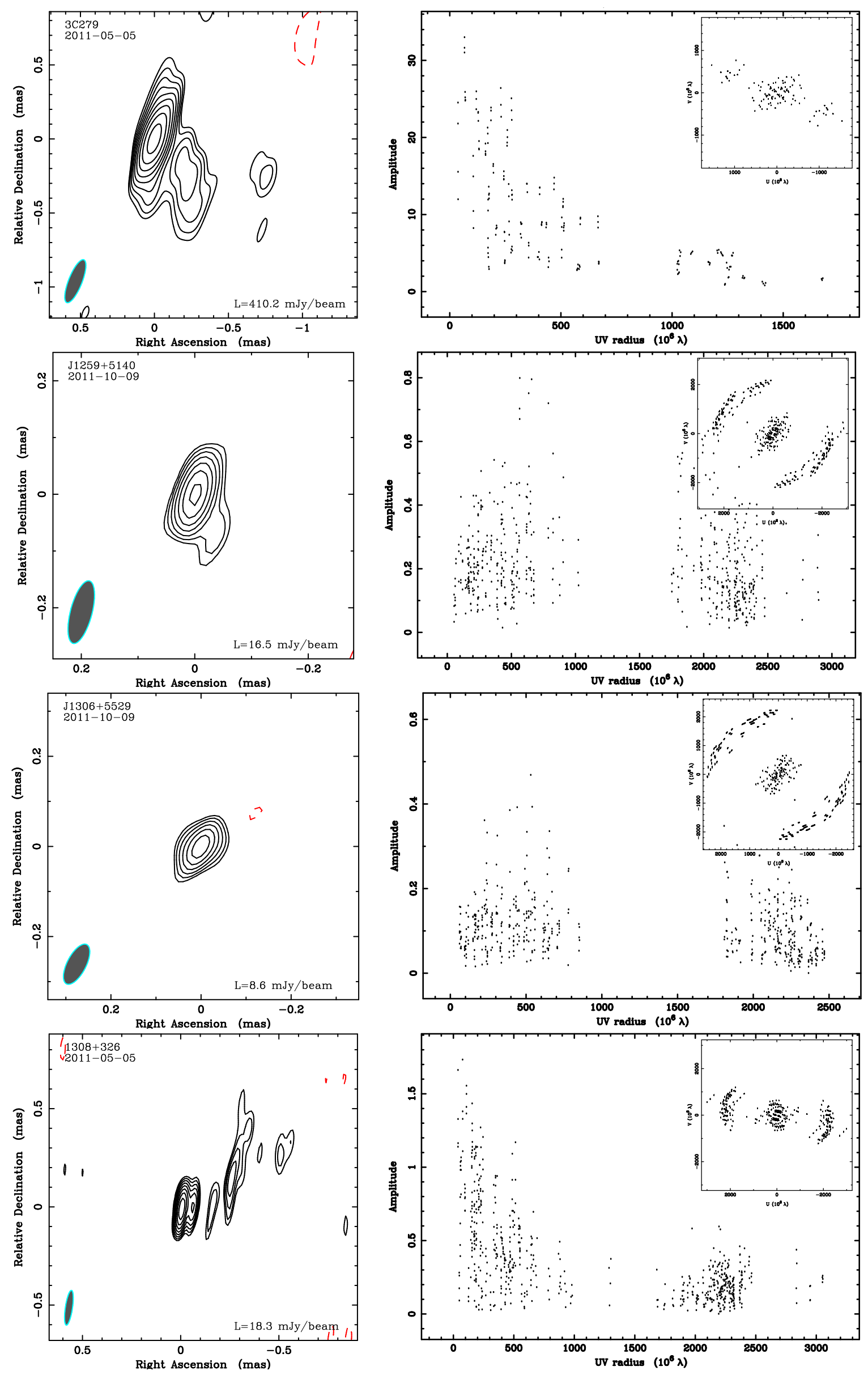

Figure 4.32: 3114 maps continued 

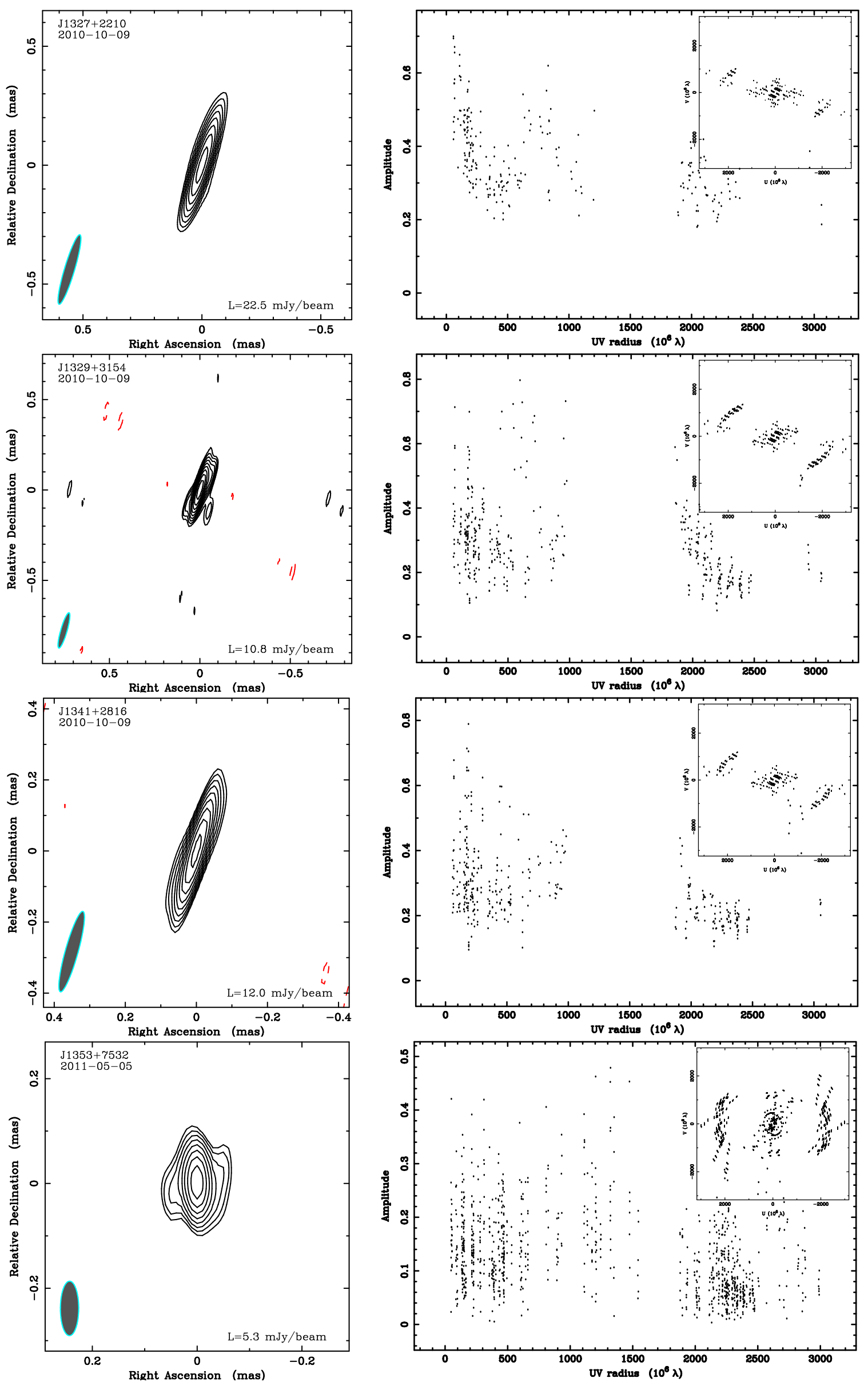

Figure 4.33: $3 \mathrm{~mm}$ maps continued 

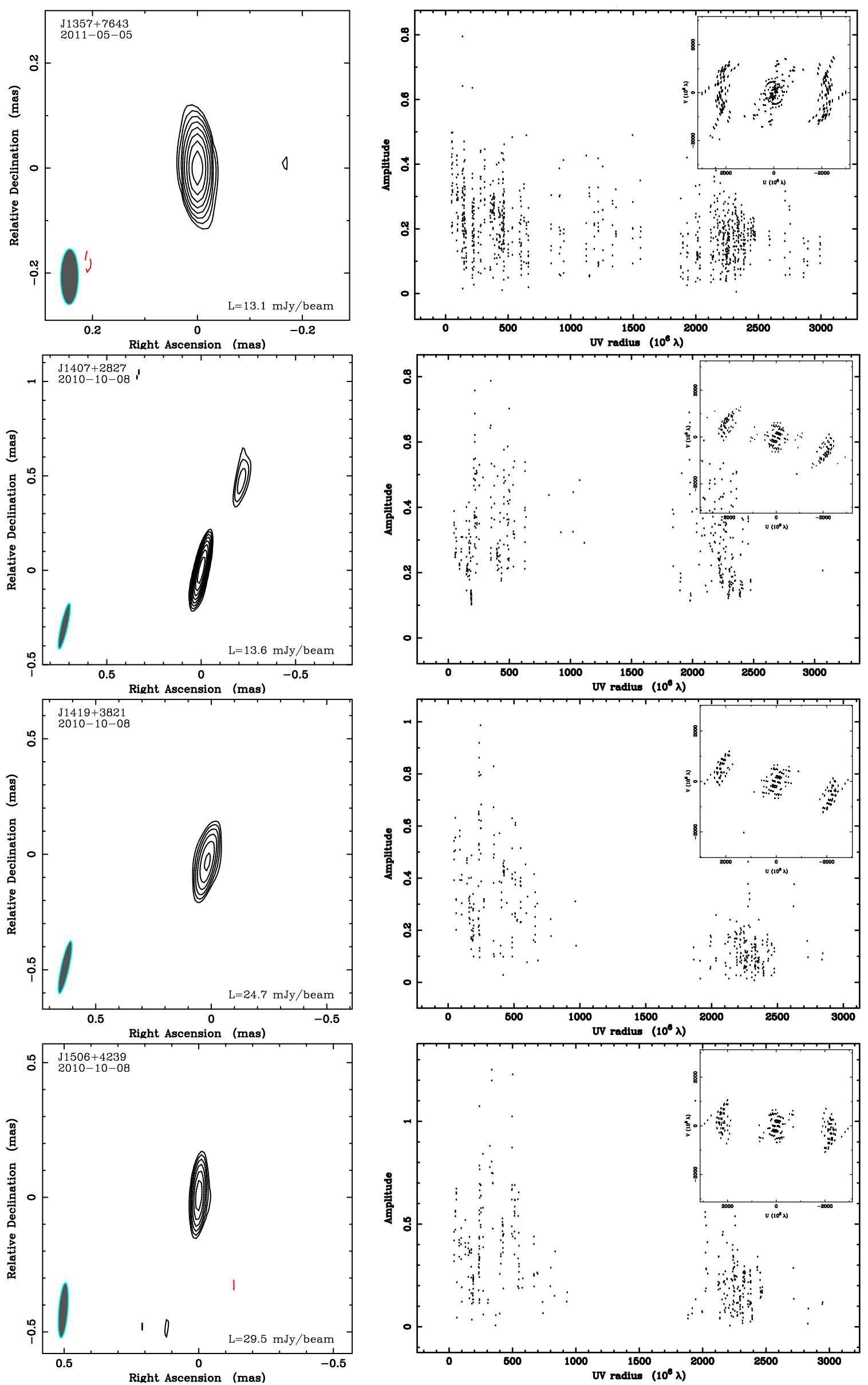

Figure 4.34: $3 \mathrm{~mm}$ maps continued 

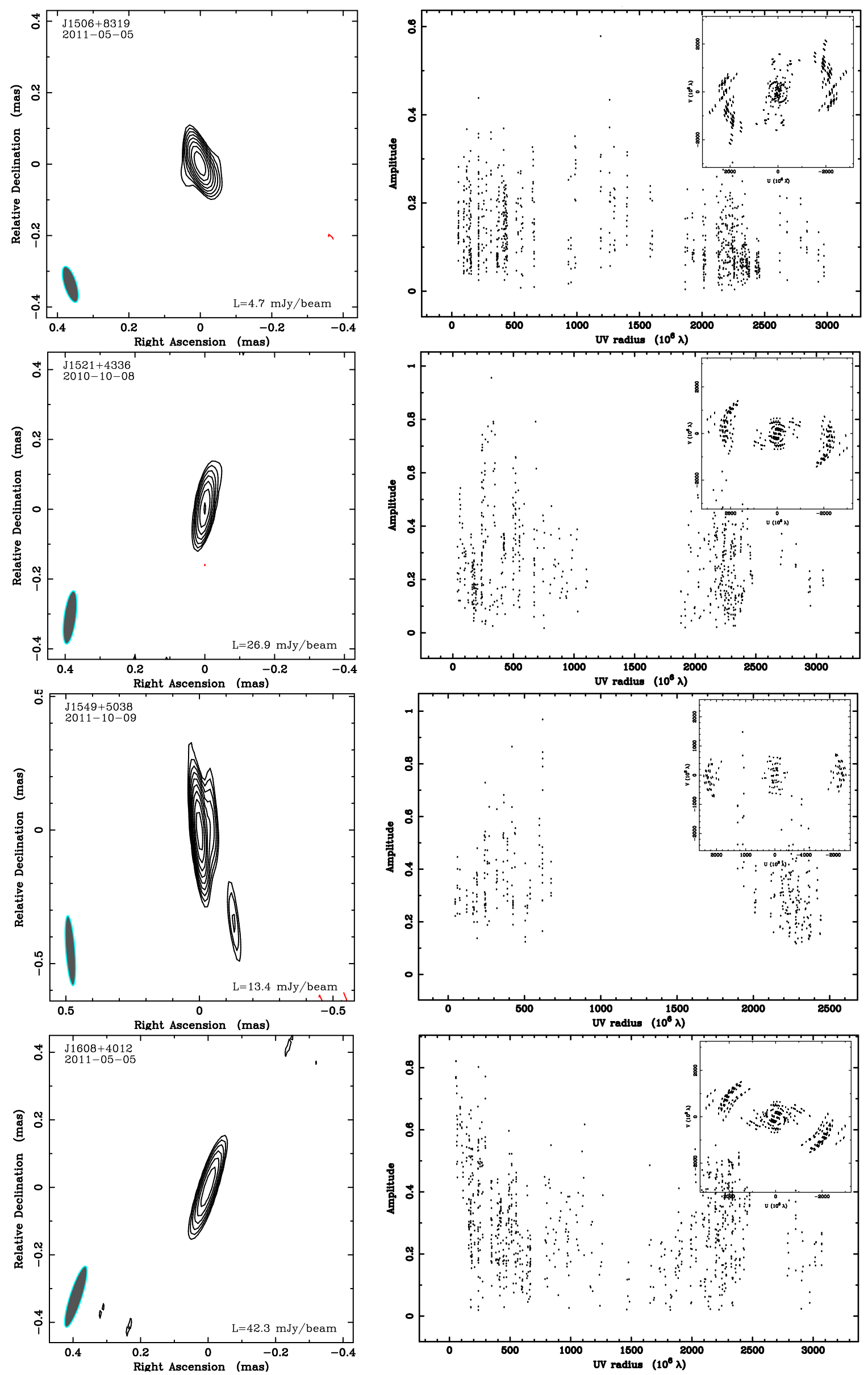

Figure 4.35: 3117 maps continued 

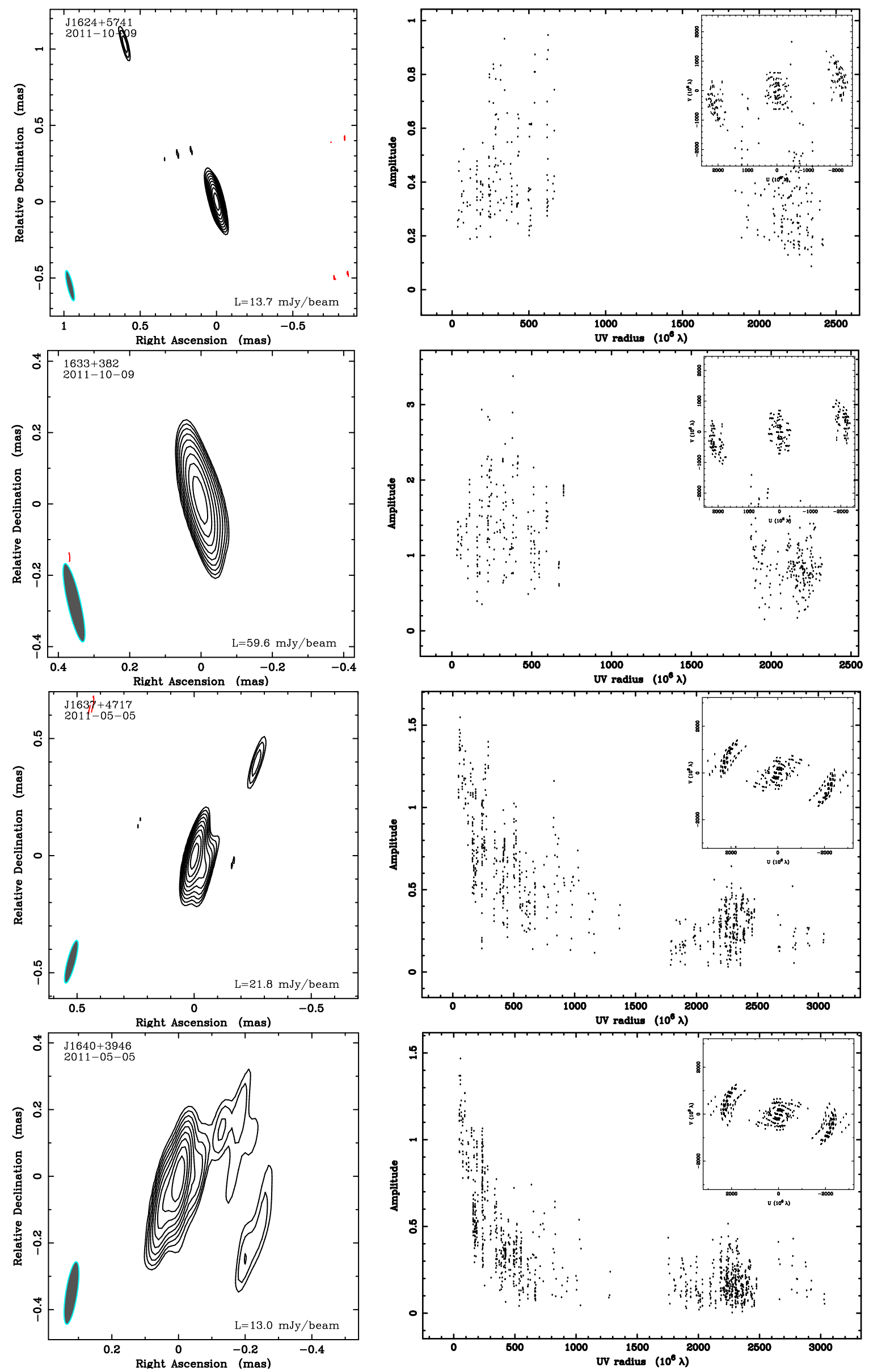

Figure 4.36: 3118 maps continued 

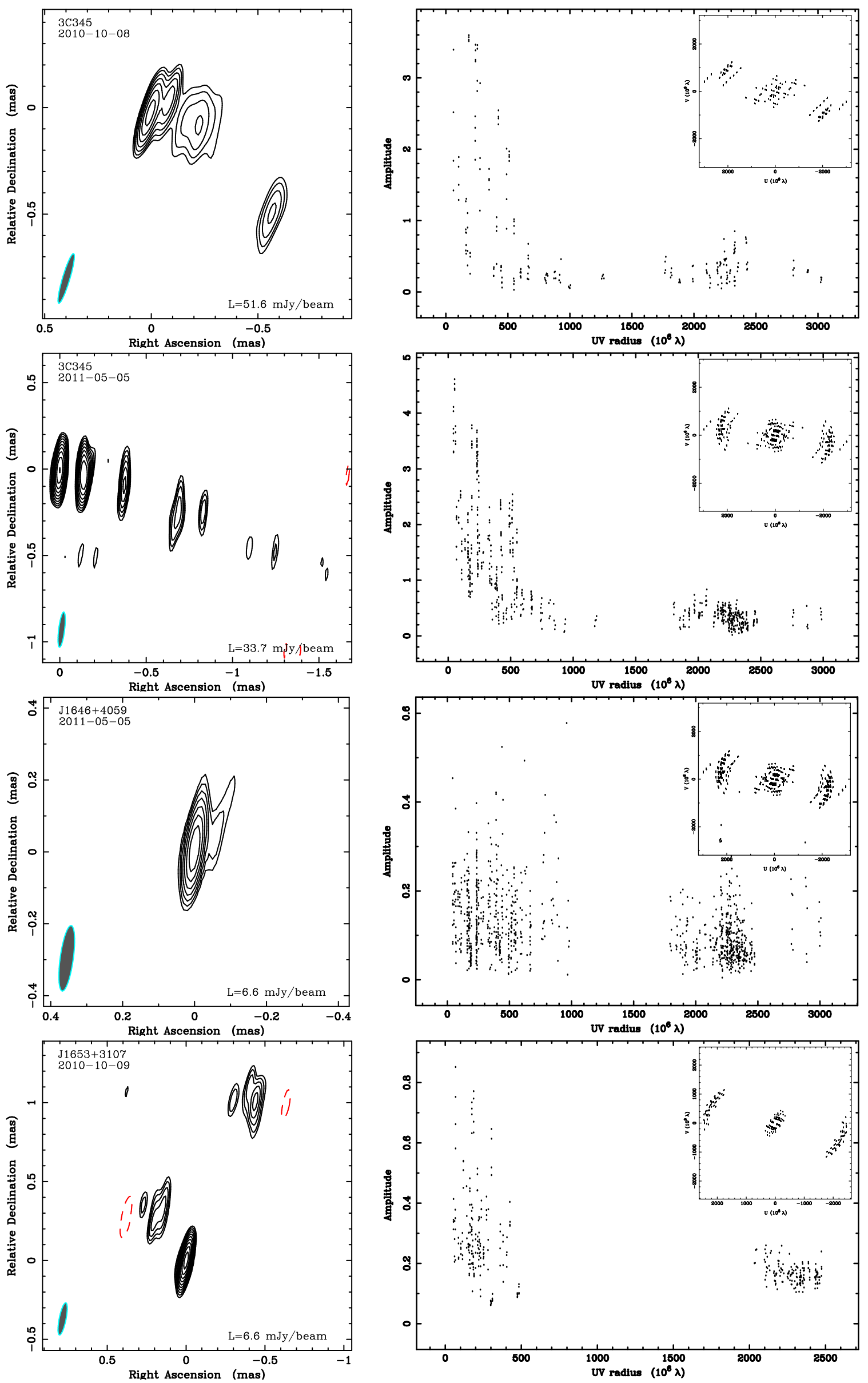

Figure 4.37: 3119 maps continued 

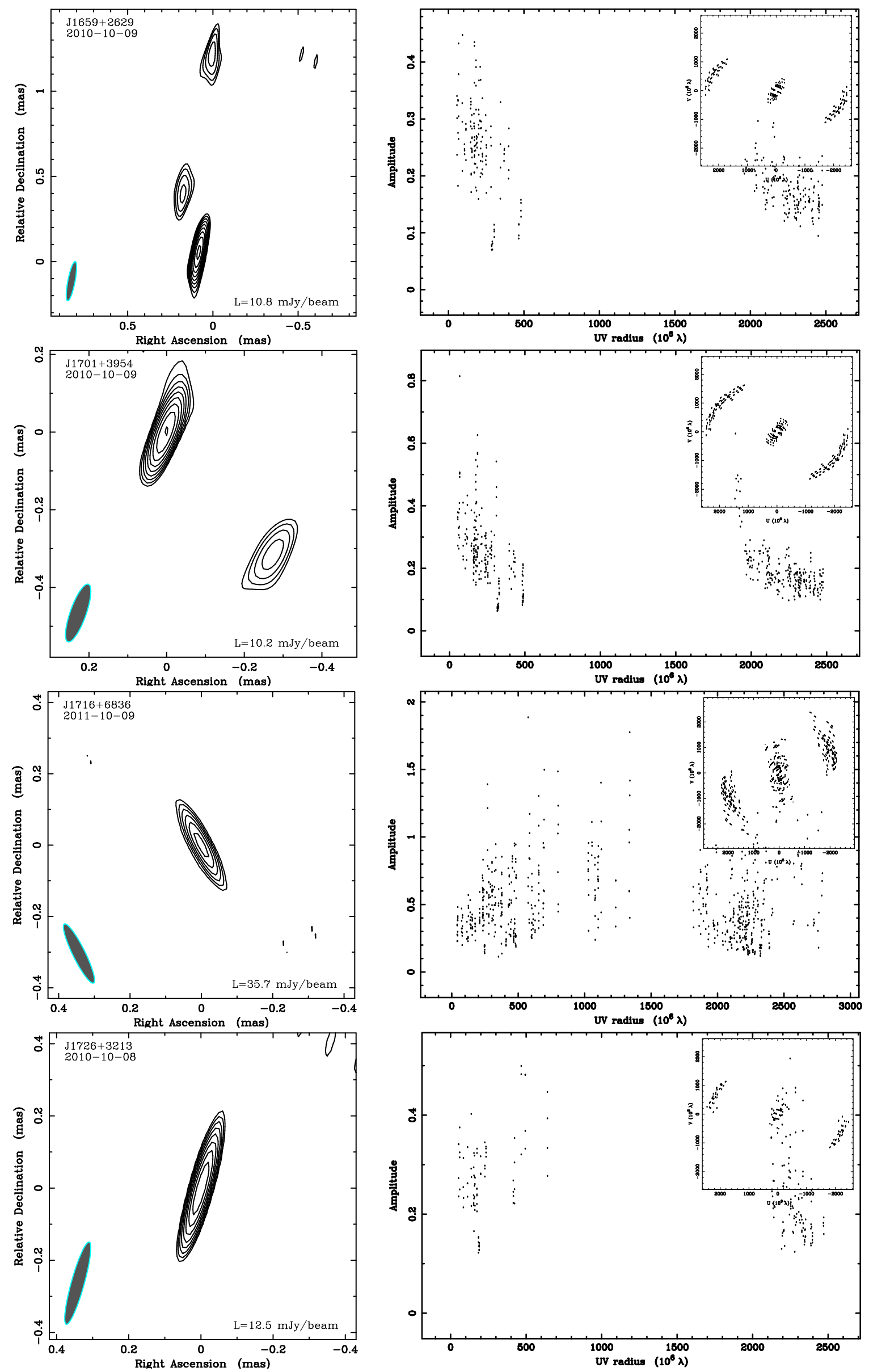

Figure 4.38: $3 \mathrm{~mm}$ maps continued 

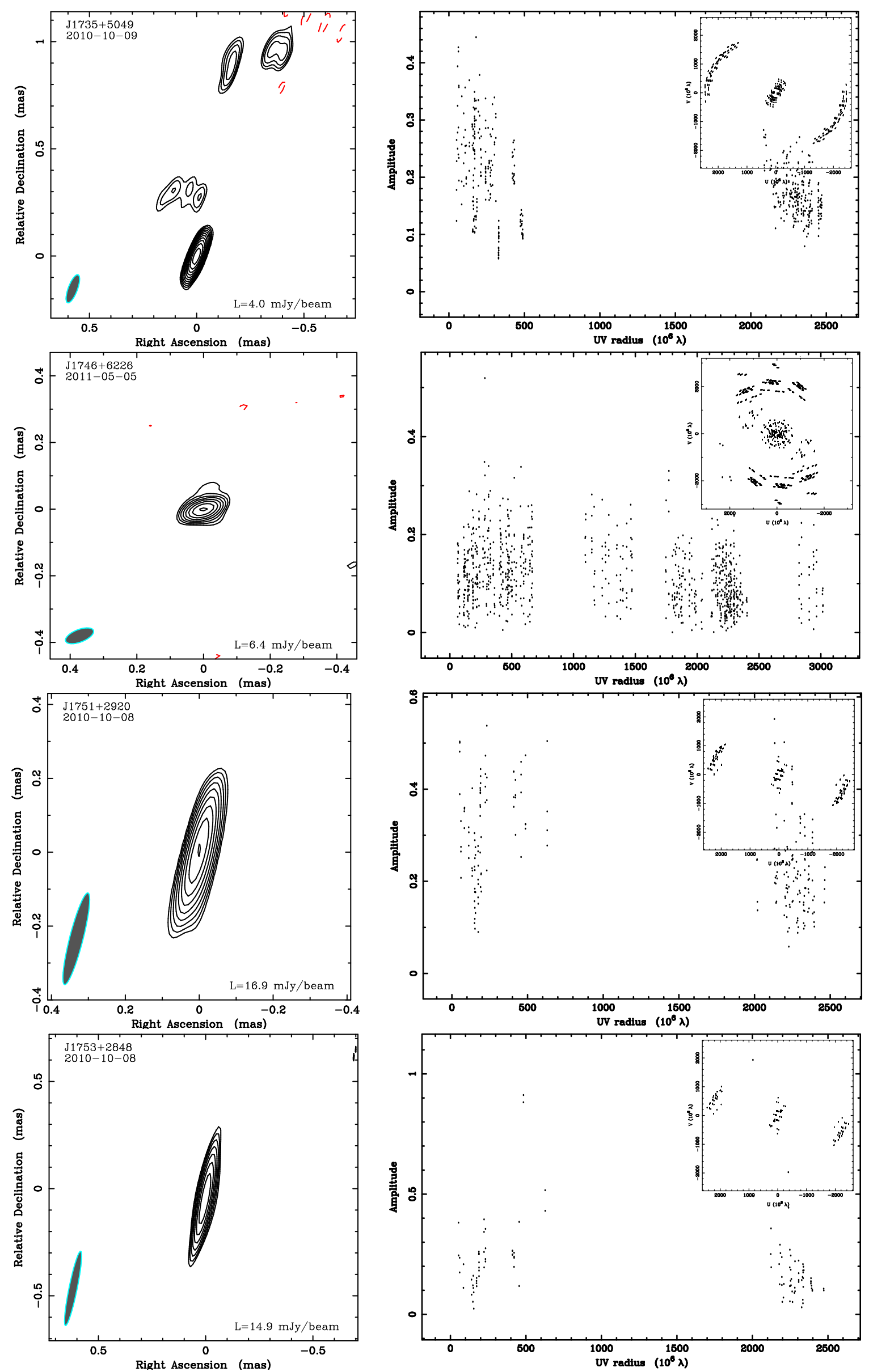

Figure 4.39: $3 \mathrm{~mm}$ maps continued 

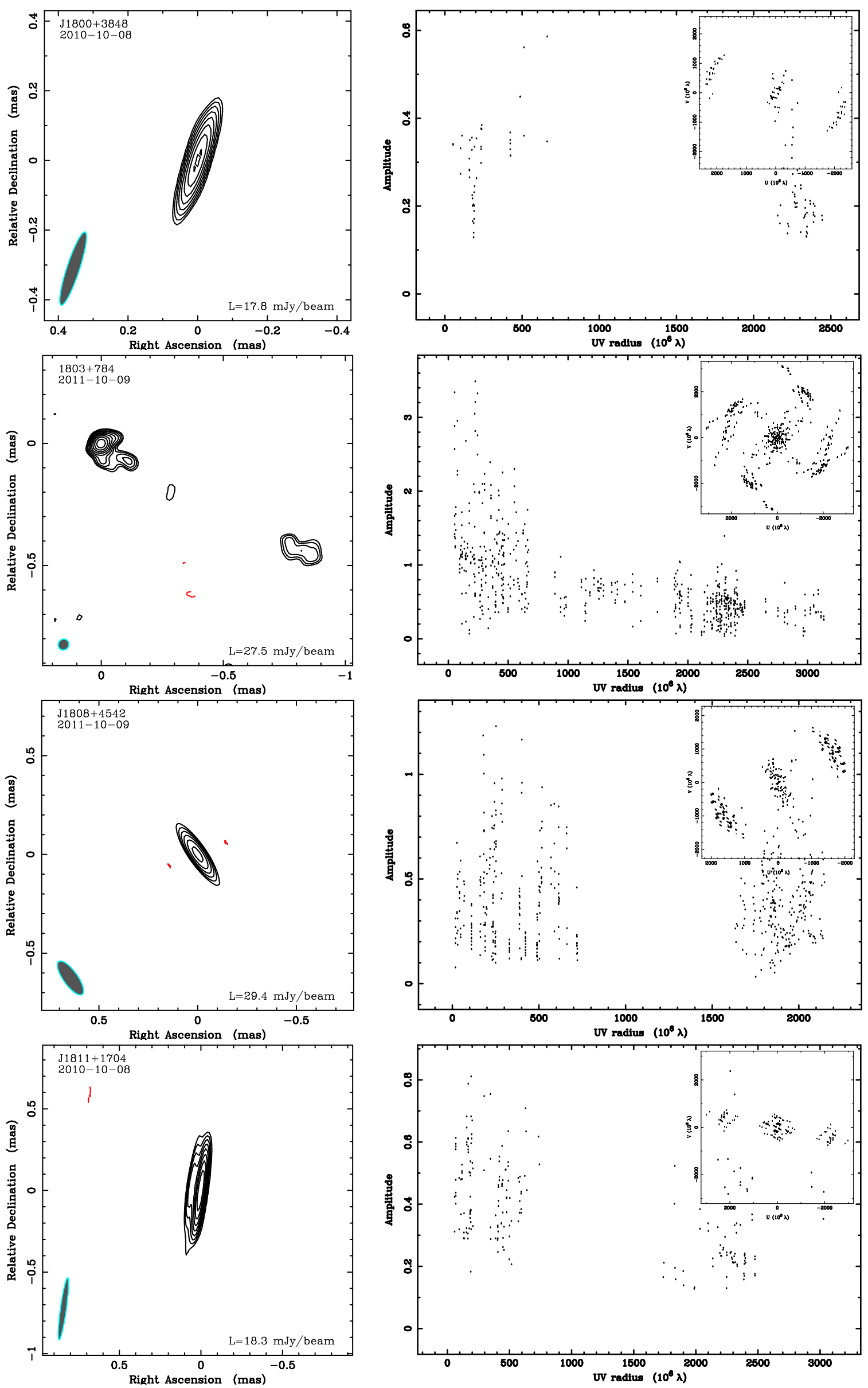

Figure 4.40: $3 \mathrm{~mm}$ maps continued 

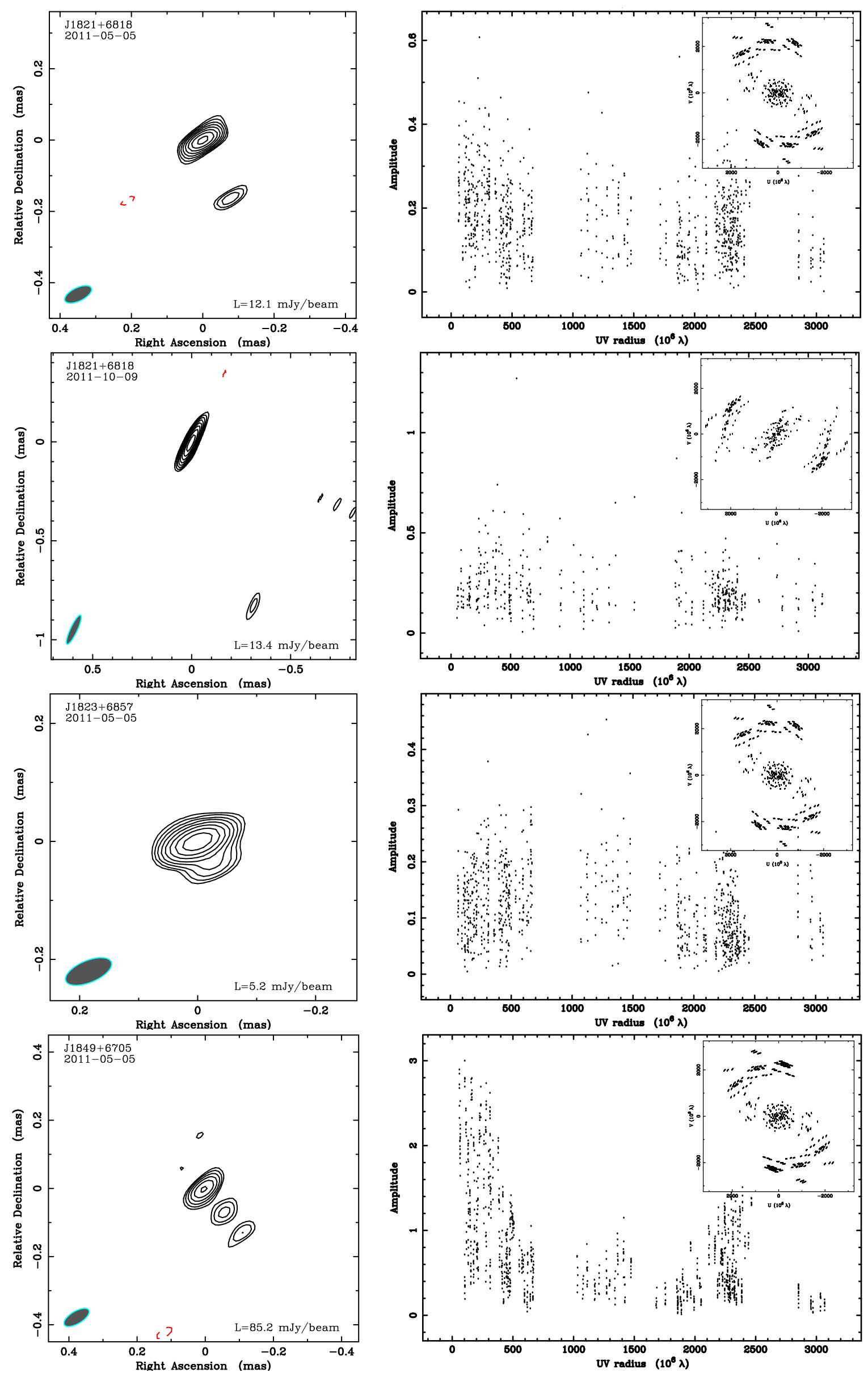

Figure 4.41: $3 \mathrm{~mm}$ maps continued 

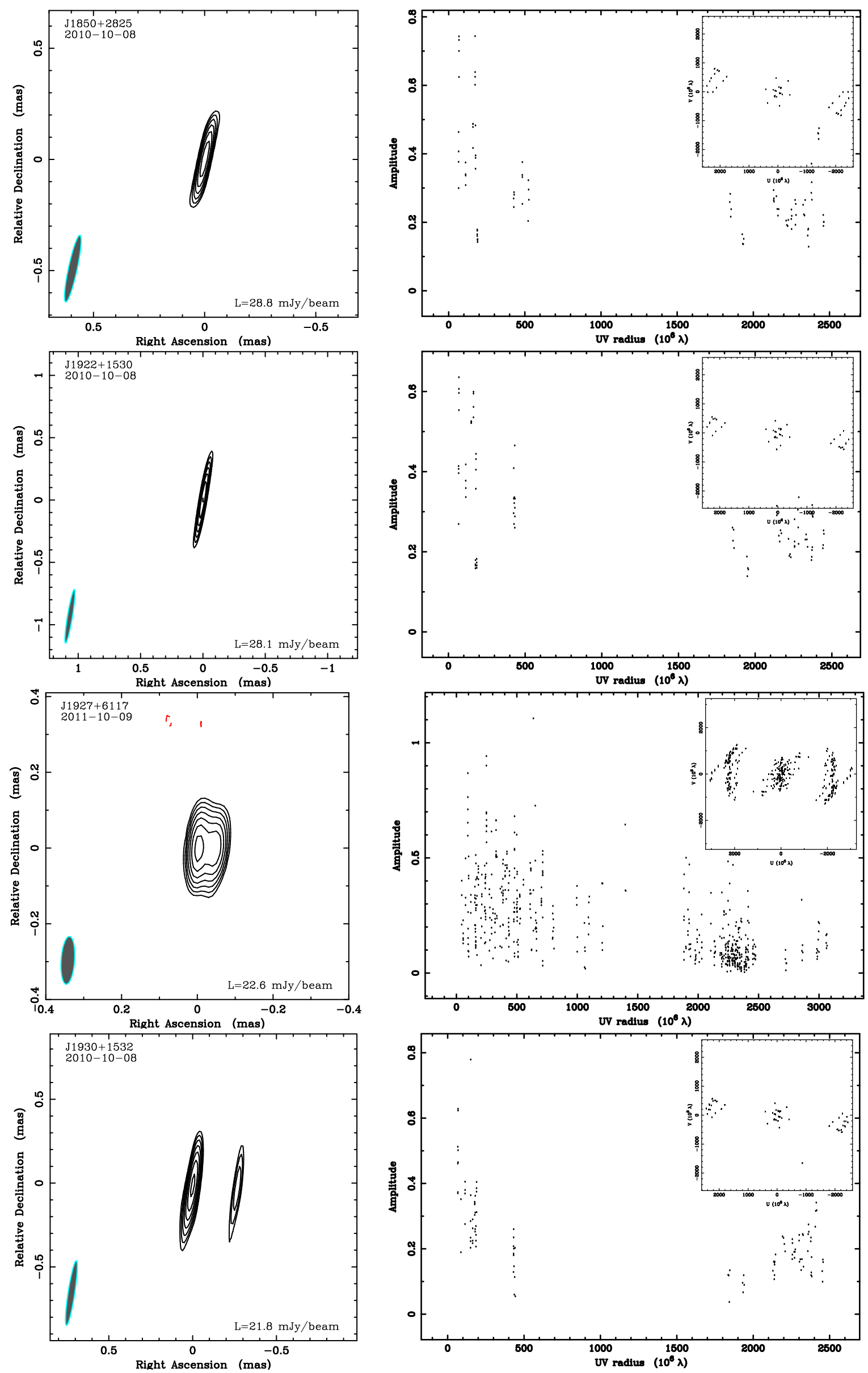

Figure 4.42: $3 \mathrm{~mm}$ maps continued 

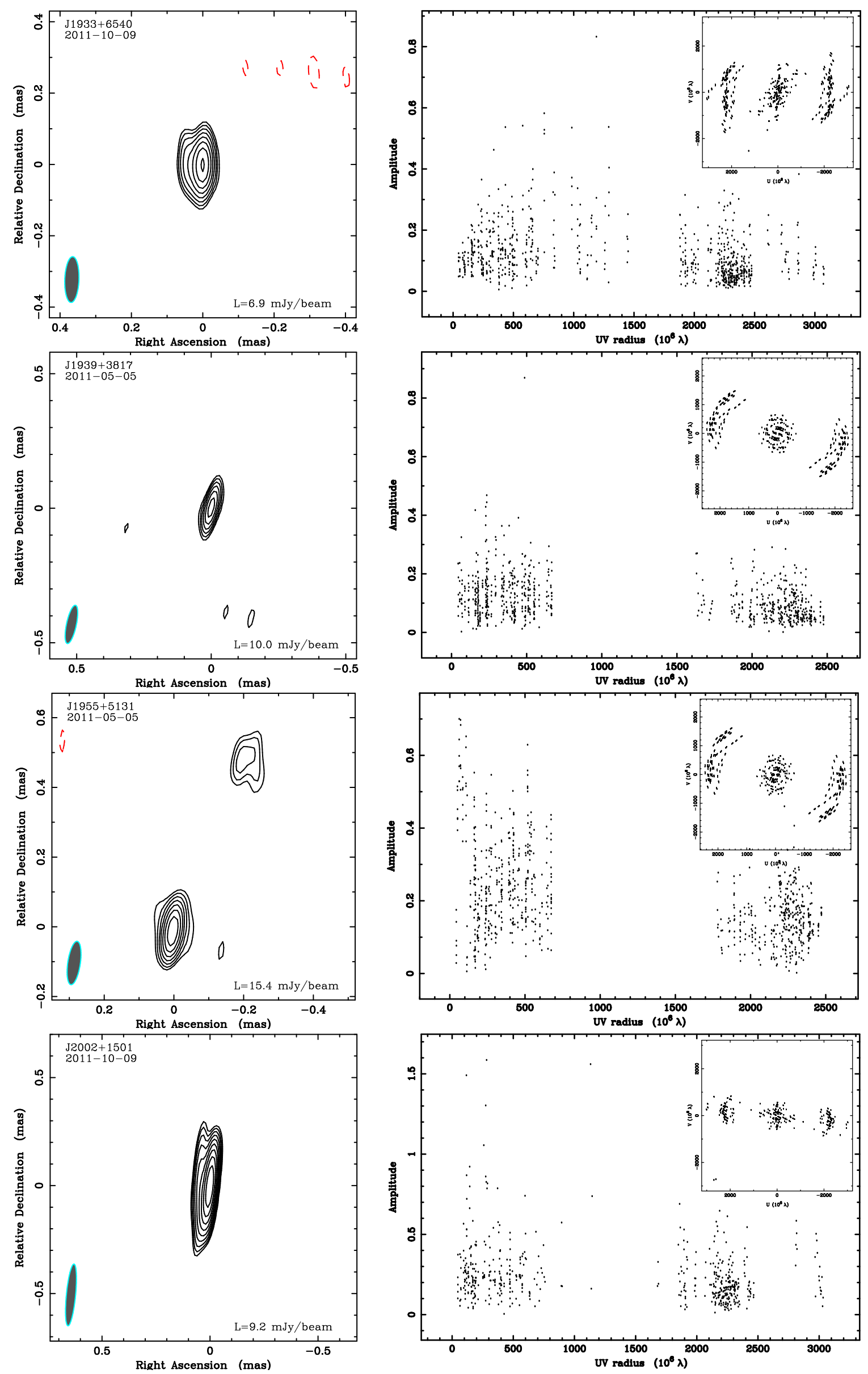

Figure 4.43: $3 \mathrm{~mm}$ maps continued 

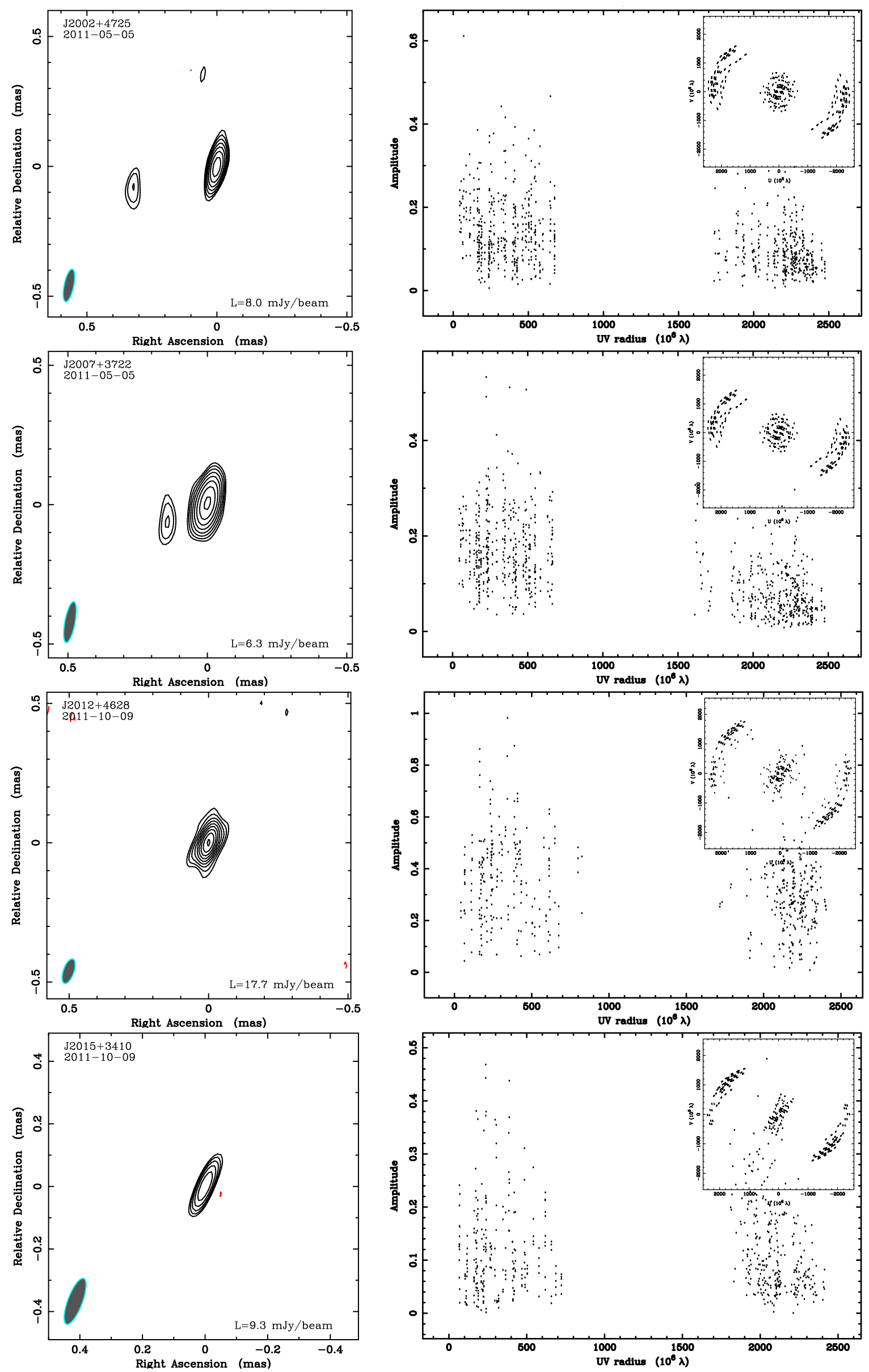

Figure 4.44: 3126 maps continued 

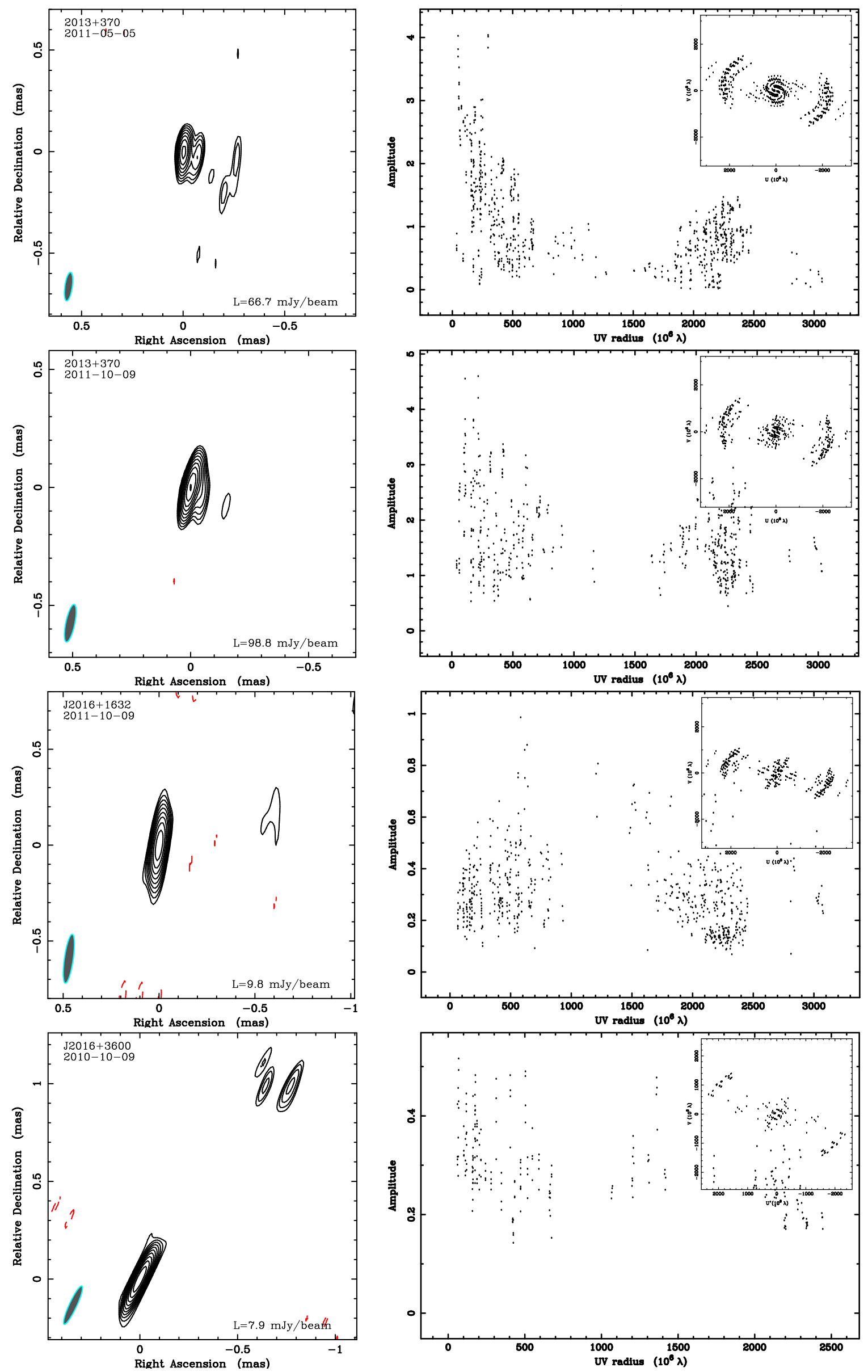

Figure 4.45: $3 \mathrm{~mm}$ maps continued 

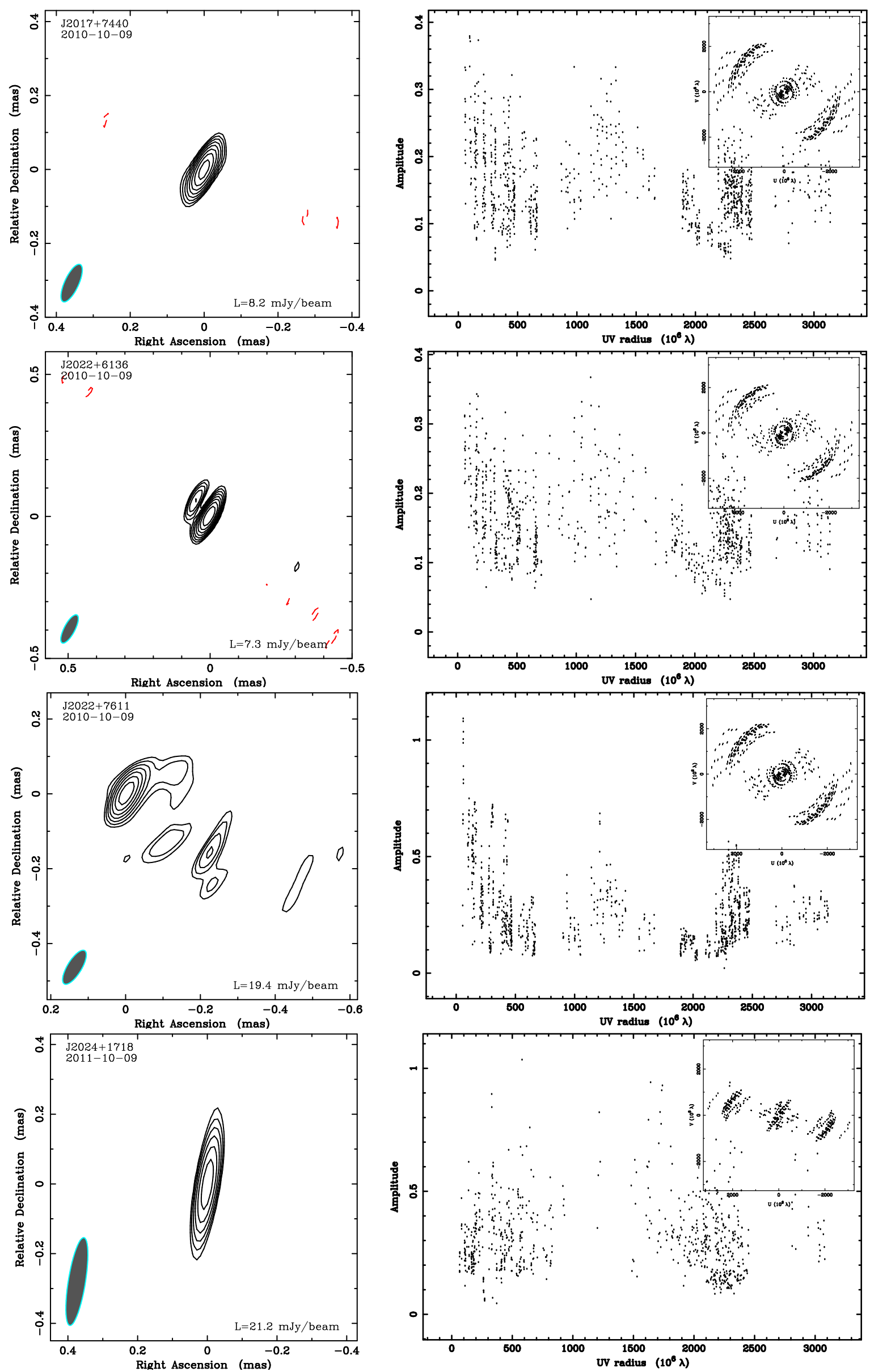

Figure 4.46: $3 \mathrm{~mm}$ maps continued 

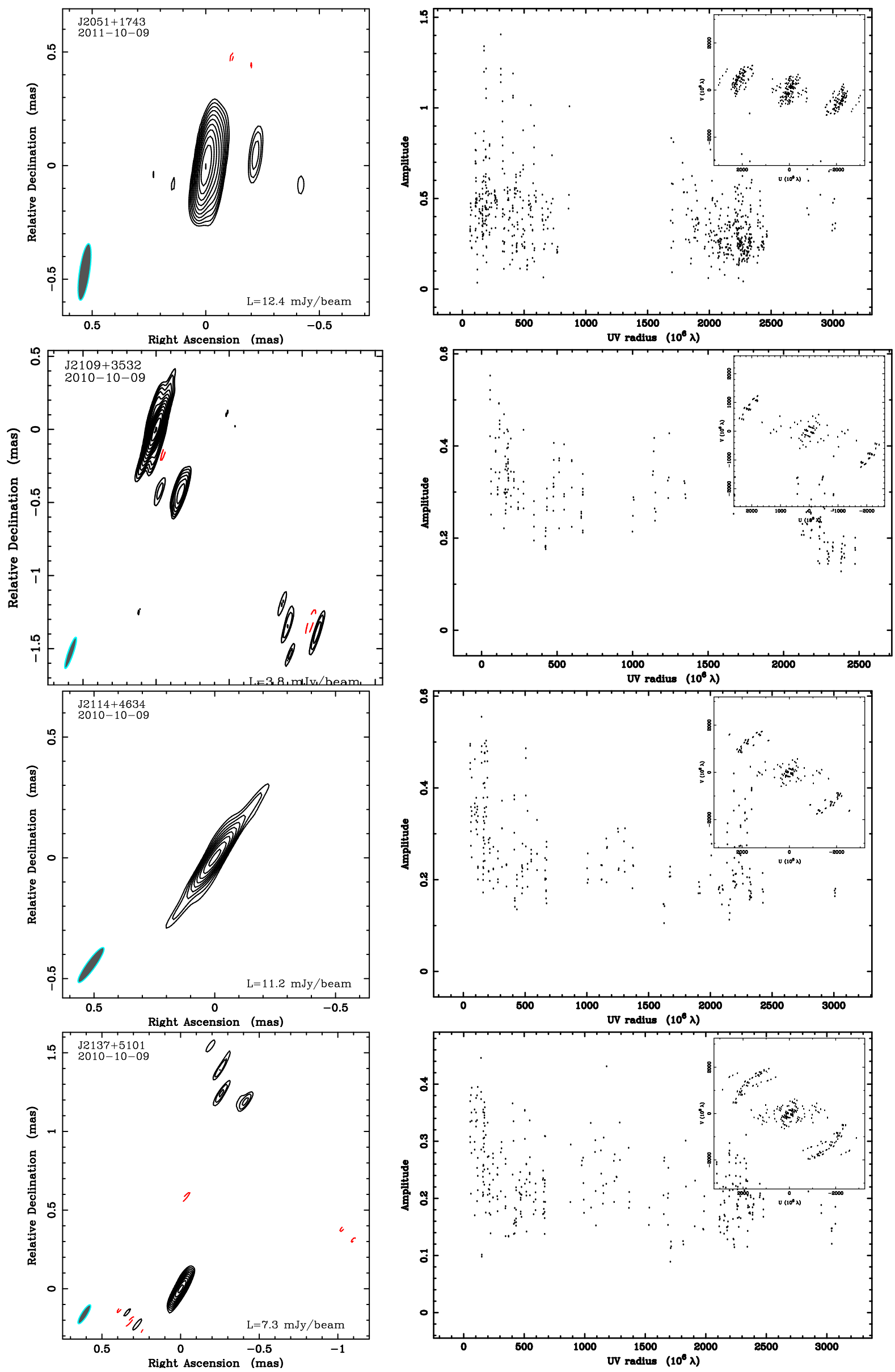

Figure 4.47: $3 \mathrm{~mm}$ maps continued 

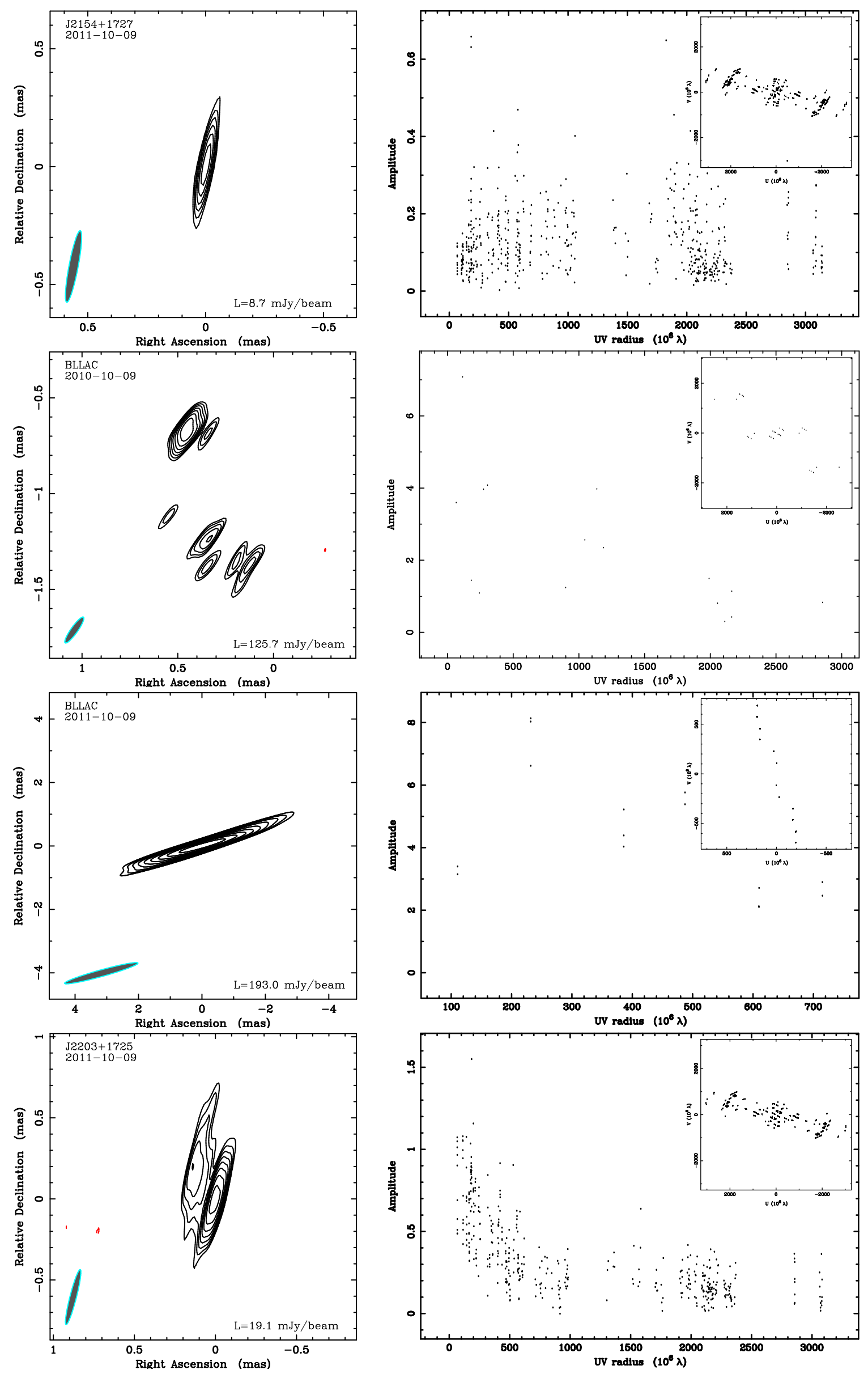

FIGURE 4.48: $3 \mathrm{~mm}$ maps continued 

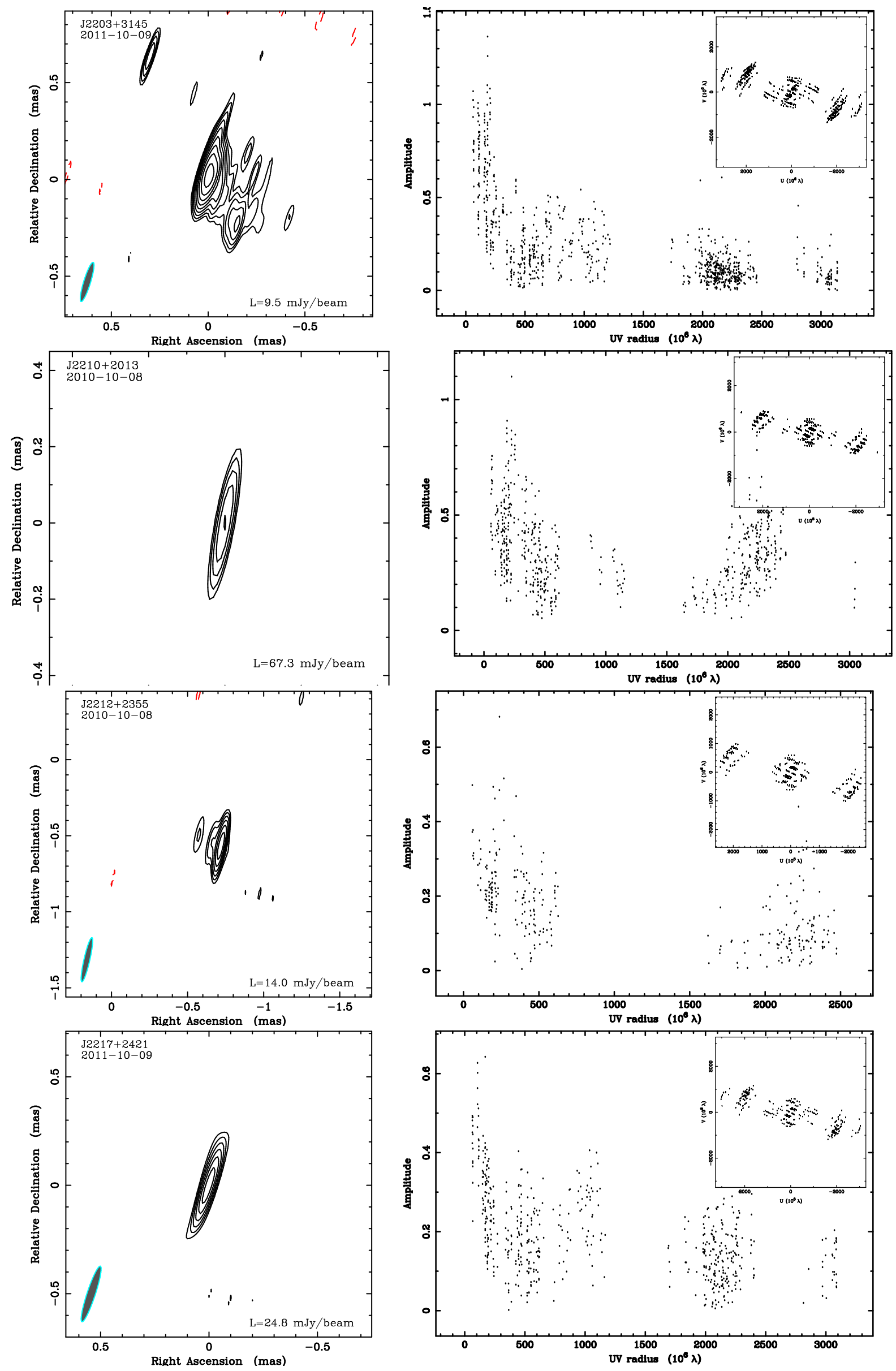

Figure 4.49: $3 \mathrm{~mm}$ maps continued 

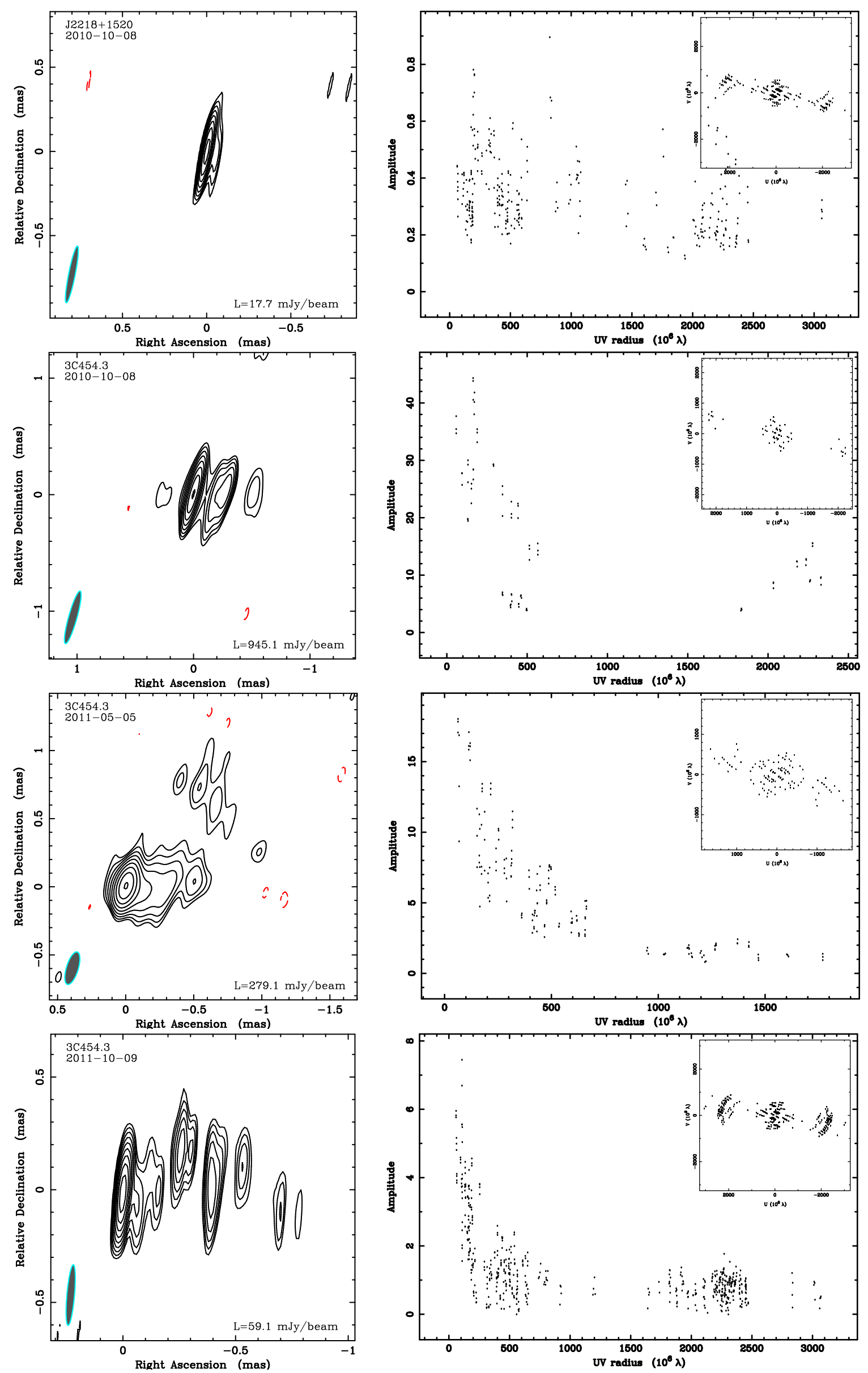

Figure 4.50: $3 \mathrm{~mm}$ maps continued 

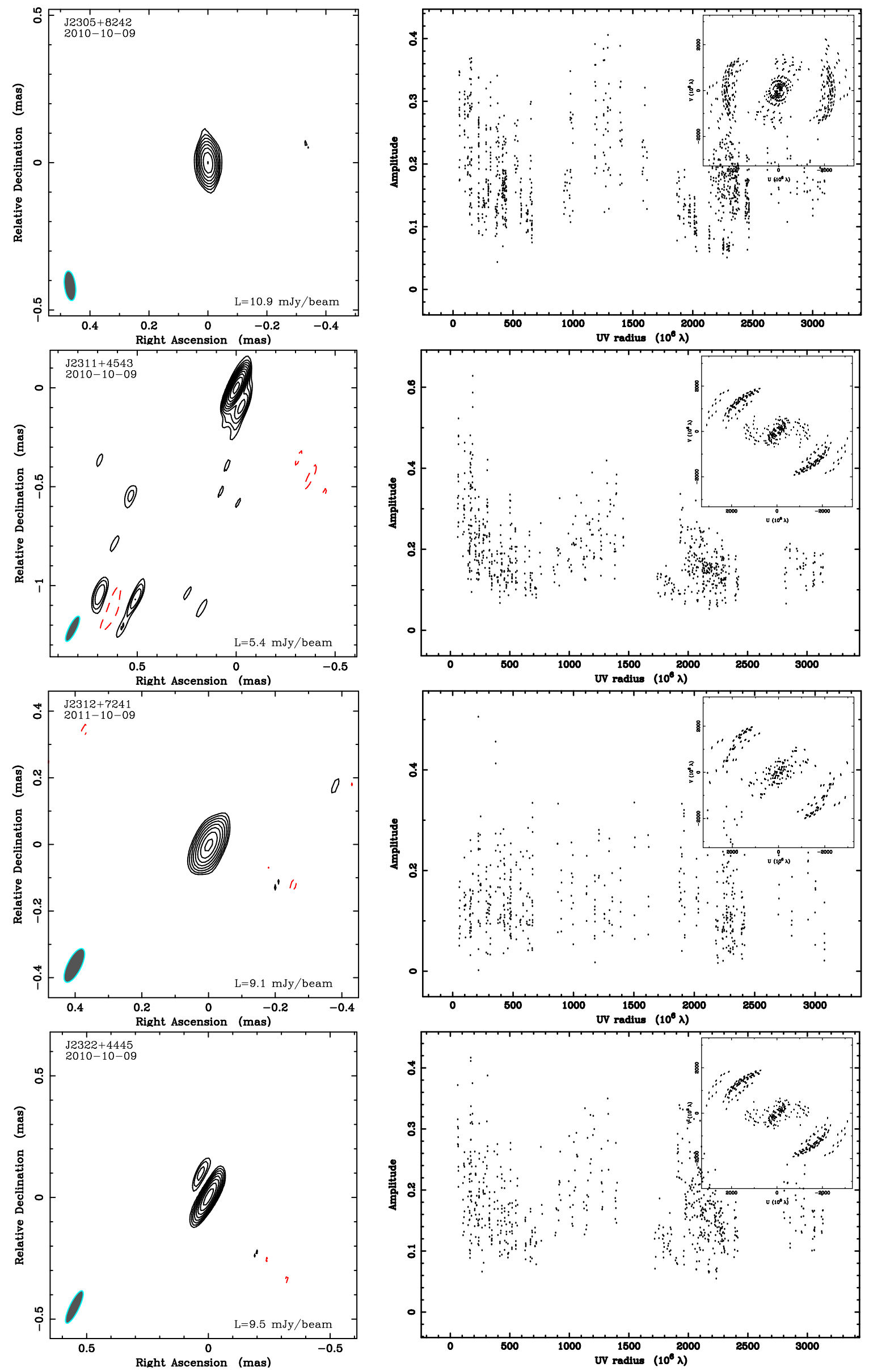

Figure 4.51: $3 \mathrm{~mm}$ maps continued 

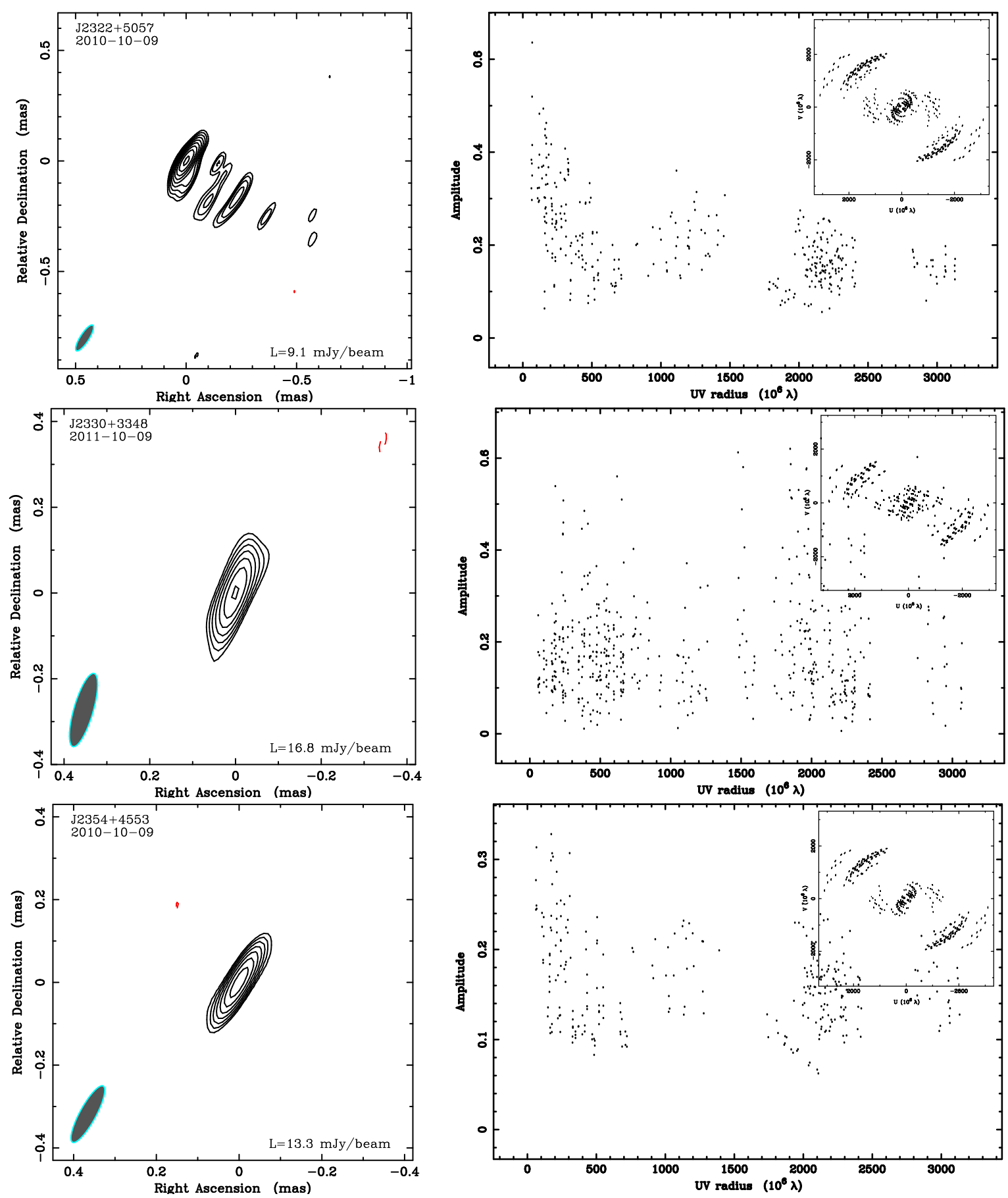

FIGURE 4.52: $3 \mathrm{~mm}$ maps continued

\subsection{Discussion}

\subsubsection{Model fit based Brightness Temperatures}

In our further analysis, we use the model fit based and visibility-based estimates of the brightness temperature $T_{\mathrm{b}}$ of the VLBI bright core (base) and the inner jet components, taking into account the resolution limits of the data at $3 \mathrm{~mm}$. The brightness temperature 
estimates for the jet cores in our sample ranges from $1.1 \times 10^{9} \mathrm{~K}$ to $1.3 \times 10^{12} \mathrm{~K}$. The distribution of $T_{\mathrm{b}}$ peaks at $10^{11} \mathrm{~K}$. The brightness temperature estimates for the nearest jet components in our sample ranges from $5.8 \times 10^{7} \mathrm{~K}$ to $4.1 \times 10^{11} \mathrm{~K}$. The median and mean of the brightness temperature distribution for the core regions is $8.6 \times 10^{10} \mathrm{~K}$ and $1.8 \times 10^{11} \mathrm{~K}$, respectively. For the inner jet components, the respective figures are is $9.1 \times 10^{9} \mathrm{~K}$ and $2.3 \times 10^{10} \mathrm{~K}$. This shows that the brightness temperature drops by approximately an order of magnitude already on sub-parsec scales in the jets, with inverse Compton, synchrotron, and adiabatic losses subsequently dominating the energy losses (cf. Marscher 1995; Lobanov \& Zensus 1999). Only $8 \%$ of the jet cores show a brightness temperature greater than $5 \times 10^{11} \mathrm{~K}$ and only $3 \%$ of the sources have a brightness temperature greater than $10^{12} \mathrm{~K}$. The distribution of $T_{\mathrm{b}}$ in different classes of AGNs in our sample are shown in figure 4.59. The model fit based brightness temperature results from this survey is investigated in detail on the background of population modelling and is discussed in Chapter 5 .

\subsubsection{Visibility based Brightness Temperatures}

We also inspect the distribution of the minimum, $T_{\mathrm{b}, \text { min }}$ and limiting, $T_{\mathrm{b}, \mathrm{lim}}$ brightness temperature of the core components (using averaged values of brightness temperature for objects with multiple observations) using equations 1.31 and 1.33 , respectively in the sample, making these estimates from the visibility amplitudes on longest baselines (Lobanov 2015). The minimum and limiting brightness temperatures are given in columns 10 and 11 , respectively, in Table 4.7. Their distribution is shown in figure 4.60. $T_{\mathrm{b}, \text { mod }}$ calculated for all the sources are either well bracketed inside $T_{\mathrm{b} \text {,min }}$ and $T_{\mathrm{b} \text {,lim }}$ or in well agreement with $T_{\mathrm{b}, \mathrm{lim}}$.

The median and mean of the limiting brightness temperature distribution for the core regions is $1.06 \times 10^{11} \mathrm{~K}$ and $3.0 \times 10^{11} \mathrm{~K}$, respectively. We find that the limiting $T_{\mathrm{b}, \mathrm{lim}}$ agrees well with $T_{\mathrm{b} \text {,mod }}$ estimated from imaging method as seen in Figure 4.53, supporting the fidelity of $T_{\mathrm{b} \text {,mod }}$ measurements obtained from model fitting. The residual logarithmic distribution of the $T_{\mathrm{b}, \mathrm{lim}} / T_{\mathrm{b} \text {,mod }}$ ratio is well approximated by the Gaussian PDF with mean value $\mu=0.001$ and standard deviation $\sigma=0.46$.

The median and mean of the minimum brightness temperature distribution for the core regions are $4.1 \times 10^{10} \mathrm{~K}$ and $6.2 \times 10^{10} \mathrm{~K}$, respectively. The residual logarithmic distribution of $T_{\mathrm{b}, \text { mod }} / T_{\mathrm{b}, \text { min }}$ ratio is approximated by the Gaussian PDF with $\mu=0.354$ and $\sigma=0.42$. The minimum brightness temperature $T_{\mathrm{b} \text {,min }}$ estimated from visibility is considerably lower than $T_{\mathrm{b} \text {,mod }}$ estimated from model fits and it is approximately a factor of $\sim 10$ lower than $T_{\mathrm{b}, \mathrm{lim}}$. This is due to the fact that $T_{\mathrm{b} \text {,min }}$ is estimated using equation 1.31 based on the zero-spacing flux as compared to $T_{\mathrm{b}, \text { lim }}$ estimated using equation 1.33 taking into account the errors on visibility amplitudes, $\sigma_{\mathrm{q}}$ also, for each source. However, a scatter seen at $T_{\mathrm{b} \text {,mod }}>10^{11.5} \mathrm{~K}$ in the $\left(\log T_{\mathrm{b}, \lim }-\log T_{\mathrm{b}, \text { mod }}\right)$ plane in figure 4.53 is due to the under estimation of the component size of the Gaussian models.

\subsubsection{Source Compactness}

The compactness of the source structure can be evaluated by comparing the single dish flux density, $S_{86}$ to the clean flux density, $S_{\text {CLEAN }}$ listed in Table 4.6 and the core flux density,

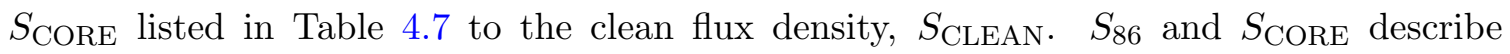



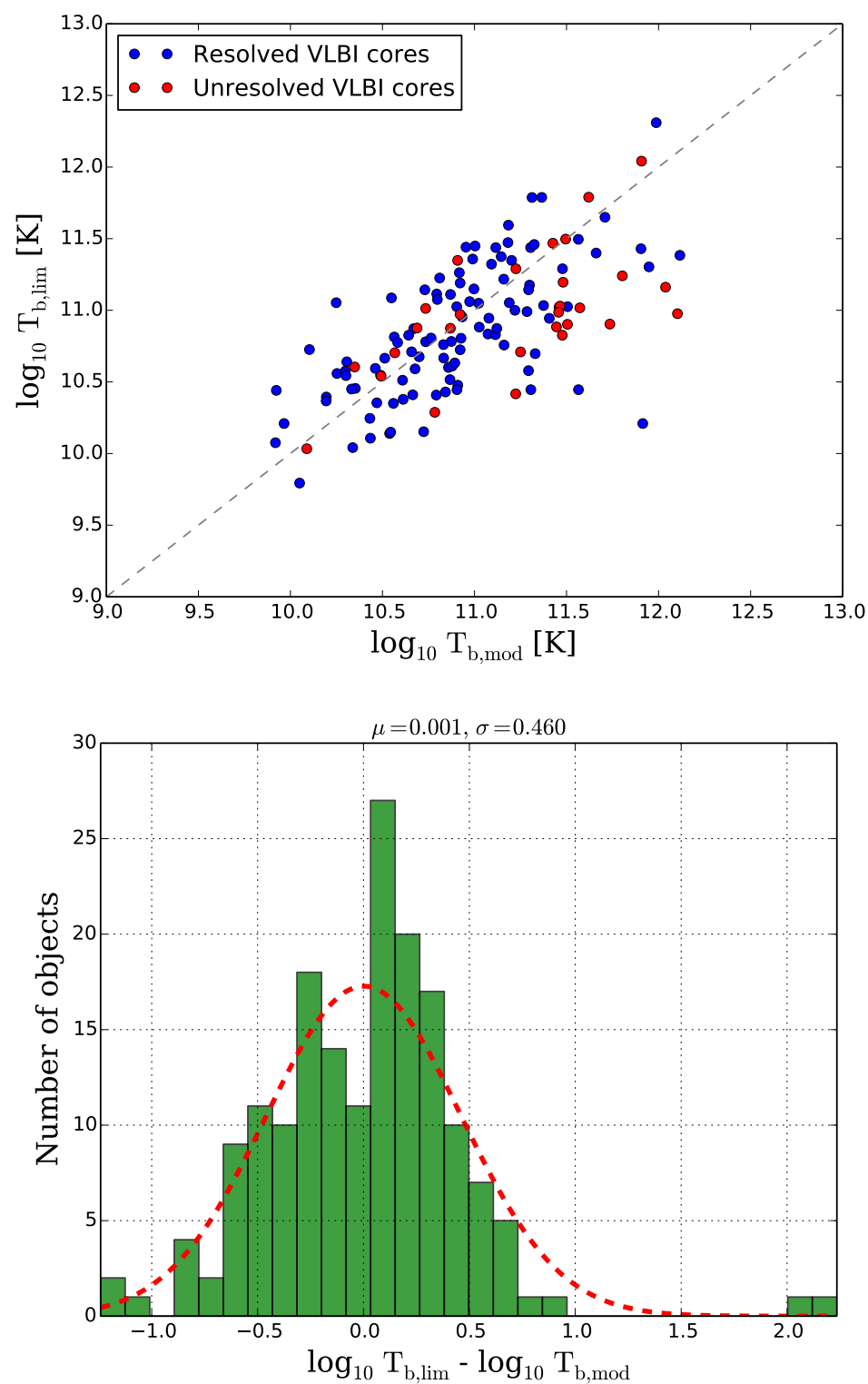

Figure 4.53: Comparison of $T_{\mathrm{b} \text {,mod }}$ measured from circular Gaussian representation of source structure and $T_{\mathrm{b} \text {,lim }}$ estimated from the interferometric visibilities at $u v$-radii within $10 \%$ of the maximum baseline $B_{\max }$ in the data for a given source (top panel). Correlation between the two distribution is illustrated by the residual logarithmic distribution of $T_{\mathrm{b} \text {,mod }} / T_{\mathrm{b} \text {,limit }}$ ratio (bottom panel) which is well approximated by the Gaussian distribution with mean value, $\mu=0.001$ and standard deviation, $\sigma=0.46$. 

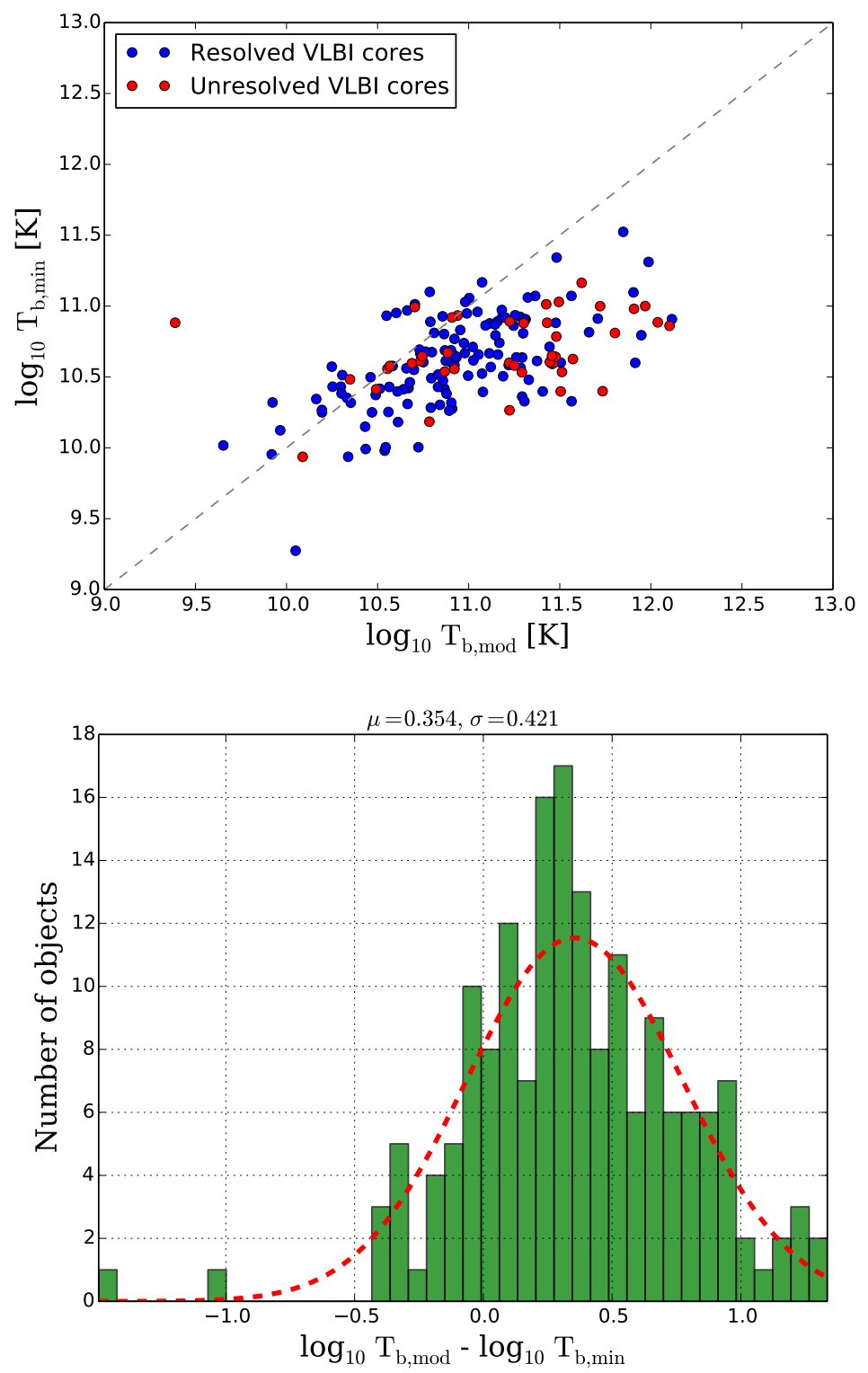

Figure 4.54: Comparison of $T_{\mathrm{b} \text {,mod }}$ measured from circular Gaussian representation of source structure and $T_{\mathrm{b} \text {, min }}$ estimated from the interferometric visibilities in the data for a given source (top panel). The residual logarithmic distribution of the $T_{\mathrm{b} \text {, mod }} / T_{\mathrm{b} \text {,min }}$ ratio is approximated by the Gaussian PDF with $\mu=0.354$ and $\sigma=0.42$ (bottom panel).

kiloparsec-scale (large scale structure) and parsec-scale (inner compact structure) of the AGN, respectively. These comparisons are presented in Figure 4.55.

To study the relation between the total and VLBI flux densities, we apply the Pearson correlation test. The Pearson correlation coefficient, $\rho$ measures the linear relationship between two datasets, assuming that each dataset is normally distributed. This varies between -1 and +1 with 0 implying no correlation. +1 implies a total positive linear correlation and -1 is a total negative linear correlation. If we have one dataset $\left(x_{1}, \ldots, x_{n}\right)$ containing $\mathrm{n}$ values and another dataset $\left(y_{1}, \ldots, y_{n}\right)$ containing $\mathrm{n}$ values, then the Pearson correlation coefficient for the sample is calculated as:

$$
\rho=\frac{\sum_{i=1}^{n}\left(x_{i}-\bar{x}\right)\left(y_{i}-\bar{y}\right)}{\sqrt{\sum_{i=1}^{n}\left(x_{i}-\bar{x}\right)^{2}} \sqrt{\sum_{i=1}^{n}\left(y_{i}-\bar{y}\right)^{2}}}
$$



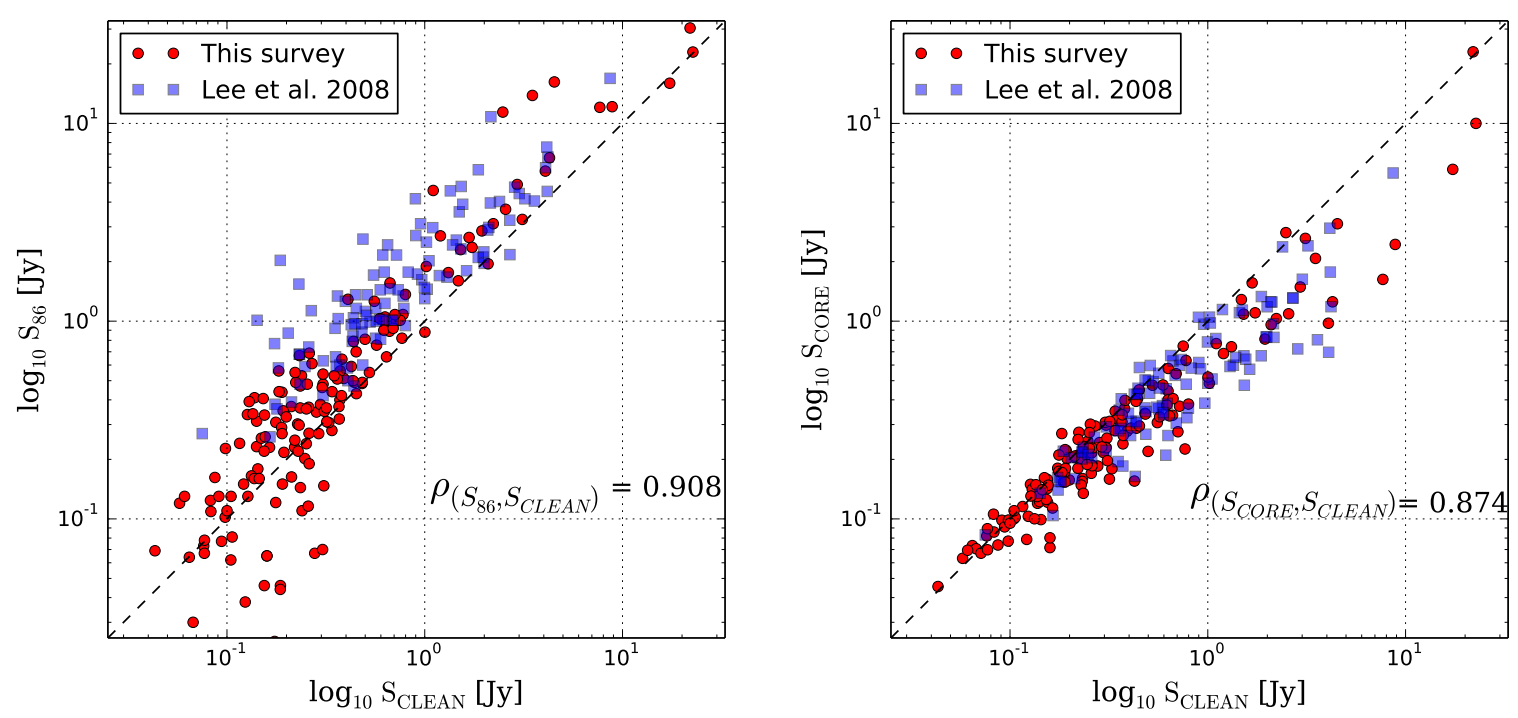

Figure 4.55: The compactness parameters $S_{86} / S_{\text {CLEAN }}$ and $S_{\text {core }} / S_{\text {CLEAN }}$ on the left and right panel respectively. The Pearson correlation coefficients, $\rho_{\left(S_{86}, S_{C L E A N}\right)}=0.908$ and $\rho_{\left(S_{C O R E}, S_{C L E A N}\right)}=0.874$ are obtained for the combined data from this survey and Lee et al. (2008).

where $n$ is the sample size, $x_{i}, y_{i}$ are the single samples indexed with $i, \bar{x}=\sum_{i=1}^{n} x_{i}$ is the sample mean for $x$ and $\bar{y}=\sum_{i=1}^{n} x_{i}$ is the sample mean for $y$.

The Pearson correlation coefficient calculated for $S_{86}$ and $S_{\text {CLEAN }}$ gives a significant value of 0.924 for this survey and 0.908 for this survey combined with the results from Lee et al. (2008). The respective plot in the left panel of figure 4.55 indicates that almost all the flux measured by a single dish (here Pv or PdB) is recovered in the VLBI clean flux. The source compactness on milli arcsec scales, $S_{\text {CLEAN }} / S_{86}$ is also shown on top left in figure 4.57 . The median of the core dominance index defined as $S_{\text {CORE }} / S_{\text {CLEAN }}$ is 0.84 , and the two are also correlated, as demonstrated in the right panel of Figure 4.55. The distribution of the core dominance index defined as $S_{\mathrm{CORE}} / S_{\mathrm{CLEAN}}$ on top right in Figure 4.57 shows that almost all the sources are core dominated and for certain extremely core dominant sources, the index is greater than unity.

Figure 4.55 shows that the stronger sources have more structures (right panel) some of which is completely resolved out even on the shortest baselines of the survey observations (left panel). A small number of cases for which $S_{\text {CLEAN }}>S_{86}$ or $S_{\text {CORE }}>S_{\text {CLEAN }}$ are observed for the weaker objects. These can be reconciled with the errors in the measurements, and they essentially imply very compact objects, with $S_{86}=S_{\text {CLEAN }}$ and $S_{\text {CORE }}=S_{\text {CLEAN }}$, respectively. The scatter seen in the case of extremely fainter sources in the lower end of $S_{86}$ vs. $S_{\text {CLEAN }}$ plot in figure 4.55 is due to uncertainties in amplitude calibration which is about $\sim 25 \%$ and $S_{\text {CLEAN }}$ which is about $\sim 20 \%$.

The distribution of the total single dish flux density of the sources, $S_{86}$ which is measured at Pico Veleta or Plateau de Bure radio telescope during the observations shown in figure 4.3 peaks at $0.42 \mathrm{Jy}$ and almost all the sources are brighter than $75 \mathrm{mJy}$, which corresponds to the flux limit of our source selection. The $S_{86}$ varies from the lowest flux 16 mJy to the maximum flux $29.43 \mathrm{Jy}$. In the same plot, $S_{86}$ distribution in this survey is compared with the previous $86 \mathrm{GHz}$ VLBI survey (Lee et al. 2008). The comparison of the $S_{86}$ distribution 


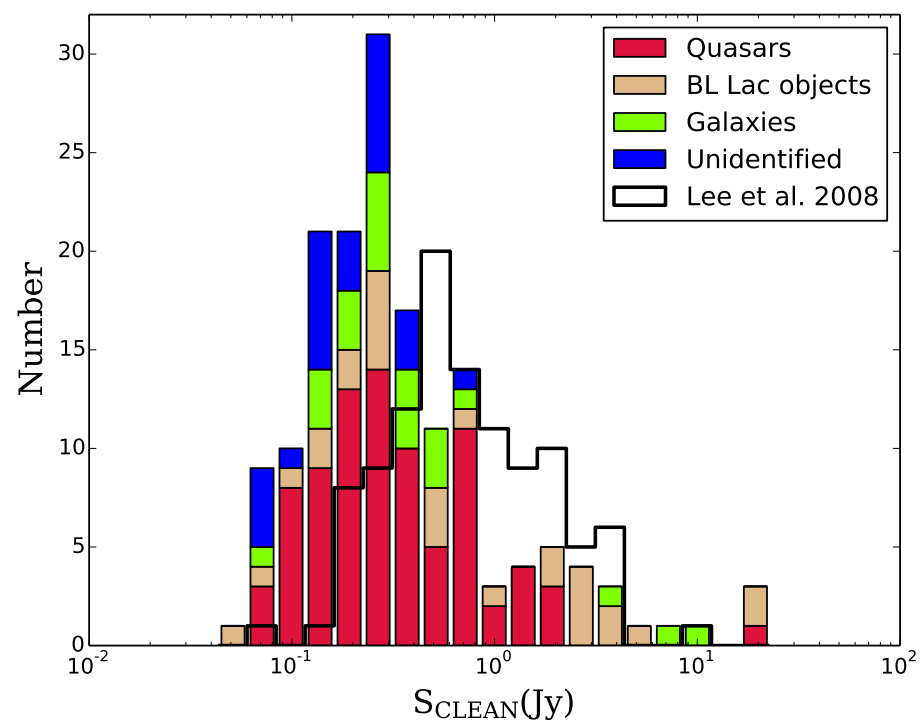

Figure 4.56: Distribution of the total CLEAN image flux density, $S_{\text {clean }}$, of the survey targets broken down along different host galaxy types and compared with respective distribution for the sources from the sample of Lee et al. (2008).

of the two surveys is the following : The $S_{86}$ distribution peaks at $42 \mathrm{mJy}$ and $1.3 \mathrm{Jy}$ for this survey and Lee et al. (2008) survey, respectively. Almost all sources are brighter than $75 \mathrm{mJy}$ and $300 \mathrm{mJy}$, for this survey and Lee et al. (2008) survey, respectively, implying that our survey includes sources which are fainter by about one order of magnitude than previous survey by Lee et al. (2008).

Figure 4.56 shows the distribution of the total CLEAN flux density $S_{\text {clean }}$ in the survey images and compares them to the respective distribution for the source sample imaged by Lee et al. (2008). $S_{\text {clean }}$ peaks at $0.27 \mathrm{Jy}$ and the median value of the clean flux density is $0.26 \mathrm{Jy}$. $S_{\text {clean }}$ distribution indicates that much of the emission at $86 \mathrm{GHz}$ is resolved out at milli arcsecond scales. The $S_{\text {clean }}$ distribution varies from the lowest flux 45 mJy to the maximum flux $2 \mathrm{Jy}$. In the same plot, $S_{\text {clean }}$ distribution in this survey is compared with the previous survey (Lee et al. 2008). The comparison of the $S_{\text {clean }}$ distribution of two surveys is the following: The $S_{\text {clean }}$ distribution peaks at $0.27 \mathrm{Jy}$ and $0.52 \mathrm{Jy}$ for this survey and Lee et al. (2008) survey, respectively. Almost all sources are brighter than 45 mJy and $300 \mathrm{mJy}$, respectively and the median is $0.26 \mathrm{Jy}$ and $0.6 \mathrm{Jy}$, respectively for this survey and Lee et al. (2008). This also implies that our survey digs into the sample of extragalactic radio sources which were unrevealed by previous surveys.

The median of the correlated flux densities corresponding to the longest baselines for each source, $S_{\mathrm{L}}$ is $0.19 \mathrm{Jy}$ and the distribution peaks at $0.20 \mathrm{Jy}$. Among 157 sources whose $S_{\mathrm{L}}$ can be measured at projected baselines longer than $2000 \mathrm{M} \lambda$, 135 sources have a $S_{\mathrm{L}}$ greater than 0.1 Jy. The median of "sub-milli arcsecond scale source compactness" defined as $S_{\mathrm{L}} / S_{\mathrm{S}}$ on milli arcsec scale is 0.74 and peaks at 0.76 . From the $S_{\mathrm{L}} / S_{\mathrm{S}}$ plot shown on botton right of figure 4.57, we can see that there are certain sources for which correlated flux density on the longer baseline is more, since they are very compact and faint. The distribution of $S_{\mathrm{L}} / S_{\mathrm{S}}$ for quasars, BL Lac objects and radio galaxies is investigated. The median and mean of $S_{\mathrm{L}} / S_{\mathrm{S}}$ for quasars is 0.48 and 0.57 , respectively and for BL Lac objects is 0.47 and 0.56 , respectively and that of radio galaxies is 0.38 and 0.48 , respectively. This implies that 
the quasars and BL Lac objects have a similar distribution whereas radio galaxies have a quite different distribution. This means that quasars and BL Lac objects are more compact than radio galaxies on sub-milli arcsecond scale. This finding is consistent with the unified scheme for AGN predicted by Urry \& Padovani (1995). Since quasars and BL Lac objects are observed close to the line of sight (Urry \& Padovani 1995) and hence Doppler boosted, we expect them to show more compactness on sub-milli arcsecond scales. But galaxies are still extended since they are observed at large viewing angles.

The flux density of the core components $S_{\text {core }}$ in the sample peaks at $0.23 \mathrm{Jy}$ and this distribution is shown on top panel of figure 4.58. The flux density of only the resolved core components in the sample peaks at $0.28 \mathrm{Jy}$. The size of the core components in this sample, $d_{\text {core }}$ on milli arcsec scale shown in middle panel of Figure 4.58 peaks at 0.03 mas. Most of the cores are smaller than 0.3 mas and greater than 0.01 mas. The size of most of the unresolved cores (shown in the bottom panel of figure 4.58) falls in the range from 0.009 mas to 0.036 mas.

\subsection{Summary}

We have used the Global Millimeter VLBI Array (GMVA) to conduct a large global $86 \mathrm{GHz}$ VLBI survey comprising 174 snapshot observations of 162 unique targets selected from a sample of compact radio sources. The survey observations have reached a typical baseline sensitivity of $0.1 \mathrm{Jy}$ and a typical image sensitivity of $5 \mathrm{mJy} /$ beam, owing to the increased recording bandwidth of the GMVA observations and the participation of large European antennas at Pico Veleta and Plateau de Bure. All of the 162 objects have been detected and imaged, thereby increasing by a factor of $\sim 2$ the total number of AGN imaged with VLBI at $86 \mathrm{GHz}$.

We have used Gaussian model fitting to represent the structure of the observed sources and estimate the flux densities and sizes of the cores and nearest jet components. For sources with extended structure detected, the model fit parameters have been also used to calculate brightness temperature in the jet components downstream of the core. The apparent brightness temperature estimates for the cores in our sample ranges from $1.1 \times$ $10^{9} \mathrm{~K}$ to $1.3 \times 10^{12} \mathrm{~K}$, with the mean brightness temperature of $1.8 \times 10^{11} \mathrm{~K}$. The brightness temperature estimates for the nearest jet components in our sample ranges from $5.8 \times 10^{7} \mathrm{~K}$ to $4.1 \times 10^{11} \mathrm{~K}$. The overall amplitude calibration error for the observations is within $25 \%$, to which extent the results of the flux densities and brightness temperatures are accurate.

We used the model fit parameters and visibility data on the longest baselines to make independent estimates of brightness temperatures at the jet base as described by the most compact and bright "VLBI core" component. These estimates are consistent with each other. $T_{\mathrm{b}, \text { mod }}$ calculated for all the sources are either well bracketed inside $T_{\mathrm{b}, \min }$ and $T_{\mathrm{b}, \mathrm{lim}}$ or in well agreement with $T_{\mathrm{b}, \mathrm{lim}}$. The median and mean of the minimum brightness temperature distribution for the core regions is $4.1 \times 10^{10} \mathrm{~K}$ and $6.2 \times 10^{10} \mathrm{~K}$, respectively. The median and mean of the limiting brightness temperature distribution for the core regions is $1.06 \times 10^{11} \mathrm{~K}$ and $3.0 \times 10^{11} \mathrm{~K}$, respectively. 

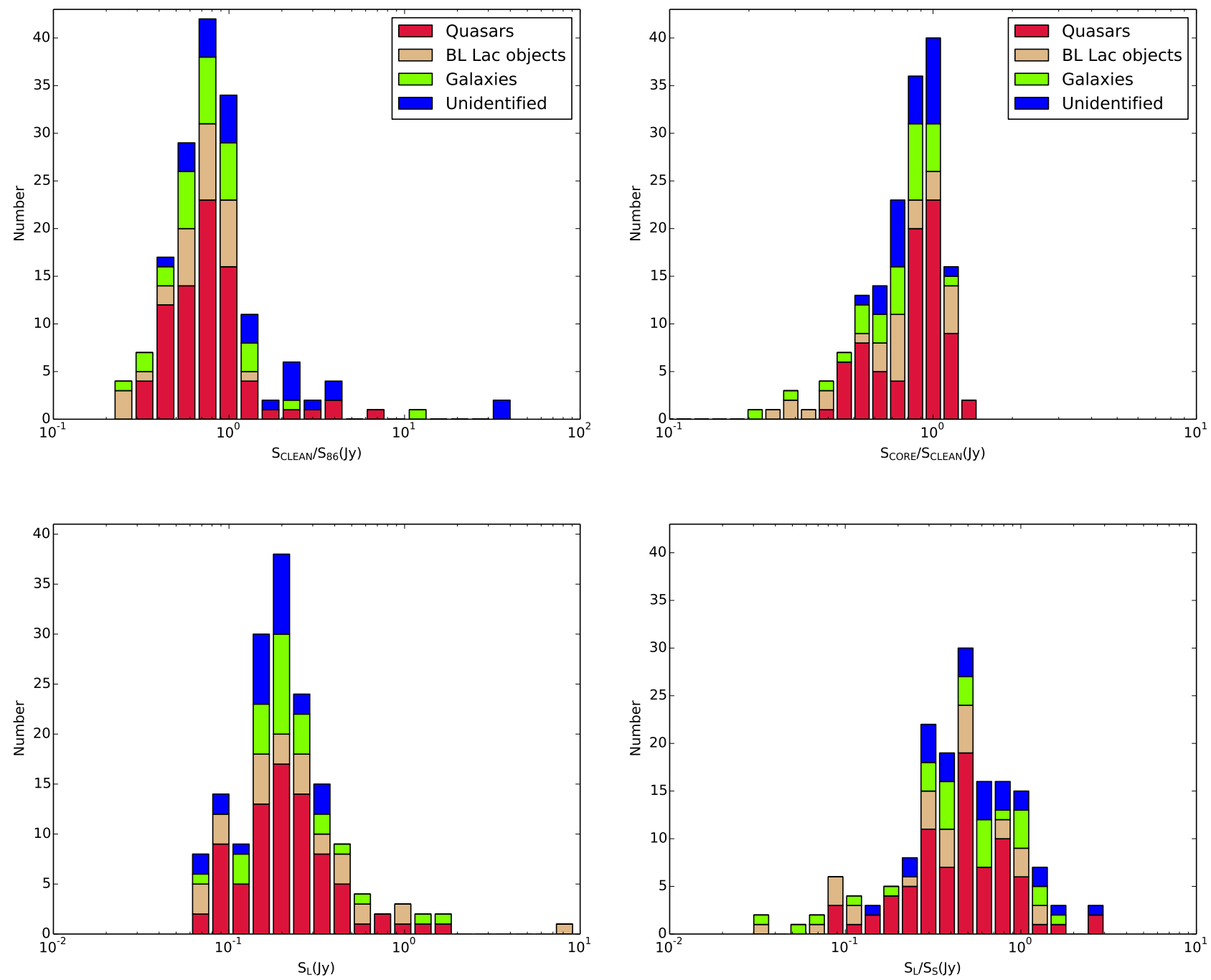

FIGURE 4.57: The distribution of the compactness indices on milli arcsecond scales $S_{\text {CLEAN }} / S_{86}$ (top left), the core dominance indices $S_{\text {CORE }} / S_{\text {CLEAN }}$ (top right), the correlated flux density on long baselines $S_{\mathrm{L}}$ (bottom left) and the compactness indices on sub-milli arcsecond scales $S_{\mathrm{L}} / S_{\mathrm{S}}$ (bottom right). 

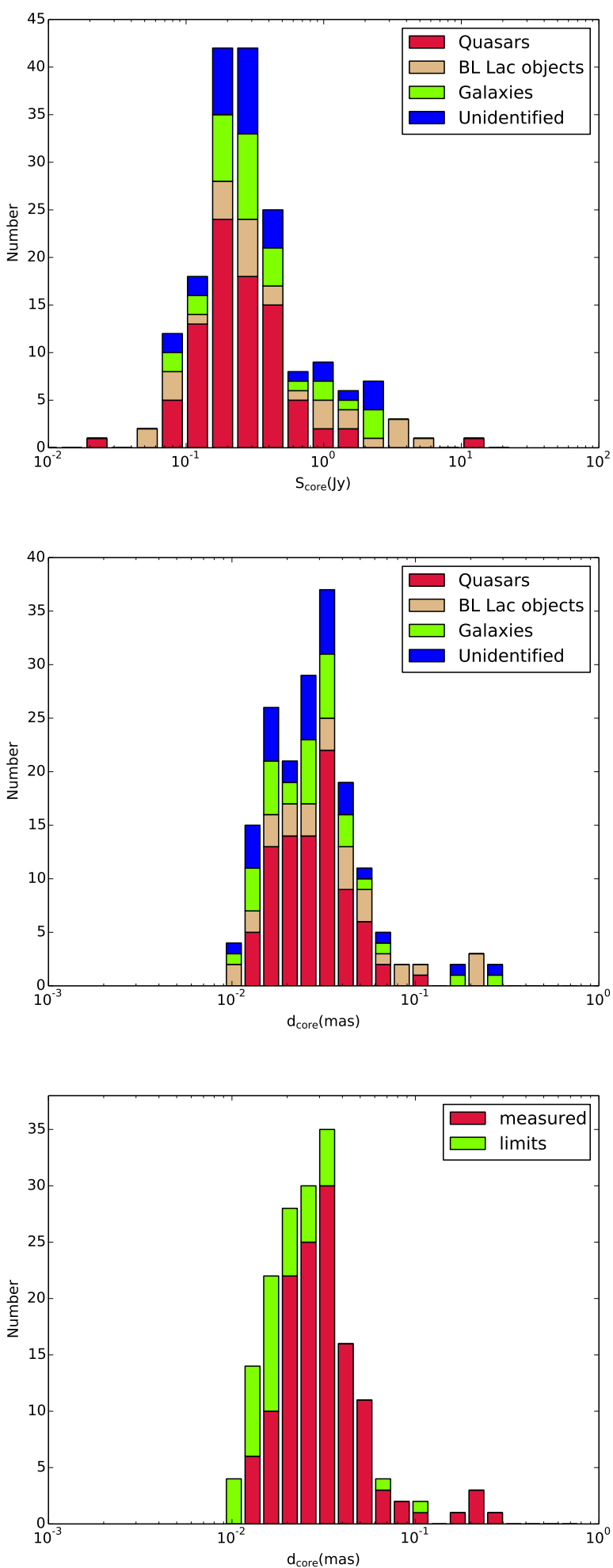

Figure 4.58: The distribution of the total flux density $S_{\mathrm{CORE}}$ (top panel) and the angular size of the core components (middle and bottom panel). In the bottom panel, the green color represents lower limits on size $d_{\min }$ estimated for unresolved core components and the red color represents measured sizes $d$ for resolved core components. 

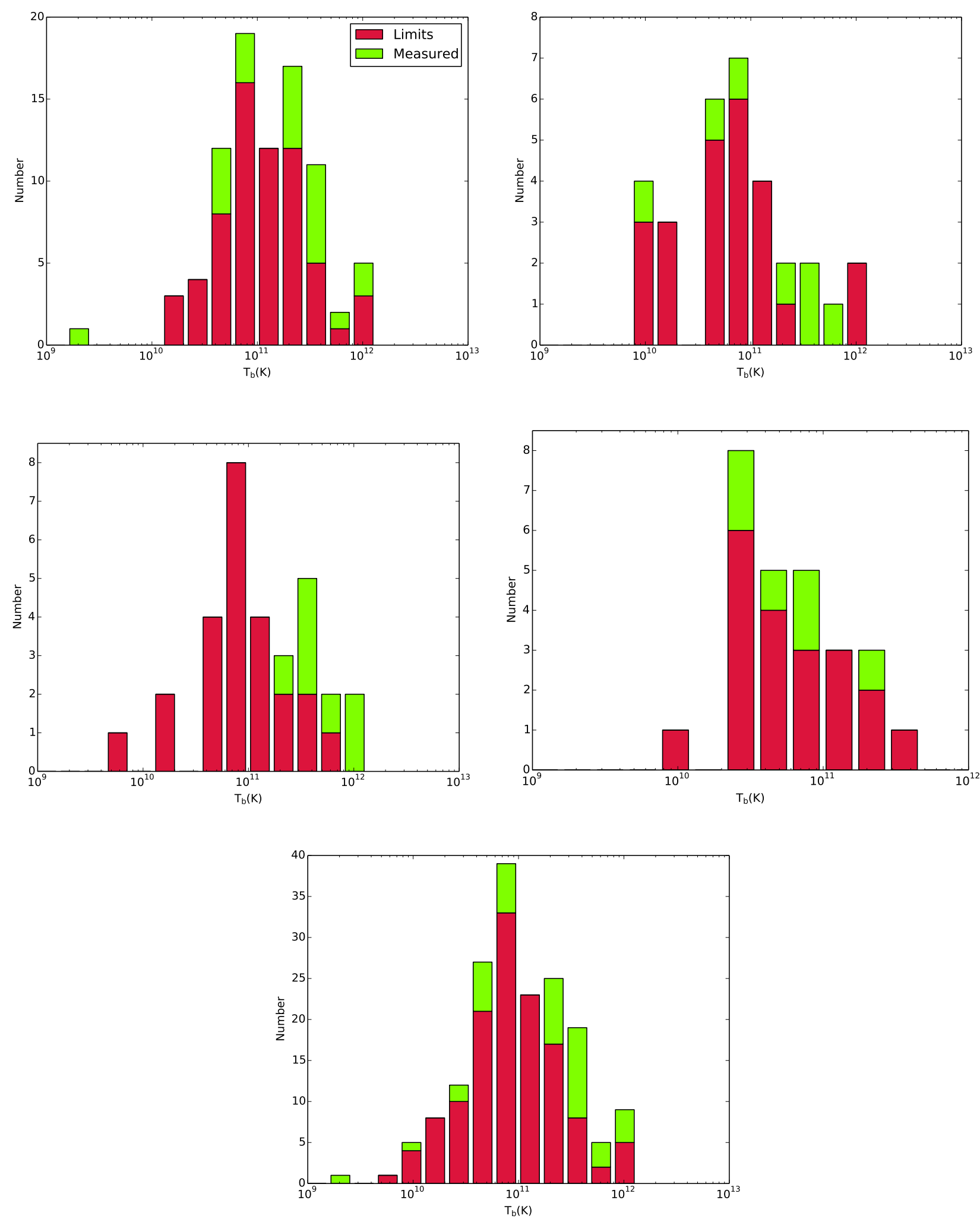

FIGURE 4.59: The distribution of the brightness temperature in the core components of different classes of the sources in this survey - quasars (top left), BL Lac objects (top right), galaxies (middle left), unidentified sources (middle right) and that of all the classes of sources together (bottom panel). The green color represents lower limits on $T_{\mathrm{b}}$ estimated for the minimum resolvable size of a core component and the red color represents measured $T_{\mathrm{b}}$ in resolved core components. 

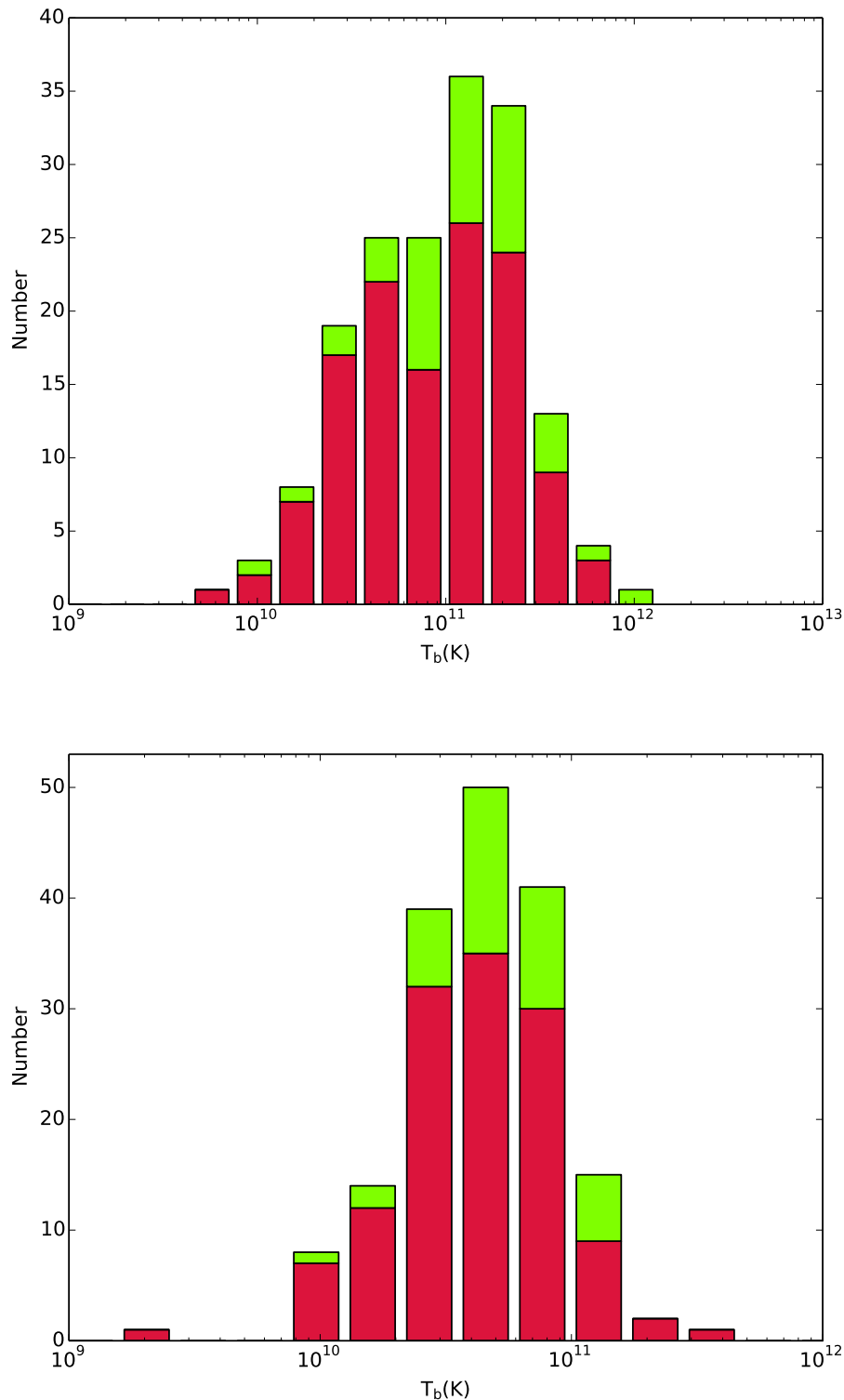

Figure 4.60: The distribution of the brightness temperature in the core components estimated from visibility. The limiting resolved $T_{\mathrm{b}, \text { lim }}$ is shown in top panel and the minimum $T_{\mathrm{b}, \text { min }}$ is shown on the bottom panel. The green color represents lower limits on size $d_{\min }$ for unresolved core components and the red color represents measured sizes $d$ for resolved core components, estimated from Gaussian model fitting. 


\section{Chapter 5}

\section{Modelling the Observed Brightness Temperatures}

This Chapter is partially published in the journal Astronomy \& Astrophysics, and is reproduced with the permission of the Editor-in-Chief. Credit: Nair et al., A\&A, 622, A92 (2019), reproduced with permission (C) ESO.

\subsection{Introduction}

It is non-trivial to measure intrinsic properties of ultracompact radio sources since their jets are highly Doppler boosted due to relativistic beaming. The observed brightness temperatures $\left(T_{\mathrm{b}}\right)$ can be applied to study the intrinsic brightness temperatures $\left(T_{0}\right)$ and other intrinsic properties of jets. The upper limit for the intrinsic brightness temperature of the extragalactic radio sources is usually taken as $10^{12} \mathrm{~K}$, known as Inverse Compton limit, beyond which a rapid inverse Compton cooling of the sources takes place by upscattering of synchrotron photons into high energy X-rays or $\gamma$ rays, thereby causes rapid electron energy losses and extinguishes the synchrotron radiation known as inverse Compton catastrophe (Kellermann \& Pauliny-Toth 1969). Readhead (1994) found a tighter upper limit of the brightness temperature for compact powerful extragalactic radio sources with the study of timescale by radiative cooling by combined synchrotron and inverse Compton radiation. The upper limit on $T_{b}$ was found as $10^{11.45} \mathrm{~K}$. Assuming if there is an equipartition between the particle energy density and the magnetic energy density (Burbidge \& Burbidge 1957), Readhead (1994) found an alternative limit to the brightness temperature in synchrotron self-absorption dominated radio sources at frequencies less than $1 \mathrm{GHz}$ and found the equipartition brightness temperature as $5 \times 10^{10} \mathrm{~K}$ for most of the radio sources. This upper limit on intrinsic brightness temperature in inner jets of AGN can be obtained by a statistical approach to model the observed brightness temperature distribution and can therefore test the theoretical values given by inverse Compton conditions and equipartition conditions.

The brightness temperature distribution can be used for obtaining estimates of the conditions in the extragalactic radio sources and to test the models proposed for the inner jets (Marscher 1995; Lobanov et al. 2000; Homan et al. 2006; Lee et al. 2016) as discussed in detail in section 2.6 in Chapter 2. In this chapter, we describe a population modelling of 
the brightness temperature distribution observed at the base (VLBI core) of the jet and in the innermost moving jet components used to obtain $T_{0}$. The theoretical discussions in this chapter are based on Lobanov et al. (2000). Evolution of the observed brightness temperature along the jet is studied in section 5.5 for the target sources with sufficient extended emission detected.

\subsection{Theory of Population Modelling of $T_{\mathrm{b}}$}

A basic population model (Lobanov et al. 2000) can be used for representing the observed brightness temperature $T_{b}$ distribution under the assumption that the jets have the same intrinsic brightness temperature, $T_{0}$, Lorentz factor, $\gamma_{\mathrm{j}}$ with the corresponding jet speed $\beta_{j}$ $=\left(1-\gamma_{j}^{-2}\right)^{1 / 2}$, and synchrotron spectral index, $\alpha$. The sources are assumed to be randomly oriented in space (within the limits of viewing angles, $\theta$ required by Doppler boosting bias). The jet is also assumed to remain straight within the spatial scales $(\sim 0.5-10 \mathrm{pc})$ probed by the observations. In such a population of jets, the measured brightness temperature, $T_{\mathrm{b}}$, is determined solely by the relativistic Doppler boosting of the jet emission where Doppler factor $\delta=\left[\gamma_{j}\left(1-\beta_{j} \cos \theta\right)\right]^{-1}$. Therefore, the observed brightness temperature $T_{\mathrm{b}}$ and intrinsic $T_{0}$ are related to each other by:

$$
T_{\mathrm{b}}=T_{0} \delta^{1 / \epsilon}
$$

where the power index $\epsilon$ is $1 /(2-\alpha)$ for a continuous jet (steady state jet) and $1 /(3-\alpha)$ for a jet with spherical blobs (or optically thin "plasmoids") i.e. moving component or feature in the jet.

The corresponding viewing angle for a source with observed brightness temperature $T_{b}$ can be found from:

$$
\cos \theta=\left(\gamma_{j}^{2}-1\right)^{-1 / 2}\left[\gamma_{j}-\delta^{-1}\right]^{\epsilon}
$$

and

$$
\sin \theta=\left(\gamma_{j}^{2}-1\right)^{-1 / 2}\left[2 \gamma_{j} \delta^{-1}-\delta^{-2}-1\right]^{1 / 2}
$$

Only approaching jets are assumed here. For a sample without Doppler boosting bias, this implies $0 \leq \theta \leq \pi / 2$, and the corresponding observed brightness temperatures ranges from

$$
T_{\text {low }}=\left.T\right|_{\theta=\pi / 2}=T_{0} \gamma_{j}^{2-\alpha}
$$

to

$$
T_{\text {high }}=\left.T\right|_{\theta=0}=T_{0}\left[\gamma_{j}-\left(\gamma_{j}^{2}-1\right)^{1 / 2}\right]^{2-\alpha} .
$$

At the same time, if the sample is biased by Doppler boosting, then $T_{\text {low }}$ should be substituted by the lowest brightness temperature, $T_{\text {lim }}$, that can be measured from observations ( $T_{\lim }>T_{\text {low }}$ in biased samples and $T_{\text {lim }} \leq T_{\text {low }}$ in unbiased samples) (Lobanov et al. 2000).

A valid distribution can therefore be derived for all $T_{0} \geq T_{\lim } \beta_{j}^{\alpha-2}$. The probability density of the brightness temperature distribution is then obtained as $p\left(T_{b}\right) d \theta=\sin \theta d \theta$, and the probability $P\left(T_{1} \leq T_{b} \leq T_{2}\right)$ is given by,

$$
P\left(T_{1}, T_{2}\right)=2 \pi \eta_{T} \int_{\theta\left(T_{2}\right)}^{\theta\left(T_{1}\right)} \sin \theta d \theta
$$


where the normalization factor

$$
\eta_{T}=\left(2 \pi \int_{0}^{\theta_{\lim }} \sin \theta d \theta\right)^{-1}=\frac{1}{2 \pi\left(1-\cos \theta_{\lim }\right)},
$$

is determined from the condition $P\left(0, \theta_{\lim }\right)=1$.

For a complete sample consisting of $N_{\text {tot }}$ objects, the source density, $\rho=\eta_{T} N_{\text {tot }}$, should be used for normalization. From equations 5.4 and 5.7,

$$
\rho=\frac{N_{\mathrm{tot}}\left(\gamma_{j}^{2}-1\right)^{1 / 2}}{2 \pi\left[\left(\gamma_{j}^{2}-1\right)^{1 / 2}-\gamma_{j}+\left(T_{0} / T_{\mathrm{lim}}\right)^{\epsilon}\right]} .
$$

Therefore, the number of objects in a bin $\left[T_{1}, T_{2}\right]$ is,

$$
N\left(T_{1}, T_{2}\right)=N_{\text {tot }} P\left(T_{1}, T_{2}\right)=\rho \int_{\theta\left(T_{2}\right)}^{\theta\left(T_{1}\right)} \sin \theta d \theta .
$$

For an incomplete sample, $N_{\text {tot }}$ is unknown. So, the only way to determine normalization factor is from the number of objects in the first bin of the observed distribution. The total number of sources required for a complete sample can be estimated as,

$$
N_{\text {tot }}=\frac{n_{1}\left(\gamma_{j}^{2}-1\right)}{T_{1}^{\epsilon}-T_{\lim }^{\epsilon}}\left[\frac{T_{\lim } T_{1}}{T_{0}}\right]^{\epsilon},
$$

where $n_{1}$ is the number of objects and $T_{1}$ is the most upper value of the brightness temperature in the first bin of the distribution. For further details on this section, we refer the reader to Lobanov et al. (2000).

\subsection{Population Modelling of Observed $T_{\mathrm{b}}$}

The probability to find a radio source with the brightness temperature, $T_{\mathrm{b}}$ in a population of sources described thoroughly in the above section is,

$$
p\left(T_{\mathrm{b}}\right) \propto\left[\frac{2 \gamma_{\mathrm{j}}\left(T_{\mathrm{b}} / T_{0}\right)^{\epsilon}-\left(T_{\mathrm{b}} / T_{0}\right)^{2 \epsilon}-1}{\gamma_{\mathrm{j}}^{2}-1}\right]^{\frac{1}{2}} .
$$

The lower end of the observed distribution of brightness temperatures depends on the sensitivity of VLBI data since the flux of the observed sample is biased by Doppler boosting (Lobanov et al. 2000). The lowest brightness temperature that can be measured from our data, $T_{\mathrm{b}, \text { sens }}$ can be obtained from

$$
T_{\mathrm{b}, \text { sens }}[\mathrm{K}]=1.65 \times 10^{5}\left(\frac{\sigma_{\mathrm{rms}}}{\mathrm{mJy} / \text { beam }}\right)\left(\frac{\text { beamsize }}{\text { mas }}\right)^{-2}
$$

where $\sigma_{\text {rms }}$ is the array sensitivity in mJy/beam and beam size $=<\sqrt{B_{\mathrm{a}} \times B_{\mathrm{b}}}>$, where $B_{\mathrm{a}}$ and $B_{\mathrm{b}}$ are the major and minor axes of the restoring beam. The typical observation time on a target source, $\Delta \mathrm{t}$ is 20 minutes and bandwidth is $128 \mathrm{MHz}$ for this survey. Therefore, 
the value of beam size for the sources in this survey is 0.12 mas and the $\sigma_{\text {rms }}$ of the array is $0.54 \mathrm{mJy} /$ beam. Thus, we have obtained a $3 \sigma$ level estimation of $T_{\mathrm{b} \text {,sens }}$ as $2.0 \times 10^{8} \mathrm{~K}$ using equation 5.12. This $T_{\mathrm{b} \text {,sens }}$ is set as the lowest brightness temperature in the modelling. $T_{\mathrm{b}, \text { sens }}$ set a limit on the viewing angle of the approaching jet given by the probability distribution described by equation 5.11 as,

$$
\theta_{\lim }<\arccos \left[\frac{\gamma_{\mathrm{j}}-\left(T_{0} / T_{\mathrm{sens}}\right)^{\epsilon}}{\left(\gamma_{\mathrm{lim}}^{2}-1\right)^{1 / 2}}\right]
$$

We normalize the results obtained from equation (5.11) to the number of objects in the lowest bin of the histogram shown in figures 5.1 and 5.2 for the modelling of the jet cores and the nearest components, respectively. For our modelling, we first make a generic assumption of $\alpha=-0.7$ (homogeneous optically thin synchrotron source) and use $\gamma_{\mathrm{j}} \approx 10$, implied from the kinematic analysis of the MOJAVE VLBI survey data (Lister et al. 2016). We assume that the jet is continuous, so $\epsilon=0.37$ is taken. For the population modelling analysis, we have included the data from Lobanov et al. (2000) and Lee et al. (2008) and the present survey yielding a final data base of 271 unique VLBI core components and 344 jet components. For objects with multiple measurements, we have used the median value of $T_{\mathrm{b}}$ in our measurements. The resulting model distributions obtained for various values of $T_{0}$ are shown in figures 5.1 and 5.2 for the VLBI cores and the inner jet components, respectively.

\subsection{Results and Discussion}

The population modelling described above yields $T_{0 \text {,core }}=\left(3.77_{-0.14}^{+0.10}\right) \times 10^{11} \mathrm{~K}$ for the VLBI cores and $T_{0 \text {,jet }}=\left(1.42_{-0.19}^{+0.16}\right) \times 10^{11} \mathrm{~K}$ for the nearest jet components. The estimated $T_{0 \text {,core }}$ is in a good agreement with the inverse Compton limit of $\simeq 5.0 \times 10^{11} \mathrm{~K}$ (Kellermann \& Pauliny-Toth 1969; Readhead 1994), beyond which the inverse Compton effect causes rapid electron energy losses and extinguishes the synchrotron radiation. The inferred $T_{0 \text {,jet }}$ of jet components are about a factor of three higher than the equipartition limit of $\simeq 5 \times 10^{10} \mathrm{~K}$ (Readhead 1994) for which the magnetic field energy and particle energy are in equilibrium. The intrinsic brightness temperature in jet components is lower by a factor of 2.7 than that of cores. The intrinsic brightness temperature obtained for the cores is within the upper limit $5.0 \times 10^{11} \mathrm{~K}$ predicted by Lobanov et al. (2000) for the population modelling of the cores.

A simultaneous fit for $T_{0}$ and $\gamma_{\mathrm{j}}$ is impeded by the implicit correlation, $T_{0} \propto \gamma_{\mathrm{j}}^{a}$ (with $a \approx 2-$ 3 ), between these two parameters, as implied by the equation (5.11). This is illustrated in figure 5.3 , from which a dependence $T_{0}[\mathrm{~K}] \approx\left(7.7 \times 10^{8}\right) \gamma_{\mathrm{j}}^{2.7}$ can be inferred for the fit to the brightness temperatures measured in the VLBI cores.

In figure $5.3, \gamma_{\mathrm{j}}$ ranges from $1.1-35$ and $T_{0}$ ranges from $10^{7} \mathrm{~K}$ to $10^{13} \mathrm{~K} . \chi^{2}$ values are presented in a color scale with blue corresponding to minimum $\chi^{2}$ values showing an agreement with the population model distribution of $T_{\mathrm{b}}$ and observed $T_{\mathrm{b}}$ whereas red corresponding to very high $\chi^{2}$ values obtained, when there is no agreement with the population model distribution of $T_{\mathrm{b}}$ and the observed $T_{\mathrm{b}}$. The blue strip shows the region where the minimum values of $\chi^{2}$ are concentrated. The minima for each gamma in the $\gamma_{\mathrm{j}}-T_{0}$ space can be seen as the narrow strip in pink color in the $2 \mathrm{D}-\chi^{2}$ distribution. The $1 \sigma$ contour levels for $\chi_{\min }^{2}$ 


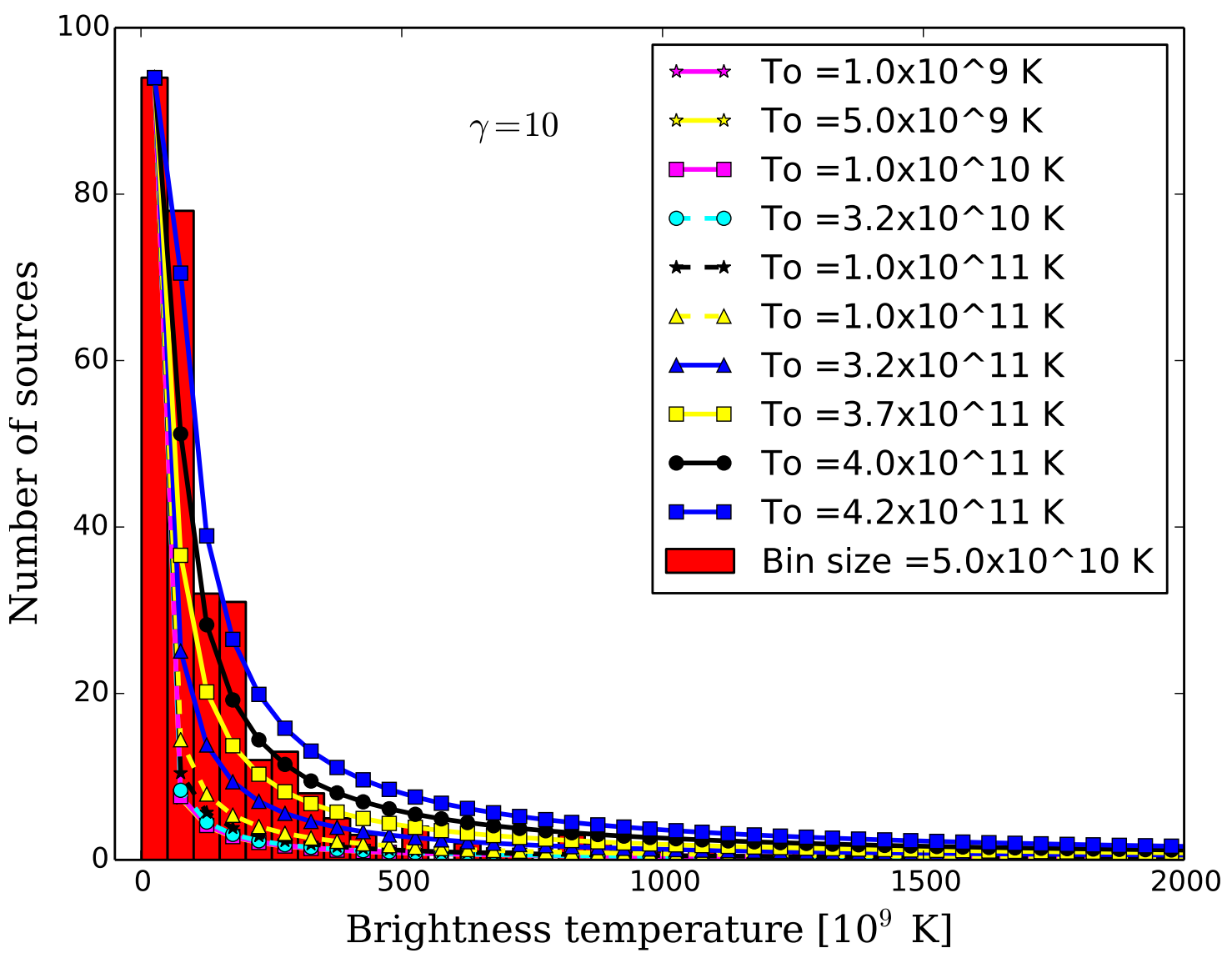

Figure 5.1: Distribution of the brightness temperatures, $T_{\mathrm{b}}$ measured in the core components and represented by the population models calculated for $\gamma_{\mathrm{j}}=10$ and different values of $T_{0}$. The best approximation of the observed $T_{\mathrm{b}}$ distribution is obtained with $T_{\mathrm{o} \text {, core }}=\left(3.77_{-0.14}^{+0.10}\right) \times 10^{11} \mathrm{~K}$. Note that for better viewing of the observed distribution, one core component with a very high $T_{\mathrm{b}}=5.5 \times 10^{12} \mathrm{~K}$ is not shown but is included in the

modelling.

i.e. $\delta \chi^{2}=\chi_{\min }^{2}+2.3$ are shown in two black dashed lines. Equation 5.11 clearly shows that the predicted distribution of $T_{\mathrm{b}}$ is valid within the range,

$$
\left(\gamma_{\mathrm{j}}-\sqrt{\gamma_{\mathrm{j}}^{2}-1}\right) \leq\left(\frac{T_{0}}{T_{\mathrm{b}}}\right)^{\epsilon} \leq\left(\gamma_{\mathrm{j}}+\sqrt{\gamma_{\mathrm{j}}^{2}-1}\right) .
$$

The region outside this range is represented in blank area in white in figure 5.3.

Our results show a clear dominance of the particle energy density over the magnetic field energy density in the core. Presumably, it is unlikely that equipartition exists in parsec scale jets where a highly collimated ejection of relativistic plasma takes place. It is natural to expect an excess of particle energy density than magnetic field energy in parsec scales of AGNs where the ejection of jet particles occur. Also, we should remember that Burbidge \& Burbidge (1957) and Scott \& Readhead (1977) originally introduced the concept of equipartition limit on $T_{\mathrm{b}}$ to minimize the excessive energy content observed in extended radio galaxies NGC 5128 and NGC 1316 with ages of $10^{8-9}$ years. The age of parsec scale jets might be just $\sim 1-100$ years, much lesser than totally evolved kiloparsec jets of age $\sim 10^{8-9}$ years. 


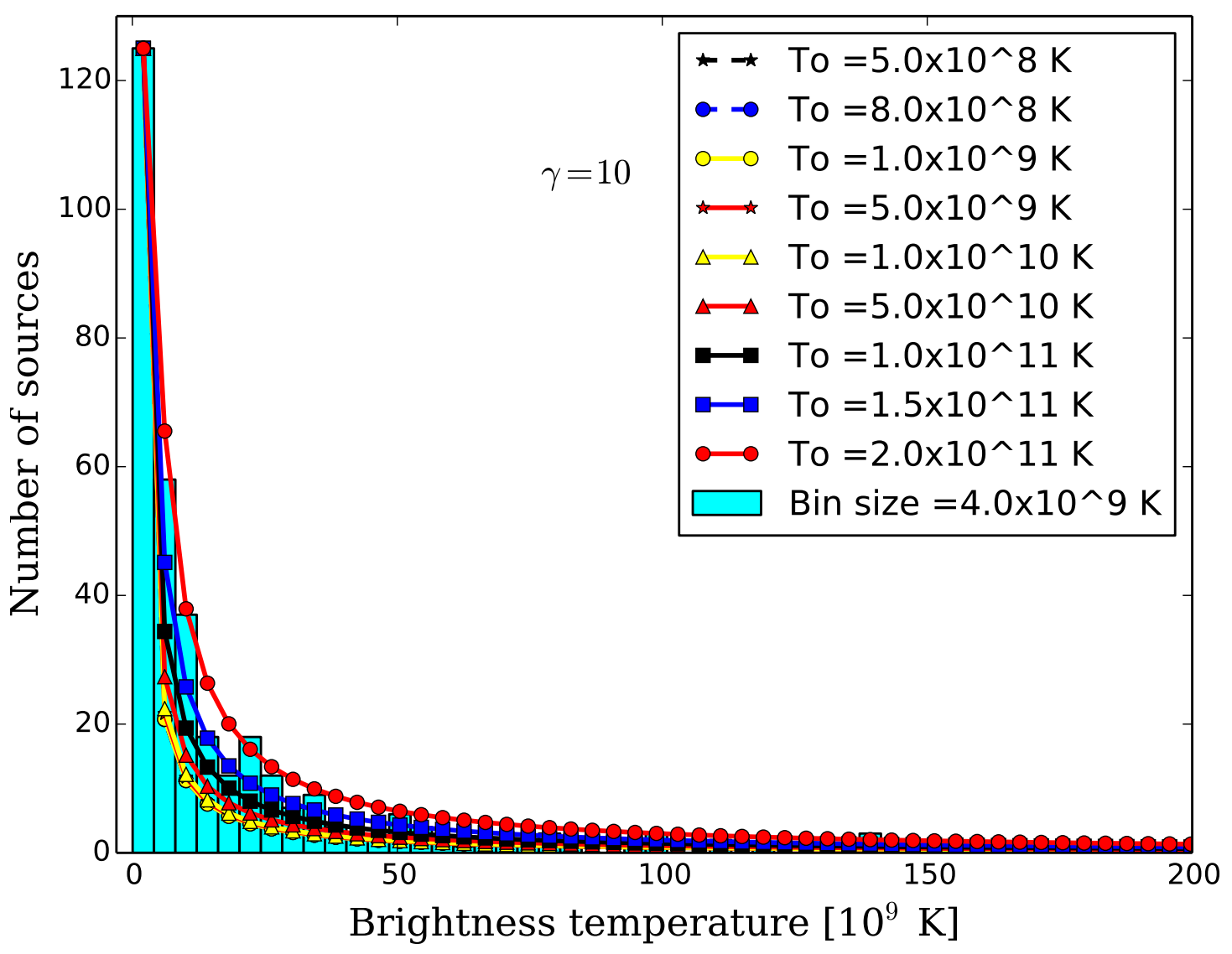

Figure 5.2: Distribution of the brightness temperatures, $T_{\mathrm{b}}$ measured in the inner jet components and represented by the population models calculated for $\gamma_{\mathrm{j}}=10$ and different values of $T_{0}$. The best approximation of the observed $T_{\mathrm{b}}$ distribution is obtained with

$$
T_{\text {o,jet }}=\left(1.42_{-0.19}^{+0.16}\right) \times 10^{11} \mathrm{~K} \text {. }
$$

The intrinsic brightness temperature we obtained is higher than the mean/median observed brightness temperature $T_{\mathrm{b}}$. This is caused by the Doppler deboosting. For a given viewing angle, $\theta$, sources with $\gamma_{\mathrm{j}}>1 / \theta$ would be deboosted so that the observed $T_{\mathrm{b}}$ will appear lesser than the intrinsic $T_{\mathrm{b}}$. Only in the case of blazars and sources very close to the line of sight, the observed $T_{\mathrm{b}}$ will appear greater than the intrinsic brightness. The 162 radio sources in our sources mostly comprise of quasars, radio galaxies and unidentified objects. There are only 26 BL Lac objects which may give a higher observed $T_{\mathrm{b}}$ than $T_{0}$. This can be further explained on the basis of Doppler deboosting given by

$$
T_{\mathrm{b}}=T_{0}\left(\gamma_{j}\left(1-\sqrt{1-\gamma_{j}^{-2} \cos \theta}\right)\right)^{1 / \epsilon},
$$

obtained by modifying relation 5.1. This gives the viewing angle,

$$
\theta=\arccos \left[\frac{1-\left(T_{\mathrm{b}} / T_{0} \gamma_{j}\right)}{\sqrt{1-\gamma_{j}^{-2}}}\right] .
$$

In the case of cores for which the mean of the observed $T_{\mathrm{b}}$ is $1.8 \times 10^{11} \mathrm{~K}$ and intrinsic $T_{0}$ is $3.77 \times 10^{11} \mathrm{~K}$, it can be easily shown that the viewing angle, $\theta=17^{\circ}$. In this case any object observed at a larger viewing angle would be deboosted resulting in a lower observed 


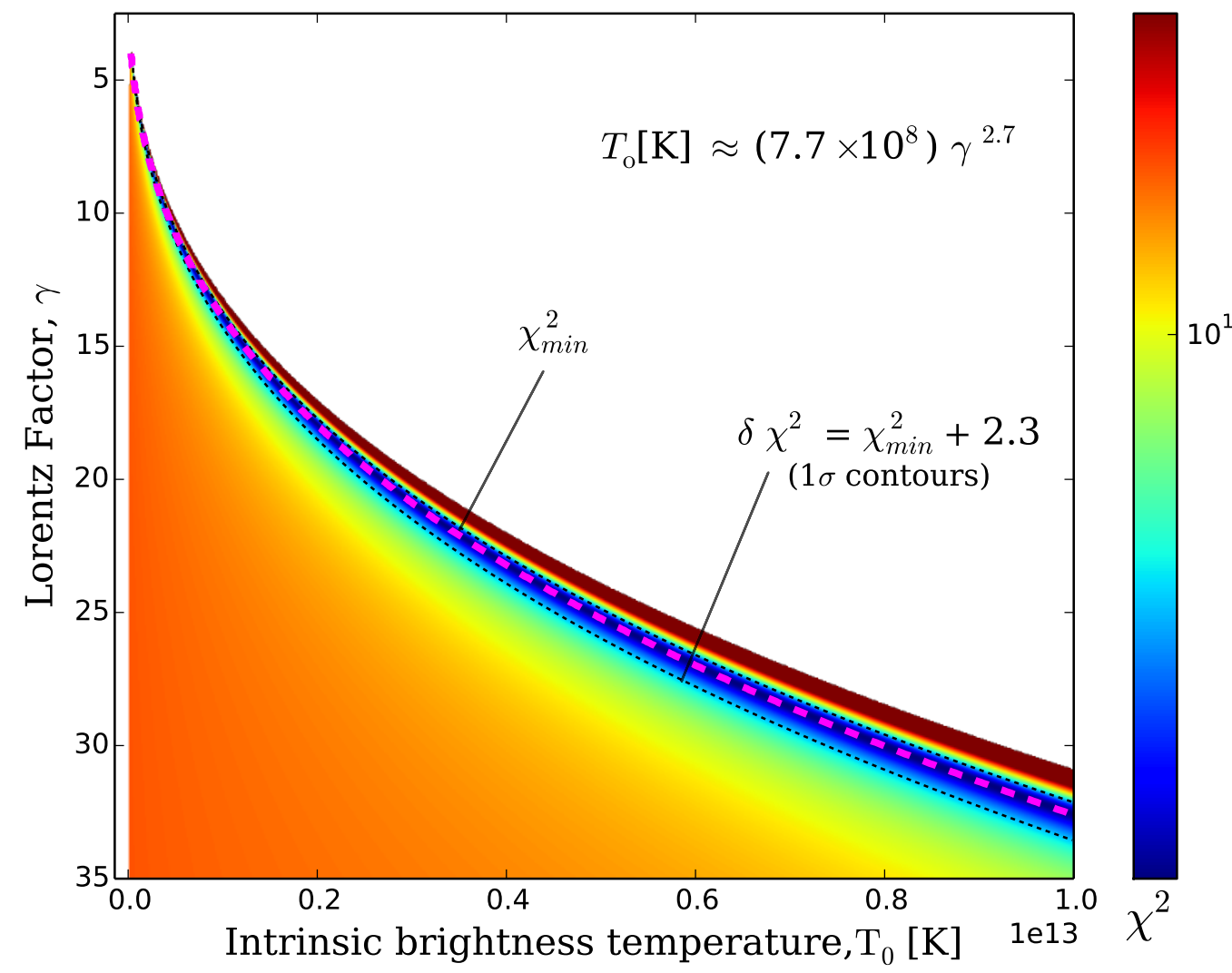

Figure 5.3: The two-dimensional $\chi^{2}$ distribution plot in the $\gamma-T_{0}$ space, calculated for the brightness temperatures measured in the VLBI cores. The blank area (in white) shows the ranges of the parameter space disallowed by the observed distribution. The distribution of the $\chi^{2}$ values indicates a $T_{0}-\gamma$ correlation, with $T_{0}[\mathrm{~K}] \approx\left(7.7 \times 10^{8}\right) \gamma_{\mathrm{j}}^{2.7}$, thus precluding a simultaneous fit for $\gamma_{\mathrm{j}}$ and $T_{0}$.

$T_{\mathrm{b}}$ than intrinsic $T_{0}$. On the other hand, only $8 \%$ of the jet cores show an observed brightness temperature greater than $5 \times 10^{11} \mathrm{~K}$. These represent the candidate sources with higher observed $T_{\mathrm{b}}$ than intrinsic $T_{0}$ in our sample which can be explained by the Doppler boosting of few BL Lac objects in the sample very close to the line of sight.

The maximum $T_{\mathrm{b}}$ that can be measured depends mainly on two factors - the baseline length and the flux density of the source. With a baseline of $\sim 10,000 \mathrm{~km}$ and a source with $\sim 1-5$ Jy flux density, our results on intrinsic brightness temperature $T_{\mathrm{b}}$ from population modelling is at the inverse Compton limit. At the same time, below the critical frequency, RadioAstron (Kardashev et al. 2013) with extremely long baselines, observed extreme brightness temperature beyond the equipartition limit or the inverse Compton limit (Kovalev et al. 2016; Johnson et al. 2016; Pilipenko et al. 2018). These high brightness temperatures are probably due to Doppler boosting (Kellermann \& Pauliny-Toth 1969), interstellar scattering (Johnson et al. 2016), Intra-Day Variable (IDV) sources (Protheroe 2003), coherent emission (Setti \& Swings 2001), transient non-equilibrium events or emission by relativistic protons (Jukes 1967; Kardashev 2000). 


\subsubsection{Comparison of Modelling Results with Low Frequency Surveys}

The approach used to derive the intrinsic brightness temperature at $86 \mathrm{GHz}$ through population modelling expressed by equation 5.11 was also applied to the data at $2 \mathrm{GHz}$ (Pushkarev \& Kovalev 2012), $8 \mathrm{GHz}$ (Pushkarev \& Kovalev 2012) and $15 \mathrm{GHz}$ (Kovalev et al. 2005). We used $\gamma_{\mathrm{j}} \approx 10$, implied from the kinematic analysis of the MOJAVE VLBI survey data (Lister et al. 2016). The modelling was applied only to the cores and the intrinsic brightness temperature for cores at different frequencies are given in Table 5.1.

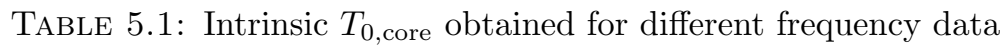

\begin{tabular}{cc}
\hline \hline $\begin{array}{c}\text { Frequency } \\
{[\mathrm{GHz}]}\end{array}$ & $\begin{array}{c}\text { Intrinsic } T_{0, \text { core }} \\
{[\mathrm{K}]}\end{array}$ \\
$(1)$ & $(2)$ \\
\hline 2 & $\left(4.59_{-0.1}^{+0.01}\right) \times 10^{11} \mathrm{~K}$ \\
8 & $\left(4.35_{-0.04}^{+0.03}\right) \times 10^{11} \mathrm{~K}$ \\
15 & $\left(4.59_{-0.04}^{+0.03}\right) \times 10^{11} \mathrm{~K}$ \\
86 & $\left(3.77_{-0.14}^{+0.10}\right) \times 10^{11} \mathrm{~K}$ \\
\hline
\end{tabular}

Columns: 1 - Frequency in Gigahertz; 2 - Intrinsic brightness temperature $T_{0, \text { core }}$ in Kelvin;

Even though, in the global picture, the intrinsic $T_{0 \text {,core }}$ obtained at $86 \mathrm{GHz}$ is lower than that of the cores at low frequencies, potential biases due to inhomogeneity of the samples studied at different frequencies should be investigated further, before reaching firm conclusions on the change of $T_{0}$ with frequency. The difference in intrinsic brightness temperatures clearly suggests that the jet emission should evolve substantially already on the sub-milliarcsecond scales. Such a decrease of $T_{0}$ will provide a strong argument in favor of the decelerating jet model or particle-cascade models as discussed by Marscher (1995). A detailed investigation of intrinsic $T_{0 \text {,core }}$ can be done by compiling the jet speeds, $\beta_{\text {app }}$ (for e.g. from $15 \mathrm{GHz}$ MOJAVE data) with observed $T_{\mathrm{b}}$ (e.g., Homan et al. 2006; Lee 2013). As mentioned in the motivation of this dissertation in Chapter 2 , a decreasing $T_{0}$ would have consequences for higher frequency VLBI.

\subsubsection{Constraints of the Population Model}

The population modelling have some limitations which will cause uncertainties on the derived $T_{0}$. This to be taken into account while investigating the physics of ultracompact radio sources. They are discussed below:

1) Since few of the observed VLBI cores are unresolved at $86 \mathrm{GHz}$, their derived brightness temperature values, in fact, represent only the lower limits. The true $T_{0}$ can be higher than what we have derived if most of the lower limits of the brightness temperatures are concentrated in the few lower bins of the observed distribution. But a $94 \%$ probability for the correlation between derived temperatures and measured flux densities is shown by Lobanov et al. (2000). Therefore, the lower limits on $T_{0}$ are distributed evenly over the entire range of derived brightness temperatures. So, we can conclude that the derived value of $T_{0}$ is not affected by the presence of lower limits of brightness temperature in our data.

2) The incompleteness of sample will affect the uncertainties of the derived $T_{0}$. From equation 5.10 , it is clear that $N_{\text {tot }} \propto \gamma_{j}^{2} T_{0}^{1 / 2.7}$. The assumption we make on $\gamma_{j}$ will affect 
the predicted total number of sources in a complete sample. If we apply equation 5.10 to evaluate the complete sample size, the assumption made about $\gamma_{j}$ affects strongly the predicted total number of sources in a complete sample. For example, if we consider $\gamma_{j}=10$, then $N_{\text {tot }} \sim 100$, which implies that the observed sample used by us in this dissertation is a complete one. Since we have a total of 271 unique sources applied in this modelling, we can safely investigate $T_{0}$ until $\gamma_{j} \sim 16$. Until $\gamma_{j} \sim 16$, our data will remain a complete sample for the frame work of this modelling. For higher $\gamma_{j}$, the number of observed objects must be increased, for the sample to be complete.

3) The model presented in this population modelling expressed by equation 5.11 is analytically computable, but the single value assumptions for $T_{0}$ and $\gamma_{j}$ put some limitations to this model. In order to account for the limitations of our population modelling results, more complex models that consider the distribution law (for example a Gaussian or power law distribution) for $T_{0}$ and $\gamma_{\mathrm{j}}$ has to be considered. This approach being not fully analytically computable, we will need Monte Carlo simulations to calculate the model for different distributions; but it will result in further improvement of the population modelling.

\subsection{Testing the Adiabatic Expansion of Jets}

The intrinsic $T_{0}$ and the observed $T_{\mathrm{b}}$ in core and jets show that the brightness temperature drops by approximately a factor of $\sim(2-10)$ already on sub-parsec scales in the jets. This evolution might occur with the inverse Compton, synchrotron, and adiabatic losses subsequently dominating the energy losses (Marscher 1995; Lobanov \& Zensus 1999). A very basic evolutionary scheme in which the changes observed in the jet emission are caused by the adiabatic energy losses in relativistic shocks is investigated below.

For four objects in our data (3C84, 0716+714, 3C454.3, and J2322+507) for which multiple jet components have been identified during the model fitting, it is possible to use the brightness temperatures of the jet components to test whether the evolution of the jet brightness on sub parsec scales could be explained by adiabatic energy losses (Marscher \& Gear 1985). For this analysis, we assume that the jet components are independent relativistic shocks embedded in the jet plasma which has a power law distribution, $N(E) \mathrm{dE} \propto E^{-s} \mathrm{dE}$ where $s$ is the energy spectral index which depends on spectral index, $\alpha$ as $\alpha=(s-1) / 2$, and pervaded by the magnetic field $B \propto d^{-a}$, where $d$ is the width of the jet and $a$ depends on the type of magnetic field ( $a=1$ for poloidal magnetic field and 2 for toroidal magnetic field).

With these assumptions, we can relate the brightness temperatures, $T_{\mathrm{b}, \mathrm{J}}$, of the jet components to the brightness temperature, $T_{\mathrm{b}, \mathrm{C}}$, of the core (Lobanov et al. 2000; Lee et al. 2008),

$$
T_{\mathrm{b}, \mathrm{J}}=T_{\mathrm{b}, \mathrm{C}}\left(\frac{d_{\mathrm{J}}}{d_{\mathrm{C}}}\right)^{-\xi}
$$

where $d_{\mathrm{J}}$ and $d_{\mathrm{C}}$ are the measured sizes of the jet component and core, respectively, and

$$
\xi=\frac{2(2 s+1)+3 a(s+1)}{6} .
$$

Assuming the synchrotron emission with spectral index $\alpha=-0.5$, we use $s=2.0$ and adopt $a=1.0$ for the description of the magnetic field in the jet. With these assumptions, we 

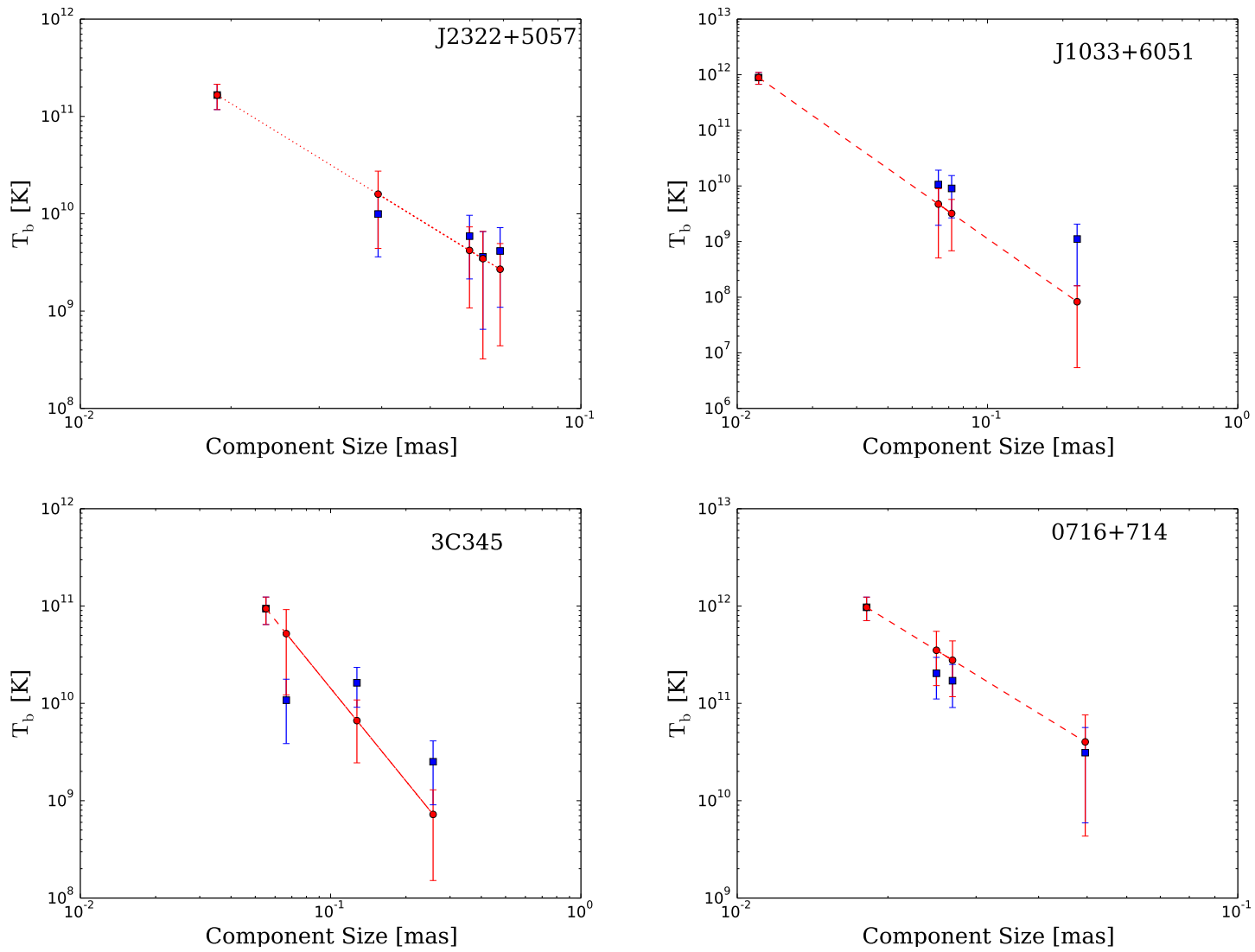

Figure 5.4: Changes of the brightness temperature as a funcion of jet width for four sources $-\mathrm{J} 2322+5037$, J1033+6051, 3C345 and 0716+714 from this survey. Blue squares denote the measured $T_{\mathrm{b}}$ from this survey. The red circles, connected with a dotted line represent theoretically expected $T_{\mathrm{b}}$ under the assumption of adiabatic jet expansion. The initial brightness temperature in each jet is assumed to be same as that measured in the

VLBI core.

calculate the predicted $T_{\mathrm{b}, \mathrm{J}}$ for individual jet components and compare them in Figure 5.4 to the measured brightness temperatures. The measured and predicted values of brightness temperature agree well, and this agreement depend only weakly on the actual choice of the jet spectral index and magnetic field distribution. This suggests that the jet components can be viewed as adiabatically expanding relativistic shocks (cf., Kadler et al. 2004; Pushkarev \& Kovalev 2012; Kravchenko et al. 2016).

\subsection{Conclusion}

A population model with a single intrinsic value of brightness temperature, $T_{0}$, is applied to reproduce the observed brightness temperature distribution. It yields $T_{0}=\left(3.77_{-0.14}^{+0.10}\right) \times$ $10^{11} \mathrm{~K}$ for the jet cores, implying that the inverse Compton losses dominate the emission. In the nearest jet components, $T_{0}=\left(1.42_{-0.19}^{+0.16}\right) \times 10^{11} \mathrm{~K}$ is found, which is slightly higher than the equipartition limit of $\sim 5 \times 10^{10} \mathrm{~K}$ expected for these jet regions. A correlation between $T_{0}$ and Lorentz factor, $\gamma_{\mathrm{j}}$ inherent to the population model description precludes fitting for $T_{0}$ and $\gamma_{\mathrm{j}}$ simultaneously. We find that a relation $T_{0}[\mathrm{~K}] \approx\left(7.7 \times 10^{8}\right) \gamma_{\mathrm{j}}^{2.7}$ is implied for this modelling framework by the survey data. The assumption of single value assumptions 
for $T_{0}$ and $\gamma_{j}$ put some limitations to this model which can be further improved by more complex models that consider the distribution law (for example a Gaussian or power law distribution) for $T_{0}$ and $\gamma_{\mathrm{j}}$.

The intrinsic $T_{0}$ and the observed $T_{\mathrm{b}}$ in core and jets show that the brightness temperature drops by approximately a factor of $\sim(2-10)$ already on sub-parsec scales in the jets. This evolution might occur with the inverse Compton, synchrotron, and adiabatic losses subsequently dominating the energy losses. A very basic evolutionary scheme in which the changes observed in the jet emission are caused by the adiabatic energy losses in relativistic shocks is investigated. For objects with sufficient structural detail detected, the adiabatic energy losses are shown to dominate the observed variations of brightness temperature along the jet, implying that the jet components can be viewed as adiabatically expanding relativistic shocks. 


\section{Chapter 6}

\section{Intrinsic Properties of Ultracompact Radio Sources}

The observed brightness temperature can be used to address the intrinsic properties of ultracompact jets. One way to explore the possibility of observed $T_{\mathrm{b}}$ is to use it along with maximum jet speed $\beta_{\text {app }}$ to derive the intrinsic brightness temperature. A method described by Kovalev et al. (2005); Homan et al. (2006) and Lee (2014) is applied to the observed $T_{\mathrm{b}}$ measurements from this survey combined with the maximum jet speeds obtained from MOJAVE (Lister \& Homan 2005; Lister et al. 2009; Homan et al. 2015; Lister et al. 2016), in order to constrain the intrinsic value of brightness temperature.

There are many inner jet models describing the ultracompact jets (Marscher 1995). The brightness temperature measurements obtained from this survey at $86 \mathrm{GHz}$ can be combined to that at the lower frequencies $(2-43 \mathrm{GHz})$ to study the brightness temperatures in source frame in the sub-parsec scale of the ultracompact radio sources and this can help to test the accelerating and decelerating jet models described in section 2.4.3 in Chapter 2. If the $T_{\mathrm{b}}$ at different frequencies is analyzed, then under the equipartition condition between the magnetic field energy and particle energy density, the core-shift (see section 2.4) can be measured (Lobanov 1998). Under this equipartition condition, the absolute distance of the VLBI core from the central engine can also be predicted. This will help us to test the inner jet models.

In this chapter, the observed $T_{\mathrm{b}}$ at $86 \mathrm{GHz}$ from this survey is compared with the maximum jet speeds at $15 \mathrm{GHz}$ in order to constrain the intrinsic brightness temperature $T_{0}$. The brightness temperature measurements obtained from this survey at $86 \mathrm{GHz}$ were combined with the database of VLBI survey at lower frequencies $(2 \mathrm{GHz}, 8 \mathrm{GHz}, 15 \mathrm{GHz}$; Pushkarev \& Kovalev 2012; Kovalev et al. 2005; Petrov et al. 2007) to study the evolution of $T_{0}$ with frequency and along the jet (Lee et al. 2016). These results and discussions are also presented in this chapter.

\subsection{Intrinsic Brightness Temperature from Jet Speeds and $T_{\mathrm{b}}$}

The highly relativistic jets are Doppler boosted to appear much brighter than their intrinsic brightness. We use the observed brightness temperature $\left(T_{\mathrm{b}}\right)$ and observed jet speeds $\left(\beta_{\mathrm{app}}\right)$ 
to constrain the intrinsic brightness temperature of a sample of the relativistic jets following the method described by Homan et al. (2006) and Lee (2014).

The physical aspects of the jet can be parameterized by the Lorentz factor $\gamma_{j}$, the intrinsic brightness temperature $T_{0}$, and the angle to the line of sight $\theta_{\mathrm{j}}$. From these intrinsic physical properties, the Doppler factor $\delta$, the apparent jet speed $\beta_{\text {app }}$, and the observed brightness temperature $T_{\mathrm{b}}$ can be calculated as:

$$
\begin{gathered}
\delta=\frac{1}{\gamma_{\mathrm{j}}\left(1-\beta \cos \theta_{\mathrm{j}}\right)}, \\
\beta_{\text {app }}=\frac{\beta \sin \theta_{\mathrm{j}}}{1-\beta \cos \theta_{\mathrm{j}}}, \\
T_{\mathrm{b}}=T_{0} \delta,
\end{gathered}
$$

where $\beta=\left(1-\gamma_{\mathrm{j}}^{-2}\right)^{1 / 2}$ is the speed of jet in the rest frame of the source (units of $c$ ).

We assume that a compact radio source contains an ideal relativistic jet, which is narrow and straight with no bends between the VLBI core and the jet components (Lee 2014). In fact, some jets are not straight and that $\theta_{\mathrm{j}}$ is not the same in the core and in the moving jet components. But if superluminal motion is found, the motion must be close to the line of sight. Then the maximum speed of the jet component can be assumed to be the same as the speed of the jet flow through the jet core. All jets are assumed to have same intrinsic brightness temperature $T_{0}$, and all sources have their viewing angles close to the critical value $\theta_{\mathrm{c}}=\arccos \beta$ for the maximal apparent speed at a given $\beta$. Then, the observed brightness temperature can be related to the maximum jet speed as:

$$
\delta \simeq \beta_{\mathrm{app}}
$$

and

$$
T_{\mathrm{b}} \simeq \beta_{\mathrm{app}} T_{0} .
$$

This resultant simple relation between the observed brightness temperature and the apparent maximum jet speed is described as a solid line in figure 6.1.

We use the observed brightness temperatures of VLBI cores at $86 \mathrm{GHz}$ from this dissertation and the apparent jet speeds taken from the $15 \mathrm{GHz}$ MOJAVE survey (Kovalev et al. 2005; Homan et al. 2006; Lee 2014). From a simulation of a relativistically beamed population of 1000 fictional compact radio sources with given $T_{0}$ and $\gamma_{\mathrm{j}}$, Homan et al. (2006) found that approximately $75 \%$ of the sources fall below and to the right of the dashed line representing the critical angle. Therefore, sources located to the right and below the dashed line are indeed inside the critical angle, and they have large Doppler factors. So we also choose the $T_{0}$ such that approximately $75 \%$ of the sources fall below and to the right of the dashed line. We found an intrinsic brightness temperature of $T_{0}=6.2 \times 10^{9} \mathrm{~K}$ at $86 \mathrm{GHz}$ using this method.

The intrinsic brightness temperature of $T_{0}=6.2 \times 10^{9} \mathrm{~K}$ is indeed less than the equipartition value of $5 \times 10^{10} \mathrm{~K}$. The difference in $T_{0}$ obtained by this method from population modelling method might be due to the strong limitation put by the assumption in this method - that the sources are observed at critical angle, which is not true for many sources. Further 


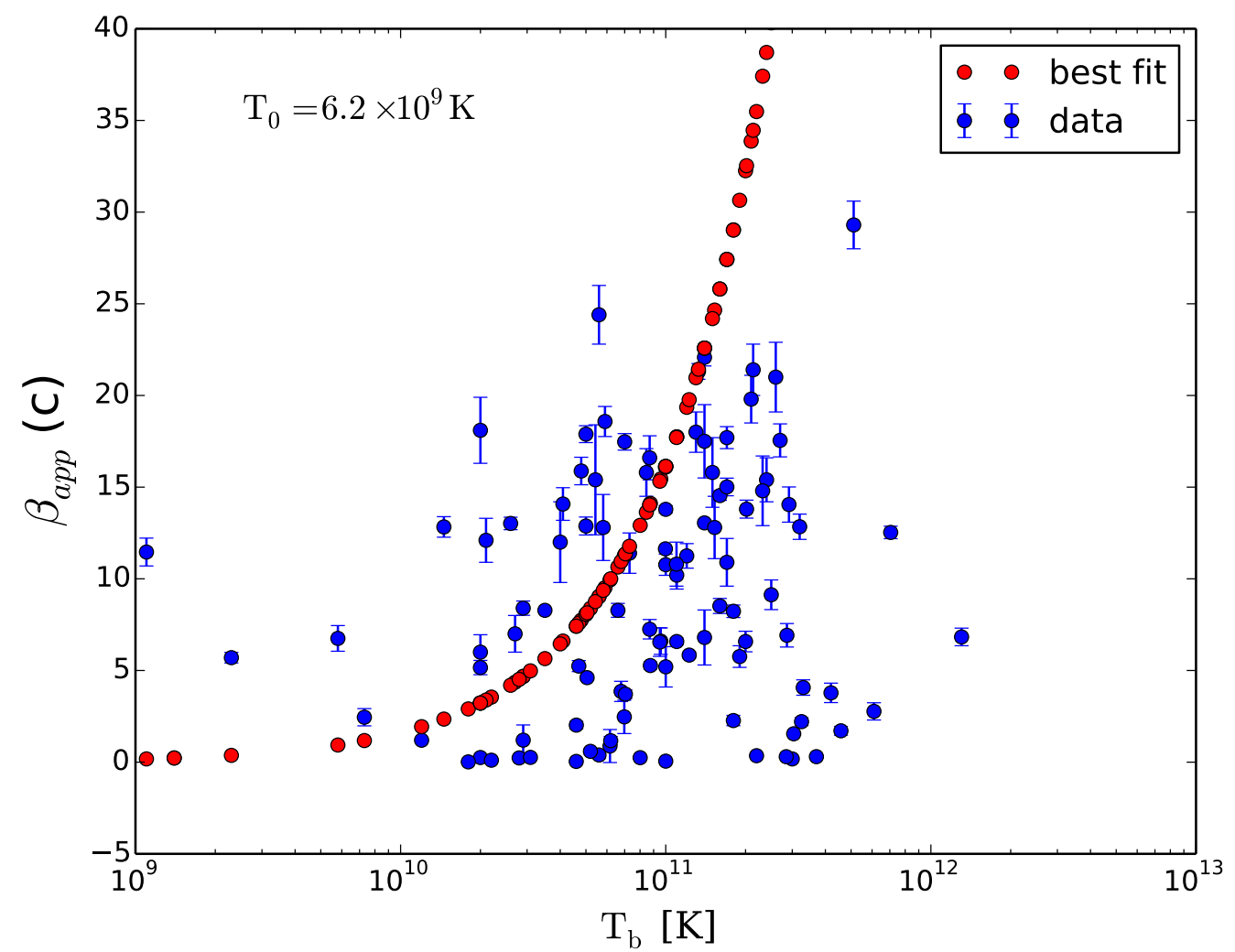

Figure 6.1: Plot of the apparent jet speed versus the observed brightness temperature for the sources in our $86 \mathrm{GHz}$ sample. The dashed line represents sources observed at the critical angle that have the intrinsic brightness temperature of $T_{0}=6.2 \times 10^{9} \mathrm{~K}$.

investigation is required on this approach. But in general, we can expect a conversion of the magnetic field energy into the kinetic energy of particles in the jet and the intrinsic brightness temperature may increase as going down stream of the jet. Homan et al. (2006) found that AGN cores at $15 \mathrm{GHz}$ are near equipartition in their median low state, yielding the intrinsic brightness temperatures of $T_{0}=3 \times 10^{10} \mathrm{~K}$ for a $75 \%$ of their sample at $15 \mathrm{GHz}$. The decrease of $T_{0}$ from $15 \mathrm{GHz}$ to $86 \mathrm{GHz}$ is clear in this approach as well. This would also give a favorable argument to the decelerating jet model or particle cascade model (Marscher 1995). In the following section the observed brightness temperatures at $86 \mathrm{GHz}$ with those at lower frequencies are analyzed together with that at lower frequencies to study the evolution of brightness temperature on sub parsec and parsec scales of ultracompact radio sources.

\subsection{Ultracompact Jets}

To study the evolution of $T_{0}$ along the jet, we follow the method described by Lee et al. (2016). We follow their method to analyze the data from this survey combined with data from the $86 \mathrm{GHz}$ survey by Lee et al. (2008). We discuss below how the absolute distance of VLBI core (base of the jet) from the central engine can be obtained based on Lee et al. (2016). 


\subsubsection{Theory - Ultracompact Jets}

The idealized model of a steady jet is followed here according to Blandford \& Königl (1979). A narrow conical jet of small opening angle $\phi$ whose axis makes an angle $\theta$ with the line of sight of the observer is assumed. The opening angle is given as:

$$
\phi_{\mathrm{o}}=\phi \csc \theta \text {. }
$$

A constant speed $\beta_{\mathrm{j}}$ is assumed for the jet and a magnetic field, $B$ varying with distance from the apex of the jet i.e. from the central engine, $r$ as $r^{-1}$ is assumed. The relativistic jet flow get accelerated by converting the internal relativistic particle energy $\gamma_{\mathrm{e}}$ to the bulk kinetic energy $\gamma_{\mathrm{k}}$. The particle energy distribution is given as $N\left(\gamma_{\mathrm{e}}\right)=N_{0} \gamma_{\mathrm{e}}^{-s}$ for $\gamma_{\min }(r)<\gamma_{\mathrm{e}}<\gamma_{\max }(r)$, where $s$ is the particle energy spectral index. For a typical spectral index $\alpha=-0.5(\alpha=(1-s) / 2)$ the particle energy distribution reduces to $N\left(\gamma_{\mathrm{e}}\right)=N_{0} \gamma_{\mathrm{e}}^{-2}$.

The total emitted synchrotron power from the emission region which extends from $r_{\min }$ to $r_{\max }$ in the jet is:

$$
L_{\mathrm{syn}}=\frac{1}{8} k_{\mathrm{e}} \Delta \gamma_{\mathrm{j}}^{2} c B^{2} r^{2} \phi^{2},
$$

where $\Delta=\ln \left(r_{\max } / r_{\min }\right)$. The relation expressed by equation 6.7 , is obtained by assuming an equipartition between the jet particle energy and the magnetic field energy (see section 2.2.2 in Chapter 2), given by $k_{\mathrm{e}} \Lambda B^{2} / 8 \pi\left[k_{\mathrm{e}} \leq 1\right.$ and $\left.\Lambda=\ln \left(\gamma_{\max } / \gamma_{\min }\right)\right]$.

If the magnetic field and particle density decrease with $r$ as $B=B_{1}\left(r_{1} / r\right)^{m}$ and $N=$ $N_{1}\left(r_{1} / r\right)^{n}$, where $B_{1}$ and $N_{1}$ are the magnetic field and the electron density at $r_{1}=1$ pc, then the optical depth of synchrotron self-absorbed region, $\tau_{s}$ is given by Rybicki \& Lightman (1979):

$$
\tau_{s}(r)=C_{2}(\alpha) N_{1}\left(\frac{e B_{1}}{2 \pi m_{e}}\right)^{\epsilon} \frac{\delta^{\epsilon} \phi_{\mathrm{o}}}{r^{(\epsilon m+n-1)} \nu^{\epsilon+1}},
$$

where $e, m_{\mathrm{e}}$ are the electron charge and mass, respectively, and $\delta$ is the Doppler factor. This is very useful to analyse VLBI cores since VLBI cores observed at any given frequency is located at a region where the optical depth to synchrotron self-absorption becomes $\tau_{s}=1$ in the jet.

Here $\epsilon=3 / 2-\alpha$, and $C_{2}(\alpha)$ is a constant at a given spectral index (Blumenthal \& Gould 1970). For a typical $\alpha=-0.5,\left(S_{\nu} \propto \nu^{\alpha}\right), C_{2}(\alpha)=8.4 \times 10^{10}$ in cgs units. The physical distance of the observed VLBI core from the central engine can be obtained by equating the optical depth $\tau_{\mathrm{s}}(r)$ to unity as,

$$
r=\left[\nu^{-1}(1+z)^{-1} B_{1}^{k_{\mathrm{b}}}\left\{6.2 \times 10^{18} C_{2}(\alpha) \delta_{\mathrm{j}}^{\epsilon} N_{1} \phi_{\mathrm{o}}\right\}^{1 /(\epsilon+1)}\right]^{1 / k_{\mathrm{r}}}
$$

where $k_{\mathrm{r}}=((3-2 \alpha) m+2 n-2) /(5-2 \alpha)$ and $k_{\mathrm{b}}=(3-2 \alpha) /(5-2 \alpha)$. According to Königl (1981), the most appropriate value to be used is $m=1$ and $m=2$ to explain the X-ray and synchrotron emission from the ultracompact jets (see section 2.4 in Chapter 2). For $m=1, n=2, k_{\mathrm{r}}=1$ and is independent of spectral index.

For the region of jet where equipartition condition is valid, the absolute position of the observed VLBI core $r$ and total synchrotron luminosity $L_{\text {syn }}$ are related as: 


$$
r=\left[\xi \mathcal{C}_{\mathrm{r}} L_{\mathrm{syn}}^{k_{\mathrm{b}} / 2}\{\nu(1+z)\}^{-1}\right]^{1 / k_{\mathrm{r}}} \mathrm{pc},
$$

with

$$
\xi=\left(\frac{8}{k_{\mathrm{e}} \Delta}\right)^{k_{\mathrm{b}} / 2}\left[6.2 \times 10^{18} C_{2}(\alpha)\right]^{1 /(\epsilon+1)}
$$

and

$$
\mathcal{C}_{\mathrm{r}}=\frac{\left(\delta_{\mathrm{j}}^{\epsilon} N_{1} \phi_{\mathrm{o}}\right)^{1 /(\epsilon+1)}}{\left(\gamma_{\mathrm{j}}^{2} \beta_{\mathrm{j}} c r_{1}^{2} \phi^{2}\right)^{k_{\mathrm{b}} / 2}},
$$

where $L_{\text {syn }}$ is in erg/s and $\nu$ is in $\mathrm{Hz}$.

\subsection{Results and Discussion}

The database of VLBI survey at lower frequencies $(2 \mathrm{GHz}, 8 \mathrm{GHz}, 15 \mathrm{GHz}$; Pushkarev \& Kovalev 2012; Kovalev et al. 2005; Petrov et al. 2007) were obtained to study the evolution of $T_{0}$ with frequency and along the jet (Lee et al. 2016). The core brightness temperatures were recalculated in the source frame correcting for redshift also. Where ever we had multi epoch measurements at any frequency, we took the median value of them to take the value near the equipartition (Lee et al. 2016).

In the top panel of figure 6.2, the observed brightness temperatures in source frame $T_{\mathrm{b}}$ is showed as a function of frequency in the rest frame of source, $\nu^{\prime}=\nu_{\mathrm{ob}}(1+z)$. The brightness temperatures at lower frequencies between 2 and $10 \mathrm{GHz}$ are increasing and then start decreasing at around $20 \mathrm{GHz}$. Equation 6.9 clearly implies that the position of core is given as $r_{\text {core }} \propto \nu^{\prime}-1 / k_{\mathrm{r}}$. This means that the position of the core is inversely proportional to the source rest frame frequency (for $m=1, n=2, k_{\mathrm{r}}=1$ ). Therefore the plot in the top panel of figure 6.2 gives a clue that the brightness temperature might be increasing from the innermost region $\left(\nu^{\prime}=400 \mathrm{GHz}\right)$ to the outer region $\left(\nu^{\prime}=10 \mathrm{GHz}\right)$ and thereafter, remains somewhat constant.

This figure indicates that brightness temperatures measured at $86 \mathrm{GHz}$ are systematically lower than that at low frequencies $(2,8$, and $15 \mathrm{GHz})$. We had a total of 210, 204, 159 and 98 sources for 2, 8, 15 and $86 \mathrm{GHz}$ data, respectively. Even though we take into consideration the uncertainties in amplitude calibration error $(25 \%)$ at $86 \mathrm{GHz}$ and the source variability of a factor of $\sim 2$, still the brightness temperatures observed at $86 \mathrm{GHz}$ are lower than other low frequencies (cf., Lee et al. 2016). Theoretical models predict $T_{0} \propto \nu^{\epsilon}$, with $\epsilon \approx 2.8$, below a critical frequency $\nu_{\text {break }}$ at which energy losses begin to dominate the emission (Marscher 1995). Above $\nu_{\text {break }}, \epsilon$ can vary from -1 to +1 , depending on the jet composition and dynamics. Previous studies (Lobanov et al. 2000; Lee et al. 2008) indicate that the value of $\nu_{\text {break }}$ is likely to be below $86 \mathrm{GHz}$ and $\nu_{\text {break }}$ can be as low as $20 \mathrm{GHz}$. This is consistent in our sample also. If $T_{0}$ starts to decrease at $86 \mathrm{GHz}$, there will be only a few sources suitable for VLBI $>230 \mathrm{GHz}$ and higher frequencies. Such a decrease of $T_{0}$ will also provide a strong argument in favor of the decelerating jet model or particle-cascade models as discussed by Marscher (1995).

In the middle panel of figure 6.3, there is a clear dependence of brightness temperature with distance from the central engine. The large scatter seen in this plot is due to the dependence of the absolute position of the core on the synchrotron luminosity (see equation 
6.10). Since the particle energy and magnetic field energy densities at $1 \mathrm{pc}$ i.e. $N_{1}$ and $B_{1}$, are different from source to source, it is difficult to determine the absolute position of the core from equation 6.9 But if the core-shift is measured (Lobanov 1998), then it is easy to use equation 6.9. All sources are assumed to have the same Lorentz factor $\gamma_{\mathrm{j}}=10$, jet opening angle $\phi_{\mathrm{o}}=1 / \gamma_{\mathrm{j}}$, and viewing angle $\theta=1 / \gamma_{\mathrm{j}}$. The magnetic field in the jets are assumed to be constant $B=1 \mathrm{G}$, and the electron density at $1 \mathrm{pc}$ is $N_{1}=5 \times 10^{3} \mathrm{~cm}^{-3}$. The observed cores of sources at low redshifts are very close to the central engine.

The synchrotron luminosity is calculated from the core flux measurements of each source over the range of rest frame frequencies, $\nu_{\min }^{\prime} \leq \nu^{\prime} \leq \nu_{\max }^{\prime}$. We fitted the spectrum of the core which gave total flux over the range of frequencies from $2 \mathrm{GHz}$ to $400 \mathrm{GHz}$. Then, the synchrotron luminosity $L_{\mathrm{syn}}$ is given by:

$$
L_{\mathrm{syn}}=4 \pi D_{\mathrm{L}}^{2} F_{t}
$$

where $D_{\mathrm{L}}$ is luminosity distance and $F_{t}$ is the integrated flux. Tables 6.1 to 6.3 list the calculated synchrotron luminosity and core flux density measurements at four frequencies. We use a Hubble constant $H_{0}=71 \mathrm{kms}^{-1} \mathrm{Mpc}^{-1}$ and a cosmological density parameter $\Omega_{\mathrm{m}}=0.27$. It is clear in the plot in the middle panel of figure 6.2 that the brightness temperature is increasing from the inner region to the outer region of sub-parsec scales. This gives a strong implication that the energy of the radiating particles is increasing as they are moving out from the central engine in the sub-parsec scale region of the jet (cf., Lee et al. 2016). The further investigation is described in the next sub section.

\subsubsection{Modelling the Radial Dependence of $T_{\mathrm{b}}$}

Since it is clear that there is an obvious radial dependence of brightness temperature, we tried to fit and investigate the observed brightness temperature $T_{\mathrm{b}}$. We follow here a method described by Lee et al. (2016). We started with a single power law fit which is shown in the top panel of figure 6.3. This gives a dependence of $T_{\mathrm{b}} \sim r^{0.5}$.

One single power law seems to fit the data, but on sub-parsec scales a number of data points fall below the best fit line. So a single power law fit may not be a good idea. Therefore, in order to understand the trend, we fitted the data with multiple power law fit as shown in the middle panel of figure 6.3. The grey lines show the restricted ranges used for multiple power law fitting. The power law fits from $0.01-0.5 \mathrm{pc}, 0.35-10 \mathrm{pc}$ and $5-100 \mathrm{pc}$ yields a power law index $0.3,0.8$ and 0.1 , respectively. In general, we can see that from the vicinity of the central engine, the brightness temperature increases slowly (power index of 0.3 ) and then rises with a steeper slope (power index of 0.8) and then slow down again and become almost constant with a power index of 0.1 . The outer region may be further investigated by compiling data from lower frequencies below $2 \mathrm{GHz}$. There is an increasing pattern in the inner region $(0.01-0.5 \mathrm{pc})$, which looks different from the outer region $(0.35-10 \mathrm{pc})$. Then we can see a slow trend very outside from $(5-100 \mathrm{pc})$. However, the large scatter of data in $5-100 \mathrm{pc}$ may make it difficult to find an increasing trend there. The multiple power law functions clearly imply a slowly increasing trends of the brightness temperature in very inside regions of $0.01-0.5 \mathrm{pc}$. Therefore, we conclude that the $T_{\mathrm{b}}$ increases with different slopes in different regions for our data.

For further analysis, we used a single function used to define such multiple power law fits in the $T_{\mathrm{b}}-r$ plane by Lee et al. (2016) to model the overall evolution of the brightness 
temperatures along the jet. The empirical model given by Lee et al. (2016) is:

$$
T_{\mathrm{b}}=T_{0}+\left(T_{\mathrm{m}}-T_{0}\right)\left\{1-(r \operatorname{csch} r)^{a}\right\},
$$

where $T_{0}$ and $T_{\mathrm{m}}$ are the minimum (or initial) and maximum brightness temperatures in kelvin, respectively, and $r$ is the distance of the VLBI core components from the central engine in parsecs.

This model assumes that $T_{0}$ is the brightness temperature near the jet base and that $T_{\mathrm{m}}$ is in the downstream of the jet. We used $T_{0}=3.742 \times 10^{10} \mathrm{~K}$, which corresponds to the mean of the observed brightness temperatures at $r=0.01-0.5 \mathrm{pc}$ in the plot shown in the middle panel of figure 6.3. The best fitting was obtained for $T_{\mathrm{m}}=(7.96 \pm 0.47) \times 10^{11} \mathrm{~K}$ and $a=0.65 \pm 0.19$, as shown in the plot in the bottom plot of figure 6.3.

This gives a clue that the brightness temperatures on sub-parsec scales are close to the equipartition temperature of $5 \times 10^{10} \mathrm{~K}$ and start to increase on sub-parsec regions, reaching the inverse Compton limit of $\sim 10^{12} \mathrm{~K}$ on parsec scales. The kind of trend on brightness temperature matches with the magnetically driven, accelerating jet model given by Vlahakis \& Königl (2004). According to Vlahakis \& Königl (2004), the mass flux is initially constant in the sub-parsec scale and then increases, and then the mass flux gets constant again in the outer region. Studies by Lee et al. (2016) has shown that the Lorentz factor also show a trend such as being constant in the inner region and then increasing in the outer region and as the mass flux gets constant, the Lorentz factor also becomes constant in the outer region. The sub-parsec-scale accelerations of relativistic AGN jets are reported in the cases of Cygnus A (Boccardi et al. 2016), M87 (Asada et al. 2014) and in many other sources.

\subsection{Conclusion}

Using a method described by Homan et al. (2006), an intrinsic brightness temperature of $T_{0}=6.2 \times 10^{9} \mathrm{~K}$ is found which is indeed less than the equipartition value of $5 \times 10^{10} \mathrm{~K}$. The difference in $T_{0}$ obtained by this method from population modelling method might be due to the strong limitation put by the assumption in this method - that the sources are observed at critical angle, which is not true for many sources. Further investigation is required on this approach. But in general, we can expect a conversion of the magnetic field energy into the kinetic energy of particles in the jet and the intrinsic brightness temperature may increase as going down stream of the jet. Homan et al. (2006) found that AGN cores at $15 \mathrm{GHz}$ are near equipartition in their median low state, yielding the intrinsic brightness temperatures of $T_{0}=3 \times 10^{10} \mathrm{~K}$ for a $75 \%$ of their sample at $15 \mathrm{GHz}$. The decrease of $T_{0}$ from $15 \mathrm{GHz}$ to $86 \mathrm{GHz}$ is clear in this approach as well. This would also give a favorable argument to the decelerating jet model or particle cascade model (Marscher 1995).

Under the equipartition condition between the magnetic field energy and particle energy density, the absolute distance of the VLBI core from the central engine can be predicted. A single power law fit in the $T_{\mathrm{b}}-r$ plane gives a dependence of $T_{\mathrm{b}} \sim r^{0.5}$. In general, we can see that from the vicinity of the central engine, the brightness temperature increases slowly (power index of 0.3 ) and then rises with a steeper slope (power index of 0.8 ) and then slow down again and become almost constant with a power index of 0.1. The multiple power law functions clearly imply a slowly increasing trends of the brightness temperature in very inside regions of $0.01-0.5 \mathrm{pc}$, then increases with steep slope and then slow down. Therefore, we conclude that the $T_{\mathrm{b}}$ increases with different slopes in different regions 
for our data. An empirical model was also applied to the data. The best fitting was obtained for $T_{\mathrm{m}}=(7.96 \pm 0.47) \times 10^{11} \mathrm{~K}$ and $a=0.65 \pm 0.19$. This gives a clue that the brightness temperatures on sub-parsec scales are close to the equipartition temperature of $5 \times 10^{10} \mathrm{~K}$ and start to increase on sub-parsec regions, reaching the inverse Compton limit of $\sim 10^{12} \mathrm{~K}$ on parsec scales. The kind of trend on brightness temperature matches with the magnetically driven, accelerating jet model given by Vlahakis \& Königl (2004). 

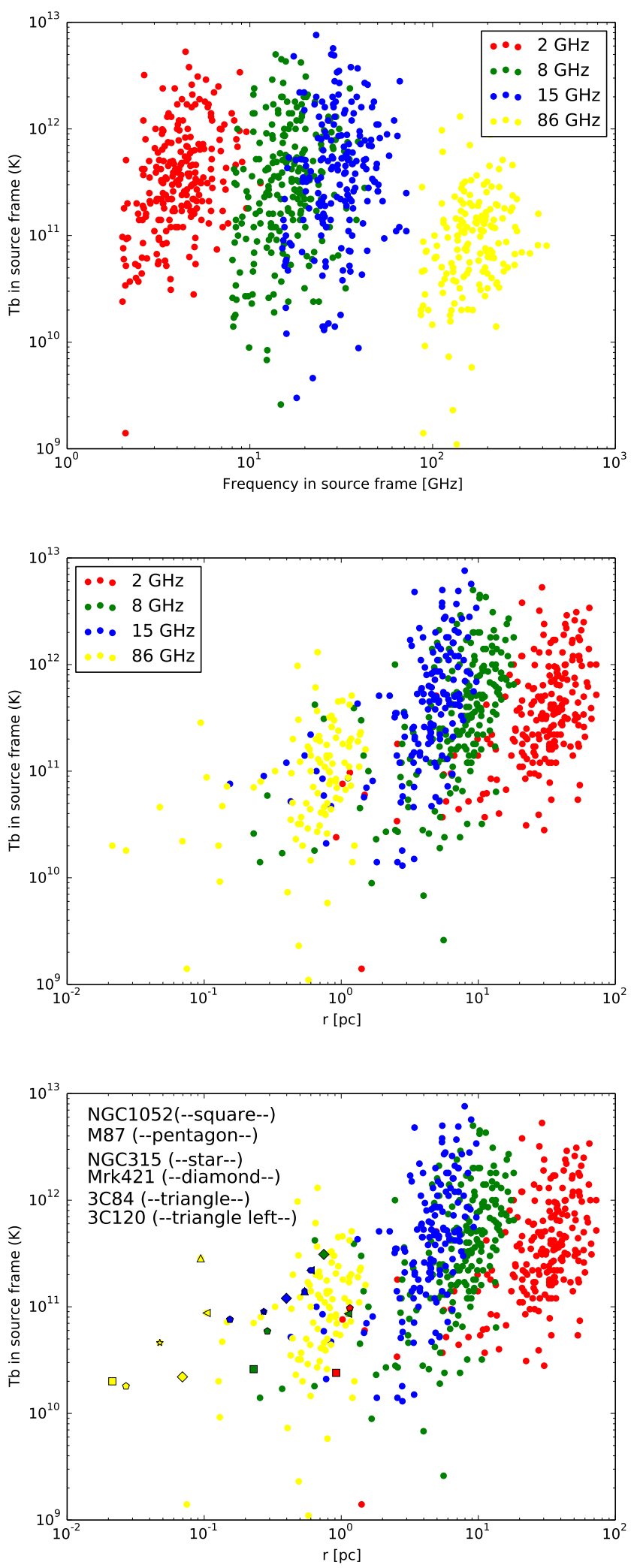

Figure 6.2: Brightness temperatures in source frame as a function of frequencies in source frame (top panel) and of absolute position of the VLBI core components (middle and bottom panel) at 2, 8, 15 and $86 \mathrm{GHz}$. The absolute position of the VLBI core is derived based on $\gamma_{\mathrm{j}}=10, \phi_{\mathrm{o}}=1 / \gamma_{\mathrm{j}}^{2}, B=1 \mathrm{G}, N_{1}=5 \times 10^{3} \mathrm{~cm}^{-3}$, and $r_{\max } / r_{\min }=100$. Nearby sources are marked as - squares for NGC 1052, pentagons for M87, stars for NGC

315, diamonds for Mrk421, triangles for 3C84 and left triangles for 3C120. 

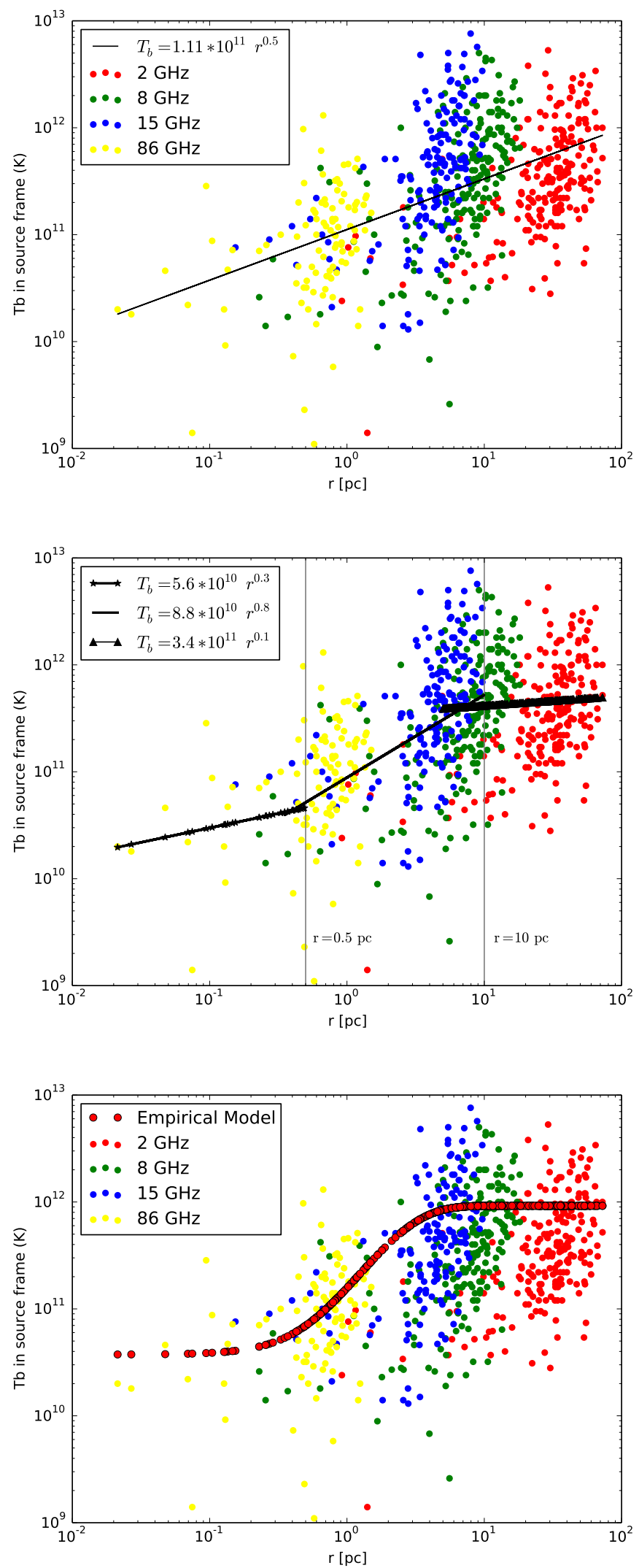

Figure 6.3: Brightness temperature $T_{\mathrm{b}}$ as a function of core position $r$ fitted with single power law (top panel), three power law functions (middle panel) and empirical model given by equation 6.14 (bottom panel). 
TABLE 6.1: Synchrotron Luminosity

\begin{tabular}{|c|c|c|c|c|c|c|}
\hline $\begin{array}{l}\text { Name } \\
(1)\end{array}$ & $\begin{array}{c}\mathrm{z} \\
(2) \\
\end{array}$ & $\begin{array}{c}S_{2 \mathrm{GHz}} \\
{[\mathrm{Jy}]} \\
(3) \\
\end{array}$ & $\begin{array}{c}S_{8 \mathrm{GHz}} \\
{[\mathrm{Jy}]} \\
(4) \\
\end{array}$ & $\begin{array}{c}S_{15 \mathrm{GHz}} \\
{[\mathrm{Jy}]} \\
(5) \\
\end{array}$ & $\begin{array}{c}S_{86 \mathrm{GHz}} \\
{[\mathrm{Jy}]} \\
(6) \\
\end{array}$ & $\begin{array}{c}L_{\text {syn }} \\
{[\mathrm{erg} / \mathrm{sec}]} \\
(7) \\
\end{array}$ \\
\hline $0003-066$ & 0.347 & 1.53 & 1.55 & 1.76 & & $2.2595112125 \mathrm{e}+45$ \\
\hline $0014+813$ & 3.382 & 0.56 & 0.54 & 0.37 & 0.0991887 & $3.94483784951 \mathrm{e}+46$ \\
\hline $0016+731$ & 1.781 & 0.68 & 0.14 & 0.51 & & $8.26868186721 \mathrm{e}+45$ \\
\hline $0055+300$ & 0.016 & $\ldots$ & $\ldots$ & 0.42 & 0.297964 & $6.14956974495 \mathrm{e}+41$ \\
\hline $0059+581$ & 0.644 & 0.57 & $\ldots$ & & 1.03038 & $4.67271724771 \mathrm{e}+45$ \\
\hline $0106+013$ & 2.107 & 1.92 & $\ldots$ & 1.87 & 0.431 & $4.69862218619 \mathrm{e}+46$ \\
\hline $0108+388$ & 0.669 & 0.5 & $\ldots$ & 0.13 & $\ldots$ & $2.46400714327 \mathrm{e}+44$ \\
\hline 0112-017 & 1.365 & 0.8 & 0.44 & 0.255 & $\ldots$ & $3.45376439768 \mathrm{e}+45$ \\
\hline 0113-118 & 0.672 & & 0.57 & 1.0 & $\ldots$ & $3.07712893155 \mathrm{e}+46$ \\
\hline $0119+041$ & 0.637 & 0.89 & 0.53 & 1.03 & $\ldots$ & $3.40191941973 \mathrm{e}+45$ \\
\hline $0119+115$ & 0.57 & 1.27 & 0.9 & 0.91 & $\ldots$ & $2.14773258174 \mathrm{e}+45$ \\
\hline 0130-171 & 1.02 & 0.69 & 0.79 & & $\ldots$ & $1.20042291451 \mathrm{e}+46$ \\
\hline $0133+476$ & 0.859 & 1.38 & 3.15 & 2.675 & 0.971 & $1.22189473574 \mathrm{e}+46$ \\
\hline 0138-097 & 0.733 & & 0.76 & 0.37 & 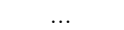 & $7.63611096946 \mathrm{e}+44$ \\
\hline $0149+218$ & 1.32 & 0.39 & $\ldots$ & 1.04 & 0.461 & $1.34600249449 \mathrm{e}+46$ \\
\hline $0201+113$ & 1.435 & 0.84 & 0.67 & 0.59 & $\ldots$ & $1.22285126312 \mathrm{e}+46$ \\
\hline $0202+149$ & 0.405 & 1.22 & 1.84 & 1.35 & $\ldots$ & $3.08120333631 \mathrm{e}+45$ \\
\hline $0202+319$ & 1.466 & 0.4 & 1.37 & 1.465 & 0.376 & $1.877145455 \mathrm{e}+46$ \\
\hline $0212+735$ & 2.367 & 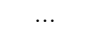 & $\ldots$ & 1.675 & 0.162 & $1.24317562599 \mathrm{e}+47$ \\
\hline $0215+015$ & 1.715 & 0.37 & $\ldots$ & 0.765 & $\ldots$ & $4.97527711843 \mathrm{e}+46$ \\
\hline $0224+671$ & 0.523 & 0.96 & 0.54 & $\ldots$ & $\ldots$ & $5.8933254669 \mathrm{e}+44$ \\
\hline $0229+131$ & 2.065 & 0.88 & 1.28 & $\ldots$ & $\ldots$ & $1.26135185756 \mathrm{e}+47$ \\
\hline $0234+285$ & 1.207 & & 3.48 & 1.61 & 1.25 & $2.37465424992 \mathrm{e}+46$ \\
\hline $0235+164$ & 0.94 & 1.08 & 1.3 & 1.46 & $\ldots$ & $1.88836272878 \mathrm{e}+46$ \\
\hline $0237-027$ & 1.116 & 0.27 & 0.49 & $\ldots$ & $\ldots$ & $1.99035062729 \mathrm{e}+46$ \\
\hline 0238-084 & 0.005 & 0.61 & 0.52 & $\ldots$ & 0.267 & $5.3102488899 \mathrm{e}+40$ \\
\hline $0239+108$ & 2.68 & 0.78 & 0.55 & $\ldots$ & $\ldots$ & $3.95688759412 \mathrm{e}+46$ \\
\hline $0316+413$ & 0.017 & & $\ldots$ & 3.63 & 1.175 & $2.71038844426 \mathrm{e}+42$ \\
\hline $0317+188$ & 0.3 & 0.51 & 0.37 & $\ldots$ & $\ldots$ & $1.77172111022 \mathrm{e}+44$ \\
\hline $0322+222$ & 2.066 & 0.77 & 0.44 & & & $1.35053674979 \mathrm{e}+46$ \\
\hline $0333+321$ & 1.261 & 0.89 & 0.87 & 1.05 & 0.384 & $1.0197678702 \mathrm{e}+46$ \\
\hline 0336-019 & 0.852 & & & 2.02 & 0.832 & $8.07255139684 \mathrm{e}+45$ \\
\hline $0338-214$ & 0.223 & 0.68 & 0.64 & & $\ldots$ & $2.64532067135 \mathrm{e}+44$ \\
\hline $0402-362$ & 1.417 & & 2.2 & 1.54 & $\ldots$ & $2.07887705784 \mathrm{e}+46$ \\
\hline 0403-132 & 0.571 & 0.7 & & 1.34 & $\ldots$ & $8.67813730423 \mathrm{e}+45$ \\
\hline $0406-127$ & 1.563 & 0.26 & 0.43 & $\ldots$ & $\ldots$ & $2.92592620016 \mathrm{e}+46$ \\
\hline 0414-189 & 1.526 & 0.29 & 0.6 & & - & $5.63608131153 \mathrm{e}+46$ \\
\hline $0415+379$ & 0.049 & 0.7 & 0.45 & 0.97 & 1.206 & $2.53169354828 \mathrm{e}+43$ \\
\hline $0420+022$ & 2.277 & $\ldots$ & $\ldots$ & 0.985 & 0.23 & $4.43343119687 \mathrm{e}+46$ \\
\hline $0420-014$ & 0.915 & . & $\ldots$ & 3.66 & 1.332 & $1.62568613845 \mathrm{e}+46$ \\
\hline $0422-380$ & 0.782 & 0.85 & 1.1 & $\ldots$ & $\ldots$ & $1.1736382904 \mathrm{e}+46$ \\
\hline $0430+052$ & 0.033 & $\ldots$ & 0.84 & 1.565 & 0.7365 & $7.22907378567 \mathrm{e}+42$ \\
\hline $0438-436$ & 2.852 & $\ldots$ & 1.8 & 1.79 & $\ldots$ & $2.150555723 \mathrm{e}+47$ \\
\hline 0440-003 & 0.844 & 0.63 & 0.45 & 0.645 & $\ldots$ & $4.21204890214 \mathrm{e}+45$ \\
\hline $0446+112$ & 1.207 & 1.5 & 1.57 & $\ldots$ & $\ldots$ & $2.9777678075 \mathrm{e}+46$ \\
\hline 0454-234 & 1.003 & 2.69 & 2.89 & 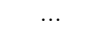 & 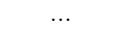 & $3.80986123167 \mathrm{e}+46$ \\
\hline 0458-020 & 2.291 & & & 0.74 & 0.501 & $4.3706135578 \mathrm{e}+46$ \\
\hline $0500+019$ & 0.584 & 1.79 & 0.58 & $\ldots$ & $\ldots$ & $4.25688290441 \mathrm{e}+44$ \\
\hline $0507+179$ & 0.416 & 0.12 & 0.47 & & $\ldots$ & $1.38063838634 \mathrm{e}+46$ \\
\hline $0521-365$ & 0.055 & 0.91 & 0.97 & 1.75 & 0.331 & $1.21866192536 \mathrm{e}+43$ \\
\hline $0537-286$ & 3.104 & 0.97 & 0.91 & 0.8 & $\ldots$ & $1.12113285315 \mathrm{e}+47$ \\
\hline $0537-441$ & 0.892 & 3.67 & 4.06 & $\ldots$ & $\ldots$ & $4.35693841941 \mathrm{e}+46$ \\
\hline 0539-057 & 0.839 & 0.7 & 0.6 & $\ldots$ & $\ldots$ & $3.68612930253 \mathrm{e}+45$ \\
\hline $0552+398$ & 2.363 & 2.92 & 3.53 & 3.21 & 0.48 & $9.43004036168 \mathrm{e}+46$ \\
\hline $0602+673$ & 1.97 & 0.84 & 0.78 & 0.93 & $\ldots$ & $4.79970822705 \mathrm{e}+46$ \\
\hline $0605-085$ & 0.872 & 0.65 & 0.74 & & $\ldots$ & $7.9425425424 \mathrm{e}+45$ \\
\hline $0607-157$ & 0.324 & 1.58 & 3.19 & 4.05 & 0.965 & $1.57148009403 \mathrm{e}+45$ \\
\hline $0615+820$ & 0.71 & 0.52 & 0.34 & 0.285 & $\ldots$ & $9.57647275318 \mathrm{e}+44$ \\
\hline $0636+680$ & 3.177 & 0.37 & 0.37 & $\ldots$ & $\ldots$ & $5.7152591541 \mathrm{e}+46$ \\
\hline $0642+449$ & 3.408 & 0.84 & 3.19 & 2.49 & 0.59 & $2.22779891984 \mathrm{e}+47$ \\
\hline $0707+476$ & 1.292 & & & 0.38 & 0.083 & $3.55335736762 \mathrm{e}+45$ \\
\hline $0716+714$ & 0.307 & 0.41 & 0.85 & $\ldots$ & 1.48869 & $1.65063985364 \mathrm{e}+45$ \\
\hline 0723-008 & 0.127 & & 0.56 & 0.82 & $\ldots$ & $5.07535079815 \mathrm{e}+44$ \\
\hline $0727-115$ & 1.591 & 2.5 & 3.4 & 2.87 & 0.64 & $3.83704379274 \mathrm{e}+46$ \\
\hline $0730+504$ & 0.72 & $\ldots$ & $\ldots$ & 1.25 & 0.148043 & $2.52280550596 \mathrm{e}+45$ \\
\hline $0735+178$ & 0.424 & $\ldots$ & 0.69 & & 0.264 & $4.77363707748 \mathrm{e}+44$ \\
\hline $0736+017$ & 0.191 & 0.58 & 0.35 & 2.26 & 0.832 & $3.97516842527 \mathrm{e}+44$ \\
\hline $0738+313$ & 0.63 & 0.49 & 0.74 & 0.28 & 0.3606 & $1.45157641474 \mathrm{e}+45$ \\
\hline $0742+103$ & 2.624 & 1.32 & 1.04 & 0.75 & & $6.36340011216 \mathrm{e}+46$ \\
\hline $0745+241$ & 0.409 & 0.31 & 0.35 & 0.67 & 0.521687 & $1.0527824497 \mathrm{e}+45$ \\
\hline $0748+126$ & 0.889 & 0.58 & 1.55 & 2.85 & 0.571 & $9.70467542586 \mathrm{e}+45$ \\
\hline $0749+540$ & 0.2 & 0.63 & 0.55 & & $\ldots$ & $1.56467042248 \mathrm{e}+44$ \\
\hline $0804+499$ & 1.432 & 0.41 & 0.48 & 0.65 & 0.14 & $6.16061341334 \mathrm{e}+45$ \\
\hline $0808+019$ & 1.039 & 0.46 & & 0.76 & - & $1.46874952404 \mathrm{e}+46$ \\
\hline $0812+367$ & 1.026 & 0.49 & 0.48 & $\ldots$ & 0.399202 & $5.03617313642 \mathrm{e}+45$ \\
\hline $0814+425$ & 0.53 & 0.78 & 0.66 & $\ldots$ & 0.311 & $9.41151782379 \mathrm{e}+44$ \\
\hline $0820+560$ & 1.417 & 0.65 & 0.37 & $\ldots$ & $\ldots$ & $4.41711280013 \mathrm{e}+45$ \\
\hline $0821+394$ & 1.216 & $\ldots$ & $\ldots$ & 1.18 & 0.257384 & $9.36206004282 \mathrm{e}+45$ \\
\hline $0823+033$ & 0.506 & & $\ldots$ & 1.1 & 0.374 & $1.1222522308 \mathrm{e}+45$ \\
\hline $0827+243$ & 0.94 & 0.51 & 0.79 & 1.505 & $\ldots$ & $3.32173029731 \mathrm{e}+46$ \\
\hline $0828+493$ & 0.548 & 0.4 & 0.19 & $\ldots$ & $\ldots$ & $1.82734960171 \mathrm{e}+44$ \\
\hline $0829+046$ & 0.18 & 0.58 & 0.49 & $\ldots$ & $\ldots$ & $1.04971670618 \mathrm{e}+44$ \\
\hline $0833+585$ & 2.101 & 0.35 & 0.68 & $\ldots$ & $\ldots$ & $1.0665878754 \mathrm{e}+47$ \\
\hline $0834+250$ & 1.122 & 0.22 & 0.34 & $\ldots$ & $\ldots$ & $1.05275364203 \mathrm{e}+46$ \\
\hline $0836+182$ & 0.28 & 0.17 & 0.1 & $\ldots$ & $\ldots$ & $2.88802047986 \mathrm{e}+43$ \\
\hline
\end{tabular}


TABLE 6.2: Synchrotron Luminosity (continued)

\begin{tabular}{|c|c|c|c|c|c|c|}
\hline $\begin{array}{l}\text { Name } \\
\text { (1) }\end{array}$ & $\begin{array}{l}z \\
(2)\end{array}$ & $\begin{array}{c}S_{2 \mathrm{GHz}} \\
{[\mathrm{Jy}]} \\
(3)\end{array}$ & $\begin{array}{c}S_{8 \mathrm{GHz}} \\
{[\mathrm{Jy}]} \\
(4)\end{array}$ & $\begin{array}{c}S_{15 \mathrm{GHz}} \\
{[\mathrm{Jy}]} \\
(5)\end{array}$ & $\begin{array}{c}S_{86 \mathrm{GHz}} \\
{[\mathrm{Jy}]} \\
(6)\end{array}$ & $\begin{array}{c}L_{\mathrm{syn}} \\
{[\mathrm{erg} / \mathrm{sec}]} \\
(7)\end{array}$ \\
\hline $0850+581$ & 1.322 & 0.4 & 0.31 & 0.06 & 0.104 & $1.97526183099 \mathrm{e}+45$ \\
\hline $0851+202$ & 0.306 & 1.6 & 1.95 & 1.26 & 0.618 & $6.2799995723 \mathrm{e}+44$ \\
\hline $0859+470$ & 1.462 & 0.35 & & 0.51 & 0.222 & $7.83251549119 \mathrm{e}+45$ \\
\hline $0859-140$ & 1.339 & . & 0.81 & 0.78 & $\ldots$ & $1.59232855803 \mathrm{e}+46$ \\
\hline $0906+015$ & 1.018 & 1.06 & & 2.17 & $\ldots$ & $4.87692553671 \mathrm{e}+46$ \\
\hline $0912+029$ & 0.427 & 0.36 & 0.55 & & & $2.30035347976 \mathrm{e}+45$ \\
\hline $0917+449$ & 2.182 & 0.25 & 0.52 & 0.865 & 0.485457 & $4.14234964 \mathrm{e}+46$ \\
\hline $0917+624$ & 1.446 & & $\ldots$ & 0.575 & 0.135 & $7.33839458653 \mathrm{e}+45$ \\
\hline 0919-260 & 2.3 & 1.25 & $\ldots$ & 1.14 & $\ldots$ & $8.09887276934 \mathrm{e}+46$ \\
\hline 0920-397 & 0.591 & 0.88 & 0.89 & $\ldots$ & $\ldots$ & $3.34988924404 \mathrm{e}+45$ \\
\hline $0923+392$ & 0.697 & 0.49 & 0.33 & 0.21 & 0.354731 & $1.46247907684 \mathrm{e}+45$ \\
\hline $0945+408$ & 1.252 & 1.55 & 0.76 & 0.77 & 0.363 & $8.19686568011 \mathrm{e}+45$ \\
\hline $0952+179$ & 1.478 & 0.31 & 0.37 & & & $1.33838639827 \mathrm{e}+46$ \\
\hline $0953+254$ & 0.712 & & & 0.92 & 0.475758 & $2.80433080846 \mathrm{e}+45$ \\
\hline $0954+658$ & 0.367 & 0.23 & 0.17 & & & $1.30288951269 \mathrm{e}+44$ \\
\hline $0955+476$ & 1.875 & 1.09 & 1.66 & 1.68 & 0.309723 & $2.95366656319 \mathrm{e}+46$ \\
\hline $1011+250$ & 1.636 & 0.41 & $\ldots$ & $\ldots$ & 0.22545 & $8.46512571617 \mathrm{e}+45$ \\
\hline $1012+232$ & 0.565 & & & 0.655 & 0.305 & $1.07502407785 \mathrm{e}+45$ \\
\hline $1014+615$ & 2.8 & 0.11 & 0.13 & $\ldots$ & $\ldots$ & $1.84428782819 \mathrm{e}+46$ \\
\hline $1020+400$ & 1.254 & 0.51 & 0.2 & $\ldots$ & $\ldots$ & $1.20532347065 \mathrm{e}+45$ \\
\hline $1021-006$ & 2.552 & 0.25 & 0.15 & $\ldots$ & & $8.20178678344 \mathrm{e}+45$ \\
\hline $1030+415$ & 1.119 & & 1.03 & $\ldots$ & 0.542177 & $8.83310314983 \mathrm{e}+45$ \\
\hline $1038+52 \mathrm{~A}$ & 0.677 & 0.46 & 0.3 & $\ldots$ & & $7.3862093029 \mathrm{e}+44$ \\
\hline $1039+811$ & 1.26 & 0.39 & 0.55 & $\ldots$ & 0.63267 & $1.24840656003 \mathrm{e}+46$ \\
\hline $1044+719$ & 1.15 & 1.54 & 0.39 & $\ldots$ & $\ldots$ & $1.42380594673 \mathrm{e}+45$ \\
\hline $1045-188$ & 0.595 & 0.65 & 0.88 & & $\ldots$ & $5.75863874357 \mathrm{e}+45$ \\
\hline $1049+215$ & 1.3 & 1.02 & 0.33 & 0.95 & $\ldots$ & $9.80821042809 \mathrm{e}+45$ \\
\hline $1053+704$ & 2.492 & 0.31 & 0.38 & & $\ldots$ & $4.39312311914 \mathrm{e}+46$ \\
\hline $1055+018$ & 0.888 & 1.24 & 1.08 & 1.37 & & $1.17012781542 \mathrm{e}+46$ \\
\hline $1101+384$ & 0.031 & & 0.29 & 0.45 & 0.264 & $2.25009952061 \mathrm{e}+42$ \\
\hline $1116+128$ & 2.118 & 0.72 & 0.71 & 0.47 & $\ldots$ & $2.71448132831 \mathrm{e}+46$ \\
\hline $1124-186$ & 1.048 & 0.92 & 0.82 & 1.24 & & $1.66715083962 \mathrm{e}+46$ \\
\hline $1128+385$ & 1.551 & $\ldots$ & 0.76 & 0.57 & 0.4377 & $1.46258787709 \mathrm{e}+46$ \\
\hline $1144+402$ & 1.089 & $\ldots$ & 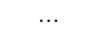 & 0.77 & 1.28576 & $1.77339465028 \mathrm{e}+46$ \\
\hline $1144-379$ & 1.048 & 1.96 & 2.34 & & $\ldots$ & $4.04236995703 \mathrm{e}+46$ \\
\hline $1145-071$ & 1.342 & 0.43 & 0.68 & 0.64 & $\ldots$ & $2.17702834267 \mathrm{e}+46$ \\
\hline $1148-001$ & 1.98 & 1.16 & 0.26 & & & $4.37585513928 \mathrm{e}+45$ \\
\hline $1150+812$ & 1.25 & 0.41 & 0.68 & 1.055 & 0.125452 & $5.08642622762 \mathrm{e}+45$ \\
\hline $1156+295$ & 0.729 & 1.22 & 2.42 & 0.91 & 1.629 & $8.95833743249 \mathrm{e}+45$ \\
\hline $1213+350$ & 0.857 & 0.63 & 0.37 & $\ldots$ & $\ldots$ & $1.35976390506 \mathrm{e}+45$ \\
\hline $1216+487$ & 1.076 & 0.55 & 0.28 & $\ldots$ & $\ldots$ & $1.49001386104 \mathrm{e}+45$ \\
\hline $1219+044$ & 0.965 & & 0.52 & 0.84 & & $3.77028864755 \mathrm{e}+46$ \\
\hline $1219+285$ & 0.102 & 0.18 & 0.16 & 0.28 & 0.155 & $1.658023935 \mathrm{e}+43$ \\
\hline $1222+037$ & 0.96 & 0.28 & 0.43 & & & $9.56275529046 \mathrm{e}+45$ \\
\hline $1226+023$ & 0.158 & & & 12.165 & 2.80378 & $7.14541352362 \mathrm{e}+44$ \\
\hline $1226+373$ & 1.515 & 0.28 & 0.2 & $\ldots$ & & $3.69640012956 \mathrm{e}+45$ \\
\hline $1228+126$ & 0.004 & 1.42 & 1.29 & 1.005 & 1.046 & $1.28784509785 \mathrm{e}+41$ \\
\hline $1237-101$ & 0.75 & 1.05 & 0.56 & $\ldots$ & $\ldots$ & $1.3174418989 \mathrm{e}+45$ \\
\hline $1240+381$ & 1.318 & 0.42 & 0.49 & $\ldots$ & $\ldots$ & $1.33578779131 \mathrm{e}+46$ \\
\hline $1243-072$ & 1.286 & 0.32 & 0.42 & $\ldots$ & $\ldots$ & $1.31218505079 \mathrm{e}+46$ \\
\hline $1244-255$ & 0.638 & 0.81 & 0.72 & 1.22 & & $5.97534717479 \mathrm{e}+45$ \\
\hline $1253-055$ & 0.537 & & & 11.42 & 10.0049 & $2.97901621391 \mathrm{e}+46$ \\
\hline $1254+571$ & 0.041 & 0.09 & 0.1 & & $\ldots$ & $1.81826198512 \mathrm{e}+42$ \\
\hline $1255-316$ & 1.924 & 1.32 & $\ldots$ & 0.85 & $\ldots$ & $3.32034696211 \mathrm{e}+46$ \\
\hline $1302-102$ & 0.286 & 1.0 & $\ldots$ & 0.33 & & $9.89675742451 \mathrm{e}+43$ \\
\hline $1308+326$ & 0.997 & 1.14 & & 1.13 & 0.51036 & $7.19574013127 \mathrm{e}+45$ \\
\hline $1308+328$ & 1.65 & 0.58 & 0.64 & & & $2.61468993284 \mathrm{e}+46$ \\
\hline $1324+224$ & 1.401 & 0.7 & 0.62 & 0.53 & 0.365388 & $1.0019826141 \mathrm{e}+46$ \\
\hline $1328+307$ & 0.846 & 3.16 & 0.76 & $\ldots$ & $\ldots$ & $1.18399291435 \mathrm{e}+45$ \\
\hline $1330+476$ & 0.668 & 0.27 & 0.49 & $\ldots$ & $\ldots$ & $7.14475462218 \mathrm{e}+45$ \\
\hline $1334-127$ & 0.539 & 2.12 & 4.07 & 4.93 & $\ldots$ & $3.66197704444 \mathrm{e}+46$ \\
\hline $1342+662$ & 0.766 & 0.37 & 0.47 & $\ldots$ & $\ldots$ & $4.64229506384 \mathrm{e}+45$ \\
\hline $1342+663$ & 1.351 & 0.58 & 0.33 & ... & $\ldots$ & $3.50084394274 \mathrm{e}+45$ \\
\hline $1354-152$ & 1.89 & & 0.25 & 0.45 & & $8.97288021584 \mathrm{e}+46$ \\
\hline $1404+286$ & 0.077 & 1.56 & 1.2 & 0.85 & 0.222228 & $1.27573719512 \mathrm{e}+43$ \\
\hline $1406-076$ & 1.493 & 0.6 & 1.31 & $\ldots$ & $\ldots$ & $1.29283093355 \mathrm{e}+47$ \\
\hline $1417+273$ & 0.539 & 0.19 & 0.31 & & & $2.37776037871 \mathrm{e}+45$ \\
\hline $1417+385$ & 1.832 & & & 0.935 & 0.274108 & $2.3241730365 \mathrm{e}+46$ \\
\hline $1420+326$ & 0.685 & 0.22 & 0.17 & & $\ldots$ & $5.60158353024 \mathrm{e}+44$ \\
\hline $1424+366$ & 1.091 & 0.39 & 0.12 & 0.28 & $\ldots$ & $1.75055302482 \mathrm{e}+45$ \\
\hline $1424-418$ & 1.522 & 0.84 & 1.14 & $\ldots$ & $\ldots$ & $5.35898461074 \mathrm{e}+46$ \\
\hline $1432+200$ & 1.382 & 0.18 & 0.14 & $\ldots$ & $\ldots$ & $2.33181500808 \mathrm{e}+45$ \\
\hline $1435+638$ & 2.066 & 0.16 & 0.21 & $\ldots$ & $\ldots$ & $1.78760676288 \mathrm{e}+46$ \\
\hline $1435-218$ & 1.187 & 0.18 & 0.64 & $\ldots$ & $\ldots$ & $1.00188558267 \mathrm{e}+47$ \\
\hline $1445-161$ & 2.417 & 0.51 & 0.34 & $\ldots$ & $\ldots$ & $1.79661250263 \mathrm{e}+46$ \\
\hline $1451-375$ & 0.314 & 0.92 & 0.46 & $\ldots$ & $\ldots$ & $1.32973631187 \mathrm{e}+44$ \\
\hline $1502+036$ & 0.413 & 0.52 & 0.75 & $\ldots$ & $\ldots$ & $2.60918211479 \mathrm{e}+45$ \\
\hline $1502+106$ & 1.839 & 1.46 & 1.39 & $\ldots$ & $\ldots$ & $5.8789931408 \mathrm{e}+46$ \\
\hline $1504+377$ & 0.674 & 0.29 & & 0.69 & $\ldots$ & $7.82186388821 \mathrm{e}+45$ \\
\hline $1504-166$ & 0.876 & $\ldots$ & 1.12 & 1.315 & & $1.80194674752 \mathrm{e}+46$ \\
\hline $1508-055$ & 1.191 & $\ldots$ & 0.41 & 0.52 & 0.503 & $8.7814023674 \mathrm{e}+45$ \\
\hline $1511-100$ & 1.513 & 0.6 & 0.4 & 0.59 & 0.336 & $1.10752814655 \mathrm{e}+46$ \\
\hline $1514-241$ & 0.048 & $\ldots$ & 0.73 & 1.61 & $\ldots$ & $8.41652070331 \mathrm{e}+44$ \\
\hline $1538+149$ & 1.365 & 0.48 & 0.55 & $\ldots$ & $\ldots$ & $1.57258746195 \mathrm{e}+46$ \\
\hline $1548+056$ & 1.422 & 2.64 & 2.13 & 2.325 & $\ldots$ & $5.01788429725 \mathrm{e}+46$ \\
\hline
\end{tabular}


TABLE 6.3: Synchrotron Luminosity (continued)

\begin{tabular}{|c|c|c|c|c|c|c|}
\hline $\begin{array}{l}\text { Name } \\
(1)\end{array}$ & $\begin{array}{c}z \\
(2) \\
\end{array}$ & $\begin{array}{c}S_{2 \mathrm{GHz}} \\
{[\mathrm{Jy}]} \\
(3) \\
\end{array}$ & $\begin{array}{c}S_{8 \mathrm{GHz}} \\
{[\mathrm{Jy}]} \\
(4) \\
\end{array}$ & $\begin{array}{c}S_{15 \mathrm{GHz}} \\
{[\mathrm{Jy}]} \\
(5) \\
\end{array}$ & $\begin{array}{c}S_{86 \mathrm{GHz}} \\
{[\mathrm{Jy}]} \\
(6) \\
\end{array}$ & $\begin{array}{c}L_{\text {syn }} \\
{[\mathrm{erg} / \mathrm{sec}]} \\
(7)\end{array}$ \\
\hline $1555+001$ & 1.772 & 0.56 & 0.7 & 0.68 & $\ldots$ & $3.39351919675 \mathrm{e}+46$ \\
\hline $1606+106$ & 0.857 & 1.9 & 2.46 & 1.45 & $\ldots$ & $1.27570069624 \mathrm{e}+46$ \\
\hline $1611+343$ & 1.401 & 2.1 & 2.33 & 2.745 & $\ldots$ & $7.83238771832 \mathrm{e}+46$ \\
\hline $1614+051$ & 3.217 & 0.51 & 0.37 & $\ldots$ & $\ldots$ & $4.26296595329 \mathrm{e}+46$ \\
\hline $1616+063$ & 2.088 & 0.53 & 0.45 & $\ldots$ & $\ldots$ & $2.19817155938 \mathrm{e}+46$ \\
\hline $1622-253$ & 0.786 & 0.78 & 1.58 & 1.44 & $\ldots$ & $2.09419801485 \mathrm{e}+46$ \\
\hline $1622-297$ & 0.815 & 1.17 & 0.72 & $\ldots$ & $\ldots$ & $2.50682711393 \mathrm{e}+45$ \\
\hline $1624+416$ & 2.55 & 0.57 & 0.38 & 0.26 & $\ldots$ & $1.89128810538 \mathrm{e}+46$ \\
\hline $1633+382$ & 1.808 & 0.95 & 0.73 & 0.87 & 1.56207 & $5.38868733908 \mathrm{e}+46$ \\
\hline $1637+574$ & 0.751 & 0.81 & 1.07 & 1.74 & 1.145 & $8.7349192872 \mathrm{e}+45$ \\
\hline $1638+398$ & 1.666 & 0.82 & 0.57 & 1.135 & 0.400088 & $1.93156867519 \mathrm{e}+46$ \\
\hline $1641+399$ & 0.593 & $\ldots$ & $\ldots$ & 2.63 & 0.929 & $4.00894689968 \mathrm{e}+45$ \\
\hline $1642+690$ & 0.751 & $\ldots$ & $\ldots$ & 0.36 & 0.597 & $3.83031424135 \mathrm{e}+45$ \\
\hline $1652+398$ & 0.033 & & & 0.4 & 0.283 & $2.4371801919 \mathrm{e}+42$ \\
\hline $1655+077$ & 0.589 & 0.58 & 0.78 & 1.745 & 0.459 & $2.5867341896 \mathrm{e}+45$ \\
\hline $1656+477$ & 0.921 & 1.09 & 0.6 & 0.68 & $\ldots$ & $3.90016342768 \mathrm{e}+45$ \\
\hline $1705+018$ & 2.576 & 0.59 & 0.69 & $\ldots$ & $\ldots$ & $8.0664652878 \mathrm{e}+46$ \\
\hline $1725+044$ & 0.293 & 0.73 & 0.31 & $\ldots$ & $\ldots$ & $6.10284124172 \mathrm{e}+43$ \\
\hline $1730-130$ & 0.902 & 2.53 & 1.91 & 6.875 & $\ldots$ & $8.30078380887 \mathrm{e}+46$ \\
\hline $1732+389$ & 0.976 & 0.34 & 0.68 & & & $2.52926564386 \mathrm{e}+46$ \\
\hline $1739+522$ & 1.379 & 0.43 & 0.52 & 0.73 & 0.979 & $2.24035385818 \mathrm{e}+46$ \\
\hline $1741-038$ & 1.057 & 3.01 & 3.96 & 4.695 & 2.404 & $4.01239706487 \mathrm{e}+46$ \\
\hline $1743+173$ & 1.702 & 0.57 & 0.36 & $\ldots$ & & $7.59344412896 \mathrm{e}+45$ \\
\hline $1745+624$ & 3.889 & & 0.6 & $\ldots$ & 0.0910988 & $7.47493766864 \mathrm{e}+46$ \\
\hline $1749+096$ & 0.32 & 1.7 & 2.58 & 3.715 & 2.375 & $3.05436950216 \mathrm{e}+45$ \\
\hline $1749+701$ & 0.742 & 0.4 & & 0.275 & $\ldots$ & $1.21812957142 \mathrm{e}+45$ \\
\hline $1758+388$ & 2.092 & 0.3 & 0.89 & 1.27 & & $2.22843703158 \mathrm{e}+47$ \\
\hline $1800+440$ & 0.663 & $\ldots$ & $\ldots$ & 0.61 & 0.432 & $2.06037122853 \mathrm{e}+45$ \\
\hline $1803+784$ & 0.68 & 1.27 & 1.52 & 1.555 & 0.9434 & $5.49399286671 \mathrm{e}+45$ \\
\hline $1807+698$ & 0.05 & 0.49 & 0.36 & 0.64 & $\ldots$ & $1.32886605883 \mathrm{e}+43$ \\
\hline $1821+107$ & 1.364 & 0.73 & 0.39 & 0.27 & & $3.60992116196 \mathrm{e}+45$ \\
\hline $1823+568$ & 0.658 & 0.4 & 0.75 & 0.85 & 0.485 & $2.99870998354 \mathrm{e}+45$ \\
\hline $1826+796$ & 0.224 & 0.17 & 0.16 & & & $6.67557540128 \mathrm{e}+43$ \\
\hline $1828+487$ & 0.692 & & & 1.385 & 1.183 & $6.14772716582 \mathrm{e}+45$ \\
\hline $1849+670$ & 0.657 & 0.33 & 0.91 & 1.61 & 0.68577 & $5.25080297915 \mathrm{e}+45$ \\
\hline $1901+319$ & 0.635 & & $\ldots$ & 0.555 & 0.212 & $1.04317517795 \mathrm{e}+45$ \\
\hline $1908-201$ & 1.119 & 1.17 & 0.99 & 2.6 & & $4.77779015603 \mathrm{e}+46$ \\
\hline $1921-293$ & 0.352 & 6.16 & 6.66 & 7.72 & 2.069 & $3.34462177991 \mathrm{e}+45$ \\
\hline $1928+738$ & 0.303 & & $\ldots$ & 1.36 & 0.502499 & $4.51955897367 \mathrm{e}+44$ \\
\hline $1936-155$ & 1.657 & 0.61 & $\ldots$ & 1.1 & $\ldots$ & $6.00613799855 \mathrm{e}+46$ \\
\hline $1937-101$ & 3.787 & 0.35 & 0.35 & 0.215 & & $5.00883857442 \mathrm{e}+46$ \\
\hline $1954+513$ & 1.223 & 0.33 & 0.37 & 0.62 & 0.2533 & $6.12606050656 \mathrm{e}+45$ \\
\hline $1954-388$ & 0.783 & 1.69 & 3.42 & 2.32 & 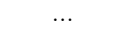 & $2.8844176769 \mathrm{e}+46$ \\
\hline $1958-179$ & 0.652 & 1.47 & 1.73 & 1.775 & $\ldots$ & $9.84326631955 \mathrm{e}+45$ \\
\hline $2000-330$ & 3.783 & 0.61 & $\ldots$ & 0.21 & $\ldots$ & $3.78957166314 \mathrm{e}+46$ \\
\hline $2007+777$ & 0.342 & 0.92 & $\ldots$ & 0.63 & & $5.01552964435 \mathrm{e}+44$ \\
\hline $2021+614$ & 0.227 & 1.12 & 1.48 & $\ldots$ & 0.119918 & $7.66596710866 \mathrm{e}+43$ \\
\hline $2029+121$ & 1.215 & 0.36 & 0.66 & 0.76 & & $2.66567134697 \mathrm{e}+46$ \\
\hline $2037+511$ & 1.687 & & & 1.69 & 0.345 & $3.30443668641 \mathrm{e}+46$ \\
\hline $2052-474$ & 1.489 & 0.88 & 0.59 & $\ldots$ & $\ldots$ & $9.661096845 \mathrm{e}+45$ \\
\hline $2059+034$ & 1.015 & 0.56 & $\ldots$ & 0.875 & $\ldots$ & $1.53314018849 \mathrm{e}+46$ \\
\hline $2113+293$ & 1.514 & 0.63 & 0.45 & 0.615 & & $1.46096276433 \mathrm{e}+46$ \\
\hline $2121+053$ & 1.941 & 2.09 & $\ldots$ & 2.15 & 0.414 & $3.95965184463 \mathrm{e}+46$ \\
\hline $2126-158$ & 3.28 & 0.77 & 0.75 & 1.11 & $\ldots$ & $1.86850354991 \mathrm{e}+47$ \\
\hline $2128-123$ & 0.501 & 1.65 & 2.92 & 0.5 & 0.237 & $7.80379037622 \mathrm{e}+44$ \\
\hline 2131-021 & 1.284 & 1.29 & 1.2 & 1.12 & $\ldots$ & $2.08244882121 \mathrm{e}+46$ \\
\hline $2136+141$ & 2.427 & 1.0 & 1.81 & 1.26 & $\ldots$ & $1.55380862094 \mathrm{e}+47$ \\
\hline $2144+092$ & 1.113 & 0.48 & $\ldots$ & 0.66 & $\ldots$ & $1.2592495401 \mathrm{e}+46$ \\
\hline $2145+067$ & 0.999 & 2.66 & 4.21 & 5.255 & $\ldots$ & $1.09340862159 \mathrm{e}+47$ \\
\hline $2155-152$ & 0.672 & 0.88 & & & 0.293 & $1.46857013493 \mathrm{e}+45$ \\
\hline $2200+420$ & 0.069 & 1.42 & 1.22 & 1.87 & 3.15059 & $1.34654842239 \mathrm{e}+44$ \\
\hline $2201+315$ & 0.298 & 0.86 & 0.78 & 2.07 & 0.611315 & $7.17554764454 \mathrm{e}+44$ \\
\hline $2209+236$ & 1.125 & 0.74 & 1.42 & 1.04 & 0.149553 & $4.76735131368 \mathrm{e}+45$ \\
\hline $2216-038$ & 0.901 & 0.37 & 1.43 & 2.025 & 0.458 & $8.28177052955 \mathrm{e}+45$ \\
\hline $2223-052$ & 1.404 & 2.39 & 3.49 & 3.74 & 0.642 & $3.0719065628 \mathrm{e}+46$ \\
\hline $2227-088$ & 1.562 & 0.77 & 0.43 & 2.03 & $\ldots$ & $6.05125021472 \mathrm{e}+46$ \\
\hline $2230+114$ & 1.037 & 2.34 & 1.74 & 2.68 & & $2.94977855987 \mathrm{e}+46$ \\
\hline $2234+282$ & 0.795 & 1.8 & 1.22 & 0.21 & 0.405 & $2.15432329908 \mathrm{e}+45$ \\
\hline $2243-123$ & 0.63 & 1.38 & 1.11 & 1.92 & $\ldots$ & $8.23533350105 \mathrm{e}+45$ \\
\hline $2250+194$ & 0.284 & 0.22 & 0.23 & & & $1.93767759114 \mathrm{e}+44$ \\
\hline $2251+158$ & 0.859 & & & 1.13 & 5.8565 & $5.99372387776 \mathrm{e}+46$ \\
\hline $2253+417$ & 1.476 & 0.47 & 0.3 & & & $4.52832534789 \mathrm{e}+45$ \\
\hline $2255-282$ & 0.927 & 0.89 & 5.99 & 5.65 & 1.868 & $3.6190737634 \mathrm{e}+46$ \\
\hline $2328+107$ & 1.489 & 0.53 & 0.26 & $\ldots$ & $\ldots$ & $2.99696729559 \mathrm{e}+45$ \\
\hline $2329-162$ & 1.153 & 0.91 & 0.93 & 0.61 & $\ldots$ & $8.7260355833 \mathrm{e}+45$ \\
\hline $2344+09 \mathrm{~A}$ & 0.673 & 0.62 & 0.68 & $\ldots$ & $\ldots$ & $3.91531537193 \mathrm{e}+45$ \\
\hline $2345-167$ & 0.576 & 0.92 & 1.52 & 1.215 & 0.365 & $1.8346977186 \mathrm{e}+45$ \\
\hline $2351+456$ & 1.986 & 0.71 & & 1.275 & $\ldots$ & $1.00656858221 \mathrm{e}+47$ \\
\hline $2352+495$ & 0.238 & 0.48 & 0.16 & $\ldots$ & $\ldots$ & $1.47032291586 \mathrm{e}+43$ \\
\hline $2355-106$ & 1.622 & 0.53 & 0.49 & $\ldots$ & $\ldots$ & $1.49761987663 \mathrm{e}+46$ \\
\hline NGC2484 & 0.043 & 0.11 & 0.04 & $\ldots$ & $\ldots$ & $1.07702176856 \mathrm{e}+41$ \\
\hline NGC3862 & 0.021 & 0.12 & 0.1 & $\ldots$ & $\ldots$ & $2.59667246568 \mathrm{e}+41$ \\
\hline NGC5675 & 0.014 & 0.08 & 0.07 & $\ldots$ & $\ldots$ & $8.88240603066 \mathrm{e}+40$ \\
\hline NGC6251 & 0.024 & 0.14 & 0.21 & $\ldots$ & $\ldots$ & $2.51577281006 \mathrm{e}+42$ \\
\hline OW-015 & 0.547 & 1.67 & 0.51 & $\ldots$ & $\ldots$ & $3.02723610003 \mathrm{e}+44$ \\
\hline
\end{tabular}




\section{Chapter 7}

\section{Conclusions \& Outlook}

We have used the Global Millimeter VLBI Array (GMVA) to conduct a large global 86 GHz VLBI survey comprising 174 snapshot observations of 162 unique targets selected from a sample of compact radio sources. The survey observations have reached the baseline sensitivity of $\approx 0.1 \mathrm{Jy}$ and image sensitivity of $\approx 5 \mathrm{mJy} / \mathrm{beam}$, owing to the increased recording bandwidth of the GMVA observations and the participation of large European antennas at Pico Veleta and Plateau de Bure. All of the 162 objects have been detected and imaged, thereby increasing by a factor of $\sim 2$ the total number of AGN imaged with VLBI at $86 \mathrm{GHz}$.

We have used Gaussian model fitting to represent the structure of the observed sources and estimate the flux densities and sizes of the cores and inner jet components. For sources with extended structure detected, the model fit parameters have been also used to calculate brightness temperature in the jet components downstream of the core. The apparent brightness temperature estimates for the cores in our sample ranges from $1.1 \times 10^{9} \mathrm{~K}$ to $5.5 \times 10^{12} \mathrm{~K}$, with the mean brightness temperature of $1.8 \times 10^{11} \mathrm{~K}$. The brightness temperature estimates for the nearest jet components in our sample ranges from $5.8 \times 10^{7} \mathrm{~K}$ to $4.1 \times 10^{11} \mathrm{~K}$. The overall amplitude calibration error for the observations is within $25 \%$, to which extent the results of the flux densities and brightness temperatures are accurate.

We used the model fit parameters and visibility data on the longest baselines to make independent estimates of brightness temperatures at the jet base as described by the most compact and bright "VLBI core" component. These estimates are consistent with each other. $T_{\mathrm{b} \text {,mod }}$ calculated for all the sources from Gaussian model fitting are either well bracketed inside $T_{\mathrm{b} \text {,min }}$ and $T_{\mathrm{b} \text {,lim }}$ or in well agreement with $T_{\mathrm{b} \text {,lim }}$ estimated directly from the visibility data. The median and mean of the minimum brightness temperature distribution for the core regions is $4.1 \times 10^{10} \mathrm{~K}$ and $6.2 \times 10^{10} \mathrm{~K}$, respectively. The median and mean of the limiting brightness temperature distribution for the core regions is $1.06 \times 10^{11} \mathrm{~K}$ and $3.0 \times 10^{11} \mathrm{~K}$, respectively.

A population model with a single intrinsic value of brightness temperature, $T_{0}$, is applied to reproduce the observed distribution. It yields $T_{0}=\left(3.77_{-0.14}^{+0.10}\right) \times 10^{11} \mathrm{~K}$ for the jet cores, implying that the inverse Compton losses dominate the emission. In the nearest jet components, $T_{0}=\left(1.42_{-0.19}^{+0.16}\right) \times 10^{11} \mathrm{~K}$ is found, which is slightly higher than the equipartition limit of $\sim 5 \times 10^{10} \mathrm{~K}$ expected for these jet regions. A correlation between $T_{0}$ and Lorentz factor, $\gamma_{\mathrm{j}}$ inherent to the population model description precludes fitting for $T_{0}$ and $\gamma_{\mathrm{j}}$ simultaneously. We find that a relation $T_{0}[\mathrm{~K}] \approx\left(7.7 \times 10^{8}\right) \gamma_{\mathrm{j}}^{2.7}$ is implied for this modelling 
framework by the survey data. The assumption of single value assumptions for $T_{0}$ and $\gamma_{j}$ put some limitations to this model which can be further improved by more complex models that consider the distribution law (for example a Gaussian or power law distribution) for $T_{0}$ and $\gamma_{\mathrm{j}}$.

The intrinsic $T_{0}$ and the observed $T_{\mathrm{b}}$ in core and jets show that the brightness temperature drops by approximately a factor of $\sim(2-10)$ already on sub-parsec scales in the jets. This evolution might occur with the inverse Compton, synchrotron, and adiabatic losses subsequently dominating the energy losses. A very basic evolutionary scheme in which the changes observed in the jet emission are caused by the adiabatic energy losses in relativistic shocks is investigated. For objects with sufficient structural detail detected, the adiabatic energy losses are shown to dominate the observed variations of brightness temperature along the jet implying that the jet components can be viewed as adiabatically expanding relativistic shocks.

Using a method described by Homan et al. (2006), an intrinsic brightness temperature of $T_{0}=6.2 \times 10^{9} \mathrm{~K}$ is found which is indeed less than the equipartition value of $5 \times 10^{10} \mathrm{~K}$. The difference in $T_{0}$ obtained by this method from population modelling method might be due to the strong limitation put by the assumption in this method - that the sources are observed at critical angle, which is not true for many sources. Further investigation is required on this approach. But in general, we can expect a conversion of the magnetic field energy into the kinetic energy of particles in the jet and the intrinsic brightness temperature may increase as going down stream of the jet. Homan et al. (2006) found that AGN cores at $15 \mathrm{GHz}$ are near equipartition in their median low state, yielding the intrinsic brightness temperatures of $T_{0}=3 \times 10^{10} \mathrm{~K}$ for a $75 \%$ of their sample at $15 \mathrm{GHz}$. The decrease of $T_{0}$ from $15 \mathrm{GHz}$ to $86 \mathrm{GHz}$ is clear in this approach as well. This would also give a favorable argument to the decelerating jet model or particle cascade model (Marscher 1995).

Under the equipartition condition between the magnetic field energy and particle energy density, the absolute distance of the VLBI core from the central engine can be predicted. A single power law fit in the $T_{\mathrm{b}}-r$ plane gives a dependence of $T_{\mathrm{b}} \sim r^{0.5}$. But, due to the fact that the large number of data points fall below the single power law fit, we further investigated the $T_{\mathrm{b}}-r$ plane with multiple power law fits. In general, the multiple power law functions clearly implies that from the vicinity of the central engine, the brightness temperature increases slowly (power index of 0.3 inside the region $0.01-0.5 \mathrm{pc}$ ) and then rises with a steeper slope (power index of 0.8 inside the region $0.35-10 \mathrm{pc}$ ) and then slows down again and become almost constant with a power index of 0.1 (inside the region $5-100 \mathrm{pc}$ ). Therefore, we conclude that the $T_{\mathrm{b}}$ increases with different slopes in different regions for our data. An empirical model was also applied to the data following the method described by Lee et al. (2016). The best fitting was obtained for the maximum brightness temperature, $T_{\mathrm{m}}=(7.96 \pm 0.47) \times 10^{11} \mathrm{~K}$ and $a=0.65 \pm 0.19$. This gives a clue that the brightness temperatures on sub-parsec scales are close to the equipartition temperature of $5 \times 10^{10} \mathrm{~K}$ and start to increase on sub-parsec regions, reaching the inverse Compton limit of $\sim 10^{12} \mathrm{~K}$ on parsec scales. The kind of trend on brightness temperature we observed matches with the magnetically driven, accelerating jet model given by Vlahakis \& Königl (2004) according to which, the mass flux is initially constant in the sub-parsec scale, and then increases, and then the mass flux gets constant again in the outer region.

The multi frequency analysis of $T_{\mathrm{b}}$ can be used to better constrain the bulk Lorentz factor and the intrinsic brightness temperature, to distinguish between the acceleration and deceleration scenario for the flow (cf., Marscher 1995), and to test several alternative acceleration 
scenarios including hydrodynamic acceleration (Bodo et al. 1985), acceleration by tangled magnetic field (Heinz \& Begelman 2000) and further investigate the possibilities of magneto hydrodynamic acceleration (Vlahakis \& Königl 2004). The multi frequency aspects of $T_{b}$ can be further studied by adding data from other low frequency AGN surveys (1.6 GHz, $5 \mathrm{GHz}, 22 \mathrm{GHz}, 43 \mathrm{GHz}$; Taylor et al. 1994; Britzen et al. 2007; Moellenbrock et al. 1996; Petrov et al. 2012) and also, by combining the brightness temperature analysis of the data provided by the space radio telescope RadioAstron (Kardashev et al. 2013) at L, C and K bands, at similar $u v$ spacings as that of this $86 \mathrm{GHz}$ survey.

Adding core shift information and gamma-ray fluxes to this $86 \mathrm{GHz}$ survey can be used to estimate the magnetic field from core shifts, which can be estimated either by synchrotron self absorption studies or an estimate of the minimum magnetic field from equipartition limit. The modeling of inner jet, based on the evolution of brightness temperature and adding MOJAVE measurements of speeds, viewing angles, opening angles and black hole masses can help to exploit a vast range of capabilities to understand the physics of ultracompact radio sources. The joint GMVA/Fermi (Fermi Gamma-ray Space Telescope) analysis of radio/ $\gamma$-ray emission in AGN can be performed to investigate the correlation between the location of gamma ray emission and the compact radio structures. In the long term, this research will help us to have a much better understanding of the acceleration, kinematic properties and collimation mechanism of the relativistic jets in a region close to the supermassive black hole. 


\section{Bibliography}

Ables, J. G. 1974, A\&AS, 15, 383

Ahn, C. P., Alexandroff, R., Allende Prieto, C., et al. 2014, ApJS, 211, 17

Alef, W. \& Porcas, R. W. 1986, A\&A, 168, 365

Alfvén, H. \& Herlofson, N. 1950, Physical Review, 78, 616

Antonucci, R. 1993, ARA\&A, 31, 473

Antonucci, R. R. J. \& Miller, J. S. 1985, ApJ, 297, 621

Asada, K., Nakamura, M., Doi, A., Nagai, H., \& Inoue, M. 2014, ApJ, 781, L2

Bañados, E., Venemans, B. P., Mazzucchelli, C., et al. 2017, ArXiv e-prints

Baczko, A.-K., Schulz, R., Kadler, M., et al. 2016, A\&A, 593, A47

Bagchi, J., Vivek, M., Vikram, V., et al. 2014, ApJ, 788, 174

Balbus, S. A. \& Hawley, J. F. 1991, ApJ, 376, 214

Bardeen, J. M. 1970, Nature, 226, 64

Barthel, P. 1989, Scientific American, 260, 20

Beasley, A. J., Dhawan, V., Doeleman, S., \& Phillips, R. B. 1997, in Millimeter-VLBI Science Workshop, ed. R. Barvainis \& R. B. Phillips, 53

Becker, R. H., White, R. L., Helfand, D. J., \& Zoonematkermani, S. 1994, ApJS, 91, 347

Beckmann, V. \& Shrader, C. 2012, in Proceedings of "An INTEGRAL view of the highenergy sky (the first 10 years)" - 9th INTEGRAL Workshop and celebration of the 10th anniversary of the launch (INTEGRAL 2012). 15-19 October 2012. Bibliotheque Nationale de France, Paris, France., 69

Bell, A. R. 1978, MNRAS, 182, 147

Best, P. N., Kauffmann, G., Heckman, T. M., et al. 2005, MNRAS, 362, 25

Biretta, J. A., Junor, W., \& Livio, M. 2002, New A Rev., 46, 239

Biretta, J. A., Owen, F. N., \& Hardee, P. E. 1983, ApJ, 274, L27

Blandford, R. D. \& Königl, A. 1979, ApJ, 232, 34

Blandford, R. D. \& McKee, C. F. 1982, ApJ, 255, 419 
Blandford, R. D., Netzer, H., Woltjer, L., Courvoisier, T. J.-L., \& Mayor, M., eds. 1990, Active Galactic Nuclei, 97

Blandford, R. D. \& Payne, D. G. 1982, MNRAS, 199, 883

Blandford, R. D. \& Rees, M. J. 1974, MNRAS, 169, 395

Blandford, R. D. \& Znajek, R. L. 1977, MNRAS, 179, 433

Blanton, M. R., Bershady, M. A., Abolfathi, B., et al. 2017, AJ, 154, 28

Blumenthal, G. R. \& Gould, R. J. 1970, Reviews of Modern Physics, 42, 237

Blundell, K. M., Fabian, A. C., Crawford, C. S., Erlund, M. C., \& Celotti, A. 2006, ApJ, 644, L13

Boccardi, B., Krichbaum, T., Bach, U., Ros, E., \& Zensus, J. A. 2015, ArXiv e-prints

Boccardi, B., Krichbaum, T. P., Bach, U., Bremer, M., \& Zensus, J. A. 2016, A\&A, 588, L9

Boccardi, B., Krichbaum, T. P., Ros, E., \& Zensus, J. A. 2017, A\&A Rev., 25, 4

Bodo, G., Ferrari, A., Massaglia, S., \& Tsinganos, K. 1985, A\&A, 149, 246

Boettcher, M., Harris, D. E., \& Krawczynski, H. 2012, Relativistic Jets from Active Galactic Nuclei

Brandie, G. W., Bridle, A. H., \& Kesteven, M. J. L. 1974, Nature, 252, 212

Bridle, A. H. \& Perley, R. A. 1984, ARA\&A, 22, 319

Britzen, S., Vermeulen, R. C., Taylor, G. B., et al. 2007, A\&A, 472, 763

Burbidge, G. R. \& Burbidge, E. M. 1957, ApJ, 125, 1

Burke, B. F. \& Graham-Smith, F. 1997, An introduction to radio astronomy

Carroll, B. W. \& Ostlie, D. A. 1996, An Introduction to Modern Astrophysics

Casadio, C., Krichbaum, T., Marscher, A., et al. 2017, Galaxies, 5, 67

Celotti, A. \& Fabian, A. C. 1993, MNRAS, 264, 228

Chiaberge, M., Capetti, A., \& Macchetto, F. D. 2005, ApJ, 625, 716

Chiaberge, M. \& Marconi, A. 2011, MNRAS, 416, 917

Christodoulou, D. \& Ruffini, R. 1971, Phys. Rev. D, 4, 3552

Clark, B. G. 1980, A\&A, 89, 377

Cohen, M. H., Cannon, W., Purcell, G. H., et al. 1971, ApJ, 170, 207

Cohen, M. H., Lister, M. L., Homan, D. C., et al. 2007, ApJ, 658, 232

Collin, S., Boisson, C., Mouchet, M., et al. 2002, A\&A, 388, 771

Condon, J. J., Cotton, W. D., Greisen, E. W., et al. 1998, AJ, 115, 1693 
Conselice, C. J., Wilkinson, A., Duncan, K., \& Mortlock, A. 2016, The Astrophysical Journal, 830, 83

Cornwell, T. 1995, in Astronomical Society of the Pacific Conference Series, Vol. 82, Very Long Baseline Interferometry and the VLBA, ed. J. A. Zensus, P. J. Diamond, \& P. J. Napier, 39

Cornwell, T., Braun, R., \& Briggs, D. S. 1999, in Astronomical Society of the Pacific Conference Series, Vol. 180, Synthesis Imaging in Radio Astronomy II, ed. G. B. Taylor, C. L. Carilli, \& R. A. Perley, 151

Cornwell, T. \& Fomalont, E. B. 1999, in Astronomical Society of the Pacific Conference Series, Vol. 180, Synthesis Imaging in Radio Astronomy II, ed. G. B. Taylor, C. L. Carilli, \& R. A. Perley, 187

Cotton, W. D. 1995, in Astronomical Society of the Pacific Conference Series, Vol. 82, Very Long Baseline Interferometry and the VLBA, ed. J. A. Zensus, P. J. Diamond, \& P. J. Napier, 189

Crane, P. \& Vernet, J. 1997, ApJ, 486, L91

Dadhich, N. 2012, ArXiv e-prints

Daly, R. A. \& Marscher, A. P. 1988, ApJ, 334, 539

de Kool, M. \& Begelman, M. C. 1989, Nature, 338, 484

De Villiers, J.-P., Hawley, J. F., Krolik, J. H., \& Hirose, S. 2005, ApJ, 620, 878

Deller, A. T., Brisken, W. F., Phillips, C. J., et al. 2011, PASP, 123, 275

Doeleman, S. S., Fish, V. L., Schenck, D. E., et al. 2012, Science, 338, 355

Dunlop, J. S., McLure, R. J., Kukula, M. J., et al. 2003, MNRAS, 340, 1095

Dunn, R. J. H., Fabian, A. C., \& Celotti, A. 2006, MNRAS, 372, 1741

Eckart, A. 2002, The mass of the Galactic Center black hole, ed. H. Falcke \& F. Hehl, $229-245$

Eftekharzadeh, S., Myers, A. D., White, M., et al. 2014, in American Astronomical Society Meeting Abstracts, Vol. 224, American Astronomical Society Meeting Abstracts, 221.01

Fan, J. H., Cheng, K. S., Zhang, L., \& Liu, C. H. 1997, A\&A, 327, 947

Fanaroff, B. L. \& Riley, J. M. 1974, MNRAS, 167, 31P

Ferrarese, L. \& Merritt, D. 2000, ApJ, 539, L9

Fomalont, E. B. 1999, in Astronomical Society of the Pacific Conference Series, Vol. 180, Synthesis Imaging in Radio Astronomy II, ed. G. B. Taylor, C. L. Carilli, \& R. A. Perley, 301

Fromm, C. M., Ros, E., Perucho, M., et al. 2013, A\&A, 551, A32

Fuhrmann, L., Larsson, S., Chiang, J., et al. 2014, MNRAS, 441, 1899

Garofalo, D. 2009, ApJ, 699, L52 
Gaskell, C. M. \& Klimek, E. S. 2003, Astronomical and Astrophysical Transactions, 22, 661

Ghisellini, G., ed. 2013, Lecture Notes in Physics, Berlin Springer Verlag, Vol. 873, Radiative Processes in High Energy Astrophysics

Ghisellini, G., Guilbert, P. W., \& Svensson, R. 1988, ApJ, 334, L5

Gopal-Krishna, Mangalam, A., \& Wiita, P. J. 2008, ApJ, 680, L13

Greisen, E. W. 1990, in Acquisition, Processing and Archiving of Astronomical Images, ed. G. Longo \& G. Sedmak, 125-142

Gubbay, J., Legg, A. J., Robertson, D. S., et al. 1969, Nature, 224, 1094

Gunn, J. E., Carr, M., Rockosi, C., et al. 1998, AJ, 116, 3040

Hada, K., Kino, M., Doi, A., et al. 2016, ApJ, 817, 131

Haiman, Z., Quataert, E., \& Bower, G. C. 2004, ApJ, 612, 698

Hardee, P. E., Mizuno, Y., Lyubarsky, Y., \& Nishikawa, K. 2010, in Bulletin of the American Astronomical Society, Vol. 42, AAS/High Energy Astrophysics Division, 706

Harris, D. E. \& Krawczynski, H. 2007, in Revista Mexicana de Astronomia y Astrofisica Conference Series, Vol. 27, Revista Mexicana de Astronomia y Astrofisica, vol. 27, 188

Hawking, S. W. \& Ellis, G. F. R. 1975, The Large Scale Structure of Space-Time

Heckman, T. M., Miley, G. K., van Breugel, W. J. M., \& Butcher, H. R. 1980, in BAAS, Vol. 12, Bulletin of the American Astronomical Society, 809

Heidt, J. \& Wagner, S. J. 1996, A\&A, 305, 42

Heinz, S. \& Begelman, M. C. 2000, ApJ, 535, 104

Helmboldt, J. F., Taylor, G. B., Tremblay, S., et al. 2007, ApJ, 658, 203

Henstock, D. R., Browne, I. W. A., Wilkinson, P. N., et al. 1995, ApJS, 100, 1

Herrnstein, J. R., Moran, J. M., Greenhill, L. J., et al. 1999, Nature, 400, 539

Hewish, A., Bell, S. J., Pilkington, J. D. H., Scott, P. F., \& Collins, R. A. 1968, Nature, 217,709

Hicks, E. K. S. \& Malkan, M. A. 2008, ApJS, 174, 31

Hirabayashi, H. \& Hirosawa, H. 2000, Advances in Space Research, 26, 589

Hodgson, J. A., Krichbaum, T. P., Marscher, A. P., et al. 2017, A\&A, 597, A80

Högbom, J. A. 1974, A\&AS, 15, 417

Homan, D. C., Kovalev, Y. Y., Lister, M. L., et al. 2006, ApJ, 642, L115

Homan, D. C., Lister, M. L., Kovalev, Y. Y., et al. 2015, ApJ, 798, 134

Horiuchi, S., Meier, D. L., Preston, R. A., \& Tingay, S. J. 2006, PASJ, 58, 211 
Hovatta, T., Leitch, E. M., Homan, D. C., et al. 2013, in European Physical Journal Web of Conferences, Vol. 61, European Physical Journal Web of Conferences, 06005

Hoyle, F. \& Burbidge, G. R. 1966, ApJ, 144, 534

Hughes, P. A. 2005, ApJ, 621, 635

Israel, F. P. 1998, A\&A Rev., 8, 237

Iwasawa, K., Fabian, A. C., Reynolds, C. S., et al. 1996, MNRAS, 282, 1038

Jackson, J. D. 1975, Classical electrodynamics

Jansky, K. G. 1933, Nature, 132, 66

Jennison, R. C. 1958, MNRAS, 118, 276

Jin, C., Done, C., \& Ward, M. 2017, MNRAS, 468, 3663

Johnson, M. D., Kovalev, Y. Y., Gwinn, C. R., et al. 2016, ApJ, 820, L10

Jorstad, S. \& Marscher, A. 2016, Galaxies, 4, 47

Jorstad, S. G., Marscher, A. P., Morozova, D. A., et al. 2017, ApJ, 846, 98

Jorstad, S. G., Marscher, A. P., Smith, P. S., et al. 2013, ApJ, 773, 147

Jukes, J. D. 1967, Nature, 216, 461

Kadler, M., Ros, E., Lobanov, A. P., Falcke, H., \& Zensus, J. A. 2004, A\&A, 426, 481

Kardashev, N. S. 2000, Astronomy Reports, 44, 719

Kardashev, N. S., Khartov, V. V., Abramov, V. V., et al. 2013, Astronomy Reports, 57, 153

Kellermann, K. I. \& Pauliny-Toth, I. I. K. 1969, ApJ, 155, L71

Kellermann, K. I., Sramek, R., Schmidt, M., Shaffer, D. B., \& Green, R. 1989, AJ, 98, 1195

Kembhavi, A. K. \& Narlikar, J. V. 1999, Quasars and active galactic nuclei : an introduction

Khachikian, E. Y. \& Weedman, D. W. 1974, ApJ, 189, L99

Komissarov, S. S., Barkov, M. V., Vlahakis, N., \& Königl, A. 2007, MNRAS, 380, 51

Königl, A. 1981, ApJ, 243, 700

Kovalev, Y. Y., Kardashev, N. S., Kellermann, K. I., et al. 2016, ApJ, 820, L9

Kovalev, Y. Y., Kellermann, K. I., Lister, M. L., et al. 2005, AJ, 130, 2473

Kovalev, Y. Y., Lister, M. L., Homan, D. C., \& Kellermann, K. I. 2007, ApJ, 668, L27

Kravchenko, E. V., Kovalev, Y. Y., Hovatta, T., \& Ramakrishnan, V. 2016, MNRAS, 462, 2747

Krichbaum, T. P., Witzel, A., Graham, D. A., et al. 1992, A\&A, 260, 33

Laing, R. A. \& Bridle, A. H. 1987, MNRAS, 228, 557 
Laing, R. A. \& Bridle, A. H. 2002, MNRAS, 336, 328

Laor, A. 1998, ApJ, 505, L83

Laor, A. 2000, ApJ, 543, L111

Lawson, C. L. \& Hanson, R. J. 1974, Solving least squares problems

Lee, J. A., Sohn, B. W., Jung, T., Byun, D.-Y., \& Lee, J. W. 2017, ApJS, 228, 22

Lee, S.-S. 2013, Journal of Korean Astronomical Society, 46, 243

Lee, S.-S. 2014, Journal of Korean Astronomical Society, 47, 303

Lee, S.-S., Lobanov, A. P., Krichbaum, T. P., et al. 2008, AJ, 136, 159

Lee, S.-S., Lobanov, A. P., Krichbaum, T. P., \& Zensus, J. A. 2016, ApJ, 826, 135

Li, L.-X. \& Paczyski, B. 2000, The Astrophysical Journal Letters, 534, L197

Lister, M. L. 2003, ApJ, 599, 105

Lister, M. L., Aller, M. F., Aller, H. D., et al. 2016, AJ, 152, 12

Lister, M. L., Cohen, M. H., Homan, D. C., et al. 2009, AJ, 138, 1874

Lister, M. L. \& Homan, D. C. 2005, AJ, 130, 1389

Lobanov, A. P. 1996, PhD thesis, New Mexico Institute of Mining and Technology

Lobanov, A. P. 1998, A\&A, 330, 79

Lobanov, A. P. 2005, arXiv:astro-ph/0503225

Lobanov, A. P. 2015, A\&A, 574, A84

Lobanov, A. P., Gómez, J. L., Bruni, G., et al. 2015, A\&A, 583, A100

Lobanov, A. P., Krichbaum, T. P., Graham, D. A., et al. 2000, A\&A, 364, 391

Lobanov, A. P., Krichbaum, T. P., Witzel, A., \& Zensus, J. A. 2006, PASJ, 58, 253

Lobanov, A. P. \& Zensus, J. A. 1999, ApJ, 521, 509

Lobanov, A. P. \& Zensus, J. A. 2001, Science, 294, 128

Lonsdale, C. J., Doeleman, S. S., \& Phillips, R. B. 1998, AJ, 116, 8

Loreta, E. 1929, Gazette Astronomique, 16, 55

Magorrian, J. \& Tremaine, S. 1999, MNRAS, 309, 447

Magorrian, J., Tremaine, S., Gebhardt, K., Richstone, D., \& Faber, S. 1996, in Bulletin of the American Astronomical Society, Vol. 28, American Astronomical Society Meeting Abstracts, 1423

Marscher, A. P. 1995, Proceedings of the National Academy of Science, 92, 11439

Marscher, A. P. \& Gear, W. K. 1985, ApJ, 298, 114

Matthews, T. A. \& Sandage, A. R. 1963, ApJ, 138, 30 
McKinney, J. C. 2005, ApJ, 630, L5

Medici, A. \& Lobanov, A. P. 2002, in Proceedings of the 6th EVN Symposium, ed. E. Ros, R. W. Porcas, A. P. Lobanov, \& J. A. Zensus, 93

Meier, D. L. 2001, ApJ, 548, L9

Meier, D. L. 2003, New A Rev., 47, 667

Meier, D. L. 2012, Black Hole Astrophysics: The Engine Paradigm

Melia, F. \& Königl, A. 1989, ApJ, 340, 162

Merritt, D. \& Quinlan, G. D. 1998, ApJ, 498, 625

Mertens, F., Lobanov, A. P., Walker, R. C., \& Hardee, P. E. 2016, A\&A, 595, A54

Michel, F. C. 1969, ApJ, 158, 727

Miyoshi, M., Moran, J., Herrnstein, J., et al. 1995, Nature, 373, 127

Mizuno, Y., Lyubarsky, Y., Nishikawa, K.-I., \& Hardee, P. E. 2012, ApJ, 757, 16

Moellenbrock, G. A., Fujisawa, K., Preston, R. A., et al. 1996, AJ, 111, 2174

Moran, J. M. 1976, Methods of Experimental Physics, 12, 228

Moser, A. L. \& Bellan, P. M. 2012, Nature, 482, 379

Nagai, H., Haga, T., Giovannini, G., et al. 2014, ApJ, 785, 53

Nair, D. G., Lobanov, A. P., Krichbaum, T. P., et al. 2019, A\&A, 622, A92

Narayan, R. \& Yi, I. 1994, ApJ, 428, L13

Narayan, R., Yi, I., \& Mahadevan, R. 1994, ArXiv Astrophysics e-prints

Nicolson, I. 1999, Unfolding our Universe, 304

Pearson, T. J. 1999, in Astronomical Society of the Pacific Conference Series, Vol. 180, Synthesis Imaging in Radio Astronomy II, ed. G. B. Taylor, C. L. Carilli, \& R. A. Perley, 335

Pearson, T. J. \& Readhead, A. C. S. 1988, ApJ, 328, 114

Pearson, T. J., Unwin, S. C., Cohen, M. H., et al. 1981, Nature, 290, 365

Penrose, R. 1969, Nuovo Cimento Rivista Serie, 1

Penrose, R. \& Floyd, R. M. 1971, Nature Physical Science, 229, 177

Perley, R. A. \& Butler, B. J. 2017, ApJS, 230, 7

Perley, R. A., Dreher, J. W., \& Cowan, J. J. 1984, ApJ, 285, L35

Peterson, B. M. 1997, An Introduction to Active Galactic Nuclei

Peterson, B. M. 2001, in Advanced Lectures on the Starburst-AGN, ed. I. Aretxaga, D. Kunth, \& R. Mújica, 3 
Peterson, B. M. \& Bentz, M. C. 2006, New A Rev., 50, 796

Peterson, B. M., Grier, C. J., Horne, K., et al. 2014, ApJ, 795, 149

Petrov, L., Hirota, T., Honma, M., et al. 2007, AJ, 133, 2487

Petrov, L., Lee, S.-S., Kim, J., et al. 2012, AJ, 144, 150

Pilipenko, S. V., Kovalev, Y. Y., Andrianov, A. S., et al. 2018, MNRAS, 474, 3523

Polatidis, A. G., Wilkinson, P. N., Xu, W., et al. 1996, VizieR Online Data Catalog, 209, 80001

Ponsonby, J. E. B. 1973, MNRAS, 163, 369

Pounds, K. \& Page, K. 2004, Nuclear Physics B Proceedings Supplements, 132, 107

Protheroe, R. J. 2003, MNRAS, 341, 230

Pushkarev, A. B. \& Kovalev, Y. Y. 2012, A\&A, 544, A34

Rantakyro, F. T., Baath, L. B., Backer, D. C., et al. 1998, A\&AS, 131, 451

Rawlings, S. \& Saunders, R. 1991, Nature, 349, 138

Readhead, A. C. S. 1994, ApJ, 426, 51

Readhead, A. C. S., Mason, C. R., Mofett, A. T., et al. 1983, Nature, 303, 504

Rees, M. J. 1966, Nature, 211, 468

Rees, M. J. 1971, Nature, 229, 312

Reynolds, C. S., Fabian, A. C., Celotti, A., \& Rees, M. J. 1996, MNRAS, 283, 873

Robson, I. 1996, Active galactic nuclei

Rogers, A. E. E., Phillips, R. B., \& Lonsdale, C. J. 1995, in Bulletin of the American Astronomical Society, Vol. 27, American Astronomical Society Meeting Abstracts, 1300

Ross, N. P., Shen, Y., Strauss, M. A., et al. 2009, ApJ, 697, 1634

Rybicki, G. B. \& Lightman, A. P. 1979, Radiative processes in astrophysics

Ryle, M. 1962, Nature, 194, 517

Ryle, M. \& Hewish, A. 1960, MNRAS, 120, 220

Sanders, D. B., Scoville, N. Z., Zensus, A., et al. 1989, A\&A, 213, L5

Scheuer, P. A. G. 1974, MNRAS, 166, 513

Scheuer, P. A. G. \& Readhead, A. C. S. 1979, Nature, 277, 182

Schinzel, F. K., Lobanov, A. P., Taylor, G. B., et al. 2012, A\&A, 537, A70

Schlickeiser, R., Biermann, P. L., \& Crusius-Waetzel, A. 1991, A\&A, 247, 283

Schmidt, M. 1963, Nature, 197, 1040

Schmidt, M. \& Green, R. F. 1983, ApJ, 269, 352 
Schwab, F. R. 1980, in Proc. SPIE, Vol. 231, 1980 International Optical Computing Conference I, ed. W. T. Rhodes, $18-25$

Schwab, F. R. \& Cotton, W. D. 1983, AJ, 88, 688

Scott, M. A. \& Readhead, A. C. S. 1977, MNRAS, 180, 539

Scott, P. F., Ryle, M., \& Hewish, A. 1961, MNRAS, 122, 95

Setti, G. \& Swings, J.-P., eds. 2001, Quasars, AGNs and Related Research Across 2000

Seyfert, C. K. 1943, ApJ, 97, 28

Shakura, N. I. \& Sunyaev, R. A. 1973, A\&A, 24, 337

Shen, Y., Strauss, M. A., Oguri, M., et al. 2007, AJ, 133, 2222

Shen, Z.-Q., Wan, T.-S., Moran, J. M., et al. 1997, AJ, 114, 1999

Shepherd, M. C., Pearson, T. J., \& Taylor, G. B. 1994, in BAAS, Vol. 26, Bulletin of the American Astronomical Society, 987-989

Sikora, M. \& Begelman, M. C. 2013, ApJ, 764, L24

Sikora, M., Begelman, M. C., Madejski, G. M., \& Lasota, J.-P. 2005, ApJ, 625, 72

Sikora, M. \& Madejski, G. 2000, ApJ, 534, 109

Sikora, M., Stawarz, Ł., \& Lasota, J.-P. 2007, ApJ, 658, 815

Sikora, M., Stawarz, Ł., \& Lasota, J.-P. 2008, New A Rev., 51, 891

Smith, J. E., Robinson, A., Alexander, D. M., et al. 2004, MNRAS, 350, 140

Soltan, A. 1982, MNRAS, 200, 115

Spruit, H. C. 2010, in Lecture Notes in Physics, Berlin Springer Verlag, Vol. 794, Lecture Notes in Physics, Berlin Springer Verlag, ed. T. Belloni, 233

Tanaka, Y., Nandra, K., Fabian, A. C., et al. 1995, Nature, 375, 659

Taylor, G. B., Carilli, C. L., \& Perley, R. A., eds. 1999, Astronomical Society of the Pacific Conference Series, Vol. 180, Synthesis Imaging in Radio Astronomy II

Taylor, G. B., Vermeulen, R. C., Pearson, T. J., et al. 1994, ApJS, 95, 345

Taylor, G. B., Vermeulen, R. C., Readhead, A. C. S., et al. 1996, ApJS, 107, 37

Tchekhovskoy, A., Narayan, R., \& McKinney, J. C. 2010, ApJ, 711, 50

Tchekhovskoy, A., Narayan, R., \& McKinney, J. C. 2011, MNRAS, 418, L79

Thakkar, D. D., Xu, W., Readhead, A. C. S., et al. 1996, VizieR Online Data Catalog, 209, 80033

Thompson, A. R., Moran, J. M., \& Swenson, Jr., G. W. 2001, Interferometry and Synthesis in Radio Astronomy, 2nd Edition

Twiss, R. Q., Carter, A. W. L., \& Little, A. G. 1960, The Observatory, 80, 153 
Urry, C. M. \& Padovani, P. 1995, PASP, 107, 803

Usher, P. D. 1975, ApJ, 198, L57

van Cittert, P. H. 1934, Physica, 1, 201

Vanden Berk, D., Wilhite, B., Kron, R., et al. 2004, in Bulletin of the American Astronomical Society, Vol. 36, American Astronomical Society Meeting Abstracts, 1548

Vermeulen, R. C. \& Cohen, M. H. 1994, ApJ, 430, 467

Visser, M. 2007, ArXiv e-prints

Vlahakis, N. \& Königl, A. 2004, ApJ, 605, 656

Walker, R. C., Benson, J. M., Unwin, S. C., et al. 2001, ApJ, 556, 756

Walker, R. C., Dhawan, V., Romney, J. D., Kellermann, K. I., \& Vermeulen, R. C. 2000, ApJ, 530, 233

Wardle, J. F. C., Homan, D. C., Ojha, R., \& Roberts, D. H. 1998, Nature, 395, 457

Wenger, M., Ochsenbein, F., Egret, D., et al. 2000, A\&AS, 143, 9

Whitney, A. R., Shapiro, I. I., Rogers, A. E. E., et al. 1971, Science, 173, 225

Xu, W., Readhead, A. C. S., Pearson, T. J., Polatidis, A. G., \& Wilkinson, P. N. 1995, ApJS, 99, 297

Zel'dovich, Y. B. \& Novikov, I. D. 1964, Soviet Physics Doklady, 9, 246

Zensus, J. A., Diamond, P. J., \& Napier, P. J., eds. 1995, Astronomical Society of the Pacific Conference Series, Vol. 82, Very Long Baseline Interferometry and the VLBA

Zernike, F. 1938, Physica, 5, 785 


\section{Thank you | Vielen Dank | Dhanyawad | Nandhi}

Now, it's time to thank the people who made this $\mathrm{PhD}$ journey possible in my life. First of all, I would like to thank my parents who is my life and soul. The hardwork of my father and the love of my mother made me what I am today! No words to thank you "Acha" and "Amma". A big hug to both of you from miles away. This dissertation is for both of you. I would like to thank my greatest motivation - my elder brother - Gopakumar. For me, the journey of $\mathrm{PhD}$ started when you started your $\mathrm{PhD}$ in Nanotechnology which aspired me to take a $\mathrm{PhD}$ degree in future in my favorite subject. From then, I started dreaming and working hard for that big day! Even though, sky watching and reading astronomy books were my passion from childhood, it was you "Gotta" who found out the astronomer in me. You gifted me "The Brief History of time" by Stephen Hawkings when I was 14 years old and told me "Read it how you like". From then, till now, your support and the basic lessons you taught me in science and life made me what I am. Thank you my dear Gotta. I wholeheartedly thank my brother "Syamchetta" who is my partner in crime! He was there in all ups and downs of my life. I learnt the philosophy of life; at the same time, the jokes of life from you. You taught me how to explore the world through travelling. A big hug and thanks to you "Syamchetta". A big thanks to my Dnyanu who share the pains and happiness in my life. Thanks, Dnyanu for sharing this incurable fervor for life.

I sincerely thank the concerned authorities of the Max-Planck Institute for Radioastronomy, Bonn, Germany for permitting me to conduct my PhD there and providing all the required facilities. I am extremely thankful to Prof Dr. Anton J. Zensus for welcoming me to VLBI group of Max-Planck Institute for Radioastronomy for conducting my research. I thank the International Max Planck Research School (IMPRS) for Astronomy and Astrophysics at the Universities of Bonn and Cologne for selecting me to be a part of their research family. It was the most happiest day in my life when I received the e-mail from Manolis saying that "You are selected to do a PhD in VLBI group".

My supervisor Dr. Andrei P. Lobanov. From the day I met you on 2nd June 2014 till today, you are there as my source of inspiration, my reference book in research who has answers to all my questions, and, as a good human being and a friendly supervisor. I acknowledge with gratefulness the valuable help and guidance extended to me by you Andrei and particularly for the freedom you gave me in the discussions, throughout this work. Thank you for telling me how to keep balance between work and life. Though I always failed to listen to your advice "to stop working at night and work only on day time" in the entire $\mathrm{PhD}$, I promise now that I will change my nocturnal life style. I thank you from the bottom of my heart for the great support you offered when I had some health issues and for positively encouraging me to work when I am down.

I also express my sincere gratitude to Prof. Dr. Eduardo Ros. Your office doors were always open for me. Thanks for helping with learning especially AIPS and Difmap in the initial days of my PhD. I thank Dr. Thomas Krichbaum who was always there to support me in research. Thanks for your comments particularly on data reduction that sharpened my thinking and polished my research. Thanks to Prof. Andreas Eckart for being my local supervisor at the University of Cologne.

I thank the staff of the observatories participating in the GMVA, the MPIfR Effelsberg $100 \mathrm{~m}$ telescope, the IRAM Plateau de Bure Interferometer, the IRAM Pico Veleta 30 
m telescope, the Metsähovi Radio Observatory, the Onsala Space Observatory, and the VLBA. I also thank Walter Alef and Alessandra Bertarini for helping in the correlation of the $86 \mathrm{GHz}$ VLBI data used in this research. Thanks to Uwe Bach and Salvador Sanchez who have helped in the observation and calibration at Effelsberg radio telescope and IRAM Pico Veleta radio telescope, respectively.

Dear Germany, thanks for your hospitality. And Bonn is indeed a place full of creativity, arts and fun. I also thank all my teachers, and friends in Germany and back in India for their valuable support and encouragement. I also express my gratitude to Shruthi, Sameer, Jayalekshmi, Anu, Athulya, Viswas, Joice, Bhavana and other friends back in India. A big thanks to all my officemates Jeff, Florent, Ioannis, Thalia, Jae-Young, Celine, Felix, Lydia and Ioannis Liodakis (a long list since I had three offices in three and half years of my PhD!). I also thank Vassilis, Bia, Shoko, Bindu, Jun, Laura, Roberto, Madhuri who were always there for me. I thank Moritz and Sebastian who helped me in converting my thesis abstract to German in less than one day. I remember with gratitude all other persons who extended a helping hand in doing this research. 


\section{Erklärung}

Ich versichere, dass ich die von mir vorgelegte Dissertation selbständig angefertigt, die benutzten Quellen und Hilfsmittel vollstndig angegeben und die Stellen der Arbeit, einschlielich Tabellen, Karten und Abbildungen, die anderen Werken im Wortlaut oder dem Sinn nach entnommen sind, in jedem Einzelfall als Entlehnung kenntlich gemacht habe; dass diese Dissertation noch keiner anderen Fakultät oder Universität zur Prüfung vorgelegen hat; dass sie, abgesehen von unten angegebenen Teilpublikationen noch nicht veröffentlicht worden ist sowie, dass ich eine solche Veröffentlichung vor Abschluss des Promotionsverfahrens nicht vornehmen werde. Die Bestimmungen dieser Promotionsordnung sind mir bekannt. Die von mir vorgelegte Dissertation ist von Prof. Dr. Andreas Eckart und Prof. Dr. J. Anton Zensus betreut worden.

Köln, den 25.02.2019 


\section{Publications/Teilpublikationen}

- Dhanya G. Nair, Andrei P. Lobanov, Thomas P. Krichbaum, Eduardo Ros, J. Anton Zensus, Yuri Y. Kovalev, Sang-Sung Lee, Florent Mertens, Yoshiaki Hagiwara, Michael Bremer, Michael Lindqvist and Pablo de Vicente, Global millimeter VLBI array survey of ultracompact extragalactic radio sources at $86 \mathrm{GHz}, 2019$, A\&A, 622, A92

- Dhanya G. Nair, Andrei P. Lobanov, Thomas P. Krichbaum, Eduardo Ros, J. Anton Zensus, 86 GHz VLBI Survey of Ultra Compact Radio Emission in Active Galactic Nuclei, 2017, 13th EVN Symposium Users Meeting Proceedings, Transactions of IAA RAS, Vol.40, pp. 3-9, book 9785931970523

- Gopalakrishnan Nair, Dhanya, Lobanov, Andrei P; Ros, Eduardo; Krichbaum, Thomas P; Zensus, J. Anton, 86 GHz VLBI survey of Ultra compact radio emission in Active Galactic Nuclei, 2016, 41st COSPAR Scientific Assembly, Bib code: $2016 \operatorname{cosp} \ldots 41 \mathrm{E} .710 \mathrm{G}$

- Nair, Dhanya G., Lobanov, Andrei P., Krichbaum, Thomas P., Ros, Eduardo and Zensus, J.Anton, 86 GHz VLBI Survey of Ultra compact Radio Emission in Active Galactic Nuclei, Young European Radio Astronomers Conference Proceedings, 2015, Space Research Review, Vol. 4, pp. 11-19, book 978-9984-648-64-4

- Dhanya G. Nair, Andrei P. Lobanov, Thomas P. Krichbaum, Eduardo Ros, J. Anton Zensus, Intrinsic Properties of Ultracompact Extragalactic Radio Sources. Manuscript in preparation to $\mathbf{A} \& \mathbf{A}$. 


\section{Curriculum Vitae}

Dr. Dhanya G. Nair

$\underline{\text { Parumala, India, April 15, } 1991 \text { (Indian citizen, female) }}$

Joint Institute for VLBI ERIC (JIVE)
Oude Hoogeveensedijk 4
7991 PD Dwingeloo
The Netherlands
e-mail: nair@jive.eu, dhanyagnair01@gmail.com
Office : +31521596 508

Current Position

Previous Position

Education

Work Experience

Ph.D. Astronomy

(GPA - 1.0, Magna Cum Laude, Excellent) Radio Sources at $86 \mathrm{GHz}$. loud sources as a function of cosmological epoch.

Bachelor of Science: Physics

(98.8\%, equivalent to $A+.2^{\text {nd }}$ rank in the university)

(NCRA), Tata Institute of Fundamental Research, Pune, India

Secondary School Leaving Certificate $\left(\mathbf{1 2}^{\text {th }}\right)$

Secondary School Leaving Certificate $\left(\mathbf{1 0}^{\text {th }}\right)$

(Grade A+ or $96 \%$ )

Research Associate
Postdoctoral Fellow

Joint Institute for VLBI ERIC (JIVE), Netherlands

Postdoctoral Fellow

Max Planck Institute for Radio Astronomy (MPIfR), Germany

February 2019 - present

June 2014 - March 2018

Max Planck Institute for Radio Astronomy \& University of Köln, Germany.

Thesis title: Global Millimeter VLBI Array Survey of Ultracompact Extragalactic

Master of Science: Physics with specialization in Astronomy and Astrophysics

July 2011- June 2013

(Grade O, outstanding or $91.67 \%$, a total of 110 grade points out of 120)

Department of Physics, University of Pune \& National Centre for Radio Astrophysics (NCRA), Tata Institute of Fundamental Research, Pune, India Thesis title: A study of the efficiency of black holes in triggering jets in radio

June 2008- May 2011

Mahatma Gandhi University and National Centre for Radio Astrophysics

Thesis title: Radio Astronomy \& Giant Metrewave Radio Telescope (GMRT).

2006-2008

Natural Science, (Grade A+ or $99.3 \%$ (state level 1st rank))

July 2013 - May 2014

National Centre for Radio Astrophysics (NCRA), Tata Institute of Fundamental 


\section{Research, Pune, India.}

Research Interests Extragalactic astrophysics, Relativistic jets in Active Galactic Nuclei (AGN), Brightness temperature studies of AGN, High Resolution Radio Interferometry

Expertise

Achievements

Python, Fortran, C/C++, Matlab, Topcat, AIPS, Difmap, LateX, Linux, Windows Observational experience at Effelsberg Radio telescope, VLBI data calibration \& Imaging

> PhD scholarship in International Max Planck Research School at Max Planck Institute for Radio Astronomy.

$>$ University $3^{\text {rd }}$ rank holder \& First Class with Distinction in M.Sc. Physics

$>$ University $2^{\text {nd }}$ rank holder \& First Class with Distinction in B.Sc. Physics

$>$ State level $\mathbf{1}^{\text {st }}$ rank holder in $12^{\text {th }}$ standard (Natural Science - Kerala State, India)

Central Sector Scholarship (Full scholarship every month) recipient from Central Government of India for Bachelor degree and Masters degree studies

Talks and Participations

$14^{\text {th }}$ European VLBI Network Symposium and Users Meeting, Oral, Oct 8-11, 2018, Granada, Spain

$>$ 42nd COSPAR Scientific Assembly, Poster, July 14-22, 2018, Caltech, Pasadena, USA

$>$ Workshop on Blazars at High Resolution, Dec 13 -15, 2017, Max Planck Institute for Radio Astronomy, Bonn, Germany.

Bonn-Dwingeloo Neighborhood VLBI Meeting 2017, Oral, July 4, 2017, Max Planck Institute for Radio Astronomy, Bonn, Germany.

$>13^{\text {th }}$ European VLBI Network Symposium and Users Meeting, Oral, Sept 20-23, 2016, Institute of Applied Astronomy of the Russian Academy of Sciences, St.Petersburg, Russia.

$>1^{\text {th }}$ Synthesis Imaging Workshop, June 1-8, 2016, National Radio Astronomy Observatory (NRAO), Socorro, USA.

$>$ Dissecting the Universe - Workshop on Results from High-Resolution VLBI, Oral, Nov 30 - Dec 2, 2015 , Max Planck Institute for Radio Astronomy, Bonn, Germany.

$>$ Bonn-Dwingeloo Neighborhood Meeting 2015 “VLBI Science”, Oral, Oct 28, 2015, ASTRON/JIVE, Dwingeloo, Netherlands.

$>\quad 4^{\text {th }}$ Young European Radio Astronomers Conference 2015 (YERAC 2015), Oral, Aug 19-21, 2015, Ventspils International Radio Astronomy Center (VIRAC), Ventspils, Latvia.

DVN Symposium 2014, $12^{\text {th }}$ European VLBI Network Symposium \& Users Meeting, Oct 7-10, 2014, Cagliari, Italy.

> Workshop on Radio Studies of Galaxies and Galaxy Systems, March 4-6, 2014, Inter University Centre for Astronomy and Astrophysics (IUCAA), Pune, India.

$>$ Workshop on Galaxy Astronomy, Oct 30 - Nov 5, 2009, Jointly organised by School of Pure and Applied Physics, Mahatma Gandhi University and Inter University Centre for Astronomy and Astrophysics (IUCAA), Kottayam, India.

$>$ Workshop on Advances in Observational Astronomy, Dec 1-3, 2008, M. G. University, India.

Extra - curricular

Classical (Carnatic) Music, Keyboard \& Harmonium

$>\quad$ Classical Dance (Bharatanatyam) 